\title{
Veränderungen von Indikatoren der „Lebensqualität“ nach Hochdosis-Chemotherapie
}

\begin{abstract}
Dissertation
Zur Erlangung des sozialwissenschaftlichen Doktorgrades der Sozialwissenschaftlichen Fakultät der Universität Göttingen
\end{abstract}

vorgelegt

von

Margret Kamm

aus Lichtenfels

Göttingen 2003 
1. Gutachter Prof. Dr. H. Friedrich

2. Gutachter Prof. Dr. P. Fassheber

Tag der mündlichen Prüfung:

9.6. 2004 
Danksagung

Mein erster Dank gilt den Patientinnen und Patienten, die bereit waren an dieser Untersuchung teilzunehmen. Ihre Offenheit und Bereitschaft in schwierigen und belastenden Lebenssituationen Einblick zu geben in Gefühle, Gedanken und Handlungen nötigen mir uneingeschränkte Hochachtung ab.

Die Deutsche Krebshilfe hat durch ihre finanzielle Unterstützung diese Arbeit ermöglicht und sie hat dazu beigetragen, dass die kontinuierliche psychosoziale Unterstützung der Patientinnen und Patienten auf Station „Holland“ der Hämatologie/Onkologie des Universitätsklinikums Göttingen möglich wurde.

Den früheren Abteilungsleitern Prof. Dr. med. W. Kuhn und Prof. Dr. med. W. Hiddemann und ihren Nachfolgern Prof. Dr. med. G. Emons und Prof. med. Dr. med. L. Trümper sei gedankt für die Ermöglichung der interdisziplinären Untersuchung.

Herrn Prof. Dr. H. Friedrich danke ich für die Betreuung der Arbeit und seine konstruktive Kritik. Mein Dank gilt ebenfalls Herrn Prof. Dr. P. Fassheber für wichtige Anregungen und Herrn Prof. Dr. med. W. Kuhn für die Durchsicht des medizinischen Teils der Arbeit. Dank auch an Herrn Prof. Dr. med. U. Rüger für seine Unterstützung.

Wichtig war die Unterstützung meiner Kollegen aus der Hämatologie/Onkologie. Mein besonderer Dank gilt Frau H. Robrecht-Kauenhoven und Prof. Dr. M. Gründel.

Für Diskussionen und Gespräche danke ich C. Mähler und E. Lübbeke-Bauer. Letztere war mir mit ihrer unermüdlichen Diskussionsbereitschaft eine große Hilfe und hat dazu beigetragen „Durststrecken“ zu überwinden.

Nicht zuletzt danke ich Edwin und Max für ihre Geduld.

Margret Kamm 
Margret Kamm

Von Bar Str. 19 a

37075 Göttingen

\section{Lebenslauf}

Geburtsdatum

Geburtsort

Familienstand

\section{Schulbildung}

1958-1962

1962-1967

1972-1976

\section{Berufsausbildung}

1967-1969

1969-1970

1970-1972

1972-1773

\section{Studium}

1981-1987

\section{Praktika}

1983

1986

\section{Berufstätigkeit}

1973-1975

1976-1977

1977-1981
31.10.1951

Korbach

Ledig, ein Kind
Grundschule

Realschule

Abendgymnasium für Erwachsene

Lehre als Zahnarzthelferin

Krankenpflegepraktikum

Ausbildung zur Krankengymnastin in Frankfurt/Höchst

Anerkennungspraktikum im Vereinskrankenhaus

Hann.Münden und im Stadtkrankenhaus Kassel

Studium der Psychologie

Abschluss: Dipl. Psychologin

Schwerpunkt: Klinische Psychologie

Thema der Dipl. Arbeit: Aspekte der Verarbeitung intentionaler und inzidenteller Bildinformation: Eine empirisch gestützte Kritik an klassischen Aufmerksamkeitsinterpretationen

Kinder- und Jugendpsychiatrie, Humboldt-Krankenhaus, Berlin Ambulanz für Schmerzbehandlung, Universität Göttingen Kinderzentrum München (Prof. Hellbrügge)

Stationärer Bereich: Frühtherapie (Bobath, Voijta, VT)

Angestellte Krankengymnastin, Praxis B. Schmidt, Hann.Münden

Schwerpunkt: Pädiatrie (für die Kinder der „Lebenshilfe“ in Hann.Münden)

Angestellt als Krankengymnastin, Medizinische Klinik der Universität Göttingen

Schwerpunkt: Herz-Kreislauf-Rehabilitation, Atemerkrankungen, Psychosomatische Erkrankungen Hauptamtliche Lehrtätigkeit an der Krankengymnastikschule der Uni Göttingen

(Schwerpunkt: Atemtherapie, Entspannungstherapie, Körperwahrnehmung, Krankengymnastik in der Med. Klinik) 
Nebenamtliche Lehrtätigkeit an der Krankengymnastikschule 2 Monate Auslandsaufenthalt(USA, Kanada)

1978-1989

1986-1987

1985-1987

1987-1988

1988-1989

1989-1991

1991-1994

Seit Jan.1991

1998-2001 Honorartätigkeit in der Ev. Familienbildungsstätte Göttingen (Kurse: Bewegungsspiele für Säuglinge/Kleinkinder; Geburtsvorbereitung für Paare; Rückbildungsgymnastik Freie Mitarbeit: Krankengymnastikpraxis H. Claus, Göttingen Schwerpunkt: Geriatrie

Tätigkeit als studentische Hilfskraft Schwerpunkt: Entwicklungspsychologie - Sprache Gedächtnis

Tätigkeit als wissenschaftliche Hilfskraft mit gleichem Schwerpunkt

Schwangerschaftsvertretung an der Krankengymnastikschule der Uni Göttingen

Ausbildungsleiterin der Krankengymnastikschule der Uni

Göttingen (Unterrichtsschwerpunkte:

Pädiatrie, Methodik/Didaktik der Gruppenarbeit, Sensomotorische Entwicklung im Säuglings-und Kleinkindalter)

Honorartätigkeit für die Erziehungsberatungsstelle Osterode: Gruppenarbeit und Einzelgespräche mit alleinerziehenden Frauen

Angestellt (Halbtagstätigkeit) als Dipl.

Psychologin/Psychotherapeutin an der Frauenklinik der Universität Göttingen

Mitarbeit in einem Drittmittelprojekt der Deutschen Krebshilfe

Weiterbildung

Gesprächspsychotherapie

Familientherapie

Psychomotorik

Seit 1999 Approbation als Psychologische Psychotherapeutin 


\section{INHALTSVERZEICHNIS}

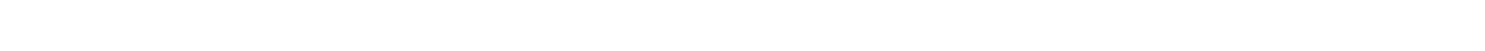

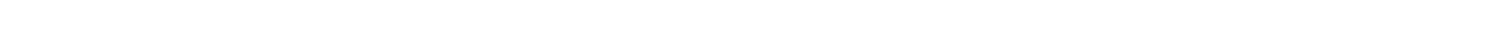

2 Konzeptualisierung von „Lebensqualität“ in der onkologischen

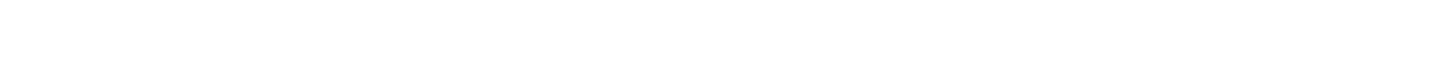

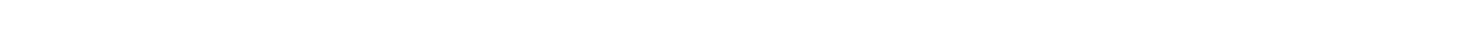

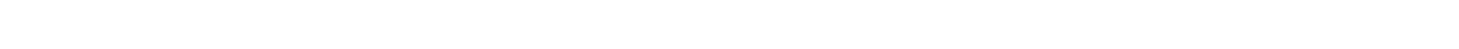

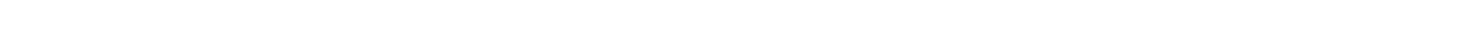

2.4 Vergleich von LQ-Ergebnissen der Wohlfahrtsforschung mit denen der onkologischen Forschung ............................................................. 31

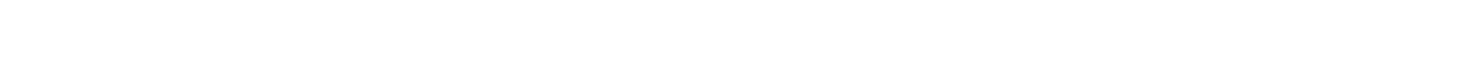

3 Ziele und klinische Relevanz der empirischen LQ-Forschung ............. 38

3.1 LQ als Zielkriterium in der Palliativmedizin .............................................

3.2 LQ zur Beschreibung von Therapieauswirkungen .....................................43

3.2.1 LQ als abhängige Variable von brusterhaltender versus radikaler

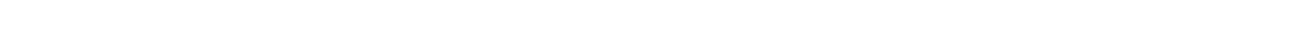

3.2.2 LQ-Werte als Hinweis für eine notwendige Intervention .........................45

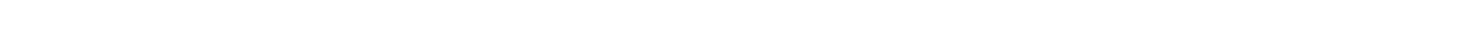

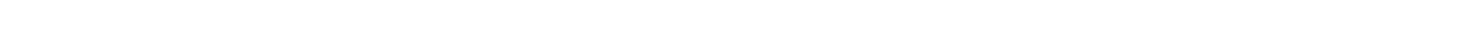



4 Stellenwert der LQ-Forschung in der Onkologie ............................... 57

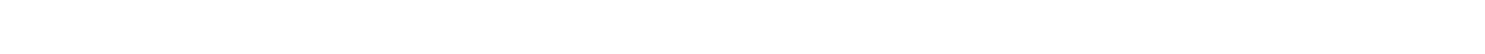

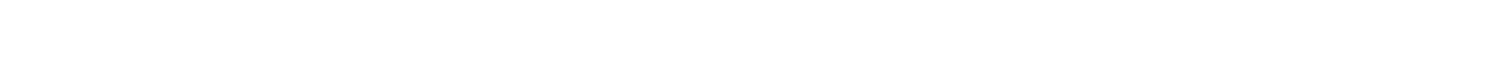

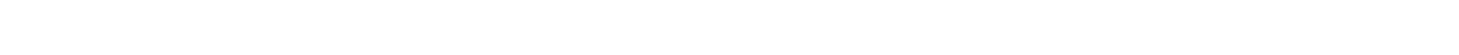

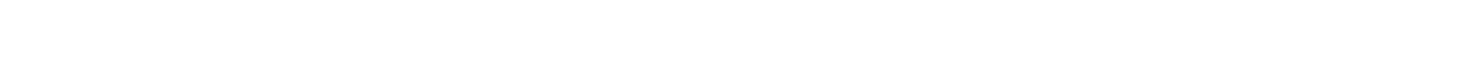

6.3 Kritische Anmerkungen zur HDC …................................................... 70

7 Beeinträchtigungen der LQ durch Krankheit und HDC ......................... 74

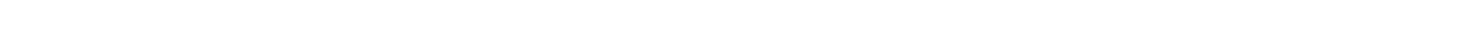

$7.2 \quad$ Krebserkrankung und psychische Störungen ..........................................75

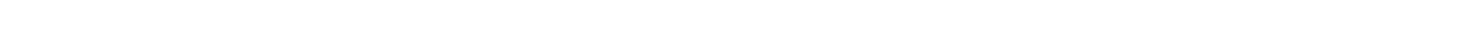

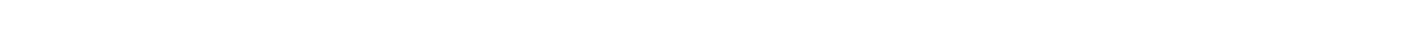

7.3.2 Prävalenz affektiver Störungen in der Onkologie ..................................80 


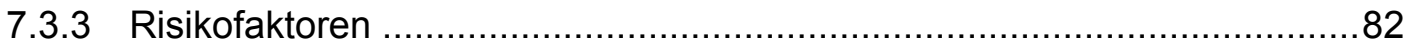

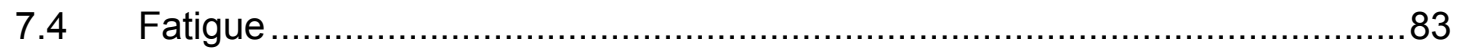



7.5.1 Wissen um die ungünstige Prognose der Erkrankung und die Nebenwirkungen der HDC .......................................................... 90

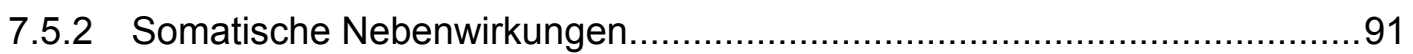

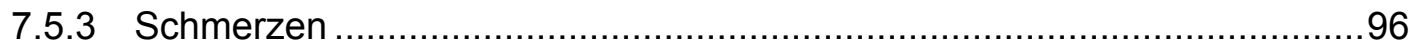

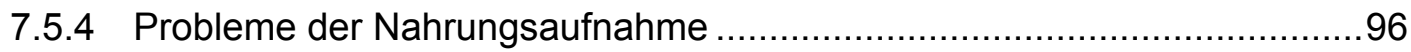

7.5.5 Bedingungen des stationären Aufenthaltes (modifizierte Isolation) ...........97

7.5.6 Zusammenfassung der erwarteten Nebenwirkungen:..............................98

8 Übersicht über die Studien zur LQ und HDC ....................................... 100

8.1 Zusammenfassung der Ergebnisse ................................................... 106

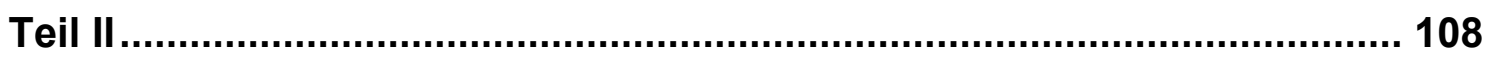

9 Fragestellung und Forschungsansatz der Studie ............................ 108

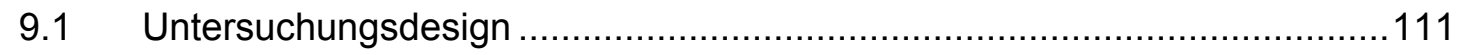

9.2 Zeitpunkte der Datenerhebung: ...........................................................111

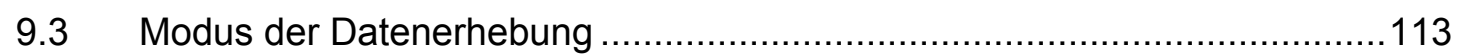

9.4 Quantitativer Teil der Datenerhebung.....................................................114

9.4.1 Standardisierte Verfahren zur Informationsgewinnung .........................114

9.4.2 „EORTC quality of life questionnaire (QLQ-C30)”..............................115

9.4.3 Hospital Anxiety and Depression Scale - Deutsche Version (HADS-D).117

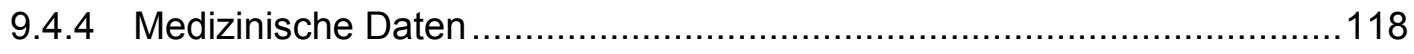

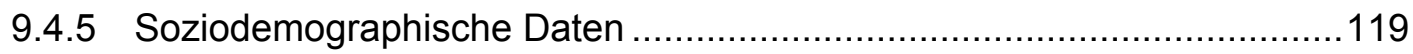

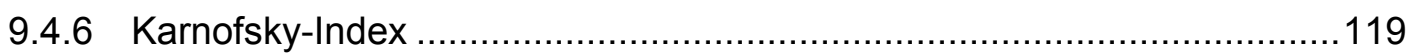

9.5 Qualitativer Teil der Datenerhebung ................................................... 119

10Darstellung der Ergebnisse .......................................................... 125

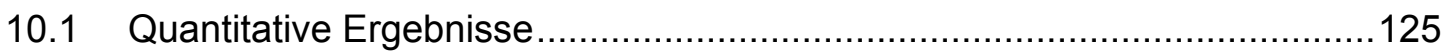

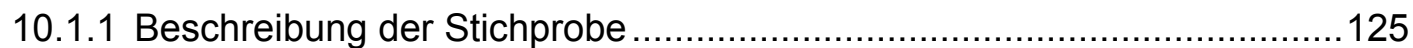

10.1.2 Deskriptive Statistik der Fragebogendaten zum Zeitpunkt T1 - T5 .........132

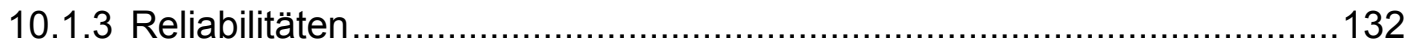

10.1.4 Hypothesen und Ergebnisse in Bezug auf die Mittelwertsverläufen der

Fragebögen ................................................................................ 136

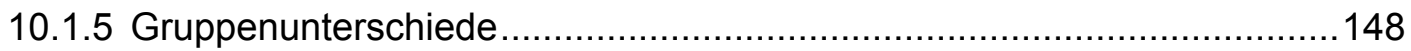

10.1.6 Zusammenfassung der quantitativen Ergebnisse ................................ 155 


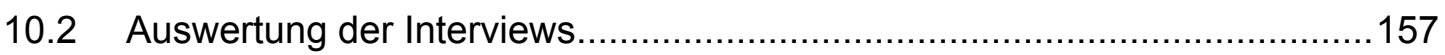

10.2.1 Hypothesen und Ergebnisse in Bezug auf die Interviewdaten .................157

10.2.2 Belastende Faktoren, hervorgerufen durch die Therapie........................ 158

10.2.3 Bewertung der Therapiebelastungen durch die Patienten .....................202

10.2.4 Übereinstimmung der erwarteten Belastungen mit den tatsächlich erlebten Belastungen ........................................................................204

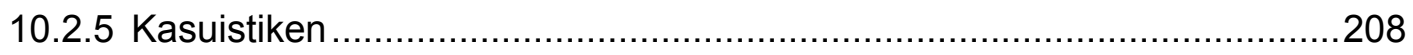

10.2.6 Bewertung der Hypothesen und Zusammenfassung ...........................217

10.2.7 Vergleich der Fragebogendaten mit denen der Daten im Interview .........220

11Schlussfolgerungen für die Patientenversorgung .......................... 222

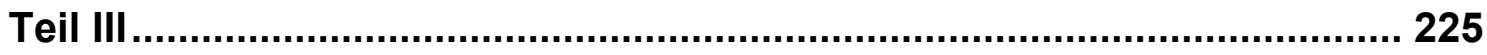

12Zusammenfassung und Diskussion................................................ 225

12.1 Zusammenfassung und Diskussion der Ergebnisse ................................225

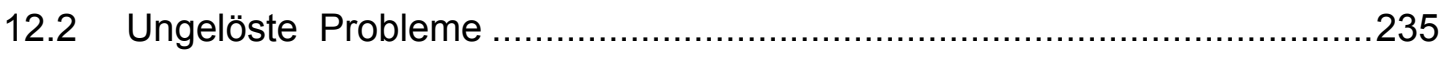

12.2.1 Methodische Probleme................................................................235

12.2.2 Problematik des LQ-Konstruktes aus klinischer Perspektive ..................237

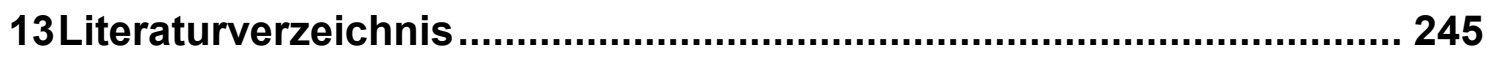

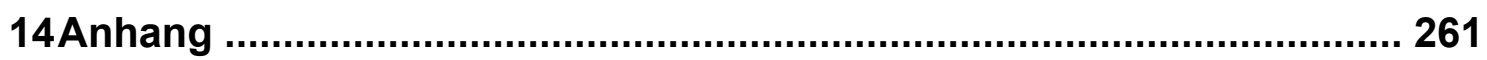




\section{Teil I}

\section{Einleitung}

„[...] jeder weiß, daß in vielen Fällen die Krankheit eben doch tödlich endet und daß dem Sterben eine lange Leidenszeit vorausgehen kann. Da kein Betroffener ausschließen kann, daß nicht doch dieses Schicksal auf inn wartet, enthält jede Krebsdiagnose faktisch eine ganz reale Todesdrohung: der eigene Tod tritt hinter dem Horizont einer weit entfernten Zukunft hervor in die Gegenwart und konfrontiert das eigene Wesen mit dem, was es am meisten fürchtet: seine Auflösung." (Gerdes 1991, S. 275)

1995 wurde in der Universitäts Frauenklinik Göttingen zum ersten Mal eine experimentelle Hochdosischemotherapie (HDC) mit autologem Stammzellsupport bei Patientinnen mit Brustkrebs durchgeführt.

Die Metapher von der „Healing Hell“" , die durch die Presse, auch die medizinische Fachpresse geisterte, gab zu diesem Zeitpunkt treffend die Hoffnungen und Befürchtungen wieder, die mit dieser Therapie verbunden waren.

Die Therapiebelastungen wurden von allen Beteiligten, Patientinnen und medizinischem Personal gleichermaßen, als sehr schwerwiegend eingeschätzt. Nicht nur die Patientinnen sondern auch das Personal sahen jeder HDC mit Unruhe entgegen, zumal es in den ersten Monaten einen Todesfall gegeben hatte, der, mit der Therapie assoziiert, als sehr belastend erlebt wurde und die Frage aufwarf, ob die Patientin ohne diese Therapie möglicherweise länger überlebt hätte. Generell ergab sich die jedwedes Interesse an Lebensqualitätsforschung konstituierende Frage, ob der Nutzen der Therapie größer ist als die Beeinträchtigung durch die Therapie.

In der Abteilung für Hämatologie/Onkologie waren schon längere Zeit Erfahrungen mit der HDC bei Lymphompatienten gesammelt worden, sowohl von ärztlicher als auch von pflegerischer Seite. Diese Erfahrungen sollten, mit dem Entschluss zu einer interdisziplinären Zusammenarbeit, auch der Gynäkologie zur Verfügung gestellt werden. Durch die „miterlebten“ Belastungen für die Patienten ${ }^{2}$ entstand bei den Mitarbeitern

\footnotetext{
${ }^{1} \mathrm{~J}$. Groopman prägte diese Metapher (Annals of Medicine, 1998 , S.34) in einem Artikel über Knochenmarkstransplantationen. Auch wenn hier ein anderes medizinisches Therapieverfahren gemeint ist, lässt sich diese Art des Verständnisses auch auf die HDC mit Stammzellsupport übertragen. Der Mediziner Groopman formuliert, was viele Patienten denken und gelegentlich auch zum Ausdruck bringen: „A grim clinical equation: the stronger the cancer, the more punishing the treatment." (ebenda)

${ }^{2}$ Wegen der Lesbarkeit des Textes wird hier die maskuline Form benutzt. Selbstverständlich sind hier auch Patientinnen mit gemeint. Diese Form wird im gesamten Text beibehalten.
} 
der Wunsch, für diese Patienten eine möglichst optimale Versorgung, die auch eine psychosoziale Unterstützung einschließen sollte, bereit zu stellen.

Von medizinischer Seite war eine Phase-III-Studie zur Evaluation von HDC ( bei NHLund Mammakarzinompatienten) im Vergleich mit konventioneller Chemotherapie geplant.

In dieser Situation wurde von Prof. Dr. W. Hiddemann und Prof. Dr. M. Gründel ein Antrag bei der "Deutschen Krebshilfe“ auf Unterstützung eines Forschungsvorhabens gestellt, mit dem sowohl die begleitende psychosoziale Forschung als auch die Betreuung der betroffenen Patienten gewährleistet werden sollte.

Das breite Konzept der Lebensqualitätsforschung, das alle möglichen Ebenen psychosozialer und medizinischer Folgen von Krankheit und Therapie umfassen kann, schien geeignet für die Umsetzung. Der Titel des Forschungsprojektes lautet: „Lebensqualität vor, während und nach Hochdosischemotherapie mit nachfolgender autologer Stammzelltransplantation bei Patientinnen mit Mammakarzinom und Patienten mit NonHodgkin Lymphomen."

Forschungsergebnisse aus deutschsprachigen Studien lagen bis dato nicht vor. Die Methodik der LQ-Forschung sollte sich an zwei Bedingungen orientieren:

- einem möglichst hohen Standard der quantitativen Forschung mit einem randomisierten Kontrollgruppendesign bei ausgewählten Krankheitsbildern und der Verwendung psychometrisch geprüfter Instrumente zur Datenerhebung. (Gruppenstatistischer Ansatz)

- $\quad$ einem subjektbezogenen, qualitativen Zugang zur Erfahrungswelt der Patienten um mit Fragebögen nicht abbildbare Prozesse zu erfassen und um zu gewährleisten, dass unmittelbar auf Ängste, Bedürfnisse, Probleme und Schwierigkeiten mit psychosozialen/psychotherapeutischen Interventionen außerhalb der Datenerhebung eingegangen werden konnte.

(Individuum zentrierter Ansatz)

Die quantitative Datenerhebung sollte sich auf die Experimental- und Kontrollgruppe, die qualitative Datenerhebung aus forschungsökonomischen Gründen nur auf die Experimentalgruppe erstrecken.

Unter anderem haben die sehr schnelllebige experimentelle onkologische Forschung, die bekannten Schwierigkeiten mit einer Randomisierung in Deutschland und organisatorische Gründe (Wechsel der Abteilungsleiter) dazu geführt, dass dieses Projekt den Erfordernissen der klinischen Praxis angepasst werden musste.

Als randomisierte Studie mit begleitender LQ-Forschung konnte das Projekt nicht durchgeführt werden. Die Studie wurde durchgeführt als explorative Längsschnittstudie mit fünf Untersuchungszeitpunkten: Alle Patienten, die in dem Zeitraum von zwei Jah- 
ren HDC erhalten haben, wurden in die Studie aufgenommen. Die Daten wurden, wie geplant, mit qualitativer und quantitativer Methodik erhoben.

Der erste Teil dieser Arbeit wird sich mit der onkologischen LQ-Forschung, ihrer Methode, ihren Konzepten, wichtigen Ergebnissen und dem gegenwärtigen Stellenwert der Forschung beschäftigen. Außerdem werden in diesem Teil die Besonderheiten der Hochdosischemotherapie und die dadurch bedingten zusätzlichen Belastungen erläutert.

Am Beginn des zweiten Teiles werden die eigenen Forschungsfragen dargelegt und begründet. Anschließend werden die Ergebnisse der vorliegenden Studie dargestellt. Die Darstellung orientiert sich an den jeweiligen Methoden der Datenerhebung. Aus den Ergebnissen werden Schlussfolgerungen für eine verbesserte Patientenversorgung gezogen.

Im dritten Teil werden Möglichkeiten und Grenzen der onkologischen LQ-Forschung aufgezeigt, unter Bezugnahme auf die Ergebnisse dieser Studie. Außerdem wird die Verwendung des Begriffes „Lebensqualität" in der psychometrischen Forschung aus der klinischen Perspektive problematisiert.

Diese Arbeit spiegelt einen Forschungs- und Erkenntnisprozess wider, dessen Ergebnisse erst im Schlusskapitel Erwähnung finden. Ausgangspunkt der Arbeit war die Vorstellung von einer Untersuchung, die im „mainstream“ der naturwissenschaftlich orientierten psychoonkologischen Forschung unter anderem die Frage beantworten sollte, ob der „Preis“ der Therapie in Form von emotionaler und physischer Belastung in einer sinnvollen Relation zum postulierten Gewinn steht, ob die Kenntnis von Therapiebelastungen in klinischen Entscheidungssituationen helfen kann.

Die intensive Beschäftigung mit der entsprechenden Literatur (und den eigenen Ergebnissen) hat zu einer völlig anderen Fragestellung geführt: nämlich, ob im Rahmen dieser Forschungslogik überhaupt sinnvolle Antworten erwartet werden können oder ob nicht diese Art von onkologischer Lebensqualitätsforschung, die weltweit mit beachtlichen personellen und finanziellen Mitteln durchgeführt wird, in die Sackgasse eines „scientistischen Selbstmissverständnisses“ (Habermas 1973) mündet. 


\section{Konzeptualisierung von „Lebensqualität“ in der onkologischen For- schung}

\subsection{Begriff „Lebensqualität“}

Der Begriff der Lebensqualität (LQ) taucht in der medizinisch-onkologischen Forschung erst spät auf. Als Schlüsselwort für medizinische Datenbanken ist er 1975 (Aaronson 1990) zum ersten Mal vermerkt.

Der Begriff der Lebensqualität wurde zuerst im Rahmen von Politik und empirischer Sozialforschung verwendet. ${ }^{3} \mathrm{LQ}$ gilt „[...] als Maß für die Güte der Lebenssituation und Versorgung einer definierten Population [...]" (Bullinger 1997, S.1). Heute ist der Begriff vielgebrauchter Slogan in allen möglichen gesellschaftlichen Bereichen: als Diskussionsgegenstand und Versprechen der Politik, als Mittel der Werbung, besonders im Rahmen der pharmakologischen Werbung für Generika und „Lifestyle“ Medikamente, als Forschungsgegenstand verschiedener Fachgebiete. In der Politik wurde und wird der Begriff zur Analyse gesellschaftlicher Bedingungen verwendet und um Zielvorstellungen zu beschreiben, die auf eine Verbesserung der Lebensbedingungen der Menschen abzielen. Hauptaugenmerk wird dabei auf den sozialen, ökonomischen und ökologischen Wandel und seine Auswirkungen auf die eigene Gesellschaft und auf Gesellschaften im internationalen Vergleich gerichtet. In den siebziger Jahren, zehn Jahre nach den ersten Untersuchungen in den USA, wurde innerhalb der empirischen Sozialforschung in West-Deutschland der Zusammenhang zwischen objektiven Lebensbedingungen (Sozialstruktur) und subjektivem Wohlbefinden (wahrgenommene Lebensqualität) ermittelt und der Versuch unternommen, zu bestimmen, durch welche Faktoren die individuelle LQ beeinflusst wird. Diese Untersuchungen wurden erstmalig durchgeführt, um die gesamtgesellschaftliche Wohlfahrtsentwicklung der BRD zu dokumentieren (Glatzer \& Zapf 1984).

LQ in der Medizin wird anders definiert: „Es handelt sich hier um die vom Patienten selbst erlebte Befindlichkeit und Funktionsfähigkeit, die Fähigkeit, Rollen im täglichen Leben zu übernehmen und die Altagstätigkeiten zur Zufriedenheit auszuführen. Zusammenfassend definiert, bezeichnet Lebensqualität das Gesamte der körperlichen,

\footnotetext{
${ }^{3}$ Ein Überblick über die Begriffs- und Wortgeschichte findet sich bei Pukrop (1997, Kapitel 1). Nach seinen Aussagen beginnt die eigentliche Verwendung des Begriffs in den späten Fünfziger Jahren: Die vorher überwiegend geführte philosophische Diskussion wurde abgelöst durch politische, soziologische, psychologische und medizinische Konzepte. Der Begriff LQ wurde dem ökonomisch-quantitativen Wachstum als Zielgröße gleichgestellt. In USA wurde der Begriff zuerst im Wahlkampf von Eisenhower verwendet. Als früheste Quelle für die Übertragung ins Deutsche gilt eine Rede Willi Brandts 1972. Nach Pukrop erlebte das Konstrukt LQ den größten Boom in Medizin bzw. Klinischer Psychologie. In der Sozialpsychologie wurden in den 80er Jahren eine ähnliche Forschung unter dem Begriff des Subjektiven Wohlbefindens verfolgt.
} 
psychischen, sozialen und funktionalen Aspekte von menschlichem Erleben und Verhalten, wie sie von der Person selbst geäußert werden." (Bullinger, 1997 S. 1)

Folgende, paradox erscheinende Entwicklung in der Medizin bildete den Ausgangspunkt für die Erforschung der LQ: medizin-technischer Fortschritt führt einerseits zur Verlängerung der Lebenszeit bei Patienten, andererseits rückt das durch ebendiese Therapieerfolge bedingte Leiden in den Mittelpunkt des Interesses. Ausnahmslos alle Autoren begründen die Notwendigkeit der Berücksichtigung von gesundheitsbezogener LQ in der Onkologie damit, dass die früher in kürzeren Zeiträumen tödlich verlaufenden Krebserkrankungen durch die effektiveren Therapien in chronische Verläufe umgewandelt wurden. Die Fortschritte in Bezug auf verlängerte Überlebenszeiten wurden/werden erkauft durch einschneidende Nebenwirkungen der Therapien und werfen die Frage auf, welche Einschränkungen (noch) akzeptabel sind um von „lebenswertem Leben“ sprechen zu können. Die Ermittlung von LQ in Abhängigkeit von Krankheit und Therapie soll dazu beitragen, beide Faktoren gegeneinander abzuwägen um zu verhindern, dass das Ausmaß der Belastung durch die Therapie die unvermeidbaren Belastungen durch die Krebserkrankung selber übersteigt.

In der medizinischen Forschung wird die reduktionistische Verwendung des Begriffes der LQ dadurch kenntlich gemacht, dass von gesundheitsbezogener LQ (Health related - HRQL) gesprochen wird, also von den Veränderungen, die unmittelbar mit Krankheit und Therapie zusammenhängen.

Der Begriff der gesundheitsbezogenen LQ in der Medizin ist unscharf und umfasst ein breites Themengebiet. Neben der Untersuchung von Beschwerden, Symptomen, Krankheitsfolgen im physischen und psychosozialen Bereich, Anpassungsleistungen, Probleme der Rehabilitation, werden auch Fragen der Arzt-Patient-Beziehung, psychosoziale und medizinisch-supportive Angebote, Zufriedenheit mit der Behandlung, bis hin zur architektonischen Gestaltung des Raumes Krankenhaus (oder RehaEinrichtung) sowie der Ernährung im Krankenhaus unter diesen Begriff gefasst.

Es bedarf deshalb einer grundsätzlichen Spezifizierung der Verwendung des Begriffes in Abhängigkeit von den formulierten Fragestellungen und damit einer Einengung auf die jeweils interessierenden Themen.

Es gibt weder eine allgemeine Theorie noch eine allgemeingültige Definition des Komplexes von gesundheitsbezogener Lebensqualität (HRLQ). ${ }^{4}$

\footnotetext{
${ }^{4}$ Siehe Pukrop (1997, S. 12): Der Autor diskutiert die evaluativen, komparativen und quantitativen Komponenten, die implizit in einer Definition von LQ enthalten sind. Er wendet sich ausdrücklich gegen eine nur deskriptive Auffassung des Qualitätsbegriffes. Nur unter der vergleichenden Annahme von höherer/niederer LQ wird LQ überhaupt messbar.
} 
Am Beginn der LQ-Forschung stand die Beurteilung des behandelnden Arztes über die beobachtbaren Nebenwirkungen seiner Behandlung und deren Auswirkungen auf die Lebensbedingungen seines Patienten und die Antwort auf die an den Patienten gerichtete Frage, „Wie geht es Ihnen?“, d.h. die Frage nach der individuellen Beurteilung des behandelten Patienten.

Zwei Traditionen lassen sich bei der Betrachtung von LQ in der Onkologie unterscheiden:

Innerhalb der ersten Tradition wird im Rahmen des naturwissenschaftlichen Forschungsparadigmas LQ aufgelöst in Variablen, die hypothesengeleitet geprüft werden können. Die Forschung im onkologischen Kontext hat sich überwiegend um die Konstruktbildung und die Entwicklung von methodischen Standards bemüht. Die Entwicklung von Verfahren, die psychometrischen Gütekriterien genügen, stand im Mittelpunkt der Bemühungen. LQ wurde zum expliziten Zielkriterium von Studien und ergänzte additiv den Wirksamkeitsnachweis von Therapien. Der quantitativen Bewertung durch das "harte“ Kriterium der Überlebenszeit wurde die qualitative Bewertung durch den Patienten zur Seite gestellt, um so eine Analyse des Nutzens zu ermöglichen. ${ }^{5}$ Diese Verfahren werden vor allem eingesetzt, um Therapievergleiche zu ermöglichen und um Hilfestellungen in Entscheidungssituationen zu leisten, in denen medizinisch gleichwertige Therapien zur Verfügung stehen.

In den letzten Jahren werden die auf diese Weise gewonnenen Daten in Beziehung gesetzt zu den Kosten der Behandlung. LQ wird so auch zu einem Faktor bei der ökonomischen Bewertung von Therapien.

Innerhalb der zweiten Tradition, die dem hermeneutischen Vorgehen in den Geistesund Sozialwissenschaften entspricht, stehen das konkrete Erleben des erkrankten Patienten und sein Bewerten des Krankheitsgeschehens in seinem biographischen Kontext im Vordergrund. Zusätzlich kann der Forscher die Äußerungen des Patienten innerhalb seines Theorierahmens interpretieren und mit Deutungsmustern versehen. In dieser Tradition erfolgt die Datenerhebung einzelfallorientiert mit qualitativen Erhebungsmethoden. Aus der Einzelfallanalyse werden verallgemeinerbare Schlüsse abgeleitet, die vor allem der Entwicklung von Unterstützungsangeboten dienen sollen. Außerdem wird bereits die Datenerhebung als „minimale Intervention“6 7 betrachtet, die den Patienten zur Reflexion über sein Erleben anregt, sein Denken und Fühlen verändert und seine Freiräume im Umgang mit der Krankheit möglicherweise erweitert.

\footnotetext{
${ }^{5}$ Zur Unterscheidung von Wirksamkeit und Nutzen in der Medizin siehe Porzsolt \& Rist, 1997

${ }^{6}$ Siehe auch Bernhard, Gusset \& Hürny (1995): Quality-of-life assessment in cancer clinical trials: an intervention by itself?

${ }^{7}$ Auch Jones et al. (1987) stellen Überlegungen an, die in die gleiche Richtung gehen.
} 
Entsprechend der jeweiligen Tradition ergeben sich ganz unterschiedliche Probleme, sowohl inhaltlicher als auch methodischer Art. Bei der Betrachtung der Beiträge zur LQ in der Onkologie hat sich das Gewicht eindeutig in Richtung der Arbeiten mit naturwissenschaftlichem Anspruch verschoben. Viele der naturwissenschaftlich orientierten Autoren betrachten den Einsatz qualitativer Forschung als Vorstufe zur Generierung von wichtigen Themen, die sich in Fragebogeninstrumente umsetzen lassen und dann einer hypothesengeleiteten Überprüfung zugänglich sind.

Im Folgenden werden wichtige Schritte der Entwicklung von LQ-Forschung im Kontext des naturwissenschaftlichen Paradigmas kurz skizziert.

\subsection{Kurzer historischer Überblick}

Am Beginn der Lebensqualitätsforschung in der Onkologie standen Globalmaße und Funktionsindizes. Karnofsky et al. (1948) waren die ersten, die neben den objektiven Parametern wie Lebenszeitverlängerung, Remissionsdauer, Verbesserung von tumorspezifischen Laborparametern, Tumorverkleinerung etc. auch subjektive Parameter wie seelische Verfassung und Allgemeinbefinden sowie die Unabhängigkeit des Patienten von ärztlicher Hilfe und Pflege zur Beurteilung chemotherapeutischer Effekte herangezogen haben. Vor allem der Aktivitäts-Index (Karnofsky-Index) wurde in der Folgezeit, bis heute, sicherlich zum meist genutzten Instrument in klinischen Studien. Der „Karnofsky-Index“ beschreibt die Leistungsfähigkeit des Patienten in einem dreistufigen Modell. In diesem werden den jeweiligen Stufen der Abhängigkeit bzw. Selbständigkeit Prozentwerte der Aktivität zugeordnet.

Dass trotz der differenzierten Beurteilung nur der Aktivitätsindex Berücksichtigung fand mag daran liegen, dass selbständige Versorgung und Leistungsfähigkeit, niemandem zur Last fallen, in der individuellen und gesellschaftlichen Bewertung einen hohen Stellenwert hatten und haben. Hinzu kommt, dass der Aktivitätsindex vom Arzt bestimmt wurde. Diese Bewertung von „außen“ entsprach dem verbreiteten Verständnis vom Patienten als „Objekt der Medizin“. Erst 20 Jahre später rückte die subjektive Bewertung der Behandlung durch den Patienten in den Vordergrund. Bullinger (1997) hält diesen Perspektivenwechsel in der Medizin - die bewusste Bezugnahme auf den Patienten und seine Gefühle über Behandlung und Erkrankung in der Evaluation - fast für eine „kleine Revolution“. Zahlreiche weitere Autoren sehen darin sogar Anzeichen für einen Paradigmenwechsels in der Medizin (u. a. Sellschopp \& Herschbach 1998). Karnofsky und Mitarbeiter verwenden in ihren Veröffentlichungen nicht den Begriff der LQ, fragen aber danach, welche Therapie geeignet ist, „useful life“ (1948) zu ermöglichen. Daneben mag die Tatsache, dass der englische Terminus „useful" sowohl mit „nützlich“ als auch mit „sinnvoll“ übersetzt werden kann, dazu beigetragen haben, dass 
Karnofskys Vorschlag in der Rezeption durchgängig auf den Funktionalitätsaspekt reduziert wurde.

Die Diskussion darum, wer der „Experte“ für die Beurteilung der LQ des Patienten ist, wurde später, auch unter dem Einfluss der Stellungnahme der WHO zur LQ, zugunsten der Selbstbeurteilung entschieden. Waren die am Einzelfall orientierten Forscher ohnehin der Ansicht, dass nur der Betroffene selbst seine LQ zu bewerten vermöge, so setzte sich auch in der psychometrischen Forschung die Ansicht durch, dass nur der Patient selber, und damit die Selbstbeurteilung eine angemessene Erhebungsmethode darstellt und Fremdbeurteilungsinstrumente nur additiv eingesetzt werden sollten. Dies ist u.a. auf die zahlreichen Untersuchungsergebnisse, die auf eine völlig differente Beurteilung von Betroffenen einerseits und Ärzten/Pflegenden andererseits hinweisen, ${ }^{8}$ zurückzuführen. Pukrop (1997) referiert zahlreiche empirische Befunde, die zeigen, dass objektive Urteile des Arztes über den Gesundheitszustand eines Patienten sich nur in geringem Maße mit der subjektiven Beurteilung der LQ durch den Patienten decken. Slevin et al. (1987, S. 110) schließen daraus, dass „[...] the doctors could not adequately measure the patient's quality of life." Das bedeutet allerdings nicht, dass nicht auch objektive Parameter (Laborwerte, Funktionstests etc.) zur Beurteilung herangezogen werden können. Im letzten Jahrzehnt wurde der Karnofsky-Index seltener verwandt und durch den Performancestatus der $\mathrm{WHO}^{9}$ abgelöst. Auch bei dieser Beschreibung geht es um eine Fremdbeurteilung des Funktionszustandes durch den Arzt. Beide Verfahren werden heute nicht mehr als angemessene Operationalisierungen von LQ akzeptiert. Zu den Globalmaßen gehören die auch heute noch vor allem in der Schmerzmessung verwendeten LASA-Skalen (Linear Analogue Self-Assessment), die1976 von Priestman und Baum zum ersten Mal eingesetzt wurden, um den subjektiven Gewinn unterschiedlicher chemotherapeutischer Regimes und hormoneller Behandlung bei Patientinnen mit fortgeschrittenem Brustkrebs zu vergleichen. Die Autoren hatten das Prinzip dieser Datenerhebung und Auswertung aus der psychopharmakologischen Forschung übernommen. Mit dieser Methode waren die Effekte der Behandlung mit Hypnotika/Tranquillizern und Placebo untersucht worden. Messmethodisch gelten diese visuellen Analogskalen in der psychologischen Forschung als akzeptabel. Die Autoren begründen ihren Ansatz im Prinzip mit den gleichen Argumenten wie es Autoren auch heute noch tun:

\footnotetext{
${ }^{8}$ siehe auch Faller, Lang \& Schilling (1998), die zur Kontrolle dieser unterschiedlichen Wahrnehmung einen Mehrebenenansatz vorschlagen.

${ }^{9}$ Der WHO-Performanzstatus beschreibt in 5 Stufen (von $0=$ keine Beschränkung bis $5=$ dauerhaft bettlägrig) die Funktionsfähigkeit von Patienten.
} 
"The whole L.A.S.A. system may be criticised for only giving a superficial view of an individual's emotions and difficulties. However, we had no intention of making an in-depth psychological study of individual patients. What we needed was a readily comprehensible, convenient, and reliable way for patients to make their own assessments as to their quality of life during and after treatment" (Priestman \& Baum 1976, S. 900). Üblicherweise wird eine Batterie von Skalen zusammengestellt, die sowohl Symptome als auch Funktionsbereiche erfassen. Auch die „Allgemeine Lebensqualität" wurde/wird auf diese Weise operationalisiert. Spätere Untersuchungen in Bezug auf die Validität und Reliabilität der visuellen Analogskalen im Vergleich mit kategorialen Skalen konnten deren Überlegenheit nicht belegen (Beckman \& Ditlev, 1987). Kritisiert wurde auch die Zusammenfassung der Einzelergebnisse zu einem Gesamt-LQ-Wert als zu undifferenziert und messmethodisch unsinnig.

Als ein weiterer Versuch LQ zu bestimmen, lässt sich die von Goldhirsch, Gelber, Simes et al. (1994) vorgeschlagene "Time Without Symptoms of disease and Toxicity" (TWiST) auffassen. Die Autoren ziehen von der Gesamtüberlebenszeit die Zeit ab, in der die Patienten unter Tumor und/oder Therapie bedingten Beschwerden leiden. Als Resultat erhalten sie eine „bereinigte“ Überlebenszeit, die als Vergleich für therapeutische Wirksamkeit herangezogen wird. TWiST als Versuch der LQ-Bewertung hat sich, verständlicherweise, nicht durchgesetzt. Außer acht gelassen wird dabei, dass Patienten in der Regel ihre LQ deutlich anders beurteilen als ein Beurteiler „von außen“, seien es nun Angehörige, Ärzte, Pflegende oder Psychologen. LQ erhält unter der Bedingung des durch die Krankheit bedrohten Lebens einen völlig anderen Charakter. Leben mit Symptomen und Beschwerden, Leben „an sich“ hat einen Wert - trotz allen Leidens. Das, was für die Betrachter unerträglich, nicht mehr lebenswert erscheint, kann für die Betroffenen durchaus sinnvolles Leben sein. Die existentielle Bedrohung der Patienten führt offenbar dazu, dass Nebenwirkungen von Therapien einen völlig anderen Stellenwert erhalten. Es sei hier nochmals betont, dass mittlerweile eine Reihe von Studien vorliegen, die diese Auffassung stützen. Patienten votieren für eine radikalere Behandlung auch wenn die Erfolgsaussichten gering sind. Sie unterscheiden sich in ihren Präferenzen deutlich von Ärzten, Krankenpflegepersonen und der allgemeinen Bevölkerung. Klinisch tätige Mitarbeiter onkologischer Abteilungen wissen, dass nur ein verschwindend geringer Anteil von Patienten Chemotherapie ablehnt. Slevin et al. (1990) folgern: "The large differences that emerged between these two groups (gemeint sind Erkrankte und nicht Erkrankte, Anmerkung der Autorin) suggest that patients' attitudes to treatment change dramatically when they are given the diagnosis of cancer. Faced with the reality of the diagnosis, as opposed to the purely theoretical possibility, patients are likely to accept any treatment that offers them some possible benefit and hope, however slight." (S.1460) 
Auf die Beurteilungsdiskrepanzen wurde auch auf $\mathrm{S} .11$ bereits hingewiesen. In den letzten Jahren finden sich nur wenige Veröffentlichungen die TWiST verwenden, und wenn dann nur zusammen mit anderen Indikatoren. Die Idee der TWiST-Methodik wurde später in der Medizinischen Ökonomik übernommen (siehe Kap. 2.5).

Danach folgte die zweite Generation von Erhebungsinstrumenten, die den Anspruch erhob, HRQL mehrdimensional und umfassender zu erfassen. ${ }^{10} \mathrm{Zu}$ diesen Instrumenten gehört der Functional Living Index-Cancer (FLIC) (Schipper et al. 1984), und die heute in Studien am meisten eingesetzten Instrumente, FACT (Functional Assessment of Cancer Therapy) von Cella et al. (1993) und QLQ (Quality of Life Questionnaire) von Aaronson et al. (1993) veröffentlicht.

Inhaltlich orientieren sich die heute gebräuchlichsten psychometrischen Verfahren, die in den achtziger und neunziger Jahren entwickelt wurden an der sehr weit reichenden und utopisch anmutenden Gesundheitsdefinition der WHO von 1948: „Health is a state of complete physical, mental and social well-being and not merely the absence of disease or infirmity" (Preamble to the Constitution of the World Health Organization as adopted by the International Health Conference, New York, 19-22 June, 1946, 1948).

Gesundheit wird nicht allein durch die Abwesenheit von Krankheit definiert, sondern als ein Zustand vollkommenen sozialen, physischen und psychischen Wohlbefindens aufgefasst. Hier werden die Aspekte physisch, psychisch und sozial als notwendige Dimensionen für die Betrachtung von LQ eingefordert. Die lediglich biologische Betrachtung von Gesundheit gilt als zu eng und dem Gegenstand nicht angemessen.

Den Instrumenten ist gemeinsam, dass sie von einer mehrdimensionalen Operationalisierung der LQ ausgehen, krankheitsspezifisch messen und den Anspruch haben psychometrischen Gütekriterien zu genügen. D.h., für jedes Instrument wurde das theoretische Konzept dargelegt und die methodische Qualität wurde mithilfe der psychologischen Testtheorie (Klassische Testtheorie) im Hinblick auf Reliabilität, interne und externe Validität sowie Sensitivität überprüft. Außerdem wurden Akzeptanz und Compliance durch die Patienten und die Anwenderfreundlichkeit (Erhebungs- und Auswertungsökonomie) berücksichtigt. Auswertungsvorschriften liegen ebenfalls vor.

Die Instrumente ermöglichen die Bestimmung von Profilen und die unabhängige Ermittlung eines Wertes der „Allgemeinen LQ“. Sie markieren die Abkehr von einem Summenwert der LQ und errechnen stattdessen Werte für die Subskalen.

\footnotetext{
${ }^{10}$ Der Sammelband von Aaronson \& Beckman (1987) enthält Aufsätze zu allen wichtigen As-
} pekten von LQ: theoretische Aspekte, Methoden, Reviews. 
Der QLQ-C30 wird in der hier vorgelegten Studie verwendet. Eine ausführlichere Beschreibung erfolgt in Teil II.

\subsection{Modelle des LQ-Konstruktes}

Da bis heute eine konsensfähige Definition von „Lebensqualität“ nicht vorliegt, begnügen sich die meisten Untersucher - wie übrigens in vielen anderen psychologischen Bereichen auch - damit, das, was sie erfassen wollen, über die Erhebungsinstrumente zu definieren. Entsprechend zahlreich sind die Messinstrumente. Während in einer Übersichtsarbeit von Gill und Feinstein (1994) 159 Instrumente zur Erhebung von LQ aufgeführt sind, gehen Bullinger (1997) und Porzsolt \& Rist (1997) von 800 bzw. über 1000 Instrumenten aus.

LQ ist in erster Linie von individuellen Werten und Erfahrungen der Betroffenen und ihrer Art der Krankheitsbewältigung abhängig. Objektiviert werden können lediglich einzelne Aspekte, vor allem diejenigen aus dem physischen Bereich der körperlichen Verfassung und der Funktions- und Leistungsfähigkeit (Schara 1990). Calman (1987) fordert, dass in der Definition von LQ drei Hauptaspekte berücksichtigt werden sollten:

- LQ kann nur individuell beurteilt werden

- Das Konzept sollte breit genug sein, um alle Lebensbereiche zu umfassen

- Persönliches Wachstum und Entwicklung sollten berücksichtigt werden

Für den letztgenannten Aspekt gibt es keinen Operationalisierungsvorschlag.

Da LQ nicht direkt beobachtbar ist, hat sie methodisch den Status eines hypothetischen Konstruktes. Idealerweise werden in einem Konstrukt relevante Variabeln, die direkt beobachtet und "gemessen“ werden können, definiert und in ihren Beziehungen zueinander beschrieben und gewichtet. Ferner sind sie empirisch hypothesengeleitet überprüfbar. Das Konstrukt LQ sollte einen Bedeutungsüberschuss enthalten und nicht nur die Einzelkomponenten beschreiben. Gerade an dieser Stelle stehen grundlegende Arbeiten noch aus. Auch die Arbeit von Pukrop (1997) zur theoretischen Explikation und empirischen Validierung am Beispiel gesunder und psychiatrischer Populationen mithilfe eines facettentheoretischen Ansatzes verweist wieder auf das bekannte Problem der mangelnden theoretischen und begrifflichen Fundierung des Terminus LQ. Dieses Problem ist ganz offensichtlich nicht auf onkologische Populationen beschränkt. Küchler \& Schreiber (1989) kommen zu folgender Gesamtbeurteilung des Konzeptes LQ:

„Lebensqualität ist ein philosophischer, ein politischer, ein ökonomischer, ein sozialwissenschaftlicher und ein medizinischer Begriff. In der Philosophie hat bereits Aristoteles das zentrale messtheoretische Problem der LQ-Forschung fomuliert: „... und oft ändert derselbe Mensch 
seine Meinung. Wird er krank, so ist es Gesundheit, und wenn er gesund ist, so ist es das Geld." Anders ausgedrückt:

- Lebensqualität bedeutet für Kranke etwas grundsätzlich anderes als für Gesunde;

- Die Bedeutung (Bewertung) einzelner Aspekte von LQ ist individuell höchst unterschiedlich;

Entsprechend ist eine allgemeinverbindliche Definition von "Lebensqualität" nicht sinnvoll (Popper, mündl. Mitt. 1989). Möglich ist jedoch eine Konzeptualisierung des Begriffs [...]. Ein solches Modell entzieht sich natürlich der direkten Messung. Hier ist eine gewisse Bescheidenheit erforderlich, die derzeit bedeutet, nicht Lebensqualität selbst, sondern Einschränkungen derselben auf Ordinal-, höchstens Intervallskalenniveau zu erfassen“ (S. 418 u. 420).

Die Autoren stellen ein Würfelmodell vor, das der Komplexität des Gegenstandes gerecht werden soll (ebenda, S.420):

\section{Abb. 1: Dimensionen der LQ im Modell}

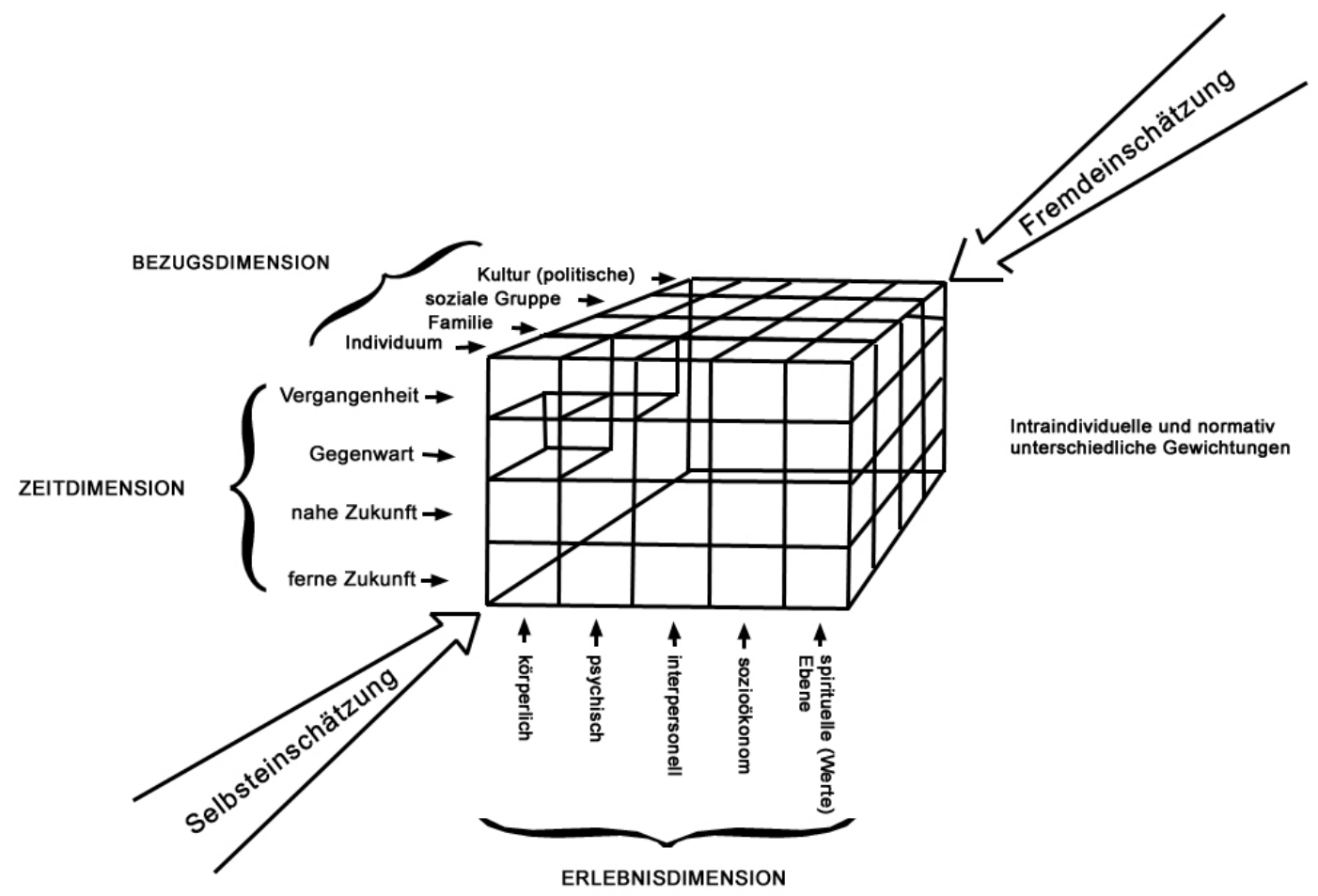

In diesem dreidimensionalen Modell werden folgende Achsen definiert:

- die Bezugsdimension mit den Eckpunkten Kultur - Individuum,

- die Zeitdimension mit den Eckpunkten Vergangenheit - ferne Zukunft,

- die Erlebnisdimension mit den Eckpunkten körperlich - spirituell.

In dem Modell wird die Perspektive der Fremd- und Selbsteinschätzung skizziert, und es wird auf interindividuelle sowie normative Gewichtungen hingewiesen. 
Das Verdienst des Modells liegt darin, dass der Rahmen um verschiedenste Einflussfaktoren von LQ gezogen wird, die Autoren erheben aber nicht den Anspruch, konkrete Vorgaben für eine „Messung“ von LQ zu liefern.

Akzeptiert man eine „gewisse Bescheidenheit“ in der Konzeptualisierung von LQ innerhalb der onkologischen Forschung, so lassen sich zwei Modelle von HRQL identifizieren, denen unterschiedliche Definitionen zugrunde liegen:

1. Das Konstrukt LQ wird definiert über verschiedene Einflussdimensionen oder Bereiche der LQ (Komponentenmodell)

2. Das Konstrukt LQ enthält als Kernstück intervenierende Variablen, die zur Ergebnisinterpretation herangezogen werden (Modell mit intervenierenden Variablen)

\section{Komponentenmodell}

Stellvertretend für viele andere soll hier für das erste Modell folgende Definition angeführt werden:

„Lebensqualität bezieht sich auf die emotionalen, funktionalen, sozialen und psychischen Aspekte menschlicher Existenz. Lebensqualität ist nicht direkt beobachtbar, sondern nur erschließbar aus verschiedenen Komponenten“" (Bullinger \& Pöppel 1989, S. 436).

In dieser Definition wird ein Konstrukt beschrieben, dessen beobachtbare Komponenten sich auf vier Bereiche erstrecken. Wichtigste Aufgabe bei Verwendung dieses Konstruktes ist die Operationalisierung der Dimensionen durch Messinstrumente. Außerdem muss eine Festlegung getroffen werden, mit welcher Gewichtung die einzelnen Komponenten in einen Gesamtwert eingehen sollen, wenn nicht eine Beschränkung auf eine Profilerstellung erfolgen soll. Wenn ein Summenwert ermittelt werden soll, werden alle Dimensionen gleich gewichtet. Diese gleiche Gewichtung ist vielfach kritisiert worden, lässt sich aber empirisch durchaus begründen (Pukrop 1997). Bei den neueren Konzepten wird in der Regel auf einen Summenwert verzichtet und die Skalenergebnisse werden als Profil dargestellt. Zusätzlich ermitteln einzelne Instrumente noch einen Gesamt-LQ-Wert, unabhängig von den Komponenten der LQ.

Konsens herrscht darüber, dass LQ als ein multidimensionales Konzept aufzufassen ist (u. a. Bullinger \& Pöppel 1989; Cella 1995; Feld 1995), welches folgende Minimalkomponenten enthalten sollte:

physische, psychische und soziale Komponenten sowie Symptome, die sich auf Erkrankung und Therapie beziehen (Feld ebenda). Sowohl objektive Parameter (z. B. Krankheitsstadium) als auch subjektive Parameter (z.B. emotionale Belastung) sollen bei der Begriffsbildung miteinbezogen werden. Aaronson (1990) möchte eine Be- 
schränkung der Komponenten auf das, was im Rahmen der Gesundheitsversorgung auch beeinflussbar ist:

1. Funktioneller Status (Selbständigkeit im Hinblick auf Alltagsverrichtungen, Mobilität, physische Voraussetzungen für die Erfüllung sozialer Rollen)

2. Krankheitssymptome oder therapieassoziierte Symptome

3. Psychische Funktion

4. Soziale Funktion

Ergänzend zu diesen "core“ Komponenten können noch spezifische Dimensionen hinzugefügt werden (z. B. bei Brustkrebserkrankungen die Dimension „Körperbild“, oder bei der Hochdosistherapie ein HDC-Modul). Die unterschiedlichen Kategorien oder Dimensionen werden dann durch Fragenkomplexe, die auf Plausibilität, Alltagsverständnis sowie Expertenurteile zurückgehen (siehe Kapitel1), weiter aufgeschlüsselt und faktorenanalytisch überprüft. Da bei der Erstellung von Fragenkatalogen unterschiedliche Gewichtungen und Interessen eine Rolle spielen, unterscheiden sich die Erhebungsinstrumente in ihren jeweiligen Skalen. Z. T. wurde zur Fragebogenkonstruktion der umgekehrte Weg beschritten.

In der Onkologie werden/wurden die in der Tabelle angeführten Instrumente am häufigsten benutzt. Die folgende Tabelle gibt eine Übersicht über die verwendeten Dimensionen.

Tabelle 1 : Übersicht über die gebräuchlichsten LQ-Erhebungsinstrumente in der Onkologie

\begin{tabular}{|c|c|c|c|c|}
\hline $\begin{array}{l}\text { Publikations- } \\
\text { jahr }\end{array}$ & Name & Autoren & Dimensionen & $\begin{array}{l}\text { Art der Daten- } \\
\text { erhebung }\end{array}$ \\
\hline 1948 & $\begin{array}{l}\text { Karnofsky- } \\
\text { Index }\end{array}$ & Karnofsky et al. & $\begin{array}{l}\text { Seelische Verfassung } \\
\text { Allgemeinbefinden } \\
\text { Selbständigkeit } \\
\text { Unabhängigkeit von ärzt- } \\
\text { licher/pflegerischer Hilfe }\end{array}$ & $\begin{array}{l}\text { Selbst- und } \\
\text { Fremdbeurteilung }\end{array}$ \\
\hline 1976 & L.A.S.A. & Priestman \& Baum & $\begin{array}{l}\text { Physische Fähigkeiten } \\
\text { Sozialer Bereich } \\
\text { Psychischer Bereich } \\
\text { Persönliche Beziehungen }\end{array}$ & $\begin{array}{l}\text { Selbstrating mit } \\
\text { visueller Analog- } \\
\text { Skala }\end{array}$ \\
\hline 1981 & $\begin{array}{l}\text { Spitzer- } \\
\text { Index }\end{array}$ & Spitzer et al. & $\begin{array}{l}\text { Aktivität } \\
\text { Selbständigkeit } \\
\text { Gesundheit } \\
\text { Unterstützung } \\
\text { Zukunftsperspektive }\end{array}$ & \begin{tabular}{|l} 
Interview \\
Fremdrating
\end{tabular} \\
\hline 1984 & FLIC & Schipper et al. & $\begin{array}{l}\text { Physisches Wohl- befin- } \\
\text { den } \\
\text { Psychologischer Status } \\
\text { Soziale Fähigkeiten } \\
\text { Familieninteraktion } \\
\text { Symptome }\end{array}$ & $\begin{array}{l}\text { Selbstbeurteilung } \\
\text { Likert-Skala }\end{array}$ \\
\hline 1993 & FACT & Cella et al. & $\begin{array}{l}\text { Physischer Bereich } \\
\text { Funktionen }\end{array}$ & $\begin{array}{l}\text { Selbstbeurteilung } \\
\text { Likert-Skala }\end{array}$ \\
\hline
\end{tabular}




\begin{tabular}{|l|l|l|l|l|}
\hline $\begin{array}{l}\text { Publikations- } \\
\text { jahr }\end{array}$ & Name & Autoren & Dimensionen & $\begin{array}{l}\text { Art der Daten- } \\
\text { erhebung }\end{array}$ \\
\hline 1993 & QLQ & Aaronson et al. & $\begin{array}{l}\text { Sozialer Bereich } \\
\text { Emotionaler Bereich } \\
\text { Beziehung zum Arzt }\end{array}$ & $\begin{array}{l}\text { Physische Funktion } \\
\text { Rollenfunktion } \\
\text { Soziale Funktion } \\
\text { Kognitive Funktion } \\
\text { Emotionale Funktion } \\
\text { Finanzielle Belastung } \\
\text { Symptome }\end{array}$ \\
\hline 1994 & Symptome & $\begin{array}{l}\text { Selbstbeurteilung } \\
\text { Likert-Skala } \\
\text { Ordinalskala }\end{array}$ \\
\hline & TWIST & Goldhirsch et al. & Fremdbeurteilung \\
\hline
\end{tabular}

Legt man die Literaturangaben in Medline (9/2001) zugrunde, so wurde in den letzten 10 Jahren in onkologischen Arbeiten der QLQ (C-30) mit großem Abstand am häufigsten zitiert, gefolgt von FLIC und FACT (QLQ 202 Angaben; FLIC 29 und FACT-G 22 Angaben). Die anderen aufgelisteten Instrumente spielen heute in der LQ-Forschung eine untergeordnete Rolle.

Der Kritik, dass all diesen Instrumenten ein äußerst subjektives und reduktionistisches Konzept zugrunde liegt, begegnen einzelne Autoren damit, dass der Katalog von Dimensionen/Bereichen ausgeweitet wird. Als Beispiel sei hier Weiss (1999) angeführt:

„Um bisher vernachlässigte bzw. auch bewusst ausgesparte Aspekte der Lebensqualität zu berücksichtigen, werden deshalb in unserer Studie [...] weitere inhaltliche Dimensionen berücksichtigt werden, so z.B. die folgenden psychologischen Konstrukte:

- Sinnerfahrung und Sinnerfüllung trotz der gegenwärtigen schweren Erkrankung

- Werteempfinden und Werteerleben

- die Fähigkeit zur Selbst-Distanzierung und Selbstreflexion als Voraussetzung dafür

- positive Grundhaltung und Lebensbejahung trotz schweren Schicksals

- die Qualität der sozialen Beziehungen/Erfahren von sozialer Unterstützung“ (S. 26)

Das Konstrukt LQ wird ergänzt nicht nur um weitere beobachtbare Variablen, sondern auch um weitere psychologische Konstrukte. Neben Weiss gibt es eine holländische Arbeitsgruppe (Sherman et al. 2000), die außerdem religiöse Werte erfassen will. Mit einem kurzen Fragebogen, dem „Duke Religious Index“ (DRI), soll die Religiosität mehrdimensional erhoben werden. Auf die psychometrische Validierung soll hier nicht eingegangen werden. Interessant ist die Schlussfolgerung, die von den Autoren gezogen wird:

„[...] clinicians may find DRI helpful as a screening tool, to determine whether various dimensions of religiosity are important to their patients. [...] Use of a brief questionnaire such as the DRI may provide pertinent information, assure the patient and family that the clinician is inter- 
ested in providing comprehensive care, and lead the way to a useful conversation about spiritual concerns." (S.107)

Vom Arzt wird im Sinne einer "ganzheitlichen“ Behandlung erwartet, auch auf die spirituellen Bedürfnisse des Patienten einzugehen. Ob eine derartige Ausweitung der Dimensionen, bezogen auf den Behandlungskontext aber überhaupt sinnvoll geschweige denn machbar ist, wird ebenso wenig problematisiert wie die naheliegende Frage, ob die geforderte Dimension nicht ausschließlich in der direkten Arzt-PatientenKommunikation zu erreichen ist.

Die tonangebenden psychoonkolgischen Arbeitsgruppen haben krankheitsspezifische Instrumente (FACT, QLQ-C30) entwickelt, die ihr Augenmerk auf die krankheitsbedingten Einschränkungen richten. Kernfragebögen werden durch Module ergänzt. Die onkologische Forschung präferiert diese modulare Strategie. ${ }^{11}$ Krankheitsspezifisch meint aber spezifisch nur bezogen auf eine Krankheitsgruppe wie z. B. Krebskrankheit oder psychiatrische Erkrankung. Bezogen auf die jeweilige Gruppe ist das Instrument dann übergreifend in dem Sinne, dass es für die übergeordnete Diagnosegruppe „Krebserkrankung“, z. B. Lungenkarzinome, Leukämien usw., eingesetzt werden kann („Core“Fragebogen). Die Module sind (krankheits-) bereichspezifisch in dem Sinne, dass sie sich auf Aspekte, die für die jeweilige Diagnosegruppe als relevant erachtet werden, beziehen. Sie können sich aber auch auf bestimmte Therapien beziehen, wie z. B. das Hochdosistherapiemodul des QLQ-C30. Das modulare Modell lässt sich unter der Annahme, dass eine Stichprobe von Patientinnen mit Mammakarzinom unter HDC untersucht werden soll, beispielsweise folgendermaßen darstellen:

\footnotetext{
${ }^{11}$ Diese Vorgabe blieb allerdings nicht unwidersprochen. Bullinger (2001) gibt dem SF-36, einem allgemeinen Instrument zur HRQL Datenerhebung, den Vorzug vor einem krankheitsspezifischen Instrument.
} 
Abb. 2: Modulares Modell am Beispiel des Mammakarzinom



Neben psychometrisch validierten Instrumenten werden unzählige ad hoc Fragebögen eingesetzt, die eine Vergleichbarkeit der Daten erschweren bzw. unmöglich machen. Neben den krankheitsspezifischen Instrumenten wurden auch Fragebögen (z. B. Depressionsinventar von Beck, Profile of Mood Skala, SF-26), die zu anderen Zwecken entwickelt wurden, in diesem Bereich eingesetzt. Das englische „Medical Research Council Cancer Commitee“ (RSCL) fordert den Routineeinsatz der „Rotterdam Symptom Checklist“ und den Einsatz der „Hospital Anxiety and Depression Scale“ (HADS), um LQ in klinischen Studien zu bestimmen.

Einige Arbeitsgruppen haben Richtlinien publiziert, die einen Mindeststandard festlegen. Der zunehmende Einsatz des QLQ-C30 ist auf die Aktivitäten der EORTC (European Organization of Research and Therapy of Cancer) Quality of Life Group zurückzuführen.

\section{Modell mit intervenierendem Konstrukt}

Während einige der Autoren die Dimensionen erweitern und Module einführen, bemühen sich andere um die Bestimmung von intervenierenden Variabeln.

Kennzeichnend für den zweiten Ansatz ist der Rückgriff auf Erklärungsmodelle aus der Stressforschung ${ }^{12}$ (oder in einem noch allgemeineren Sinne auf sozialpsychologische Theorien von Adaptation und Dissonanzreduktion). Ähnlich wie in der Stressforschung wird davon ausgegangen, dass nicht alle Stressoren gleich wirken, sondern Verarbeitungsprozesse darüber entscheiden, ob der jeweilige „Stress“ (Eustress, Disstress) negative, keine oder positive Auswirkungen hat.

Die zweite Definition steht exemplarisch für diese Art von Konstrukt:

\footnotetext{
${ }^{12}$ Die meisten Autoren berufen sich auf das „klassische“ Modell von Lazarus \& Folkman (1984). In diesem Modell wird auf die Wechselwirkung zwischen Person und Umwelt verwiesen und der Versuch unternommen, jenseits von Reiz-Reaktions- oder Persönlichkeitsmodellen zu beschreiben, wie Menschen belastende Situationen bewältigen.
} 
„[...]Life Quality is the patients appraisal of and satisfaction with their current level of functioning compared to what they perceive to be possible or ideal" ( Cella \& Tulsky, 1993, S. 327).

In dieser Definition wird der Schwerpunkt auf die Bewertung durch den Patienten und seine Zufriedenheit mit der jeweiligen Lebenssituation unter Krankheitsbedingungen gelegt. Nicht objektivierbare Einschränkungen und Belastungen stehen im Vordergrund, sondern Vergleichsprozesse, deren Resultate sich verändern (können). Anlass für die Annahme eines intervenierenden Konstruktes waren Befunde, die keinen oder nur geringe Zusammenhänge zwischen objektiven Bedingungen und subjektiver LQ belegen konnten.

Befunde zeigten das unerwartete Resultat, dass Krebskranke ihre LQ oftmals besser bewerteten als Gesunde (Henrich \& Herschbach, 1998). Erklärt wurden diese Befunde damit, dass durch die bedrohliche Erkrankung Menschen ihre etablierten Werte verändern, ihre Ansprüche an Gesundheit reduzieren und sich in sozialen Vergleichsprozessen mit anderen, denen es genauso „schlecht“ und/oder „schlechter“ geht, „trösten“. Diese Veränderung von Wertmaßstäben oder, wie Autoren (Bernhard et al., 1995; Muthny \& Bermejo, 1993) es genannt haben, der Prozess des Reframing ${ }^{13}$, macht Vergleiche mit einer gesunden Population sehr fragwürdig. Die Konzeptualisierung, die Art der Datenerhebung, die Erhebungsinstrumente und vor allem auch die situativen Bedingungen (Erstdiagnose, Rezidiv, Progress, Remission, Stadium des „sich geheilt fühlens“) scheinen großen Anteil daran zu haben, dass die Forschung nicht nur zu inkonsistenten sondern zueinander diametral entgegengesetzten Aussagen kommt. Die folgenden Zitate, die sich beliebig erweitern ließen, sollen diese Aussage exemplarisch belegen:

HRQL ist im Vergleich zu Gesunden deutlich reduziert (u. a. Krischke \& Petermann, 2001) oder, Krebskranke bewerten ihre HRQL besser als Gesunde (u. a. Muthny, Koch \& Stump, 1993; Harrer, Mosheim, Richter et al., 1993).

Herschbach (2002) stellt in der folgenden Abbildung Ergebnisse zusammen:

${ }^{13}$ Reframing ist ein Begriff aus der familientherapeutischen Tradition. Gemeint ist damit, dass Bedingungen (z. B. familiäre), die nicht verändert werden können, in neuem Licht betrachtet werden. Mit "kognitiver Umstrukturierung“ ist das Gleiche gemeint. 


\section{Abb. 3: Lebensqualität bei verschiedenen Erkrankungen im Vergleich mit der deutschen} Normpopulation (S.143)

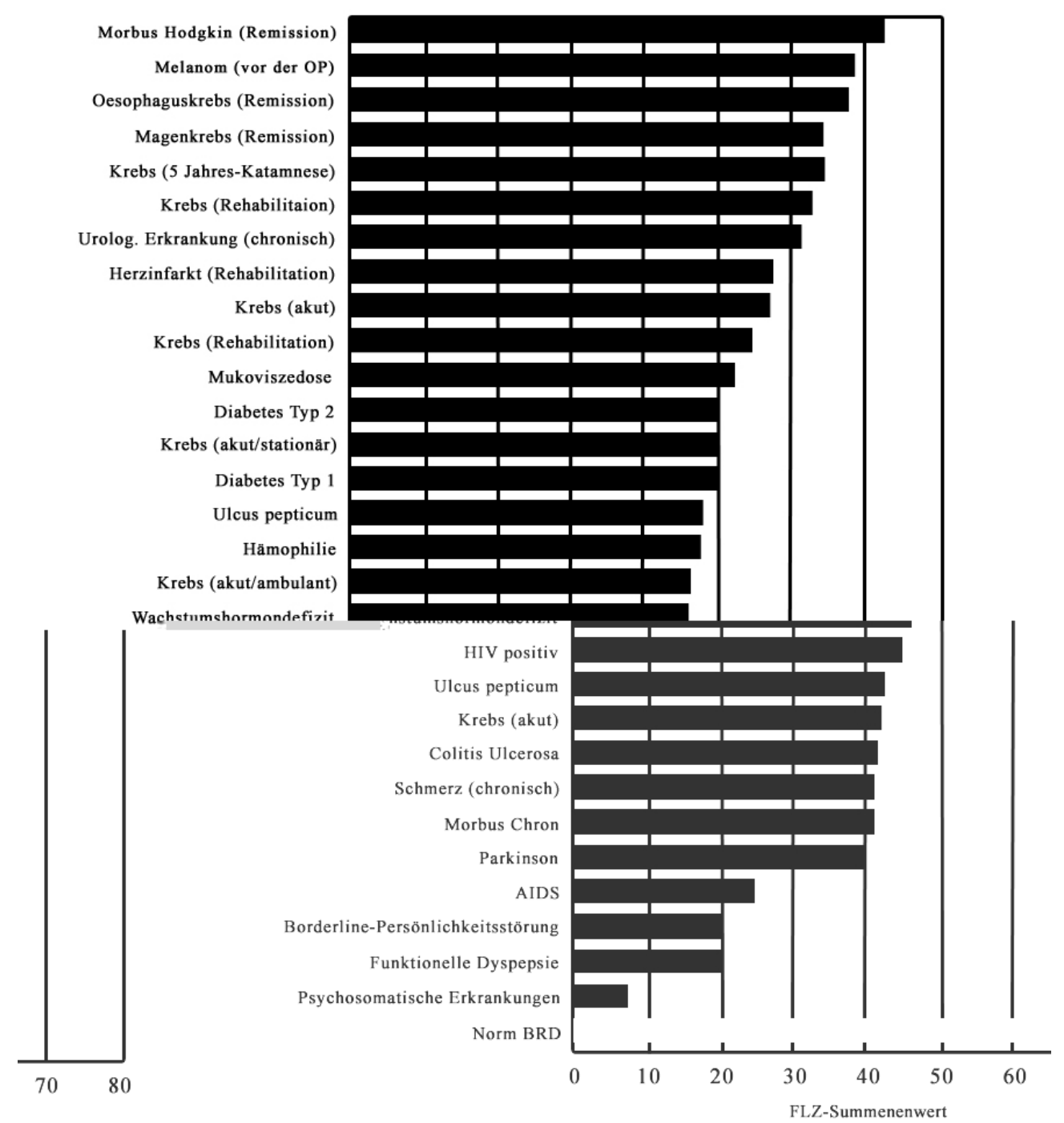

Herschbachs Anliegen besteht u. a. darin zu belegen, dass es sich beim „Zufriedenheitsparadox" respektive "Wohlbefindensparadox" (siehe auch S. 31) nicht um einen Methodenartefakt der psychometrischen Forschung handelt. Er argumentiert, dass Menschen die Möglichkeit haben, sich auch mit objektiv negativen Bedingungen zu arrangieren: „Es spricht viel empirische Evidenz dafür, dass es eine generalisierte Tendenz im Menschen gibt, sich selbst und seine Lebenssituation eher positiv als negativ wahrzunehmen [...] Die theoretische und empirische Evidenz, die vorgelegt wurde, legt nahe, dass eine Kombination von Faktoren dafür verantwortlich zu machen ist. Auf der einen Seite scheint der Mensch mit Eigenschaften ausgestattet zu sein, die inn die Realität grundsätzlich positiv getönt wahrnehmen lassen. Hinzu kommen Anpassungsmechanismen, die inn in die Lage versetzen, mit 
widrigen Umständen fertig zu werden. Dazu gehört die Fähigkeit, auch aus negativen Erfahrungen einen produktiven Sinn zu ziehen. Hinzu kommt, dass man durch Vergleiche mit anderen Menschen oder auch mit der eigenen Vergangenheit immer wieder seine Wertmaßstäbe an die Realität anpasst. [...] Es scheint fast, dass die genannten Eigenschaften und Mechanismen eine fundamentale Funktion für das Überleben haben“ (S. 143).

Während Herschbach diese Befunde positiv wertet, kommt Pukrop (1997) zu einer ganz anderen Wertung. Nach Durchsicht der Forschungsergebnisse zur LQ und Lebenszufriedenheit ergibt sich für inn, kurz zusammengefasst, folgende "deprimierende“ Feststellung: LQ im Lichte der intervenierenden Prozesse bedeutet, dass die in unserer Leistungsgesellschaft vorherrschenden Normen einen Anpassungsdruck ausüben in Richtung Zufriedenheit. Andere Einschätzungen werden als persönliches Versagen gewertet. Diese „resignative“ Anpassung äußert sich dann in „Pseudozufriedenheit“. Pukrop zieht für die psychometrische Forschung daraus die Folgerung, dass der Schwerpunkt auf den subjektiven Konstruktionen von Ist-Zuständen liegen sollte und ipsativen Längsschnittuntersuchungen zugunsten von Gruppenvergleichen der Vorzug gegeben werden sollte.

LQ-Forschung in der Onkologie benutzt bevorzugt Krankheitsverarbeitung (synonym Krankheitsbewältigung) oder Coping ${ }^{14}$ als intervenierendes Konstrukt. Hürny (1997) beschreibt Lebensqualität als das Resultat der Krankheitsverarbeitung, der Adaptation des Patienten und seiner Umgebung in bezug auf Krankheit und Behandlung. Das Ausmaß der geglückten Anpassung bestimmt im Wesentlichen das physische, psychische und soziale Wohlbefinden.

\section{Exkurs}

Krankheitsverarbeitung wird hier synonym mit den oben genannten Begriffen gebraucht. Krankheitsverarbeitung wird betrachtet als Mediator zwischen der durch die Krankheit objektiv veränderten somatischen und psychosozialen Situation und deren Bewertung, die zur Einschätzung der Lebensqualität führt. Das Konstrukt Krankheitsverarbeitung ähnelt in seiner Komplexität und damit auch gleichzeitig in seiner konzeptuellen Unschärfe dem der Lebensqualität. Faller (1998) hat sich in seinem Buch „Krankheitsverarbeitung bei Krebskranken“ ausführlich mit den verschiedenen Aspekten auseinandergesetzt und liefert eine profunde Übersicht über wichtige Forschungsergebnisse. Faller geht von der Frage aus, wie es Menschen, die auf traumatische Weise von einer lebensbedrohlichen Erkrankung heimgesucht werden, schaffen, mit dieser Bedrohung umzugehen. Wie erleben diese Menschen ihre Erkrankung? Welches sind die be-

\footnotetext{
${ }^{14}$ In der Literatur werden die Begriffe häufig synonym verwendet. Coping bezieht sich nicht nur auf Krankheitsereignisse sondern generell auf belastende Lebensereignisse. In Anlehnung an Lazarus \& Folkman (1984) definiert Heim (1988, S. 8/9) Krankheitsverarbeitung als die Gesamtheit der Prozesse, die darauf abzielt, bestehende oder erwartete Belastungen im Zusammenhang mit der Krankheit emotional, kognitiv oder aktional aufzufangen, auszugleichen oder zu meistern. Krankheitsverarbeitung ist zielorientiert, und der Ausgangspunkt ist die wahrgenommene Belastung. Die Verarbeitungsprozesse können im tiefenpsychologischen oder Copingkontext betrachtet werden. Wesentliche Vorannahmen sind: die Verarbeitung hat Prozesscharakter, emotionale, kognitive und Handlungsebene sind betroffen, individuelle und interaktionelle Komponenten greifen ineinander.

Einen Überblick über den Stand der Copingforschung geben Schüßler \& Leibing (Hg.)(1994).
} 
gleitenden Gefühle und Gedanken und wie wird mit den Belastungen umgegangen? Seine Definition von Krankheitsverarbeitung lautet: „Krankheitsverarbeitung soll die emotionalen Belastungen, die eine Krankheit mit sich bringt, mildern und ausgleichen. Krankheitsverarbeitung soll negatives Befinden auflösen und Wohlbefinden fördern" (S. 80). In Fallers Vorstellung von Krankheitsverarbeitung werden sowohl Abwehr als auch Coping integriert. Er stellt die beiden Modelle gegenüber.

Tabelle 2: Krankheitsverarbeitung (S. 37)

\begin{tabular}{|l|l|}
\hline Abwehr & Coping \\
\hline Herkunft des Modells aus der Psychoanalyse & Herkunft des Modells aus der Stressforschung \\
\hline $\begin{array}{l}\text { Ziel: } \\
\text { Unbewusstbleiben von bedrohlichen Gefüh- } \\
\text { len und Phantasien }\end{array}$ & $\begin{array}{l}\text { Ziel: } \\
\text { Realitätsbewältigung }\end{array}$ \\
\hline Sichert die Funktionsfähigkeit des Ich & Sichert die Realitätsanpassung des Individuums \\
\hline Erfolg unbewusst & Erfolg bewusst \\
\hline
\end{tabular}

Der Autor beschreibt den Zusammenhang zwischen Abwehr und Coping:

„Wenn ein Mensch von einer plötzlich eintretenden, traumatischen Bedrohung betroffen wird, kann er zunächst überfordert sein, realitätsangemessenes Bewältigungsverhalten zu zeigen [...] In dieser Situation bedarf es einer Notfallreaktion, die das Ich kurzfristig entlastet und erst einmal wieder in den Stand versetzt realitätsbezogen zu handeln. Im Sinne einer Notfallreaktion können Verleugnungsprozesse fungieren. Sie verhindern die Überflutung des Ich mit Emotionen, die nicht mehr bewältigt werden können, z. B. mit Todesangst [...] Indem sie auch vor inneren Ängsten abschirmen, halten sie dem Individuum sozusagen den Rücken frei; sie mildern die durch das Krankheitsereignis ausgelösten inneren Ängste [...] Mit zunehmend erfolgreicher Verarbeitung der realen Situation kann das Ich dann langfristig nach und nach Abwehrformen durch Coping-Verhalten ersetzen, auf übermäßige Abwehr verzichten“ (ebenda S.37). Hervorgehoben wird in diesem Ansatz die sinnvolle Funktion von Abwehr zum Schutz vor nicht zu bewältigenden Emotionen. Abwehr kann in bestimmten Situationen als funktional und angemessen gelten. Als Besonderheit bei der Krankheitsbewältigung von Krebspatienten stellt Faller heraus, dass trotz der schweren emotionalen Belastungen die Betroffenen alles daran setzen, ein positives Selbstbild aufrecht zu erhalten. Zumindest erscheint es dem Außenstehenden so. Er verweist auf die Notwendigkeit, Verleugnung als differenziert zu sehen, nicht als Alles-oderNichts-Phänomen. Andere Autoren sprechen in diesem Zusammenhang von doppelter Buchführung und Zweigleisigkeit des Denkens und Fühlens. Weisman (1972) hat dafür den Begriff des "middle knowledge" geprägt, der das Pendeln zwischen Akzeptieren und Verleugnen zusammenfasst. Das Wissen um den Zustand ist zwar da, muss aber wegen seiner Bedrohlichkeit zeitweilig ausgeblendet werden. Faller fragt danach, ob nicht Illusionen, Räume zwischen Realität und Phantasie generell notwendig und hilfreich sind bei der Bewältigung einer Krebskrankheit. Für die Bewältigung scheint zentral zu sein, dass etwas gegen die Erkrankung getan werden kann. Bei belastenden Therapien ist die Hoffnung für das seelische Erleben wichtiger als die körperliche Beeinträchtigung. Krankheitsverarbeitung ist ein prozesshaftes Geschehen, dass dem körperlichen Verlauf der Erkrankung folgt. Die Krisenmomente haben immer mit der Konfrontation in Bezug auf Fortschreiten, Neuauftreten und damit dem partiellen Verlust von Hoffnung auf Gesundung zu tun. Eine weitere wichtige Arbeit zum Verständnis von Krankheitsverarbeitung ist die von Bolund (1990). Ähnlich wie Faller bezieht sie sich auf die theoretische Tradition psychodynamischer Konzepte und das behaviorale Modell der Stressforschung. Ausgangspunkt der Krankheitsverarbeitung ist die Krebserkrankung als persönliche Katastrophe, als Krise. Krebs steht als kulturelles Symbol für Gefahr, Leid und Schmerz, Schuld und Scham, Ekel, Isolation, Nutzlosigkeit, Nichtwissen, Chaos und Angst. Krise bedeutet den Verlust von Sicherheit und Kontrolle. Die meisten Patienten kommen trotzdem zurecht, ohne dass psychosoziale Interventionen notwendig werden. Idealerweise kann der Patient auf eigene Ressourcen zurückgreifen und wird dabei unterstützt von ihm nahe stehenden Menschen. Schwierig wird das „zurecht kommen“ dann, wenn das Trauma der Krebserkrankung koinzidiert mit anderen schwierigen Lebenssituationen und Ereignissen. Bolund entwickelt ein Modell, das die verschiedenen Anteile bei der Krankheitsverarbeitung anschaulich macht und auch diagnostische Hilfestellung leisten kann. Der Faktor Zeit spielt sowohl bei Faller als auch bei Bolund eine wichtige Rolle. 
Die folgende Graphik beschreibt, in Anlehnung an Bolund (1990 S.16), das Modell des Umgangs mit der Bedrohung durch eine Krebserkrankung:

\section{Abb. 4: Umgang mit Bedrohung}

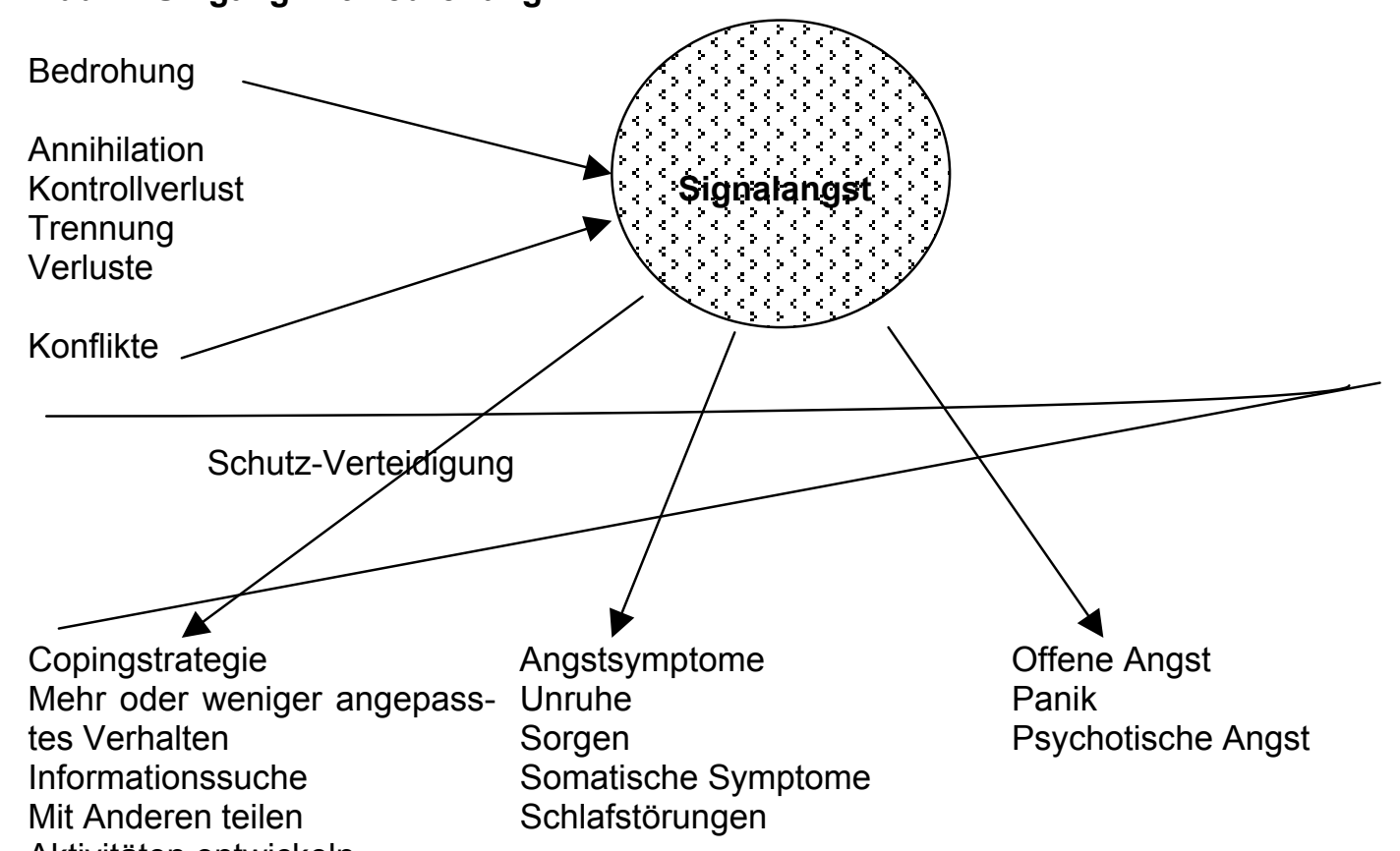

Aktivitäten entwickeln

Verleugnen

Resignieren

Abhängigkeit/Regredieren

Die Angst der Patienten wird verstärkt, wenn die behandelnden Ärzte oder andere Mitglieder des Behandlungsteams widersprüchliches Verhalten zeigen oder dann, wenn in einer Situation Vertrauen und Sicherheit fehlen. Das Umgehen mit der Angst benötigt sehr viel an Energie, die für andere Alltagsanforderungen nicht mehr zur Verfügung steht. Bolund nimmt eine systemische Perspektive ein, wenn sie nicht nur die Patientenseite mit ihren sozialen Bezügen sondern auch das Gesundheitssystem in den Blick nimmt: „The important factors in coping should not only be seen as the strength or weakness of the individual patient but also as the adequacy of the surrounding social structure in its contribution to consistency and meaning. In times of illness the health care system is the most important structure, that upon which your health, life or death depends" (ebenda S.24). Das Modell liefert Anhaltspunkte dafür, wie hilfsbedürftig Patienten sind: Je weniger eigene Schutzmöglichkeiten, desto wichtiger professionelle Hilfe von außen.

Schwierigkeiten bereitet im Rahmen dieses Modells die Indentifizierung adaptiver und maladaptiver Verarbeitungsstrategien, sofern sie nicht im Zeitverlauf betrachtet werden. Gerade im Kontext von lebensbedrohlichen Erkrankungen können Strategien, die bei chronischen Erkrankungen z. B. rheumatischen Erkrankungen oder chronischen Schmerzsyndromen ungünstig sind - bspw. Passivität, Rückzug, Regression - zeitweilig zum Selbstschutz notwendig sein. Allerdings ist dies abhängig vom Individuum, vom Krankheitsstadium, vom Ausmaß der Bedrohung und vom Zeitablauf der Erkrankung. In der Onkologie wurde versucht, Verarbeitungsstrategien zu identifizieren, die mit längeren Überlebenszeiten korrelieren und deswegen als „geglückt“ gelten und/oder die zu einer positiven Bewertung der LQ führen. 
Nicht nur in der vulgärpsychologischen Argumentation wird immer noch davon ausgegangen, dass es diese Strategien gibt. Dazu zählen in erster Linie „Kampfgeist“, „Positiv Denken“ und generell „Aktivität“. An dieser Stelle sei dazu nur soviel gesagt: Bislang hat sich keine der Strategien als eindeutig überlebensrelevant bestätigt. ${ }^{15}$ Die Ergebnisse der Forschung sind widersprüchlich und die am bekanntesten gewordene Studie von Spiegel et al. (1989), der bei Brustkrebspatientinnen einen signifikanten Überlebenseffekt durch psychologische Intervention gefunden hat, wurde methodisch kritisiert und konnte bislang nicht repliziert werden. Auch der Sinn von „Kampfgeist“ und „Positiv Denken“ muss hinterfragt werden in Situationen, in denen es nichts zu kämpfen gibt, bzw. in Situationen, in denen es realitätsgerechter wäre, Verluste zu betrauern und Aggressionen auszudrücken, kurz, auch negative Emotionen zu akzeptieren. Allerdings ist Stärkung von Autonomie, dem Entgegenwirken von Kontrollverlust, durchaus eine präventive Rolle gegen Depressivität und Angstüberwältigung zuzusprechen. Die einzige Strategie, die vermutlich schädliche Auswirkungen hat, ist die Aufgabe jeglicher Hoffnung, die einen Zustand von Hilflosigkeit und Handlungsunfähigkeit („helplessness“ - „hopelessness“) zur Folge hat. Hier gibt es allerdings Hinweise, dass diese Befindlichkeit mit kürzeren Überlebenszeiten korreliert ist und dass Kontrollverlust mit schlechterer Anpassung einhergeht (Everson et al. 1996). Diese Hinweise decken sich mit der klinischen Erfahrung.

Im Gegensatz zu anderen chronischen Erkrankungen, die nicht unmittelbar mit Sterben assoziiert werden, scheinen bei Krebserkrankungen andere/zusätzliche Faktoren von Bedeutung zu sein. Regelhaft findet sich eine unterschiedliche Bewertung der emotionalen Belastung durch externe Beobachter. Von Außenstehenden werden die Belastungen meist wesentlich gravierender eingeschätzt. Faller et al. (1992) weisen auf die Tradition hin, diese Diskrepanz zwischen Selbst- und Fremdurteil als Abwehrvorgang zu interpretieren. ${ }^{16}$ Im Schlussteil dieser Arbeit wird dieser Zusammenhang kritisch zu würdigen sein.

Bezogen auf fast alle Phasen der Erkrankung spielt für Patienten Belastungen und Symptome für die Beurteilung ihrer LQ eine untergeordnete Rolle, sofern sie eine Heilung bzw. Verlängerung der Lebenszeit versprechen. Heute scheint die Frage nach den Einbußen an LQ durch Therapie eher eine akademische Frage zu sein bzw. eine Frage der Nicht-Betroffenen, die von der Mehrzahl der Erkrankten nicht gestellt wird, insbesondere nicht in der Situation der Ersterkrankung. Die Mehrzahl der Patienten hofft auf Heilung (oder Lebensverlängerung), ohne nach dem Preis zu fragen.

${ }^{15}$ Siehe Faller (1998): Krankheitsverarbeitung bei Krebs

${ }^{16}$ Zum Problem von Bewältigung und Abwehr siehe Kächele \& Steffens (1988) 
In der Literatur von Krebskranken, in der die Biographien und die Schritte im Umgang mit der Erkrankung nachgezeichnet werden, findet sich als entscheidender Hinweis der Aspekt der „Hoffnung“. In der wissenschaftlichen Literatur wird Hoffnung nur als eine Strategie unter anderen thematisiert. Krankheitsverarbeitungsstrategien sind sowohl zeitgebunden als auch abhängig von Persönlichkeitsvariablen. ${ }^{17}$ Hoffnung scheint hingegen einen universelleren Charakter zu haben, scheint zu bestimmen, welchen belastenden Prozeduren sich Patienten unterziehen, selbst wenn die Erfolgschancen nicht sehr groß scheinen. Für Aulbert (1998) ist Hoffnung die wichtigste Triebkraft für die Adaptation an die Erkrankung: „[...] wichtigster Faktor, ohne den Lebensqualität in dieser Situation überhaupt nicht möglich wäre, ist die Hoffnung."

Hoffnung ändert sich:

- „Ist es beispielsweise zuerst die Hoffnung, dass sich die Diagnose Krebs nicht bestätigt,

- so wird es dann die Hoffnung, dass die Therapie Erfolg haben möge,

- oder auch nur, dass die Krankheit nicht so schnell fortschreiten möge.

- Hoffnung kann zu einem späteren Zeitpunkt auch sein, dass die Krankheit nicht von quälenden Schmerzen begleitet wird,

- Oder zuletzt, dass man in seiner letzten Stunde nicht allein sein möge.

Auf diese Weise birgt jede dieser genannten Hoffnungen ein Stück Lebensqualität in sich."(S. 104/105) Solange Behandlung dazu angetan ist, Hoffnung aufrechtzuerhalten, solange werden auch belastende Behandlungsauswirkungen ertragen.

Innerhalb des Prozessmodells von Krankheit haben v. Kerekjarto et al. (1989, S.18-29) versucht einen konzeptuellen Beitrag zu leisten. LQ ist nach ihrer Auffassung das Ergebnis von Anpassungsprozessen in Interaktion mit den jeweiligen Lebensumständen. Daraus ergibt sich zwangsläufig, dass es sich um ein dynamisches, kein statisches Konzept handelt. Ihre Divergenzhypothese sagt aus, dass die objektiven und subjektiven Indikatoren der LQ für sich allein genommen nur einen begrenzten Zugang zur Ermittlung von LQ ermöglichen. Objektive Einschränkungen, Behinderungen etc. führen nicht zwangsläufig zu einer reduzierten LQ. Erst die Betrachtung der Art und Weise, wie Veränderungen subjektiv bewertet werden, erweitert diesen Zugang. Ergänzend formulieren sie die Prozesshypothese: Die Bewertung von LQ ist ein ständiger Anpassungsprozess, der Veränderungen unterworfen ist. Wie dieser Anpassungsprozess gemeistert wird, hängt u.a. ab von der Persönlichkeitsstruktur, früheren Erfahrungen und sozialer Unterstützung. Alle bisherigen Modelle beschränken sich auf Beschreibungen. Auch diesem Modell mangelt es an konkreten Vorgaben zur Bestim-

\footnotetext{
${ }^{17}$ Siehe auch Faller (1998), der die Annahmen der Variabilität mit den empirischen Befunden kontrastiert und diskutiert.
} 
mung der Beziehungen der Variablen untereinander und bei der Gestaltung einer empirischen Überprüfung.

Die Problematik des Kontruktes LQ wird bedingt durch den Zwang, ergänzend immer weitere psychologische Konstrukte zur Erklärung heranzuziehen, die ihrerseits genauso unzureichend definiert sind. Nimmt man die möglichen Konstruktionselemente ernst, ergeben sich eine Unmenge von möglichen Variabeln mit einer noch größeren Menge von möglichen Beziehungen untereinander. Das Ziel eines Konstruktes, empirische Daten zu ordnen und ihnen Bedeutung zu verleihen, wird so kaum erreichbar werden. Während Modell 1 sich auf die Bestimmung der Dimensionen/Bereiche konzentriert, liegt das Augenmerk im Modell 2 auf der Identifizierung der intervenierenden Variablen (Konstrukte). In diesem Modell sind die Komplexität und damit auch die Schwierigkeit der Bestimmung auf das intervenierende Konstrukt verschoben. In der Forschungspraxis vermischen sich beide Modelle in der Weise, dass für die Operationalisierung der Erhebungsinstrumente Modell 1 herangezogen wird, zur Erklärung der Ergebnisse allerdings auf Modell 2 rekurriert wird.

Zusammenfassend die graphische Darstellung der beiden Modelle:

Abb. 5: Modell 1

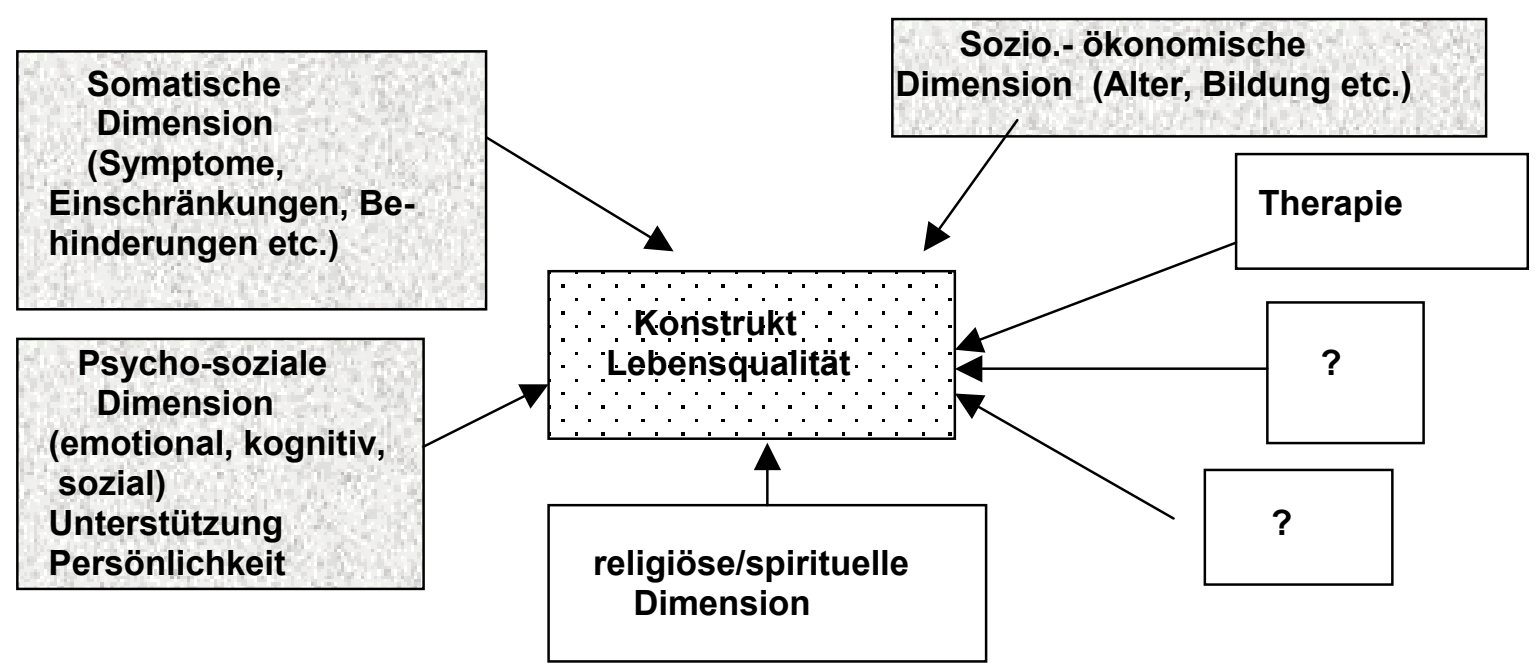

Abb. 6: Modell 2 (einfachste Variante ohne Interaktionsprozesse)

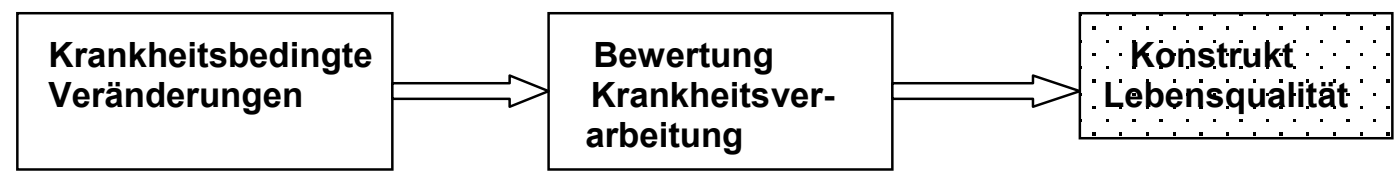

Pukrop (1997) charakterisiert das vorherrschende Modell als nominal-operational. Nominal bezieht sich auf die deskriptiv inhaltliche Festlegung relevanter Bereiche der LQ, 
operational bezieht sich auf die Bewertungsprozesse der $L Q$, bei denen interne und externe normative Standards eine Rolle spielen. Den externen und internen Normen fügt Bullinger (1991) noch eine klinische Norm für den medizinischen Bereich hinzu. Diese Charakterisierung bezieht sich auf beide Modelle. Neben diesen wichtigsten Modellen gibt es ein weiteres Modell aus der psychologischen Forschung, das sich in der onkologisch psychometrischen Forschung nicht durchsetzen konnte, dessen Einfluss aber implizit mitschwingt. Das Modell geht auf Bradburn zurück und wird von Baltrusch \& Waltz (1987) als Zwei-Faktoren-Modell der LQ bezeichnet. LQ wird hier gedacht als psychische Balance zwischen negativen und positiven Affekten. Individuelle und sozioökonomische Faktoren können sowohl gratifizierend als auch belastend erlebt werden. Zentrale Variablen in diesem Modell sind Selbstachtung (Selbstwert) und Depression. Durch die Krankheit wird die personale Integrität (Kohärenz) gestört und es kann zu psychosozialer Morbidität kommen. Beim Prozess der psychosozialen Gesundung wird persönlichen und sozialen Ressourcen (soziale Unterstützung) eine wichtige Funktion zugeschrieben.

Die Entwicklung in der Onkologie spiegelt die Diskussion wider, die auch außerhalb der medizinischen LQ-Forschung stattgefunden hat.

1. Die Frage nach der Urteilsinstanz: Selbstbeurteilung vs. Fremdbeurteilung

2. Die Frage danach, ob eine allgemeine Beurteilung besser ist als bereichsspezifische Werte: globale vs. spezifische Beurteilung

3. Die Frage nach geeigneten Indikatoren: subjektiv vs. objektiv (intersubjektiv)

4. Die Frage, wie die erhobenen Daten dargestellt werden: Summenwert vs. Profil

5. Die Frage nach der Art, in der die Items formuliert werden: funktional vs subjektiv

Bezieht man die Forschung zur LQ außerhalb der Medizin in sein Blickfeld mit ein, so lassen sich Ergebnisse finden, die sowohl Ähnlichkeiten als auch Unterschiede mit denen in der onkologischen Forschung aufweisen und die mit Rückgriff auf verschiedene Theorietraditionen erklärt wurden:

- Motivationstheorien

- Kompetenztheorien (Soziale Lerntheorien)

- Persönlichkeitstheorien

Auf die nähere Erläuterung der einzelnen Theorien soll hier verzichtet werden. Die ausführliche Darstellung und der Nachweis der empirischen Ergebnisse findet sich bei Pukrop (ebenda, Kapitel 8). 
Die Unterschiede und Gemeinsamkeiten der Ergebnisse zwischen Wohlfahrtsforschung und onkologischer LQ-Forschung werden im nächsten Abschnitt aufgezeigt.

\subsection{Vergleich von LQ-Ergebnissen der Wohlfahrtsforschung mit denen der onkologischen Forschung}

Auf die Ergebnisse kann hier nur in aller Kürze verwiesen werden.

\section{Verallgemeinerbare Ergebnisse aus der Wohlfahrtsforschung}

Folgende Ergebnisse aus der Wohlfahrtsforschung lassen sich, mit gewissen Einschränkungen, verallgemeinern:

- Demographische und objektive Faktoren (Alter, Familienstand, sozioökonomischer Status, Wohnort, mit Einschränkungen auch der objektive Gesundheitszustand) haben kaum Auswirkungen auf die LQ eine Ausnahme findet sich für die Stichprobe mit folgender Charakterisierung: weiblich - alt - arm - niedriger Bildungsstand - verwitwet. Hier fällt die Beurteilung der LQ negativer aus.

- Zwei Drittel der untersuchten Personen sind - weitgehend stichproben- unabhängig - mit ihrer LQ zufrieden. Unzufriedenheit wird mehr mit der öffentlichen Sphäre in Zusammenhang gebracht, während die meisten angeben, mit ihrer Privatsphäre zufrieden zu sein.

- Ob die Personen tatsächlich zufrieden sind oder aber nur Zufriedenheit äußern, ist nicht zu entschlüsseln. Mithilfe von Adaptations- und Dissonanztheorien wurde erklärt, wie diese weitgehend konsistenten Ergebnisse zustande kommen.

- Die Vorhersage der LQ kann über bekannte Persönlichkeitsmerkmale relativ treffsicher erfolgen (Trait bzw. top down Modelle). Zwei Varianten lassen sich finden:

- das Zufriedenheitsparadoxon (trotz schlechter objektiver Bedingungen zufrieden)

- das Unzufriedenheitsdilemma (trotz guter Bedingungen unzufrieden)

$\mathrm{Zu}$ den vorhergenannten Befunden gibt es einzelne, die von diesen generellen abweichen. Trotzdem kann von einer deutlichen Tendenz gesprochen werden.

Versucht man die LQ-Ergebnisse aus der Onkologie zu verallgemeinern, so zeigen sich andere Trends. ${ }^{18}$

\footnotetext{
${ }^{18}$ Diese Angaben beziehen sich auf zahlreiche onkologische Studien. Auf den Einzelnachweis wird hier verzichtet, die Argumente können aber z. B. für den Bereich der Behandlung mit HDC anhand der tabellarischen Studienliste nachvollzogen werden. Weitere Nachweise finden sich im Kapitel über die Verwendung empirischer LQ-Ergebnisse in der Onkologie.
} 
Verallgemeinerbare Ergebnisse aus der onkologischen Forschung

Folgende Trends aus der onkologischen LQ-Forschung lassen sich verallgemeinern:

- Werden funktionale Maße miteinbezogen, so zeigt sich durchaus eine Abhängigkeit vom objektiven Zustand (Krankheitszustand).

- Bei der Beurteilung der LQ scheinen Krankheitsereignisse (Diagnose, Rezidiv, Progress) kurzfristig Einfluss auszuüben.

- Gravierende Therapien und ihre Nebenwirkungen spiegeln sich in Funktionsund Symptomwerten.

- Aber - bei chronischen Verläufen wird der Zusammenhang zwischen Symptomen/Beschwerden/Einschränkungen und LQ geringer.

- Der Zusammenhang zwischen subjektiven und objektiven (und intersubjektiv überprüfbaren) Indikatoren der LQ bleibt auch für die Population der Krebskranken gering.

- Widersprüchlich bleiben die Ergebnisse im Vergleich mit gesunden Populationen: lange Zeit wurde der Stellungnahme, dass Krebskranke ihre LQ nicht schlechter bzw. sogar besser beurteilen nicht widersprochen. Neuerdings mehren sich allerdings Studien, die den umgekehrten Trend belegen wollen. Deutlich ist, dass Gesunde und Kranke zwar gleichermaßen Gesundheit als wichtigsten Bereich an die erste Stelle setzen, die Zusammenhänge aber trotzdem unterschiedlich sind. ${ }^{19}$ Lässt man Einzelergebnisse außer Acht so gilt noch die vor langer Zeit getroffene Aussage von de Haes \& Knippenberg (1985): „It has been generally accepted on the basis of intuition and empirical study that cancer and cancer treatment cause a major disruption of life emotionally, socially and physically. This assumption is confirmed in descriptive and comparative studies to some extent. However, the results often do not point in the expected direction." (S. 811).

Wegen dieser Schwierigkeiten nehmen einzelne Autoren kurvilineare

${ }^{19}$ Zum Unterschied in der Beurteilung der Wichtigkeit von Gesundheit bei kranken und gesunden Populationen führt Pukrop (ebenda, S. 37) aus: Verschiedene Autoren haben die Reihenfolge der Kategorien untersucht. Weltweit wird von ca. 80\% der Befragten Gesundheit als wichtigster Lebensbereich genannt. Es besteht aber ein offensichtlicher Widerspruch zwischen der empfundenen Wichtigkeit und der statistisch nachweisbaren Bedeutung: „Die Gesundheit wird beispielsweise bei nicht vorhandenen Einschränkungen eher als selbstverständliches Gut betrachtet, das daher nur geringen statistischen Einfluss auf die allgemeine LQ im Sinne des subjektiven Wohlbefindens ausübt. Beim Lebensstandard verhält es sich dagegen genau anders herum: Eine zu offensichtliche materialistische Werthaltung ist sozial unerwünscht und geht daher nicht in ein direktes Wichtigkeitsurteil mit ein, spielt objektiv (im Sinne eines höheren Regressionskoeffizienten) jedoch eine wesentlich größere Rolle." 
Zusammenhänge zwischen Erkrankungsstadien und LQ an.

Verallgemeinerbare empirische Belege sind hier aber nicht zu finden.

- Es bleibt festzuhalten, dass auch für die Stichproben mit Krebskranken die Zwei-Drittel-Regel gilt: meistens findet sich eine schiefe Verteilung zugunsten höherer LQ-Werte.

Zum Abschluss des konzeptuellen Teils soll auf kritische Anmerkungen hingewiesen werden.

\subsection{Kritische Anmerkungen}

Das Spektrum der kritischen Argumente bzgl. der Messbarkeit und Messung reicht weit und bezieht sich auf definitorische, inhaltliche und methodische Aspekte. Außerdem wird die Verwendung von LQ-Ergebnissen kritisch gesehen. Die Kritik an der LQForschung soll kurz dargestellt werden, ausgehend von theoretisch übergeordneten Gesichtspunkten bis hin zu technisch-instrumentellen Problemen und der Anwendungspraxis.

Am weitreichendsten sind Kritiken, die eine quantifizierende Erhebung von LQ generell ablehnen. Hier spielen unterschiedliche Menschenbilder und wissenschaftliche Methoden - naturwissenschaftlich-quantifizierbar vs anthropologisch-qualifizierbar - eine zentrale Rolle. In diesen Kontext gehört auch die Frage, ob LQ überhaupt nomothetisch erfasst werden kann oder ob lediglich eine idiographische Erfassung möglich ist. Autoren wie Schara (1990) lehnen eine vermeintlich objektive, erst recht eine quantitative Erfassung in Gänze ab. Ihrer Ansicht nach kann nur durch einen hermeneutischen Zugang $L Q$ für das jeweilige Individuum erschlossen werden.

Andere Autoren (Viehues 1990; Raspe 1990) wählen einen anderen Ausgangspunkt für ihre Kritik. Im Zentrum steht dabei die Auffassung von LQ. Nur dann, wenn Leid als rein negatives Phänomen, als sinnloser, möglicherweise kompensierbarer Defekt der menschlichen Existenz definiert wird, ist ein quantifizierbarer Begriff von LQ möglich. Wenn allerdings auch eine positive Sinngebung von Leid akzeptiert wird, ist ein solcher Begriff ausgeschlossen (Viehues ebenda, S.22). Raspe lehnt ebenfalls eine negative Definition von $L Q$ ab, die sich an der An- oder Abwesenheit von Beschwerden, Nebenwirkungen, seelischen Problemen usw. orientiert. Er argumentiert gegen die reduktionistische Verwendung von LQ in der Onkologie. Der fragmentierten, gesundheitsbezogenen LQ (physische LQ) stellt er die Forderung nach einer „erlebensnahen“ Auffassung, einer „Gestalt“ von Leben und seiner Qualität gegenüber. Diese ganzheitliche Betrachtung vermag auch Gegensätzliches und Disparates zu integrieren. Raspe plädiert dafür, den „seichten“ Begriff der LQ aus der wissenschaftlichen Diskussion zu verbannen. Auf den zwangsläufig normativen Gehalt des Begriffes LQ 
wurde bereits verwiesen. Raspe gehört auch hier zu den schärfsten Kritikern: LQ ist für inn ein Wertebegriff. Urteile über LQ beziehen sich demnach auf unterschiedliche Systemebenen, deren Bestimmung schwierig ist. Prekär ist somit auch der implizierte und unreflektierte Werteaspekt, der alles, was vom Ideal einer potenten vitalen Existenzweise abweicht, in pubertärer Anthropologie mit Last- und Unwert identifiziert (ebenda S. 31/32).

Andere Autoren beziehen sich auf eine untergeordnete Ebene von Kritik und stellen die methodischen Probleme in den Vordergrund.

Eine Theorie der LQ gibt es nicht und vermutlich wird es sie in naher Zukunft auch nicht geben. Die „bescheidenen“ konzeptuellen Ansätze haben bislang die empirischen Befunde nicht plausibel einzuordnen vermocht. Das Konstrukt LQ muss selbst noch validiert werden, damit man überhaupt weiß, was man damit messen kann. Die Autoren, die LQ für messbar halten, widersprechen dieser Auffassung. Sie gehen davon aus, dass es möglich, ist von LQ als einem einheitlichen Phänomen auszugehen. Begründet wird diese Auffassung mit statistischen Argumenten: in empirischen Untersuchungen findet sich bei faktorenanalytischen Untersuchungen meist eine starke Hauptkomponente, die für einen gemeinsamen Faktor bei unterschiedlichen Dimensionen spricht. Nach Pukrop (1997) haben zahlreiche Untersuchungen (außerhalb des onkologischen Bereichs) einen Primärfaktor ergeben, der ca. 60\% der Varianz aufklärt. Der Autor folgert, dass „[...] trotz eines zum Teil sehr uneinheitlichen Konzeptes doch alle ein ähnliches Konstrukt erfassen. Es muss allerdings deutlich daraufhin gewiesen werden, dass es sich bei den referierten Ergebnissen im wesentlichen nur um subjektive Wohlbefindensmaße und keine objektiven LQ-Parameter handelt." (S.31)

Auch in den onkologischen Studien gibt es einen Primärfaktor (die physische Funktion, subjektiv beurteilt). Eine Varianzaufklärung durch einen Primärfaktor von $60 \%$ findet sich aber selten. Auch auf dieser Ebene formuliert Raspe die entschiedenste Kritik: Leben erscheint in der LQ Forschung als eine Ansammlung von Mosaiksteinchen, nicht als ganzes Bild. Erstaunlicherweise fehlen neben politischen und ökologischen immer religiöse Bezüge. Alle empirische Evidenz weist im Gegenteil darauf hin, dass LQ nicht als eine latente Variable aufgefasst werden kann. Das, was analytisch-methodisch getrennt worden ist, wächst in der statistischen Auswertung nicht von selbst zusammen. Er warnt vor einem naiven LQ-Verständnis analog zu Lebensmittel-, Wasser- und Luft"Qualität“ (1990a).

Die Messung der LQ hat ein ganzes Jahrzehnt im Zentrum der Forschungsbemühungen gestanden. In Bezug auf Messinstrumente sind viele ältere Kritiken, lässt man die mangelnde theoretische Fundierung außer Acht, heute hinfällig. Bei den heute gebräuchlichen Messinstrumenten kann davon ausgegangen werden, dass diese Instru- 
mente im Rahmen ihres jeweiligen Konzeptes mehr oder weniger weitgehend valide, reliabel und sensitiv sind. Generell ergeben sich die gleichen methodischen Probleme wie bei den Messungen anderer subjektiver Variablen: Die Antworten sind abhängig von der Form, der Präsentation und dem Kontext der Datenerhebung. Bei geschlossenen Fragen muss eine Entscheidung über die Art der Skalierung getroffen werden. Bei kategorialen Skalen müssen die Untersuchungen über Auswirkungen von Anzahl und Formulierung der Kategorien beachtet werden, um Antworttendenzen und mangelnde Differenzierung zu verringern.

Feld (1995) stellt kritische Überlegungen an, die sich aber mehr auf pragmatische Aspekte beziehen: fehlender "gold standard“; Unsicherheit, wie gemessen und analysiert werden soll; wechselnde Compliance zwischen Patienten und Untersuchern, schädliche Auswirkungen auf die Beteiligung an klinischen Versuchen; Erfordernis von Ressourcen für die Messung, die anderweitig genutzt werden könnten. Trotzdem kommt er zu dem Schluss, dass LQ-Messungen dort besonders wichtig sind, wo die erwarteten Überlebenszeiteffekte zwischen Therapien gering sind. Weitere methodische Probleme entstehen durch den ungeklärten Einfluss von „response shift“. Gerade bei Längsschnittuntersuchungen wird ein erheblicher Einfluss angenommen. Soziale Erwünschtheit könnte durch ergänzende Instrumente kontrolliert werden. ${ }^{20}$ Sehr unterschiedlich sind die Angaben über fehlende Daten. In der Münchener Qualitätssicherungsstudie äußerten sich Patienten ausgesprochen positiv über das Interesse an ihrer Befindlichkeit, das über medizinische Daten hinausging. Generell lässt sich verallgemeinern, dass Patienten bereitwillig über ihre Sicht der Behandlungssituation berichten. Interaktive Fragebogenerhebungen und Interviews werden gerne wahr genommen. Moynihan (1998), der sich in seiner Arbeit mit dem Problem der fehlenden Daten in LQ-Studien beschäftigt, verweist ebenfalls darauf, dass "Interestingly studies show that in general, patients are pleased to be induced in QL studies." (S 10) Sigle \& Porzsolt (1996) berichten über eine außergewöhnlich gute Compliance $(98,9 \%)$ bei der Erprobung der elektronischen Version des QLQ-C30. Anders sieht es allerdings aus bei postalischen und telefonischen Untersuchungen. Maughan et al. (2002) berichten über eine große Studie, die drei Chemotherapieregimes vergleicht (905 Patienten mit Darmkrebs). Weniger als die Hälfte der überlebenden Patienten füllt die Fragebögen (QLQ-C30 und HADS) aus. Die Autoren machen dafür eine schlechte Organisation verantwortlich. Wenn das so ist, lässt sich zumindest daraus ableiten, dass von den Untersuchern die LQ-Daten im Vergleich mit den medizinischen Daten vernachlässigt werden. Einen Bias in Abhängigkeit vom Patiententyp können die Autoren nicht ausmachen. Dieses Ergebnis bestä-

20 „Soziale Erwünschtheit“ spielt im Zusammenhang mit LQ-Forschung nach Pukrop (1997) eine untergeordnete Rolle. 
tigt die von Fayers et al. (1997) durchgeführte Literatursichtung: auch er kam zu dem Ergebnis, dass nur die Hälfte der Patienten die Fragebögen zurück schickt. Dass die Ergebnisse in Bezug auf Compliance so unterschiedlich ausfallen, liegt mit Sicherheit weniger an den Patienten als am Erhebungsinstrumentarium und der Art und Weise der Datenerhebung. Während englische und amerikanische Autoren dies für ein ernstzunehmendes Problem halten, berichten deutsche Autoren über eine ausgesprochen gute Compliance bei der Datenerhebung.

Wie soll mit den erhobenen Daten verfahren werden? Die bloße Deskription reicht meist nicht aus, vor allem dann nicht, wenn unerwartete Ergebnisse auftauchen. Auf einzelne Beispiele wird im nächsten Kapitel eingegangen. Aus der mangelnden theoretischen Fundierung ergeben sich gravierende Interpretationsprobleme. Absolute Werteangaben sind sinnlos. Interpretieren lassen sich nur Vergleichsdaten, entweder im Längsschnitt (intraindividueller Vergleich) oder im Gruppenvergleich (interindividueller Vergleich) oder beides kombiniert. Es ist nicht möglich, abzulesen, was ein Allgemeiner LQ-Wert inhaltlich bedeutet. Aus den genannten Gründen sind Vergleiche mit gesunden Populationen sehr fragwürdig.

Dem Aspekt der klinischen Relevanz ist ein eigenes Kapitel gewidmet. Pukrop (1997 S. 207) teilt in unterschiedliche Zweckkategorien ein. Er unterscheidet diskriminative, prädiktive und evaluative Zwecke. An dieser Stelle soll, in Erweiterung der Zweckkategorien von Pukrop, noch auf die ideologische und legitimatorische Kategorie hingewiesen werden. ${ }^{21}$ Die beiden letztgenannten werden, verständlicherweise, nicht explizit genannt, spielen aber eine wichtige Rolle. Als Beleg dafür ließen sich unzählige Werbebroschüren aber auch wissenschaftliche Broschüren, die der Produktwerbung dienen und die häufig auf Kongressen und Tagungen verteilt werden, anführen. Aber auch in wissenschaftlichen Publikationen findet sich das Ausweichen auf LQ als Surrogatkriterium. Dies scheint vor allem dann der Fall zu sein, wenn sich bei der Effizienzprüfung von unterschiedlichen Therapien keine oder nur geringfügige (z. B. ein Monat) Überlebenszeiteffekte ergeben. Wenn die allgemeine LQ durch belastende Therapien nicht so

${ }^{21}$ Der Begriff LQ spielt in vier Bereichen der Medizinethik eine wichtige Rolle:

1. Behandlungsverzicht/Abbruch bei schwerstbehinderten Neugeborenen

2. Bei der Diskussion um „Sterbehilfe" bei Schwerkranken (Schmerzen)

3. Bei der onkologischen Therapie (Behandlungsverzicht, „Sterbehilfe“, Patientenverfügungen)

4. Gesundheitspolitische Allokation

Sass (1990) warnt vor ideologischen, kulturellen oder subkulturellen Scheinkriterien in der LQDiskussion. Er fordert, dass sich alle in der Medizin Handelnden ihrer Kriterien der Bewertung bewusst werden. Akzeptabel sind nur Argumentationen die sich an der „Unantastbarkeit des Lebens“, des "rationalen menschlichen Vermögens“ und dem „interpersonellen Kommunikationsvermögen" orientieren.

Meines Wissens gibt es keine Studien zur LQ in der Onkologie die direkt für sich reklamieren Entscheidungshilfe bei den unter 3. genannten Themen zu bieten. 
stark beeinträchtigt wurde wie erwartet, darf das alleine nicht als Argument für eine Anwendung bei ansonsten nicht belegter Wirksamkeit herangezogen werden.

So wichtig die Unterscheidung zwischen Wirksamkeit und Nutzen auch sein mag, so bleibt doch eine eindeutige Hierarchie der Argumente bestehen. Die meisten Patienten lassen keinen Zweifel daran, dass die Überlebenszeit dass primäre Argument ist. Diese Argumentation wird noch von einer anderen Forschungsrichtung gestützt: Jacoby et al. (1999) untersuchen die Bedeutung des „informed consent" bei knochenmarkstransplantierten Patienten. Ausgangspunkt ihrer Untersuchung ist die Frage danach, ob in der Situation einer lebensbedrohlichen Erkrankung mit nur einer potentiell kurativen Alternative, die Selbstbestimmung des Patienten nur eine Fiktion ist. Ähnlich wie in der von ihnen referierten Literatur, entscheiden sich die Patienten ihrer Studie auf der Basis von Erfolgserwartungen und im Vertrauen auf den ärztlichen Rat bezüglich der Therapie. Die Autoren fassen zusammen: „Research studies on informed consent for experimental cancer treatments in general, show that patients tend not to recall information on risks and complications. The studies of BMT patients in particular, conclude that patients do not give much weight to such information in their decision making process. The survival motive, the disease are the most salient factors for participating in the treatment." (S. 711)

Gerade wegen der Interpretationsunschärfen können LQ-Daten missbräuchlich verwendet und für unterschiedliche Interessen instrumentalisiert werden.

Im nächsten Kapitel werden die Ziele und Anwendungsbereiche der LQ-Forschung in der Onkologie näher beleuchtet. 


\section{Ziele und klinische Relevanz der empirischen LQ-Forschung}

Ergebnisse der LQ-Forschung können den unterschiedlichsten Zwecken dienen. Neben der übergeordneten Zweckdefinition in evaluativ, prädiktiv und diskriminativ, sollen hier spezifischere Ziele und ihre Umsetzung erläutert werden. Von den zahlreichen Zielen, die in ganzen Katalogen zusammengestellt wurden (siehe u.a. Feld 1995), sind die Folgenden für die klinische Praxis relevant. Interessant ist vor allem, wie sich die Zielformulierung umsetzen lässt und inwieweit die Ergebnisse tatsächlich genutzt werden können. Die folgenden Fragestellungen lassen sich den oben genannten Zweckdefinitionen zuordnen:

Diskriminativer Zweck:

1. $L Q$ ist das einzige Zielkriterium im Zusammenhang mit palliativmedizinischer Versorgung in der letzten Lebensphase. Die Ergebnisse dienen ausschließlich der individuellen Patientenversorgung.

2. Die Datenerhebung dient der ausführlichen Beschreibung von Therapieauswirkungen, Krankheitsverläufen, Therapieerfolgen, Rehabilitationsverläufen usw. Auch hier ist das Hauptziel die Verbesserung der Patientenversorgung und die Entwicklung von Interventionsprogrammen.

Prädiktiver Zweck:

3. LQ dient als Prognosefaktor hinsichtlich von Morbidität und Mortalität. Evaluativer Zweck:

4. LQ dient als Kriteriumsvariable der Evaluation und Optimierung von therapeutischen Maßnahmen.

5. Die LQ-Daten werden für Kosten-Nutzen-Analysen (CUA) verwendet. ${ }^{22}$

\subsection{LQ als Zielkriterium in der Palliativmedizin}

LQ ist das wesentliche, einzige und unbestrittene Zielkriterium in der letzten Lebensphase, auch unter Inkaufnahme einer Verkürzung der Lebenszeit, z.B. durch Morphingaben zur Schmerzreduktion. „Der Mittelpunkt der Betreuung ist der Patient und seine Familie. LQ steht im Zentrum der Betreuung, nicht mehr Quantität. Symptomkontrolle, Kommunikation, Rehabilitation und Begleitung in der Sterbephase sind die Schwerpunkte in der Palliativbetreuung" (Bausewein \& Hartenstein, 2000 S. 334). LQ in der palliativen Phase steht nicht nur am Ausgangspunkt der onkologischen LQ Forschung sondern hat auch, bezogen auf die Anzahl der Betroffenen, höchste Priorität. In Deutschland erkranken jedes Jahr 340000 Menschen an Krebs, 45\% davon werden kurativ behandelt. Von diesen $45 \%$ erleiden 15\% ein Rezidiv. 55\% werden von der Diagnosestellung an palliativ behandelt

22 Die Kosten für eine HDC beim metastasierten Mammakarzinom sind um den Faktor 50-75 höher als bei konventioneller Chemotherapie (Richter, 1997) 
(Bausewein \& Hartenstein, 2000). Aulbert (1993) grenzt die Frage nach der LQ in der palliativen Situation auf folgende Fragestellungen ein:

- Wie einschneidend darf eine Therapie sein, um was zu erreichen?

- Ist doch noch eine Heilung zu erzwingen?

- Um welchen Preis kann Lebensverlängerung erreicht werden?

- Wie kann ich möglichst effektiv Schmerzen und Beschwerden lindern?

- Muß ich überhaupt therapieren?

Aulbert (ebenda) formuliert als Ziel: „Wir wollen nicht dem Leben (um jeden Preis) Zeit hinzufügen, sondern der verbleibenden Zeit Leben (Lebensqualität) geben." (S.6/7) Argumente für mehr LQ haben in der Palliativmedizin einen herausragenden Stellenwert. Die Forderung, mehr palliativmedizinische Lehrstühle und Abteilungen an den Universitätskliniken einzurichten, findet, im Vergleich zu anderen Ländern mit zwanzig Jahren Verspätung, in Deutschland Gehör und damit auch die Forderung nach palliativer LQForschung.

Akzeptiert man die Fragestellungen von Aulbert als die zentralen der Palliativmedizin, muss beantwortet werden, auf welche Weise nach einer Antwort gesucht werden soll. Die Schwierigkeiten beginnen mit dem Begriff der Palliativmedizin. Dem Lehrbuch der Palliativmedizin (Radbruch \& Zech, 1990 S.2) sind die folgenden Modelle entnommen, die aktuelle begriffliche Vorstellungen kontrastieren mit dem, was sich Pallitativmediziner wünschen:

\begin{tabular}{|l|l|}
\hline Kurative Therapie & $\begin{array}{l}\text { Palliative } \\
\text { Therapie }\end{array}$ \\
\hline
\end{tabular}

Diagnose

Therapieplanung bei unheilbaren

Erkrankungen: IST-Zustand („Papierkorbalternative“23)

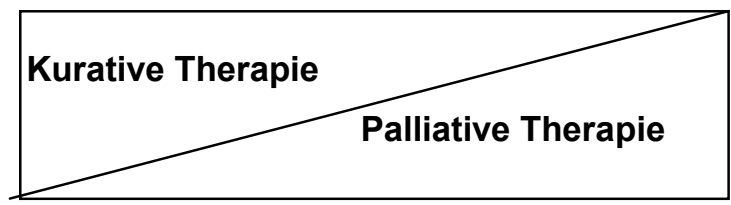

Tod

Diagnose

Tod

Therapieplanung bei unheilbaren

Erkrankungen: SOLL-Zustand

Die Wortbedeutung von palliativer Medizin ist eindeutig festgelegt: eine kurative Behandlung ist nicht (mehr) möglich, die Linderung der Beschwerden steht im Vordergrund. Es bedarf eines Verständigungsprozesses zwischen Arzt und Patient über die Zieldefinition medizinischen Handelns oder deren Veränderung. Auch aufgeklärte, gut informierte Patienten definieren in der Regel die palliative Phase anders als Palliativmediziner. Für Patienten ist es die Phase, die der Sterbephase unmittelbar voraus-

\footnotetext{
${ }^{23}$ Papierkorbalternative meint, dass bis zu einem bestimmten Zeitpunkt alles für eine Heilung getan wird und dann wird „nur noch“ symptomatisch behandelt. Leider ist von manchen Ärzten immer noch zu hören, dass „hier jetzt nichts mehr getan werden kann“.
} 
geht. Auch Patienten, die kognitiv alle Begründungen und Definitionen erfasst haben, können in diesem Stadium Hoffnung auf Heilung aufrecht erhalten, auch wenn das „irrational“ (?) erscheinen mag: «Vielleicht gehört man zu denjenigen, denen das Wunder einer Spontanheilung zuteil wird» - «vielleicht überlebt man lange genug, bis die medizinische Forschung den Schlüssel zur Heilung gefunden hat». (Äußerungen von Patienten, die in dieser oder ähnlicher Formulierung häufig zu hören sind).

Nach der konservativen ärztlichen Definition beginnt die palliative Phase, wenn aufgrund statistischer Evidenz keine Heilung mehr möglich ist. Diese Phase kann in Abhängigkeit vom Krankheitsbild unterschiedlich lang sein und völlig unterschiedlich verlaufen. Für einen Patienten mit Fernmetastasen, der vielleicht (noch) keine Beschwerden hat und in seinen physischen Funktionen nicht beeinträchtigt ist, kann sein als palliativ definierter Zustand nicht erfahrbar werden.

Auch wenn die Zustandsbeschreibung der oben genannten Autoren mehr als ein Jahrzehnt zurückliegt, scheint sich die Behandlungspraxis, zumindest außerhalb spezialisierter Palliativeinrichtungen, immer noch am „Papierkorbmodell“ zu orientieren. Orientiert an der medizinischen Praxis müsste deshalb, auch für den Bereich der Forschung, die palliative Phase noch in eine todesnahe Phase und in eine Phase, in der nur noch ganz bedingt kurative Ziele verfolgt werden können, unterteilt werden.

Die von Aulbert aufgeworfenen Fragen lassen sich auf zwei Kernfragen verdichten:

Die Frage nach dem „Preis“ für Lebensverlängerung, und die Frage danach, wie Beschwerden am wirksamsten gelindert werden können. Die erste Frage kann nicht mit Bezug auf Ergebnisse der psychometrischen LQ-Forschung beantwortet werden. Es bedarf eines intimen Dialoges zwischen Arzt und Patient, in dem vor allem die Bedeutung geplanter Maßnahmen für den Patienten eruiert werden müssen. Ohne biographische Kenntnisse über den Patienten und ohne eine tragende, „ummantelnde“ Beziehung ist dieser Dialog nicht möglich. Die zweite Frage kann mit Bezug auf Ergebnisse aus der psychometrischen LQ-Forschung annähernd beantwortet werden, sofern akzeptiert wird, dass die Erforschung von Nebenwirkungen einen wichtigen Bereich der LQ-Forschung ausmacht.

Im Folgenden soll die Praxis der LQ-Forschung im palliativen Bereich durch Beispiele aus der Forschung verdeutlicht werden. Die standardisierte Datenerhebung mithilfe psychometrisch fundierter Instrumente der Selbstbeurteilung ist in diesem Feld allerdings ausgesprochen selten und wird umso problematischer und ethisch fragwürdiger, je mehr sich ein Patient dem Sterben nähert. Häufiger erfolgt die Datenerhebung als Fremdbeurteilung durch Ärzte/Pflegepersonal/Angehörige oder aber durch das direkte, unstrukturierte Gespräch mit dem Patienten. 
Aus dem englischsprachigen Raum stehen zwei psychometrisch überprüfte Erhebungsinstrumente (Spitzer-Index und Hospice Quality of Life Index) zur Verfügung, die ausdrücklich für diesen Bereich entwickelt wurden. Beides sind Fremdbeurteilungsinstrumente, mit denen der Arzt die Datenerhebung durchführt.

Osoba (2000) kritisiert in seinem Review, dass Studien zur LQ supportiver Behandlung zu kurz kommen. Die wenigen Studien der palliativen Behandlungssituation beziehen sich meist auf Aspekte der Symptomkontrolle (Anorexie und Gewichtsverlust, Übelkeit und Erbrechen, Anämie, Fatigue und Schmerzen) und nicht auf das breitere Konzept der HRQL. Nach seiner Aussage ist es notwendig, wissenschaftlich zu belegen, dass Symptomkontrolle Auswirkungen hat auf die vom Patienten selbst eingeschätzte LQ. Er zitiert Beispiele, die diesen Zusammenhang nicht belegen können: In einer Doppelblindstudie wurden 206 Patienten mit Megestrol oder Placebo zur Verbesserung der Tumor bedingten Anorexie behandelt. Obwohl in der Megestrolgruppe eine signifikante Gewichtszunahme registriert wurde, zeigte sich in den Skalen des QLQ-C30 keine Verbesserung für die Megestrolgruppe sondern die Werte verschlechterten sich (Simons et al. 1996; zitiert nach Osoba ebenda). In der zweiten Studie wurden 243 Patienten mit ausgedehntem Lungenkarzinom nach Chemo- und Radiotherapie mit Plazebo oder Megestrol behandelt. Obwohl Appetit und Gewicht signifikant besser und Übelkeit signifikant vermindert waren, zeigte sich kein Unterschied in der LQ. Osoba zitiert auch Studien, in denen ein Zusammenhang zwischen Symptomen und LQMessung nachgewiesen wird. Welche Schlussfolgerungen lassen sich daraus ziehen? Ähnlich wie in anderen Feldern der LQ-Forschung ist der Zusammenhang zwischen den objektiven Indikatoren (Symptomen) und der subjektiven LQ inkonsistent. Trotzdem wird die Symptomkontrolle vorrangiges Ziel bleiben. Aulbert (1990, S. 89) listen die Symptome auf, die anhand zahlreicher Patientenstichproben ermittelt wurden: Schmerzen (70,3\%) - Mundtrockenheit (67,5\%) - Anorexie (60,9\%) - Schwäche $(46,8 \%)$ - Verstopfung $(44,7 \%)$ - Luftnot $(42,3 \%)$ - Übelkeit $(36,3 \%)$ - Schlaflosigkeit $(34,2 \%)$ - Schwitzen $(25,3 \%)$ - Schluckbeschwerden $(23,2 \%)$ - urologische Symptome $(21,3 \%)$ - neuropsychiatrische Symptome $(19,8 \%)$ - Erbrechen $(18,5 \%)$ - dermatologische Symtpome $(16,3 \%)$ - Dyspepsie (11,3\%) - Diarrhö (7,6\%). Es bedarf keiner großen Phantasie, um sich vorzustellen, wie quälend diese Symptome sein können, auch wenn es keinen linearen Zusammenhang mit LQ-Werten gibt. Und kein ernstzunehmender Arzt würde auf die Idee kommen, bei Patienten, die deutlich machen, dass sie mit ihrer Lebensqualität zufrieden sind, die Symptomkontrolle zu vernachlässigen. Meier et al. (1998) untersuchten in einer Studie in den USA Gründe, die von Patienten angeführt werden, wenn sie ihre behandelnden Ärzte um Sterbehilfe bitten. Nach den Angaben der Ärzte, bitten 52\% der Patienten um Sterbehilfe wegen der Schmer- 
zen/Symtpome, $47 \%$ der Patienten weil ihnen der Sinn des Lebens („loss of meaning in life") verloren gegangen ist. In einer anderen Studie von Cameron et al. (2002) werden die Belastungen von pflegenden Angehörigen krebskranker Patienten untersucht. Diese Studie kommt ebenfalls zu dem Ergebnis, dass die Belastungen und der emotionale Stress der Angehörigen unmittelbar mit der Anzahl und der Schwere von Symptomen der Patienten zusammenhängen.

In einer kürzlich veröffentlichten Studie befassen sich Donnelly, Rybicki \& Walsh (2001) u. a. mit den methodischen Schwierigkeiten der LQ-Messung in der palliativen Phase bei fortgeschrittener Karzinomerkrankung. Sie vergleichen verschiedene Instrumente (FACT-G, QLI, Performanz Status, VAS und Kategoriale Skalen) an einer, auf die Mortalitätsstatistik bezogenen, repräsentativen Stichprobe in den USA. Teilgenommen haben 157 Patienten des „Cleveland Clinic Palliative Medicine Program“ von denen erwartet wurde, dass sie die nächsten vier Wochen überleben. Die Untersuchung umfasst einen Zeitraum von 4 Wochen. Weniger als die Hälfte der Patienten sind überhaupt in der Lage (oder gewillt? d. V.) an allen 3 Zeitpunkten die Fragebögen auszufüllen. Die Autoren halten, auch bei der Gruppe, die durchgängig die Fragebögen ausfüllt, die längeren Fragebögen (FACT-G und QLI) nicht für praktikabel. Die Daten der „übrig gebliebenen“ Patienten in ihrer umfangreichen Stichprobe zeigen keine Veränderung oder sogar eine leichte Verbesserung (nicht signifikant) der LQ: „The QOL measure reveals that, surprisingly, most patients with advanced disease had a neutral or positive outlook on their QOL, which was stable in patients who were able to complete the study during the study period despite progressive disease" (S.365) (Hervorhebung d. V.). Die Autoren schließen daraus, dass sich durch die Besonderheit der palliativen Stichprobe völlig unrealistische Verzerrungen in Richtung einer konstanten/verbesserten LQ ergeben, da die Patienten mit am weitesten fortgeschrittener Erkrankung oder die Patienten, die vor Studienende starben, unberücksichtigt blieben. Ob es sich um eine unrealistische Verzerrung handelt oder ob ganz andere Faktoren für dieses Ergebnis verantwortlich sind, geht nicht eindeutig aus der Studie hervor. Dennoch zieht der Autor aus dieser Studie methodische Schlussfolgerungen, ohne nachvollziehbar zu überprüfen, ob und ggf. welche inhaltlichen Probleme involviert sind. Die Hälfte der aufgeforderten Patienten, die sich geweigert haben, die Fragebogenbatterien auszufüllen, sind vielleicht gar nicht die am stärksten beeinträchtigten oder diejenigen, deren LQ besonders „schlecht“ ist. Vielleicht halten sie, angesichts ihrer Situation, nur die Fragestellungen für irrelevant oder absurd bzw. für eine Zumutung.

Problematisch bleibt in jedem Fall die Frage, inwieweit Schwerstkranken das Ausfüllen von Fragebögen zugemutet werden kann, vor allem dann, wenn die Datenerhebung 
nicht in einem schützenden Beziehungskontext stattfindet sondern sogar, wie nicht selten beschrieben, per Telefonbefragungen durchgeführt wird!

Obwohl LQ-Argumenten theoretisch ein hoher Stellenwert eingeräumt wird, spielen sie für Therapieentscheidungen in der Palliativsituation eine untergeordnete Rolle. Studien, die diesen Eindruck der Verfasserin stützen, werden von Detmar et al. (2002) referiert. Die Autoren kommen zu folgendem Ergebnis: „In the presence of tumor progression or serious toxicity, HRQL considerations played little or no role in treatment decisions. Furthermore, approximately $70 \%$ of patients without evidence of tumor progression or toxicity, but with seriously impaired HRQL, continued to receive their treatment as planned [...] HRQL considerations seem to play a relatively minor role in decisions regarding modification or discontinuation of palliative chemotherapy." (S. 1056)

Diesen Tatbestand erklären die Autoren auf folgende Weise:

1. Die Beendigung der aktiven Behandlung widerspricht dem vorherrschenden Ethos in der klinischen Medizin. Wenn Interventionen nicht mehr angemessen erscheinen, führen sie zu Gefühlen von Hilflosigkeit.

2. Die wenigen randomisierten Studien in der Palliativsituation, die aktive Behandlung mit der bestmöglichen supportiven Therapie vergleichen, führen zu widersprüchlichen Ergebnissen. Es ist eindeutig nicht so, dass sich alle Patienten, die nur supportiv behandelt werden, emotional besser fühlen, als die Patienten, die zusätzlich die Nebenwirkungen von Chemotherapien ertragen müssen.

3. Die Patienten selber akzeptieren die Einschränkungen der HRQL zugunsten einer minimalen Chance auf längeres Überleben.

\subsection{LQ zur Beschreibung von Therapieauswirkungen}

Die Beschreibung von LQ in Abhängigkeit von Krankheit und Therapie hat bislang den breitesten Raum eingenommen, es liegen unzählige Studien dazu vor. Alle möglichen Studientypen sind hier einzuordnen. Am häufigsten wurden Querschnittsstudien durchgeführt. Dabei stand die LQ in Abhängigkeit von unterschiedlichen Therapien und bei unterschiedlichen Patientengruppen im Mittelpunkt. Für den Bereich der HDC-Therapie findet sich eine tabellarische Auflistung der durchgeführten Studien auf S. 101ff. Durch Längsschnittstudien sollten aktuelle und spätere Nebenwirkungen und kritische Zeitpunkte im Behandlungs- und Rehabilitationsverlauf erfasst werden, um den sich daraus ergebenden Behandlungsbedürfnissen der Patienten gerecht zu werden. So verstandene LQ-Forschung war/ist eng verknüpft mit der Entwicklung von psycho-sozialen Unterstützungsbemühungen. Ziel ist neben dem Erkenntnisgewinn vor allem eine verbesserte Patientenversorgung. Neben den Hinweisen auf mögliche medizinische Ver- 
besserungen soll die Fokussierung auf psychosoziale Aspekte auch Hinweise auf notwendige Schwerpunkte bei der psychosozialen Versorgung begründen. Da es wegen der Fülle des Materials völlig unmöglich ist, die Ergebnisse auch nur annähernd wiederzugeben, sollen hier zwei Beispiele betrachtet werden:

- LQ und die Auswirkungen unterschiedlicher chirurgischer Behandlungen beim Mammakarzinom

- LQ zur Ermittlung des individuellen Interventionsbedarfs

\subsubsection{LQ als abhängige Variable von brusterhaltender versus radikaler Operation}

Die chirurgische Behandlung hat sich von der radikalen Mastektomie über die modifizierte Mastektomie bis zum heute angestrebten brusterhaltendem Vorgehen (unter Berücksichtigung der Indikationskriterien) entwickelt. Den weniger destruktiven Verfahren wird mit Verweis auf die LQ eindeutig der Vorzug gegeben. LQ-Messungen in den letzten Jahren haben das unerwartete Ergebnis erbracht, dass sich LQ bei mastektomierten und brusterhaltend operierten Frauen nicht unterscheidet ${ }^{24}$ - mit der Ausnahme, dass mastektomierte Frauen mehr Scheu haben ihren Körper nackt zu zeigen und mit den figuralen Aspekten ihres Körperbildes unzufriedener sind. Ganz et al. (1992) untersuchen in ihrer Studie die Frage: „Is There a Difference in Psychological Adjustment or Quality of Life in the Year after surgery?“. Die Autoren begründen ihre Studie mit der „intuitiven“ Annahme, dass eine Mastektomie schwieriger zu bewältigen sei als eine brusterhaltende Operation. Sie operationalisieren LQ mit der FLIC-Skala und dem KarnofsyIndex. Zwischen den beiden Gruppen zeigen sich keine Unterschiede. Dieses Ergebnis wird von den Autoren kommentiert: „The life-threatening nature of the breast cancer diagnosis and disruption caused by surgery are similar for patients having either procedure, and these factors may outweigh the cosmetic differences between the two primary treatments". (S. 1729) Nach diesen Ergebnissen, die sich erweitern lassen (früheste Arbeit von de Haes \& Knippenberg, 1985), ist die selbstbeurteilte LQ nicht als Kriterium der Bewertung der unterschiedlichen Operationen geeignet. Ein weiterer Artikel von Tasmuth et al. (1995) vergleicht ebenfalls die unterschiedlichen Operationen im Hinblick auf Schmerzen und andere Symptome. Die Autoren untersuchten die Langzeitfolgewirkungen in Bezug auf Armbeschwerden (Schmerzen, Beweglichkeit, Lymphödem). In ihrer Studie, an der 92\% der aufgeforderten 569 Patientinnen teilnahmen, sind vor allem Schmerzen (auch

\footnotetext{
${ }^{24}$ Zum gleichen Ergebnis kommt bei einer völlig anderen Stichprobe Sugarbaker (1982): Die Hypothese, dass die LQ von Sarkompatienten, die sich einer Gliedmaßenamputation unterziehen mussten, schlechter ist als die der Patienten mit Gliedmaßen erhaltender Therapie und Bestrahlung, konnte nicht bestätigt werden.
} 
sensible Störungen, Ödeme, Muskelschwäche) in der konservativ behandelten Gruppe signifikant ausgeprägter. Die Autoren halten dies für ein bemerkenswertes Ergebnis: „In previous studies the impact of the type of breast surgery on the Quality of Life has been comparable except for body image, which has been better after breast conserving treatment, and sensory loss and paraesthesia, which are more common after radical mastectomies." (ebenda S. 457) (Hervorheb. d. V.). Interessanter als die Ergebnisse selber ist der Kommentar in den editorischen Anmerkungen. Die Herausgeber der "Annals of Oncology“ warnen davor, dieses Ergebnis in die öffentliche Diskussion einzubringen. Sie stellen die methodische Güte, die ja eigentlich vor der Veröffentlichung geprüft sein sollte, in Frage und argumentieren im Hinblick auf den möglichen Missbrauch dieser Daten: Nachdem es Jahre gedauert hat, bis akzeptiert wurde, dass beide Verfahren im Hinblick auf die Überlebenszeit gleichwertig sind, soll dieser Prozess nicht gefährdet werden. Die Autoren befürchten, die Ergebnisse könnten aus ökonomischen Gründen aufgegriffen werden, da radikale Operationen sehr viel kostengünstiger sind als Operationen mit anschließender obligatorischer Bestrahlung. Unter der Annahme, dass die Ergebnisse der Autoren verallgemeinert werden können, müssen die Folgewirkungen der brusterhaltenden Operation mit Bestrahlung ernst genommen werden. Aber wer soll entscheiden, ob ein unbeschädigteres Körperbild oder weniger chronische Schmerzen (vorausgesetzt die Befunde sind verallgemeinerbar) für die betroffenen Frauen von Bedeutung sind, zumal es sich immer um unsichere Angaben handelt.

Für die Schwierigkeit, LQ-Ergebnisse in die Praxis zu übertragen, lassen sich beliebig viele weitere Beispiele finden.

\subsubsection{LQ-Werte als Hinweis für eine notwendige Intervention}

Die Bereitstellung von Lebensqualitätsverläufen soll es dem Arzt im Einzelfall ermöglichen, Patienten einzuordnen und inm Hinweise auf psychosozialen Handlungsbedarf geben (Sellschopp \& Herschbach 1998). Die Autoren halten es für notwendig, für jede Diagnosegruppe, spezifiziert nach Alter, Geschlecht und Krankheitszustand eine Art Normprofil zu ermitteln, mit dem einzelne Patientenprofile dann verglichen werden, um bei Abweichungen, deren Höhe noch definiert werden müsste, mit entsprechenden Interventionsangeboten zu reagieren.

Die LQ Arbeitsgruppe der Nordic Myeloma Study Group (NMSG) arbeitet mit dieser Art von „Normprofil“ (Wisloff \& Gulbrandsen 2000). Die Arbeitsgruppe verwendet den QLQ-C30. Das Normprofil bezieht sich allerdings nicht, wie Sellschopp \& Herschbach (1998) fordern, auf das Durchschnittsprofil der entsprechenden Diagnosegruppe sondern auf eine Bevölkerungsstichprobe. Die Autoren stellen in einem EffektgrößenDiagramm die Skalenmittelwerte für den QLQ-C30 im Vergleich mit der Referenzpopu- 
lation graphisch dar. Die Nulllinie charakterisiert die Referenzpopulation. Für jede Skala wird die Abweichung von der Nulllinie markiert und die Effektgrößen werden als gering - mäßig - ausgeprägt, entsprechend konventionellen statistischen Kriterien definiert.

Die Autoren ignorieren bei dieser Art des Vorgehens eine wichtige Vorannahme des QLQ-C30: Sie setzen den als krankheitsspezifisches Instrument entwickelten Fragebogen in einer repräsentativen Bevölkerungsstichprobe ein.

Würden die Forderungen von Sellschopp \& Herschbach (1998) beachtet, wäre theoretisch der Vergleich des „Normprofils“ für eine Diagnosegruppe mit einem individuellen Profil denkbar. Abgesehen davon, dass es praktisch völlig unmöglich ist, diese Normprofile zu erstellen, sind die psychometrischen Fragebögen auch nicht darauf ausgerichtet, punktuell individuelle Profile zu ermitteln. Die Autoren des QLQ-C30 warnen ausdrücklich davor, den Fragebogen für individuelle Querschnittsmessungen zu verwenden. Allenfalls intraindividuelle Vergleiche sind gestattet. Für die Praxis sind aufwendige Fragebögen kaum geeignet, der Einsatz ist nur im Rahmen von Studien möglich. Neue Hinweise auf Interventionsbedarf haben sich durch psychometrische LQForschung nicht ergeben, sondern Erkenntnisse sind im Kontext dieser Forschung gewonnen worden.

\subsection{LQ als Prognosefaktor}

Die Tatsache, dass LQ-Messungen einen prognostischen Werte in Bezug auf Überlebenszeiten haben, zumindest innerhalb von Krankheitsgruppen und nach der Einteilung in definierte Krankheitsstadien, ist vielfach durch Studien belegt (zu den neueren Studien gehören, u.a. die von Kaasa et al., 1989; Ganz et al., 1991a; Ruckedeschel et al., 1991; Coates et al., 1992; Feld 1995; Coates \& Gebski, 1996; Coates, Porzsolt \& Osoba, 1997; Porzsolt \& Rist, 1997). Gerade die Frage nach der Allgemeinen LQ, z. B. operationalisiert durch die Frage: «Wie schätzen Sie Ihre allgemeine Lebensqualität ein?», führt zu prognostisch verwertbarer Information. Obwohl gerade dieser „AntwortWert" am unbestimmtesten ist und die unterschiedlichsten intervenierenden Konstrukte, die nicht aus dem Fragebogen erschlossen werden können, in die individuelle Beurteilung eingehen. Wenn onkologische Patienten ihre LQ schlechter bewerten, scheinen sie, unter Berücksichtigung der oben formulierten Grenzen, eine kürzere Lebenserwartung zu haben. Dieser Allgemeine LQ-Wert ist genauso aussagekräftig wie differenziertere Profilbeschreibungen und hat sich in gruppenstatistischen Auswertungen bewiesen.

Ganz et al. (1991a) untersuchen mit dem FLIC eine Patientenstichprobe $(N=40)$ mit metastasiertem Lungenkarzinom. Sie halbieren die Stichprobe nach hohen/niedrigen 
FLIC-Werten und ermitteln die Überlebenszeiten. In Abhängigkeit von einem hohen/niedrigen Summenwert im FLIC gibt es signifikante Überlebenseffekte: die Gruppe mit niedrigen Werten überlebt durchschnittlich 11,9 Wochen, die Gruppe mit hohen Werten überlebt 24 Wochen. Auch Coates \& Gebski (1996) überprüfen den prognostischen Wert von LQ an einer großen Stichprobe (Patientinnen mit metastasiertem Mammkarzinom N=240; Patienten mit metastasiertem malignen Melanom N=150). Eingesetzt werden der Spitzer-Index und die LASA-Skalen. Sowohl der totale QLI-Wert als auch die einzelnen 5 Skalenwerte sind signifikante Pädiktoren der Überlebenszeit. Bei den LASA-Skalen erweisen sich die Werte „physical well-being“ und „appetite“ als beste Prädiktoren für die Mammakarzinompatientinnen. Die Autoren schließen: „A simple explanation might be that QL score provides a better measure of the extent of the underlying disease, and thus its prognosis, than other clinically recorded parameters. Even this explanation would, of course, be sufficient to justify the measurement of QL." (S. 63).

In Deutschland haben Porzsolt u.a. ebenfalls den Zusammenhang zwischen LQ und Überlebenszeit im Rahmen einer umfangreichen internationalen Multicenterstudie (mehr als 700 Patienten) untersucht. Der Autor folgert mit Bezug auf die Ergebnisse: „Die Ergebnisse zeigen eindeutig, dass die Lebensqualität einen harten prognostischen Faktor für die Vorhersage der verbleibenden Lebenszeit darstellt. Durch die Messung der Lebensqualität können Informationen gewonnen werden, die mit keinem anderen Parameter zu erhalten sind." (Porzsolt \& Rist, 1997 S.20).

Welche Konsequenzen daraus sind denkbar? Bislang gibt es keine Studien, die direkte Konsequenzen aufzeigen. Es gibt auch keine Studie, die eine Vorhersage von Responseraten wagt. Coates und Gebski (ebenda) überlegen, in welche Richtung Konsequenzen gedacht werden können: „New studies are required to examine the association between QL and survival, in order to rationally approach the design of intervention studies aimed at prolonging of survival through improvement of quality of life." (S. 67)

Psychoonkologische Forschungsbemühungen, die LQ als Zielkriterium zur Wirksamkeitsüberprüfung von psychoonkologischen Interventionen definieren und die einen Überlebenseffekt durch diese Maßnahmen belegen wollen, zielen in diese Richtung. Die bisherigen Ergebnisse sind widersprüchlich.

Neuerdings werden LQ-Indikatoren auch im Rahmen deskriptiver und analytischer Epidemiologie in Deutschland genutzt (Bullinger 2001). 


\subsection{Evaluation}

Zunehmend wichtiger geworden ist die LQ-Forschung bei der Evaluation von therapeutischen Maßnahmen. LQ-Indikatoren werden den klassischen Evalutationskriterien Überlebenszeit, partielle/totale Remission ${ }^{25}$ und Therapieverlauf hinzugefügt. Gerade auch im Zusammenhang mit Evidenz Basierter Medizin (EBM) wird die Forderung nach Berücksichtigung von LQ gestellt (Porzsolt 2000). Nicht nur bei der Bewertung medizinischer Therapien ist LQ ein wichtiger Indikator sondern vor allem auch bei der Bewertung von psychosozialen, psychoedukativen und psychotherapeutischen Interventionen. ${ }^{26}$ Dass damit die Daten auch eine wichtige Rolle aus der Perspektive der Qualitätssicherung spielen/spielen können, liegt auf der Hand. Hier soll auf die Bewertung von nicht-medizinischen Interventionen eingegangen werden. Schulz et al. (2001) führen aus, dass über einen langen Zeitraum überwiegend die Effekte von psychosozialen Interventionen auf Nebenwirkungen und emotionale Probleme untersucht wurden. Von den in ihrer Übersicht angegebenen 47 Studien benutzen nur 6 Studien LQ als Kriterium. Beim gegenwärtigen Wissensstand kann nicht davon ausgegangen werden, dass durch psychosoziale Interventionen das Überleben verlängert werden kann, auch wenn es einzelne Studien gibt, die diesen Zusammenhang postulieren. ${ }^{27}$ Ein anderes Bild zeigt sich bei der Auswahl anderer Autoren. Zunehmend mehr Interventionsstudien nutzen LQ als Kriteriumsvariable, operationalisiert über Fragebögen. In der Regel handelt es sich bei den Interventionen um supportive Angebote und/oder psycho-edukative Interventionen. Längere psychodynamische Therapien sind nach Keller (2001) nur bei unter $5 \%$ der behandlungsbedürftigen Patienten indiziert. Keller gibt einen Überblick über Interventionsstudien mit standardisierten Instrumenten. Sie berücksichtigt ausschließlich randomisierte Kontrollgruppendesigns. Eine weitere, kürzlich veröffentlichte umfangreiche Arbeit von Rehse (2001) unterzieht 37 Studien, die zwischen 1979 -1999 veröffentlicht wurden, einer Metaanalyse. In der Regel wird LQ operationalisiert durch Fragebögen (z. B. POMS, FLIC, QLQ-C30, FACT u.a.), deren Ergebnisse im PräPosttest (Kontrollgruppendesign) verglichen werden. Sie beschreibt alle Studien mit ihren Interventionen und Ergebnissen und ermöglicht dadurch einen fundierten Überblick über die Forschungslandschaft. Hier soll nur kurz auf die allgemeinen Ergebnisse

\footnotetext{
${ }^{25}$ Remission gehört zwar zu den klassischen Effektivitätsparametern, wird aber als Surrogatkriterium kritisiert. Remissionen sind nicht zwangsläufig auch mit verlängerter Lebenszeit assoziiert.

${ }^{26}$ Siehe die Übersicht von Keller (2001): Effekte psychosozialer Interventionen auf Lebensqualität und Krankheitsverlauf von Krebspatienten.

${ }^{27}$ Siehe den Aufsatz von Schwarz (2001): Psyche und Krebsentstehung und die ausführliche Literaturübersicht von Faller (1997): Beeinflussen psychologische Faktoren die Überlebenszeit bei Krebskranken Teil I und von Faller et al. (1997) Teil II
} 
(die Arbeit liefert eine Fülle interessanter Einzelergebnisse) verwiesen werden: psychosoziale Interventionen sind effektiv in Bezug auf eine verbesserte Lebensqualität. Längerfristige Interventionen scheinen mit besseren Effekten einherzugehen. Je höher die methodische Güte der Studie, desto geringer sind die Effekte (und umgekehrt).

\subsection{Kosten-Nutzen-Analysen}

Wegen der hohen Bedeutung und den möglicherweise gravierenden Auswirkungen auf zukünftige Gesundheitspolitik, soll dieser Aspekt ausführlicher betrachtet werden. Gerade in der Onkologie sind die Kosten überproportional angestiegen.

Das Sozialgesetzbuch V (SGB V) fordert, dass Qualität und Wirksamkeit medizinischer Maßnahmen dem allgemeinen Stand medizinischer Erkenntnisse entsprechen müssen. „Sie sollen ausreichend, zweckmäßig und wirtschaftlich sein und dürfen das Maß des Notwendigen nicht überschreiten ( $\S 12$ ). Daneben haben die Krankenkassen zusammen mit den Leistungserbringern auch auf eine humane Krankenbehandlung hinzuwirken (§ 70)“ (Glaeske 1997, S. 29).

Ergänzt wird diese Forderung durch den Grundsatz der Beitragsstabilität in § 71 SGB V. Bei steigenden Kosten kann eine Beitragsstabilität nur durch Rationalisierung oder Rationierung der Gesundheitsleistungen erreicht werden. Der Anwendungsbereich für Kosten-Nutzen-Analysen in der Medizin wird von Walshe \& Diehl (1998) in einer Tabelle zusammengestellt:

Tabelle 3: Anwendungsbereiche von Kosten-Nutzen-Analysen (S. 945)

\begin{tabular}{|l|}
\hline Anwendungsbereiche von Kosten- \\
Nutzen-Analysen in der Medizin \\
\hline Unterstützung bei der Entscheidung, ob eine \\
bestimmte medizinische Leistung erbracht werden soll \\
Unterstützung bei der Entscheidung, in \\
welchem mengenmäßigen Ausmaß eine \\
medizinische Leistung erbracht werden soll \\
Unterstützung bei der Auswahlentscheidung \\
zwischen alternativen medizinischen \\
Leistungen
\end{tabular}

Im Rahmen von Wirtschaftlichkeitsanalysen gehört die Berücksichtigung von LQ in den Bereich der Kosten-Nutzwert-Analyse: „Die Nutzwertanalyse ist eine sozioökonomische Untersuchung, in welcher die Kosten monetär, die Konsequenzen jedoch als Nutzen resp. Nutzwert ausgedrückt werden." (Szucs 1996, S. 49) In den Nutzwert geht die Beurteilung der Betroffenen bzw. der potentiell Betroffenen ein. Die CUA (cost-utility-analysis) ist die einzige Analysemethode, bei der nicht-monetäre Größen in die Berechnung einbezogen werden. Mithilfe von CUAs soll das Verhältnis von „Gewinn an Gesundheit“ und 
„den dafür benötigten Kosten“ ermittelt werden. Durch die Orientierung an der LQ der Patienten respektive dem Gesundheitszustand ist es möglich, eine Vielzahl von subjektiven Variablen zu berücksichtigen. Die unterschiedlichen Effektivitätsparameter werden durch Standardisierungs- und Gewichtungsverfahren in einheitliche Nutzwerte überführt (Walshe \& Diehl 1998). Der Nutzwert berücksichtigt sowohl die durch die Behandlung gewonnene zusätzliche Lebenszeit als auch die gesundheitsbezogene LQ in der gewonnenen Lebenszeit. Objektive und subjektive Parameter werden kombiniert. CUA liefert Informationen, die für Entscheidungsprozesse aus der sozialen Perspektive (Ressourcenverteilung) genutzt werden können! CUAs können keine Entscheidungen an sich treffen.

Die Methodik der Datengewinnung unterscheidet sich erheblich von denen der bisher vorgestellten Verfahren. Da in der öffentlichen Diskussion häufig Bezug genommen wird auf ökonomische Argumentationen sollen hier in Kürze die Grundzüge dargestellt werden. $^{28}$

Verschiedene Modelle, auf welche Weise die LQ in die Kosten-Nutzen-Analyse einbezogen werden kann, wurden vorgestellt. Es gibt zur Zeit keinen Konsens, welches Verfahren am Besten geeignet ist.

In der Arbeit von König et al. (1999) werden in einer Tabelle die methodischen Schritte der Nutzwertbestimmung in ihrer hierarchischen Ordnung aufgelistet:

Tabelle 4: Nutzwertbestimmung (S. 668)

\begin{tabular}{|ll|}
\hline $\begin{array}{l}\text { Methodische Schritte der Nutzwertbestimmung } \\
\text { (hierarchische Ordnung) }\end{array}$ \\
\hline 1. & Messung der gesundheitsbezogenen Lebensqualität \\
\hline 2. & $\begin{array}{l}\text { Zusammenfassung der gesundheitsbezogenen Lebensqualität zu } \\
\text { einem Index }\end{array}$ \\
\hline 3. & $\begin{array}{l}\text { Zusammenfassung des gesundheitsbezogenen } \\
\text { Lebensqualitätsindexes und der Überlebenszeit zu einem Index }\end{array}$ \\
\hline 4. & $\begin{array}{l}\text { Multiplikative Form der Zusammenfassung des } \\
\text { gesundheitsbezogenen Lebensqualitätsindexes und der } \\
\text { Überlebenszeit }\end{array}$ \\
\hline
\end{tabular}

In dieser Tabelle wird nicht vorgeschrieben, auf welche Weise gesundheitsbezogene LQ gemessen werden soll. Zwei Methoden, wie Lebensqualität und -quantität verbunden werden können, sind bekannter geworden: QALY (Quality-adjusted-life-years) und Q-TWiST (Quality - Time without Symptoms and Therapy). Vom pragmatischen Standpunkt (Einfachheit und Praktizierbarkeit) ist die Ermittlung von (QALYs) die Methode der Wahl. QALYs werden ermittelt, wenn es um Informationen für Entschei-

${ }^{28}$ Eine ausführliche Darstellung der Probleme, Methoden, Anwendungsmöglichkeiten stellen die Autoren Gold, Russell, Siegel \& Weinstein (Hrsg.) in: „Cost-Effectiveness in Health and Medicine" vor (1996). 
dungsfindungen bei der Auswahl verschiedener Therapieverfahren geht. Im Kontext von QALYs werden die Begriffe "health status“, „functional status“ und „HRQL" synonym verwendet. Die Basis für die Berechnung von QALYs bildet die Bestimmung von Gesundheitszuständen, die mit einem Nutzwert (Score) $)^{29}$ charakterisiert werden. Für jedes Individuum (oder für Populationen, Subgruppen etc.) können die Gesundheitszustände über die gesamte Lebensspanne oder Abschnitte der Lebensspanne beschrieben werden. Den Gesundheitszuständen wird ein numerischer Wert zugeordnet, der zwischen $O$ und 1 liegen sollte. 0 entspricht dabei dem Zustand Tod, 1 entspricht dem Zustand vollkommener Gesundheit. ${ }^{30}$ Der höhere Wert kennzeichnet immer einen besseren Gesundheitszustand. Vorteil dieser Analyse ist, dass medizinische Wirkungen in unterschiedlichen Bereichen miteinander verglichen werden können. Zur Veranschaulichung von Nutzwerten sei die folgende Tabelle angeführt:

Tabelle 5: Beispiele von Nutzwerten (aus Szucs 1996, S. 50)

\begin{tabular}{|l|c|}
\hline Gesundheits- & Nutzwert \\
zustand & \\
\hline Gesund & 1,00 \\
Postmenopausales Syndrom & 0,99 \\
Milde Angina pectoris & 0,99 \\
Schweres postphlebitisches Syndrom & 0,98 \\
Herzinsuffizienz NYHA II & 0,90 \\
Status nach Nierentransplantation & 0,84 \\
Status nach Schlaganfall & 0,79 \\
Herzinsuffizienz NYHAIII und IV & 0,70 \\
Schwere Angina pectoris & 0,50 \\
Blindheit & 0,39 \\
Herzinsuffizienz NYHA IV hospitalisiert & 0,30 \\
Intrakranielle Blutung & 0,29 \\
Tod & 0,00 \\
\hline
\end{tabular}

Nutzwerte liegen nur für einen kleinen Teil des Krankheitsspektrums vor. Ihre Ermittlung ist methodisch und inhaltlich schwierig. Die Problematik der Nutzwerte wird anhand der Tabelle offensichtlich. Der bewertete Krankheitszustand muss notwendigerweise sehr grob sein und kann den unterschiedlichen Bedingungen auch in gleich definierten Krankheitsstadien kaum entsprechen. Für ökonomische Analysen können wiederum zu komplexe Differenzierungen nicht verwendet werde. Der Status nach Schlaganfall wird, um nur ein Beispiel herauszugreifen, mit 0,79 bewertet. Das klinische Bild nach Schlaganfall kann gravierend variieren und die Konsequenzen sind individuell kaum vergleichbar. Ein Pianist der durch eine Hemiparese für immer die Fä-

\footnotetext{
${ }^{29}$ Nutzwert - Präferenzen - Utility werden in der Literatur synonym verwendet

${ }^{30}$ Empirische Untersuchungen haben gezeigt, dass es für die Befragten Gesundheitszustände gibt die schlimmer sind als der Tod. Theoretisch sind also Werte kleiner Null denkbar.
} 
higkeit einbüßt seinen Beruf auszuüben, mag den Nutzwert völlig anders beurteilen als jemand, der durch eine leichte motorische Aphasie in Beruf und Privatleben nur unwesentlich beeinträchtigt wird. Nutzwerte werden ermittelt durch zwei unterschiedliche Methoden:

- Präferenzbasierte Verfahren

- Nicht-Präferenzbasierte Verfahren.

Die Nicht-Präferenzbasierten Verfahren entsprechen den Verfahren, wie sie in der klinisch orientierten LQ-Forschung verwendet werden, also den psychometrisch validierten Fragebögen, die als Selbstbeurteilungsinstrumente verwendet werden.

Das Hauptproblem dieser Daten besteht in der Bewertung. Die aufsummierten Ratings zu einem Gesamtscore spiegeln nicht die Bedeutung der einzelnen Scores wider. Veränderte Scores sind nicht gleichzusetzen mit einem veränderten Gesundheitszustand bzw. LQ. Die neueren Instrumente verzichten deshalb auf einen Gesamtscore und bilden Profile ab, oder sie beschränken sich auf eine Skala. Im Rahmen von QALYs werden die präferenzbasierten Methoden vorgezogen. Für die Ermittlung der präferenzbasierten Scores gibt es wiederum eine Reihe unterschiedlicher Methoden, z. B. „standard gamble”, „time-tradeoff”, „willingness to pay”, visuelle Analogskalen (z.B. EuroQol), die ihrerseits wieder bestimmten methodischen und inhaltlichen Anforderungen (Reliabilität, Validität, Neutralität, Kompetenz) genügen müssen. Die Erläuterung dieser Methoden würde den Rahmen der Arbeit sprengen. Allen ist gemeinsam, dass Zeiten (oder ein anderer „Wert“) in optimaler Gesundheit mit denen in reduzierter Gesundheit verglichen werden.

Ein wichtiger Unterschied besteht allerdings bei den verschiedenen Ermittlungsverfahren. Werte, die mit VAS und TTO ermittelt werden, heißen value scores. Werte, die im Rahmen von spieltheoretischen Verfahren ermittelt werden, d. h. unter der Annahme von ungewissem Ausgang, Wahrscheinlichkeiten, Risiken, die nicht eindeutig bestimmbar sind usw. werden utilities genannt. Utilities entsprechen der realen Situation während value scores einer künstlichen Situation entsprechen. Zur Vereinfachung sei hier das TTOModell beschrieben. Das Zeitintervall in reduziertem Gesundheitszustand wird solange verkürzt, bis es im Empfinden einem Jahr im optimalen Zustand äquivalent ist. Z. B.: Ein Jahr in reduziertem Zustand entspricht einem halben Jahr im optimalen Zustand. Der Nutzwert für den hier angenommenen reduzierten Gesundheitszustand würde dann in diesem Beispiel dem Wert von 0,5 entsprechen. 
Abb. 8: Darstellung von QALY (adaptiert von Gold et al., 1996, S.88)

optimaler Gesundheitszustand/HRQL

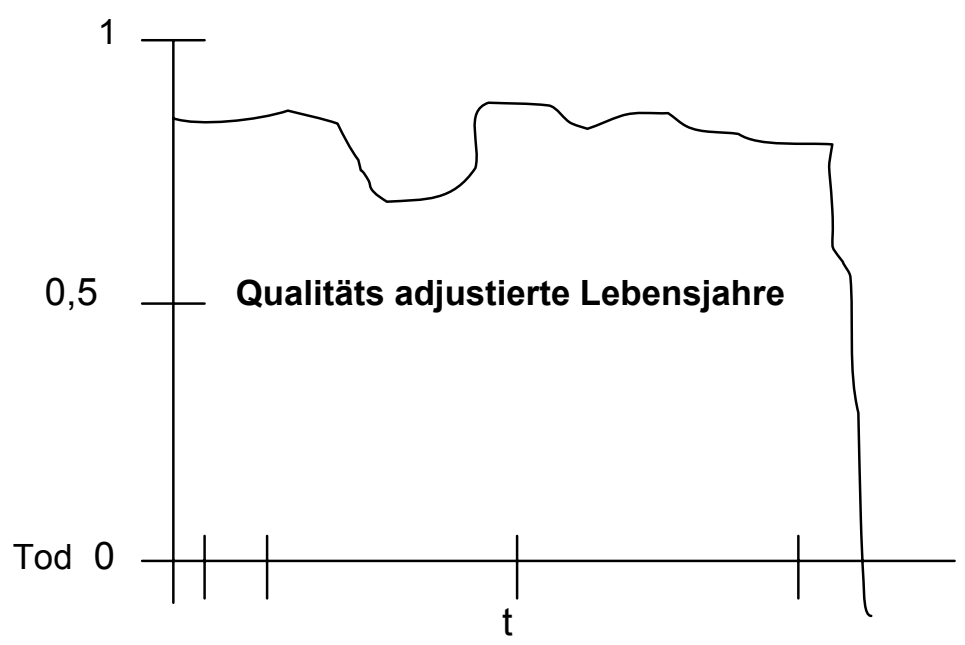

Lebenspanne

Die Kurve kann sowohl einen individuellen Verlauf über die gesamte Lebensspanne bis zum Tode beschreiben als auch den Mittelwertsverlauf einer Population darstellen. Wichtig für die Ermittlung von QALYs ist die Frage, wer die Nutzwerte bestimmen soll. Generell ist die Beantwortung der Frage abhängig vom Studiendesign. In Frage kommen Betroffene (Patienten), Experten (Angehörige der Gesundheitsberufe) und repräsentative Bevölkerungsstichproben. Wenn es um die soziale und gesundheitspolitische Perspektive geht, wird von den Gesundheitsökonomen die Ermittlung der Scores durch eine Bevölkerungsstichprobe vorgezogen. Geht es um Therapievergleich, wird Patientenstichproben der Vorzug gegeben. Aus empirischen Studien ist bekannt, dass die Urteile zwischen Beurteilergruppen sowohl relativ gut übereinstimmen als auch völlig differieren können. ${ }^{31}$ Generell besteht eine Tendenz darin, dass Erkrankte höhere Werte für Gesundheitszustände vergeben als Gesunde (Raisch 2000).

Die QALYs werden auf folgende Weise ermittelt:

Im ersten Schritt werden spezielle prospektive Zeitintervalle, die sich auf den geplanten Nutzen der Therapie beziehen, definiert. Jeder Patient (oder ein anderer Beurteiler) kann für die jeweiligen Intervalle einen Nutzenscore bestimmen. Der Nutzenscore wird mit der Zeitdauer dieses Intervalls multipliziert. Aus den individuellen Werten wird dann der Mittelwert der Gesamtstichprobe errechnet. Jedes gewonnene Lebensjahr wird mit der für dieses Jahr erreichten LQ (zusammengesetzt aus den verschiedenen Zeitintervallen) gewichtet. Die zusätzlichen Lebensjahre werden dann gewichtet aufsummiert und ergeben die QALYs. Das Resultat der QALY-Methode ist ein Schätzwert, der die

\footnotetext{
${ }^{31}$ Siehe Gold, Patrick \& Torrance et al. (1996):Identifying and Valuing Outcomes
} 
gewonnenen Lebensjahre repräsentiert. Der Wert kann mit denen anderer Studien verglichen werden. QALY wird in Kosten-Nutzwert-Analysen und in Entscheidungsprozessen auf politischer Ebene, die einen einzigen Messwert erfordern, benutzt.

Im nächsten Schritt, nach der Ermittlung von Nutzwerten, werden Kosten für medizinische Interventionen, bezogen auf jedes QALY, berechnet. Nicht alle geretteten Lebensjahre sind äquivalent (Szucs, 1996). Auch hier genüge zur Veranschaulichung, eine der ermittelten Ranglisten (league tables):

Tabelle 6: Rangliste für ausgewählte medizinische Interventionen (ebenda S. 50)

\begin{tabular}{|l|c|}
\hline Interventionen & $\begin{array}{l}\text { Kosten pro } \\
\text { gerettetes } \\
\text { Lebensjahr } \\
(\$, \text { median) }\end{array}$ \\
\hline Pädiatrische Impfungen & $<0$ \\
Grippeschutzimpfung & 600 \\
Arzneimittel (Medianwert) & 5000 \\
Pneumokokkenimpfung & 12000 \\
Koronarer Bypass bei Dreigefäßerkrankungen & 15000 \\
Brustkrebsreihenuntersuchung & 17000 \\
Nierentransplantation & 22000 \\
Neonatale Intensivmedizin (Geburtsgewicht<1kg) & 22000 \\
Koronarer Bypass bei Eingefäßerkrankung & 55000 \\
Hämodialyse & 85000 \\
\hline
\end{tabular}

Anhand solcher Tabellen werden Mittelzuweisungen diskutiert und in anderen Ländern (z. B. England, US Bundesstaat Oregon) werden damit direkte Rationierungen von Gesundheitsleistungen begründet.

Im onkologischen Rahmen wird QALYs aus folgenden Gründen wenig genutzt:

1. Lange serielle Befragungszeiträume sind erforderlich

2. Problematik der fehlenden Daten bei Längsschnittstudien: neben den üblichen „drop outs“ durch mangelnde Compliance, Umzug etc. muss in onkologischen Studien in Abhängigkeit von der Krebsart mit erheblichen Ausfällen durch Todesfälle gerechnet werden.

3. Die Bewertung der Daten ist problematisch, da die Schätzwerte der KaplanMeier-Kurve ${ }^{32}$ und der QALY-Scores statistisch nicht voneinander unabhängig sind (bei der Bestimmung der QALY-Scores gehen die gleichen Überlebenszeitschätzungen mit ein).

Q-TWiST ist eine Weiterentwicklung der TWiSt-Methode wie sie auf S. 12 beschrieben wurde. In Q-TWIST ist das Endprodukt im Vergleich mit QALYs Kontext abhängig. Höchstwert ist die möglichst beste Gesundheit in einem bestimmten Kontext. Die ande-

\footnotetext{
${ }^{32}$ Kaplan-Meier-Kurven: Überlebenszeitkurven, die auf der Grundlage von empirischen Daten geschätzt werden.
} 
ren Gesundheitszustände werden in Relation zu dieser bestmöglichen Gesundheit betrachtet. Die Anwendbarkeit ist im Vergleich zu QALY auf einen Krankheitsbereich beschränkt. Die Definition von Gesundheitszuständen ist hier ebenfalls sehr schwierig, wird aber auf eine geringe Anzahl von Zuständen beschränkt. Diese Methode wird in klinischen Studien verwendet, wenn unterschiedliche Behandlungen verglichen werden sollen. Die Anwendung in der Onkologie ist nur im adjuvanten Setting möglich. Die Behandlungszeit und die Folgezeit werden in drei klinisch relevante Gesundheitszustände aufgeteilt. Für jeden Zustand wird ein Nutzenscore bestimmt. Die Mittelwerte der Zeit, die der Patient in einem bestimmten Zustand verbringt werden geschätzt und diese Schätzung wird kombiniert mit dem Nutzenscore. Die Methode erfordert, wie QALY, drei Schritte der Ermittlung des Vergleichsergebnisses:

1. Klinische Gesundheitszustände, die sich auf Unterschiede der LQ bei verschiedenen Behandlungen beziehen, müssen definiert werden.

2. Das Gesamtüberleben wird aufgeteilt auf die verschiedenen vorher definierten Gesundheitszustände.

3. Vergleich der Regimes in Bezug auf das qualitätsangepasste Überleben. Q-TWIST kann retrospektiv angewendet werden, ohne dass vom Patienten LQ-Daten erhoben wurden. Die verschiedenen Gesundheitszustände werden bestimmt und gleichgesetzt mit LQ. Eine inhaltliche Spezifikation von LQ erfolgt nur über die folgenden Merkmale für bestimmte Zeiten:

a. LQ, die beeinträchtigt wird durch die Nebenwirkungen der Therapie (TOX - Toxizität)

b. LQ, ohne Nebenwirkungen und Symptome (TWiST)

C. LQ, Metastasierung, Progress bis zum Tode (Rel - Relapse)

Die verschiedenen Zeitintervalle (TOX - TWiST - REL) werden graphisch präsentiert anhand von Kaplan-Meier-Überlebenskurven. Unterschiedliche Behandlungsmodalitäten können in einer gemeinsamen Graphik dargestellt und verglichen werden. Patienten könnten theoretisch auswählen, ob innen die Zeit ohne Toxizität (TOX) wichtiger ist als die Zeit mit Rezidiv/Progress (Relapse - Rel). Hauptproblem von Q-TWiST ist wie bei QALYs auch, die Definition von Gesundheitszuständen. Gesundheitszustände sind selten statisch und unterliegen erheblichen intra- und interindividuellen Schwankungen. Für die palliative Situation existieren die TWiST-Phasen per definitionem praktisch nicht. Die drei angenommenen Gesundheitszustände mit besserer oder minderer LQ synonym zu setzen, ist simplifizierend und deckt sich nicht mit der Patientenwahrnehmung, wie in zahlreichen Studien gut belegt ist: „Ärzte sehen die Lebensqualität durch die Krankheit oft erheblich negativer, durch die Therapie aber meist deutlich positiver beeinflusst als der Kranke selber" (Helmchen, 1990, S. 107). Auch die retrospektive Analyse ist me- 
thodisch problematisch. Ein weiteres Problem ergibt sich durch die Nutzenscores: häufig wurden willkürliche Werte gesetzt, um das Funktionieren der Methode anhand von Entscheidungsbäumen zu belegen. Die Verwendung von spieltheoretischen Methoden oder „Time Trade Off-Verfahren“ durch Patienten zur Bestimmung der Scores ist wünschenswert aber äußerst aufwendig und deshalb in klinischen Studien kaum durchführbar. Die wenigen Arbeiten in der Onkologie sind meistens theoretische Arbeiten, die ihre Parameter aus den unterschiedlichsten Quellen beziehen (Expertenurteile, Beobachtungsstudien, Therapiestudien unterschiedlichster methodischer Qualität und Metaanalysen). Eine der wenigen Arbeiten ist von de Haas \& Stiggelbout (1996). Die Autoren untersuchen verschiedene Patientenstichproben (Colon $\mathrm{Ca}, \mathrm{Ca}$ Testes) mit den drei genannten Methoden, um die mit der jeweiligen Methode erhaltenen Daten untereinander zu vergleichen. Wie erwartet, ergeben sich, methodenabhängig, unterschiedliche Bewertungen.

Die beiden skizzierten Methoden integrieren Überlebenszeit und LQ, allerdings in unterschiedlicher Weise. Q-Twist beschränkt sich auf einen Vergleich im definierten Kontext einer bestimmten Erkrankung und deren Behandlung, während QALY einen Wert ermittelt, der zu allgemeinen Vergleichen ganz unterschiedlicher Therapien herangezogen werden kann 


\section{Stellenwert der LQ-Forschung in der Onkologie}

Obwohl LQ heute als Schlagwort in der Onkologie allgegenwärtig ist, muss die Frage gestellt werden, wie sich das Interesse in Klinik und Forschung tatsächlich bestimmen lässt.

Generell sind die Veröffentlichungen in der Medizin zum Thema LQ von 0,1\% 1980 auf 0,9\% 2001 angestiegen (Kaufman 2001). In einer aktuellen Übersicht von Kaufman (ebenda) wird darauf verwiesen, dass die Publikationsrate der HRQOL-Publikationen stärker gestiegen ist als die allgemeine Publikationsrate, dass aber die absoluten Zahlen immer noch sehr gering sind. Die randomisierten Interventionsstudien, in denen HRQOL gemessen wird, haben von 4,6\% 1990 auf 12,6\% im gleichen Zeitraum zugenommen.

Kaufman betrachtet es als einen Meilenstein, der sich auf zukünftige Forschungsinteressen wegweisend auswirken wird, dass die US Food and Drug Administration (FDA) der neuen Technologie „laser transmyocardial revascularization“ die Zulassung erteilt hat, nicht zuletzt aufgrund von HRQOL-Daten. Diese Aufwertung von LQ-Ergebnissen wird andere Forschungen, die sich mit Therapie befassen, seiner Ansicht nach unter Zugzwang setzen. Ähnlich argumentiert Weber (1997), der die Zulassung von Gemcitabine (Zytostaticum) für die Behandlung des Pankreaskarzinom aufgrund von nur unerheblich verlängerter Lebenszeit (1 Monat) aber eines deutlicher ausfallenden Effektes der „Clinical Benefit Response“ hervorhebt: „Diese Untersuchungsergebnisse wurden auch der als konservativ bekannten und methodisch strengen Food and Drug Administration vorgelegt. Die Anerkennung durch diese Behörde macht uns wiederum Mut, auf unserem Weg zur Ermittlung neuer Parameter und Indikatoren für die Quantifizierung der Lebensqualität weiter zu gehen, [...]“ (ebenda, S. 27).

Inzwischen gibt es mehrere nationale und internationale Arbeitsgruppen, die es sich zum Ziel gesetzt haben, Richtlinien für LQ-Messungen zu erarbeiten. Aktuell ist die FDA und die European Agency for the Evaluation of Medical Products dabei, Leitlinien für die Entwicklung und psychometrische Überprüfung von Instrumenten zu entwickeln - vor allem auch dafür, wie die Integration von LQ-Daten und anderen Daten erfolgen soll und welche Schlussfolgerungen daraus gezogen werden können.

Die Arbeitsgruppe „Life Quality“ der EORTC spielt im onkologischen Feld eine herausragende Rolle. 1981 wurde diese Arbeitsgruppe gegründet. Sie hat erreicht, dass onkologische Fachgesellschaften ihre Mitglieder auffordern, in ihren Studienprotokollen die LQ-Messung mit zu berücksichtigen. Außerdem ist der Trend zu beobachten, dass Ethikkomissionen, die bei Forschungsanträgen konsultiert werden müssen, ebenfalls eine Berücksichtigung von LQ-Parametern fordern. 1986 hat das New England Jour- 
nal, eine der renommiertesten medizinischen Fachzeitschriften, LQ als Kriterium innerhalb der pharmazeutischen Forschung akzeptiert. 1994 wurde die „International Society for Quality of Life Research“ gegründet, die eine eigene Fachzeitschrift herausgibt. In einer 1999 veröffentlichten Studie von König et al. wurde untersucht, inwieweit von Ärzten LQ als Effektmaß in klinischen Studien akzeptiert wird und ob Ärzte mit der Methodik der Datenerhebung in diesem Kontext vertraut sind. Die Befragung war nicht repräsentativ, sondern bezog überwiegend onkologische Studienleiter in die Befragung ein. 90\% der Ärzte hielten LQ für ein brauchbares Effektmaß, 68\% akzeptierten die Zusammenfassung von LQ und Überlebenszeit, aber nur $44 \%$ akzeptierten QALYs allerdings kannte nur die Hälfte der befragten Ärzte das Konzept der LQ-Erhebung. Daraus schließen die Autoren, dass LQ wenig Berücksichtigung in der klinischen Arbeit findet. Ähnliche Ergebnisse zeigte eine zeitgleich durchgeführte Studie in Österreich. Eine ältere kanadische Studie (1992/93) zeigte ebenfalls, dass die überwiegende Zahl der untersuchten Onkologen LQ für ein wichtiges Konzept hielten, aber zwei Drittel hatten damit keine Erfahrungen und berücksichtigten LQ nicht bei klinischen Entscheidungen. Andere Autoren, die direkt oder indirekt der Frage nachgehen, ob Ärzte Ergebnisse aus der LQ-Forschung in ihrer klinischen Arbeit mit einbeziehen, kommen ebenfalls zu dem Ergebnis, dass der Einfluss auf klinisches Handeln gering ist. Moynihan (1998), der sich mit dem Problem der fehlenden Daten in LQ-Studien beschäftigt und die entsprechende Literatur auswertet, folgert: „There is a general impression that QL information is not valued as highly as biological information on patient management including chemotherapy use. [...] do physicians actually make use of QL information? [...] This aspect of the research process is seldom revealed" (S. 9). Der Autor vermutet, dass der Bezug auf LQ-Argumente lediglich einem Lippenbekenntnis gleich kommt.

1997 erschien eine Untersuchung von dem Pionier der Psychoonkologie J. Holland und seinen Kollegen (Batel-Copel, Kornblith, Batel \& Holland) mit dem Titel „Do Oncologists have an increasing interest in the Quality of Life of their patients?". Die Bedeutung von LQ leiten die Autoren aus der Nutzung von LQ als additivem Zielkriterium im Rahmen von Phase II und III Studien ab. ${ }^{33}$ Zu diesem Zwecke durchsuchten die Autoren die vier bedeutendsten onkologischen Zeitschriften: „Journal of Clinical Oncology“ - "Cancer“ - „British Journal of Cancer“ - „European Journal of Cancer“ nach Studien, die LQ-Daten beinhalten. Die Studiensuche war äußerst ernüchternd: zwar stieg der Anteil von 1980 bis 1995 an, dabei wurde aber überwiegend der Performanzstatus als Indikator verwendet (15\% - 56\%), während gesundheitsbezogene LQ nur einen minimalen Anstieg (0\% -3\%) aufwies. Von den 13 Studien für 1995 werden nur 3 als ak-

\footnotetext{
${ }^{33}$ Phase II und III dienen der Wirksamkeitsbeurteilung von Therapien
} 
zeptabel hinsichtlich der Methodik der Datenerhebung beurteilt. Folgt man der Argumentation der Autoren, dass sich die praktische Relevanz für Onkologen niederschlägt durch die Einbeziehung von LQ in klinische Wirksamkeitsstudien, dann kann zu diesem Zeitpunkt von einer weitergehenden Bedeutung in der onkologischen Forschung keine Rede sein.

Um Entwicklungstrends in den letzten fünf Jahren auszumachen, wurde eine Medlinesuche (1995 bis 9/2001, Stichwörter: Lebensqualität und Onkologie/klinische Studien) durchgeführt. Insgesamt sind dort 196 Titel verzeichnet. Das Bild fällt deutlich positiver aus als das von Batel-Copel et al. gezeichnete: Nur wenige Untersuchungen benutzen LQ lediglich als Stichwort ohne ausdrückliche Verwendung von entsprechenden Erhebungsinstrumenten. Die alleinige Verwendung des Performanzstatus als LQ-Indikator ist fast völlig verschwunden. Psychometrisch überprüfte Instrumente gehören mittlerweile zum Standard. Studien, die einen ausschließlich qualitativen Ansatz verfolgen, sind nicht vermerkt. Ad hoc Fragebögen sind kaum noch zu finden. Einzelne Studien verwenden Tagebuchaufzeichnungen in Kombination mit anderen Instrumenten. Der QLQ-C30 wird mit großem Abstand am meisten benutzt. Danach folgt der FACT. Der FLIC wird in dieser Auflistung nur zweimal verwendet. In 11 Studien wird die Toxizität allein als LQ-Indikator beschrieben. Daneben finden sich 5 Studien, die ausschließlich Schmerz registrieren. Der Spitzer-Index wird nur wenige Male verwendet. Der SF-36, der als generisches Erhebungsinstrument konzipiert ist und deshalb häufig außerhalb des onkologischen Bereiches benutzt wird, findet hier fünfmal Verwendung. In einzelnen Fällen werden auch die Rotterdam Symptom Checklist, die POMS (Profile of Mood Status), VAS und HADS als LQ-Instrumente eingesetzt. Auffällig ist, dass die Datenerhebung mittlerweile häufiger im Rahmen von randomisierten Studien stattfindet. Also den Studien, die nach den Forderungen der EBM aufgrund ihrer methodischen Güte am aussagekräftigsten sind. ${ }^{34}$

Während ca. $25 \%$ der Studien LQ in Abhängigkeit von Interventionen (am häufigsten Chemotherapien) beschreiben, liegt der Anteil der Studien, die ausdrücklich Therapien vergleichen, bei ca. 10\%. Die Ergebnisse dieser Arbeiten führen dazu, dass explizit oder implizit Therapien anhand von LQ bewertet werden. Als Beispiele sollen hier zwei Studien genannt werden. Beide Studien überprüfen die Effekte von Chemotherapie und Überlebenszeit, beides sind randomisierte Phase III Studien.

In der Studie von Fossa et al. (2000) werden Prostatakarzinompatienten (M1) mit schlechter Prognose den Therapiearmen „Orchiectomy alleine“ und „Orchiectomy mit

\footnotetext{
${ }^{34}$ Diese Auflistung hat nur beschreibenden Charakter. Über die inhaltliche und methodische Qualität ist damit keine Aussage gemacht. Das würde eine umfangreiche Metaanalyse voraussetzen.
} 
Chemotherapie (Mitomycin C)“ zugeteilt. In beiden Armen verbessern sich die Symptome Schmerz und Urinausscheidungsfunktion durch die Therapie. In der Gruppe mit Chemotherapie verschlechtert sich der Skalenwert für die "Allgemeine LQ" signifikant. Außerdem verschlechtern sich eine Reihe von Funktions- und Symptomwerten. Die Überlebenszeit unterscheidet sich nicht. Die Autoren kommen zu dem Schluss, dass die adjuvante Gabe von Mitomycin C nicht zu empfehlen ist.

Jerkeman et al. (1999) untersuchen die Chemotherapiekombinationen CHOP vs MACOB-B bei fortgeschrittener Lymphomerkrankung. 400 Lymphompatienten (Stadien IIIV) werden den Therapiearmen zugewiesen. 95 Patienten werden mit dem QLO-C30 untersucht. Für die 5-Jahres-Überlebenszeit ergaben sich keine Unterschiede. Patienten die MACOB-B erhielten waren in ihrer physischen Funktion (PF-Skala) und der Allgemeinen Lebensqualität stärker beeinträchtigt. Außerdem war die Toxizität für diese Gruppe höher. Die Autoren folgern aus den Ergebnissen, dass CHOP als Therapie der Wahl beibehalten werden sollte.

Diese beiden Beispiele zeigen, dass LQ-Untersuchungen durchaus in der Lage sind, bei der Auswahl zwischen Therapiealternativen zu helfen. Allerdings würde bereits die unterschiedliche Toxizität als Argument für diese oder jene Therapieentscheidung ausreichen.

Als ein weiteres Indiz für die Bedeutung der Lebensqualitätsforschung kann gewertet werden, dass 1996 zum ersten Mal der Forschungspreis eines Pharma-Unternehmens, der „Lilly-Quality of Life Preis“, in Deutschland ausgewiesen wurde. Zweimal wurde er für LQ-Forschung in der Onkologie verliehen. Eine ganze Reihe von Büchern sind in Deutschland ebenfalls zu diesem Themenbereich erschienen. Die Durchsicht der verzeichneten deutschsprachigen Bücher zeigt folgenden Trend: Anfang der 90er Jahre beziehen sich die Titel noch auf ein weites Spektrum außermedizinischer Themen. Danach folgt eine Einengung auf medizinische Themen, die dann sehr eindeutig in Richtung onkologische Forschung (überwiegend medizinische Dissertationen) geht.

Noch gängiger als im wissenschaftlichen Kontext erscheint zurzeit der Stellenwert von LQ-Ergebnissen auf populär-wissenschaftlicher Ebene. Dieser Trend ist vor allem innerhalb der Werbung abzulesen. Es ist kaum eine Zeitschrift/Broschüre der Gesundheitsindustrie in den Wartezimmern von Ärzten, Zahnärzten und Kliniken (oder Apothekerzeitschriften) zu finden, in der keine Lebensqualitätsargumente bemüht werden. Nach eigener klinischer Erfahrung im Akut-Krankenhaus werden hier jedoch kaum LQArgumente von betroffenen Patienten benutzt. Wenn sie doch benutzt werden, dann am ehesten von dem kleinen Teil von Patienten, die sich auch für Selbsthilfegruppen interessieren bzw. dort Mitglieder sind. 


\section{Fazit}

Welches Fazit lässt sich aus den angeführten Überlegungen und Befunden ziehen? Ist den Mitgliedern der EORTC Quality of Life Study Group (Küchler et al. 2000) zuzustimmen, wenn sie den gegenwärtigen Stand und die Bedeutung in folgenden Thesen zusammenfassen?

Thesen (ebenda, S. 34)

- LQ ist nach der Überlebenszeit das wichtigste Behandlungsziel für Krebspatienten

- Gesundheitsbezogene LQ ist heute mit naturwissenschaftlichen Methoden zuverlässig messbar

- Auswirkungen von Krankheit und Therapie lassen sich systematisch darstellen undvergleichen

- Patienten erleben die LQ-Messung als einen Teil der Humanisierung in der onkologischen Behandlung

- LQ-Messung stellt einen Beitrag zur Qualitätssicherung dar

Niemand wird der These, dass LQ nach der Überlebenszeit das wichtigste Kriterium für Patienten ist, widersprechen. Im Hinblick auf die Interpretation der empirischen Befunde wird allerdings die fehlende theoretische Explikation, der Mangel an einer einheitlichen Definition und das Fehlen von Hypothesen über das Zusammenwirken von einzelnen Dimensionen/Variablen sehr schnell deutlich. Sinnvolle Ergebnisinterpretation ist nur dann möglich, wenn durch den Kontext der Datenerhebung, sowohl für Forscher als auch für Patienten, zumindest der Referenzrahmen definiert wird. Implizit legen zahlreiche Instrumente den Referenzrahmen „körperlicher Funktionszustand“ nahe. Hohe Korrelationen mit allgemeinen LQ-Werten weisen daraufhin, dass Patienten dieser Vorgabe folgen.

Auch die Zweckbestimmung der LQ-Daten sollte transparent gemacht werden, damit Patienten nicht LQ mit „lebenswert“ resp. „unwertem Leben“ gleichsetzen.

Gesundheitsbezogene LQ ist mit naturwissenschaftlichen Methoden nicht zuverlässig messbar. Das, was gemessen werden kann, sind einzelne Aspekte - Beschwerden, Funktionen, Symptome, Einschränkungen usw., die mit LQ assoziiert werden. Schuldig geblieben ist die psychometrische LQ-Forschung bislang den Beleg dafür, dass LQ ein sinnvoller Begriff ist, der mehr enthält als die Summation von Einzelaspekten. Die Definition erfolgt, je nach individuellem Interesse des Forschers, über die Erhebungsinstrumente. Durch unterschiedliche Operationalisierungen und Besonderheiten der jeweiligen Stichproben sind inkonsistente Ergebnisse nicht verwunderlich. Auch bei gleichen Instrumenten erschwert die Kontextabhängigkeit Vergleiche und Verallgemeinerungen. Bei der begrifflichen Unschärfe scheint der Verfasserin das Vorgehen der häufig kritisierten englischen Gesundheitsbehörde konsequenter: Unter dem Stichwort LQ 
wird gefordert, dass neben einer Symptomcheckliste ein Screeninginstrument bezüglich Angst und Depressivität eingesetzt wird. Dieses Vorgehen ermöglicht es dann auch, dem Vorschlag von Aaronson (1990) zu folgen, nämlich nur das zu erheben, was im klinischen Kontext auch als beeinflussbar gilt. Diese Bescheidung auf „weniger“ würde den Umgang mit den Daten erleichtern und konzeptioneller Kritik entgegen wirken. Das kritisierte Verfahren, primär über Fragebögen Auskünfte über das Krankheitsempfinden einholen zu wollen ist ja nicht nur deshalb so problematisch, weil es entscheidende Elemente dieses Empfindens nicht wirklich erfassen kann, sondern weil es auch der besonderen Leistung der Patienten nicht gerecht wird und somit sowohl bei den Forschern als auch bei den Patienten Unbehagen hinterlassen kann.

Zumindest für den deutschsprachigen Raum gilt, dass Patienten sehr bereitwillig an den Fragebogenuntersuchungen teilnehmen. Hervorgehoben wird von den Patienten immer wieder, wie wichtig es für sie ist, ihre Sicht darzulegen und ihre Fragen mit Mitgliedern - am liebsten mit den behandelnden Ärzten - des Behandlungsteams zu besprechen. Wenn dies im Kontext der Datenerhebung möglich ist, dann lässt sich vielleicht von „Humanisierung“ sprechen. Das anonyme Ausfüllen von Fragebögen ohne einen interaktiven Kontext trägt sicherlich nicht zur Humanisierung bei. Nicht die Messung selber ist ein Indiz für Humanisierung, sondern einzig und allein der Kontext der Datenerhebung und Datenverwendung. Dazu gehört in besonderer Weise, dass die sich aus den Auskünften ergebenden Schlussfolgerungen mit den Patienten eingehend erörtert werden. Dieser Aspekt wird überhaupt nur in einer einzigen Arbeit erwähnt.

Im diesem eingeschränkten Sinne leistet LQ-Messung einen Beitrag zur Qualitätssicherung. In der Tat lassen sich Schlussfolgerungen aus den Ergebnissen ziehen, die z. B. die Ausprägung von Symptomen bei gleichen Therapieverfahren in unterschiedlichen Einrichtungen oder unterschiedliche Therapien in gleichen Einrichtungen miteinander vergleichen.

Nicht erwähnt wird in den Thesen die prognostische Bedeutung von „Allgemeiner LQ“ sofern sie eng mit der physischen Funktion korreliert. Diese Bedeutung ist durch zahlreiche Studien gut belegt. Allerdings ist es dabei notwendig, den Einfluss von soziodemographischen Variablen zu kontrollieren da diese ebenfalls einen Einfluss haben und als Moderatorvariable wirken können.

All das schmälert keineswegs die Bedeutung der Ergebnisse, die unter dem Etikett der LQ-Forschung gewonnen wurden. Diese Forschung hat einen entscheidenden Beitrag dazu geleistet, Belastungen als Folgewirkungen von Krankheit und Therapie zu verringern. Verbesserungen haben sich am unmittelbarsten ergeben in Bezug auf eine 
effektivere Symptombehandlung. An erster Stelle sind hier Schmerztherapie ${ }^{35}$ und AntiEmese-Behandlung zu nennen. Neuerdings ist Fatigue als belastende Konsequenz ebenfalls in das Blickfeld gerückt.

Auch das Aufzeigen von psychosozialen Belastungen hat im Kontext dieser Forschung eine größere Aufmerksamkeit erfahren. Die Umsetzung dieser Erkenntnisse hat allerdings in den verschiedenen medizinischen Sektoren ganz unterschiedliche Auswirkungen gezeitigt. Während die Erkenntnisse der LQ-Forschung die Praxis der Rehabilitationsbehandlung verändert haben, sind ihre Auswirkungen auf den Bereich der akuten und ambulanten Behandlung verschwindend gering.

Auf dieser Ebene ist die LQ-Forschung uneingeschränkt positiv zu werten. Die Problematik der unscharfen und beliebigen Verwendung des Begriffes liegt auf einer anderen Ebene und wird im Schlussteil behandelt

\footnotetext{
${ }^{35}$ Auch wenn Deutschland im Vergleich mit anderen europäischen Ländern in der Schmerzthe-
} rapie hinterher hinkt, haben sich in den letzten zehn Jahren Verbesserungen abgezeichnet. 


\section{Hochdosischemotherapie (HDC)}

Bei der HDC handelt es sich um ein Verfahren, das seit ca. 20 Jahren angewendet wird (Morrison \& Peterson 1999). Wie der Name sagt, werden dabei bewährte und/oder neue Zytostatika deutlich höher dosiert (bis zur dreifachen Dosis) als es bei konventionellen Chemotherapien üblich ist. Die Wirksamkeit von Chemotherapie setzt voraus, dass die Tumorzellen chemosensitiv sind. Auch chemosensitive Tumorzellen haben die Möglichkeit, gegen die Zytostatika resistent zu werden, und machen dadurch Therapieeffekte zunichte. Der Wirkungsvorstellung bei der HDC liegt die Hypothese zugrunde, dass die Resistenzentwicklung von Tumorzellen erschwert wird, wenn die Dosierung der Chemotherapie möglichst hoch gewählt wird. ${ }^{36}$

Die Vorstellung: „More is better“ (Hudis \& Münster 1999) geht auf die Laborbeobachtungen zurück, dass einzelne Substanzen (z. B. Alkylantien und Antrazykline) die Wirkstoffresistenz von Tumorzellen überwinden können (Crown 1995; Van Hoff et al. 1986). Dosiseskalation und Hochdosistherapie sind die Übersetzung dieser Beobachtung in die Klinik. Durch Knochenmarks- oder Stammzelltransplantationen kann die konventionelle Dosis auf das Mehrfache gesteigert werden.

Auch in vivo gibt es Hinweise auf eine Beziehung zwischen angewendeter Dosis und Effektivität der Behandlung, wobei allerdings die Eindeutigkeit der DosisWirkungsbeziehung nicht im gleichen Maß wie im Laborexperiment vorhanden ist.

Eine der ersten Studien, in der das Verfahren und seine Begründung dargestellt wurden, erschien bereits 1966 (Redon et al. 1966, Literaturangabe in Hudis \& Münster 1999). Bei der konventionellen Zytostatikagabe wird die Dosis begrenzt durch die toxische Wirkung auf das hämatopoetische (blutbildende) System. Zytostatika schädigen alle sich schnell teilenden Zellen, zu denen auch die des blutbildenden Systems gehören. Bei einer höheren Dosierung, bei der die Zellen des hämatopoetischen Systems geschädigt bzw. zerstört werden, müssen Ersatzmöglichkeiten gefunden werden, um die Bildung der Blutzellen wieder zu ermöglichen. Früher war die HDC-Therapie deshalb immer an eine allogene (Fremdspende) oder autologe (eigene Spende) Knochenmarkstransplantation gebunden.

\footnotetext{
${ }^{36}$ Pinedo (1995) beschreibt die eigenen Forschungsbemühungen und wirft wichtige Fragen auf. Er geht von dem immer wieder zu beobachtendem Phänomen aus, dass Chemotherapien zwar zu Remissionen führen, aber nicht zu längeren Überlebenszeiten. Er verweist auf einen Aspekt bei der Mobilisierung von Stammzellen vor ihrer Entnahme, der hier erwähnt werden soll: Da häufig (auch in dieser Studie) konventionell dosierte Chemotherapiezyklen zur Mobilisierung von Stammzellen verabreicht werden, können diese Dosierungen möglicherweise die Resistenzentwicklung schon vor der eigentlichen HDC „triggern“. Die Gabe von Wachstums- faktoren reicht seiner Ansicht nach für den Gewinn von Stammzellen aus (vgl. Drug Resistance. Illustration Of The Complexity Of Translational Research. Steiner Award Lecture 1995)
} 
Zu Beginn des Einsatzes der HDC war die Anwendung des Verfahrens wegen der hohen Mortalitätsraten, technischen Schwierigkeiten, immensen Kosten und des Mangels an geeigneten Spendern zahlenmäßig sehr beschränkt. Zur Ausweitung kam es in einem ersten Schritt nach der Einführung der autologen Knochenmarkstransplantation und in einem zweiten, als periphere Blutstammzellen gewonnen werden konnten, die mithilfe eines relativ wenig aufwendigen technischen Verfahrens aus dem zirkulierendem Blut entnommen werden. Die gewonnenen Blutstammzellen werden kryokonserviert und 1-2 Tage nach der HDC-Konditionierungstherapie retransfundiert. Durch die HDC werden Blutzellen (Leukozyten, Lymphozyten und Thrombozyten, weniger die Erythrozyten) weitgehend zerstört und die Patienten kommen in die Phase der Aplasie. Hierbei handelt es sich um einen Zustand, in dem wichtige Blutzellen vermindert sind oder fehlen und damit für die Patienten das Risiko von Infekten, Blutungen und anderen Störungen sehr hoch sind. Der Zeitraum, in dem ausreichend neue Blutzellen gebildet werden, gilt als kritisch: Infekte können hier sehr schnell zur Lebensbedrohung werden. Die Aplasiephase dauert zwischen 8-14 Tage und kann durch die Gabe von Wachstumsfaktoren ${ }^{37}$ um 1-3 Tage verkürzt werden (Trümper 1998/99).

Wachstumsfaktoren üben vor allem einen Einfluss auf die schnellere Bildung von Leukozyten aus (Fields et al. 1998). Durch die Stammzellrückgabe (und durch Wachstumsfaktoren) soll die toxische Wirkung auf das Blutsystem kompensiert werden. Bei dieser Therapie limitieren nicht die toxischen Auswirkungen auf das blutbildende System die Dosis, sondern toxische Auswirkungen auf andere Organsysteme. Die Aplasie wird bewusst therapeutisch erzeugt, während alle anderen Organsysteme möglichst wenig und wenn, dann nicht dauerhaft durch die Therapie geschädigt werden sollen.

\subsection{Anwendungsbereich}

Besonders häufig wird die HDC bei den Non-Hodgkin-Lymphomen (NHL) $)^{38}$ und beim Mammakarzinom angewendet, weil hier die Prognose durch konventionelle Chemotherapien in den letzten 20 Jahren nur unwesentlich verbessert werden konnten.

Von den jährlich 55.000 neu diagnostizierten Patienten mit NHL in den USA stirbt die Mehrheit noch immer an dieser Erkrankung, trotz effektiver Standardtherapien (Morri-

\footnotetext{
${ }^{37}$ Wachstumsfaktoren (in den meisten Studien wird G-CSF - Granulozyten-Kolonie als stimulierender Faktor - eingesetzt) beeinflussen die Aktivität der myeloisch hämatopetischen Zellen. Bei einer Zerstörung der Zellen durch Chemotherapie reduzieren sie das Infektions- risiko, indem sie zu einer schnelleren Rekonstituierung des Blutbildes führen. Wachstumsfaktoren werden, je nach Ziel, täglich subkutan gespritzt. Als Nebenwirkung tritt bei einem Teil der Patienten ein dumpfer Knochenschmerz auf, dessen Genese noch nicht geklärt ist.

${ }^{38}$ Beard, Lorrigan \& Sampson (2000) konstatieren, dass die Inzidenzrate von HodgkinLymphomen abnimmt, während sie bei NHL um jährlich 3\% zunimmt. Zu ähnlichen Aussagen kommen auch Monnereau et al. 1998.
} 
son \& Peterson 1999). Bei den hämatologischen Erkrankungen wird HDC eingesetzt u.a. bei akuten und chronischen Leukämien, multiplen Myelomen, aggressiven NonHodgkin und Hodgkin Lymphomen. Bei den hochmalignen Lymphomen ist die Anwendung beschränkt auf Hochrisikopatienten mit einer initial großen Tumormasse, die auf die primäre Chemotherapie nur unzureichend reagiert haben bzw. die Patienten, die ein Rezidiv erlitten haben (Mills et al. 1995; Gianni et al. 1996; Schmitz 1997, Schmitz 1997a; Kaiser et al. 1997; Smith \& Nademanee 1995), also bei den Patientengruppen, die eine besonders ungünstige Prognose haben.

Für die an Mammakarzinom erkrankten Frauen gilt ähnliches: sobald es zu einer regionalen Metastasierung in den Lymphknoten gekommen ist, verschlechtert sich die Prognose deutlich. Ob der medizinische Fortschritt die Mortalität positiv beeinflusst, oder „nur“ zur Verringerung der Morbidität beigetragen hat, ist nach der Datenlage offensichtlich auch für die Experten nicht eindeutig zu beantworten. Unklar bleibt, ob bei steigender Inzidenz verlängerte Überlebenszeiten auf die bessere Früherkennung oder auf die adjuvante Behandlung zurückzuführen sind. Im Konsensusstatement der NIH (National Institute of Health) (2000) wird festgestellt, dass Frauen heute ein besseres Langzeitüberleben haben als jemals zuvor, und zwar dank der systemischen adjuvanten Therapien (Hormontherapie, Chemotherapie): „Chemotherapy has been shown to substantially improve long-term, relapse-free, and overall survival in both premenopausal and postmenopausal women up to the age 70 years with node-positive and node-negative diesease" (S. 5). Es gibt allerdings auch eine Reihe von weniger optimistischen Stellungnahmen. Im „Lancet“ vom Januar 2000 warnen die Herausgeber vor einer allzu optimistischen Sichtweise. Die erwartete Mortalitätsreduktion durch neue Chemotherapien halten sie für vorschnell. Unbestritten ist, dass Behandlungsergebnisse in kontrollierten Studien generell besser ausfallen. Für das Mammakarzinom und seine Behandlung in Deutschland zeichnen die Autoren der Münchener Feldstudie zur Qualitätssicherung in der Onkologie (Engel et al. 2001) ein düsteres Bild: Entgegen den Aussagen von großen Metaanalysen, die von einer Verbesserung der Überlebenszeit zwischen 5 und $10 \%$ sprechen, gibt es für den untersuchten Großraum München in den letzten 20 Jahren keine Verbesserung der Überlebensrate. Wegen eines fehlenden Krebsregisters für Gesamtdeutschland, gibt es nur Aussagen über die untersuchten Modellregionen.

Patientinnen mit mehr als 9 befallenen axillären Lymphknoten gehören zur HochRisikogruppe. Bei 90\% der Patientinnen mit 10 und mehr befallenen Lymphknoten, kommt es innerhalb der 5 postoperativen Jahre zu einem Rezidiv. ${ }^{39}$

\footnotetext{
${ }^{39}$ Die Anzahl der befallenen Lymphknoten ist als Prognosefaktor unbestritten. Problematisch bleibt die Risikoeinschätzung der nodalnegativen Frauen. Auch hier kommt es bei $60-70 \%$ der Patientinnen zum Rezidiv. Kongressbericht Mammakarzinom, Im Focus Onkologie 6/2000.
} 
In Deutschland sterben jedes Jahr etwa 19.000 Frauen an einem Mammakarzinom. Pro Jahr werden ca. 46.000 Neuerkrankungen festgestellt. Die Mortalität/Inzidenz-Rate beträgt damit für Deutschland $40 \%$, d.h. $40 \%$ der neuerkrankten Frauen sterben an ihrem Mammakarzinom. Im internationalen Vergleich steht Deutschland ungleich schlechter da als z. B. die USA mit einer Mortalität/Inzidenz-Rate von $26 \%{ }^{40}$ Für das ungünstigere Ergebnis in Deutschland werden sowohl mangelnde und fehlerhafte Früherkennung als auch fehlende optimale Behandlungsstandards verantwortlich gemacht.

Die Indikation für eine HDC in den USA betraf überwiegend Frauen mit Mammakarzinom, die der Hochrisikogruppe zugerechnet wurden (Hudis \& Münster 1999), da hier die Prognose besonders schlecht ist und konventionelle Chemotherapien daran wenig ändern können. In den neunziger Jahren sind in den USA nach unterschiedlichen Schätzungen 41000 - 44000 Patientinnen mit Mammakarzinom (Mello \& Brennan 2001; Baynes 2001) durch HDC behandelt worden.

Bei soliden Malignomerkrankungen wird HDC als ultima ratio eingesetzt, wenn andere therapeutische Verfahren versagt haben, mehr in der Hoffnung auf Remission als auf kurative Effekte.

\subsection{Effektivität der HDC}

Bei den niedrig malignen Lymphomen bietet die HDC mit autologer Stammzelltransplantation die derzeit einzig erkennbare kurative Perspektive und gilt als Standard bei Patienten unter 60 Jahren (Hiddemann et al. 1997). ${ }^{41}$ Positive Effekte aus Phase-IIStudien $^{42}$ sind von mehreren Autoren beschrieben (u. a. Haas et al. 1994; Rohatiner et al. 1994; Schouten et al. 1994; Bierman et al. 1994).

Einige kleinere randomisierte Studien wurden vorgelegt von Linch et al. (1993), Philip et al. (1995) und Schmitz et al. (1999).

Verlängerte Überlebenszeiten werden von folgende Autoren beschrieben: Lazarus (1998), Fields et al. (1998), Vahdat \& Antman (1997), Linch et al. (1993), Philip et al. (1995) und Schmitz et al. (1999).

Die Ergebnisse bei den Lymphomen sind nicht eindeutig. De Groot et al. (1995) finden in ihrer randomisierten Studie mit NHL Patienten keinen Überlebensvorteil.

${ }^{40}$ Die Angaben sind aus dem Kongressbericht „Mammakarzinom“, Focus Onkologie, 6/2000.

${ }^{41}$ Der derzeitige Stand wird in einem Review von Beard et al. 2000 beschrieben.

42 Die Phasen der Studien sind wie folgt definiert:

Phase-I-Studie: Ausschließlich zur Bestimmung der Toxizität

Phase-II-Studie: Effektivitätsvergleich und Toxizität

Phase-III-Studie: Prospektiv randomisierte Studie zur Effektivitätsermittlung 
Auch bei niedrig malignen NHL stehen Ergebnisse aus prospektiv randomisierten Studien noch aus: „[...] there is a problematic lack of randomized phase III trials that would aid in answering important questions regarding the role of transplantation in these disease processes." (Morrison \& Peterson 1999, S. 84) Eine vorsichtige Würdigung versucht Sweetenhamn (2000) und hofft auf eine Klärung der Effektivitätsfrage durch die noch laufenden Studien.

In Bezug auf die Wirksamkeit beim Mammakarzinom gab es in der Planungsphase dieser Studie optimistische Hinweise hinsichtlich der Überlegenheit der HDC auf die rezidivfreie Zeit und das Gesamtüberleben. Einige Autoren sahen in der HDC die Chance, einen Durchbruch bei den bisher enttäuschenden Prognosen zu erreichen.

Bei Crown (1995) findet sich eine Übersicht über die Studien, bei denen eine positive Dosis-Wirkungsbeziehung beschrieben wurde. Der Autor verweist gleichzeitig auf Studien, die andere Faktoren als ebenso wichtig ausmachen: Dosis pro Kurs, Gesamtdosis, Behandlungsintervalle.

In einer der ersten deutschsprachigen Übersichten geht Frickhofen (1993) davon aus, dass, wegen der verbesserten technischen Möglichkeiten und der Senkung der Mortalität nahezu auf das Niveau konventioneller Chemotherapien sowie des Einsatzes von Wachstumsfaktoren, die HDC in Zukunft in großem Umfang ambulant durchgeführt werden kann. Er warnt aber auch vor einer Überschätzung und diskutiert die Vor- und Nachteile autologer vs. allogener Transplantationen. ${ }^{43}$

Der Stand der Forschung in Bezug auf die Effektivität der HDC wie er in Deutschland gesehen wurde, findet sich in einer Übersicht von Nitz (2000).

Tabelle 7: Phase-III-Studien und Phase-II-Studien zur adjuvanten HDC des Mammakarzinom (S.214)

\begin{tabular}{|l|l|l|l|}
\hline Autor & Induktion & HDC & Kontrolle \\
\hline $\begin{array}{l}\text { Peters } \\
\text { (Phase 2) }\end{array}$ & 5 Jahre 71\% & 5 Jahre 32\% & signifikant \\
\hline $\begin{array}{l}\text { Gianni } \\
\text { (Phase 2) }\end{array}$ & 5 Jahre 57\% & 5 Jahre 41\% & signifikant \\
\hline $\begin{array}{l}\text { Rodenhuis } \\
\text { (Phase 2) }\end{array}$ & 4 Jahre 54\% & 4 Jahre 54\% & nicht signifikant \\
\hline $\begin{array}{l}\text { Peters (n=783) } \\
\text { Phase 3) }\end{array}$ & 36 Monate 80\% & 36 Monate 72\% & nicht signifikant \\
\hline $\begin{array}{l}\text { Bergh (n=525) } \\
\text { Phase 3) }\end{array}$ & 36 Monate 60\% & 36 Monate 68\% & nicht signifikant \\
\hline $\begin{array}{l}\text { Bezwoda (n=154) } \\
\text { (Phase 3) }\end{array}$ & 5 Jahre ca. 75\% & 5 Jahre ca. 31\% & nicht signifikant \\
\hline
\end{tabular}

${ }^{43}$ Frickhofen merkt an, dass bei autologer Transplantation der GvL Effekt (Graft versus Leukemia effect), der bei allogenem Transplantat das Wachstum von Tumorzellen kontrollieren kann, und die „immune surveillance" fehlen.

Es besteht ausserdem die Gefahr, dass Tumorzellen retransfundiert werden. Technisch gibt es mittlerweile Reinigungsverfahren, die das verhindern können. In kaum einer der Studien werden dazu Anmerkungen gemacht. 
In dieser Übersicht sind die randomisierten Studien und die Nachbeobachtungszeiträume angeführt. Die Interpretation von Nitz stützt sich auf die letzte Spalte der Tabelle: von den 6 Studien ergeben nur zwei einen Überlebensvorteil der HDC im Vergleich mit konventioneller Chemotherapie. Die Einwände von U. Nitz gegen eine vorschnelle Interpretation der bisherigen Ergebnisse (Vergleichbarkeit ist erschwert, da die konventionellen Therapien in ständiger Weiterentwicklung sind) sind schwer nachzuvollziehen. Bessere Wirkungen konventioneller Therapie machen einen Wirkungsnachweis der HDC noch schwieriger. Auch zur Frage der Wirksamkeit beim metastasierten Mammakarzinom gibt es mittlerweile einige abgeschlossene Studien. Die aufschlussreichste ist die von Stadtmauerer et al. (2000). Für diese Studie wurden nur die Patientinnen ausgewählt, die mit einer dokumentierbaren partiellen oder kompletten Remission auf eine 4-6 Zyklen umfassende, konventionell dosierte Chemotherapie reagiert hatten. Bei diesen Patientinnen wurde davon ausgegangen, dass eine Chemosensitivität angenommen werden konnte. Verglichen wurden die HDC-Gruppe ( $\mathrm{N}=110)$ mit der konventionell weiterbehandelten Gruppe $(\mathrm{N}=89)$ in Bezug auf Gesamtüberleben und krankheitsfreies Überleben nach 3 Jahren. Die Autoren verneinen eindeutig den Überlebensvorteil. Sie dokumentieren die wesentlich stärker ausgeprägten Nebenwirkungen der HDC. Unter Bezugnahme auf diese Ergebnisse folgert Lippmann (2000) in den editorischen Bemerkungen der gleichen Ausgabe des New England Journal: "We should now acknowledge that, to a reasonable degree of probability, this form of treatment for women with metastatic breast cancer has been proved to be ineffective and should be abandoned in favor of well-justified alternative experimental approaches" (S. 1119-1120).

Der Bericht über das 36. ASCO Meeting (American Society of Clinical Oncology vom 20. - 23. Mai 2000) führt nur noch 4 laufende Studien mit HDC beim Mammakarzinom an. In allen Studien wird jetzt Taxotere (eines der neueren zytostatischen Medikamente) in Kombination mit anderen Substanzen verwendet. Eine der Studien beim fortgeschrittenen Mammakarzinom untersucht den Effekt von Taxotere im Vergleich mit anderen Regimes zur Stammzellmobilisation (Borges et al. 2000). Eine Phase-I-Studie untersucht die Toxizität von Taxotere/Melphalan/Carboplatin bei einer gemischten Gruppe mit einem überwiegendem Anteil von Mammakarzinompatientinnen (Nieto et al. 2000). Eine Phase-II-Studie untersucht Hochrisikopatientinnen mit Mammakarzinom in der adjuvanten Situation. Bislang lässt sich nur berichten, dass 58 von 60 Patientinnen nach 8 Monaten rezidivfrei sind (Martinelli et al. 2000). Eine Phase-II-Studie mit sequentieller HDC beim inflammatorischen Mammakarzinom untersucht den Einfluss der Therapie auf die Responserate. Die Autoren berichten „exzellente“ Responseraten, 
für eine Aussage über krankheitsfreies Überleben sei es jedoch noch zu früh (Palangie et al. 2000).

Äußerst ernüchternde Ergebnisse ihrer Studie präsentieren Braun et al. (2000): Sie untersuchten 59 Patienten mit Mammakarzinom der Hoch-Risiko-Gruppe (> 4 LK oder inflammatorische) vor der HDC auf das Vorhandensein von Mikrometastasen im Knochenmark. Die Untersuchung erfolgte immunzytochemisch mit dem monoklonalen Antizytokeratinantikörper (CK). Vor der HDC wurden 29\% CK-positive Patientinnen ermittelt. Nach der Therapie mit Taxanen und Antrazyklinen ergab sich noch ein Anteil von 48,3\% CK-positiven Patientinnen. Von den Patientinnen, die vorher CK-negativ waren, waren nach der Therapie 36,7\% CK-positiv. Dass die Gegenwart der CK-positiven Zellen nach der Therapie mit einer schlechteren Prognose assoziiert war, entsprach den Erwartungen.

Eine aktuelle Stellungnahme zur adjuvanten Hochdosistherapie beim Mammakarzinom findet sich im NIH Consensus Statement (2000): „There is currently no convincing evidence to demonstrate that more dose-intensive treatment regimens (e.g., high-dose chemotherapy with peripheral stem cell support) results in improved outcomes to the administration of polychemotherapy program at standard dose levels" (S. 5).

\subsection{Kritische Anmerkungen zur HDC}

Selbstverständlich ist die Frage nach der Wirksamkeit der wichtigste Aspekt, den die Kritiker der HDC anführen. Im vorhergehenden Abschnitt wurde gezeigt, wie schwierig sich die evidenzbasierte Beurteilung gestalten kann. In renommierten Fachzeitschriften gab es, parallel zu positiven Darstellungen, schon seit Jahren warnende Hinweise. In fast allen Veröffentlichungen der letzten Jahre zum Thema HDC wird auch von denjenigen, die eine sehr optimistische Sichtweise vertreten, gefordert, dass die Behandlung ausschließlich in methodisch gut fundierten, prospektiv randomisierten Studien durchgeführt werden soll (Hudis \& Münster 1999; Van der Wall et al. 1995; Kennedy 1995). Auch Ende 2001 liegen eindeutige Belege für die HDC bei NHL noch nicht vor. Allerdings bietet sich für die Behandlung beim Mammakarzinom und NHL mit HDC ein unterschiedliches Bild:

Beim Mammakarzinom geht die Tendenz dahin, die Wirksamkeit zu verneinen. Schockierend war für die Fachöffentlichkeit, dass ausgerechnet die Studie (Bezwoda et al. 1995), die als methodisch vorbildlich galt und die als einzige prospektiv randomisierte Studie einen eindeutigen Überlebensvorteil für Mammakarzinom-patientinnen erbracht 
hatte - und dadurch für viele Folgestudien als Begründung diente - sich fünf Jahre später als Fälschung herausgestellt hat. ${ }^{44}$

2001 sind 3 Reviews/Metaanalysen veröffentlicht worden, deren Autoren (Mello \& Brennan; Tan \& Swain; Bergh et al.) die eindeutige Aussage treffen, dass die randomisierten Studien nur den Schluss zulassen, dass HDC konventionellen Therapien beim Gesamtüberleben nicht überlegen ist. Baynes (2001) kommt in seinem Review zu keiner eindeutigen Aussage und Aapro (2001), der sich in seinem Artikel mit der Stellungnahme der deutschsprachigen Gynäkologen, Konsensusmeeting St. Gallen, beschäftigt, hält die Frage ebenfalls noch für offen. Mittlerweile ist auch eine randomisierte deutsche Studie von Schneeweiss et al. (2001) publiziert worden. Auch hier findet sich kein Überlebensvorteil.

Sieht man einmal von den zögerlichen Stellungnahmen von Baynes und Aapro ab und betrachtet man die klinische Praxis in den letzten Monaten, dann lässt sich nur folgern, dass HDC beim Mammakarzinom sich als nicht effektiv in Bezug auf verlängertes Überleben erwiesen hat.

Die Stellungnahmen zur Wirksamkeit bei NHL sind wie folgt:

Von den 4 veröffentlichten Reviews/Metaanalysen 2001 kommen alle zum gleichen Ergebnis: Die Ergebnisse aus randomisierten Studien sind widersprüchlich und lassen noch keine eindeutigen Schlussfolgerungen zu (Brandt et al.; Kimby et al.; Mink \& Armitage; Tan \& Bartlett).

Neben den Fragen nach der Wirksamkeit wurden allerdings auch Fragen nach den tumorbiologischen Begründungen für diesen Ansatz gestellt. „Must We Kill to Cure?” Diese provokative Frage (Schipper et al. 1995) zielt darauf ab, die Wirkungsvorstellungen und Modelle derjenigen zu hinterfragen, die HDC als zukunftsweisende Therapieoption ansehen. Die Autoren kritisieren das „killing paradigma“ und fordern ein Regulationsmodell, welches Krebs als einen dynamischen, maladaptiven Prozess beschreibt, der im Träger entsteht, sich konstant entwickelt und potentiell reversibel ist. Sie stellen die zentrale Annahme des „Killing-Modells“ infrage: dass es notwendig und möglich ist, die letzte Tumorzelle zu vernichten, um eine Heilung zu erreichen. Die Autoren verweisen u.a. auf die klinischen Ergebnisse von erhöhter Behandlungsintensität der cytotoxischen Therapien, die zwar zu erhöhten Remissionsraten, nicht aber zur Verlängerung

\footnotetext{
${ }^{44}$ In „Lancet“ vom 18. März 2000 beschäftigen sich mehrere Stellungnahmen mit dem Fälschungsskandal. R. Horton stellt die Frage: „Was kommt nach Bezwoda?“ Er spricht sich aus für ein internationales Register, dass eine zeitnähere Datenkontrolle wichtiger randomisierter Studien ermöglicht, um negative Folgewirkungen falscher (und gefälschter) Ergebnisse schneller aufzudecken (Commentaries, Lancet, S.942-945, Vol.355, 2000).

Die Öffentlichkeit wurde über die Fälschung informiert in einem Artikel der New York Times von

D. Grady: Breast cancer researcher admits falsifying data. Feb 5, 2000:9.
} 
der Überlebenszeit geführt haben, von wenigen Ausnahmen abgesehen. Schipper et al. (ebenda) fordert aufgrund der Daten ein Paradigma, das auf zellulärer und interzellulärer Kommunikation und biologischer Kontrolle beruht und sich radikal von dem alten Verständnis viraler und mikrobiotischer Erkrankungen unterscheiden muss.

Während z. B. bei der Vernichtung eines antibiotikasensiblen Bakteriums durch eine Antibiose die Höhe der Dosierung das entscheidende Kriterium für die effektive Vernichtung ist, spielen bei der Tumorzellvernichtung zum größten Teil unbekannte Faktoren eine wesentlich bedeutsamere Rolle. Das „Killing-Paradigma“ sehen die Autoren als überholt an.

Hochdosischemotherapie ist durch beeindruckende technologische Verbesserungen möglich geworden. Eine Analyse für Nord-Amerika ergab eine Reduzierung der Mortalitätsrate (Todesfälle nach Therapie innerhalb von 100 Tage) von 20\% 1989, auf $5 \%$ 1995 (Antman et al. 1997). Diese beeindruckenden Verbesserungen dürfen aber nicht als Ersatz für den Effektivitätsnachweis herhalten.

Hossfeld (1998) kommt zu der abschließenden Bewertung, dass sich die HDC bei Patienten mit Lymphomen und soliden Tumoren derzeit zwischen zwei Polen bewegt: Optimisten behaupten, „mehr sei besser“, die Skeptiker sprechen von „der letzten grünen Wiese der Chemotherapie“[...] „Fahren wir fort, die Patienten im Rahmen von monomanen Phase-II-Studien oder in Heilversuchen mit Hochdosis-Therapie zu behandeln, werden wir die Antwort nie erfahren“" (S.4).

Fakt ist, dass trotz der Forderung HDC nur innerhalb von Studien anzuwenden, die Praxis anders aussieht. Die Gründe, warum randomisierte Studien seltener als geplant durchgeführt werden können, sind vielschichtig. Im onkologischen Feld kommt eine nicht zu unterschätzende spezifische Problematik hinzu: Die ungünstige Prognose, um die viele Patientinnen und Patienten heute wissen, setzt eine Dynamik in Gang, das „Neueste“ von Ärzten einzufordern. Allein die Chance auf (geringfügige) prognostische Verbesserungen, die häufig genug in unseriösen Berichten der Medien publiziert werden, lassen die „Nachfrage“ nach diesen Therapien in die Höhe schnellen, und die Behandlungs- und Forschungspraxis passt sich dem an bzw. leistet dem unter Umständen Vorschub. Prospektiv randomisierte Studien sind dann nur noch schwer durchführbar, da alle Beteiligten unter hohem Handlungsdruck stehen und Patienten in ihrer Angst Therapien einfordern, von denen sie gehört haben und die sie als „vielversprechend“ bewerten. Ärzte und Patienten sind dann gefangen im gemeinsamen Dilemma onkologischer Forschung und Therapie: Aus therapeutischer Sicht besteht der Wunsch, die Situation der Patientinnen und Patienten mit prognostisch ungünstig eingeschätzten Krankheitsverläufen zu verbessern und nicht auf Effektivitätsnachweise 
aus prospektiv randomisierten Studien warten zu müssen. Auf Patientenseite besteht das Verlangen, nach jedem Strohhalm zu greifen, auch dann, wenn eine Wirksamkeit noch nicht ausreichend belegt ist und unter Umständen sogar negative Auswirkungen hat.

Das Zusammenwirken beider Seiten verhindert dann geradezu eine wissenschaftliche Begründung für oder gegen den Nutzen der neuen Therapie.

Nicht übersehen werden darf die große Not der Patienten angesichts der bedrohlichen Perspektive. Ein Patient hat dies stellvertretend für andere folgendermaßen formuliert: „Selbst eine Chance von einem Prozent ist besser als gar keine Chance." Wie hoch der „Preis“ ist, spielt dann keine Rolle mehr.

Im nächsten Abschnitt wird auf die physischen und psychischen Auswirkungen von Krankheit und Therapie eingegangen 


\section{Beeinträchtigungen der LQ durch Krankheit und HDC}

Bevor die besonderen Belastungen durch HDC erläutert werden, beschäftigt sich der folgende Text mit den Belastungen, die generell auf Patienten mit Krebserkrankungen zukommen können.

\subsection{Emotionale Belastungen}

Angst ist das alles überschattende Gefühl, das auf den „Sturz aus der normalen Wirklichkeit“ (Gerdes 1985) folgt. Der „biographische Bruch“, den die Erkrankung kurzfristig oder dauerhaft verursacht, löst dieses allumfassende Gefühl aus, das in Selbstschilderungen von Patienten immer wieder beschrieben und in empirischen Befunden zu emotionalen Reaktionen auf die Erkrankung an erster Stelle genannt wird. Unabhängig von der medizinischen Prognose sehen sich Patienten mit dem möglichen Tod konfrontiert, mit tiefgreifenden, eventuell verstümmelnden Eingriffen und Stigmatisierungen, mit Schmerzen, mit dem Ausgeliefertsein an das medizinische System, mit familiären und beruflichen Rollenveränderungen, mit der Infragestellung des Selbstbildes.

Dazu können Schuldgefühle kommen, die sich auf die Vergangenheit beziehen und die Angst vor einer Bedrohung der Zukunftsperspektive.

Negative Assoziationen werden mobilisiert. An Krebs zu erkranken bedeutet für die meisten Menschen, von der am meisten gefürchteten Erkrankung betroffen zu werden. Trotz oder wegen der Allgegenwart der Thematik in der Presse steigt die Krebsangst in der Bevölkerung kontinuierlich an (Verres 1986). Daneben scheint ein nicht unbeträchtlicher Teil der Patientinnen und Patienten im familiären und sozialen Umfeld Erfahrungen mit Krebserkrankungen gemacht zu haben, die oft negativ geprägt sind. In einer älteren Studie (Jonasch 1985, zitiert nach Schlömer-Doll \& Doll 2000) geben 80\% der befragten Studienteilnehmer an, Krebserkrankungen im Familien- und/oder Bekanntenkreis miterlebt zu haben. Von diesen Menschen hatten aber nur 11,9\% eine Heilung erlebt. Negative Erfahrungen überwiegen nicht nur, sie haben auch nachhaltige Auswirkungen. Auch in der klinischen Praxis zeigt sich, dass Patienten mit negativen Erfahrungen in viel stärkerem Maß als andere um die Kontrolle ihrer Ängste ringen müssen. Ängste sind damit in erster Linie auf reale Belastungen gerichtet. Neben der Angst spielen depressive Reaktionen eine herausragende Rolle, insbesondere wenn die Krankheit einen chronischen Verlauf nimmt und Patienten wissen, dass sie vermutlich nicht geheilt werden können. 


\subsection{Krebserkrankung und psychische Störungen}

Es ist unbestritten, dass Krebserkrankungen „psychische Störungen“ auslösen können. Jedoch sind die Befunde in Bezug auf Art und Häufigkeit sehr unterschiedlich. Wichtig ist, dass der größere Teil der Krebspatientinnen und -patienten die Erkrankung ohne psychische Störungen bewältigt.

In der Praxis ist es außerordentlich wichtig, Patientinnen und Patienten nicht durch zusätzliche psychiatrische Etikettierungen zu stigmatisieren. Leider gibt es bis heute kein Klassifizierungssystem, das dem onkologischen Bereich gerecht würde. ${ }^{45}$ Diese Lücke ist für die praktische Arbeit mit Krebspatienten nicht zu unterschätzen. Diejenigen, die mit onkologischen Patienten arbeiten, registrieren die psychischen Ausnahmezustände, die zum Teil sehr ausgeprägten Emotionen, scheuen sich aber oft, diese mit psychiatrischen Etikettierungen zu versehen, wie es zur Rechtfertigung von Interventionen vorgeschrieben ist. Solange kein spezifisches Klassifizierungssystem besteht, muss auf die von den meisten Patienten als kränkend empfundenen Klassifikationen aus der Psychiatrie zurückgegriffen werden. Nur so kann die psychosoziale Unterstützung und eventuelle psychotherapeutisch/ psychiatrische Behandlung zur Verfügung gestellt werden.

Schwierigkeiten treten bei der Diagnostik von Angst und depressiven Störungen bzw. bei der Kategorisierung als „Anpassungsstörung“ an die Erkrankung auf. Schwierigkeiten deshalb, weil Verlustgefühle und Traurigkeit, Angst und Verzweiflung realitätsangemessen und „normal“ sind und die Abgrenzung zum psychopathologischen Bereich unscharf und damit letztendlich fehlerhaft bleibt. Wann hört eine „normale Trauerreaktion" auf und wo beginnt die Depression? Wann ist Angst angemessen und einfühlbar und wo mischt sie sich mit neurotischen Ängsten und wird behandlungsbedürftig? Leichter zu diagnostizieren sind dagegen die hirnorganisch verursachten emotionalen Störungen.

Trotz wiederholt nachgewiesener hoher Prävalenzraten gibt es nach wie vor kaum systematische Diagnostik und wenig Angebote der psychosozialen und psychotherapeutischen Unterstützung im Akutkrankenhaus.

Hautzinger \& Janssen (1995) führen die folgenden „psychischen Störungen“ bei Krebskranken in ihrer Übersicht auf:

1. Psychische Vorerkrankungen:

\footnotetext{
${ }^{45}$ Angelino \& Treisman (2001) unterscheiden Major Depression und Demoralisation. Demoralisation ist die normale psychologische Antwort auf einen bedrohlichen Stressor. Demoralisation wird gleichgesetzt mit Trauerreaktion oder reaktiver Depression. Demoralisation spricht an auf supportive Psychotherapie, Hoffnung und therapeutischen Optimismus, während Versuche medikamentöser Beeinflussung wenig erfolgreich sind.
} 
- Latente psychische Erkrankungen (spezifische Konfliktlabilität, Persönlichkeitseigenheiten) können durch das Krebsleiden bzw. dessen Folgen manifest werden.

- Manifeste psychische Erkrankungen bestanden zeitlich vor dem Krebsleiden und haben Einfluss auf die Anpassung an die Krankheitssituation und deren traumatisierende Wirkung.

2. Psychische Begleiterkrankungen, ausgelöst durch ein Krebsleiden bzw. dessen Therapie bei vorbestehender (unspezifischer) Vulnerabilität.

3. Psychische Folgeerkrankungen: diese Krankheitsbilder wären ohne Belastung nicht entstanden, sie sind durch diese verursacht, nicht nur ausgelöst.

3.1. Psycho-vegetative Begleiteffekte bzw. Nebenwirkungen bei Chemotherapie und Strahlenbehandlung.

3.2. Unspezifische Reaktionen auf schwere Belastungen als Anpassungsstörungen (ICD 43),

- akute Belastungsreaktion (Krisenreaktion, psychischer Schock, ICD 43.0)

- posttraumatische Belastungsstörung (traumatische Neurose, ICD 43.1)

- Anpassungsstörungen (ICD 43.2 bis 43.9 - individuelle Disposition oder Vulnerabilität spielt eine größere Rolle als bei 43.0 und 1)

- (posttraumatische) Persönlichkeitsstörung nach Extrembelastung (ICD 62)

3.3 Bei speziellen Tumorlokalisationen zu erwartende psychosoziale Beeinträchtigungen und Störungen:

- Beeinträchtigte Sexualität bei Mamma-Ca, Prostata-Ca, Hodentumoren etc.

- Soziale Isolation bei Gesichts- und Kehlkopftumoren, etc.

4. Störungen in der Primärgruppe (Familie).

Zur Diagnostik von affektiven Störungen stehen die psychiatrischen Klassifikationssysteme, zur Ergänzung bzw. zu Forschungszwecken stehen lediglich Fragebogeninstrumente zur Verfügung. Die am weitesten verbreiteten Klassifikationssysteme sind das DSM III und, für den deutschsprachigen Raum, die ICD 10 (internationale Klassifikation psychiatrischer Störungen).

Die ICD 10 (Dilling et al. 1993) bezieht sich nicht auf spezifische Entstehungstheorien, sondern verwendet deskriptiv phänomenologische Einteilungskriterien.

Die am häufigsten verwendeten Kategorien der ICD10 für die Diagnostik und, daraus abgeleitet, die Einleitung von psychotherapeutischen und/oder psychopharmakologischen Interventionen sind die Folgenden:

F 32 depressive Episoden

„[...] gedrückte Stimmung, Interessenverlust, Freudlosigkeit, Verminderung des Antriebs, Verminderung der Energie führt zu erhöhter Ermüdbarkeit und Aktivitätseinschränkung."(S.139) 
Andere häufige Symptome sind:

1. Verminderte Konzentration und mangelnde Aufmerksamkeit

2. Vermindertes Selbstwertgefühl und geringes Selbstvertrauen

3. Schuldgefühle und Gefühle von Wertlosigkeit

4. Negative und pessimistische Zukunftsperspektiven

5. Suizidgedanken, erfolgte Selbstverletzung oder Suizidhandlungen

6. Schlafstörungen

7. Verminderter Appetit

Angst bei einer Krebserkrankung ist zunächst Realangst, die sich aus verschiedenen Quellen speist. Auch bei günstiger Prognose bleibt ein Teil Ungewissheit über den weiteren Verlauf. Die Angst, ausgelöst durch die Krebserkrankung, ist schwer zuzuordnen. Nicht kontrollierbare Angst kann mit der Kategorie F 1 „generalisierte Angststörung“ beschrieben werden. Unter den diagnostischen Leitlinien wird genannt:

„Der Patient muss primäre Symptome von Angst an den meisten Tagen, mindestens mehrere Wochen [...] aufweisen. In der Regel sind folgende Einzelsymptome festzustellen:

1. Befürchtungen (Sorge über zukünftiges Unglück, Nervosität, Konzentrationsschwierigkeiten usw.)

2. Motorische Spannung (Körperliche Unruhe, Spannungskopfschmerz, Zittern, Unfähigkeit sich zu entspannen)

3. Vegetative Übererregbarkeit (Benommenheit, Schwitzen, Tachykardie oder Tachypnoe, Oberbauchbeschwerden, Schwindelgefühl, Mundtrockenheit etc.)“ (S. 162)

Die Kategorie der Anpassungsstörung mit der Spezifizierung durch die vorherrschenden Symptome ist häufig zur Diagnostizierung geeignet. Sie setzt die betroffenen Patienten nicht noch einer zusätzlichen Stigmatisierung aus, weil deutlich gemacht werden kann, dass es sich um eine Reaktion auf eine existentielle Ausnahmesituation handelt. Die meisten Patienten fühlen sich bereits durch die Krebserkrankung stigmatisiert; Schamgefühle sind bei vielen vorhanden, auch wenn sie rational als unsinnig bewertet werden. Wenn eine weitere „Beurteilung“ als ängstlich, depressiv, labil etc. erfolgt oder als solche erlebt wird, kann das verständlicherweise leicht zur Ablehnung von psychotherapeutischen Unterstützungsangeboten führen.

Bei der Spezifizierung dieser Kategorie wird „ein außergewöhnlich belastendes Lebensereignis, das eine akute Belastungsreaktion hervorruft oder eine besondere Veränderung im Leben, die zu einer anhaltenden, unangenehmen Situation geführt hat [...]" (S.167), in Zusammenhang gebracht mit den von Patienten empfundenen Schwierigkeiten.

Unter anderem kann sich die Belastung äußern in depressiver Stimmung, Angst, Besorgnis und dem Gefühl, unmöglich zurechtzukommen, nicht vorausplanen zu können. 
Die Belastung kann so ausgeprägt sein, dass die Patienten die alltäglichen Routineleistungen nicht mehr oder nur noch eingeschränkt erbringen können.

Wie bereits bemerkt, führt der Einbruch einer Krebserkrankung zu einer Lebenskrise, einem Bruch in der Biographie, der massive Angst- und Bedrohungsgefühle mit sich bringt. Verschiedene Autoren vergleichen die psychischen Belastungen mit denen eines Verfolgungstraumas. Von jedem Erkrankten werden enorme Anpassungsleistungen verlangt. Die oben definierten „Anpassungsstörungen“ wären ohne den auslösenden Stressor „Krebserkrankung“ nicht aufgetreten.

Selten wird eine Posttraumatische Belastungsstörung diagnostiziert. In der Übersicht von Schmitt (2000) sind die bisherigen Studien zur Posttraumatischen Belastungsstörung bei Krebserkrankung aufgelistet. Bisher liegen nur wenige Querschnittstudien, meist mit geringen Fallzahlen, vor. ${ }^{46}$

Wie aus der obigen Beschreibung der vitalen depressiven Symptome abzuleiten ist, wird die Differentialdiagnostik zusätzlich erschwert durch die Unmöglichkeit der Zuordnung der Symptome (Schlaflosigkeit, mangelnder Appetit, sexuelle Inappetenz, Erschöpfung und Antriebslosigkeit) zur psychopathologischen Symptomatik.

Gerade diese Symptome können durch die Erkrankung selber oder durch deren Therapie verursacht werden. Eine ganze Reihe von Medikamenten, die in der Krebstherapie verwendet werden, haben nachweislich depressive oder stimmungsverändernde Nebenwirkungen (z. B. Kortikosteroide, Vincristin, Interferon u.a. Eine ausführliche Liste siehe bei Zettl \& Hartlapp1997). Holland (1990) verweist, abgesehen von der direkten Hirnmetastasierung, auf weitere physische Faktoren, die zu Depressivität führen können: Neben paraneoplastischen Syndromen können auch metabolische Encephalopathien auftreten, die zu Veränderungen führen. Ungeklärt ist, warum Patienten mit Pankreaskarzinom vermehrt unter Depressionen leiden.

Das unter onkologischen Patientinnen weit verbreitete „Fatigue-Syndrom“ hat eine enge Beziehung zu depressiven Entwicklungen (Aass et al. 1997) (siehe auch Kap. 5.4 Fatigue).

Zunehmend gibt es Dokumentationen von zentral-nervösen Nebenwirkungen der chemotherapeutischen Behandlung. Unter der Behandlung klagen Patienten häufig über Konzentrations- und Gedächtnisstörungen oder generell über intellektuelle Leistungs-

\footnotetext{
${ }^{46}$ Nach den eigenen Erfahrungen der Verfasserin ist mit dem Auftreten einer Posttraumatischen Belastungsstörung ( $F 43.1$ nach ICD-10) weit weniger häufig zu rechnen als mit dem Auftreten von Depressiver Störung oder einer Angstsymptomatik.

Cordova et al. (2000) überprüfen faktorenanalytisch ob die PTSD-Checkliste sinnvoll auf onkologische Patienten anwendbar ist. Im Vergleich mit den DSM-IV Kriterien für das PostTraumatische-Syndrom fällt die Übereinstimmung eher unzureichend aus.
} 
einbußen (siehe auch Kap. 6.5.2). Eine reaktive depressive Entwicklung aufgrund der wahrgenommenen Leistungseinschränkungen ist also durchaus auch denkbar.

\subsection{Empirische Befunde}

\subsubsection{Diagnostische Probleme}

Studien zeigen, dass erhebliche Schwierigkeiten bestehen, depressive Störungen bei onkologischen Patienten zu diagnostizieren (Fritsche et al. 1998; Hardman et al. 1989; Derogatis 1983). Ärzte lassen sich bei ihrer Einschätzung von emotionalen Störungen zumeist von offensichtlichen Äußerungen, wie Traurigkeit, Weinen, wechselnden emotionalen Zuständen leiten. Außerdem fließen in die Beurteilung medizinische Aspekte ein wie der Performanzstatus, das Krankheitsstadium und die Tumorgröße. Wichtige Gedanken und Gefühle, die sich auf Schuld, Suizidgedanken, Sinnlosigkeit, Leere und Hoffnungslosigkeit beziehen, werden wenig beachtet. Differentialdiagnostisch ist die Exploration dieser Gefühle wichtig.

Als Gründe für die Nichterkennung emotionaler Probleme werden angeführt:

1. Die depressive Symptomatik kann auch durch den physischen Zustand bestimmt werden oder durch andere nicht-psychische Faktoren ( Bukeberg et al. 1986; Berard et al. 1996; Valente \& Saunders 1997; Pinder et al. 1993; Valentine et al. 1998; Passik et al. 1998)

2. Es besteht die Schwierigkeit, Depression von normaler Trauerreaktion abzugrenzen (Bukeberg et al., ebenda.)

Maguire (1990) unterscheidet Barrieren auf Seiten der Patienten von denen auf Seiten der Professionellen, um die Gründe für die „Hidden Morbidity“ zu erklären. Patienten glauben, dass ihre psychologischen und sozialen Probleme die notwendige Konsequenz aus Erkrankung und Therapie sind, gegen die nichts getan werden kann. Das führt dazu, dass Patienten es nicht für legitim halten, auch noch über psychische Probleme zu klagen. Weniger als $1 / 4$ der Patienten mit emotionalen Problemen spricht spontan über diese Probleme. Die anderen wollen das Behandlungsteam nicht verärgern und fürchten eine Stigmatisierung, wenn sie ihre Probleme preisgeben. Dazu kommt der oft vorhandene Zeitdruck seitens der Ärzte, der die Patienten veranlasst, sich in der Arzt-Patient-Kommunikation auf physische Aspekte der Erkrankung und des Überlebens zu beschränken. Seitens der Professionellen macht Maguire folgende wesentliche Barrieren aus: die Vermeidung direkten Nachfragens und der Gebrauch von dis- 
tanzschaffenden Verhaltensweisen, um Zeit zu sparen und um sich vor schwierigen Themen zu schützen. ${ }^{47}$

10 Jahre später kommen Stiefel et al. (2001) zum gleichen Ergebnis. Depression scheint bei todkranken Patienten für unausweichlich gehalten zu werden. Sie folgern: „It is unfortunate that health care professionals and the public agree that depressive illness should be treated in the physically healthy but remain skeptical about the treatment of depression in patients with severe somatic diseases" (ebenda, S. 479).

Abschließend ist zu sagen, dass es in der medizinischen Praxis gegenwärtig zwei unterschiedliche Tendenzen gibt, wie „Depression“ erfasst wird:

- Ärzte tendieren dazu, sich auf generelle Phänomene (z. B. Traurigkeit, Weinen) zu beziehen

- $\quad$ psychiatrisch/psychotherapeutisch ausgebildete Therapeuten bevorzugen spezifisch diagnostische Kriterien

\subsubsection{Prävalenz affektiver Störungen in der Onkologie}

In den epidemiologischen Studien zur Bestimmung von Prävalenzraten affektiver Störungen wurden unterschiedliche Methoden und Instrumente verwendet. Es wurden verschiedene Erkrankungsgruppen in verschiedenen Krankheitsstadien einbezogen. Die Datenerhebung fand im ambulanten oder stationären Setting statt. Deshalb verwundert es nicht, dass die Angaben in den verschiedenen Arbeiten erheblich differieren. Die Angaben reichen von einem Minimum von ca. $15 \%{ }^{48}$ bis zu einem Maximum von $60 \%{ }^{49}$ und sind in Stichproben mit palliativen Patienten noch größer. ${ }^{50}$ Eine Übersicht über Depression in der palliativen Behandlung wurde von der European Assoziation for Palliative Care (EAPC) zusammengestellt (Stiefel et al. 2001). Die Arbeitsgruppe konstatiert, dass fehlende Entdeckung und fehlende Behandlung nach wie vor ein großes Problem in dieser Patientengruppe sind.

So unterschiedlich die Zahlen auch sein mögen, bleibt doch festzuhalten, dass die Anzahl psychischer Störungen bei onkologischen Patienten verglichen mit der Anzahl in der Allgemeinbevölkerung (Schepank 1987) weit höher liegt, jedoch nach Härter

\footnotetext{
${ }^{47}$ Das gilt nicht nur für Ärzte, die in der Onkologie arbeiten, sondern auch für psychosoziale Mitarbeiter ohne spezielle Ausbildung und regelmäßige Supervision.

48 14\% bei ambulanten Patienten mit Mamma Ca, HNO-Tumoren, Lymphomen (Berard et al. 1996); $16 \%$ bei einer ambulanten Stichprobe mit unterschiedlichen Krebserkrankungen (Bredart et al. 1999); 20-25\% unterschiedliche Krebserkrankungen (Valente \& Saunders 1997); 22\% unterschiedliche Krebserkrankungen (Aass et al. 1997); 25\% (Pirl \& Roth 1999); 28\% (Hosaka \& Aoki 1996); 33\% bei neudiagnostizierten Patienten (Ford et al. 1995); 34\% (Farber et al. 1984)

${ }^{49}$ 44\% (Derogatis, 1983); 40\% (Alexander et al., 1993); 44\% (Hosaka \& Aoki, 1996); 55\% (Aragona et al.,1997)

${ }^{50}$ Vgl. auch die tabellarische Übersicht von Prävalenzraten psychischer Symptome in Abhängigkeit vom Krankheitsstadium (Schwarz \& Krauß 2000)
} 
(2000) nicht anders als bei anderen schweren somatischen Erkrankungen. Razavi \& Stiefel (1994) kommen in ihrer Übersichtsarbeit zu dem Ergebnis, dass psychiatrische Störungen doppelt so häufig vorkommen wie bei anderen körperlichen Erkrankungen und dreimal so häufig wie in der Normalbevölkerung. Sie erläutern auch die Problematik der Suizidalität und verweisen hier auf unterschiedliche Ergebnisse ${ }^{51}$. Die Ergebnisse sind inkonsistent und reichen von der Stellungnahme „kein Unterschied“ bis hin zu einer höheren Suizidrate im ersten Jahr nach der Diagnose. Fox et al. (1982) beschreibt ein relativ erhöhtes Suizidrisiko bei Männern um den Faktor 2.3, bei Frauen um 0.9. Zu ähnlichen Ergebnissen kommt auch Storm et al. (1992). ${ }^{52}$ Verres (1990) hält dagegen, dass Selbstmordversuche bei aufgeklärten Tumorpatienten nicht häufiger vorkommen als bei der gesunden Normalbevölkerung. In die gleiche Richtung gehen die Ausführungen von Faller (1998).

Bolund (1990) verweist auf die Einstellung gesunder Personen, auch der von Ärzten und Pflegepersonal, die Selbstmord als eine rationale Lösung ansehen bei schweren, zum Tode führenden Erkrankungen. Sie konstatiert, dass zwar viele Patienten über Selbstmord als einer Möglichkeit des Ausweges und der Rückgewinnung von Kontrolle nachdenken, dass aber nur wenige Krebspatienten tatsächlich Selbstmord begehen. Sie betont, dass man bei der Analyse von Suiziden bei Krebskranken kein simples Erklärungsmuster finden kann, sondern eine Reihe von Mosaiksteinchen aus medizinischen, psychiatrischen, psychischen und psychosozialen Problemen. Suizid sollte weder als die heroische Wahl der Starken, noch als die Flucht der Schwachen betrachtet werden, sondern als Signal, dass der Sinn des Lebens angesichts der lebensbedrohlichen Erkrankung verloren gegangen ist.

Auch nach den Erfahrungen der Verfasserin sind Selbstmordgedanken und der Wunsch, darüber zu sprechen, bei Patienten häufig. Selbstmordversuche bzw. vollendete Suizide treten dagegen extrem selten auf. ${ }^{53}$ Als Schutz wirkt eine tragfähige Beziehung, in der über Selbstmordphantasien auch gesprochen werden darf und die es ermöglicht, nach anderen Auswegen und Problemlösungen zu suchen.

\footnotetext{
${ }^{51}$ Siehe auch Kapfhammer (1996): Depression und Suizid bei Krebskranken. TW Neurologie Psychiatrie 10(4)281-283.

${ }^{52}$ Faller (1998) nennt als Risikofaktoren: fortgeschrittene Erkrankung mit schlechter Prognose und unkontrollierbarer Schmerz. „[...] Schmerz, der als unkontrollierbar erfahren wird, Verlust wahrgenommener Selbstbestimmung über das eigene Leben, Hilflosigkeit und Abhängigkeit. Dies gilt naturgemäß insbesondere für Patienten, denen aufgrund ihrer Persönlichkeit Autonomie unabdingbar ist und die es schwer ertragen können, auf andere angewiesen zu sein." (S. 35)

${ }^{53}$ In den letzten 10 Jahren gab es in der Universitäts Frauenklinik Göttingen einen einzigen Suizid einer onkologischen Patientin.
} 
In den Studien wird häufig keine Unterscheidung zwischen Angst, Depressivität und Anpassungsstörung getroffen, sondern es wird meist allgemein von psychischer Störung gesprochen.

Unabhängig vom theoretischen Hintergrund ist sicherlich zu akzeptieren, dass Krebspatienten einen Zustand extremer Hilflosigkeit und damit verbunden das Gefühl des Kontrollverlustes erleiden. Angst und Depression können als zwei Aspekte des erfahrenen Kontrollverlustes angesehen werden.

Bei den Studien, die explizit eine Unterscheidung vornehmen, zeigt sich, dass der Anteil an Angststörung deutlich höher ist als der Anteil an depressiven Störungen (u. a. Aass et al. 1997; Ford et al. 1995). ${ }^{54}$

Leider liegen nur wenige Langzeitstudien vor, die Angst und Depressivität in Abhängigkeit von der Krankheitsentwicklung erfassen. Wichtig erscheinen die Befunde von Thomas et al. (1997), die bei 31\% der Langzeitüberlebenden noch eine deutliche Angstsymptomatik feststellen. Die Studie von Ford et al. (1995) verweist darauf, dass beim Vergleich zwischen neu diagnostizierten Krebspatienten und einer Nachuntersuchung nach 6 Monaten, die Angst nur geringfügig abgenommen hat.

Ratsak (1993) geht im Gegenteil davon aus, dass Angst im Verlauf der Erkrankung zunimmt. Holland (1990) ermittelt eine Zunahme von Angst nach Abschluss der Radiotherapie. Angst steht außerdem im Zusammenhang mit physischen Faktoren. Neben schlechter Symptomkontrolle bei Schmerzen tritt Angst verstärkt auf bei abnormen Stoffwechselzuständen wie Hypoxie, Sepsis, Hypoglykämie, Lungenembolie, Delirium, Herzproblemen und bei Blutungen. Ein deutlicher Zusammenhang zwischen Depressivität und Schmerzen wurde häufig nachgewiesen. Die Studien können aber keine Aussage treffen, ob Schmerzen ursächlich für die Entwicklung von Depressivität verantwortlich gemacht werden können. Eine gegenseitige Verschlimmerung der Symptomatik ist aber unbestritten (Stiefel et al. 2001).

\subsubsection{Risikofaktoren}

Weitgehend übereinstimmend wird in verschiedenen Studien darauf verwiesen, dass eine psychiatrische Vorgeschichte das Risiko, mit einer affektiven Störung auf die Belastung durch die Krebserkrankung zu reagieren, erhöht (u. a. Valentine et al. 1998; Leopold et al. 1998; Aass et al. 1997; Hardman et al. 1989).

Der Performanzstatus und die physische Situation (Übelkeit, Erbrechen, Fatigue) sind eng mit affektiven Störungen assoziiert (Aass et al. ebenda; Pinder et al. 1996; Hardman et al. 1989). Es gibt eine signifikante Beziehung zwischen Schmerzempfinden

\footnotetext{
${ }^{54}$ Siehe auch Spiegel et al. 1994: Common psychiatric disorders in cancer patients II. Anxiety and confusional states. Supp Care Cancer 2:233-237.
} 
(Leopold et al. 1998) und der Einschätzung der Betroffenen, ob sie sich in einem fortgeschrittenen Krankheitsstadium befinden oder ob die Therapie kurativ oder palliativ ausgerichtet ist (Bredart et al. 1999; Breitbart 1995; Alexander et al. 1993; Hardman et al. 1989). Veränderungen der sozialen und/oder familiären Rolle zeigen ebenfalls eine Beziehung zu affektiven Störungen. Aass et al. (ebenda) finden einen Zusammenhang zwischen Krankheitsprogress/Metastasierung und Depression, dagegen keinen Zusammenhang mit Angst. Bei hospitalisierten Patienten ist die Störungsprävalenz größer (Aass et al. ebenda).

Der Einfluss des Geschlechts bleibt unklar: Aass et al. (ebenda) finden keinen Zusammenhang, während Bredart et al. (ebenda) einen deutlichen Zusammenhang zwischen weiblichem Geschlecht und Störungen ausmachen können. Während Angst keine Beziehung zu soziodemographischen Variablen und Krankheitsfaktoren zeigt (Pinder et al. 1993), scheint Depression eher mit niedrigem sozioökonomischem Status in Verbindung zu stehen.

Härter (2000) nennt für die Entwicklung depressiver Störungen bei Krebspatienten folgende Risikofaktoren:

- soziale Isolation

- $\quad$ kurz zurückliegende Verlusterlebnisse

- Tendenz zum Pessimismus

- Sozioökonomische Belastungen

- Depressive Vorgeschichte

- Alkohol- oder Drogenmissbrauch

- Suizidversuche in der Vorgeschichte

- Wenig kontrollierbare Schmerzzustände (S. 278)

Aufgrund der Daten sollte unbestritten sein, dass eine Diagnostik und die Einleitung therapeutischer Maßnahmen notwendig sind, um die Belastungen für diese Patientengruppen nicht unnötig zu verschlimmern.

Wie bereits erwähnt wird Fatigue von einigen Autoren in engem Zusammenhang mit Depressivität gesehen. Der nächste Abschnitt geht auf das Phänomen „Fatigue“ ausführlicher ein.

\subsection{Fatigue}

Ein weiterer Komplex subjektiver Beschwerden wird unter dem Begriff „Fatigue“ zusammengefasst. Fatigue soll hier ausführlich dargestellt werden und zwar aus zweierlei Gründen: 
1. Fatigue stellt ein gravierendes Problem für Patienten nach HDC dar, sowohl als unmittelbare Reaktion auf die Therapie als auch in der späteren Zeit im Sinne einer chronischen Spätauswirkung

2. Fatigue ist bislang kaum untersucht worden

Fatigue ist ein nichtspezifisches, multidimensionales Konstrukt, das mit Gefühlen von Müdigkeit, Schwäche und Energieverlust einhergeht. ${ }^{55}$ Fatigue kann durch Schonen und Ausruhen nicht überwunden werden. Fatigue ist ein weit verbreitetes Symptom, das viele Krebspatienten während der Therapie und auch später als sehr belastend beschreiben. $60-90 \%$ aller Tumorpatienten sind von Fatigue in unterschiedlichem Ausmaß betroffen (Rüffer \& Flechtner 2001).

Während Fatigue früher überwiegend im Bereich der Arbeitsmedizin untersucht wurde, ist es erst in den letzten Jahren als weitere Belastung von Krebspatienten in das Bewusstsein gerückt. Zunächst hat sich vor allem die Pflegewissenschaft des Phänomens angenommen. Später wurde Fatigue dann auch zum Gegenstand der psychoonkologischen Forschung, hier vor allem im Bereich der psychometrischen Lebensqualitätsforschung. Das erste internationale Symposium hat 1999 stattgefunden. Cella et al. (1998) gehen davon aus, dass Fatigue ähnlich negative Auswirkungen auf die Lebensqualität von betroffenen Patienten hat wie chronische Schmerzen.

Schwarz et al. (2001) beschreiben Fatigue folgendermaßen:

„Fatigue kann Krankheitsvorläufer, Kranheitssymptom oder Ergebnis medizinischer Eingriffe sein und kennzeichnet ein besonderes Ausmaß bzw. eine spezifische Qualität von Müdigkeit und Erschöpfung. Fatigue ist ein häufiges Symptom bei vielen physischen, insbesondere chronischen Krankheiten und bei Depression, außerdem ist es ein Leitsymptom des Allgemeinen Erschöpfungssyndroms (General Fatigue Syndrome). Mehrwöchige Erschöpfungsphasen sind eine häufige Neben- und Nachwirkung chirurgischer, chemo- und radiotherapeutischer Behandlungen; Medikamente wie Analgetika, Psychopharmaka und Schlafmittel können tagsüber Fatigue hervorrufen. Durch Fatigue wird neben der Selbstversorgung auch die Lebensqualität von betroffenen Patienten beeinträchtigt“ (S. 14).

Wie oben erwähnt, wird von den meisten Autoren eine Mehrdimensionalität der Fatigue angenommen. Die Beschwerden können den Dimensionen physisch, kognitiv, motivational, emotional und sozial zugeordnet werden.

Als Ursachen für das Fatigue-Syndrom bei Tumorpatienten kommen, mit jeweils unterschiedlichem Anteil, je nach Art der Tumorerkrankung und dem Krankheitsstadium, folgende Faktoren infrage:

- Direkte Auswirkungen des Tumors: Zellzerfall, metabolische Veränderungen, Anämie, Zytokinproduktion, Kachexie, Erbrechen, Diarrhö, Dyspnoe

${ }^{55}$ Einen aktuellen Forschungsüberblick gibt A. Glaus 1998 in: Fatigue in patients with cancer. 
- Auswirkungen der Tumortherapie: Folgen der Zytotoxizität von Radio- und Chemotherapie, Folgen von operativen Eingriffen, Nebenwirkungen der supportiven medikamentösen Therapie

- Psychosoziale Prozesse: Umgang mit der Erkrankung, Anpassungs- und Abwehrmöglichkeiten, direkte und indirekte Folgen für soziale Beziehungen und soziale Situation

Da Fatique ein weitverbreitetes Symptom auch in der Allgemeinbevölkerung ist, haben Schwarz et al. (2001) in ihrer Studie eine umfangreiche repräsentative Stichprobe der Bevölkerung mit Stichproben aus Allgemeinarztpraxen verglichen, mit dem Ziel, Normwerte zu erstellen. Erst mithilfe der Normwerte lassen sich aussagekräftige Vergleiche anstellen, die möglicherweise bei einer sozialmedizinischen Begutachtung von Krebspatienten eine Rolle spielen könnten. Bisher gibt es den Begriff „Fatigue“ nicht als eigenständige Diagnose/Symptom im Rahmen der sozialmedizinischen Begutachtung der gesetzlichen Rentenversicherung (Weis, Bartsch \& Woltemate 2000). Gerade im Zusammenhang mit der beruflichen Integration nach Erkrankung und Rückkehr in den Arbeitsprozess, ob zu Hause oder außerhalb, spielt das Ausmaß von Fatigue eine Rolle. In diesem Zusammenhang liefert die Arbeit von Schwarz et al. einen wichtigen Beitrag. Generell lässt sich für die Bevölkerungsstichprobe feststellen, dass Frauen höhere Werte angeben und bei älteren Menschen die Werte höher sind als bei jüngeren. Für die verschiedenen Patientengruppen treffen diese Aussagen nicht zu.

Glaus (2000) berichtet ebenfalls über eine Studie, die zu einer Abgrenzung von Fatigue bei Gesunden und Krebskranken beitragen will. Mithilfe von qualitativer Methodik wurden unstrukturierte Interviews erhoben und Themen identifiziert, die der Kategorie Fatigue zugeordnet wurden. Als wesentlicher Unterschied zwischen den beiden Gruppen zeigte sich, dass Kranke Fatigue auch als Schwäche bezeichnen während in der Beschreibung der Gesunden Schwäche nicht als Teil ihrer Müdigkeit enthalten war. Sie folgert, dass der Unterschied zwischen Fatigue bei Kranken und Gesunden sich nicht quantitativ fassen lässt, sondern dass die Qualität der Müdigkeitsgefühle aus einem „angenehmen Gefühl ein lästiges Leiden“ werden lässt. Schwarz et al. diskutieren, welche kritischen Werte für die Abgrenzung zwischen unauffälligen und auffälligen Fatigue-Werten infrage kommen. Die Autoren haben Fatigue-Werte mit dem „Multidimensional Fatigue Inventory“ nach Smets et al. in einer für Deutschland repräsentativen Bevölkerungsstichprobe erhoben. Die Auswertung zeigt eine Abhängigkeit der Werte von Geschlecht und Alter. Bei Männern und Frauen gibt es eine lineare Zunahme in Abhängigkeit vom Alter. Kein Zusammenhang war zwischen sozialer Schicht, Wohnort und Fatigue festzustellen. 
In einer französischen Studie (Henry-Amar \& Joly 2001) geben die Autoren Wertebereiche zur Beurteilung von Fatigue bezogen auf den Skalenwert im QLQ-C30. Die Werte werden mithilfe einer vierstufigen Likert-Skala gewonnen und dann in eine Skala von 0 - 100 transformiert. Dabei bedeutet 0 keinerlei Fatigue während 100 den höchst möglich vorstellbaren Wert von Fatigue beschreibt. Der Wert von 0-19 gilt als unauffällig, der Wert von 20 - 39 als fraglich und ein Wert von größer 40 als eindeutig auffällig. Ob diese „Cut-off-Werte“ brauchbar sind, wird sich erst in der Zukunft zeigen. In einer Interviewstudie von Kuuppelmkaki \& Lauri (1998) wurden palliative Patienten nach ihren Erfahrungen mit „Leiden“ im Krankheitskontext befragt. Neben Chemotherapie und Schmerzen wird Fatigue genannt. In einer weiteren Studie (Stone et al. 1999), ebenfalls mit palliativen Krebspatienten und einer nicht-krebskranken Kontrollgruppe, wird verglichen, ob sich das Auftreten von Fatigue in den Kollektiven unterscheidet. In der Patientengruppe leiden $75 \%$ der Patienten an ausgeprägten Formen von Fatigue. Es gibt keine Zusammenhänge mit Alter, Geschlecht, Diagnose, Vorhandensein oder Nicht-Vorhandensein von Metastasen und deren Lokalisation, Anämie, Schmerzmitteldosierung, hämatologischen oder biochemischen Indizes, Ernährungszustand und Gemütszustand. Allerdings gibt es einen signifikanten Zusammenhang zwischen Fatigue, Schmerzen und Dyspnoe. In der nicht-krebskranken Kontrollgruppe gab es dagegen einen Zusammenhang zwischen Fatigue, Angst und Depression. Das Ergebnis für die Gruppe der Krebskranken steht im Widerspruch zu den Ergebnissen anderer Studien (Aas et al. 1997; Akechi et al. 1999; Blesch \& Paice 1998; Glaus 1998; Hayes 1991), in denen sich signifikante Zusammenhänge zwischen Angst, Depression und Fatigue finden.

Eine sehr schwerwiegende Ausprägung von Fatigue ist fast immer in der Aplasiephase nach der HDC-Behandlung zu beobachten. Untersuchungen, die Patientinnen und Patienten nach Knochenmarks- und Blutstammzelltransplantationen betreffen, bestätigen das Vorhandensein und das Persistieren von Fatigue lange Zeit nach Abschluss (mehrere Jahre) der Behandlung. Für diese Patientengruppe wird Fatigue mittlerweile als wichtiges Folgeproblem anerkannt.

Wie bereits erwähnt, nimmt Fatigue insofern eine Sonderstellung ein, als verursachende Faktoren ungeklärt sind. Infrage kommen sowohl physische als auch psychosoziale Faktoren. Unter den somatischen Faktoren wird vor allem ein niedriger Hb-Wert als mit ursächlich diskutiert. Eindeutig ist dieser Zusammenhang allerdings in den bisherigen Studien nicht nachgewiesen.

Der Zusammenhang zwischen Fatigue und Chemotherapie bei Brustkrebspatientinnen ist ebenfalls nicht konsistent. Mast (1998) und Woo et al (1998) finden in ihren Studien signifikante Zusammenhänge. Andrykowski et al.(1998), Berlund et al. (1991) und Bo- 
wer et al. (2000) finden keine oder nur schwache Zusammenhänge in Bezug auf Chemotherapie. In der Radiologie wird Fatigue als gängige Nebenwirkung genannt unter der zwei Drittel aller Patienten leiden (Feyer et al. 2000).

Deutliche Zusammenhänge finden sich zwischen verschiedenen psychologischen Konstrukten wie Angst, Depressivität, emotionale Störung, Unsicherheit in Bezug auf das weitere Krankheitsgeschehen, Schlafprobleme, Menopausensymptome und Fatigue (Hann et al. 1997; Andrykowski et al. 1998; Broeckel et al. 1998; Mast 1998; Blesch et al. 1991).

Andere Erkrankungen, unabhängig von der Karzinomerkrankung, wie z. B. Diabetes, Bluthochdruck und Arthritis, gehen ebenfalls mit höherer Fatigue einher. Einen sehr deutlichen Zusammenhang gibt es zwischen Schmerz und Fatigue (Blesch et al. 1991; Spiegel et al. 1994; Bower et al. 2000).

Wegen des Zusammenhangs zwischen Fatigue und psychischen Störungen werden in der Psychoonkologie unterschiedliche Modellvorstellungen diskutiert.

Reuter \& Härter (2000) präferieren Depression als das umfassendere Konzept, da alle Fatigue-Symptome auch bei der Depression nachweisbar sind:

Abb. 9: Überschneidungen von Depression und Fatigue (S.42)

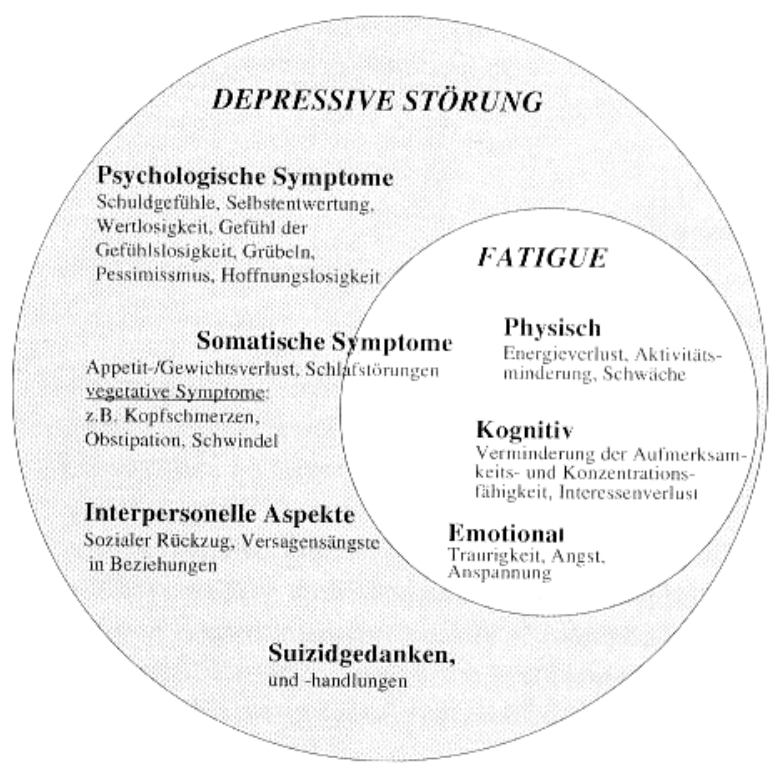

Nach diesem Modell geht Fatigue in der depressiven Störung auf und ist nicht als gesondertes Störungsbild zu betrachten. Die Autoren wenden sich gegen die Tendenz in der Onkologie, Fatigue als eigenständige diagnostische Entität zu betrachten. Sie fürchten, dass diese Betrachtung dazu führt, depressive Störungen zu verkennen. 
Feyer et al. (2000) ziehen ein anderes Modell vor, das den physischen Aspekten der Tumorerkrankung mehr Gewicht verleiht:

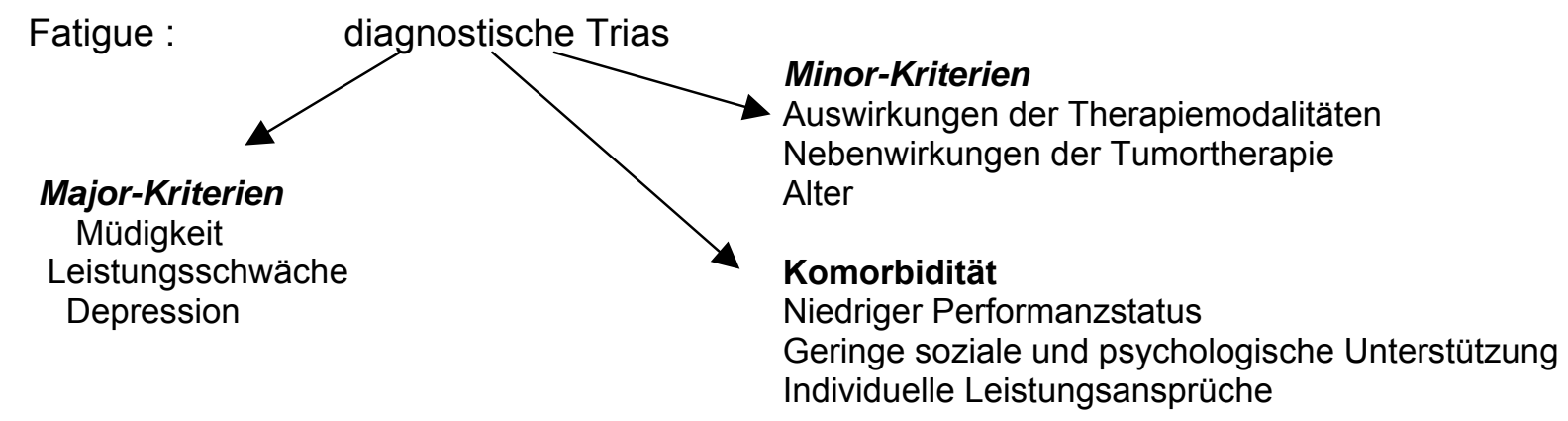

Neben diesen beschreibenden Modellen wird von Glaus (2000) ein Entwicklungsmodell für Fatigue vorgestellt.
Abb. 10: Entwicklungsmodell Fatigue
(S. 10)

\begin{tabular}{|c|c|}
\hline Stufe & Konditionierende Faktoren \\
\hline Nozizeption & $\begin{array}{l}\text { Spezifität (Ursachen, zentral, peripher, allgemein) } \\
\text { Krebsspezifische Einwirkungen auf Organfunktionen (primäre Fatigue) } \\
\text { Ausschüttung von Zytokinen, Krebsart- und -stadium-spezifisch (sekundäre } \\
\text { Fatigue) } \\
\text { (z.B. Interferone, Interleukine, Tumor-Nekrose-Faktor) } \\
\text { Energietransformationsprozesse (z. B. Muskelabbau, Energieverschleiß, } \\
\text { Anorexie, Ernährungsdefizit) } \\
\text { Immunologische Abwehr gegen den Tumor, paraneoplastische Syndrome } \\
\text { Metabolische Störungen (z. B. Hyperkalzämie, Hyponatriämie, Hypoglykä- } \\
\text { mie) } \\
\text { Endokrine Störungen (z. B. Hypoadrenokortizismus, Hypothyreoidismus) } \\
\text { Zirkulatorische Anpassungsinsuffizienz (z. B. eingeschränkter Sauerstoff- } \\
\text { transport) } \\
\text { Behandlung (Toxizität, Abbauprodukte, Zelltod, Heilung) } \\
\text { Andere Symptome, Nebenwirkungen (z. B. Nausea, Emesis, Anorexie, } \\
\text { Schmerz, Immobilität, Dyspnoe, Anämie) } \\
\text { Krankheitsverarbeitung, Coping-Prozeß (Depression, Angst, Isolation) }\end{array}$ \\
\hline$\stackrel{\text { Perzeption }}{\downarrow}$ & $\begin{array}{l}\text { Wahrnehmung, Regulation der Fatigue-Schwelle } \\
\text { Emotionaler Zustand, psychologische und soziale Unterstützung, Glaube, } \\
\text { Persönlichkeit, Biographie, Allgemeinbefinden }\end{array}$ \\
\hline $\begin{array}{c}\text { Expression } \\
\nabla\end{array}$ & $\begin{array}{l}\text { Manifestation der Fatigue nach außen } \\
\text { physisch affektiv kognitiv }\end{array}$ \\
\hline
\end{tabular}

In diesem Modell werden Auslösefaktoren angenommen, die dann auf der Ebene der Wahrnehmung moduliert werden und sich im Verhalten ausdrücken. Vorteil dieses Modells ist die Ableitung von Interventionen, die auf den unterschiedlichen Ebenen ansetzen können und jeweils unterschiedliche Faktoren ins Blickfeld nehmen. 
Während speziell für die Fatigue-Messung entwickelte Fragebögen die unterschiedlichen Faktoren und einen Allgemeinen Fatiguewert ermitteln wollen, beschränken sich die Fragen in den Instrumenten zur LQ-Erhebung in der Regel auf einen Allgemeinen Fatiguewert, der sich, wie im QLQ-C30, auf die Fragen „Waren Sie müde?" und „Mussten Sie ausruhen?“ bezieht. Diese Vereinfachung ist gerechtfertigt, da auch bei den differenzierteren Erfassungsinstrumenten die physische, soziale, kognitive und emotionale Skala ausreichend hoch mit dem allgemeinen Wert korrelieren.

Entscheidend ist, dass unabhängig von den Modellvorstellungen bei der Beurteilung von Fatigue jeweils alle Ebenen (psychisch, physisch, sozial) einbezogen werden. Für die Beeinflussung von Fatigue ist ein multimodaler Therapieansatz notwendig, auch wenn die Gewichte unterschiedlich verteilt sein mögen. Aus dem Modell von Glaus lässt sich ableiten, wie wichtig eine frühzeitige Einflussnahme auf die Faktoren in der akuten Erkrankungsphase ist. Ähnlich wie beim Schmerz können „Spuren“ zurückbleiben, auch wenn die auslösenden Faktoren nicht mehr vorhanden sind. Effektive Symptombekämpfung wirkt einer späteren Verfestigung von Fatigue entgegen. Prins et al. (2001) haben eine randomisierte Multicenterstudie über die Wirksamkeit kognitiver Verhaltenstherapie bei Chronischem Fatigue Syndrom publiziert. Patienten mit somatischen Erkrankungen waren ausgeschlossen. Im Modell der Autoren spielen Kontrollüberzeugungen und somatische Attributionen eine zentrale Rolle. Kognitive VT war in dieser Studie signifikant wirksamer als „nur“ supportive Therapie. Allerdings war hier „supportive Therapie“ als Selbsthilfegruppe konzipiert. Für onkologische Patienten liegen keine vergleichbaren Programme vor. Interessant wäre die modifizierte Anwendung bei onkologischen Patienten. Somatische Attribuierungen sind bei dieser Gruppe durchaus angemessen - deswegen müsste der Schwerpunkt auf Kontrollüberzeugungen gelegt werden. Psychotherapeutische Interventionen sind in diesem Bereich bislang wenig erforscht.

Die zusätzlich durch die HDC induzierten Belastungen werden im nächsten Abschnitt dargestellt.

\subsection{Belastungen durch HDC}

Bezogen auf die Therapie werden den Patienten weitere und vergleichsweise schwerwiegende Belastungen zugemutet, die sich auf verschiedene Phasen der Behandlung und Rehabilitationszeit beziehen.

Vor der Behandlung ist vor allem der Hinweis auf die schlechte Prognose und die "Gefährlichkeit“ der HDC zu akzeptieren. 


\subsubsection{Wissen um die ungünstige Prognose der Erkrankung und die Nebenwir- kungen der HDC}

Neben den „üblichen“, bereits beschriebenen Ängsten, die mit Diagnose und Erkrankung einhergehen, sind die Patienten weiteren Belastungen ausgesetzt: In den Aufklärungsgesprächen über die Möglichkeit einer HDC muss sowohl die schlechte Prognose als auch die höhere Toxizität durch die HDC-Therapie thematisiert werden.

In der Einwilligungserklärung einer vergleichenden Multicenterstudie werden den Mammakarzinom Patientinnen die Prognose ohne HDC und die Nebenwirkungen der HDC erklärt:

„[...] Das Fünfjahresüberleben ohne Rezidiv herkömmlicher Therapie liegt bei ungefähr 30\%. Die Hochdosischemotherapie kann ein Mehrfaches der Stärke einer Standardchemotherapie beinhalten und damit bei bestimmten Krebsarten in einer Gruppe von Patienten die letzten verbliebenen Krebszellen abtöten und somit zu einer Langzeitheilung führen. Die Wirksamkeit dieses Prinzips wurde bisher am besten in der Behandlung von Leukämien und Lymphomen unter Beweis gestellt. Vorläufige Daten bei der Behandlung des Mammakarzinoms sind als positiv anzusehen, bedürfen aber noch weiterer Bestätigung. Die Hauptnebenwirkung der Chemotherapie betrifft die Blutproduktion und kann zu schweren Verringerungen der Leukozytenzahl (Weiße Zellen) und Thrombozyten (Plättchen) und somit zu schweren, eventuell lebensbedrohlichen Infektionen und Blutungen führen. Diese Nebenwirkung kann zum Teil umgangen werden, indem den Patienten vor der Hochdosisbehandlung Blutstammzellen (die Fabrikzellen der Blutproduktion) aus dem Blut entnommen und eingefroren werden. Diese Stammzellen werden nach Abschluss der Hochdosisbehandlung zur schnelleren Erholung der Blutbildung zurückgegeben. [...J" (unveröffentlichte, aktuell verwendete Einverständnis- erklärung der Abtl. Hämatologie/Onkologie/Gynäkologie der Universitätsklinik Göttingen).

Die Aufklärungs-/Einwilligungstexte für andere Patientengruppen enthalten ähnlich lautende Informationen. Thematisiert wird die begrenzte Wirkung herkömmlicher Therapien, im oben genannten Text die Fünfjahresüberlebensrate von 1/3 der Patienten. Gemessen an den Heilungswünschen der Patienten wird das Überleben von nur einem Drittel der Patienten als sehr wenig vielversprechend aufgefasst. Der erhoffte Wirkungseffekt der HDC wird dagegen völlig offen gelassen: „und (kann) somit zu einer Langzeitheilung führen“ (siehe oben). Allein das Wort Langzeitheilung lässt den Patienten viel Spielraum für Hoffnungen. Auffällig wenige Patienten wollen Genaueres wissen. Die Vorstellung (oder bei den Patienten die eigenen Erfahrungen mit Chemotherapie) einer Therapie mit mehrfacher Stärke aber auch mehrfachen Nebenwirkungen wie mehr Übelkeit usw. löst mehr Ängste aus. In den Texten besteht die Verpflichtung, auf Nebenwirkungen hinzuweisen. Die Beschreibung der Auswirkungen auf die Blutproduktion, auf eventuell entstehende lebensbedrohliche Infekte und mögliche Blutungen 
schürt die Vorstellung, dass es sich um eine äußerst gefährliche Therapie handelt. Patienten haben kaum konkrete Vorstellungen, was Aplasie bedeutet, und gehen so davon aus, dass es etwas „sehr Schlimmes, Gefährliches“ sein muss.

Von den wenigen Untersuchungen (und aus eigener Erfahrung) ist bekannt, dass zwei Tendenzen bei Patienten und Ärzten vorherrschen (Hillner et al. 1992; Weeks et al. 1998; Slevin et al. 1990):

1. Die Wirkung von Chemotherapie wird generell in Bezug auf ihre Effektivität überbewertet, d.h. auch die Chance einer Heilung wird überschätzt.

2. Die Gefährlichkeit der Therapie wird ebenfalls überschätzt, sowohl von Ärzten als auch Patienten.

So setzen denn auch etliche der Patienten auf „Alles oder Nichts“ und müssen erhebliche Abwehrleistungen vollbringen, um gegen ihre Todesängste anzugehen. Dagegen gesetzt wird die Hoffnung auf Heilung. Es scheint außerdem die Vorstellung vorzuherrschen, dass die Chance, den Krebs zu zerstören und das damit verbundene Leiden zu lindern, umso größer ist, je aggressiver die Therapie ist. ${ }^{56}$

Hoffnung ist der wesentliche Faktor, der es den Patienten ermöglicht, belastende Therapien in Erwägung zu ziehen und die damit verbundenen Nebenwirkungen zu akzeptieren.

\subsubsection{Somatische Nebenwirkungen}

Die Beeinträchtigungen des Befindens durch die einschneidenden Behandlungen liegen auf der Hand. Die Toxizität der Chemotherapieregimes und der Ganzkörperbestrahlung wirken sich im Bereich der verschiedenen Organsysteme aus.

Die im Rahmen der HDC-Regimes bei den Mammakarzinompatientinnen verwendeten Medikamente sind Mitoxantrone, Thiotepa und Cyclosphosphamid, Cyclosphosphamid bei den NHL-Patienten und -Patientinnen.

Die Nebenwirkungen für die verwendeten Zytostatika werden in der Roten Liste (1998) angeführt. Für alle Medikamente gilt gleichermaßen die Auswirkung auf das blutbildende System. Auch Schleimhautschädigung in den verschiedenen Bereichen gilt für alle. Bei Thiotepa werden zusätzlich Appetitlosigkeit und Übelkeit sowie die Gefahr von Entzündungen im Magen-Darm-Trakt als wichtige Wirkung genannt. Ovulation und Spermatogenese werden gestört. Es können Symptome wie Schwindel und Kopfschmerzen auftreten, die denen beim organischen Hirnsyndrom ähneln. Bei Mitoxantrone wird auf die Veränderung der Nierenfunktion und ebenfalls auf Komplikationen im Magen-DarmTrakt verwiesen. Bei Cyclosphosphamid sind Darm und Blase gefährdet, darüber hin-

\footnotetext{
${ }^{56}$ In diesem Kontext spielt die Diskussion um einen „informed consent“ eine wichtige Rolle.
} 
aus wird eine Herzmuskelschädigung als mögliche Nebenwirkung genannt. Bei vorangegangener Bestrahlung ist auch das Lungengewebe gefährdet.

Van der Wall et al. (1995) listen für die am häufigsten verwendeten Medikamente und Medikamentenkombinationen Nebenwirkungen auf und unternehmen eine Herleitung der Nebenwirkungen durch die spezifischen Wirkprozesse und Metabolismen der Stoffe. Für Cyclophosphamid heben sie die besondere Cardiotoxizität hervor, die sich bei $25 \%$ der Patienten entwickelt. Die Cardiotoxizität ist dosislimitierend für die HDC. Daneben kommt es bei $12-35 \%$ der Patienten zu einer hämorrhagischen Cystitis, da ca. $10 \%$ des Stoffes über den Urin ausgeschieden wird.

Bei Thiotepa ist die zentral-nervöse Toxizität dosislimitierend. Daneben ist mit Mucositis, Ösophagitis und Enterocolitis zu rechnen. Die Hauttoxizität, die sich durch akute Erytheme äußern kann, führt unter Umständen zu einer Verdunkelung der Haut, die Monate fortbestehen kann, aber reversibel ist. Übelkeit und Erbrechen sind mit den Standardmitteln gut beherrschbar.

Bei Mitoxantron sind Stomatitis und Mucositis dosislimitierend. Übelkeit, Erbrechen und Durchfall sind eher gering ausgeprägt. Anzeichen einer geringgradigen Lebertoxizität sind häufig. Cardiotoxizität tritt nur bei ca. 3\% der Patientinnen auf und nur bei cardiovadkulärer Vorschädigung oder bei vorhergehender Antrazyklinbehandlung. ${ }^{57}$

Einen Vergleich zwischen den auftretenden Nebenwirkungen bei HDC und konventioneller Chemotherapie, angewandt beim metastasierten Mammakarzinom, liefern Stadmaurer et al. (2000) in einer prospektiv randomisierten Studie. Die Nebenwirkungen sind in der folgenden Tabelle zusammengestellt.

\footnotetext{
${ }^{57}$ Van der Walt et al weisen darauf hin, dass die Angaben über die Ausprägungen der verschiedenen Toxizitäten anhand der publizierten Literatur nur schwer zu bestimmen seien. Zu unterschiedlich seien die Bedingungen und die Auswahl der Patienten. Weitere Beschreibungen von Nebenwirkungen finden sich auch bei Crown (1995).
} 
Tabelle 8: Inzidenz von Nebenwirkungen (adaptiert von Stadtmaurer et al., ebenda)

\begin{tabular}{|l|c|c|}
\hline Nebenwirkung & $\begin{array}{l}\text { HDC mit Stammzell- } \\
\text { transplantation }\end{array}$ & $\begin{array}{c}\text { Konventionelle } \\
\text { Chemotherapie }\end{array}$ \\
\hline Leukopenie & $96 \%$ & $52 \%$ \\
\hline Thrombozytopenie & $95 \%$ & $5 \%$ \\
\hline Anämie & $69 \%$ & $6 \%$ \\
\hline Infektion & $31 \%$ & $3 \%$ \\
\hline Diarrhö & $25 \%$ & $1 \%$ \\
\hline Hepatische Komplikationen & $9 \%$ & $1 \%$ \\
\hline Erbrechen & $8 \%$ & $1 \%$ \\
\hline Cardiologische Komplikationen & $8 \%$ & $0 \%$ \\
\hline Lungenkomplikationen & $7 \%$ & $1 \%$ \\
\hline Neurologische Komplikationen & $6 \%$ & $0 \%$ \\
\hline Mukositis & $5 \%$ & $2 \%$ \\
\hline
\end{tabular}

Die Tabelle verdeutlicht die gravierenden Unterschiede zwischen HDC und konventioneller Chemotherapie. Die deutlichsten Unterschiede finden sich beim blutbildenden System. Auch die Infektionsrate ist wesentlich höher. Erstaunlicherweise sind nur wenige Patientinnen von Mukositis betroffen. Die Erfahrungen mit eigenen Patientinnen aus der Vorphase der von uns durchgeführten Studie sind in diesem Punkt völlig anders: Mukositis, bis hin zu Ulcerationen, waren eher die Regel als die Ausnahme.

Die akuten Nebenwirkungen der HDC sind inzwischen bekannt und gut dokumentiert. Völlig anders verhält es sich mit chronischen und späteren Auswirkungen. Die Neurotoxizität der Therapie macht sich oft erst nach Monaten bemerkbar. ${ }^{58}$ Sie ist nicht mehr lebensbedrohlich, aber sie beeinträchtigt sehr stark die Lebensqualität im Alltag der Patienten. Im Gegensatz zu den akuten Nebenwirkungen sind die späteren nicht reversibel (Markman 1996; Meyers et al.1994).

Als Spätnebenwirkung - wobei „spät“ in diesem Falle sehr relativ ist - wurde auch das Auftreten von Zweitmalignomen, vor allem die Myeloische Leukämie und das Myelodysplastische Syndrom, beschrieben. Pedersen-Bjergaard, Andersen \& Christiansen (2000) beobachten, dass $27 \%$ der Patienten, die in ihrer Studie eine therapieverursachte hämatologische Zweitneoplasie entwickeln, dies bereits im ersten Jahr nach HDC tun. Mittlerweile scheint aber Konsens zu bestehen, dass dieses Risiko bei Mammakarzinompatientinnen eher gering ist und dass bei NHL- Patienten die chemotherapeutische Vorbehandlung und/oder die Ganzkörperbestrahlung von Bedeutung ist

${ }^{58}$ Einen kurzen deutschsprachigen Überblick gibt Poppelreuter 2001. 
für die unzweifelhaft erhöhte Rate an Zweitmalignomen (Kollmannsberger et al. 1998; Krishan et al. 2000).

Eine weitere Nebenwirkung, die allerdings erst nach Entlassung aus der Klinik auftritt, scheint insbesondere bei Mammakarzinompatientinnen sehr häufig zu sein: das „delayed pulmonary toxicity syndrome“, eine milde Form der interstitiellen Pneumonie, die mit Steroiden gut zu behandeln ist. Sie führt nicht zum Tode, kann aber Atembeschwerden erklären, über die Patientinnen häufiger klagen (Chap et al. 1997; Wilcynski et al. 1998).

Als Belastungsfaktor für die Mammakarzinompatientinnen kommt eine Radiatio der Brust nach Abschluss der HDC hinzu. Es gibt erstaunlich wenig Arbeiten, die sich mit den akuten und den späteren Folgen der Bestrahlung nach Brustkrebs beschäftigen. Bekannt sind die Hautrötungen, die unter der Bestrahlung häufig auftreten und, in sehr seltenen Fällen, Verbrennungen höheren Grades. In einer Studie von Grayden (1994) werden 53 Frauen mit Brustkrebs im frühen Stadium nach Lumpektomie (Teilentfernung) oder brusterhaltender Operation untersucht, die eine Radiotherapie erhalten haben. Ca. 7 Wochen nach Abschluss werden die Frauen nach ihrer Befindlichkeit, ihren Alltagsaktivitäten und Beschwerden befragt. Die untersuchten Frauen zeigen keine Einschränkungen in ihren Alltagsaktivitäten, keine wesentlichen emotionalen Auffälligkeiten und insgesamt wenig andere Symptome. Trotzdem leidet ein erheblicher Teil der Patientinnen unter Fatigue. Fatigue ist in dieser Stichprobe assoziiert mit niedrigerem Funktionsstatus und mehr physischen Symptomen.

Eine ältere Arbeit von Berglund et al. (1991) vergleicht die Auswirkungen von adjuvanter Chemotherapie oder Radiotherapie (Randomisierung erfolgte nach der Operation) 2-10 Jahre nach Abschluss der Therapie bei 448 Patientinnen mit Brustkrebs ohne Rezidiv. Insgesamt sind die Unterschiede zwischen den Gruppen gering. Allerdings haben die radiotherapierten Patientinnen signifikant mehr Probleme mit Ausdauer und Angst sowie Beschwerden mit der Operationsnarbe. Die allgemeine Lebensqualität wird von der Radiotherapiegruppe tendenziell etwas niedriger eingeschätzt.

Eine weitere Studie (Tasmuth et al. 1995) untersucht Beschwerden nach Mastektomie ohne Nachbehandlung und Beschwerden nach brusterhaltender Operation mit Radiatio. In dieser Arbeit beschreiben die Frauen der brusterhaltend operierten Gruppe signifikant mehr Schmerzen als die Gruppe der mastektomierten Patientinnen ohne Radiatio.

In unserer Stichprobe sind sowohl Patientinnen mit Mastektomie als auch Patientinnen, die brusterhaltend operiert wurden. Alle erhielten aber eine Radiotherapie im Anschluss an die HDC. 
Die NHL-Patienten werden vor der HDC einer Ganzkörperbestrahlung unterzogen, die ihrerseits erhebliche Nebenwirkungen und Spätfolgen haben kann.

Die unmittelbaren Nebenwirkungen mischen sich mit denen der HDC und sind nur durch den früheren Beginn, zeitgleich mit der Radiotherapie, abzugrenzen. Da die Ganzkörperbestrahlung bei den hämatologisch-onkologischen Erkrankungen routinemäßig vor der HDC eingesetzt wird, beziehen sich die Beschreibungen meist auf den Gesamtkomplex der Behandlung.

Lange bekannt sind die Auswirkungen auf die Fortpflanzungsfunktionen. Die Behandlung kann zur Infertilität führen. Bei Frauen kommt es zu ovariellen Störungen (Amenorrhöe, Östrogenmangel, Menopausensymptome). Bei Männern wird die Spermatogenese gestört und es kommt zum Androgenmangel. Einen Überblick über endokrine Probleme insgesamt geben Reincke et al. 2001.

Eine Studie von Andrykowski et al. (1990) zeigt eine Zunahme kognitiver Dysfunktionen in den Bereichen Reaktionszeit, Aufmerksamkeit, Konzentration, Problemlösen und Erfassung von Begründungszusammenhängen bei steigender TBI-Dosis (total body irradiation).

Drei aktuelle Studien beschäftigen sich mit der akuten Toxizität vor der HDC: bei einem erheblichen Teil der Patienten stellen Szekely et al. (1999) mildere Ausprägungen von Kopfschmerzen, Übelkeit und Erbrechen fest.

Bei Buchali et al. (2000) überwiegt ebenfalls die gastrointestinale Toxizität (Nausea, Vomiting, Appetitlosigkeit, milde Ösophagitis und Diarrhöe). Daneben kommt es bei einem kleinen Teil der Patienten zu leichten Hauterythemen. Außerdem finden sich auch Beschwerden über Kopfschmerzen und Fatigue. Bemerkenswert ist, dass in dieser Studie Frauen doppelt so häufig über Fatigue klagen wie Männer.

Die dritte Studie von Wenz et al. (1999) untersucht, ob sich eine akute zentral-nervöse Neurotoxizität nachweisen lässt. Die Ergebnisse zeigen, dass der mit einer neuropsychologischen Testbatterie erfasste Zustand sich durch die Radiatio nicht verschlechtert hat.

Eine weitere Nebenwirkung, die durch die Bestrahlung verursacht werden kann (unmittelbar aber auch erst Jahre später), besteht in einer Schädigung der Augenfunktion. Bei ca. einem Drittel der Patienten, die sich einer Ganzkörperbestrahlung unterzogen haben treten Katarakte (Trübung der Linse - grauer Star) auf (Zierhut et al. 2000). Einen deutschsprachigen Überblick über die verschiedenen Probleme, somatische und psychosoziale, geben Bartsch \& Mertelsmann (Hrsg.) (2001). 


\subsubsection{Schmerzen}

Obwohl Schmerzen bei der HDC eine wichtige Rolle spielen und ein großer Teil der Patienten, vor allem in der stationären Phase, damit rechnen muss, soll dieser Punkt hier aus folgenden Gründen nur kurz erwähnt werden:

- Im Falle der HDC Therapie besteht für die Patienten ein eindeutiger Zusammenhang zwischen Schmerzursache und Schmerzerleben. Den Patienten wird mitgeteilt, dass Schmerzen eine indirekte Folge der HDC sind. Vor allem während der Aplasie kann es zu entzündlichen Veränderungen (Schleimhäute) kommen, die mit Schmerzen einhergehen. Obwohl die Schmerzen ein größeres Ausmaß annehmen können, werden sie von den Patienten deutlich anders erlebt als Schmerzen, die mit der Krebserkrankung selber in Verbindung gebracht werden. Krebs - Schmerz - fortgeschrittene Erkrankung - Leiden - Tod werden von vielen Patienten in einer Assoziationskette verknüpft und können zu einer erheblichen Schmerzverstärkung führen. Die Schmerzen in dieser Behandlungssituation werden anders attribuiert, haben einen anderen Stellenwert und werden relativ gut ertragen.

- Die Patienten gehen davon aus, dass während des stationären Aufenthaltes eine wirksame Schmerzbekämpfung stattfindet, bei der unter Umständen auf eine intravenöse Morphingabe zurückgegriffen wird. Schmerzen werden zwar gefürchtet, spielen aber, im Vergleich mit der Furcht vor Infektionen, keine zentrale Rolle. Nach der Entlassung, in der Nachsorgephase, verändert sich die Ursachenattribuierung von Schmerzen, und sie rücken wieder ins Zentrum der Wahrnehmung.

\subsubsection{Probleme der Nahrungsaufnahme}

Verschiedene Faktoren führen zu einer erschwerten Nahrungsaufnahme: Mucositis und Stomatitis mit begleitenden Schmerzen, Übelkeit und Erbrechen, Durchfall und deutliche Veränderungen des Geschmacks- und Geruchsempfindens. Besonders der letzte Aspekt ist auch bei konventioneller Chemotherapie bekannt. Es gibt wenig Untersuchungen zu den Geruchs- und Geschmacksveränderungen, obwohl sie in der Praxis bekannt sind und für Patienten ein großes Problem darstellen. In der Arbeit von Mattson et al. (1992) wurden zehn Patienten untersucht. Alle zeigten deutliche Störungen bis hin zu völligem Geschmacksverlust. Nach einem Jahr war bei acht Patienten das Geschmacksvermögen wieder hergestellt. Im Review von Comeau, Epstein und Migas (2001) wird auf das Probleme der Mangelernährung, auch für Responseraten und Prognose insgesamt, hingewiesen. 
Durch die Informationsgespräche wissen die Patienten, dass eine zeitweilige künstliche Ernährung notwendig werden kann.

Nebenwirkungen, die speziell auf die HDC zurückzuführen sind, sollen in der folgenden Aufstellung zusammengefasst werden. Die Nebenwirkungen gelten vor allem für die stationäre Phase. Eine Alopezie wurde bei allen Patienten bereits in der Vorphase der HDC herbeigeführt (durch die Mobilisationszyklen bei den Mammakarzinom Patientinnen und durch die CHOP oder Beam Therapien bei der NHL-Gruppe) und wird deshalb in der Zusammenfassung nicht besonders erwähnt.

Das gesamte hämatopoetische System ist betroffen. Die Aplasie entspricht quasi der Toxizität vierten Grades. Durch die Gabe von Wachstumsfaktoren wird diese Phase verkürzt und dementsprechend mindern sich auch die Belastungen durch Verkürzung dieser Phase. In jedem Fall wird prophylaktisch antibiotisch und antimykotisch behandelt.

\subsubsection{Bedingungen des stationären Aufenthaltes (modifizierte Isolation)}

$\mathrm{Zu}$ den Besonderheiten der Behandlung gehört der Schutz vor Infektionen. Sowohl Patienten als auch Personal müssen dieser erhöhten Schutzbedürftigkeit Rechnung tragen. In Studien zur HDC wird die "Isolation“ als weiterer Belastungsfaktor genannt. Der Begriff der „Isolation“ ist für Patienten mit autologer Stammzelltransplantation nur sehr eingeschränkt zu verwenden. Die dabei praktizierten „Isolationsbedingungen“ in der Aplasiephase unterscheiden sich grundlegend von denen, die bei allogenen Knochenmarkstransplantationen (Umkehrisolation oder Life island) üblich sind. Diese Phase ist wesentlich kürzer (wenige Tage bis zu zwei Wochen, während sie bei der allogenen KMT über Monate bestehen bleiben). ${ }^{59}$ Nach dem Eindruck aus der Vorphase der Studie wird diese milde Form der Isolation als angenehm erlebt.

Die Patienten werden darauf hingewiesen, dass zur Verminderung der Ansteckungsgefahr nur wichtige Bezugspersonen als Besucher erwünscht sind. Die Patienten sollten auch die Anzahl der Besucher möglichst gering halten. Letztlich bleibt es aber den Patienten überlassen, darüber zu befinden, wen und wie viel Besucher zugelassen werden. Die Patienten sind in ihrer Bewegungsfreiheit nicht auf ihre Zimmer beschränkt, sondern können sich, mit Mundschutz, auch außerhalb aufhalten. Sie können im Garten oder im Kliniksgebäude spazieren gehen. Erfahrungsgemäß beschränken die Patienten von sich aus die Zahl der Besucher auf die wichtigsten Bezugspersonen, da bei innen ausnahmslos die Angst vor einer Infektion sehr groß ist. Daneben ist bei vielen

\footnotetext{
${ }^{59}$ Eine Übersicht über Forschungen zur Auswirkungen von Isolation unter den verschärften Bedingungen der KMT findet sich bei Neuser (1989). Sein Fazit lautet, dass die Anpassung in der Regel gut gelingt.
} 
die Erschöpfung so extrem, dass der Wunsch nach Ruhe und „In-Ruhe-gelassenwerden“ vorherrscht. In dieser Weise praktizierte „Isolation“ wird als Entlastung empfunden und kommt dem Regressionsbedürfnis der meisten Patienten entgegen.

Über die Belastungen der nachstationären Phase ist wenig bekannt. Angelsächsische Studien gehen davon aus, dass nach einem Jahr wieder ein Zustand wie vor der Therapie erreicht wird und dass dann die meisten Patienten wieder arbeitsfähig sind. Für Deutschland ist kürzlich die erste Studie über den Rehabilitationsverlauf von jüngeren NHL Patienten veröffentlicht worden (Weis et al. 2001), die in der Klinik für Tumorbiologie an der stationären Rehabilitation über mehrere Wochen teilgenommen haben.

\subsubsection{Zusammenfassung der erwarteten Nebenwirkungen:}

Toxizitäten in folgenden Bereichen sind zu erwarten (abgeleitet von den Nebenwirkungsprofilen der Medikamente und den klinischen Erfahrungen aus der Vorphase der Studie):

1. Stomatitis und Mukositis sind bei allen Patienten zu erwarten. Auch hier variiert der Grad der Beeinträchtigung von $0-4$.

2. Geringgradiges, nicht infektionsbedingtes Fieber (febrile Neutropenie) ist bei der Mehrzahl der Patienten zu erwarten.

3. Infektionen sind als Komplikation zu werten und sollten möglichst nicht auftreten.

4. PNS und ZNS Toxizität sind akut nicht zu erwarten. Sie treten meist als Spätnebenwirkungen in der Rehabilitationsphase auf.

5. Schmerzen unterschiedlicher Genese treten fast in jedem Falle auf und werden medikamentös behandelt. An erster Stelle stehen die durch Stomatitis und Mukositis verursachten Schmerzen. Die Schmerzintensität variiert von Grad 2 - 4 und muss mit entsprechenden Medikamenten (unter Umständen ist eine Morphingabe notwendig) behandelt werden. Daneben können als Nebenwirkungen der Wachstumsfaktoren Schmerzen in Knochen und Gelenken auftreten.

6. Allergien sind bei einer Minderzahl der Patienten zu erwarten.

7. Ein von allen betroffenen Patienten gleichermaßen beschriebenes Phänomen sind eine als extrem empfundene völlige Kraftlosigkeit und Erschöpfung.

8. Erbrechen, Übelkeit, Diarrhöe und Appetitlosigkeit unterschiedlicher Ausprägung sind bei allen Patienten zu erwarten. Wie bei jeder Chemotherapie wird eine intensive Antiemesebehandlung prophylaktisch durchgeführt.

Dass die Patienten besonderer medizinischer und pflegerischer Aufmerksamkeit bedürfen, steht außer Frage. Die Tatsache, dass behandlungsbedingte Mortalität durch 
wachsende Erfahrung reduzierbar ist, verleiht dieser Forderung besonderes Gewicht. Entscheidenden Anteil an der Verringerung der Mortalität haben prophylaktische Maßnahmen (Antibiose, Antimykose, Antiemese, Wachstumsfaktoren, Wechsel des Zentralen Venenkatheders, Ermunterung zu körperlicher Aktivität usw.), therapeutische Maßnahmen bei Komplikationen und schützende Umgebungsbedingungen.

Um einen Überblick zu geben, werden die Studien zu HDC und LQ im nächsten Abschnitt mit ihren wichtigsten Ergebnissen tabellarisch aufgelistet 


\section{8 Übersicht über die Studien zur LQ und HDC}

Einen Überblick über die früheren Studien zur LQ und HDC (in der Regel Patienten mit allogener oder autologer Knochenmarkstransplantation) für den Zeitraum von 1977 bis 1992 liefert Korth (1995). In seiner tabellarischen Übersicht sind 31 retrospektive psychologische Studien, 15 Kasuistiken und 8 prospektive psychologische Studien aufgelistet.

Um einen Überblick über die aktuellere Forschungslandschaft zu geben, sind die späteren Studien mit ihren Fragestellungen in der nachfolgenden Tabelle zusammengefasst. Die Literaturrecherche in „Medline“60 ergab für den Zeitraum 1992 - 12/2001 31 Titel zu den Stichworten „High-dose chemotherapy and life quality and oncology“. Von diesen Artikeln sind 13 als Reviews ausgewiesen. Ein Teil der Studien bezieht LQ lediglich auf die Nebenwirkungen, ohne den Begriff weiter zu operationalisieren. Drei Studien befassen sich mit Entscheidungssituationen in Bezug auf die Akzeptanz von Nebenwirkungen in Relation zum erwarteten Lebenszeitgewinn. Nicht alle Studien setzen psychometrisch überprüfte Erhebungsinstrumente zur Untersuchung der Lebensqualität ein. Die Recherche in „Medline“ wurde ergänzt durch Querverweise in verschiedenen Studien. Die Zusammenstellung erhebt jedoch keinen Anspruch auf Vollständigkeit.

Tabelle 8: Übersicht Reviews

\begin{tabular}{|c|c|c|c|}
\hline Autoren & Titel & Fragestellung und Anmerkungen & \begin{tabular}{|l|}
$\begin{array}{l}\text { Diagnose- } \\
\text { gruppe }\end{array}$ \\
\end{tabular} \\
\hline $\begin{array}{l}\text { Wisloff et } \\
\text { al. } \\
1999\end{array}$ & $\begin{array}{l}\text { Therapeutic options in the treatment of } \\
\text { multiple myeloma: pharmacoeconomic } \\
\text { and quality-of-life considerations. }\end{array}$ & $\begin{array}{l}\text { Vergleich der Standard- und der neuen } \\
\text { Chemotherapieregimes unter Einschluss } \\
\text { von HDC mit autologer Stammzelltrans- } \\
\text { plantation, Kostenvergleich, Frage nach } \\
\text { der Lebenszeit-verlängerung. }\end{array}$ & \begin{tabular}{|l|} 
Multiple \\
Myelome
\end{tabular} \\
\hline $\begin{array}{l}\text { Leitgeb } \\
1998\end{array}$ & $\begin{array}{l}\text { The development of the hematopietic } \\
\text { growth-factors had a major influence } \\
\text { on the treatment of malignant dis- } \\
\text { eases. Quality of Life gets increasingly } \\
\text { important in the setting of high-dose } \\
\text { chemotherapy and stem cell } \\
\text { transplantation. }\end{array}$ & $\begin{array}{l}\text { Untersuchung des Einflusses von } \\
\text { Wachstumsfaktoren ( G-CSF, EPO) auf } \\
\text { Nebenwirkungen der HDC. } \\
\text { Der Autor fragt nicht nach der Effektivität } \\
\text { in Bezug auf Lebenszeitverlängerung. }\end{array}$ & allgemein \\
\hline $\begin{array}{l}\text { Sprangers } \\
1998\end{array}$ & $\begin{array}{l}\text { The European Organization for Re- } \\
\text { search and Treatment of Cancer ap- } \\
\text { proach to developing questionnaire } \\
\text { modules: an update and overview. } \\
\text { EORTC Quality of Life Study Group. }\end{array}$ & $\begin{array}{l}\text { Ergänzend zu früheren Veröffentlichun- } \\
\text { gen werden neue Module vorgestellt } \\
\text { darunter das Modul „high-dose- } \\
\text { chemotherapy“. Forderungen in Bezug } \\
\text { auf methodische Qualität werden aufge- } \\
\text { stellt. }\end{array}$ & allgemein \\
\hline
\end{tabular}

60 „Medline“ ist die Bezeichnung für eine der renommiertesten internationalen medizinischen Datenbänke. 


\begin{tabular}{|c|c|c|c|}
\hline Autoren & Titel & Fragestellung und Anmerkungen & \begin{tabular}{|l}
$\begin{array}{l}\text { Diagnose- } \\
\text { gruppe }\end{array}$ \\
\end{tabular} \\
\hline $\begin{array}{l}\text { Lazarus } \\
1998\end{array}$ & $\begin{array}{l}\text { Hematopoietic progenitor cell trans- } \\
\text { plantation in breast cancer: current } \\
\text { status and future directions. }\end{array}$ & $\begin{array}{l}\text { Der Autor würdigt die Erfolge neuer } \\
\text { Technologien in Bezug auf Minderung } \\
\text { von Mortalität und Nebenwirkungen. Er } \\
\text { verweist auf die erhöhten Responsera- } \\
\text { ten bei gleichbleibender Überlebenszeit. }\end{array}$ & $\begin{array}{l}\text { Mamma- } \\
\text { karzinom }\end{array}$ \\
\hline $\begin{array}{l}\text { Lucey \& } \\
\text { Westphal } \\
1998\end{array}$ & $\begin{array}{l}\text { New approach to administrative medi- } \\
\text { cal decision-making: evidence-based } \\
\text { medicine using high-dose chemother- } \\
\text { apy/bone marrow transplant for breast } \\
\text { cancer. }\end{array}$ & $\begin{array}{l}\text { Die Autoren setzen sich kritisch mit der } \\
\text { gegenwärtigen unkontrollierten Anwen- } \\
\text { dungspraxis der HDC auseinander. } \\
\text { Kostengesichtspunkte sowie neue Be- } \\
\text { wertungskriterien für die Anwendung } \\
\text { werden untersucht. }\end{array}$ & $\begin{array}{l}\text { Mamma- } \\
\text { karzinom }\end{array}$ \\
\hline $\begin{array}{l}\text { Vahdat \& } \\
\text { Antman } \\
1997\end{array}$ & $\begin{array}{l}\text { High-dose chemotherapy with autolo- } \\
\text { gous stem cell support for breast can- } \\
\text { cer. }\end{array}$ & $\begin{array}{l}\text { Überblick, in dem Remissionsraten und } \\
\text { rezidivfreie Intervalle sehr optimistisch } \\
\text { dargestellt werden. }\end{array}$ & $\begin{array}{l}\text { Mamma- } \\
\text { karzinom } \\
\text { lokal fort- } \\
\text { geschritten, } \\
\text { inflamma- } \\
\text { torisch }\end{array}$ \\
\hline $\begin{array}{l}\text { Gilbert } \\
1996\end{array}$ & $\begin{array}{l}\text { Peripheral blood progenitor cell trans- } \\
\text { plantation for breast cancer: Pharma- } \\
\text { coeconomic considerations. }\end{array}$ & $\begin{array}{l}\text { Würdigung des Effektes der Wachstums- } \\
\text { faktoren in Bezug auf verringerte Ne- } \\
\text { benwirkungen, kürzere Krankenhaus- } \\
\text { aufenthalte und Möglichkeiten der ambu- } \\
\text { lanten Durchführung von HDC. Hinter- } \\
\text { fragung des generellen Nutzens fehlt. }\end{array}$ & $\begin{array}{l}\text { Mamma- } \\
\text { karzinom }\end{array}$ \\
\hline $\begin{array}{l}\text { Hoekman } \\
\text { \& Vermor- } \\
\text { ken } \\
1996\end{array}$ & $\begin{array}{l}\text { Incidence and prevention of } \\
\text { nonhaematological toxicity of high- } \\
\text { dose chemotherapy. }\end{array}$ & $\begin{array}{l}\text { Die Autoren untersuchen die Toxizität, } \\
\text { die sich nicht auf das Blut bezieht, und } \\
\text { geben eine detaillierte Übersicht über } \\
\text { Nebenwirkungen. Außerdem wird auf } \\
\text { Prävention und Behandlung dieser Ne- } \\
\text { benwirkungen eingegangen. }\end{array}$ & allgemein \\
\hline $\begin{array}{l}\text { Burns et } \\
\text { al. } \\
1995\end{array}$ & $\begin{array}{l}\text { Critical pathway for administering } \\
\text { high-dose chemotherapy followed by } \\
\text { peripheral blood stem cell rescue in } \\
\text { the outpatient setting. }\end{array}$ & $\begin{array}{l}\text { Frage, wie Patientinnen im ambulanten } \\
\text { Setting unterstützt werden können. }\end{array}$ & $\begin{array}{l}\text { Mamma- } \\
\text { karzinom } \\
\text { metasta- } \\
\text { siert }\end{array}$ \\
\hline $\begin{array}{l}\text { Smith \& } \\
\text { Nade- } \\
\text { manee } \\
1995\end{array}$ & $\begin{array}{l}\text { Bone marrow transplantation: the City } \\
\text { of Hope experience. }\end{array}$ & $\begin{array}{l}\text { Die Autoren vergleichen verschiedene } \\
\text { Chemotherapieregimes bei NHL. } \\
\text { HDC wird optimistisch beurteilt. }\end{array}$ & $\mathrm{NHL}$ \\
\hline $\begin{array}{l}\text { Shapiro \& } \\
\text { Henderson } \\
1994\end{array}$ & Adjuvant therapy of breast cancer. & $\begin{array}{l}\text { Einer der frühesten Reviewartikel, der } \\
\text { HDC verhalten optimistisch beurteilt. }\end{array}$ & $\begin{array}{l}\text { Mamma- } \\
\text { karzinom }\end{array}$ \\
\hline $\begin{array}{l}\text { Gis- } \\
\text { selbrecht } \\
1994\end{array}$ & $\begin{array}{l}\text { Chemotherapy dose intensity facili- } \\
\text { tated by use of lenograstim - implica- } \\
\text { tions for quality of life and survival. }\end{array}$ & $\begin{array}{l}\text { Beschäftigt sich mit den positiven Aus- } \\
\text { wirkungen von Wachstumsfaktoren, } \\
\text { ohne die Problematik der generellen } \\
\text { Effektivität in Bezug auf Lebenszeitver- } \\
\text { längerung zu vernachlässigen. }\end{array}$ & \begin{tabular}{|l} 
solide \\
Tumore
\end{tabular} \\
\hline
\end{tabular}

Die Betrachtung der Reviews zeigt, dass, mit einer Ausnahme (Sprangers 1998), LQ als unspezifischer, allgemeiner Begriff für alle möglichen Aspekte der Behandlung verwendet wird. Meistens wird LQ bezogen auf die Intensität und Dauer von Nebenwirkungen. Drei dieser Reviews fassen die positiven Effekte von Wachstumsfaktoren zusammen und verwenden in diesem Zusammenhang den Begriff der LQ. In die gleiche 
Richtung weist die Übersicht über ein verbessertes Management der Toxizität. Die Möglichkeit der Anwendung im ambulanten Setting wird ebenfalls als günstig in Bezug auf LQ interpretiert. Eine kritische Betrachtung und die Frage nach der Effektivität in Bezug auf die Überlebenszeit wird von vier Autoren/-gruppen vorgenommen.

In der nächsten Tabelle sind die Querschnittsstudien (mit und ohne Randomisierung) mit ihren jeweiligen Fragestellungen und kurzen Ergebnissen zusammengestellt, vermerkt ist auch die Methode der Datenerhebung und die untersuchte Tumorart:

Tabelle 9: Studien zu HDC und LQ

\begin{tabular}{|c|c|c|c|c|}
\hline Autor & Fragestellung & Tumor & Methodik & Ergebnisse \\
\hline $\begin{array}{l}\text { Vellenga } \\
\text { et al. } \\
2001\end{array}$ & $\begin{array}{l}\text { Wie unterscheiden sich } \\
\text { autologe Blutstammzell- } \\
\text { transplantation und auto- } \\
\text { loge Knochenmarks- } \\
\text { transpl. hinsichtlich Blut- } \\
\text { bildrekonstitution, LQ } \\
\text { und Kosten? }\end{array}$ & $\begin{array}{l}\text { Lymphom- } \\
\text { patienten mit } \\
\text { Rezidiv }\end{array}$ & $\begin{array}{l}\text { Querschnittstudie } \\
\text { N=204 } \\
2 \text { Gruppen werden } \\
\text { verglichen }\end{array}$ & $\begin{array}{l}\text { Die autologe Blutstamm- } \\
\text { zelltransplant. ist in jeder } \\
\text { Hinsicht überlegen. }\end{array}$ \\
\hline $\begin{array}{l}\text { Mounier } \\
\text { et al. } \\
2000\end{array}$ & $\begin{array}{l}\text { Q-TWiST-Analyse: } \\
\text { Vergleich von HDC mit } \\
\text { sequentieller Chemothe- } \\
\text { rapie }\end{array}$ & $\begin{array}{l}\text { Aggress. } \\
\text { Lymphom } \\
\text { Erste Kom- } \\
\text { plettremission }\end{array}$ & $\begin{array}{l}\text { Retrospektive Ana- } \\
\text { lyse }\end{array}$ & $\begin{array}{l}\text { Überlebenszeitschätzugen } \\
\text { differieren nicht. } \\
\text { HDC bringt für Hochrisiko- } \\
\text { patienten einen Vorteil } \\
\text { bezüglich der krankheits- } \\
\text { freien Zeit. }\end{array}$ \\
\hline $\begin{array}{l}\text { Bonomi } \\
\text { et al. } \\
2000\end{array}$ & $\begin{array}{l}\text { Wie unterscheiden sich } \\
\text { Überlebenszeit und } \\
\text { Lebensqualität bei } \\
\text { hoch/niedrig dosiertem } \\
\text { Paclitaxel/Cisplatin im } \\
\text { Vergleich mit Etopo- } \\
\text { sid/Cisplatin? }\end{array}$ & $\begin{array}{l}\text { Lungen- } \\
\text { karzinom }\end{array}$ & $\begin{array}{l}\text { Multicenterstudie } \\
\text { randomisiert, } \\
3 \text { Treatment- } \\
\text { Gruppen } \\
\text { N=599 } \\
\text { Fragebogen }\end{array}$ & $\begin{array}{l}\text { Längere Überlebenszeit } \\
\text { mit Paclitaxel/Cisplatin, } \\
\text { kein Unterschied zwischen } \\
\text { hoch und niedrig dosierter } \\
\text { Gruppe } \\
\text { QOL-Scores : kein signifi- } \\
\text { kanter Unterschied in den } \\
\text { drei Gruppen. }\end{array}$ \\
\hline $\begin{array}{l}\text { Winer et al. } \\
1999\end{array}$ & $\begin{array}{l}\text { Wie ist die LQ ein Jahr } \\
\text { nach HDC Therapie? }\end{array}$ & $\begin{array}{l}\text { Mamma- } \\
\text { karzinom }\end{array}$ & $\begin{array}{l}82 \text { Pat. werden nach } \\
\text { einem Jahr befragt } \\
\text { mit den Fragebö- } \\
\text { gen: FLIC, SDS und } \\
\text { einem selbst- } \\
\text { entworfenen Frage- } \\
\text { bogen zur Sexuali- } \\
\text { tät }\end{array}$ & $\begin{array}{l}\text { Persistierende Symptome: } \\
\text { Schlaflosigkeit, Erschöp- } \\
\text { fung, Schmerzen, } 1 / 3 \text { klagt } \\
\text { über verminderte sexuelle } \\
\text { Aktivität. Der Mittelwert der } \\
\text { Wiederaufnahme der Be- } \\
\text { rufstätigkeit liegt bei } 48 \\
\text { Wochen. Die Patientinnen } \\
\text { beschreiben ihre LQ als } \\
\text { relativ gut. }\end{array}$ \\
\hline $\begin{array}{l}\text { Dimeo et al. } \\
2000\end{array}$ & $\begin{array}{l}\text { Wirkt sich Fahrradergo- } \\
\text { metertraining auf psy- } \\
\text { chologische Belastung } \\
\text { und physische Sympto- } \\
\text { me aus? }\end{array}$ & keine Angabe & $\begin{array}{l}\text { HDC-Patienten: } \\
\mathrm{N}=27 \text { (Training) } \\
\mathrm{N}=32 \text { (Kontrolle) } \\
\text { Fragebögen bei } \\
\text { Entlassung }\end{array}$ & $\begin{array}{l}\text { signifikante Verbesserun- } \\
\text { gen in der Trainingsgrup- } \\
\text { pe: geringere Aufenthalts- } \\
\text { dauer, weniger psychische } \\
\text { Belastung, geringere Er- } \\
\text { schöpfung. }\end{array}$ \\
\hline $\begin{array}{l}\text { Ahles et al. } \\
1999\end{array}$ & $\begin{array}{l}\text { Welche Auswirkungen } \\
\text { hat Massage auf die } \\
\text { Beschwerden von HDC } \\
\text { Patientinnen? }\end{array}$ & $\begin{array}{l}\text { Mamma- } \\
\text { karzinom }\end{array}$ & $\begin{array}{l}\text { HDC-Patientinnen: } \\
\text { Behandlungs- } \\
\text { gruppe und Kon- } \\
\text { trollgruppe (rando- } \\
\text { misiert) } \\
\text { Fragebögen }\end{array}$ & $\begin{array}{l}\text { In der Behandlungsgruppe } \\
\text { kommt es zu einer signifi- } \\
\text { kanten Reduzierung von } \\
\text { Distress, Müdigkeit, Nau- } \\
\text { sea und Angst. }\end{array}$ \\
\hline
\end{tabular}




\begin{tabular}{|c|c|c|c|c|}
\hline Autor & Fragestellung & Tumor & Methodik & Ergebnisse \\
\hline $\begin{array}{l}\text { Wettergren } \\
\text { et al. } 1999\end{array}$ & $\begin{array}{l}\text { Post-Traumatisches } \\
\text { Stress-Syndrom in Be- } \\
\text { ziehung zu LQ und } \\
\text { "sense of coherence" }\end{array}$ & $\begin{array}{l}\text { Maligne hä- } \\
\text { matologi- } \\
\text { sche Erkran- } \\
\text { kungen }\end{array}$ & $\begin{array}{l}\mathrm{N}=20 \\
\text { Fragebögen }\end{array}$ & $\begin{array}{l}\text { Hohe PTSS-Werte, die mit } \\
\text { der Zeit zwar abnehmen, } \\
\text { aber höher sind als bei } \\
\text { anderen onkologischen } \\
\text { Stichproben. } \\
\text { Vermeidung korreliert sig- } \\
\text { nifikant mit Angst und De- } \\
\text { pression. }\end{array}$ \\
\hline $\begin{array}{l}\text { Jacobson } \\
\text { et al. } 1998\end{array}$ & $\begin{array}{l}\text { Post-Traumatisches } \\
\text { Stress-Syndrom nach } \\
\text { ABMT }\end{array}$ & $\begin{array}{l}\text { Mammakar- } \\
\text { zinom }\end{array}$ & $\begin{array}{l}2 \text { Patientinnen } \\
\text { 2-62 Monate nach } \\
\text { Therapie ohne Re- } \\
\text { zidiv }\end{array}$ & $\begin{array}{l}12-19 \% \text { PTSD (DSM-IV) } \\
\text { Zusammenhang mit: niedr. } \\
\text { Ausbildungsstand, fort- } \\
\text { geschr. Erkrankung und } \\
\text { längerem Krankenhaus- } \\
\text { aufenthalt }\end{array}$ \\
\hline $\begin{array}{l}\text { van Dam } \\
\text { et al. } 1998\end{array}$ & $\begin{array}{l}\text { Wird die kognitive Funk- } \\
\text { tion durch HDC beein- } \\
\text { trächtigt? }\end{array}$ & $\begin{array}{l}\text { Mammakar- } \\
\text { zinom } \\
\text { Hochrisiko- } \\
\text { Patientinnen }\end{array}$ & $\begin{array}{l}\mathrm{N}=34 \text { (HDC) } \\
\mathrm{N}=36 \text { (konvent.) } \\
\mathrm{N}=34 \text { (unbeh. Kon- } \\
\text { trollgruppe) } \\
\text { Neuropsycho- } \\
\text { logische Test- } \\
\text { batterie, } 2 \text { Jahre } \\
\text { nach Therapie- } \\
\text { abschluss }\end{array}$ & $\begin{array}{l}\text { Signifikante Unterschiede } \\
\text { zwischen allen drei Grup- } \\
\text { pen. Die kognitive Funktion } \\
\text { ist in der HDC-Gruppe } \\
\text { stärker beeinträchtigt als in } \\
\text { der Gruppe mit konventio- } \\
\text { neller Therapie. }\end{array}$ \\
\hline $\begin{array}{l}\text { Hecht et al. } \\
1997\end{array}$ & $\begin{array}{l}\text { Auftreten und Ursache } \\
\text { von persistierender } \\
\text { Übelkeit und Erbrechen. }\end{array}$ & $\begin{array}{l}\text { Mammakar- } \\
\text { zinom } \\
\text { Hochrisiko- } \\
\text { Patientinnen }\end{array}$ & $\begin{array}{l}\text { Beobachtungsstudie } \\
\mathrm{N}=38 \text { Befragung } \\
\text { hinsichtlich Übel- } \\
\text { keit/Erbrechen }\end{array}$ & $\begin{array}{l}\text { Bei einem Teil der Patien- } \\
\text { tinnen persistiert die Sym- } \\
\text { ptomatik trotz antiemeti- } \\
\text { scher Medikation. } \\
\text { Die Autoren machen eine } \\
\text { Gastroparese als Ausdruck } \\
\text { der gastrointestinalen To- } \\
\text { xizität verantwortlich. }\end{array}$ \\
\hline $\begin{array}{l}\text { Andrykowski } \\
\text { et al. } \\
1997\end{array}$ & $\begin{array}{l}\text { Energielevel und Schlaf- } \\
\text { störungen nach BMT. }\end{array}$ & $\begin{array}{l}\text { Gemischte } \\
\text { Gruppe }\end{array}$ & $\begin{array}{l}\text { Multicenterstudie } \\
2 \text { Messzeitpunkte } \\
\mathrm{N}=137 \\
\text { Fragebogen und } \\
\text { Telefoninterview }\end{array}$ & $\begin{array}{l}50-70 \% \text { klagen über er- } \\
\text { niedrigtes Energieniveau } \\
\text { und Schlafprobleme. 15- } \\
20 \% \text { davon schwerwie- } \\
\text { gend. } \\
\text { Deutlicher Zusammenhang } \\
\text { zwischen den Symptomen, } \\
\text { fortgeschr. Alter und weibl. } \\
\text { Geschlecht. }\end{array}$ \\
\hline $\begin{array}{l}\text { Fromm et al. } \\
1996\end{array}$ & $\begin{array}{l}\text { Positive und negative } \\
\text { Effekte von BMT und die } \\
\text { Konsequenzen für die } \\
\text { LQ. }\end{array}$ & $\begin{array}{l}\text { Gemischte } \\
\text { Gruppe }\end{array}$ & $\begin{array}{l}\mathrm{N}=137 \\
\text { Interviews } \\
\text { Thematische Analy- } \\
\text { se }\end{array}$ & $\begin{array}{l}\text { Fragebogeninstrumente } \\
\text { können positive Aspekte } \\
\text { von Veränderung nach } \\
\text { BMT vermutlich nicht er- } \\
\text { fassen. }\end{array}$ \\
\hline $\begin{array}{l}\text { Bush et al. } \\
1995\end{array}$ & $\begin{array}{l}\text { Wie ist die Lebensquali- } \\
\text { tät bei Langzeitüberle- } \\
\text { benden nach KMT? }\end{array}$ & $\begin{array}{l}\text { Gemischte } \\
\text { Gruppe }\end{array}$ & $\begin{array}{l}\mathrm{N}=125 \\
\mathrm{Im} \text { Mittel hatten die } \\
\text { Patienten } 10 \text { Jahre } \\
\text { überlebt. } \\
\text { Fragebögen }\end{array}$ & $\begin{array}{l}\text { Langzeitüberlebende: LQ } \\
\text { gut bis sehr gut, bei gleich- } \\
\text { zeitig vorhandenen Sym- } \\
\text { ptomen in milder Ausprä- } \\
\text { gung. Minderheit mit nied- } \\
\text { riger LQ. }\end{array}$ \\
\hline $\begin{array}{l}\text { Andrykowski, } \\
\text { Greiner \& } \\
\text { Altmaier } \\
1995 \text { (a) }\end{array}$ & $\begin{array}{l}\text { LQ nach HDC } \\
\text { Vergleich allogen vs. } \\
\text { autolog. }\end{array}$ & $\begin{array}{l}\text { Maligne hä- } \\
\text { matologische } \\
\text { Erkrankun- } \\
\text { gen }\end{array}$ & $\begin{array}{l}\text { Multicenterstudie } \\
\text { Querschnitts- } \\
\text { analyse } \\
2 \text { Gruppen } \\
\text { autolog } 54 \% \\
\text { allogen } 46 \% \\
\text { Fragebögen }\end{array}$ & $\begin{array}{l}\text { LQ der allogen Transplan- } \\
\text { tierten ist niedriger. Niedri- } \\
\text { ge LQ korreliert mit for- } \\
\text { geschr. Erkrankung und } \\
\text { niedrigem Bildungsniveau. }\end{array}$ \\
\hline $\begin{array}{l}\text { Andrykowski, } \\
\text { Brady, Grei- } \\
\text { ner et al. } \\
1995 \text { (b) }\end{array}$ & $\begin{array}{l}\text { Situation nach BMT - } \\
\text { Übereinstimmung mit } \\
\text { Erwartungen und infor- } \\
\text { med consent? }\end{array}$ & $\begin{array}{l}\text { Gemischte } \\
\text { Gruppe }\end{array}$ & $\begin{array}{l}\text { Multicenterstudie } \\
\mathrm{N}=172 \\
\text { Fragebögen und } \\
\text { Interview, ca. } 44 \\
\text { Monate (Mittelwert) } \\
\text { nach BMT }\end{array}$ & $\begin{array}{l}\text { Eine Minderheit ist zum } \\
\text { „normalen“ Leben zurück- } \\
\text { gekehrt. Einschränkungen: } \\
\text { physisch, kognitiv, sexuell, } \\
\text { interpersonell. } \\
\text { Positive Bewertung der } \\
\text { Behandlung. }\end{array}$ \\
\hline
\end{tabular}




\begin{tabular}{|l|l|l|l|l|}
\hline Autor & Fragestellung & Tumor & Methodik & Ergebnisse \\
\hline $\begin{array}{l}\text { Henon et al. } \\
1995\end{array}$ & $\begin{array}{l}\text { Vergleich dreier Chemo- } \\
\text { therapiegruppen hin- } \\
\text { sichtlich Überleben, LQ } \\
\text { und Kosten. }\end{array}$ & $\begin{array}{l}\text { Multiple Mye- } \\
\text { lome }\end{array}$ & $\begin{array}{l}\text { 3 Gruppen: zwei } \\
\text { regimes HDC } \\
\text { N=12 und N=10 } \\
\text { konven.Therapie } \\
\text { N=15 } \\
\text { Fragebögen }\end{array}$ & $\begin{array}{l}\text { Intensive Therapie erhöht } \\
\text { die Überlebenszeit bei } \\
\text { befriedigender LQ und } \\
\text { begründbaren Kosten. }\end{array}$ \\
\hline $\begin{array}{l}\text { Hillner et al. } \\
1992\end{array}$ & $\begin{array}{l}\text { Theoretische Arbeit, die } \\
\text { mit hypothetischer Ko- } \\
\text { horte von Frauen mit } \\
\text { metastasiertem Brust- } \\
\text { krebs operiert um die } \\
\text { Akzeptanz von HDC zu } \\
\text { erfassen. }\end{array}$ & $\begin{array}{l}\text { Mammakar- } \\
\text { zinom }\end{array}$ & $\begin{array}{l}\text { 2 hypothetische } \\
\text { Therapieoptionen: } \\
\text { konventionelle The- } \\
\text { rapie/ HDC Frage- } \\
\text { bögen }\end{array}$ & $\begin{array}{l}\text { HDC wird präferiert, ohne } \\
\text { Rücksicht auf die Kosten. }\end{array}$ \\
& & & \\
& & & \\
\hline
\end{tabular}

Diese Studien verwenden (überwiegend psychometrisch überprüfte) Fragebögen. Drei Studien vergleichen LQ von Patienten mit HDC und konventioneller Chemotherapie. Eine Studie vergleicht allogene mit autologer Transplantation. Drei Studien geben eine Zustandsbeschreibung nach HDC in Bezug auf LQ-Indikatoren. Zwei Studien beschreiben den Zustand im Hinblick auf persistierende Symptome. Zwei Studien untersuchen den Effekt von Interventionen (Massage, Training) auf die LQ. Eine Studie untersucht die Auswirkungen von HDC und konventioneller Chemotherapie im Vergleich mit unbehandelter Kontrollgruppe auf neuropsychologische Leistungen. Eine Studie untersucht gesunde Frauen mit der Vorgabe zu entscheiden, welche Therapie sie unter Akzeptanz welcher Nebenwirkungen auswählen würden. In einer Studie werden die Daten durch Interviews erhoben und inhaltsanalytisch ausgewertet, in einer weiteren Studie werden Fragebögen mit Interviews kombiniert. Zuletzt sollen noch die prospektiven Studien und ihre Ergebnisse zusammengestellt werden:

Tabelle 10: Prospektive Studien

\begin{tabular}{|l|l|l|l|l|}
\hline Autor & Fragestellung & Tumor & Methodik & Ergebnisse \\
\hline $\begin{array}{l}\text { Carslson } \\
\text { et al. } \\
2001\end{array}$ & $\begin{array}{l}\text { Auswirkung von HDC auf } \\
\text { Lebensqualiät. }\end{array}$ & $\begin{array}{l}\text { Mamma- } \\
\text { Karzinom, } \\
\text { metastasiert }\end{array}$ & $\begin{array}{l}\text { N=50 } \\
\text { Vor HDC - 1 } \\
\text { Jahr danach } \\
\text { FLIC } \\
7 \text { Meßzeit-punkte }\end{array}$ & $\begin{array}{l}\text { Krankheitsbürde wird zu allen } \\
\text { Zeitpunkten als am schwierigs- } \\
\text { ten empfunden. } \\
\text { Angst vor der Zukunft, Verlust } \\
\text { von sex. Interesse, Therapie- } \\
\text { angst. Belastungen generell } \\
\text { vor und kurz nach Therapie } \\
\text { am größten, Angst/Depression } \\
\text { bleibt ausgeprägt. }\end{array}$ \\
\hline $\begin{array}{l}\text { Macquart- } \\
\text { Moulin et al. }\end{array}$ & $\begin{array}{l}\text { LQ Veränderungen durch } \\
\text { sequentielle HDC - Akzep- } \\
\text { tanz der Therapie. }\end{array}$ & $\begin{array}{l}\text { Inflammator. } \\
\text { Mamma- } \\
\text { karzinom }\end{array}$ & $\begin{array}{l}\text { N=95 } \\
5 \text { Meßzeitp. } \\
\text { T5 ein Jahr nach } \\
\text { HDC } \\
\text { QLQ-C30 und Ad } \\
\text { hoc Fragen }\end{array}$ & $\begin{array}{l}\text { Nach der 3. HDC erhebliche } \\
\text { Belastungen, die sich auch im } \\
\text { QLQ-C30 zeigen. } \\
\text { Nach einem Jahr Rückkehr zu } \\
\text { den Ausgangswerten, } \\
\text { Ausnahme: phys. Funktion }\end{array}$ \\
\hline $\begin{array}{l}\text { Courneya et } \\
\text { al. } \\
2000\end{array}$ & $\begin{array}{l}\text { Wirkt sich Training auf LQ } \\
\text { nach HDC aus? }\end{array}$ & gemischt & $\begin{array}{l}\text { N=25 } \\
\text { Wöchentliche } \\
\text { Messung im } \\
\text { Krankenhaus } \\
\text { Fragebögen }\end{array}$ & $\begin{array}{l}\text { Signifikante Effekte durch } \\
\text { Training auf alle LQ- } \\
\text { Indikatoren, auf Depression } \\
\text { und Angst, auf die Dauer des } \\
\text { stationären Aufenthaltes. }\end{array}$ \\
& & & &
\end{tabular}




\begin{tabular}{|c|c|c|c|c|}
\hline Autor & Fragestellung & Tumor & Methodik & Ergebnisse \\
\hline $\begin{array}{l}\text { Fife et al. } \\
2000\end{array}$ & $\begin{array}{l}\text { Belastung wann am größ- } \\
\text { ten? Welche Variablen sind } \\
\text { verant-wortlich? Gibt es } \\
\text { Prädiktorvariablen für Inter- } \\
\text { ventionen? }\end{array}$ & Gemischt & \begin{tabular}{|l|}
$\mathrm{N}=100$ \\
6 Meßzeit- \\
punkte: vor $-7 / 14$ \\
Tage nach \\
Transplant. $-1-$ \\
$3-12$ nach Ent- \\
lassung \\
\end{tabular} & $\begin{array}{l}\text { Psychosoz. Vulnerabilität vor- } \\
\text { her am größten. Ausmaß der } \\
\text { persönlichen Kontrollüberzeu- } \\
\text { gung als wichtige Variable. } \\
\text { Der emotionale Zustand vorher } \\
\text { ist ein wichtiger Prädiktor. }\end{array}$ \\
\hline $\begin{array}{l}\text { Hjermstad et } \\
\text { al. } \\
1999\end{array}$ & $\begin{array}{l}\text { Vergleich Lymphom- } \\
\text { patienten mit unterschiedli- } \\
\text { chen chemoth. Behandlun- } \\
\text { gen in Bezug auf gesund- } \\
\text { heitsbezogene LQ nach } \\
\text { einem Jahr. }\end{array}$ & $\begin{array}{l}\text { Lymphome } \\
\text { Leukämie }\end{array}$ & $\begin{array}{l}2 \text { HDC Gruppen: } \\
\text { allogen } \mathrm{N}=41 \\
\text { autolog } \mathrm{N}=51 \\
\text { kombin. Che- } \\
\text { moth. (CT) N= } 85 \\
\\
\text { Prä/Post (nach } \\
\text { einem Jahr) } \\
\text { QLQ-C30 }\end{array}$ & $\begin{array}{l}\text { Die kognitive Funktion ist in } \\
\text { der CT-Gruppe signifikant } \\
\text { besser nach einem Jahr. } \\
\text { Vor der Behandlung sind die } \\
\text { Gruppenunterschiede größer } \\
\text { als nach einem Jahr. } \\
\text { Das Veränderungsmuster in } \\
\text { den Gruppen unterscheidet } \\
\text { sich. }\end{array}$ \\
\hline $\begin{array}{l}\text { Larsen } \\
\text { et al. } \\
1996\end{array}$ & $\begin{array}{l}\text { Wie wird die LQ durch HDC } \\
\text { beeinflusst? }\end{array}$ & $\begin{array}{l}\text { Mamma- } \\
\text { karzinom }\end{array}$ & $\begin{array}{l}\mathrm{N}=8 \\
\text { Erhebung zu drei } \\
\text { Zeitpunkten mit- } \\
\text { hilfe von Frage- } \\
\text { bögen: SIP, } \\
\text { QUAL }\end{array}$ & $\begin{array}{l}\text { Der physische Zustand ist am } \\
\text { schlechtesten bei der Entlas- } \\
\text { sung, der emotionale Zustand } \\
\text { ist während der gesamten } \\
\text { Untersuchungsperiode beein- } \\
\text { trächtigt. }\end{array}$ \\
\hline $\begin{array}{l}\text { Ahles et al. } \\
1996\end{array}$ & $\begin{array}{l}\text { Neurologische und psycho- } \\
\text { logische Auswirkungen vor, } \\
\text { während und nach HDC. }\end{array}$ & $\begin{array}{l}\text { Mamma- } \\
\text { karzinom } \\
\text { Maligne Häma- } \\
\text { tol. } \\
\text { Erkrankungen }\end{array}$ & $\begin{array}{l}\mathrm{N}=34 \\
\text { Fragebögen } \\
\text { neuropsycho- } \\
\text { logische Test- } \\
\text { batterie }\end{array}$ & $\begin{array}{l}\text { Neuropsychologisch kommt es } \\
\text { zur Verschlechterung der Er- } \\
\text { gebnisse für beide Gruppen. } \\
\text { Die psycholog. Situation ver- } \\
\text { bessert sich im Verlauf der } \\
\text { Behandlung. } \\
\text { Ma Ca Patientinnen haben zu } \\
\text { Beginn ein niedrigeres } \\
\text { Angstniveau. }\end{array}$ \\
\hline $\begin{array}{l}\text { McQuellon } \\
\text { et al. } \\
1995\end{array}$ & $\begin{array}{l}\text { Frauen mit früherkanntem } \\
\text { Brustkrebs werden nach } \\
\text { hypothetischen Therapieop- } \\
\text { tionen befragt und die LQ } \\
\text { wird vor und nach HDC } \\
\text { erfasst. }\end{array}$ & $\begin{array}{l}\text { Mamma- } \\
\text { karzinom }\end{array}$ & $\begin{array}{l}115 \text { Frauen mit } \\
\text { Ma-Ca, alle Pati- } \\
\text { entinnen mastek- } \\
\text { tomiert/ lumpek- } \\
\text { tomiert und radio- } \\
\text { therapiert } \\
76 \text { adjuvante } \\
\text { Chemotherapie } \\
24 \text { HDC } \\
\text { prae-post nach } 1 \\
\text { Jahr } \\
\text { FACT, POMS } \\
\text { u.a. } \\
\end{array}$ & $\begin{array}{l}\text { Ein erheblicher Anteil von } \\
\text { Patientinnen akzeptiert das } \\
\text { Risiko erhöhter Toxizität bei } \\
\text { nur minimalem Effekt auf die } \\
\text { Gesamtüberlebenszeit. } \\
\text { Generell gute Anpassung nach } \\
\text { HDC. } \\
30 \% \text { haben Probleme in Bezug } \\
\text { auf Sexualität, Fatigue und } \\
\text { Depressivität. }\end{array}$ \\
\hline $\begin{array}{l}\text { Syrjala et al. } \\
1993\end{array}$ & $\begin{array}{l}\text { Erholungsverlauf nach auto- } \\
\text { loger KMT - physische und } \\
\text { psychosoziale Funktionen. }\end{array}$ & Gemischte & $\begin{array}{l}\mathrm{N}=67 \\
4 \text { Zeitpunkte bis } \\
\text { ca. } 4 \text { Jahre nach } \\
\text { KMT } \\
\text { Fragebögen }\end{array}$ & $\begin{array}{l}\text { Nach } 90 \text { Tagen fühlen sich die } \\
\text { Patienten am stärksten beein- } \\
\text { trächtigt, nach einem Jahr ist } \\
\text { ein Zustand wie vorher er- } \\
\text { reicht. }\end{array}$ \\
\hline $\begin{array}{l}\text { Chao et al. } \\
1992\end{array}$ & $\begin{array}{l}\text { Veränderung der LQ im } \\
\text { zeitlichen Verlauf nach } \\
\text { autologer KMT. }\end{array}$ & $\begin{array}{l}\text { Maligne } \\
\text { Hämatolo- } \\
\text { gische Erkran- } \\
\text { kungen }\end{array}$ & $\begin{array}{l}\mathrm{N}=58 \\
\text { Alle } 90 \text { Tage, ein } \\
\text { Jahr lang } \\
\text { Fragebögen }\end{array}$ & $\begin{array}{l}\text { Nach einem Jahr bestehen bei } \\
32-55 \% \text { der Patienten noch } \\
\text { Probleme in Bezug auf Ar- } \\
\text { beitsfähigkeit, Sexualität, Kör- } \\
\text { perbild. }\end{array}$ \\
\hline
\end{tabular}




\subsection{Zusammenfassung der Ergebnisse}

Von zwei Ausnahmen abgesehen werden die Daten der prospektiven Studien mithilfe von Fragebögen erhoben und deskriptiv ausgewertet. Gruppen- und Zeiteffekte werden berechnet. Generell sind sich alle Autoren darüber einig, dass die invasiven medizinischen Prozeduren und die aus der Toxizität der Behandlung resultierenden Belastungen neue Anpassungsstrategien erfordern. Bislang ist der Zeitraum von bis zu vier Jahren nach HDC mit autologer Stammzelltransplantation untersucht worden. Die Anpassung nach einer HDC ist gut, legt man die Selbstbeschreibungen der Patienten aus den Fragebögen zugrunde. Eine Minderheit von Patienten, bei der noch Symptome vorhanden sind, teilt diese Beurteilung trotz der Beeinträchtigungen mit den anderen.

Die größte emotionale Belastung besteht für Patienten zum Zeitpunkt unmittelbar vor Beginn der HDC (Andrykowski 1994; Fife et al. 2000) und ist vermutlich als hohe Erwartungsangst zu werten.

Obwohl die physische Belastung durch die Aplasiephase zunimmt, nehmen Angst und Depressivität ab. Der physische Zustand wird bei Entlassung am schlechtesten beurteilt. Nach der Entlassung aus dem Krankenhaus nehmen sowohl physische als auch psychische Symptome mit der Zeit ab. Nach einem Jahr ist jedoch bei den Patienten, die über physische Symptome klagen, noch erheblicher emotionaler Distress vorhanden (Fife et al. 2000). Nach einem Jahr finden sich unter den Patienten noch ca. 30\% mit Beeinträchtigungen in folgenden Bereichen:

- Sexualität $t^{61}$

- Selbstwert/Aussehen

- Fatigue ${ }^{62}$

- Depressivität

- Arbeitsfähigkeit

Die Ergebnisse in Bezug auf persistierende Beschwerden decken sich in den vorliegenden Studien weitgehend.

Die Patienten, denen ein positives Reframing der Behandlungssituation gelingt (,Zweite Geburt“, „Neue Lebenschance“), reagieren mit deutlich weniger Angst, Depressivität und Unsicherheit (Fife et al. 2000).

Fife et al. stellen eine signifikante Beziehung zwischen Angst und Depressivität vor Behandlungsbeginn und der späteren emotionalen Reaktion und Anpassung an die Belastung fest. Je mehr vermeidendes Coping Patienten zeigen, desto größer ist die

\footnotetext{
${ }^{61}$ Die Sexualität wird erheblich beeinträchtigt. Siehe auch die Studien von Bush et al. 1995; Wingard et al. 1992.

${ }^{62}$ Fatigue scheint über einen längeren Zeitraum zu persistieren. Van Dam et al. (1998) stellen einen deutlichen Unterschied zur Kontrollgruppe noch nach 2 Jahren fest.
} 
Angst. Andrykowski et al. (1995a) ermitteln einen Zusammenhang zwischen LQ, niedrigem Bildungsniveau und fortgeschrittenem Krankheitsprozess.

Eine Studie, die über die oben genannten Zeiträume hinausgeht, liegt für den Bereich HDC mit autologer Stammzelltransplantation noch nicht vor.

Vergleichbar erscheinen, ausgehend von den noch erheblicheren Behandlungsbelastungen, die Ergebnisse der Studie an Langzeitüberlebenden (Mittelwert 10 Jahre) von Bush et al. (1995) mit KMT-Patienten ( $N=125)$. Die Befragten beschreiben, abgesehen von einer kleinen Gruppe, ihre LQ als gut bis ausgezeichnet trotz bestehender Symptome! Die meisten betrachten sich nach so langer Zeit als geheilt. Sie sind überwiegend produktiv, psychisch stabil und haben die Erkrankung gut verarbeitet. Beschwerden beziehen sich auf folgende Bereiche: sexuelle Dysfunktion, emotionale Probleme, Fatigue, Augenprobleme, Schlafstörungen, Schmerzen und kognitive Einschränkungen (Gedächtnis und Konzentration).

Wenn auch die Fragestellung der eigenen Studie nicht unmittelbar berührt wird, so sind doch noch einige weitere Studien im Kontext von Lebensqualität interessant. Einige Autoren untersuchen den prognostischen Wert von LQ-Indikatoren:

Broers et al. (1998) : kein Einfluss psychologischer Variabeln ( tendenziell ist niedriger Selbstwert mit kürzerem Überleben verbunden).

Murphy et al. (1996): kein Einfluss psychologischer Variablen. In der Folgeanalyse der gleichen Stichprobe findet sich zu einem späteren Zeitpunkt ebenfalls kein Einfluss.

In den folgenden Studien findet sich ein Zusammenhang mit psychologischen Variablen:

Andrykowsy (1994): eine höhere physische Funktion verbunden mit der angstvollen Beschäftigung mit der Erkrankung korreliert mit kürzerer Überlebenszeit.

Colon et al. (1991): geringe Depressivität vor der Behandlung ist verbunden mit längerer Überlebenszeit (nach einem Jahr).

Die Ergebnisse lassen derzeit keine Verallgemeinerungen zu. 


\section{Teil II}

\section{$9 \quad$ Fragestellung und Forschungsansatz der Studie}

Wie im Kapitel Konzeptualisierung der LQ in der Onkologie ausgeführt, besteht die Schwierigkeit des Konstrukes LQ darin, eindeutige Beziehungen zwischen Allgemeiner LQ und einzelnen Komponenten von LQ zu definieren. Aus diesem Grunde ist ein deduktives, Hypothesen testendes Vorgehen im strengen Sinne nicht möglich. Als heuristischer Rahmen für diese Studie wurde das Modell von Abbey und Andrews (1985) gewählt und angepasst an die Bedingungen der Studie. Aus folgenden Gründen ist das Modell dazu gut geeignet:

Im Modell der Autoren sind sowohl Persönlichkeitsfaktoren als auch Umwelteinflüsse integriert. Attribuierungsprozesse und soziale Unterstützung gehen als Stressmoderatoren in die Wahrnehmung und Bewertung der Ausgangssituation ein. Wichtige intervenierende Variabeln sind Depression und Angst. Das Modell geht von Lebensqualitätskonzepten aus, nicht von einem einheitlichen Konstrukt.

Modell adaptiert von Abbey \& Andrews 1985

Abb. 11: Verbindung von Sozialpsychologischen Konzepten mit dem LQ-Konzept (S. 4)

\begin{tabular}{|c|c|c|}
\hline Sozialpsychologische & & Lebensqualitätskonzepte \\
\hline Konzepte & Konzepte & \\
\hline Stress & Depression & Allgemeine LQ \\
\hline Lebensgestaltung & Angst & Komponenten LQ \\
\hline Internale Konrolle & & Selbstwert \\
\hline Externale Kontrolle & & Negative Affekte \\
\hline Soziale Unterstützung & & Positive Affekte \\
\hline & & Kognitionen \\
\hline
\end{tabular}

Das Modell dient als heuristischer Rahmen. Wie bereits erwähnt, lautet das Studienziel:

„Das Ziel der Studie besteht darin, an einer definierten Patientengruppe unter der standardisierten Therapiebedingungen der Hochdosischemotherapie mit nachfolgender autologer Stammzelltransplantation, mithilfe der therapiebegleitenden „Lebensquali- 
tätsforschung" ein genaues Bild des Belastungsprofiles und der moderierenden

Faktoren zu gewinnen. Die Analyse soll dazu beitragen, die während und nach der Therapie aufgetretenen Einschränkungen zu bewerten und Anhaltspunkte dafür liefern, wie der Behandlungsverlauf am besten unterstützt werden kann."

Zunächst soll noch einmal auf die in der Einleitung formulierten Grundsätze hingewiesen werden:

1. Gemäß der gegenwärtig herrschenden Forschungspraxis wird ein quantifizierender Zugang mit psychometrisch überprüften Fragebögen gewählt.

2. Zusätzlich wird ein hermeneutischer Zugang gewählt, der einen subjektbezogenen Zugang ermöglichen soll.

3. Den Mitarbeitern schien eine Untersuchung nur dann vertretbar, wenn Patienten nicht nur als „Datenlieferanten“ gesehen wurden sondern wenn gleichzeitig ein Unterstützungsangebot offeriert wurde. Das Angebot hatte supportiven Charakter und sollte in erster Linie der Stabilisierung des Ich dienen und im Sinne der Angstreduktion wirken. Auf konfrontative und aufdeckende Interventionen wurde verzichtet.

Die Studie ist als prospektive, explorative Längsschnittstudie mit fünf Erhebungszeitpunkten konzipiert. Es wird angenommen, dass die Belastungen (und damit auch LQ) vom Untersuchungszeitpunkt abhängig sind. Im Zeitverlauf werden Veränderungen erwartet. Die Daten sollen in einem psychoonkolgischen Betreuungskontext erhoben werden. Die HDC-Therapie wird als unäbhängige Variable betrachtet, deren Auswirkungen auf die abhängigen biomedizinischen und psychologischen Variablen untersucht werden sollen. Neben dem Studienteil, der sich standardisierter Verfahren zur Datenerhebung bedient, werden in einem qualitativen Teil durch semistrukturierte Interviews Daten erhoben, um sich den komplexen Phänomenen zu nähern, die unter dem Stichwort LQ zusammengefasst werden. Die Interviews sollen Antworten geben auf Fragen, die durch die standardisierten Instrumente nicht beantwortet werden können, und sie sollen dazu dienen, Hypothesen in Bezug auf die inhaltliche Bedeutung von LQ in diesem spezifischen Kontext zu generieren. Da es bei der Untersuchung von LQ nicht um Forschungsneuland geht, waren entsprechende Vorannahmen unumgänglich und sind im Sinne einer Interessenfokussierung eingegangen. Quantitative Studien können im Gegensatz zu qualitativen nichts darüber aussagen, wie Patienten die belastenden Situationen wahrnehmen noch welche Prozesse bei den einzelnen Patienten ablaufen. 
Die beiden Forschungsmethoden werden nicht als gegensätzlich betrachtet sondern sollen sich ergänzen. Pope \& Mays $(1995)^{63}$ verweisen auf eine der gebräuchlichen Gegenüberstellungen:

Tabelle 12: Gegenüberstellung qualitativer und quantitativer Wissenschaft (S. 42)

\begin{tabular}{|lcc|}
\hline \multicolumn{3}{l}{$\begin{array}{l}\text { The overstated dichotomy between quantitative and } \\
\text { qualitative social science }\end{array}$} \\
\hline & Qualitative & Quantitative \\
\hline Social theory: & Action & Structure \\
Methods: & Observation, interview & Experiment, survey \\
Question: & What is X (classification) & How many X's (enumeration) \\
Reasoning: & Inductive & Deductive \\
Sampling method: & Theoretical & Statistical \\
Strength: & Validity & Reliability \\
\hline
\end{tabular}

Die Autoren diskutieren die beiden Ansätze und ihren Nutzen für den Forschungsgegenstand Gesundheit/Gesundheitssystem und schließen: „In addition, qualitative work can reach aspects of complex behaviours, attitudes, and interactions which quantitative methods cannot" (ebenda S.45).

In einem Forschungsfeld mit widersprüchlichen Ergebnissen scheint es geboten, den Versuch zu unternehmen, sich dem Phänomen LQ nicht nur psychometrisch sondern vor allem auch qualitativ zu nähern. Diese Ansicht wird auch von Vertretern der LQForschung auf dem Weltkongress Psycho-Oncology in Melbourne 2001 vertreten: „Bei den Studien zum Thema Lebensqualität zeigte sich wieder, dass erst durch die quantitative und qualitative Erfassung der Daten (bspw. Fragebogen ergänzt durch Interviews) eine umfassende Beurteilung der Situation des Patienten möglich ist". (Schumacher, Kongressbericht der dapo e.v., 25.2.2002; www.dapo-ev.de/ipos.html)

Durch die Kombination unterschiedlicher Erhebungsmethoden sollen die Vorteile beider Methoden:

- Validität, Reliabilität, Objektivität, Vergleichbarkeit

- Datenerhebung im Beziehungskontext, Möglichkeit der Nachfrage, Erfassung biographischer Aspekte, subjektive Theorien, Fremdbeobachtung in Bezug auf Bewältigung und Abwehr

genutzt werden. Systematische Fehler, die sich daraus ergeben könnten (z. B. Evozierung, Reaktanz, Soziale Erwünschtheit) müssen zugunsten der Vorteile in Kauf genommen werden.

Die Kombination unterschiedlichen Datenmaterials dient der Erweiterung der Perspektive auf den Forschungsgegenstand.

${ }^{63}$ Der Artikel von Pope \& Mays ist der erste einer siebenteiligen Serie des British Medical Journal 1995, in der qualitative Methodik vorgestellt wird und Anwendungsbeispiele im medizinischen Bereich beschrieben werden. 


\subsection{Untersuchungsdesign}

Die Untersuchung wird als prospektive Studie mit 5 Untersuchungszeitpunkten durchgeführt.

Zeitachse für Mammakarzinom Patientinnen

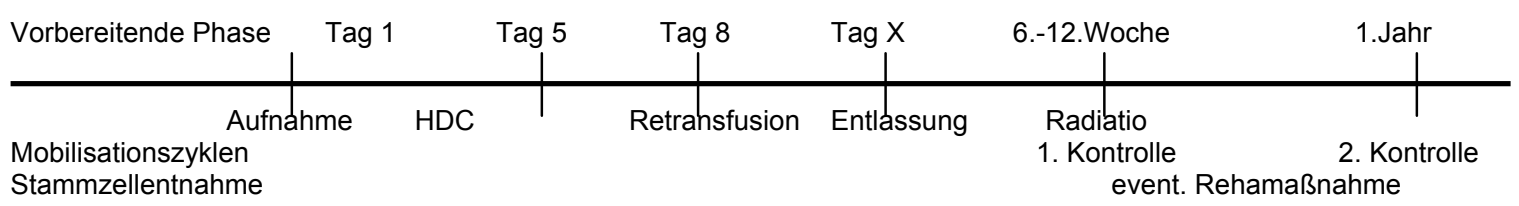

Zeitachse für NHL Patientinnen und Patienten

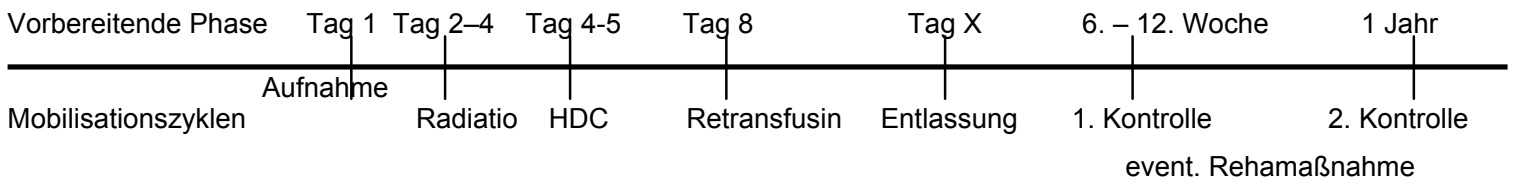

Für die Patientinnen und Patienten, die nicht diesen beiden Diagnosegruppen zugeordnet werden können, entspricht die Zeitachse der beim Mammakarzinom. Die Patienten der 3. Diagnosegruppe werden in der Regel nicht nachbestrahlt.

Für alle Patientinnen und Patienten gilt, dass mehrere Zyklen Chemotherapie vorausgegangen sind (Mobilisationszyklen) und Wachstumsfaktoren verabreicht wurden (siehe Anhang Medizinisches Datenblatt). Die vorhergehenden Behandlungen wurden bei einem Teil der Gruppe ambulant durchgeführt. Unmittelbar vor Beginn der HDC bzw. der Ganzkörperbestrahlung wurden die Patientinnen und Patienten stationär aufgenommen. Nach der Rekonstituierung des Blutbildes und wenn keine schwerwiegenden Beeinträchtigungen vorlagen, wurden die Patientinnen und Patienten entlassen. Die Zeitpunkte der Datenerhebung sind u.a. in der folgenden Tabelle zusammengestellt:

\subsection{Zeitpunkte der Datenerhebung:}

Bei der Auswahl der Messzeitpunkte mussten verschiedene Aspekte berücksichtigt werden. Zeitpunkte, die mit einer besonderen Belastung bzw. mit einer Änderung der Belastungen einhergingen, sollten markiert werden. Autoren haben darauf hingewiesen, dass die Ergebnisse hinsichtlich des subjektiven Erlebens von Nebenwirkungen der Chemotherapie sehr stark vom Zeitpunkt der Erhebung, d.h. seinem zeitlichen Abstand zur HDC, determiniert werden (Hürny et al. 1994; Osoba 1996; Pater et al. 1998).

Während des Klinikaufenthaltes waren die Zeitpunkte relativ fixiert, nach der Entlassung mussten Kompromisse eingegangen werden, da viele der Patienten von außerhalb kamen und die Kontakte mit den Untersuchern sich nach den Möglichkeiten der 
Patienten richten mussten, d.h., die Untersuchung erfolgte im Kontext von Nachsorgeterminen. Die Zeitfenster der standardisierten Instrumente waren vorgegeben (letzte Woche). Die Zeitfenster der Interviews zu T4 und T5 waren wesentlich weiter gefasst und sollten Auskunft über den gesamten Zeitraum zwischen den Datenerhebungen geben.

T1 sollte als „baseline“ Bezugspunkt dienen. T2 sollte vor allem die unmittelbar durch die HDC verursachten Nebenwirkungen erfassen, während T3 sich auf die Aplasie bezogenen Beschwerden bezieht. Eine scharfe Trennung ist hier allerdings erfahrungsgemäss nicht möglich. Mit beiden Zeitpunkten sollen akute Nebenwirkungen erfasst werden. T4 bezieht sich auf den Erholungszeitraum und damit auf die Frage, inwieweit akute Beschwerden persistieren. Definitionsgemäß werden die noch nach mehr als 90 Tagen bestehenden Nebenwirkungen als chronische Nebenwirkungen definiert. Der Übergang zu den Spätnebenwirkungen ist fließend und soll mit T5 erfasst werden.

\section{Tabelle 13: Messzeitpunkte}

\begin{tabular}{|l|l|}
\hline T 1: & $\begin{array}{l}\text { Zu Beginn des stationären Aufenthaltes, unmittelbar vor dem Beginn der HDC oder der } \\
\text { Radio-HDC }\end{array}$ \\
\hline T 2: & $\begin{array}{l}8-10 \text { Tage nach Gabe der myeloablativen Therapie, nach der Stammzellrücktransfu- } \\
\text { sion } \\
\text { in der Aplasiephase }\end{array}$ \\
\hline T 3: & $\begin{array}{l}\text { Zum Zeitpunkt der Rekonstituierung des Blutbildes, unmittelbar vor der stationären } \\
\text { Entlassung }\end{array}$ \\
\hline T4: & $\begin{array}{l}\text { Etwa zum Zeitpunkt der ersten Kontrolle, } 6-8 \text { Wochen nach der stationären Entlas- } \\
\text { sung }\end{array}$ \\
\hline T 5: & ein Jahr nach Gabe der HDC \\
\hline
\end{tabular}

Die Auswahl der Erhebungsinstrumente soll im folgenden Abschnitt dargestellt werden. Als erstes werden in der folgenden Tabelle Messzeitpunkte und Erhebungsinstrumente einander zugeordnet. 
Tabelle 14: Messzeitpunkte und zugehörige Instrumente der Datenerhebung

\begin{tabular}{|l|l|}
\hline Messzeitpunkte & Erhebungsinstrumente/Daten \\
\hline T 1 & soziodemographische Daten \\
& Tumorklassifikation und anamnestische Daten \\
& QLQ- C30 \\
& HADS-D \\
& Karnofsky-Index \\
& Semi-strukturiertes Interview \\
\hline T 2 & QLQ- C30 \\
& HADS-D \\
& Karnofsky-Index \\
& Semi-strukturiertes Interview \\
\hline T 3 & QLQ- C30 \\
& HADS-D \\
& Karnofsky-Index \\
& Semi-strukturiertes Interview \\
\hline T4 & QLQ- C30 \\
& HADS-D \\
& Karnofsky-Index \\
& Semi-strukturiertes Interview \\
\hline T 5 & QLQ- C30 \\
& HADS-D \\
& Karnofsky-Index \\
& Semi-strukturiertes Interview \\
\hline
\end{tabular}

Im Folgenden sollen die Methoden und Instrumente der Datenerhebung beschrieben und ihre Auswahl begründet werden.

\subsection{Modus der Datenerhebung}

Die Daten wurden von den psycho-sozialen Mitarbeitern der beiden beteiligten Stationen erhoben. ${ }^{63}$ Nach der stationären Aufnahme wurden die Patientinnen und Patienten persönlich über die Studie informiert und um ihre Mitarbeit gebeten. Eine schriftliche Information wurde zur Ergänzung ausgehändigt. Dass für alle Patientinnen und Patienten eine schriftliche Einverständniserklärung vorliegt, versteht sich von selbst.

Wegen der besseren Kontaktmöglichkeit und des höheren Zeitaufwandes erfolgte zuerst die nicht-standardisierte Befragung. Patientinnen und Patienten hatten Gelegenheit, sich ausführlich zu äußern und Fragen zu stellen zum Ablauf auf Station, zur bevorstehenden Therapie und zu anderen sie bewegenden Themen. Diese Art der Datenerhebung war bewusst als minimale Intervention intendiert, um den Aspekt der Unterstützung und nicht nur den der Einforderung von Daten deutlich zu machen.

Die Fragebögen wurden in Anwesenheit der Mitarbeiter ausgefüllt. Unklarheiten in Bezug auf Fragen konnten, wenn nötig, gleich beseitigt werden. Zu den späteren Zeitpunkten, als die Patientinnen und Patienten mit den Fragebögen bereits vertraut wa-

${ }^{63}$ An dieser Stelle noch einmal ein besonderer Dank an meine Kollegin H. RobrechtKauenhoven und meinen Kollegen Prof. Dr. M. Gründel. 
ren, wurden die Bögen nach dem Interview ausgegeben und konnten später ausgefüllt werden. Zwischen den Terminen der Datenerhebung standen die Mitarbeiter den Patientinnen und Patienten auf Wunsch zur Verfügung.

\subsection{Quantitativer Teil der Datenerhebung}

Der quantitative Teil der Datenerhebung verwendet den QLQ-C30 zur Operationalisierung von LQ und zusätzlich die HADS-D (Screeninginstrument zur Ermittlung von Angst und Depressivität). Der LQ-Fragebogen fokussiert zum einen auf körperliche Symptome, die bei der HDC eine wichtige Rolle spielen, zum anderen erfasst er die vom Patienten wahrgenommenen funktionalen Einschränkungen (siehe S. 199ff). Seelische Befindlichkeit wird mit dem QLQ nur unzureichend erfasst. Da ein hohes Angstniveau der Patienten vor der Behandlung erwartet wurde und depressive Störungen und Angst kontrolliert werden sollten, war ein zusätzlicher Fragebogen gerechtfertigt, der sich speziell auf die Entdeckung dieser „Störungen“ bezieht. Die bereits vorliegenden Untersuchungen haben übereinstimmend ergeben, dass bei den Patienten mit einer hohen Erwartungsangst zu rechnen ist. Nur einem Teil der Patienten gelingt eine weitgehende Angstabwehr. Wie der weitere Verlauf von Angst aussieht, lässt sich nicht eindeutig beantworten und soll in der Studie exploriert werden. Mit einem größeren Anteil von Patienten, die depressiv auffällig werden, ist zu Beginn der Therapie nicht zu rechnen. Zur Erlangung dieser Therapie bedarf es unüblicher Anstrengungen und der Beurteilung durch die Ärzte als hinreichend belastungsfähig. Deswegen wird erwartet, dass sich Depressivität, wenn überhaupt, erst als spätere Störung manifestiert.

Neben den beiden Selbstbeurteilungsinstrumenten wurde noch der Karnofsky-Index als Fremdbeurteilungsinstrument eingesetzt, um für den Bereich der körperlichen Funktion, die durch die HDC zumindest kurzfristig erheblich beeinträchtigt wird, einen Vergleich der Beurteilungsperspektiven zu ermöglichen.

Abgeleitet aus den bisherigen Untersuchungen der LQ im Zusammenhang mit einer HDC wird erwartet, dass sich Funktionen, Beschwerden/Symptome und die Einschätzung der Allgemeinen Lebensqualität (QL) im Zeitverlauf verändern.

\subsubsection{Standardisierte Verfahren zur Informationsgewinnung}

In der folgenden Tabelle ist die Art/das Instrument der Datengewinnung aufgelistet. Im danach folgenden Text werden die Instrumente beschrieben und ihre Auswahl wird begründet. 
Tabelle 15: Standardisierte Datengewinnung

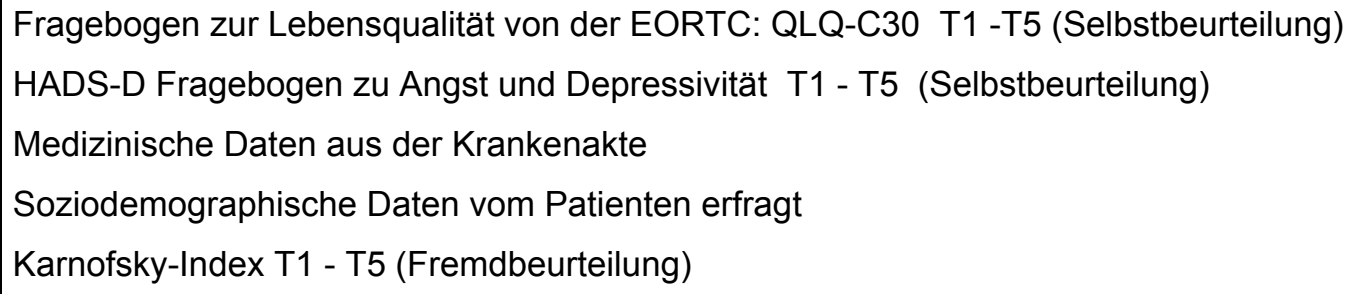

\subsection{2 „EORTC quality of life questionnaire (QLQ-C30)”}

Dieser Fragebogen wurde von der EORTC Arbeitsgruppe „Life quality“ zur standardisierten, interkulturellen Erfassung von gesundheitsbezogener LQ herausgegeben. Die jetzige Fassung (siehe Anhang) wird folgendermaßen beschrieben:

Der Fragebogen enthält neun Skalen, fünf davon sind sogenannte funktionelle Skalen, die sich auf die physische Situation, die Rollenfunktion, Kognitionen, Emotionen und die soziale Funktion beziehen. Drei Symptomskalen sollen Müdigkeit, Schmerzen Übelkeit und Erbrechen erfassen. Die Skalen enthalten Likert-skalierte Items in vierfacher Abstufung. Daneben gibt es eine allgemeine Skala zur Gesundheits- und Lebensqualität, außerdem zahlreiche Einzelitems, die sich auf klinische Variablen beziehen. Die Validität sehen die Autoren durch folgende Ergebnisse als bestätigt an:

- Die Interkorrelationen der Items sind hoch. Zwischen den Skalen sind die Korrelationen mäßig, was als Ausdruck unterschiedlicher Komponenten des Lebensqualitätskonstruktes gewertet wird.

- Die funktionellen Skalen diskriminieren deutlich zwischen Patienten in unterschiedlichen Krankheitsstadien (nach der Stadiendefinition der EORTC).

- Statistisch signifikante Veränderungen ergaben sich in der erwarteten Richtung bei Patienten, bei denen sich der Zustand während der Behandlung verschlechterte.

Positiv werten die Autoren, dass Reliabilität und Validität konsistent sind, auch unter unterschiedlichen kulturellen Bedingungen. Der Fragebogen wurde bis 1995 bereits in 24 Sprachen übersetzt.

Mehrere Autoren kommen zu dem Schluss, dass es sich beim EORTC QLQ-C30 um ein reliables und valides Messinstrument handelt, das auch in multikulturellen Forschungsprojekten eingesetzt werden kann (u. a. Aaronson et al. 1993; Kaasa et al. 1995).

Der „core questionnaire“ soll LQ, unabhängig von der Art der Krebserkrankung, erfassen. Der Fragebogen kann ergänzt werden durch Diagnose- und/oder behandlungsspezifische Module, die nach vorgegebenen Regeln entwickelt werden sollen/worden sind. Mittlerweile liegt auch ein „Hochdosis-Modul“ vor. Für den Einsatz des QLQ wird 
ein Studienkoordinator gefordert, der dafür sorgt, dass die Datenerhebung in die Kliniksroutine integriert wird. Der Fragebogen kann auch als strukturiertes Interview in interaktiver Form eingesetzt werden.

Krischke und Petermann (1995) führen den QLQ-C30 in ihrer Übersichtsarbeit unter dem Stichwort „International eingesetzte und validierte Verfahren zur Erhebung der LQ" auf. Sie unterziehen den QLQ einer kritischen Analyse und kommen zum Ergebnis, dass einige der Skalen signifikant untereinander korrelieren und nicht unabhängig voneinander sind. Auch der Globalwert korreliert hoch mit den Subskalen. Entgegen den Erwartungen zeigen sich zwischen Patienten mit unterschiedlichem ECOGStatus ${ }^{64}$ nur geringe Unterschiede in der LQ.

Die Autoren kommen trotz der kritischen Anmerkungen zu dem Schluss, „dass sich der QLQ-C30 als ein psychometrisch robustes Lebensqualitätsinstrument erwiesen hat, der therapeutisch bedingte Veränderungen erfassen kann“ (ebenda, S.49).

Die Kritiken in Bezug auf psychometrische Qualität wurden von der Arbeitsgruppe aufgenommen und 1997 wurde von der Studiengruppe der EORTC (Osoba et al. 1997) eine veränderte Fassung vorgelegt, die jetzt eine „global life quality - Skala“ (QL) enthält und in der die dichotomen Items der Skala „RF“ (Rollenfunktion) umformuliert und ebenfalls vierstufig präsentiert wurden.

Im Vergleich mit anderen Fragebogeninstrumenten (SF-36, FACT) schneidet der QLQ in Bezug auf konvergente Validität gleich gut ab, wird aber von den Patienten am besten akzeptiert (Glick et al. 1998). Weitere Validitätsprüfungen von McLachlan et al. (1998) an einer Stichprobe von Brustkrebspatientinnen weisen auf eine gute Übereinstimmung mit dem Konstrukt hin. Auch die Studien von Apolone et al. (1998) und Montazeri et al. (1998) bestätigen, dass die psychometrischen Eigenschaften zufriedenstellend sind.

Der prognostische Wert wurde in mehreren Studien, die zu unterschiedlichen Ergebnissen kommen, überprüft. Dancy et al. (1997) betätigen die Vorhersagemöglichkeit des Überlebens durch den globalen QLQ (QL), Blazeby et al. (2000) verweisen darauf, dass nur die „physical function scale“ von signifikanter prognostischer Bedeutung ist. Coates et al. (1997) untersuchen den prognostischen Wert bei fortgeschrittener Erkrankung. An dieser prospektiven Studie nehmen Patienten aus Krankenhäusern in verschiedenen Ländern teil. Diese Studie belegt einen signifikanten und unabhängigen Zusammenhang zwischen Aspekten von LQ, wie sie der QLQ-C30 erfasst, und Überlebenszeit. Die Autoren weisen darauf hin, dass die prognostische Information durch

${ }^{64}$ Beschreibt den unterschiedlichen physischen Zustand mit Bezug auf Krankheitsstadien. 
die beiden Einzelitems zur allgemeinen und körperlichen LQ (Skala QL) bereits erschöpfend erfasst wird.

Der Fragebogen wurde für die verschiedensten Fragestellungen erprobt. Stichwortartig sollen hier einige Punkte erwähnt werden:

Am häufigsten wurde die Auswirkung von Chemotherapie beschrieben, außerdem wurden Chemotherapieregimes verglichen, sowohl bei unterschiedlichen als auch bei gleichen Tumorarten und unterschiedlichen Regimes. Der QLQ wurde häufig im Rahmen von Interventionsstudien eingesetzt (siehe S. 45f).

Elektronische Versionen wurden getestet, um festzustellen, wie akzeptabel ein solches Vorgehen für Patienten ist und wie hoch die Kosten der elektronischen Version sind, um daraus Schlussfolgerungen bzgl. der Eignung des Verfahren für den Routineeinsatz zu ziehen.

In den ersten Jahren überwogen die Veröffentlichungen, die sich mit den psychometrischen Gütekriterien befassten.

Ausschlaggebend für die Verwendung des QLQ-C30 in dieser Studie war, dass der Fragebogen mittlerweile zum Standardinstrument avanciert ist und der Fragebogen von den großen Fachgesellschaften in onkologischen Therapiestudien obligat gefordert wird. Mittlerweile liegt eine Anzahl von Vergleichstudien vor. Der Fragebogen ist geeignet für die Beschreibung von (Langzeit-) Therapieverläufen. In der Planungsphase hat sich herausgestellt, dass „unsere“ Patienten den Fragebogen gut akzeptieren konnten. Die Auswertung erfolgt nach den Richtlinien der Autoren so wie im Handbuch beschrieben. Positiv ist auch zu werten, dass die EORTC Arbeitsgruppe „life quality“ die Nutzer des QLQ-C30 verpflichtet ihre Daten an die zentrale LQ-Datenbank weiterzuleiten. Von der Arbeitsgruppe werden in regelmäßigen Abständen „up-dates“ verfasst.

\subsubsection{Hospital Anxiety and Depression Scale - Deutsche Version (HADS-D)}

Die HADS-Skalen wurden von Zigmond und Snaith (1983) als Screeninginstrument zur Erfassung von Angst und Depressivität für internistische Patienten entwickelt. Validität, Reliabilität und Akzeptanz der Skalen sind befriedigend. Durch die Kürze ist der Einsatz in der Kliniksroutine möglich. Folgeuntersuchungen haben die Ergebnisse bestätigt (u. a. Bjelland et al. 2002, Hermann et al. 1991, Ibbotson et al 1994). Hermann et al. (ebenda) legen eine deutsche Version der Skala vor. Die Autoren kommen ebenfalls zu einer positiven Bewertung in Bezug auf psychometrische Kriterien, Differenzierungsfähigkeit und Anwendbarkeit. Sie heben hervor, dass der Verzicht auf die Verwendung von somatischen Indikatoren eine Verfälschung der Skalenwerte durch gleichzeitig bestehende somatische Symptome ausschließt. Um ein Screening zu ermöglichen, werden drei Wertebereiche angegeben - unauffällig/suspekt/auffällig -, die eine orien- 
tierende Klassifizierung der Patienten ermöglichen. Im Unterschied zu der Originalarbeit (Zigmond \& Snaith 1983) findet sich bei der Überprüfung der deutschen Version eine relativ hohe Korrelation zwischen der Angst- und Depressionsskala. Die Autoren diskutieren diese Ergebnisse auf dem Hintergrund von theoretischen Vorstellungen über Angst und Depressivität und bieten als eine Erklärung an, dass beide Symptome häufig nebeneinander vorkommen. ${ }^{65}$

In der englischen Studie von Ibbotson et al. (ebenda) wird der HADS im Vergleich mit anderen Instrumenten („The General Health questionnaire 28“; „Rotterdam Symptom Checklist") auf seine Tauglichkeit im onkologischen Bereich, im Hinblick auf Angst und Depressivität, in Abhängigkeit von Krankheitszustand und Behandlung überprüft. Auch hier kommen die Autoren zu dem Ergebnis, dass es sich bei der HADS um ein brauchbares Instrument handelt, welches in der Gesamtstichprobe am besten diejenigen Patienten identifiziert, die unter affektiven Störungen leiden. Lediglich in der Gruppe der Patienten mit progredientem Krankheitsverlauf ist die HADS der „Rotterdam Symptom Checklist" unterlegen. Hosaka \& Aoki (1996) vergleichen SDS, POMS und HADS mit einem psychiatrischen Interview und bestätigen ebenfalls die Differenzierungsfähigkeit dieses Fragebogens. In einer italienischen Studie von Costantini et al. (1999) wird der HADS mithilfe des strukturierten klinischen Interviews aus dem DSM-III-R auf seine Diskriminierungsfähigkeit überprüft. Die Autoren warnen davor, die beiden Skalen des HADS getrennt voneinander zu bewerten. Den Gesamtscore beider Skalen halten sie für einen validen Indikator für emotionale Belastung.

Da die Inzidenz von affektiven Störungen, an erster Stelle Angst und Depressivität, hoch ist und davon auszugehen ist, dass die Befindlichkeit ein wichtiger Aspekt bei der Beurteilung der LQ ist, sollte ein entsprechendes Screeninginstrument zum Einsatz kommen und die Patientinnen und Patienten mit auffälligen Werten sollten unmittelbar ein Unterstützungs-/Therapieangebot erhalten.

\subsubsection{Medizinische Daten}

Im Rahmen der allgemeinen medizinischen Dokumentation wurden neben einer spezifizierten Diagnose, die auf Krankheitsstadium und Prognose hinweist, anamnestische Daten, Verlaufsdaten und Zustand zum Zeitpunkt T5, ein Jahr nach Abschluss der

\footnotetext{
${ }^{65}$ Es gibt sicher auch ganz andere Ansätze um diesen Zusammenhang zu erklären. In Rahmen anderer Modelle (z. B. kognitive Erklärungsmodelle) werden Angst und Depressivität als zwei Seiten der gleichen Störung aufgefasst. Außerdem ist das Gefühl von Hilflosigkeit für beide Störungen gleichermaßen wichtig. Andere Modelle, die sich auf hierarchische Faktorenanalysen beziehen, postulieren ein 3-Faktor-Modell mit dem übergeordneten Faktor „Psychologischer Disstress“ und den untergeordneten Faktoren „Anhedonie“ und „Autonomic Anxiety“.
} 
HDC, des weiteren die Behandlung und der Krankheitsverlauf bis zum Zeitpunkt der HDC mit/ohne Radiatio erhoben.

\subsubsection{Soziodemographische Daten}

Hier wurden die üblichen Daten erfasst, um Besonderheiten der Stichprobe zu bemerken bzw. die Vergleichbarkeit mit anderen Stichproben zu ermöglichen.

Bei der Untersuchung von onkologischen Stichproben ist das deshalb zwingend, weil der Einfluss sozio-demographischer Variablen auf den Krankheitsverlauf und auf Überlebenszeiten gut belegt ist (Tschuschke 2002, Marmot \& Wilkinson 2001, Balfour \& Kaplan 1998). ${ }^{66}$ Arme erkranken häufiger und ihre Überlebenszeit ist, im Vergleich zu Erkrankten höherer sozialer Schichten, bei gleicher Tumorart und gleichem Erkrankungsstadium, kürzer.

\subsubsection{Karnofsky-Index}

Der Karnofsky-Index ist eine einfache, zehnstufige Skala zur Fremdbeurteilung des physischen Zustandes (siehe Kapitel I). Der Karnofsy-Index ist weit verbreitet und wird in Therapiestudien oft als eines der Einschlusskriterien für die Patientenauswahl angeführt.

\subsection{Qualitativer Teil der Datenerhebung}

Durch die Interviews sollen die Erfahrungen der Patienten in dieser spezifischen Situation erfasst werden. Es soll der Versuch unternommen werden, die Kontextlosigkeit von LQ-Datensammlungen ebenso wie die Fixierung auf negative Aspekte von Krankheit und Therapie zu überwinden.

Durch das Interviewverfahren sollen folgende Grundgedanken der LQ-Forschung umgesetzt werden:

1. Es geht um Befindlichkeit, etwas, das sich auch in den sorgfältigst zusammengestellten Laborparametern nur indirekt und unvollständig widerspiegelt und

2. es geht um die subjektive Sicht des Patienten, der schließlich und endlich - wenn nicht die einzige - so doch in vielen Bereichen die einzige valide Informationsquelle für eben diese Befindlichkeit darstellt (Küchler 1991 S. 23).

Das Interview sollte offen genug sein, um dem Patienten die Möglichkeit zu geben, all die Dinge zu äußern, die ihm wichtig erscheinen. Das hier verwendete Interview - Leitfadeninterview oder semistrukturiertes Interview - gehört, im Vergleich mit anderen

\footnotetext{
${ }^{66}$ Tschuschke gibt in Kap. 1.2 seines Buches „Psychoonkologie“ eine kurze Literaturübersicht zum Zusammenhang von sozialer Schicht und Krebserkrankung.
} 
Interviewvarianten (narratives Interview, klinisches Interview usw.) zu den teilstandardisierten Interviews (Hopf 2000).

Die im semi-strukturierten Interview formulierten Fragen sollen Auskunft geben über verschiedene Aspekte des Umgangs mit lebensbedrohlicher Erkrankung, eingreifender Therapie und deren Folgewirkung. In die Auswahl der Fragen sind implizite Annahmen, Alltagsannahmen und Erfahrungen, die sich aus der langjährigen psychoonkologischen Tätigkeit ergeben haben, eingeflossen. Alle Patienten dieser Stichprobe wissen, dass eine Heilung ihrer Erkrankung nur schwer möglich ist. Die Wahl eines aggressiven Therapieverfahrens erscheint in dieser Situation als einzige erfolgversprechende Möglichkeit der Krankheitsbekämpfung. Die Bedrohung wird abgewehrt oder kontrolliert durch die Annahme der effektiven Bekämpfung der Erkrankung durch die einschneidende Therapie, die die Hoffnung auf Heilung oder Lebensverlängerung aufrechterhält. Belastung wird ausbalanciert durch Hoffnung auf Besserung. Hoffnung wird aufgefasst als der wesentliche interne Kontrollmechanismus, der negative Affekte ausbalanciert. Hinzu kommt die externale Kontrolle durch die medizinische Behandlung. Die Vorannahmen ergeben sich aus dem Modell von Abbey \& Andrews (siehe S. 111). Durch die Fragen des Interviewleitfadens sollen, neben den Belastungen, die moderierenden Faktoren entschlüsselt werden:

- Belastungsfaktoren und die Bewertung dieser Belastungen

- Belastungsausgleichende Faktoren

- Bewertung der Behandlung

Der Interviewleitfaden entstand in der Vorphase der Studie und spiegelt die Aspekte wider, die von den Autoren ${ }^{67}$ der LQ in einem erweiterten Sinne zugeordnet werden. Zu den belastenden Faktoren werden somatische und psychische „Symptome“ gezählt, belastende Interaktionen zwischen Patienten und medizinischem Personal und institutionelle Bedingungen, die den Patienten die Behandlungszeit und den Genesungsverlauf erschweren.

Als entlastend wird all das gewertet, was von den Patienten zur Erleichterung ihrer Situation angeführt wird. Dazu gehören die jeweiligen persönlichen Ressourcen der Patienten (u. a. Strategien zur Krankheitsbewältigung), familiäre und soziale Ressourcen sowie stützende Interaktionen zwischen Patienten und Behandlungsteam. Die Frage nach der Nutzung von Rehabilitationsmaßnahmen und der Wiedereingliederung in den normalen Lebens- und Arbeitsprozess gehört ebenfalls zu diesem Komplex.

\footnotetext{
${ }^{67}$ Der Leitfaden entstand in der Diskussion mit Frau H. Robrecht-Kauenhoven, Prof. Dr. M. Gründel und der Autorin.
} 
Bei der Frage nach Bewertung der Behandlung interessiert sowohl die persönliche Bilanz der Patienten als auch die implizite und explizite Bewertung der Behandlungsmaßnahmen. Indirekt soll so das Urteil der Patienten in Bezug Behandlungsqualität erfasst werden.

Die Interviews wurden von erfahrenen Psychotherapeutinnen und einem Psychotherapeuten durchgeführt, die in einem kontinuierlichen Diskussionszusammenhang standen und an einer gemeinsamen Supervision teilnahmen. Soweit möglich, sollte dem Bewältigungsbedürfnis der Patienten Rechnung getragen werden: „Aus forschungsethischer Perspektive ist es sogar geboten, die Methoden nicht allein nach Kriterien der Erkenntnisgewinnung auszuwählen, sondern dabei auch die Verantwortung für mögliche Reaktionen der Untersuchten im Blick zu behalten. Qualitative Interviews haben auch in dieser Hinsicht eine andere "Qualität" als beispielsweise Fragebögen, weil sie einen Rahmen bieten, in dem Aufgewühltes wieder und neu eingeordnet werden kann [...]“ (Gründel 1995 S.38). Selbstverständlich wurde all das vermieden, was zu einer Ich-Schwächung, zur Verunsicherung und Verstärkung von Angst hätte führen können. Küchler (1991 S. 32/33) geht auf die Vorund Nachteile von Interviewverfahren ein:

1. Unter humanistischen Gründen/Gesichtspunkten ist festzuhalten, dass Interviewverfahren dem Gegenstand Lebensqualität gerade in der Onkologie durch das Prinzip der Intersubjektivität, also der zwischenmenschlichen Begegnung, am gerechtesten werden.

2. Unter therapeutischen Gesichtspunkten lässt sich feststellen, dass sich besonders die psychische Belastung für den Patienten, die sich oftmals nicht vorhersehbar an einzelnen Themenbereichen entwickeln kann - bedingt entweder durch die Krankheits- und Therapiesituation oder durch die Untersuchung selbst - im Interview am ehesten auffangen lässt.

3. Unter methodischen Gesichtspunkten liegt der Hauptvorteil von Interviewverfahren darin, dass sie schwer messbare Lebensqualitätsaspekte, eben wirklich qualitative Aspekte, wie z. B. Beziehungsfähigkeit, Abwehrstruktur, Religiosität, auch Sexualität, zum einen durch den Kontextbezug mit der Möglichkeit der Querfragen, zum anderen aber auch durch die Einbeziehung des non-verbalen Verhaltens am besten erfassen lässt.

Nachteilig bewertet er dagegen die Datenerhebungs- und Auswertungsobjektivität und vor allem auch den hohen zeitlichen Aufwand sowohl bei der Datenerhebung als auch bei der Auswertung und der Interpretation.

Die Datenerhebung, Auswertung und Interpretation orientiert sich an der qualitativen Inhaltsanalyse nach Mayring (1995). Diese Orientierung wurde gewählt, weil sie am geeignetsten erschien in Kombination mit psychometrischer Forschung zur LQ.

Flick (2000) charakterisiert in seiner Tabelle „Verfahren der Textinterpretation“ die Qualitative Inhaltsanalyse im Vergleich mit anderen Verfahren: 
Tabelle 16: Verfahren der Textinterpretation: Kodierung und Kategorisierung (adaptiert nach Flick, S. 234):

\begin{tabular}{|c|c|}
\hline \multicolumn{2}{|l|}{ Qualitative Inhaltsanalyse } \\
\hline Kriterien & \\
\hline Offenheit für den jeweiligen Text durch: & - $\quad$ explizierende Inhaltsanalyse \\
\hline $\begin{array}{l}\text { Strukturierung (z. B. Vertiefung) des Gegens- } \\
\text { tandes durch: }\end{array}$ & $\begin{array}{ll}\text { - } & \text { zusammenfassende Inhaltsanalyse } \\
\text { - } & \text { strukturierende Inhaltsanalyse } \\
\end{array}$ \\
\hline $\begin{array}{l}\text { Beitrag zur allgemeinen Entwicklung der Me- } \\
\text { thode der Interpretation }\end{array}$ & $\begin{array}{ll} & \text { stark regelgeleitetes Verfahren zur } \\
& \text { Reduktion von Datenmengen } \\
\end{array}$ \\
\hline Anwendungsbereich & $\begin{array}{l}\text { - große Datenmengen in verschiedens- } \\
\text { ten Inhaltsbereichen }\end{array}$ \\
\hline Probleme der Anwendung & $\begin{array}{l}\text { - } \quad \text { Anwendung der schematischen Re- } \\
\text { geln ist häufig schwierig }\end{array}$ \\
\hline Grenzen der Methode & $\begin{array}{l}\text { - Stark an quantitativer Methodik orien- } \\
\text { tiert }\end{array}$ \\
\hline
\end{tabular}

Der Auszug aus der Tabelle macht deutlich, warum dieses Verfahren gewählt wurde: Für diese Studie gilt, dass theoretische Vorannahmen vorhanden sind und in die Auswahl der „Themen“ mit eingehen. Kategorien werden aus den theoretischen Modellen abgeleitet und nicht aus dem Material gewonnen. Gleichzeitig ist das Verfahren offen für neue Kategorien. Die Stichprobengröße und die Interviewanzahl sind festgelegt und führen zwangsläufig zu einer großen Textmenge. Aus pragmatischen Gründen war es bei diesem Projekt notwendig, die Trennung von Planung, Datenerhebung und Auswertung/Interpretation aufrecht zu erhalten. ${ }^{68}$ Die Daten wurden parallel erhoben. Beide Datenmengen wurden auf deduktivem Wege gewonnen. ${ }^{69}$

Bezogen auf diese Studie folgt der Ablauf der qualitativen Inhaltsanalyse in Anlehnung an Mayring folgendem Schema:

- Festlegung des Materials: der Interviewleitfaden wurde nach ausführlicher Diskussion und einer Testphase in der vorliegenden Version festgelegt.

- Analyse der Erhebungssituation: hier wurde bestimmt, dass die für die jeweiligen Stationen zuständigen psychosozialen Mitarbeiter alle Daten erheben. Vorausgesetzt wurden ein gleicher Ausbildungsstand und ein gleicher Erfah-

\footnotetext{
${ }^{68}$ Wichtige Ausnahme: Stellte sich bei der Datenerhebung heraus, dass Patienten in einer Weise belastet waren auf die in entlastender Weise Einfluss genommen werden konnte, so wurde dieser „Befund“ dem Behandlungsteam mitgeteilt und Hilfsmaßnahmen eingeleitet.

${ }^{69}$ Flick (2000, S.283/284): „Die Probleme bei der Kombination von qualitativer und quantitativer Forschung sind bislang noch nicht befriedigend gelöst. Versuche der Integration beider Ansätze laufen häufig auf ein Nacheinander (mit unterschiedlichem Vorzeichen), Nebeneinander(mit unterschiedlichem Ausmaß der Unabhängigkeit beider Strategien) oder eine Über- bzw. Unterordnung (ebenfalls mit unterschiedlichem Vorzeichen) hinaus. Die Integration bleibt oft auf die Ebene des Forschungsdesigns begrenzt - die kombinierte Verwendung verschiedener Methoden mit unterschiedlichem Ausmaß der Bezugnahme aufeinander."
} 
rungshintergrund, Vertrautheit mit der Stationssituation und eine gemeinsame Supervision. Festgelegt waren Zeitpunkte und Orte.

- Formale Charakterisierung des Materials: während des Interviews wurde soweit als möglich die wörtliche Mitschrift bevorzugt. Im Anschluss an das Interview wurde der handschriftliche Text mit Kontextnotizen versehen und Textinhalte, die nur stichwortartig mitgeschrieben werden konnten, wurden ergänzt und der Text wurde zur Auswertung in „winword“ eingegeben.

- Fragestellungen der Analyse wurden vorab geklärt: siehe die übergeordneten Fragestellungen des Interviewleitfadens und die Differenzierung in untergeordnete Fragen.

- Analysetechnik: strukturierende Inhaltsanalyse

- Kodiereinheiten (kleinster Textteil): Wörter als die kleinste bedeutungstragende Einheit/Metapher: z. B. „Hölle“ zur Charakterisierung der Belastung durch HDC

- Kontexteinheiten (größte Textbestandteile): Sätze, Abschnitte: z. B. „Das einzige, was der Heilung im Wege steht, ist , dass ich nicht mehr richtig glücklich bin. Wenn ich glücklich bin, kann mich die Krankheit nicht mehr erwischen. Aber ich bin so unglücklich gewesen in den letzten Jahren in meiner Beziehung zu meinem Mann." Dieser Absatz kann sowohl als Ausdruck der subjektiven Krankheitstheorie der Patientin gewertet werden, als auch als Hinweis auf wichtige Aspekte ihrer Biographie dienen.

Konkret wurden alle relevanten Textstellen zu Fragen und übergeordneten Themen in der folgenden Weise erfasst:

\begin{tabular}{|c|l|l|}
\hline \multicolumn{3}{|l|}{ Fragestellung, Thema etc. (z. B. Hoffnung?) } \\
\hline Pat.Nr. & Text (Wörter, Zitate, Ausschnitte) & Kategorie \\
\hline 1 & $\begin{array}{l}\text {,...die Voraussetzungen sind gut, um der Sache ein für alle mal ein } \\
\text { Ende zu machen.“ }\end{array}$ & $\begin{array}{l}\text { Hoffnung } \\
\text { auf Hei- } \\
\text { lung }\end{array}$ \\
\hline 2 & $\cdots \cdots$ & $\cdots \cdots . .$. \\
\hline
\end{tabular}

In einem inhaltsanalytischen Prozess werden aufgrund des theoretischen Vorverständnisses Materialien/Zitate aus den Texten entnommen. Diese Zitate (Ankerbeispiele) werden in einem ersten Abstraktionsschritt in ein Kategoriensystem eingeordnet. Im nächsten Schritt, auf der nächst höheren Abstraktionsstufe, werden dann die endgültigen Kategorien oder Dimensionen formuliert. In der dann stattfindenden Analyse wird 
geprüft, welche Dimensionen sich für eine Generalisierung im Hinblick auf die Erklärung des Phänomens LQ eignen.

Wo es sinnvoll erscheint, werden die Häufigkeiten der zu einer Kategorie gehörenden Beobachtungen/Äußerungen mitgeteilt.

Die Darstellung der Interviewergebnisse spiegelt die Auswertungsschritte wider. Um die Einzelergebnisse zu einer "Gestalt" zusammenzufügen, werden exemplarisch drei Kasuistiken vorgestellt, die dem Prinzip der kontrastiven Fallauswahl folgen.

Im nächsten Teil werden die Ergebnisse getrennt nach der Erhebungsmethodik dargestellt. 


\section{Darstellung der Ergebnisse}

In dem folgenden Abschnitt wird zuerst die Stichprobe beschrieben, danach folgt die Beschreibung der Fragebogendaten (QLQ-C30, HADS-D) und die Fremdbeurteilung des Funktionszustandes, am Ende erfolgt die Auswertung der Interviews.

\subsection{Quantitative Ergebnisse}

\subsubsection{Beschreibung der Stichprobe}

Die Patientinnen und Patienten wurden rekrutiert bei ihrer Aufnahme in die Frauenklinik und in die Hämatologie/Onkologie. Durch die Teilnahme an der regelmäßig wöchentlich stattfindenden „Stammzellkonferenz" konnten die vorgesehenen Patientinnen und Patienten für die geplanten HDC-Therapien weitgehend vollständig erfasst werden.

Die Datenerhebung wurde im April 1998 begonnen und im Dezember 2000 beendet.

Der jüngste Patient der Studie war 27 Jahre, der älteste Patient 61 Jahre alt.

Im ersten Jahr wurden nur Patientinnen und Patienten mit Mammakarzinom und NHL aufgenommen, später auch Patientinnen und Patienten mit anderen Diagnosen.

Von den Patientinnen und Patienten, von denen wir Kenntnis erhielten und die wir um ihre Teilnahme gebeten hatten, hat keine/keiner die Teilnahme verweigert.

Vier der Patientinnen und Patienten, die ihr Einverständnis zur Teilnahme bekundet hatten, wurden nicht berücksichtigt:

Die jüngste Patientin sprach mangelhaft Deutsch und ihre Kooperationsmöglichkeit war sehr gering. Nach der Krankenhausentlassung brach der Kontakt ab. Die Informationen, die wir erhalten hatten, waren sehr dürftig und wir bezweifelten, dass die Patientin die Fragen wirklich verstanden hatte.

Die zweitjüngste Patientin wurde vor Beginn der Hochdosischemotherapie während der Induktionsphase psychotisch und musste daher ausgeschlossen werden. Bei der Patientin konnte die Therapie nicht wie geplant durchgeführt werden. Die Anamnese zeigte, dass es bereits verschiedene psychotische Episoden im Leben der Patientin gegeben hatte.

Ein weiterer Patient stimmte der Teilnahme zwar zu, lehnte aber das Ausfüllen der Fragebögen ab. Die Interviews für T1-T3 liegen vor, wurden in der Gesamtauswertung aber nicht erfasst, da vollständige Datensätze angestrebt wurden, außer bei den Patienten, bei denen die Datenerhebung durch Krankheitsprogress, Rezidiv oder Tod verhindert wurde.

Der vierte ausgeschiedene Patient erschien nach der ersten Kontaktaufnahme nicht zur Therapie. 
Sieht man von den genannten Patienten ab, war die Bereitschaft zur Teilnahme außerordentlich groß und die Kooperationsbereitschaft der Patientinnen und Patienten ausgezeichnet. Der Kontakt blieb bei den Patienten, die zur Nachsorge kamen oder die wegen eines Rezidivs weiter behandelt wurden, über einen langen Zeitraum erhalten.

Schwieriger war es, die Patientinnen und Patienten zu T4 (nach der Entlassung aus dem Krankenhaus) zu befragen: hier war es gelegentlich notwendig die Teilnehmer und Teilnehmerinnen telefonisch und brieflich zu ermuntern weiter mitzumachen.

Der Untersuchungstermin T5, ein Jahr nach Abschluss der Hochdosischemotherapie, wurde ausnahmslos gern wahrgenommen und es mussten keine besonderen Motivationsanstrengungen unternommen werden. Im Gegenteil, es bestand ein großes Bedürfnis, über die Erfahrungen zu berichten.

Von den 34 ausgewerteten Patientinnen und Patienten nahmen 30 an allen fünf Erhebungen teil. Für die Rezidivpatientinnen und Patienten wurde die Datenerhebung modifiziert, z. T. wurde auf die Fragebögen verzichtet und telefonisch um Auskunft gebeten, wenn die Patienten zu krank waren, um nach Göttingen zu kommen, oder wenn sie in anderen Krankenhäusern weiter behandelt wurden.

4 Patientinnen und Patienten sind im Verlauf der Studie, nach dem Zeitpunkt der Entlassung aus dem Krankenhaus, verstorben. Eine der Patientinnen verstarb kurz nach der Entlassung aus dem Krankenhaus, die zweite wenige Wochen nach T4. Für diese beiden Patientinnen liegen vollständige Daten bis einschließlich T3 bzw. T4 vor. Zwei Patienten sind nach T4 verstorben.

Für die Patientinnen und Patienten waren von medizinischer Seite keine expliziten Einoder Ausschlusskriterien formuliert, abgesehen von einem ausreichenden Allgemeinzustand, fehlenden organischen Funktionsstörungen und einem Höchstalter, das ca. 60 Jahre nicht überschreiten sollte.

Bei der Altersverteilung der Stichprobe sind die Patientinnen und Patienten im mittleren Alter in der Überzahl. Das entspricht keiner repräsentativen Verteilung bei den entsprechenden Diagnosegruppen, sondern ist eine Auswahl von Patienten, die einerseits als belastbar genug angesehen werden, um die Therapie durchzustehen, und die andererseits eine sehr schlechte Prognose haben. Allerdings ist die Stichprobe repräsentativ (ökologische Repräsentativität) für Patienten, die eine HDC Therapie bekommen, also eine selegierte Gruppe von Patienten, die gut informiert sind (oder deren Ärzte sie informiert haben) über Verfahren, die noch nicht als Routinebehandlung angesehen werden.

Die Altersverteilung ist in der folgenden Tabelle und nachfolgenden Graphik beschrieben: 
Tabelle 17: Altersmittelwerte und Standardabweichungen

\begin{tabular}{|l|c|c|}
\hline Alter & Mittelwerte & $\begin{array}{l}\text { Standardab- } \\
\text { weichung }\end{array}$ \\
\hline Gesamt & 46,56 & 8,27 \\
\hline Frauen & 46,46 & 7,20 \\
\hline Männer & 46,8 & 10,31 \\
\hline
\end{tabular}

Abb. 12: Altersverteilung der Stichprobe

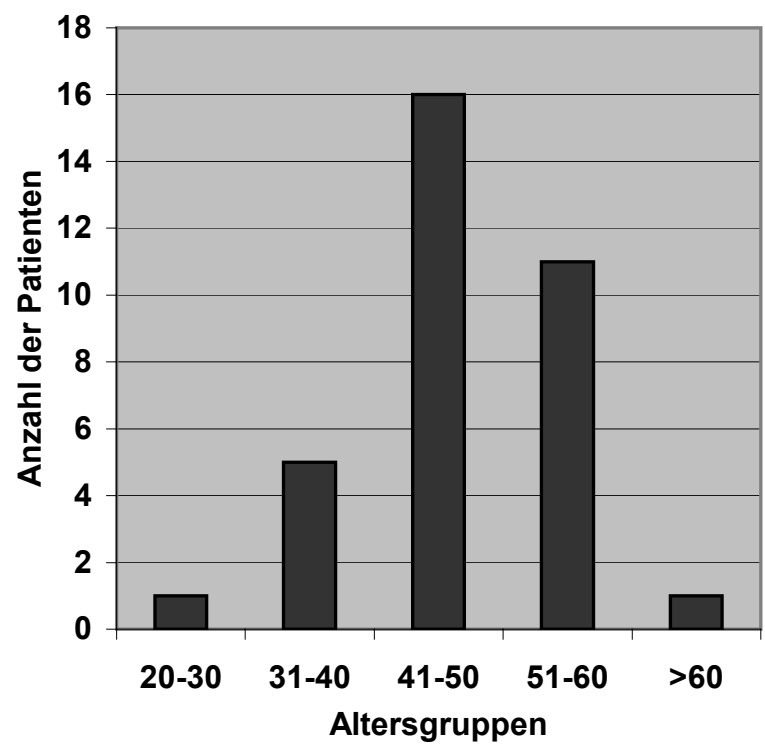

\subsubsection{Geschlechterverteilung}

Durch die Teilnahme von Brustkrebspatientinnen an der Studie ist die Geschlechterverteilung ungleich: 23 Frauen und 11 Männer nahmen an der Studie teil. Lediglich in der Diagnosegruppe NHL sind ca. gleich viele Männer und Frauen vertreten

\subsubsection{Diagnosen und ihre Verteilung}

Die größte Patientengruppe ist die der Patienten mit Non-Hodgkin-Lymphomen und anderen lymphatisch-hämatologischen Erkrankungen. An zweiter Stelle kommen die Patientinnen mit Mammakarzinom. Diese zweite Gruppe ist wesentlich kleiner als erwartet, da zwischenzeitlich, ca. ein halbes Jahr nach Beginn der Studie, die HDCTherapie bei dieser Gruppe als nicht erfolgversprechend abgesetzt wurde. 
Tabelle 18: Verteilung der Diagnosen

\begin{tabular}{|l|c|}
\hline Diagnosen & N \\
\hline NHL & 15 \\
\hline Mamma Karzinom & 8 \\
\hline sonstige Lymphome & 3 \\
\hline Plasmocytom & 2 \\
\hline Lungen/Bronchial Karzinom & 2 \\
\hline Osteosarkom & 1 \\
\hline AML & 1 \\
\hline PNET & 1 \\
\hline Immunozytom & 1 \\
\hline Gesamt & 34 \\
\hline
\end{tabular}

Für die spätere Gruppenanalyse wurden die Patientinnen und Patienten in 3 Gruppen zusammengefasst. Überlegt wurde, welche Zusammenfassung medizinisch am ehesten zu rechtfertigen wäre. Die Gruppe „Sonstige Erkrankungen“ ist die Gruppe, die in Bezug auf die Symptomatik und den Krankheitsverlauf am heterogensten ist. Gemeinsam ist allen in dieser Gruppe die ungünstige Prognose und die Einstufung der HDC als Heilversuch. ${ }^{70}$

Zusammenfassung der Diagnosen in Gruppen:

Gruppe 1: Mammakarzinom

Gruppe 2: NHL und Lymphome

Gruppe 3: Sonstige Erkrankungen

\section{Abb. 13: Diagnosen und ihre Verteilung}

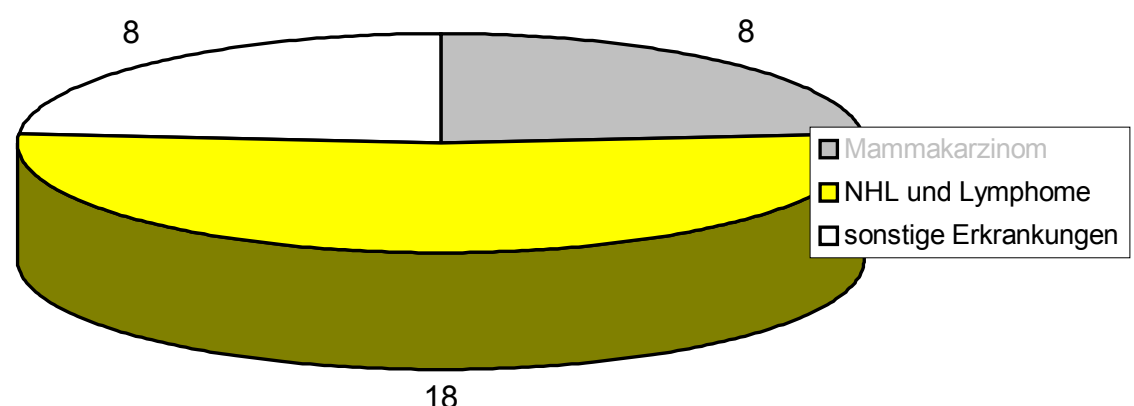

\subsubsection{Krankheitsdauer}

Die mittlere Krankheitsdauer für die einzelnen Gruppen ist in der Tabelle aufgeführt, ebenso wie die Streuung. Die Gruppe der Mammakarzinompatientinnen hat die kürzes-

70 Als Heilversuch gilt eine Behandlung dann, wenn keine andere evidenzbasierte Therapie verfügbar ist. 
te Krankheitsdauer, gefolgt von der Gruppe „Sonstige Erkrankungen“. Die NHL-Gruppe ist am längsten erkrankt.

Tabelle 19: Krankheitsdauer (Monate)

\begin{tabular}{|l|c|c|}
\hline Diagnosegruppen & MW & SD \\
\hline Mammakarzinom & 7,25 & 3,07 \\
\hline NHL & 28,39 & 32,89 \\
\hline
\end{tabular}

Die Krankheitsdauer der Gruppe „sonstige“ wird nicht in der Tabelle aufgeführt. Die Zusammenfassung der Daten ist wenig sinnvoll, da die beiden Patienten mit Plasmozytom den Mittelwert verfälschen. Die Plasmozytompatienten sind durchschnittlich 149,5 Monate erkrankt. Der andere Teil dieser Gruppe im Durchschnitt 9,5 Monate.

\subsubsection{Vorherige Therapien}

Mastektomie: Die Patientinnen wurden in der Regel mit 3-4 Zyklen EC (Epirubicin/Cyclosphamid) behandelt. Eine Patientin erhielt noch 3 Zyklen Taxol, da sich unter EC Therapie ein Lokalrezidiv entwickelt hatte.

NHL-Gruppe: Die Patienten erhielten vor der HDC mehrere Zyklen CHOP und Dexa Beam Behandlungen.

Sonstige: Jeder Patient erhielt mehrere Chemotherapien mit unterschiedlichen Medikamentenkombinationen.

Ausführliche Beschreibung siehe Anhang „Medizinische Daten“.

\subsubsection{Sozioökonomischer Status}

Der sozioökonomische Status sollte berücksichtigt werden, da es Einflüsse in Bezug auf Verlauf und Überlebenszeit bei Krebserkrankungen gibt. Diese Stichprobe ist ausgesprochen homogen, es sind, mit einer Ausnahme, überwiegend Patienten in weitgehend gesicherten materiellen Verhältnissen enthalten.

In dieser Stichprobe überwiegen Patienten mit Familie. 
Tabelle 20: Familienstand

\begin{tabular}{|l|c|}
\hline & $\mathrm{N}$ \\
\hline ledig & 2 \\
\hline verheiratet/feste Partnerschaft & 28 \\
\hline geschieden/getrennt & 4 \\
\hline verwitwet & 0 \\
\hline \multicolumn{2}{|c|}{} \\
\hline Kinder & 22 \\
\hline Keine Kinder & 12 \\
\hline
\end{tabular}

Tabelle 21: Schulbildung und Berufsausbildung

\begin{tabular}{|l|c|l|c|}
\hline & $\mathrm{N}$ & & $\mathrm{N}$ \\
\hline Hauptschule ohne Abschluss & 2 & Keine Ausbildung & 3 \\
\hline Hauptschule mit Abschluss & 12 & Lehre & 20 \\
\hline Realschule/Fachschule & 13 & Fachschule & 5 \\
\hline Abitur & 7 & Fachhochschule/Universität & 6 \\
\hline
\end{tabular}

Tabelle 22: Berufsgruppe

\begin{tabular}{|l|c|}
\hline & $\mathrm{N}$ \\
\hline (Fach)Arbeiter & 10 \\
\hline Angestellte & 12 \\
\hline Beamte & 2 \\
\hline Selbständige & 2 \\
\hline & \\
\hline Rentner & 1 \\
\hline Studierende & 1 \\
\hline
\end{tabular}

\subsubsection{Medizinische Daten zum Zeitpunkt T5}

Für die medizinische Beurteilung ist der Krankheitszustand nach einem Jahr von herausragender Bedeutung: Von 34 Patientinnen und Patienten haben 12 ein Rezidiv oder einen Krankheitsprogress erlitten. Unter den Patientinnen und Patienten mit Rezidiven gab es vier Todesfälle: 2 Patienten waren zu T4 verstorben, zwei weitere Patienten zu T5. Von den vier Todesfällen waren zwei Frauen und zwei Männer betroffen. Die Todesfälle verteilen sich auf die Diagnosen Kleinzelliges Bronchialkazinom, Mammakarzinom, Mantelzellymphom und Primitiver neuroektodermaler Tumor (PNET).

Insgesamt ist die Verteilung der 12 Patienten wie folgt:

Tabelle 23: Verteilung der Rezidivpatienten $(\mathrm{N}=12)$

\begin{tabular}{|l|l|l|}
\hline Diagnosegruppen & + & $0^{\wedge}$ \\
\hline Mammakarzinom & 3 & \\
\hline NHL & 1 & 4 \\
\hline Sonstige & 2 & 2 \\
\hline
\end{tabular}


Im Hinblick auf die berufliche Integration ergeben sich nach einem Jahr folgende Daten: ca. ein Viertel der Patienten $(\mathrm{N}=6)$ ist wieder im gleichen Umfang berufstätig wie zuvor. Eine dieser Patientinnen befindet sich noch im Stadium der stufenweisen Wiedereingliederung.

Tabelle 24: Veränderung der Beschäftigung

\begin{tabular}{|l|l|l|l|l|l|}
\hline .Zeitpunkt & Anzahl & ganztags & Teilzeit & berentet & keine \\
\hline Vor der HDC & 34 & $18(53 \%)$ & $12(35 \%)$ & $2(6 \%)$ & $2(6 \%)$ \\
\hline T5 & 22 & $6(27 \%)$ & $1(4 \%)$ & $13(59 \%)$ & $2(10 \%)$ \\
\hline
\end{tabular}

Von den 13 berenteten Patienten war eine Patientin bereits vor der Erkrankung frühberentet. Von den 3 Patientinnen, die keiner Berufstätigkeit nachgehen, war eine zuvor „nur“ Hausfrau, eine Patientin ist entlassen worden und eine Patientin war vorher im eigenen Betrieb tätig.

Zum letzten Zeitpunkt wurden auch ausgeprägte Symptome erfasst, die durch die Fragebögen nicht erfasst werden:

Tabelle 25: Symptome/Beschwerden

(berücksichtigt werden nur die Patienten, die kein Rezidiv haben)

\begin{tabular}{|l|c|}
\hline & $\mathrm{N}$ \\
\hline Ausgeprägte Polyneuropathie & 1 \\
\hline Behandlungsbedürftige Depression/Angsterkrankung & 3 \\
\hline Schwere Allergie & 2 \\
\hline Schmerzen & 3 \\
\hline Sensibilitätsstörungen & 1 \\
\hline
\end{tabular}

Zu den in der Tabelle genannten Patientinnen mit behandlungsbedürftiger Depression/Angsterkrankung kommen noch zwei weitere Patientinnen hinzu, die bereits vor der Therapie psychotherapeutisch behandelt wurden und die nach der Therapie die Behandlung fortgeführt haben. Zwei weiteren Patientinnen wurde aufgrund des Intervieweindrucks zu T5 psychosoziale Hilfe angeraten.

Im nächsten Abschnitt werden die Fragebogendaten analysiert. Der Längsschnittanalyse ist eine deskriptive Statisik und eine Reliabilitätsanalyse (Ausnahme KarnofskyIndex) vorangestellt. Berücksichtigt werden nur die Patienten ohne Rezidiv. 


\subsubsection{Deskriptive Statistik der Fragebogendaten zum Zeitpunkt T1 - T5}

In der folgenden Tabelle sind die Reliabilitäten für alle Skalen (mit Ausnahme der EinItem-Skalen) des QLQ-C30 und der HADS-D angegeben. Die Abkürzungen der Skalen werden in der ersten Tabelle erläutert.

\subsubsection{Reliabilitäten}

\section{Tabelle 26: Reliabilitätsanalyse zum Zeitpunkt T1 -T2 - T3 - T4 - T5 - QLQ-C30}

Abkürzungen der Skalen des QLQ-C30: PF-physische Funktion; RF-Rollenfunktion; EFemotionale Funktion; CF-kognitive Funktion; SF-soziale Funktion; QL-allgemeine LQ; FAFatigue; NV-Übelkeit/Erbrechen; PA-Schmerz; DY-Kurzatmigkeit; SL-Schlafstörungen; APAppetitverlust; CO-Verstopfung; DI-Durchfall; FI-Finanzielle Belastungen

Skalen des HADS-D: A - Angst ; D - Depressivität

\begin{tabular}{|c|c|c|c|c|c|}
\hline & \multicolumn{5}{|l|}{ Cronbachs $\alpha$} \\
\hline $\begin{array}{l}\text { Skalen } \\
\text { (Items) }\end{array}$ & $\mathrm{T} 1(\mathrm{~N}=34)$ & $\mathrm{T} 2(\mathrm{~N}=34)$ & T3 $(N=34)$ & $\mathrm{T} 4(\mathrm{~N}=32)$ & $\mathrm{T} 5(\mathrm{~N}=22)$ \\
\hline $\begin{array}{|ll|}\text { PF } & 1-5 \\
\end{array}$ & .74 & 85 & .77 & .79 & .83 \\
\hline RF 6,7 & .82 & 85 & .89 & .88 & .77 \\
\hline EF $21-24$ & .89 & 85 & .73 & .88 & .86 \\
\hline CF 20,25 & .61 & .57 & .53 & .80 & .91 \\
\hline SF 26,27 & .68 & .69 & .72 & .77 & .85 \\
\hline QL 29,30 & .65 & .90 & .90 & .88 & .95 \\
\hline FA $\quad 10,12,18$ & 78 & 88 & 83 & 89 & 92 \\
\hline NV 14,15 & .72 & 79 & .85 & .58 & .79 \\
\hline PA 9,19 & .94 & .90 & .83 & .95 & .89 \\
\hline & & & & & \\
\hline$A$ & .13 & .70 & .81 & .91 & .83 \\
\hline $\mathrm{D}$ & .71 & 87 & .86 & .87 & .87 \\
\hline
\end{tabular}

Die Reliabilitäten für die Skalen des QLQ-C30 unterscheiden sich zwar zu den verschiedenen Zeitpunkten, sind aber mit Ausnahme der CF-Skala zu T1,T2 und T3, der QL-Skala zu T1 und der NV-Skala zu T4 zufriedenstellend. Auch die RF-Skala, die in der Literatur hinsichtlich ihrer Reliabilität häufig als problematisch beurteilt wird, ist in dieser Untersuchung hinreichend reliabel. Die Cronbachs $\alpha$-Koeffizienten dieser Stichprobe liegen für fast alle Skalen höher als die, die von Sigle \& Porzsolt (1996) für ihre umfangreiche deutsche Stichprobe angegeben wurden.

Die Reliabilitätswerte des HADS-D sind ebenfalls zufriedenstellend im Vergleich mit den im Handbuch angegebenen Werten: Cronbachs $\alpha$-Koeffizient für die Angstskala .80 und für die Depressionsskala .81 (Herrmann et al. 1995). 


\subsubsection{Faktorenanalyse}

Für den QLQ-C30 und den HADS-D wurde eine Faktorenanalyse ${ }^{71}$ durchgeführt um die erhaltenen Stichprobendaten mit denen in der Literatur zu vergleichen. Für den QLQ-C30 war die Faktorstruktur über alle Zeitpunkte ähnlich. Mit der Hauptkomponentenanalyse wurden mehrere Faktoren extrahiert, gemäß Kaiserkriterium mit allen Werten über 1 (Summe der quadrierten Faktorladungen). Für alle Zeitpunkte ergibt sich eine drei bzw. vierfaktorielle Lösung mit einer starken Hauptkomponente. Auf dem 1. Faktor laden alle Funktionsskalen und fast alle Symptomskalen. Nur zu T2 und T3 laden einige der Symptomskalen auf gesonderten Faktoren. Wie auch die Korrelationsmatrix zeigt, bestimmt ein Hauptfaktor im Wesentlichen die Ergebnisse des QLQ-C30. Eine Unterscheidung in Symptom- und Funktionsbereich ist zwar inhaltlich gerechtfertigt, wird statistisch aber nicht gestützt.

Für den HADS-D ergibt die Komponentenmatrix für den Zeitpunkt T3 eine dreifaktorielle, für T4 eine zweifaktorielle Lösung. Alle Items, bis auf A6 und D7, laden am höchsten auf dem 1. Faktor. Die Reproduktion der Skalen A und D ist nicht möglich. Zu T1, T2 und T5 ergibt sich eine mehr als vierfaktorielle Lösung, die nicht zu einer sinnvollen Itemreduktion führt.

Die Ergebnisse der Faktorenanalyse sind im Anhang „CD-Diss“ dokumentiert.

\subsubsection{Beziehung der Variablen untereinander}

Von Interesse für die inhaltlichen Fragestellungen ist die Beziehung der Variablen untereinander. Die Korrelationsmatrix zeigt, dass unter allen Funktionsskalen (Ausnahme SF) und der Symptomskala FA ein signifikanter Zusammenhang über alle Zeitpunkte besteht. Einzelne Symptome wechseln in ihren Beziehungen zu den Funktionsskalen zu T2 und T3.

Beispielhaft soll hier die Korrelation von QL mit den anderen Skalen aufgelistet werden.

Tabelle 27: Korrelation der „Allgemeinen Lebensqualität“ (QL) mit weiteren Skalen: (Pearson Korrelation sig. (2-seitig); ${ }^{*}=$ alpha<.05)

\begin{tabular}{|c|c|c|c|c|c|c|c|c|c|c|c|c|c|c|}
\hline$\overline{Q L}$ & PF & RF & EF & CF & SF & FA & NV & DY & PA & $\mathbf{S L}$ & $\mathrm{CO}$ & AP & A-HADS & D- HADS \\
\hline $\mathrm{T} 1$ & .65 * & .49 * & .66 * & .48 * & .33 & -.73 * & -.26 & $-.41 *$ & -.41 * & -.18 & -.14 & -.27 & -.47 * & -.30 \\
\hline T2 & .67 * & $.63^{*}$ & .57 ** & $.62 *$ & .48 * & -.58 * & -.34 & -.42 * & -.42 * & $-.45^{\star}$ & -.34 * & -.33 & -.57 * & -.32 \\
\hline T3 & .48 * & $.47 *$ & $.63 *$ & $.60 *$ & .31 & -.50 * & -.43 * & -.30 & -.27 & -.42 * & -.37 * & -.38 * & $-.40^{*}$ & $-.45^{*}$ \\
\hline $\mathrm{T} 4$ & $.77^{*}$ & .77 * & .66 * & .59 * & .57 * & -.73 * & -.51 * & -.74 * & -.55 * & -.35 * & -.26 & -.50 * & -.51 * & -.66 * \\
\hline T5 & .75 * & .66 * & .70 * & $.63^{*}$ & $.88^{*}$ & -.83 * & -.44 * & -.56 * & -.38 * & -.53 * & -.52 * & -.55 * & -.56 * & -.61 * \\
\hline
\end{tabular}

${ }^{71}$ Die geringe Fallzahl erschwert eine faktorenanalytische Auswertung. Aus diesem Grunde ist nicht zu erwarten, dass die von den Autoren angegebene Faktorstruktur reproduziert werden kann. 
Wie aus der Tabelle hervorgeht, korrelieren die Funktionsskalen und Symptomskalen untereinander über den Zeitverlauf mit QL. Auch der A- und D-Wert (Ausnahme T1, T2) des HADS korreliert mit QL. Lediglich in der Phase des Krankenhausaufenthaltes besteht kein Zusammenhang zwischen den Symptomskalen NV und AP (T2), DY und PA (T3) und der Allgemeinen Lebensqualität, obwohl gerade zu diesen Zeitpunkten die Symptome besonders ausgeprägt sind. Dieses Ergebnis lässt sich möglicherweise als Ausdruck eines Reframing-Prozess interpretieren: diese Symptome werden als selbstverständlich, als zur Behandlung zugehörig akzeptiert und gehen nicht in die Beurteilung von QL ein. Vor und nach der stationären Behandlung haben sie eine andere Bedeutung und beeinflussen die QL. Der D-Wert des HADS korreliert nicht vor der Behandlung und zu T2 mit QL. Zu allen späteren Zeitpunkten besteht eine signifikante Korrelation. Wie bereits mit der FA gezeigt, sind die einzelnen Skalen/Variablen voneinander abhängig. Das lässt sich im Sinne eines bestimmenden Hauptfaktors, der „Lebensqualität“ interpretieren. Diese Interpretation wird gestützt durch Ergebnisse der FA, auf die im vorherigen Abschnitt hingewiesen wurde.

\subsubsection{Zusammenhang: Selbstbeurteilung und Fremdbeurteilung}

Aus der Literatur ist bekannt (siehe S. 12), dass sich die Ergebnisse der Selbst- und Fremdbeurteilung nicht decken. Von besonderem Interesse ist hier der Zusammenhang zwischen der Skala „Physische Funktion“ und dem Karnofsky-Index. Beide sollen, aus unterschiedlicher Perspektive, das Gleiche messen: die körperliche Funktionsfähigkeit/Belastbarkeit.

Tabelle 28: Korrelationsmatrix Karnofsky-Index - PF

(Pearson Korrelation Sig. (2-seitig)) ${ }^{*}=\alpha>.05$

\begin{tabular}{|c|c|c|c|c|c|}
\hline & Karn T1 & Karn T2 & Karn T3 & Karn T4 & Karn T5 \\
\hline PF & $.36^{*}$ & $.76^{*}$ & .17 & $.59^{*}$ & $.72^{*}$ \\
\hline
\end{tabular}

Bezogen auf die vorliegenden Daten besteht mit der Ausnahme zu T3 eine enge Beziehung zwischen Fremd- und Selbstbeurteilung der physischen Funktion. Die gute Übereinstimmung hängt vermutlich damit zusammen, dass den Fremdbeurteilern die Patienten vertraut sind und in die Urteile des Karnofsky-Index zwangsläufig auch Beobachtungen einfließen, die nicht nur dem Moment der Datenerhebung entsprechen. Auch die Diskrepanz zu T3 ist plausibel zu erklären: in die Urteile der Patienten mag sich Erleichterung über die durchgestandene Therapie mischen und daraus resultiert eine günstigere Einschätzung der körperlichen Funktion. Diese Vermutung wird durch 
die Interviewäußerungen zu T4 gestützt: Nach der Entlassung wurde der eingeschränkte Funktionszustand vielen Patienten erst bewusst.

Zwischen den Skalen des HADS und der EF-Skala des QLQ-C30 liegt über alle Zeitpunkte eine signifikante Korrelation vor, wie die folgende Tabelle zeigt:

Tabelle 29: Korrelationsmatrix Skalen HADS - EF

(Pearson Korrelation Sig. (2-seitig)) ${ }^{*}=\alpha>.05$

\begin{tabular}{|l|l|l|l|l|l|}
\hline & EF T1 & EF T2 & EF T3 & EF T4 & EF T5 \\
\hline A-HADS & $-.80^{*}$ & $-.71^{*}$ & $-.71^{*}$ & $-.78^{*}$ & $-.69^{*}$ \\
\hline D-HADS & $-.41^{*}$ & $-.67^{*}$ & -.65 & $-.68^{*}$ & $-.66^{*}$ \\
\hline
\end{tabular}

\subsubsection{Zusammenfassende Beurteilung der verwendeten Instrumente}

Die Analyse der Stichprobendaten im Hinblick auf psychometrische Eigenschaften der verwendeten Instrumente zeigt nicht in allen Aspekten eine Übereinstimmung mit den Angaben der Autoren dieser Instrumente und weiteren Studien. Die Instrumente QLQC30 und HADS-D erfüllen die Anforderungen hinsichtlich der Reliabilitätswerte (Cronbach`s alpha $\geq .70$ ). Beide Instrumente sind für Messwiederholungen geeignet. Die Übereinstimmung zwischen A-HADS und Emotionaler Belastung (EF) ist ein Indiz für deren konvergente Validität.

Die Items A4 - A6 - D7, die auf einen gesonderten Faktor laden und sich nicht einordnen lassen, lauten:

A4: Ich kann behaglich dasitzen und mich entspannen

A6: Ich fühle mich rastlos, muss immer in Bewegung sein

D7: Ich kann mich an einem guten Buch, einer Radio- oder Fernsehsendung freuen

Diese A-Items werden von Patienten anders beantwortet als die Items, in denen Angst explizit thematisiert wird. Interessanterweise findet sich auch in der Studie von Hinz \& Schwarz (2001), dass sich das Item A6 nicht einordnen lässt. Das D7-Item unterscheidet sich hinsichtlich seines semantischen Gehaltes ebenfalls von den anderen DItems. Die hohe Korrelation zwischen A- und D-Skala in dieser Studie (wie auch bei Hinz \& Schwarz ebenda) deutet auf einen gemeinsamen Faktor hin, der sich als psychischer Disstress benennen lässt.

\section{Zusammenfassung:}

\section{Psychometrische Eigenschaften der verwendeten Fragebögen}

Der QLQ-C30 erweist sich hinsichtlich seiner psychometrischen Qualität als brauchbares Instrument zur Längsschnittuntersuchung. Die Faktorstruktur weist nicht auf einen 
einheitlichen Symptom- und Funktionsbereich hin. Das Symptom Fatigue (FA) ist am ehesten dem Funktionsbereich zuzuordnen. Hier ergeben sich, in Abhängigkeit von den Besonderheiten dieser Stichprobe, Unterschiede zu anderen Untersuchungen. Das Symptom Schmerz (PA) lädt zu unterschiedlichen Zeitpunkten auf unterschiedlichen Faktoren.

Die unterschiedlichen Beurteilerperspektiven (Fremdbeurteilung/Karnofsky-Index und Selbstbeurteilung/Körperliche Funktion PF) stimmen in dieser Untersuchung gut überein.

Der HADS-D zur Erfassung von Angst und Depressivität erweist sich ebenfalls als reliables Messinstrument.

\subsubsection{Hypothesen und Ergebnisse in Bezug auf die Mittelwertsverläufen der} Fragebögen

Verlauf der Skalenwerte des QLQ-C30, Verlauf des Karnofsky-Index und der Skalenwerte des HADS-D

Wie in der Fragestellung formuliert, soll der Einfluss der HDC auf die „Lebensqualität“, operationalisiert über den Fragebogen OLQ-C30, den HADS-D und den KarnofskyIndex, erfasst werden.

Nach den bisher vorliegenden Studienergebnissen werden die folgenden allgemein gehaltenen psychologischen Hypothesen formuliert:

- Die „Lebensqualität“ (QLQ-C30) verändert sich in Abhängigkeit vom Zeitpunkt der Messung in folgender Weise:

Zum Zeitpunkt vor der HDC sind die Symptomwerte am niedrigsten und der Funktionszustand ist im Vergleich zu den drei folgenden Zeitpunkten am höchsten. Durch die HDC verschlechtern sich die Werte in der stationären Zeit um danach, im Verlaufe der Rehabilitation, wieder anzusteigen. Nach einem Jahr soll das Ausgangsniveau wieder erreicht sein (u. a. McQuellon et al. 1996). Die Kurve der Funktionswerte zeigt einen U-förmigen Verlauf. Die Symptomwerte verhalten sich spiegelbildlich dazu.

Folgende Ausnahmen lassen sich anhand bisher vorliegender Ergebnisse formulieren:

1. Fatigue ist nach den bisherigen Untersuchungen das am längsten persistierende Symptom. Deswegen wird erwartet, dass der FA-Wert (QLQ-C30) auch nach einem Jahr noch erhöht ist (allerdings niedriger als in der stationären Phase). 
2. Der Verlauf der EF-Werte (Emotionale Funktion) wird in annähernd vergleichbaren Untersuchungen sehr unterschiedlich beschrieben. Während die Autoren die EF-Skala für differenzierungsfähig halten im Hinblick auf Krankheitsstadium und Messwiederholung, zeigen spätere Untersuchungen diesen Effekt nicht (U. a. Genre et al. 2002). Eine Vorhersage ist aus diesem Grunde nicht möglich.

- Der Karnofsky-Index zeigt ebenfalls einen U-förmigen Verlauf. Die Funktionswerte des QLQ-C30, insbesondere die PF-Werte (Körperliche Funktion) und der Karnofsky-Index sind in ihrem Verlauf ähnlich.

- Der A-Wert (HADS-D) ist vor der Behandlung am höchsten, fällt zu T2 ab um nach der Entlassung wieder anzusteigen (u.a. Carlson et al. 2001).

Der D-Wert (HADS-D) ist generell niedrig, fällt nach der Behandlung ab, um dann wieder moderat anzusteigen (u.a. Carlson et al. 2001).

\section{Differenzielle Einflüsse}

- Die Werte des HADS-D werden von der Variablen „Geschlecht“ beeinflusst. Für Angst und Depressivität ist der Einfluss des Geschlechts bekannt (Herrmann et al. 1995). Die Angst- und Depressionswerte für Frauen liegen generell höher als die der Männer. Auch für die Werte des QLQ-C-30 wird ein solcher Einfluss angenommen (Heinonen et al. 2001; Andrykowski et al. 1997). In den wenigen Untersuchungen, die bislang die Variable "Geschlecht“ beachtet haben, kommt es zu widersprüchlichen Ergebnissen (Hensel et al. 2002; Weis et al. 2001).

- Bei den Patienten der Rezidivgruppe zeigt sich nach Therapieende generell einen ungünstigerer Verlauf aller Skalenwerte (trotz der geringen Stichprobengröße soll dieser Effekt berücksichtigt werden). Diese Hypothese wird aus klinischen Beobachtungen abgeleitet. Untersuchungen liegen nur für eine kleine Stichprobe vor (Carlson et al. 2001).

Die These der Zeitabhängigkeit der LQ wird geprüft über eine mehrfaktorielle Varianzanalyse mit Messwiederholung. Bei einem signifikanten Haupteffekt (Zeit) werden die Effekte mit T-Tests für gepaarte Stichproben überprüft (Nonparametrische Tests führten zum gleichen Ergebnis) um zu ermitteln, zwischen welchen Zeitpunkten signifikante Unterschiede vorliegen. Korrekterweise müsste, da es sich um eine Messwiederholung handelt, eine Fehleradjustierung erfolgen. Die geringe Stichprobengröße lässt das nicht zu. 
Um den Einfluss von „Geschlecht“ und „Rezidiv“ zu überprüfen, werden die mehrfaktoriellen Varianzanalysen jeweils mit der Interaktionsvariablen „Sex" und mit der Interaktionsvariablen „Rezidiv“ durchgeführt.

Tabelle 30: Ergebnisse der VA

(signifikant ${ }^{*}=p<.05$ )

\begin{tabular}{|c|c|c|c|c|c|c|c|c|}
\hline Skala & $\begin{array}{ll}T 1 & \\
\text { MW } & \text { (SD) } \\
\mathrm{N}=34\end{array}$ & $\begin{array}{l}\text { T2 } \\
\text { MW } \quad \text { (SD) } \\
\mathrm{N}=34\end{array}$ & $\begin{array}{l}\mathrm{T3} \\
\mathrm{MW} \text { (SD) } \\
\mathrm{N}=34\end{array}$ & \begin{tabular}{|l} 
T4 \\
MW \\
(SD) \\
$\mathrm{N}=32$
\end{tabular} & $\begin{array}{l}\text { T5 } \\
\text { MW (SD) } \\
\mathrm{N}=22\end{array}$ & \begin{tabular}{|l} 
Haupteffekt \\
Zeit \\
$F_{(4,80)}$ \\
-Werte
\end{tabular} & $\begin{array}{l}\text { Haupteffekt } \\
\text { Sex } \\
F_{(1,20)} \\
\text {-Werte }\end{array}$ & $\begin{array}{l}\text { Zeit *Sex } \\
\text { Interaktions- } \\
\text { effekt } \\
F_{(4,80)} \text {-Werte }\end{array}$ \\
\hline PF & \begin{tabular}{|l|}
79.41 \\
$(19.51)$
\end{tabular} & \begin{tabular}{|l|}
53.33 \\
$(29.31)$
\end{tabular} & $\begin{array}{l}63.33 \\
(24.36)\end{array}$ & \begin{tabular}{|l|}
66.67 \\
$(23.89)$
\end{tabular} & $\begin{array}{l}73.18 \\
(21.83)\end{array}$ & 5.35 * & 2.33 & 2.07 \\
\hline RF & $\begin{array}{l}61.76 \\
(31.39)\end{array}$ & $\begin{array}{l}33.82 \\
(34.45)\end{array}$ & $\begin{array}{l}29.41 \\
(34.59)\end{array}$ & $\begin{array}{l}44.79 \\
(35.78)\end{array}$ & $\begin{array}{l}62.88 \\
(31.26)\end{array}$ & 3.16 * & 4.08 & 0.79 \\
\hline EF & $\begin{array}{l}53.68 \\
(30.65)\end{array}$ & $\begin{array}{l}58.33 \\
(28.50) \\
\end{array}$ & $\begin{array}{l}59.31 \\
(24.60)\end{array}$ & $\begin{array}{l}56.25 \\
(29.10)\end{array}$ & $\begin{array}{l}54.36 \\
(25.54)\end{array}$ & 1.37 & 4.51 * & 0.73 \\
\hline CF & $\begin{array}{l}78.92 \\
(26.37)\end{array}$ & $\begin{array}{l}63.24 \\
(29.52)\end{array}$ & $\begin{array}{l}65.20 \\
(26.71)\end{array}$ & $\begin{array}{l}69.27 \\
(31.43)\end{array}$ & $\begin{array}{l}73.48 \\
(33.59)\end{array}$ & 1.35 & $5.23^{*}$ & 0.77 \\
\hline SF & \begin{tabular}{|l|}
61.76 \\
$(31.66)$ \\
\end{tabular} & \begin{tabular}{|l|}
46.08 \\
$(33.06)$ \\
\end{tabular} & $\begin{array}{l}48.04 \\
(33.27) \\
\end{array}$ & $\begin{array}{l}63.02 \\
(31.60) \\
\end{array}$ & $\begin{array}{l}65.15 \\
(32.49) \\
\end{array}$ & 2.60 * & 9.62 * & 0.89 \\
\hline QL & \begin{tabular}{|l|}
63.97 \\
$(16.63)$ \\
\end{tabular} & \begin{tabular}{|l|}
43.14 \\
$(22.61)$
\end{tabular} & \begin{tabular}{|l}
40.44 \\
$(20.73)$
\end{tabular} & \begin{tabular}{|l|}
51.30 \\
$(22.51)$
\end{tabular} & \begin{tabular}{|l|}
59.09 \\
$(23.70)$ \\
\end{tabular} & 4.46 * & 6.41 * & 0.22 \\
\hline FA & \begin{tabular}{|l|}
38.56 \\
$(25.98)$
\end{tabular} & \begin{tabular}{|l|}
68.95 \\
$(29.31)$ \\
\end{tabular} & $\begin{array}{l}70.59 \\
(25.05)\end{array}$ & \begin{tabular}{|l|}
54.51 \\
$(30.83)$
\end{tabular} & $\begin{array}{l}45.71 \\
(28.79)\end{array}$ & 4.97 * & 2.96 * & 0.42 \\
\hline NV & $\begin{array}{l}5.39 \\
(15.74)\end{array}$ & $\begin{array}{l}55.88 \\
(35.51)\end{array}$ & $\begin{array}{l}47.06 \\
(37.93)\end{array}$ & $\begin{array}{l}11.46 \\
(22.18)\end{array}$ & $\begin{array}{l}14.77 \\
(23.84)\end{array}$ & 14.00 * & 3.22 & 1.61 \\
\hline PA & $\begin{array}{l}16.18 \\
(30.56)\end{array}$ & $\begin{array}{l}26.47 \\
(36.50)\end{array}$ & $\begin{array}{l}46.09 \\
(37.62)\end{array}$ & $\begin{array}{l}28.13 \\
(37.97)\end{array}$ & $\begin{array}{l}23.86 \\
(26.01)\end{array}$ & 4.44 * & 2.36 & 0.60 \\
\hline DY & $\begin{array}{l}24.51 \\
(37.88) \\
\end{array}$ & $\begin{array}{l}26.47 \\
(36.50) \\
\end{array}$ & $\begin{array}{l}32.35 \\
(38.91) \\
\end{array}$ & $\begin{array}{l}43.75 \\
(42.70) \\
\end{array}$ & $\begin{array}{l}34.85 \\
(37.77) \\
\end{array}$ & 0.75 & 2.33 & 0.35 \\
\hline SL & $\begin{array}{l}31.37 \\
(33.78)\end{array}$ & $\begin{array}{l}50 \\
(35.06)\end{array}$ & $\begin{array}{l}46.09 \\
(33.85)\end{array}$ & $\begin{array}{l}32.29 \\
(36.40)\end{array}$ & $\begin{array}{l}30.30 \\
(33.98)\end{array}$ & 2.25 & 0.57 & 0.57 \\
\hline AP & $\begin{array}{l}11.77 \\
(24.46)\end{array}$ & $\begin{array}{l}63.73 \\
(39.67) \\
\end{array}$ & $\begin{array}{l}78.43 \\
(34.70)\end{array}$ & $\begin{array}{l}29.17 \\
(35.67)\end{array}$ & $\begin{array}{l}9.09 \\
(21.04)\end{array}$ & 21.12 * & 0.40 & 0.69 \\
\hline $\mathrm{CO}$ & $\begin{array}{l}9.80 \\
(25.33)\end{array}$ & $\begin{array}{l}18.63 \\
(30.91)\end{array}$ & $\begin{array}{l}17.65 \\
(30.96)\end{array}$ & $\begin{array}{l}7.29 \\
(20.28)\end{array}$ & $\begin{array}{l}2.27 \\
(10.66)\end{array}$ & 1.27 & 0.89 & 0.98 \\
\hline DI & $\begin{array}{l}12.75 \\
(27.23) \\
\end{array}$ & \begin{tabular}{|l}
47.06 \\
$(43.51)$
\end{tabular} & $\begin{array}{l}48.04 \\
(38.66)\end{array}$ & $\begin{array}{l}11.46 \\
(21.77) \\
\end{array}$ & $\begin{array}{l}18.94 \\
(29.23)\end{array}$ & 4.53 * & 0.20 & 0.74 \\
\hline $\mathrm{FI}$ & $\begin{array}{l}23.53 \\
(30.18) \\
\end{array}$ & $\begin{array}{l}29.41 \\
(37.39) \\
\end{array}$ & $\begin{array}{l}23.53 \\
(31.28) \\
\end{array}$ & $\begin{array}{l}20.83 \\
(29.02) \\
\end{array}$ & $\begin{array}{l}20.46 \\
(29.07) \\
\end{array}$ & 0.90 & 0.89 & 0.18 \\
\hline $\begin{array}{l}\text { A- } \\
\text { HADS }\end{array}$ & $\begin{array}{l}7.68 \\
(3.66)\end{array}$ & $\begin{array}{l}6.59 \\
(3.63)\end{array}$ & $\begin{array}{l}5.64 \\
(4.07)\end{array}$ & $\begin{array}{l}6.69 \\
(4.88)\end{array}$ & $\begin{array}{l}7.09 \\
(3.87)\end{array}$ & 1.65 & 5.27 * & 0.55 \\
\hline $\begin{array}{l}\text { D- } \\
\text { HADS }\end{array}$ & $\begin{array}{l}4.62 \\
(3.08)\end{array}$ & $\begin{array}{l}6.18 \\
(4.55)\end{array}$ & $\begin{array}{l}4.71 \\
(3.81)\end{array}$ & $\begin{array}{l}4.91 \\
(3.83)\end{array}$ & $\begin{array}{l}4.32 \\
(3.32)\end{array}$ & 1.33 & $4.46^{*}$ & 0.76 \\
\hline $\begin{array}{l}\text { Karn.- } \\
\text { Index }\end{array}$ & $\begin{array}{l}8.82 \\
(0.90)\end{array}$ & $\begin{array}{l}7.03 \\
(1.59)\end{array}$ & $\begin{array}{l}7.04 \\
(1.54)\end{array}$ & $\begin{array}{l}7.97 \\
(1.16)\end{array}$ & $\begin{array}{l}8.05 \\
(0.90)\end{array}$ & 5.39 * & 10.26 * & 1.88 \\
\hline
\end{tabular}

Wie aus der Tabelle hervorgeht, ergeben sich für die Funktionsskalen PF, RF, SF und QL sowie für die Symptomskalen FA, NV, PA, AP und DI signifikante Haupteffekte „Zeit“. Das Gleiche gilt für den Karnofsky-Index. Kein Haupteffekte „Zeit“ ist nachweisbar für die Funktionsskalen EF und CF, die Symptomskalen DY, SL und CO. Auch 
die Skala FI und die A- und D-Werte des HADS-D zeigen keine signifikante Veränderung im Zeitverlauf.

Um festzustellen, zwischen welchen einzelnen Zeitpunkten die Unterschiede signifikant sind, wurden T-Tests durchgeführt. Die Ergebnisse für die Skalen mit signifikantem Haupteffekt (PF, RF, SF, QL, FA, NV, PA, AP, DI, Karnofsky-Index) werden in der folgenden Tabelle mitgeteilt:

Tabelle 31: T-Test bei gepaarten Stichproben

\begin{tabular}{|c|c|c|c|c|c|}
\hline & t-Werte $\quad{ }^{*}$ & $\mathbf{p}<.05)$ (zweis & itig) & & \\
\hline Skala & $\mathrm{T} 1 / \mathrm{T} 2(\mathrm{df}=33)$ & T2/T3 (df=33) & T3/T4 (df=31) & T4/T5 (df=21) & $\mathrm{T1} / \mathrm{T} 5(\mathrm{df}=21)$ \\
\hline PF & 5.80 * & -2.03 * & -1.11 & -1.13 & 1.44 \\
\hline RF & 4.29 * & .77 & -2.42 & -1.71 & -.24 \\
\hline SF & 3.72 * & -.36 & -3.16 * & .32 & -.67 \\
\hline $\mathbf{Q L}$ & 5,52 * & .73 & -2.73 * & -1.06 & .30 \\
\hline FA & -6.28 * & -.39 & 2.73 * & .85 & -.73 \\
\hline NV & -7.85 * & 1.31 & 5.57 * & -1.31 & -2.80 * \\
\hline $\mathbf{P A}$ & -1.84 & -2.58 * & 3.07 * & .36 & -.73 \\
\hline AP & -6.95 * & -2.33 * & 6.54 * & 1.63 & .00 \\
\hline DI & -4.42 * & -.12 & 5.30 * & -.89 & -.68 \\
\hline $\begin{array}{c}\text { Karnofsky- } \\
\text { index }\end{array}$ & 6.08 * & .00 & $-4.94 *$ & -.16 & 2.56 * \\
\hline
\end{tabular}

Bevor die Verläufe der Skalenwerte diskutiert werden, sollen durch die Abbildungen die Zeitverläufe veranschaulicht werden.

\subsubsection{Funktionsskalen QLQ-C30}

In der folgenden Abbildung sind die Verläufe der Funktionsskalen, mit Ausnahme der Allgemeinen $L Q(Q L)$, dargestellt. 
Abb. 14: QLQ-C30 Funktionsskalen

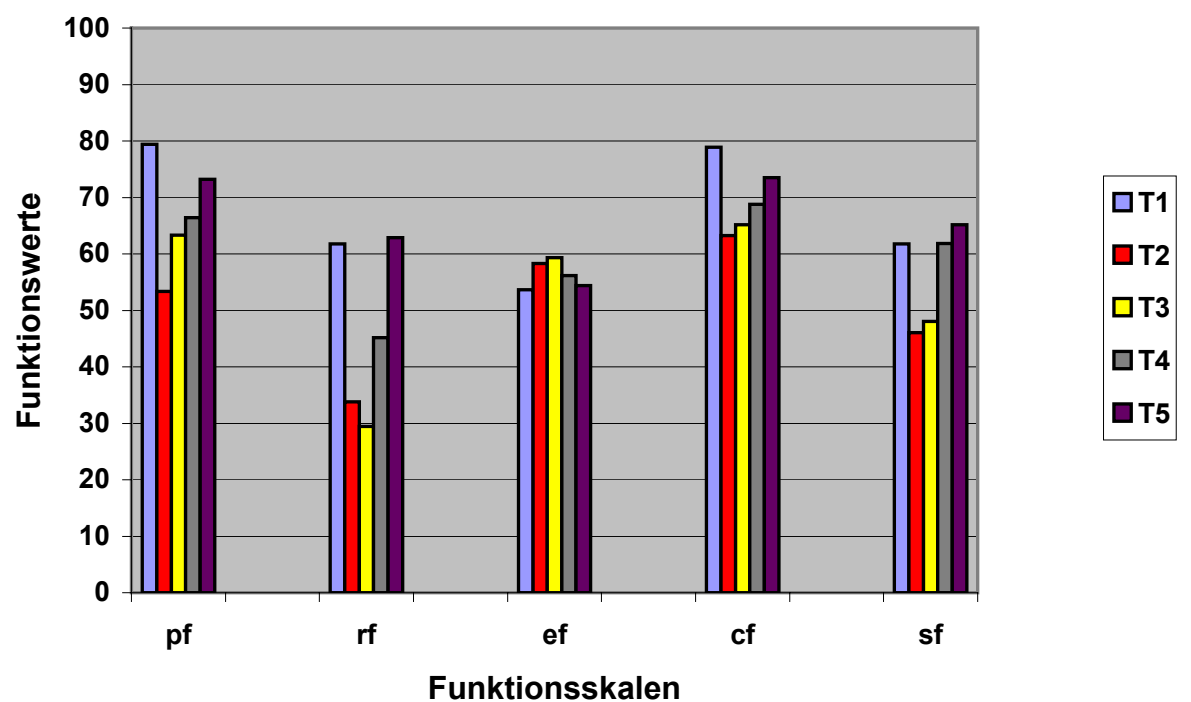

Die Werte der Funktionsskalen PF, RF und SF sind abhängig vom Messzeitpunkt. Alle drei Skalenwerte nehmen nach der HDC signifikant ab. Der PF-Wert hat sich zu T3 vor der Entlassung aus der Klinik - wieder signifikant gebessert. SF und RF verbessern sich signifikant nach der Entlassung zu T4. Für keine der genannten Skalen besteht zwischen T1 und T5 ein signifikanter Unterschied. Die Skalenverläufe entsprechen den Annahmen und bestätigen die psychologische Hypothese. SF und RF sind in dieser Stichprobe sehr ähnlich beurteilt worden. Die SF und RF nehmen in der stationären Phase ab, steigen nach der Entlassung wieder auf das Ausgangsniveau an und bleiben auf diesem Niveau. Dieser Verlauf ist plausibel: Die Patientinnen und Patienten kehren in die Familie zurück, werden umsorgt, die sozialen Kontakte werden durch die Besucher zu Hause wieder aufgenommen und alle streben einen relativ „normalen“ Alltag an. In dieser Stichprobe beklagt keine der Patientinnen und keiner der Patienten Kontaktabbrüche oder Vereinsamung.

Die Skala EF bildet keine signifikanten Zeiteffekte ab. Der Kurvenverlauf ist umgekehrt U-förmig. Die Mittelwertsunterschiede liegen unter 10, d.h. auch im klinisch irrelevanten Bereich. Der Verlauf der EF-Werte entspricht in dieser Untersuchung dem Verlauf der Angstwerte des HADS-D. Die hohe Korrelation zwischen beiden Skalen weist darauf hin, dass offensichtlich die emotionale Funktion wesentlich durch Angst mitbestimmt wird. Die fehlende Abbildung von Zeiteffekten durch die EF-Skala liegt in dieser Studie am unterschiedlichen Antwortverhalten von Frauen und Männern (s. u.). 
CF zeigt eine deutliche Abnahme der Funktionswerte zwischen T1 und T2 (T-Test zeigt einen signifikanten Unterschied), die ein klinisch relevantes Ausmaß erreichen, auch wenn die VA keinen signifikanten Haupteffekt nachweist. Danach steigt der Wert wieder kontinuierlich an (keine signifikanten Unterschiede), bleibt aber unter dem Ausgangsniveau. Auch dieser Verlauf entspricht den Erwartungen. Generell klagen Patienten häufig, auch bei den konventionellen Chemotherapien, über Konzentrations- und Gedächtnisprobleme. Von den wenigen Untersuchungen (siehe S. 96) ist bekannt, dass sich diese Klagen durch Performanztests nicht objektivieren lassen. Es gibt aber Hinweise, dass emotionale Einflüsse die kognitive Leistung beeinflussen. Dieser Zusammenhang ist aus der Depressionsforschung geläufig. Die Arbeit von van Dam et al. (Siehe S. 105) verweist auf einen negativen Langzeiteffekt (2 Jahre nach Therapieende) der HDC, der sich aber in dieser Stichprobe, nach einem Jahr, (noch) nicht nachweisen lässt.

Da die QL (Allgemeine LQ) eine Skala ist, die quasi Funktions- und Symptomwerte zusammenfassend bewerten soll, wird sie hier wegen ihrer übergeordneten Bedeutung gesondert dargestellt:

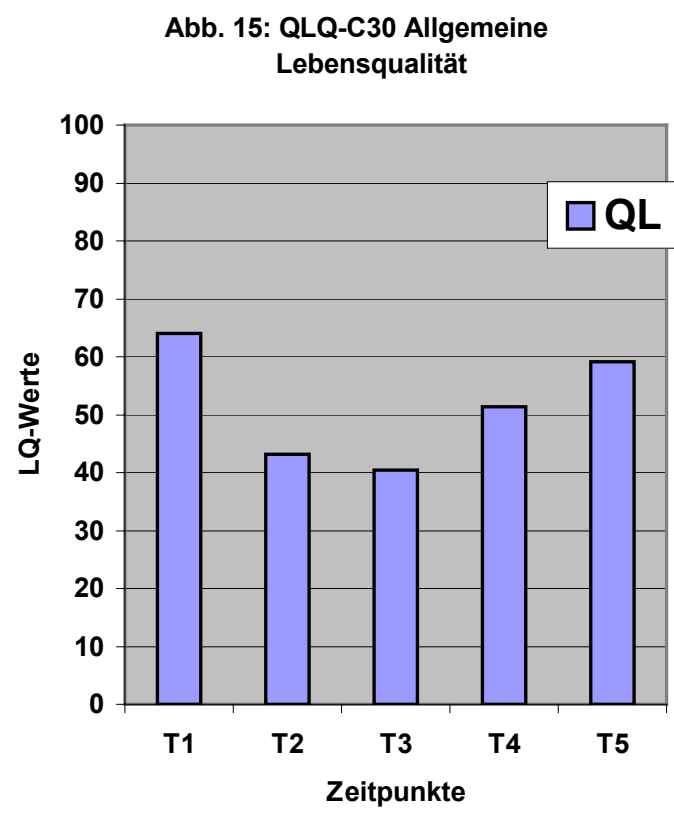

Der Verlauf der QL-Werte ist hypothesenkonform. Die Werte der QL-Skala verändern sich signifikant in Abhängigkeit vom Zeitpunkt der Messung. Zwischen T1 und T2 kommt es zur signifikanten Abnahme, zwischen T3 und T4 zur signifikanten Verbesserung der Werte. T4 und T5 unterscheiden sich nicht, ebenso wenig T1 und T5, obwohl der Wert zu T5 tendenziell niedriger liegt. 
Auch dieser Verlauf entspricht der Vorhersage und legt die Annahme nahe, dass nach einem Jahr annährend wieder die Ausgangssituation erreicht ist, auch wenn der T5Wert niedriger liegt. Dass nach einem Jahr Rezidivfreiheit die Werte „nur" annähernd das Ausgangsniveau erreichen mag daran liegen, dass die Patienten sich in ihrer Leistungsfähigkeit noch eingeschränkt fühlen und dass sie einen so langwierigen Rehabilitationsprozess nicht erwartet hatten (siehe Interviewauswertungen). Im Vergleich mit anderen Stichproben ist der QL-Wert mit maximal 63.97 eher niedrig. In zahlreichen anderen Untersuchungen finden sich Werte um 80.

\subsubsection{Symptomskalen des QLQ-C30}

Die Werte der Symptomskalen sind in der folgenden Abbildung dargestellt:

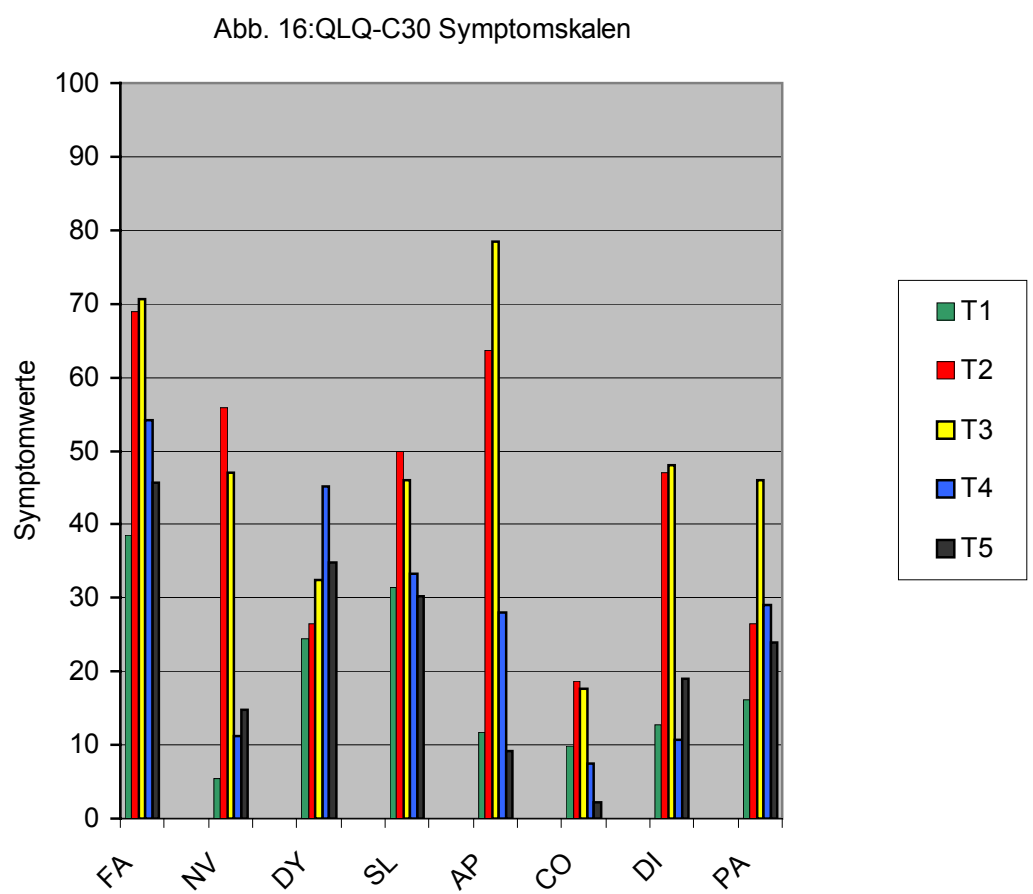

Skalen

Alle Werte, bis auf DI und NV, zeigen den erwarteten Kurvenverlauf.

Wie erwartet nehmen die Symptome FA (Erschöpfung), NV (Übelkeit/Erbrechen), PA (Schmerz), AP (Appetitverlust) und DI (Durchfall) signifikant durch die HDC zu. PA erreicht zu T3 seinen Höhepunkt. Der signifikante Unterschied zwischen T2und T3 ist plausibel, da die Schmerzintensität gesteigert wird durch die entzündlichen Prozesse in der Aplasiephase und deshalb nicht unmittelbar zu T2 am ausgeprägtesten ist. Nach der Entlassung bessern sich die genannten Symptome. Für NV ist der Anstieg zu T2 am höchsten, als unmittelbare Reaktion auf die Therapie. Der Werte für NV liegt zu T5 noch signifikant über dem Ausgangswert, wenn auch auf niedrigem Niveau. Der Sym- 
ptomwert für FA bleibt auf hohem Niveau. Das Ergebnis entspricht dem anderer Studien (siehe tabellarische Übersicht S. 105, 107f).

Die Symptome DY (Atemnot), SL (Schlafstörungen) und CO (Verstopfung) zeigen keine signifikanten Effekte in der VA, trotz der klinisch relevanten Mittelwertsunterschiede. Fünf von sieben Symptomwerten sind tendenziell nach einem Jahr noch höher als vor Therapiebeginn. CO spielt aufgrund der Therapie und des Krankheitsspektrums im gesamten Zeitverlauf eine untergeordnete Rolle.

Insgesamt sind die Kurvenverläufe Hypothesen konform sie decken sich auch mit den klinischen Erwartungen.

\subsubsection{Karnofsky-Index}

Der Karnofsky-Index gibt ein Urteil aus der Fremdbeobachterperspektive ab. Inhaltlich entspricht der Karnofsky-Index der PF-Skala des QLQ-C30.

Abb. 17: Verlauf des Karnofsky-Index

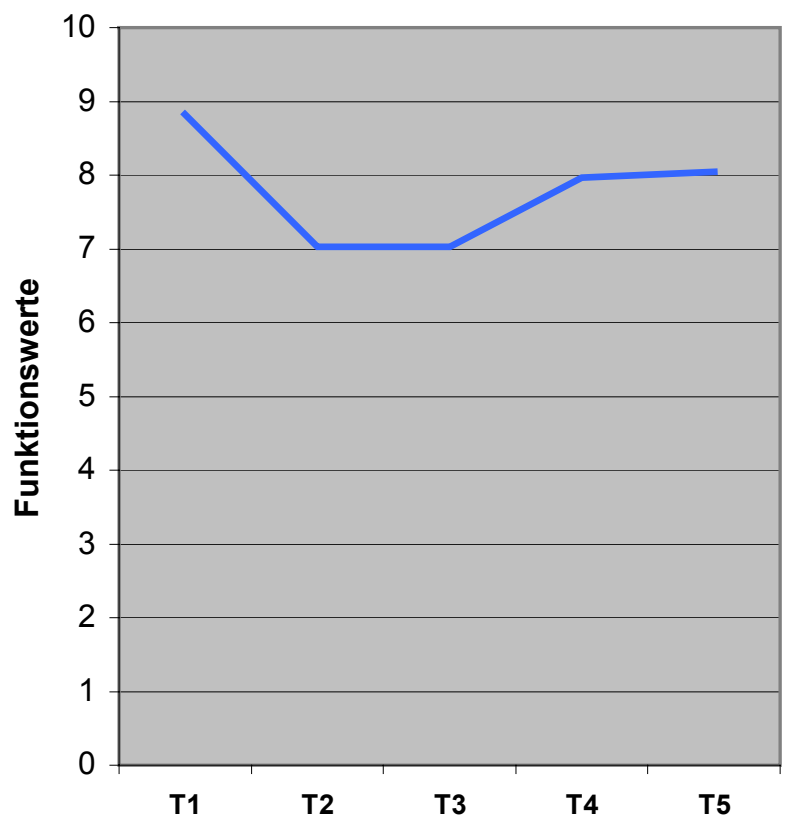

Die VA ergab einen signifikanten Haupteffekt „Zeit“. Nach der HDC kommt es zum Abfall des Wertes. Danach, während der stationären Zeit wird der Funktionszustand annähernd als gleichbleibend beurteilt. Zwischen T3 und T4 kommt es zum signifikanten Anstieg während sich zwischen T4 und T5 keine Verbesserung mehr abzeichnet. Bemerkenswert ist, dass aus der Fremdbeurteilerperspektive der Funktionszustand zu T5 signifikant schlechter ist als der Zustand vor der HDC. Während die Kurvenform der Vorhersage entspricht, wurde der niedrigere Wert zu T5 nicht erwartet. 


\subsubsection{Angst und Depressivität im HADS-D}

In der VA zeigt sich kein signifikanter Haupteffekt „Zeit“ (Signifikanter Haupteffekt „Sex“, siehe Abschnitt Gruppenunterschiede). Bei der beschreibenden Darstellung zeigen sich erhebliche Mittelwertsunterschiede für $A$ und $D$ die im T-Test zwischen den Zeitpunkten T1/T2 und T2/T3 signifikant sind. Da zumindest für den Unterschied zwischen T1/T2 eine gerichtete Hypothese vorliegt, ist diese Prüfung gerechtfertigt. Der hohe A-Wert entspricht der Vorhersage der erhöhten Erwartungsangst zu T1. Allerdings ist dieser Unterschied ausschließlich durch das Antwortverhalten der Frauen bedingt (siehe differenzielle Effekte) Auch die Abnahme der Angstwerte bis zur Entlassung aus der Klinik und der danach erfolgende moderate Anstieg sind erwartungsgemäß.

Die Kurven liegen im Bereich unauffälliger Werte für Depression und fraglich/unauffälliger Werte für Angst. Der Vergleich der Mittelwerte dieser Stichprobe mit den Ergebnissen der repräsentativen Bevölkerungsstichprobe von Hinz \& Schwarz (2001) ergibt zu allen Zeitpunkten höhere Werte für D und A in dieser Stichprobe.

Tabelle 32: T-Test bei gepaarten Stichproben

\begin{tabular}{|c|c|c|c|c|c|}
\hline & \multicolumn{5}{|c|}{ df t-Werte $\quad\left({ }^{*}=p<.005\right)$ (zweiseitig) } \\
\hline Skala & $\mathrm{T} 1 / \mathrm{T} 2(\mathrm{df}=33)$ & $\mathrm{T} 2 / \mathrm{T} 3(\mathrm{df}=33)$ & $\mathrm{T} 3 / \mathrm{T} 4(\mathrm{df}=31)$ & $\mathrm{T} 4 / \mathrm{T} 5(\mathrm{df}=21)$ & $\mathrm{T} 1 / \mathrm{T} 5(\mathrm{df}=21)$ \\
\hline A-HADS & $2.1^{*}$ & 2.46 * & -1.19 & -.82 & -1.57 \\
\hline D-HADS & $2.68 *$ & $-2.86^{*}$ & -.20 & .57 & .20 \\
\hline
\end{tabular}

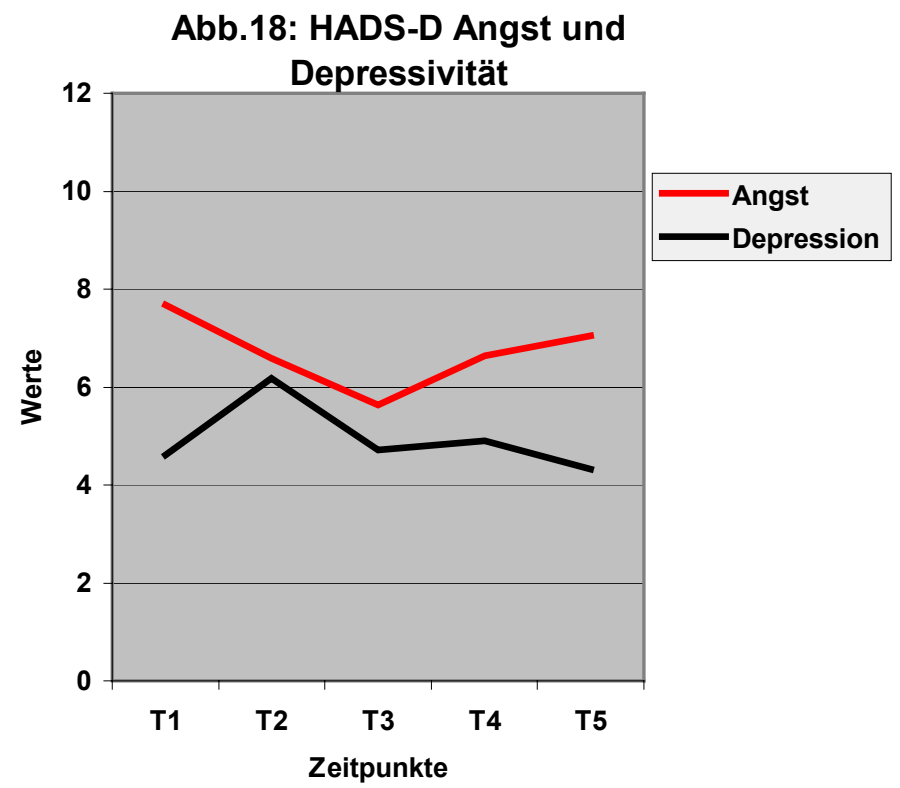


Wie erwartet, zeigen die Kurven für die Angst- und Depressionswerte einen unterschiedlichen Verlauf: Das Kurvenniveau der Angst liegt zu jedem Zeitpunkt höher. Zu Beginn ist die Angst am größten, fällt dann zum Entlassungszeitpunkt T3 deutlich ab und steigt dann wieder steil an bis T4. Danach ist der Anstieg abgeflachter und bleibt etwas unter dem Ausgangsniveau (nicht signifikant). Der Verlauf der Angstkurve spiegelt die deutliche Entlastung nach überstandener HDC wider. Der Anstieg des Angstniveaus auf ein mittleres Niveau ist kennzeichnend für Krebspatientinnen und -patienten, die dauerhaft (oder zumindest vorübergehend) mit der Unsicherheit über den weiteren Verlauf leben müssen. Der Anstieg nach Abschluss der Therapie und Entlassung aus dem Krankenhaus zeigt sich in vielen anderen Untersuchungen und wird als Rezidivangst gewertet.

Die Kurve für die Depressionswerte liegt generell im unauffälligen Bereich. Sie steigt zu T2 signifikant an, fällt zu T3 signifikant ab und erreicht zu T5 einen Werte unterhalb des Ausgangswertes. Depressivität ist für diese Stichprobe und den untersuchten Zeitabschnitt von untergeordneter Bedeutung. Das hat vermutlich mit Selektionseffekten zu tun: Patientinnen und Patienten, die sich einer HDC aussetzen, müssen über ein hohes Maß an Hoffnung verfügen. Außerdem wird die Behandlung nur ausgewählten Patientinnen und Patienten angeboten, denen die Ärzte eine entsprechende Belastbarkeit zuschreiben und denen sie zutrauen, dass sie die Behandlungsbedingungen meistern können. Aus den Vorgeschichten geht hervor, dass einige unserer Studienteilnehmer beharrlich und aktiv, manchmal auch gegen den Rat ihrer Hausärzte, auf die Suche nach Therapiemöglichkeiten gegangen sind. Der Anstieg von Depressivität zu T2 spiegelt die physischen Einschränkungen, die dadurch erlebten Aktivitätseinschränkungen und den erfahrenen Kontrollverlust wider. Absolut gesehen, spielen die veränderten Werte keine Rolle.

In der folgenden Tabelle sind die Patienten aufgelistet, die nach den Kriterien der Autoren fragliche/auffällige Angstwerte/Depressionswerte aufweisen und die deswegen einer weiteren Diagnostik bedürfen: 
Tabelle 33: Anzahl der Patienten, die zu den Zeitpunkten T1 - T5 folgenden Kategorien zugerechnet werden können HADS-D

\begin{tabular}{|c|c|c|c|c|c|c|c|}
\hline \multirow{2}{*}{$\begin{array}{c}\text { Zeitpunkte } \\
\mathrm{N}\end{array}$} & \multicolumn{3}{|c|}{ Angst } & \multirow{2}{*}{$\begin{array}{c}\text { Zeitpunkte } \\
\qquad \mathrm{N}\end{array}$} & \multicolumn{3}{|c|}{ Depressivität } \\
\hline & $\begin{array}{c}\text { unauffällig } \\
\leq 7\end{array}$ & $\begin{array}{c}\text { fraglich } \\
8-10 \\
\end{array}$ & $\begin{array}{c}\text { auffällig } \\
\geq 11\end{array}$ & & $\begin{array}{c}\text { unauffällig } \\
\leq 7\end{array}$ & $\begin{array}{c}\text { fraglich } \\
8-10 \\
\end{array}$ & $\begin{array}{c}\text { auffällig } \\
\geq 11\end{array}$ \\
\hline T1 34 & 17 & 6 & 11 & T1 34 & 27 & 6 & 1 \\
\hline T2 34 & 21 & 7 & 6 & T2 34 & 21 & 7 & 6 \\
\hline T3 34 & 23 & 9 & 2 & T3 34 & 24 & 8 & 2 \\
\hline T4 32 & 19 & 5 & 8 & T4 32 & 26 & 1 & 5 \\
\hline T5 29 & 16 & 8 & 5 & T5 28 & 23 & 2 & 3 \\
\hline
\end{tabular}

Zu T1 haben 50\% der Patienten erhöhte Angstwerte. Während des stationären Aufenthaltes nimmt dieser Anteil ab, um nach einem Jahr wieder anzusteigen: von 29 Patienten haben 13 (45\%) zu T5 fragliche/auffällige Angstwerte, die einer diagnostischen Abklärung bedürfen. Von den 5 Patienten mit auffälligen A-Werten sind drei krankheitsfrei, zwei haben ein Rezidiv erlitten. In Bezug auf Depressivität ist der Anteil der Patienten mit hohen Werten wesentlich geringer: 3 von 22 Patienten liegen im fraglichen/auffälligen Bereich (14\%). Im Vergleich mit den Werten einer repräsentativen Bevölkerungsstichprobe (Hinz \& Schwarz 2001) liegen aber die relativen Häufigkeiten deutlich höher.

Nach einem Jahr sind drei Patientinnen in psychotherapeutischer/psychiatrischer Behandlung, zusätzlich zu den beiden Patientinnen die bereits vor der Erkrankung in Behandlung waren und die nach der Therapie ihre Behandlung wieder aufgenommen haben. Zwei der Patientinnen gehören zur Rezidivgruppe. Bei allen liegt eine gemischte Symptomatik von Angst und Depressivität vor, bei der allerdings die Angstsymptomatik im Vordergrund steht. Die Angst bezieht sich in erster Linie auf die Angst vor einem Fortschreiten der Erkrankung. 


\section{Zusammenfassung \\ Mittelwertsverläufe von Funktions- und Symptomskalen}

Die Mittelwertsverläufe der Funktionskurven entsprechen im Wesentlichen den Erwartungen. Während der stationären Zeit nehmen die Funktionswerte und der Allgemeine Wert der LQ, ab um dann in der Rehabilitationsphase wieder anzusteigen. Der Ausgangswert von T1 wird allerdings nicht ganz erreicht, was dafür spricht, dass der Einjahreszeitraum für eine vollständige Rehabilitation zu kurz ist. Die Skala für die emotionale Funktion stellt eine Ausnahme dar: Zeiteffekte sind nicht nachweisbar, der Kurvenverlauf verweist auf eine tendenziell bessere Beurteilung der EF in der stationären Phase.

Die Symptomwerte verändern sich durch den Einfluss der Therapie am stärksten. Die meisten Symptomwerte zeigen eine signifikante Verschlechterung nach der HDC an. Der Erholungsverlauf ist tendenziell wie erwartet, allerdings wird der Ausgangszustand nur annähernd wieder erreicht. Der Wert für Fatigue (FA) bleibt auf hohem Niveau.

\section{Karnofsky-Index}

Der Karnofsky-Index verringert sich signifikant durch die Therapie. Während der Klinikzeit wird die Belastungsfähigkeit als gleichbleibend niedrig eingeschätzt. Nach der signifikanten Verbesserung zu T4 bleibt der Wert auch nach einem Jahr auf dem gleichem Niveau. Der niedrigere Wert zu T5 entspricht nicht den Erwartungen.

\section{Angst und Depressivität im HADS-D}

Der Verlauf der Mittelwerte für $A$ und $D$ ist annähernd wie erwartet. Angst spielt zu allen Zeitpunkten eine größere Rolle als Depressivität. $A$ und D Werte sind durchgängig, im Vergleich zur Normalbevölkerung, erhöht. Erhöhte Angstwerte (fragliche/auffällige) zeigen sich bei nahezu der Hälfte aller Patienten zu T1 und T3. Der Anteil von Patienten mit erhöhten Depressionswerten ist unmittelbar nach der HDC und nach einem Jahr am größten. Der HADS-D weist auf Patienten hin, die einer diagnostischen Abklärung bedürfen.

\subsubsection{Finanzielle Belastungen durch Erkrankung und Therapie}

Die finanziellen Belastungen durch die Erkrankung werden ebenfalls im QLQ-C30 erfragt. Auf eine graphische Darstellung wird verzichtet, da sich über den Zeitverlauf kei- 
ne Veränderungen ergeben und insgesamt die finanziellen Belastungen als gering bewertet werden. Die finanzielle Belastung wird lediglich während des Klinikaufenthaltes geringfügig größer eingeschätzt. Dieses Ergebnis spiegelt sicherlich die relativ gute soziale Absicherung in Deutschland wider, außerdem machen sich auch hier Selektionseffekte der Stichprobe (siehe soziodemographische Daten, S. 139f) bemerkbar. Zu T5 werden die finanziellen Belastungen als am geringsten eingeschätzt. $\mathrm{Zu}$ diesem Zeitpunkt hat sich geklärt, ob die Patientinnen und Patienten wieder in den Beruf zurückkehren konnten oder ob sie auf Zeit oder dauerhaft berentet wurden.

\section{Zusammenfassung}

Finanzielle Belastung (QLQ-C30)

Durch die Therapie kommt es nicht zu vermehrter finanzieller Belastung der Patienten.

\subsubsection{Gruppenunterschiede}

Der klinische Eindruck legt (trotz der geringen Stichprobengröße) eine Differenzierung unter verschiedenen Gesichtspunkten (Geschlecht, Rezidiv/kein Rezidiv) nahe, auf die im folgenden Kapitel eingegangen wird.

Von großem Interesse war die Frage ob sich zwischen den Patienten, die im Verlaufe des ersten Jahres nach HDC ein Rezidiv erleiden und denen, die krankheitsfrei sind, Unterschiede zeigen. Wider Erwarten zeigt sich kein einziger signifikanter Effekt zwischen diesen beiden Gruppen obwohl die Mittelwerte tendenzielle Unterschiede fast aller Skalen ab T2 in der erwarteten Richtung (geringere Funktions- und höhere Symptomwerte) zeigen. Lediglich die Skala PF liegt knapp unter der Signifikanzgrenze.

Signifikante Unterschiede für Männer und Frauen zeigen sich dagegen in den mehreren Skalen. Der Effekt der Variablen „Sex“ ist trotz der geringen Stichprobengröße nachweisbar.

\subsubsection{Unterschiede zwischen den Geschlechtern}

Der Vergleich zwischen Frauen und Männer ergibt für folgende Skalen einen signifikanten Haupteffekt „Sex“ (siehe Tabelle S. 145): EF, CF, SF, QL, FA, A-HADS, DHADS und Karnofsky-Index. Generell liegen die Werte der Funktionsskalen des QLQ-C30 bei den Frauen niedriger als die Werte der Männer. Die Symptomwerte der Frauen sind dagegen tendenziell höher, aber nur die Werte der FA-Skala sind bei den Frauen signifikant größer. Zu welchen Zeitpunkten sich Männer und Frauen in den genannten Skalenwerten unterscheiden wird mit T-Tests für unabhängige Stichproben überprüft. Die Mittelwerte, Standardabweichungen und T-Werte sind in der folgenden Tabelle aufgeführt: 
Tabelle 28: T-Tests Gruppenunterschiede Geschlecht (Signifikanz $\left.{ }^{*}=p<.05\right)($ zweiseitig)

\begin{tabular}{|c|c|c|c|c|c|}
\hline Variable & Zeitpunkte & $\begin{array}{c}q \\
\text { MW (SD) }\end{array}$ & $\begin{array}{c}\hat{\partial} \\
\mathbf{M W}(\mathrm{SD})\end{array}$ & T-Wert & df \\
\hline \multirow{5}{*}{ A-HADS } & T1 & $9.26(3.09)$ & $4.36(2.30)$ & 4.66 * & 32 \\
\hline & T2 & $7.35(3.85)$ & $5.0(2.61)$ & 1.82 & 32 \\
\hline & T3 & $6.78(4.18)$ & $3.27(2.73)$ & $2.54 *$ & 32 \\
\hline & T4 & $8.38(5.01)$ & $3.45(2.46)$ & 3.05 * & 30 \\
\hline & T5 & 7.94 (3.67) & $4.2(3.35)$ & 2.04 & 20 \\
\hline \multirow{5}{*}{ D-HADS } & T1 & $5.39(3.06)$ & $3(2.57)$ & 2.24 * & 32 \\
\hline & T2 & $7.52(4.76)$ & $3.36(2.38)$ & $2.73^{*}$ & 32 \\
\hline & T3 & $6.13(3.79)$ & $1.73(1.35)$ & 3.71 * & 32 \\
\hline & T4 & 6.29 (3.91) & $2.27(1.79)$ & 3.21 * & 30 \\
\hline & T5 & $4.50(3.50)$ & $3.4(2.61)$ & .70 & 20 \\
\hline \multirow{5}{*}{$\begin{array}{c}\text { Karnofsky- } \\
\text { Index }\end{array}$} & T1 & $8.65(1.03)$ & $9.18(0.40)$ & -1.63 & 32 \\
\hline & T2 & $6.61(1.62)$ & $7.91(1.14)$ & -2.40 * & 32 \\
\hline & T3 & $6.57(1.38)$ & $8(1.41)$ & -2.82 * & 32 \\
\hline & T4 & 7.59 (1.1) & $8.73(0.9)$ & $-2.96 *$ & 30 \\
\hline & T5 & $8(0.94)$ & $8.2(0.84)$ & -0.43 & 20 \\
\hline \multirow{5}{*}{ EF } & T1 & $43.48(29.19)$ & 75 (22.05) & $3.17^{*}$ & 32 \\
\hline & T2 & $48.55(28.83)$ & $78.79(13.10)$ & $-3.3^{*}$ & 32 \\
\hline & T3 & $50.72(23.29)$ & $77.27(16.70)$ & $-3.38 *$ & 32 \\
\hline & T4 & $48.41(29.18)$ & $71.21(23.38)$ & -2.24 * & 30 \\
\hline & T5 & $52.70(25.30)$ & $60(28.51)$ & -.55 & 20 \\
\hline \multirow{5}{*}{ CF } & T1 & $73.91(27.44)$ & $89.40(31.06)$ & -1.64 & 32 \\
\hline & T2 & $57.97(27.92)$ & $74.24(31.06)$ & -1.53 & 32 \\
\hline & T3 & $57.25(26.51)$ & $81.82(18.94)$ & $-2.75 *$ & 32 \\
\hline & T4 & $61.11(34.30$ & $84.85(17.41)$ & -2.14 * & 30 \\
\hline & T5 & 70.59 (36.10) & $83.33(23.57$ & -.74 & 20 \\
\hline \multirow{5}{*}{ SF } & T1 & $53.62(30.13)$ & $78.79(28.76)$ & -2.31 * & 32 \\
\hline & T2 & 35.51 (29.0) & $68.18(32.88)$ & $-2.95^{*}$ & 32 \\
\hline & T3 & $40.58(32.5)$ & $63.64(30.57)$ & -1.97 & 32 \\
\hline & T4 & $54.76(32.97)$ & $78.79(22.47)$ & $-2.16^{*}$ & 30 \\
\hline & T5 & $62.74(35.12)$ & $73.33(22.36)$ & -0.63 & 20 \\
\hline \multirow{5}{*}{ QL } & T1 & $59.06(15.87)$ & $74.24(13.67)$ & $-2.77^{*}$ & 32 \\
\hline & T2 & $36.59(21.13)$ & $56.82(20.01)$ & -2.65 * & 32 \\
\hline & T3 & $34.78(18.06)$ & $52.27(21.76)$ & -2.47 * & 32 \\
\hline & T4 & $45.63(21.51)$ & $62.12(21.20)$ & -2.07 * & 30 \\
\hline & T5 & $56.86(23.24)$ & $66.67(26.35)$ & -0.98 & 20 \\
\hline & T1 & $44.44(25.73)$ & $26.26(22.92)$ & 2.0 & 32 \\
\hline & T2 & $71.98(29.18)$ & $62.63(29.93)$ & .87 & 32 \\
\hline
\end{tabular}




\begin{tabular}{|c|c|c|c|c|c|}
\hline \multirow{2}{*}{ Variable } & Zeitpunkte & $\begin{array}{c}+ \\
\text { MW (SD) }\end{array}$ & $\begin{array}{c}\hat{\sigma} \\
\text { MW (SD) }\end{array}$ & T-Wert & df \\
\hline \multirow{2}{*}{ FA } & T3 & $79.23(19.62)$ & $52.53(26.34)$ & $\mathbf{3 . 3 2}^{*}$ & 32 \\
\cline { 2 - 6 } & T4 & $59.79(31.52)$ & $44.44(28.11)$ & 1.36 & 30 \\
\cline { 2 - 6 } & T5 & $47.06(29.80)$ & $41.11(27.67)$ & .40 & 20 \\
\hline
\end{tabular}

In den folgenden Abbildungen sind die Mittelwertsverläufe der Skalen für Männer und Frauen dargestellt. Die Unterschiede betreffen alle Funktionsskalen (mit Ausnahme von PF) und die Symptomskala FA, sowie Angst und Depressivität (HADS-D) und den Karnofsky-Index:

Abb. 19: SF im Vergleich

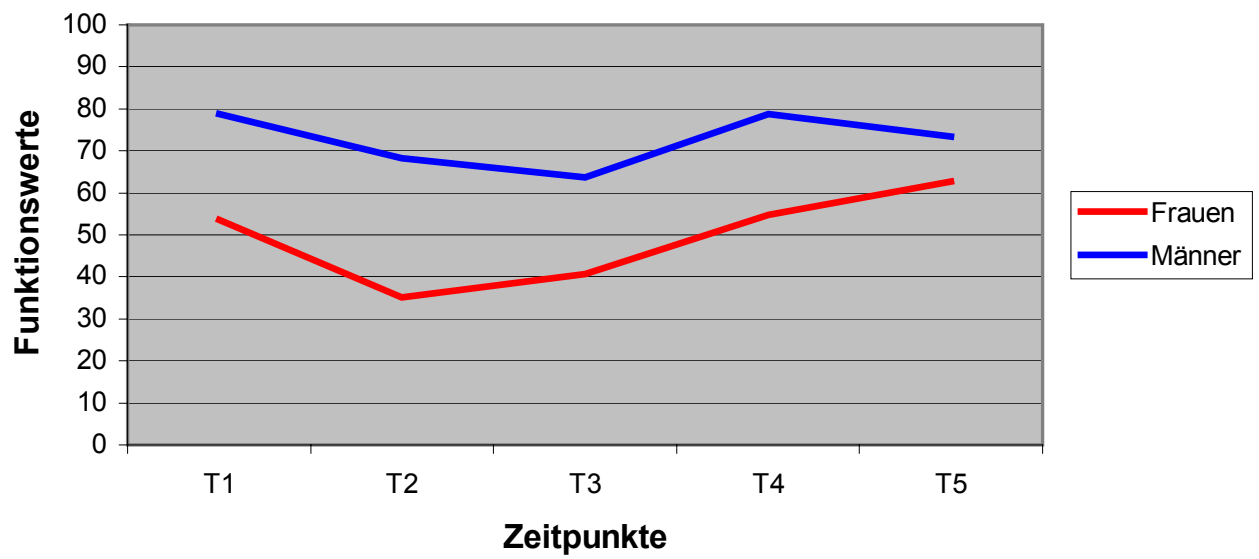

Die Soziale Funktion wird von den Frauen durchgängig niedriger eingeschätzt. Allerdings zeigt die Kurve der Frauen ab T2 eine steigende Tendenz und liegt zu T5 tendenziell über dem Ausgangswert. Die Kurve der Männer fällt zu T3 ab, steigt nach der Entlassung steil an und fällt danach tendenziell unter das Niveau des Ausgangswertes. 
Abb. 20: $C F$ im Vergleich

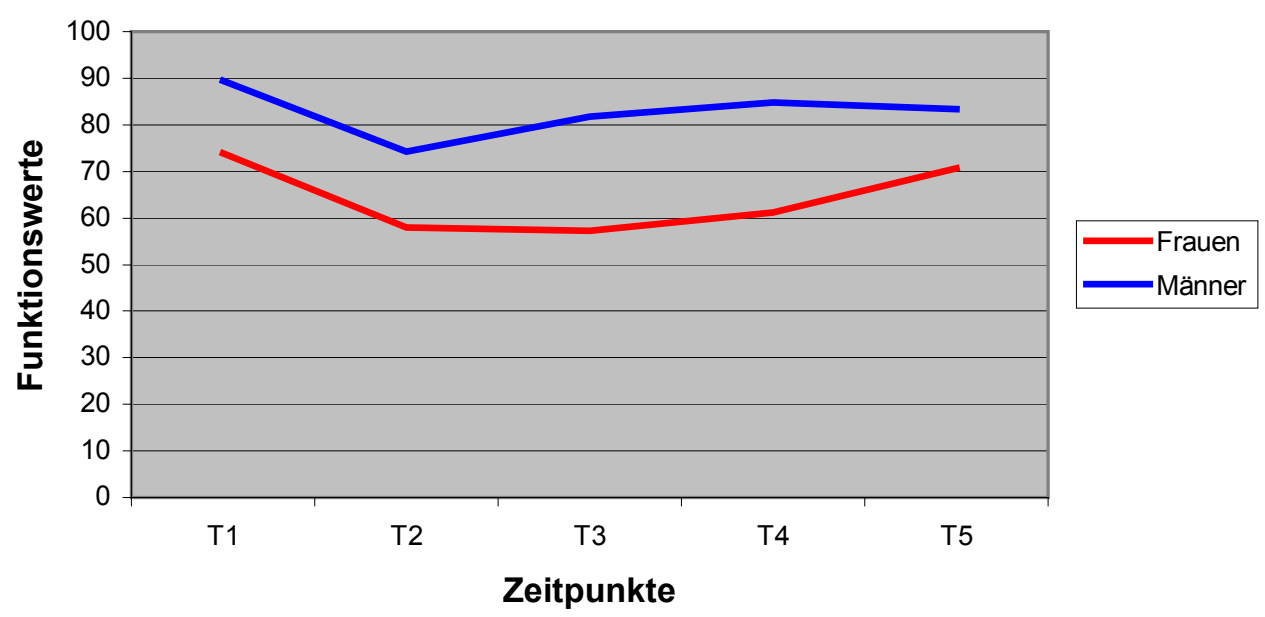

Die Kognitive Funktion zeigt für beide Geschlechter bis zu T4 eine ähnliche Verlaufskurve, bei negativerer Beurteilung durch die Frauen. Zu T5 haben beide Gruppen wieder fast das Ausgangsniveau erreicht.

Abb. 21: FA im Vergleich

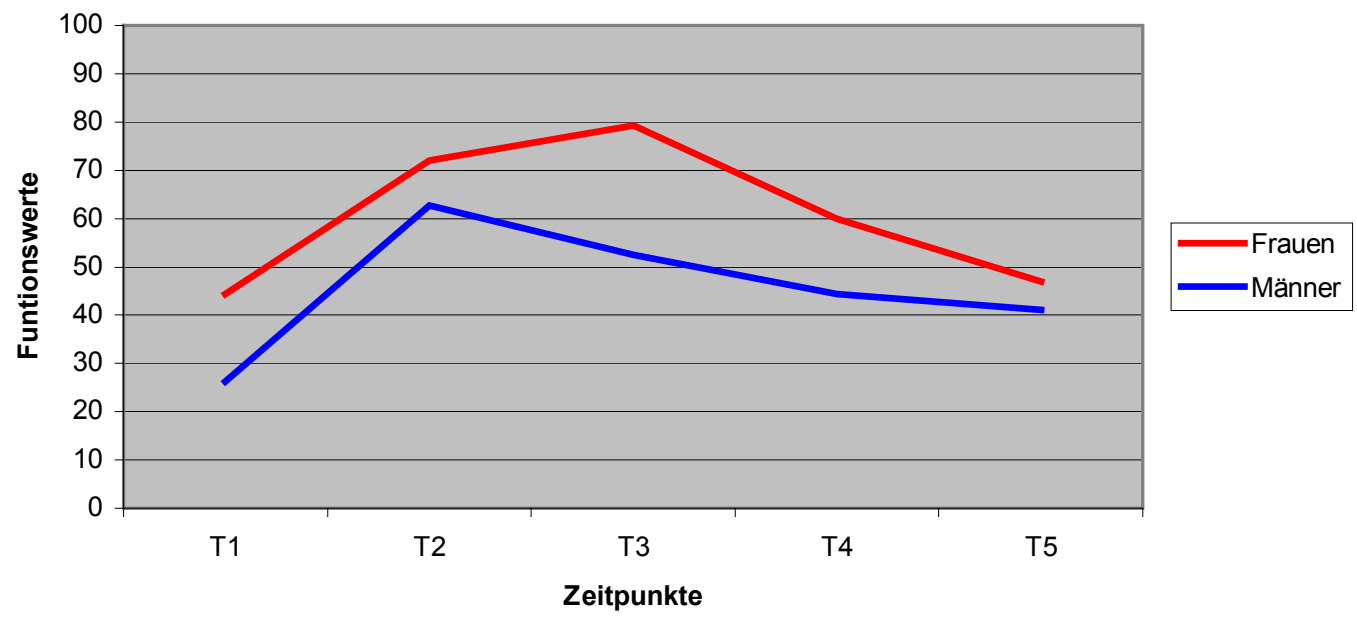

Fatigue ist der einzige Symptomwert, der sich bei Frauen und Männern signifikant unterscheidet. Die Kurvenverläufe sind ähnlich. Die Erholung tritt bei den Männern früher ein, ist aber weniger deutlich. Die Frauen beschreiben zu T3 die höchste Symptomausprägung (signifikant größer als die der Männer). Die Männer beschreiben zu T5 einen höheren Wert im Vergleich zu T1. Die Frauen erreichen zwar zwischenzeitlich höhere Werte, erholen sich aber offensichtlich besser. 
Abb. 22: QL im Vergleich

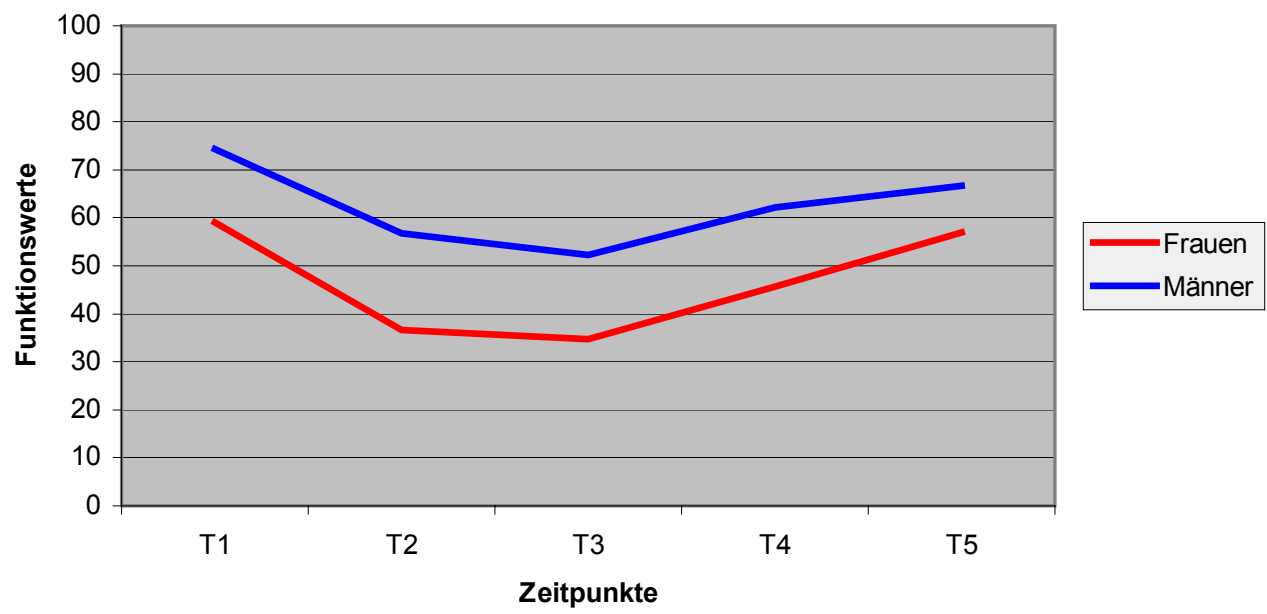

Die Kurvenverläufe der Allgemeinen Lebensqualität sind ähnlich, allerdings auf signifikant unterschiedlichem Niveau (T1-T4). Männer bewerten ihre QL besser.

Abb. 23 : Karnofsky-Index im Vergleich

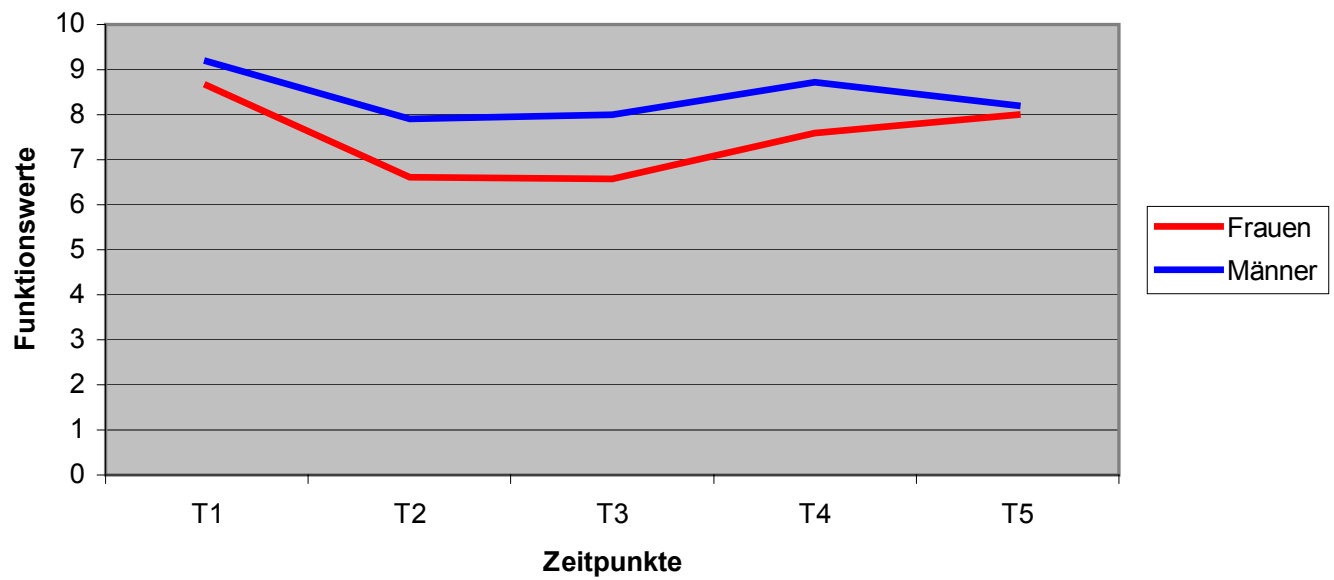

Beim Karnofsky-Index zeigt sich eine deutlichere Verschlechterung bei geringfügig niedrigerem Ausgangsniveau für die Frauen. Bei den Frauen steigt der Wert kontinuierlich in der Rehabilitationsphase an, bei den Männern fällt er nach T4 wieder ab und wird fast so ungünstig beurteilt wie in der stationären Phase. Männer stellen offensichtlich zu T5 ihre Funktionseinschränkungen stärker in den Vordergrund während Frauen die Erholungstendenzen betonen. 
Abb. 24: EF im Vergleich

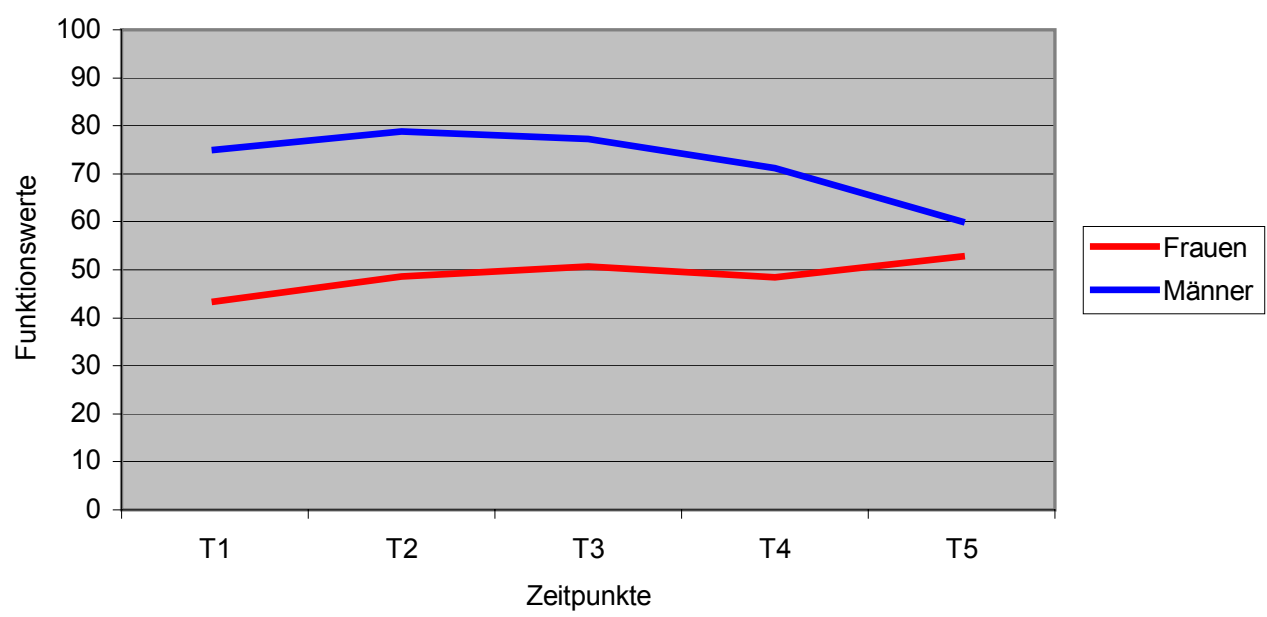

Die EF-Skala zeigt signifikante Unterschiede zwischen den Geschlechtern, aber keine Zeit abhängigen Unterschiede. Auffällig ist, dass die Frauen ihre emotionale Funktion generell als wesentlich schlechter beschreiben, aber eine deutlichere Erholungstendenz zeigen.

Abb. 25: D-HADS im Vergleich

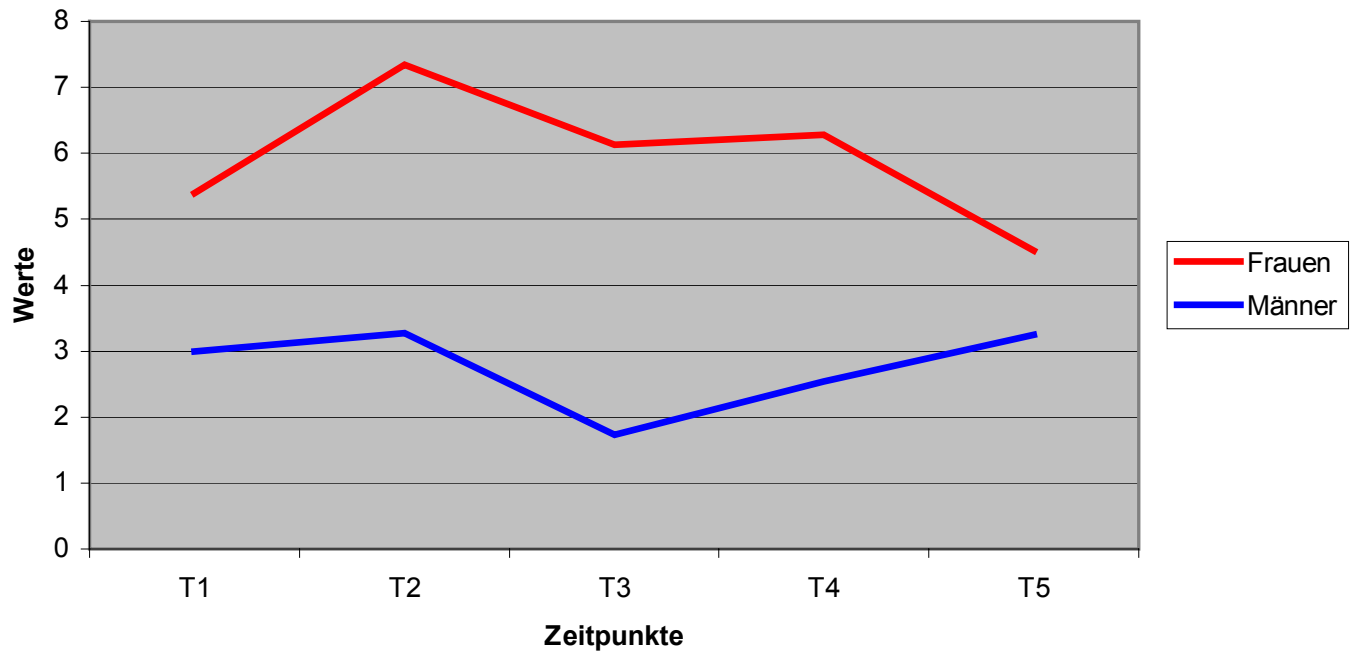

Der Verlauf des Depressionswertes ist zu den ersten vier Zeitpunkten ähnlich, wenn auch unterschiedlich steil. Zu T5 zeichnet sich ein gegenläufiger Trend ab: die Werte der Männer sind gestiegen, die der Frauen abgefallen. 
Die Kurven der D-Werte liegen insgesamt im Bereich „unauffälliger“ Werte. Mit der einen Ausnahme, dass zu T2 der Wert der Frauen im „fraglich auffälligen“ Bereich liegt.

Abb. 26: A-HADS im Vergleich

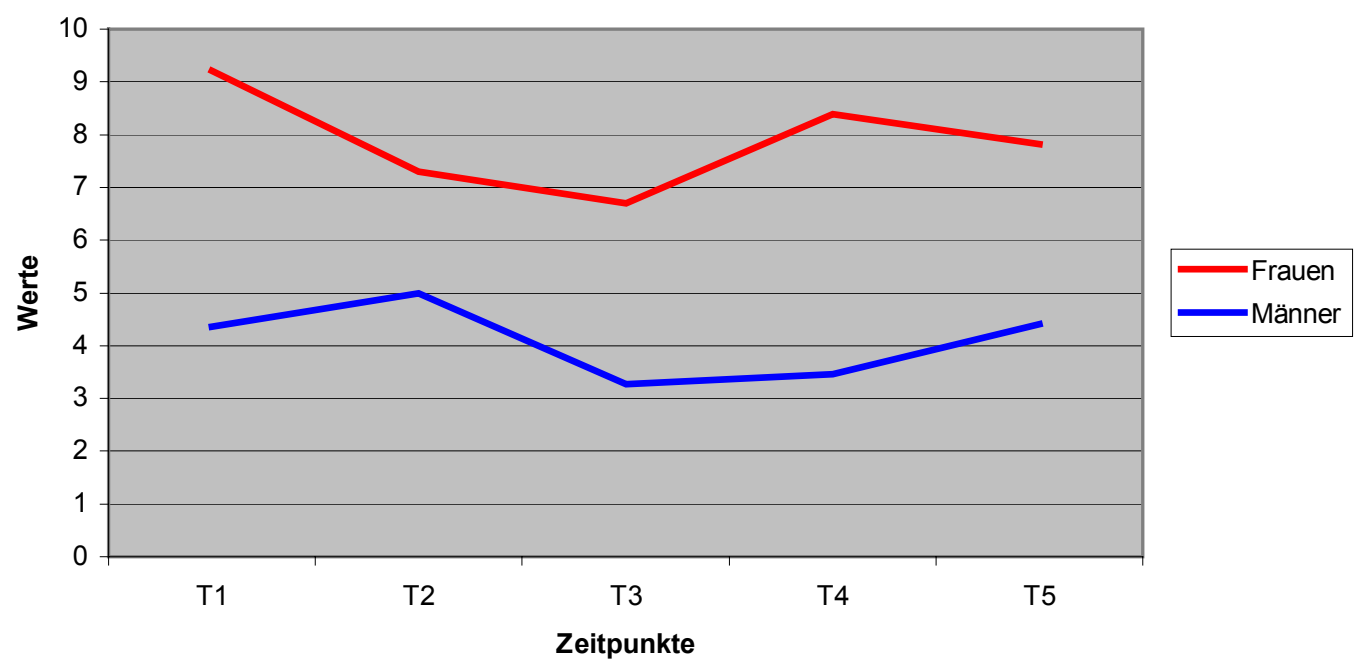

Die Mittelwerte der Angst verlaufen teilweise gegenläufig: vor der Behandlung zeigen die Frauen einen deutlich höheren Wert, der dann kontinuierlich abfällt. Nach der Entlassung steigt der Wert wieder an und fällt dann zu T5 wieder ab, bis unter das Ausgangsniveau. Bei den Männern ist der Ausgangswert deutlich niedriger, er steigt nach der HDC an, fällt zum Entlassungszeitpunkt wieder ab auf den niedrigsten Wert, um dann kontinuierlich, bei unterschiedlich steilem Anstieg, wieder zuzunehmen bis auf das Ausgangsniveau. Die Mittelwerte der Männer liegen, im Gegensatz zu denen der Frauen, zu keinem Zeitpunkt im fraglichen/auffälligen Bereich.

Die Mittelwerte der Frauen ändern sich signifikant im Zeitverlauf während für die Männer kein signifikanter Zeiteffekt auszumachen ist. Die Hypothese der hohen Erwartungsangst lässt sich nur für die Gruppe der Frauen bestätigen.

Von den 17 Patientinnen und Patienten, die mit ihren Angstwerten zu T1 im fraglichen/auffälligen Bereich liegen, sind 14 Frauen (82\%). Drei Männer (18\%) haben Werte, die fraglich/ auffällig sind. Die höchsten Angstwerte $(\mathrm{N}=10)$ haben ausschließlich Frauen.

- Ein Teil der Frauen nimmt ganz offensichtlich Angst stärker wahr (im Handbuch für den HADS-D wird auf dieses Ergebnis, das sich in verschiedenen Stichproben nachweisen ließ, hingewiesen), während bei den Männern die Angstabwehr besser gelingt bzw. Angst nicht geäußert wird.

Die unterschiedlichen Angstäußerungen von Frauen und Männern entsprechen dem klinischen Eindruck. 


\section{Dauer der stationären Unterbringung}

Da die Symptomausprägung bei den Frauen tendenziell größer war, stellte sich die Frage, ob sich das in einem längeren Aufenthalt im Krankenhaus widerspiegelt. Die Frauen waren im Mittel 21,74 Tage stationär untergebracht, die Männer 19,82 Tage. Der Unterschied von ca. 2 Tagen ist nicht signifikant.

\section{Zusammenfassung}

\section{Geschlechtsunterschiede}

Das unterschiedliche Antwortverhalten von Frauen und Männern ist bekannt. Das gesundheitliche Paradoxon, das Frauen mehr Beschwerden und Einschränkungen beklagen, aber trotzdem länger leben, scheint sich auch in dieser Studie zu spiegeln. Ausgehend von den Ergebnissen, erleiden 26\% der Frauen und 54\% der Männer ein Rezidiv. Zu T5 sind 9\% der Frauen und 18\% der Männer gestorben. Dass diese Aussagen bei der kleinen Stichprobe mit der ungleichen Geschlechterverteilung nur als Tendenz und Aufforderung zu genauerer Beobachtung verstanden werden kann, ist selbstverständlich.

Für diese Stichprobe legen die Daten, bei Berücksichtigung des gesamten Verlaufes, nahe dass den Frauen die Krankheitsanpassung, relativ gesehen, besser gelingt, auch wenn sie bei der Betrachtung der absoluten Werte ungünstiger abschneiden und z. T. stärkeren Schwankungen ausgesetzt sind.

\subsubsection{Zusammenfassung der quantitativen Ergebnisse}

Methodische Aspekte

- Der QLQ-C30 ist für Verlaufsuntersuchungen geeignet und bildet Veränderungen im Funktions- und Symptombereich zufriedenstellend ab.

- Die erwarteten Veränderungen in Bezug auf emotionale Belastungen und Angst für die Gesamtgruppe werden nicht abgebildet. Geschuldet ist dieser fehlende Effekt dem unterschiedlichen Antwortverhalten von Frauen und Männern. Die durchgängig hohe Korrelation zwischen A (HADS-D) und EF (QLQ-C30) weist darauf hin, dass Ähnliches gemessen wird.

Inhaltliche Aspekte

- Generell entsprechen die Veränderungen im Zeitverlauf denen in den englischsprachigen Studien und damit weitgehend den Erwartungen. Die Zeiteffekte zwischen T1 und T2/3 sind in der Regel signifikant und spiegeln die höheren Symptom- und teilweise verminderten Funktionswerte wider. Nach der Entlassung kommt es zur Erholung und der Ausgangswert wird annähernd 
wieder erreicht (wenig signifikante Unterschiede zwischen T1 und T5). Für den Karnofsky-Index liegt der Wert zu T5 signifikant niedriger als zu T1. Die Allgemeine LQ wird nach einem Jahr tendenziell niedriger eingeschätzt. Eine erstaunlich gute Übereinstimmung ergibt sich mit der einzigen deutschen Studie von Weis et al. (2001) zum Zeitpunkt T5. Weis et al. haben Patienten $(\mathrm{N}=77)$, die in der Klinik für Tumorbiologie zur Rehabilitation waren, nachuntersucht ( $M=27,4$ Monate, $S D \pm 9,96$ Monate nach Transplantation). Die Werte für die Gruppe der autolog Transplantierten sind fast identisch mit denen dieser Stichprobe. ${ }^{73}$ Allerdings muss beachtet werden, dass für die Patienten der Stichprobe von Weis et al. die HDC teilweise mehr als doppelt so lange zurückliegt im Vergleich mit den Patienten dieser Stichprobe.

- Die hohe Erwartungsangst vor der Therapie bildet sich nicht in der EF-Skala ab. Am deutlichsten stützen die Ergebnisse der relativen Häufigkeiten des HADS-D diese Annahme: 50\% der Patienten haben vor der Therapie fraglich auffällige/auffällige Angstwerte. Frauen haben die höheren A-Werte. Einer Teilgruppe der Stichprobe gelingt eine komplette Angstabwehr über den gesamten Zeitraum (durchgängig niedrige Angstwerte im HADS-D). Bemerkenswert sind die ausgeprägten interindividuellen Unterschiede!

- Angst spielt eine größere Rolle als Depressivität (HADS-D). Der Angstwert erreicht zu T5 das gleiche Niveau wie in der Stichprobe von Weis et al. (2001). Interessanterweise ist trotz des gleichen Mittelwertes die Anzahl der Patienten mit fraglichen/auffälligen Angstwerten in dieser Stichprobe größer (45\% vs. ca. $35 \%)$. Vermutlich spielen hier Zeiteffekte eine Rolle. Mit zunehmendem Abstand zur Therapie tritt die Rezidivangst wieder in den Hintergrund bzw. sie kann besser abgewehrt werden, sofern keine physischen Veränderungen einen Rezidivverdacht nähren. Der Depressionswert liegt in dieser Stichprobe deutlich niedriger. Auch das könnte durch die unterschiedlichen Erhebungszeitpunkte bedingt sein.

- Für die Phase nach der Entlassung, zu T4, liegen die wenigsten Vergleichsdaten aus anderen Studien vor. Die QLQ-Werte dieser Studie legen die Annahme nahe, dass sich das Befinden deutlich gebessert hat.

- Eine Minderheit der Patienten ist nach einem Jahr ins Berufsleben zurückgekehrt. Zwei Patienten haben, nach zwischenzeitlichen Arbeitsversuchen, Dauerrente beantragt. Dieses Ergebnis widerspricht den Angaben anderer Autoren,

\footnotetext{
${ }^{73}$ Möglicherweise handelt es sich bei dem fast identischen Ergebnis zu unterschiedlichen Zeitpunkten um einen Methodenartefakt. Die lediglich vierstufige Skala des QLQ-C30 lässt genauere Differenzierungen im Zeitverlauf, beim Wegfall der dramatischeren Symptome, nicht zu.
} 
dass nach einem Jahr mindestens zwei Drittel der transplantierten Patienten wieder in den Beruf integriert sind (zit. nach Weis et al. 2001). In der Studie von Weis et al. sind es allerdings auch weniger Patienten, die wieder berufstätig sind. Diese Studie und die Studie von Weis et al. stimmen überein, unterscheiden sich aber von denen anderer Länder (vor allem USA, England). Weis et al. interpretieren diesen Unterschied als Negativselektion von Patienten, die aufgrund ihres körperlichen Zustandes, eine stationäre Rehabilitation benötigen. Das Ergebnis dieser Stichprobe bestätigt die Annahme von Weis et al. nicht, sondern liefert Hinweise darauf, dass soziale Faktoren (Absicherung) ausschlaggebend sein könnten. In dieser Studie nehmen kaum Männer an einer Rehabilitation teil.

- Der HADS-D ist als Screeninginstrument geeignet und liefert Hinweise auf Patienten, deren Befindlichkeit einer weiteren Abklärung bedarf.

- Gruppenanalysen sind notwendig und sinnvoll. Trotz des gemeinsamen Therapiefaktors sind differenzielle Effekte des Geschlechts nachzuweisen. Die Geschlechter unterscheiden sich tendenziell in fast allen Variablenverläufen. Frauen haben stärkere Beeinträchtigungen oder nehmen Beeinträchtigungen anders/intensiver wahr und berichten entsprechend darüber. Bemerkenswerterweise finden sich die signifikanten Unterschiede zwischen den Geschlechtern bei den Funktionsskalen, nicht im Symptombereich. Die einzige Symptomskala (FA) mit signifikantem Geschlechtseffekt lässt sich anhand der faktorenanalytischen Auswertung eher dem Funktionsbereich zuordnen. Relativ gesehen ist die Besserung bei den Frauen ausgeprägter als bei den Männern. Über eine bessere psychosoziale Anpassung der Frauen nach Erkrankung und Therapie berichten auch Mumm et al. (2001).

\subsection{Auswertung der Interviews}

\subsubsection{Hypothesen und Ergebnisse in Bezug auf die Interviewdaten}

Die Interviewdaten fokussieren auf individuelle Konstanten im Verlauf von Therapieund Rehabilitationsprozess. Folgende allgemeine psychologische Hypothesen werden formuliert:

- Die Therapiebelastungen werden unabhängig von erwarteten Belastungen in Kauf genommen zugunsten einer erhöhten Chance auf Heilung/Besserung.

- Die Bewertung des Therapienutzens ist unabhängig von realen Belastungen und dem Ergebnis der Therapie. 
- Angst, in unterschiedlicher Ausprägung und inhaltlicher Orientierung, spielt zu allen Zeitpunkten eine wichtige Rolle.

- Neben der Hoffnung auf Heilung ist soziale Unterstützung (Familie, Klinikmitarbeiter) wichtig für die Bewältigung der Therapiebelastungen.

- Krankheitsanpassung wird mitbestimmt von situativen Einflüssen und den Möglichkeiten der internalen und externalen Kontrolle dieser Einflüsse.

- Ein Jahr nach Abschluss der Therapie sind die Patienten, die bis zu diesem Zeitpunkt nicht wieder erkrankt sind, zur Normalität zurückgekehrt.

Die Auswertung der Interviews orientiert sich an der folgenden Gliederung:

1. Belastenden Faktoren

2. Belastungsausgleichenden Faktoren

3. Bewertung der HDC durch die Patienten

4. Kasuistiken

10.2.2 Belastende Faktoren, hervorgerufen durch die Therapie

Befürchtungen und Ängste vor der Therapie

\subsubsection{Befürchtungen und Ängste vor der Therapie}

Wie im Abschnitt 6.1 ausgeführt ist Angst das zentrale Gefühl, dem sich Krebspatienten stellen müssen. Für die Patienten dieser Stichprobe kommt zu Angst durch die Erkrankung noch die Angst vor der Therapie hinzu. Aus anderen Untersuchungen ist bekannt, dass die emotionale Belastung unmittelbar vor der Therapie von den Patienten am höchsten eingeschätzt wird. Hier spielt eine Überschätzung der Gefährlichkeit der Therapie eine wichtige Rolle. Hinzu kommt für die meisten Patienten eine neue stationäre Aufenthaltssituation, unbekannte Personen (Pflegepersonal, Ärzte, Mitpatienten) und Unsicherheit in Bezug auf das Prozedere.

Die Patienten beantworten die Frage nach Ängsten und Befürchtungen wie folgt:

\begin{tabular}{|l|l|ll|}
\hline \multicolumn{2}{|l|}{ Tabelle 35: Ängste } \\
\hline Textzitate (Patientennummer) & Kategorie & N & (\%) \\
\hline $\begin{array}{l}\text { "Ich war völlig verunsichert und wusste vor Angst } \\
\text { nicht, was ich tun sollte“. „Ich habe nur immer ge- } \\
\text { hört, dass man an der Therapie sterben kann“. „Im- } \\
\text { mer nur Tod“. ,Alles wird auf Null gefahren, ich }\end{array}$ & & 9 & $(26)$ \\
\hline
\end{tabular}




\begin{tabular}{|c|c|c|}
\hline wusste ja nicht, was das bedeutet". (4) & & \\
\hline $\begin{array}{l}\text { "Trotzdem hatte ich sehr viel Angst, wie ich das wohl } \\
\text { vertrage und ich war zwei Tage, bevor ich hier auf- } \\
\text { genommen wurde, fix und fertig, hatte Alpträume } \\
\text { und konnte nicht mehr schlafen. Angst habe ich } \\
\text { auch vor dem Gift hier". (19) }\end{array}$ & $\begin{array}{l}\text { Angst vor aktuellen Neben- } \\
\text { wirkungen }\end{array}$ & $6 \quad(17)$ \\
\hline $\begin{array}{l}\text { „Befürchtungen habe ich natürlich, Sekundärschä- } \\
\text { den zu erleiden. Soll ich Ihnen mal ein paar nennen } \\
\text {.... (16) }\end{array}$ & $\begin{array}{l}\text { Angst vor Spätnebenwirkun- } \\
\text { gen }\end{array}$ & $9 \quad(26)$ \\
\hline „Außerdem sagen viele, das hilft nicht. (1) & $\begin{array}{l}\text { Angst davor, dass die Thera- } \\
\text { pie nicht wirkt }\end{array}$ & (6) \\
\hline $\begin{array}{l}\text { "Über Befürchtungen habe ich mir nicht so viele } \\
\text { Gedanken gemacht, die habe ich verdrängt". (12) }\end{array}$ & $\begin{array}{l}\text { Angst, die aktiv „verdrängt“ } \\
\text { wird }\end{array}$ & $(17)$ \\
\hline $\begin{array}{l}\text { "Habe keine Angst oder Befürchtungen. Angst habe } \\
\text { ich nie gehabt, auch nicht zu Anfang“. (27) }\end{array}$ & $\begin{array}{l}\text { Fehlende Angst bzw. kein } \\
\text { Angsterleben }\end{array}$ & \\
\hline
\end{tabular}

(bei den Prozentangaben wurde gerundet. Die Anzahl von 35 Textzitaten ergibt sich dadurch, dass ein Patient mit zwei Äußerungen in unterschiedlichen Kategorien vertreten ist)

Da die Frage von den Patienten eindeutig beantwortet wird, bedarf es hier keiner zusätzlichen Abstraktionsebene. Klinisch ist die Unterscheidung sinnvoll, ob sich Patienten den Angstaffekten schutzlos ausgeliefert fühlen oder ob sie Angstaffekte nicht wahrnehmen (passiver Modus) oder ob innen die Kontrolle der Angstaffekte gelingt (aktiver Modus). Die Patienten, die den passiven Modus „wählen“ benötigen je nachdem ob und wie die Angstaffekte wahrgenommen werden sehr unterschiedliche Unterstützung. Nur einem kleineren Teil der Patienten gelingt die aktive Angstkontrolle so gut, dass sie sich nicht wesentlich beeinträchtigt fühlen.
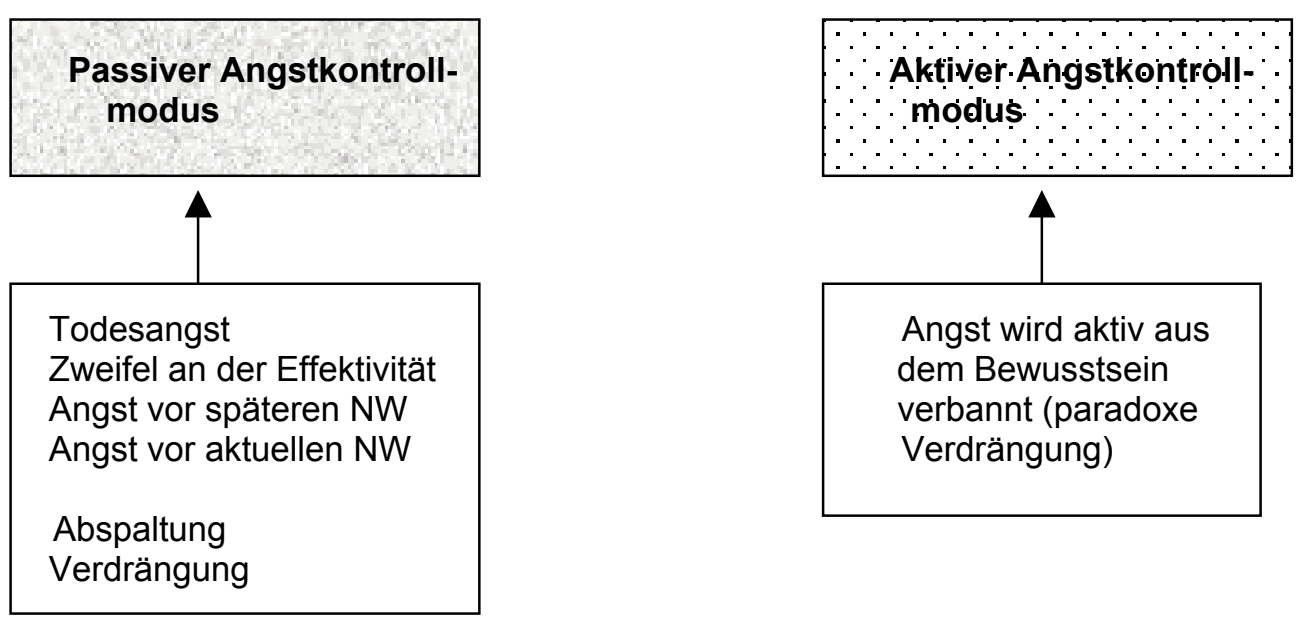

Der größere Teil der Patienten (ca. 75\%) leidet unter Angst und sieht sich Ängsten, zumindest zeitweilig, schutzlos ausgesetzt. Die Angst durch die Therapie sterben zu können, steht bei einigen Patienten im Vordergrund. Die meisten Patienten fürchten aktuelle oder spätere Nebenwirkungen. Übelkeit wird besonders dann gefürchtet, wenn frühere Therapien aus diesem Grunde als sehr belastend erinnert werden. Die Bezeichnung "Gift", die von mehreren Patienten verwendet wird, fasst die bedrohlichen Assoziationen zusammen. 
Eine Patientin berichtet von einem Traum, in dem die Todesangst sehr präsent war: „Die Befürchtung ist ganz klar, dass ich an der Behandlung sterben könnte. Ich trau mich nicht zu fragen, wie viele daran schon gestorben sind. Ich hatte einen Traum und da habe ich es Prof. X gefragt und er hat gesagt 23. Das hat mich ziemlich geschockt“. (Patient 9). Diese hohe Zahl ist nicht zutreffend und entbehrt auch jeder realen Grundlage. Die Schilderung verweist jedoch darauf, wie die Patientin in ihren Angstphantasien die Gefahr überhöht. Eine weitere Patientin, deren Freund vor einigen Monaten gestorben ist, steht der Therapie äußerst ambivalent gegenüber. Sie äußert Todeswünsche, die ihr aber gleichzeitig viel Sorgen machen und die nicht als Suizidalität im eigentlichen Sinne gewertet werden können: „Scheißspiel. Abends denke ich immer an J (den verstorbenen Freund), gebe inm einen Kuss und sage: hol mich doch zu dir“. (Patient 7). Die Patientin unterzieht sich der Therapie, weil sie gesund werden will, gleichzeitig sehnt sie sich in ihrer Trauer nach Ruhe und Vereinigung mit dem Freund. Ihre Schilderung dient auch dazu, sich durch die Reaktion des Gegenübers auf die sichere Seite zu stellen, zu hören, dass ihre Wünsche durchaus „einfühlbar“ sind, um sich dann von diesen „Todeswünschen“ distanzieren zu können.

Ein hoher Anteil von Patienten benötigt zur Angstkontrolle Hilfe von außen (holding function): Sichernde Andere, die eine beruhigende Wirkung haben und Regression gestatten, parallel dazu Informationen zur Stärkung von kognitiver Kontrolle, Konkretisierung der Angst, Verweis auf Möglichkeiten des medizinischen Personals und im Falle des Versagens dieser Hilfen oder wenn die Ängste ein „überflutendes“ Ausmaß erreichen, medikamentöse Hilfe (Anxiolytika, Sedativa) als kurzzeitige Schutz- und Hilfsmaßnahme.

Ebenfalls sehr große Schwierigkeiten haben die beiden Patientinnen, die an der Wirksamkeit der Therapie zweifeln. In beiden Fällen sind diese Zweifel von außen induziert worden: „Außerdem sagen viele, das hilft nicht. Schwarze Gedanken tauchen immer wieder auf". (Patient 1)

„Wichtig wäre, mir den Mut zu geben, dass ich mich ganz dahinter stellen kann, das ist schwer, das fehlt mir." (Patient 30)

Die Patientinnen beschreiben das, was, meist ohne Absicht, im klinischen Alltag passieren kann, wenn es um experimentelle Therapien geht und diese Therapien nicht vom gesamten Behandlungsteam gut geheißen werden. Unausgesprochen werden Zweifel transportiert und unterminieren die Erfolgshoffnungen der Patienten. Hoffnung kann dann kaum als Gegengewicht zu den Zweifeln aufrechterhalten werden und für die Patienten ist die durch Zweifel an der Wirksamkeit der Therapie gekennzeichnete Situation äußerst quälend. Hoffnung auf Wirksamkeit ist der wichtigste Faktor in Bezug auf die Kontrolle der durch Krankheit und Therapie bedingten Ängste. 
10.2.2.2 Seelische und körperliche Befindlichkeit während und nach der Therapie - Bewertung des eigenen Zustandes -

Die Äußerungen der Patienten auf die Frage nach ihrem Befinden werden für die jeweiligen Untersuchungszeitpunkte zusammengestellt. Die ursprüngliche Absicht, jeweils psychische und körperliche Befindlichkeit zu differenzieren, hat sich, mit Ausnahme von T3, als unnötig herausgestellt. Die Angaben für beide Dimensionen stimmen relativ gut überein. Da die Symptome im quantitativen Teil beschrieben wurden, wird hier auf eine detaillierte Auflistung verzichtet. Lediglich für T2 wird zur Veranschaulichung eine ausführliche Schilderung der negativen Bewertung gewählt. Bei den weiteren Beschreibungen soll der Erlebensaspekt der Beschwerden im Vordergrund stehen.

\begin{tabular}{|c|c|c|}
\hline \multicolumn{3}{|l|}{ Tabelle 36: Bewertung der Befindlichkeit T2 } \\
\hline Textzitate (Patientennummer) & Kategorie & $\mathrm{N}=34 \quad(\%)$ \\
\hline $\begin{array}{l}\text { „Es geht mir ganz gut, ich stehe auf, mache meine Spazier- } \\
\text { gänge draußen, kann unbeeinträchtigt essen. Es geht mir } \\
\text { psychisch und körperlich gut“. (15) }\end{array}$ & positiv & $(18)$ \\
\hline $\begin{array}{l}\text { „Nach der Stammzellrückgabe ging es sofort mit der Übelkeit } \\
\text { los... Schlappheit folgte sofort. Mit Magen und Darm ging es } \\
\text { auch sofort bergab und es musste sofort gespritzt werden... } \\
\text { Abneigung gegen Essen wurde immer stärker, ging immer } \\
\text { weniger. Ab dem } 2.7 \text {. musste ich künstlich ernährt werden. } \\
\text { Der Morphinperfusor wurde von } 1 \text { auf } 2 \text { auf } 2,5 \text { hochgestellt... } \\
\text { Manchmal hatte ich nachts alle } 10 \text { Minuten Durchfall...nachts } \\
\text { hatte ich enorme Schlafschwierigkeiten. Fieber habe ich über } \\
\text { die ganze Zeit, zwischen } 37.0 \text { und } 38.0 \text { Grad. Hatte große } \\
\text { Angst, konnte weder sitzen, stehen, liegen. War in großer } \\
\text { Unruhe....Bis dann diese große Ruhe kam mit den Tabletten, } \\
\text { das fand ich einfach nur gut... Ich war zu fertig, um Angst zu } \\
\text { empfinden, außer an diesem einen Vormittag, wo ich soviel } \\
\text { geweint habe. Sonst war ich einfach so fertig, dass ich nicht } \\
\text { mal über mich nachdenken konnte.“ (26) }\end{array}$ & negativ & $(41)$ \\
\hline $\begin{array}{l}\text { "Eigentlich geht es mir körperlich gut, bis auf die ständige } \\
\text { Übelkeit und den Durchfall. Seelisch habe ich zwischendurch } \\
\text { ziemliche Tiefs“. (11) }\end{array}$ & ambitendent & $(41)$ \\
\hline
\end{tabular}

Die Kategorisierung wurde dadurch erschwert, dass einzelne Patienten konstatierten, es ginge ihnen gut, dann aber eine ganze Reihe von schwerwiegenden Beschwerden (u.a. Angsträume, Durchfall, Erbrechen) schilderten. Diese Patienten wurden in die Kategorie „ambitendent“ eingeordnet. Viele Patienten berichten von Schlafproblemen. Die Zunahme von Schlafstörungen, die ja auch als Indikator für innere Unruhe, Anspannung und allgemein emotionale Belastung gewertet werden, können auch mit anderen Faktoren zusammenhängen: mehr nächtliche Störungen durch intensivere Überwachung, häufigere Infusionswechsel, Schmerzen und Verlust von Tages- und 
Nachtrhythmik, weil die meisten Patientinnen und Patienten ihre Tage überwiegend im Bett verbringen.

\begin{tabular}{|c|c|c|}
\hline \multicolumn{3}{|l|}{ Tabelle 37: Seelische Befindlichkeit T3 } \\
\hline Textzitate (Patientennummer) & Kategorie & $\mathrm{N}=34 \quad(\%)$ \\
\hline $\begin{array}{l}\text { "Mir geht es gut. Ich fühle mich zwar geschwächt und müde, } \\
\text { aber mir geht es gut. Nachts kann ich zwar nicht schlafen } \\
\text {...Fühle mich stabiler und ausgeglichener". (15) }\end{array}$ & positiv & $(47)$ \\
\hline $\begin{array}{l}\text { "Eine Nacht habe ich dann die Tablette verlangt, musste ein- } \\
\text { fach schlafen. Angst stand die ganze Zeit nicht zur Debatte. Ist } \\
\text { das eine Form der Abwehr? Typisch, die ganze Zeit durch- } \\
\text { gehalten und dann kam der Zusammenbruch. Während der } 4 \\
\text { Wochen hätte ich nicht sagen können es war die Hölle, dann } \\
\text { hätte ich das nicht durchgehalten. Also ich war letzten Freitag } \\
\text { und Samstag richtig depressiv, das war ganz schön haarig. } \\
\text { Habe dann erst angefangen Freunden zu sagen, es war die } \\
\text { Hölle..." (13) }\end{array}$ & negativ & $(11)$ \\
\hline $\begin{array}{l}\text { „Im Bett fühle ich mich am wohlsten. Der Appetitmangel be- } \\
\text { lastet mich... Seelisch geht es mir recht gut. Bei der schlechten } \\
\text { Prognose ist es ja auch schon viel, es soweit geschafft zu ha- } \\
\text { ben." (18) }\end{array}$ & ambitendent & $(42)$ \\
\hline
\end{tabular}

\section{Tabelle 38: Körperliche Befindlichkeit T3}

\begin{tabular}{|c|c|c|}
\hline Textzitate & Kategorie & $\mathrm{N}=34 \quad(\%)$ \\
\hline $\begin{array}{l}\text { "Es geht mir wieder gut. Jetzt kann ich wieder alles essen und } \\
\text { habe großen Appetit... Die Mundschleimhaut war total zerstört, } \\
\text { Probleme mit dem Gehör, das Denken war verlangsamt". (22) }\end{array}$ & positiv & (32) \\
\hline $\begin{array}{l}\text { "Die Schmerzen waren gestern grauenvoll. Hals und Po, bei- } \\
\text { des. Der Schüttelfrost, das war ein solches Elend...Rotz und } \\
\text { Wasser habe ich geheult". (16) }\end{array}$ & negativ & (23) \\
\hline $\begin{array}{l}\text { "Mir geht es wieder besser...Leukos steigen wieder an. Aber } \\
\text { ich sage mal, vom Rachenraum bis zum After war es ein } \\
\text { Trümmerfeld, da ging mit Essen und Schlucken nichts mehr...". } \\
\text { (20) }\end{array}$ & ambitendent & (45) \\
\hline
\end{tabular}

Seelisch geht es der Hälfte der Patienten gut. Ein Teil äußert Stolz darüber, die Therapie geschafft zu haben. Symptome werden in diesem Kontext nicht als so gravierend empfunden. Es gibt allerdings auch vereinzelt Äußerungen, dass die Belastungen jetzt erst richtig ins Bewusstsein rücken.

Zum vierten Zeitpunkt ändert sich der Trend. In den Interviews die meisten Patienten eine negative Befindlichkeit, nur wenige beurteilen ihre Befindlichkeit als gut. Die Befindlichkeit wird schlechter bewertet als unmittelbar vor der Entlassung aus dem Krankenhaus. Vermutlich spielen hier Prozesse von Enttäuschung und Ungeduld eine Rolle. Patienten äußern, dass sie mit einer so langen Zeit der Erholung nicht gerechnet haben. 


\begin{tabular}{|l|l|l|l|}
\hline \multicolumn{2}{|l|}{ Tabelle 39: Befindlichkeit T4 } & Kategorie & N=33 (\%) \\
\hline Textzitate (Patientennummer) & 3 & (9) \\
\hline $\begin{array}{l}\text { "Ich habe mich gut erholt, es sind keine Probleme aufgetaucht. } \\
\text { Sofort nach der Entlassung bin ich im Urlaub gewesen, bin } \\
\text { nach Mallorca geflogen. Es ist alles gut gegangen und den } \\
\text { Urlaub konnte ich genießen. Seit einem Monat gehe ich schon } \\
\text { wieder arbeiten, Vollzeit und mache auch so wie früher } \\
\text { Schichtdienst." (12) }\end{array}$ & & & \\
\hline $\begin{array}{l}\text { "Angst!! Psychisch im Moment ganz schlecht. Bin kurzatmig. } \\
\text { "Bin sehr viel müde, kaputt, fange an zu frieren, ich schlafe } \\
\text { ganz viel. Wenn ich ein bisschen was mache habe ich } \\
\text { Schmerzen, starke Hüftschmerzen. Geschmacksverlust.“ (4) }\end{array}$ & negativ & 19 & (57) \\
\hline $\begin{array}{l}\text { "Psychisch habe ich mich gut erholt, da bin ich eigentlich ganz } \\
\text { gut drauf. Aber körperlich kann ich nicht so, wie ich gerne wür- } \\
\text { de. Das macht mich dann wieder unzufrieden... Und dann habe } \\
\text { ich jetzt ziemlich Rückenschmerzen, wo keiner weiß, woher die } \\
\text { kommen. Na ja wenigstens ist jetzt der Port raus, der hatte ja } \\
\text { so gedrückt und war nie richtig benutzbar... Insgesamt will man } \\
\text { mehr als geht. Es geht schon aufwärts, aber man stößt perma- } \\
\text { nent an seine Grenzen. Wenn ich vormittags einen Termin } \\
\text { habe, dann brauche ich mir für den Nachmittag nichts mehr } \\
\text { vorzunehmen, weil ich es sowieso nicht mehr schaffen würde... } \\
\text { (19) }\end{array}$ & & & \\
\hline
\end{tabular}

Ein Jahr nach Behandlung ergibt sich wiederum ein neues Bild. Berücksichtigt werden nur die Äußerungen der Patienten ohne Rezidiv, da sich sonst Krankheitsauswirkungen und Therapieeffekte vermischen würden.

Nur 5 Patientinnen und Patienten (22\%) beantworten die Frage nach dem derzeitigem Befinden mit eindeutig gut. 6 (28\%) beklagen eine schlechte Befindlichkeit und 11 sprechen von einer differenzierten Befindlichkeit (50\%). Bei zwei Patientinnen war die Zuordnung zur letzten Kategorie außerordentlich schwierig. Zwar lautet die verbale Aussage „Sagen wir mal so: ich bin zufrieden“ oder im anderen Fall „Mir geht es ganz gut", was danach folgt, ist aber ein Text, der ausschließlich Beschwerden enthält. Beim Lesen bleibt der Eindruck zurück, dass esden Betreffenden nicht gut geht. 


\begin{tabular}{|c|c|c|}
\hline \multicolumn{3}{|l|}{ Tabelle 40: Befindlichkeit T5 } \\
\hline Textzitate (Patientennummer) & Kategorie & $\mathrm{N}=22 \quad(\%)$ \\
\hline $\begin{array}{l}\text { „Mir geht es jetzt wieder gut...Aber, es hat wirklich ein Jahr } \\
\text { gebraucht... Körperliche Beschwerden habe ich überhaupt } \\
\text { nicht mehr.“ (13) }\end{array}$ & positiv & (22) \\
\hline $\begin{array}{l}\text { „Na ja, also körperlich geht es mir schlecht. Nicht ganz } \\
\text { schlecht, das will ich nicht sagen, aber eben schlecht. Das ist } \\
\text { so alles, an vielen Stellen. Am schlimmsten sind im Moment } \\
\text { die Bronchien, die immer total zu sind. Die ganze Haut tut mir } \\
\text { oft weh, die ist so ledrig. Mit den Schleimhäuten hab ich auch } \\
\text { noch Schwierigkeiten; in den Händen hab ich oft so was wie } \\
\text { Krämpfe, die Augen tun weh und mit den Zähnen kamen auch } \\
\text { ab November noch Schmerzen dazu. Nur mit der Kraft, das ist } \\
\text { nicht mehr so schlimm wie vorher... Seelisch geht es auch } \\
\text { nicht so gut. Ich mache mir halt viel Gedanken, dass das alles } \\
\text { so lange dauert. Unzufrieden ist das richtige Wort, ja, dass bin } \\
\text { ich oft...aber ich werde dann nörgelig. Manchmal unduld- } \\
\text { sam,...Es war und ist ein langes Warten, dass es besser wird. } \\
\text { Und dann vor allem nervt mich, dass immer noch was dazu } \\
\text { kommt. Das Immunsystem ist kaputt.“ (17) }\end{array}$ & negativ & (28) \\
\hline $\begin{array}{l}\text { „Körperlich geht es mir ganz gut und psychisch auch. Natürlich } \\
\text { bin ich nicht mehr so belastbar wie früher. Gelenkprobleme } \\
\text { habe ich immer noch. Die Arbeit schaffe ich, aber es ist } \\
\text { schwer. Man reißt sich ja zusammen...Das Hauptproblem ist } \\
\text { die Mattigkeit.“ (12) }\end{array}$ & ambitendent & $(50)$ \\
\hline
\end{tabular}

Untersucht man die Äußerungen zur Befindlichkeit im Hinblick auf die genannten Gründe, dann werden, neben den eigentlichen körperlichen Beschwerden, immer wieder, mit ähnlichen Formulierungen, folgende Punkte genannt:

\begin{tabular}{|l|l|}
\hline \multicolumn{2}{|l|}{ Tabelle 41 : Einflussfaktoren Befindlichkeit } \\
\hline \multicolumn{1}{|c|}{ (Zitatausschnitte) } \\
\hline Schwarze Gedanken & \multicolumn{1}{|c|}{ T3 } \\
Wenig Hoffnung & Zweifel an der Wirksamkeit \\
Bedrohlicher Traum & Unendliche Schlappheit \\
Ärger über Beschwerden & Appetitmangel \\
Erschrocken über Schwäche & Ungeduld, Schlaflosigkeit \\
Dachte ich sterbe, Schlafschwierigkeiten, Unru- & Große Anspannung wegen der Untersuchung \\
he, zu fertig um Angst zu empfinden & Kaum Kraft, bis zu Toilette zu gehen, hätte nie \\
War ganz tief unten mit der Kraft und allem, & Ging mir beschissen schwer ist \\
schlimmste Nächte & Wie durch den Wolf gedreht \\
Angst es nicht zu schaffen & Permanente Übelkeit \\
Durchhänger & Total träge, kein Hungergefühl \\
Nicht fähig zu denken geschweige denn zu & Gedanke, was ist wenn in zwei Jahren Metas- \\
handeln. Ich bin wie im Trance & tasen kommen \\
Viel Angst & Dreht sich wie ein Karussell: ob ich gesund \\
Langeweile & bin...? \\
\hline
\end{tabular}




\begin{tabular}{|l|l|}
\hline T4 & T5 \\
\hline Gleiches Niveau wie 87jährige Mutter & Mit dem Schwächegefühl kann ich mich nicht \\
Ging schlechter als in der Klinik & abfinden, \\
Abgenommen & wieder massivste Ängste, immer müde - im- \\
Viel Angst vor Rezidiv, Schmerzen & mer kaputt \\
Mental runter, Atemnot, Leistungsfähigkeit ist & Bin überhaupt nicht belastbar \\
ziemlich am Ende & Innere Ruhe fehlt.. ziemliche Ängste wieder ins \\
Nicht so belastbar & Berufsleben rein zu gehen \\
Völlig fertig, weil Kontrolluntersuchung anstand, & ..Beine wollen einfach nicht mehr, wie bei einer \\
meist in Gedanken bei der Krankheit & alten Frau \\
Hitzewallungen wie in den Wechseljahren & abgenommen durch Schmerzen \\
Schlafstörungen & nicht in der Lage zu arbeiten \\
Angstgefühle & wenn was überstanden ist, kommt hinterher \\
Tiefes Loch, Schwächegefühl zu Hause beson- & der Zusammenbruch \\
ders schlimm & Depression gehabt \\
völlig teilnahmslos: ich habe kaum gesprochen & Ausgeprägte Polyneuropathie als Folge \\
..bin doch noch jung, ..damit werde ich nicht & \\
fertig (Leistungsschwäche) & \\
schwer depressiv - suizidal & \\
Entzugserscheinungen & \\
Enttäuscht, dass es nicht besser aufwärts ge- & \\
gangen ist & \\
\hline
\end{tabular}

Zu allen Zeitpunkten ist Angst das am häufigsten benannte Gefühl. Zu den späteren Zeitpunkten wird die Angst, es nicht zu schaffen, abgelöst von der Angst vor einem Rezidiv. Nach dem Klinikaufenthalt steht neben der Angst für die meisten Patienten die spürbare Leistungseinbuße im Vordergrund. Die Enttäuschung über den langsamen Genesungsverlauf wird ebenfalls thematisiert.

Neben den hier genannten allgemeinen Beeinträchtigungen werden die spezifischen Beeinträchtigungen Fatigue und Sexualität im nächsten Abschnitt gesondert betrachtet.

\subsubsection{Spezielle Beeinträchtigungen: Fatigue und Sexualität}

$\mathrm{Zu}$ den persistierenden Beeinträchtigungen, die immer wieder von Patienten genannt werden, gehört an erster Stelle Fatigue.

Beschwerden über eine veränderte Sexualität werden in der Regel nur auf direktes Befragen geäußert.

\section{Fatigue}

Fatigue ist das Symptom, das ein Jahr nach Abschluss der HDC noch erhebliche Probleme bereitet. Die Ergebnisse in dieser Stichprobe stimmen mit den Literaturangaben überein. 


\begin{tabular}{|l|ll|}
\hline \multicolumn{2}{|l|}{ Tabelle 42: Angaben über Fatigue } \\
\hline & $\mathrm{N}=22$ (gesamt) \\
\hline Fatigue vorhanden & $\mathrm{N}=15 \quad(66 \%)$ \\
\hline Nicht vorhanden & $\mathrm{N}=7 \quad(34 \%)$ \\
\hline
\end{tabular}

Die folgenden Zitate sollen den „Charakter“ von Fatigue verdeutlichen:

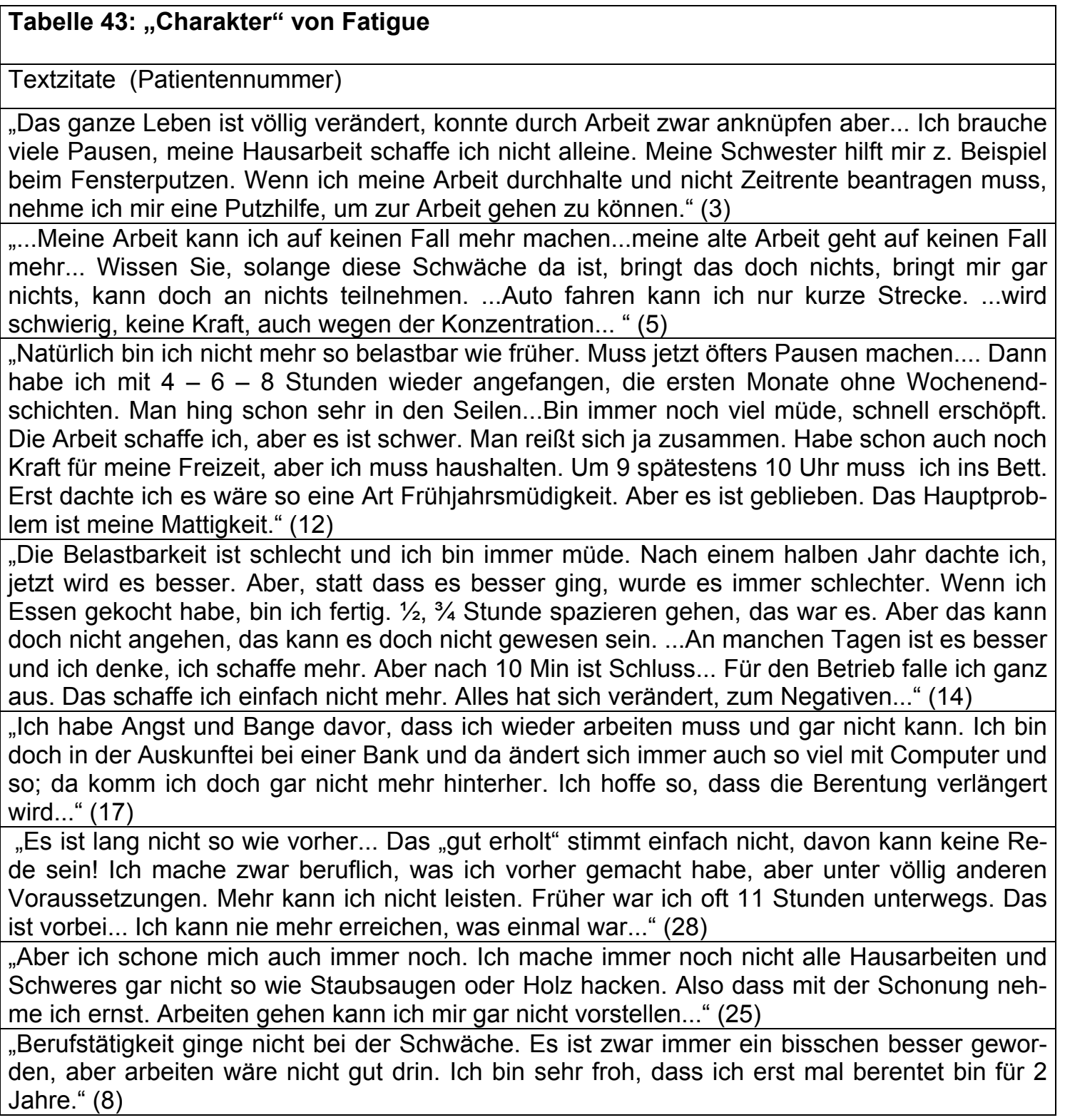

Alle Patienten vergleichen mit ihrem früheren Zustand. Das, was früher gut zu schaffen war, geht heute nur noch mit Anstrengung. Diejenigen, die noch zeitberentet sind, können sich nicht vorstellen, den Arbeitsanforderungen gewachsen zu sein. Einige haben deswegen Angst vor der erneuten Begutachtung. Diejenigen, die bereits wieder angefangen haben zu arbeiten, betonen ihre verringerte Belastbarkeit und ihre geringere Stresstoleranz im Vergleich zu früher. Alle mussten ihr Leben umstellen, umorganisie- 
ren und mehr Hilfe in Anspruch nehmen, um mit den veränderten Belastungen im Alltag zu Recht zu kommen. Den Beschreibungen ist gemeinsam, dass sie Charakterisierungen wie - nicht belastbar - schnell am Ende - keine Kraft mehr - müde - erschöpft - enorme Schwäche - verwenden, also Bezeichnungen, die den gleichen oder ähnlichen Zustand beschreiben. Diejenigen, die ein hohes Ausmaß von Fatigue beschreiben, schätzen auch ihre gesamte Befindlichkeit negativer ein. Mit einer Ausnahme, befinden sich in dieser Gruppe auch diejenigen Patientinnen, denen wegen Angst und Depressivität eine Psychotherapie angeraten wurde. Phänomenologisch stützen diese Ergebnisse die theoretische Annahme von Fatigue als einer Erscheinungsform von Depressivität (siehe S. 88).

\section{Beeinträchtigung von Sexualität durch die HDC}

Wie durch zahlreiche Studien ${ }^{72}$ bekannt, wird gerade auch die Sexualität erheblich beeinflusst durch Chemotherapien allgemein und HDC im Besonderen. Frauen kommen durch die Therapie abrupt in die Menopause (je nach Alter und Therapie vorübergehend oder dauerhaft) mit ihren begleitenden Symptomen, die sehr viel ausgeprägter sein können als bei einem längeren und milderen Übergang durch die Wechseljahre. Bei Brustkrebs ist dieser Effekt ausdrücklich indiziert und wird als therapeutisch wirksam angesehen. Auch die Ganzkörperbestrahlung wirkt sich negativ aus. In den Studien, die Sexualität nach HDC untersucht haben, finden sich hohe Inzidenzangaben bezüglich gestörter Sexualität. Dabei stehen Libidoverlust und Potenzminderung bei Frauen und Männern im Vordergrund. Bei den Frauen kann es zu Veränderungen im Bereich der Vagina kommen, die zu Schmerzen beim Geschlechtsverkehr führen können, bei den Männern kann es von leichteren Erektionsproblemen bis zur völligen Impotenz kommen.

Die vorliegende Studie weist ein methodisches Problem auf: obwohl ausdrücklich vorgesehen, wurde nur knapp die Hälfte der Patienten $(N=15)$ nach sexuellen Veränderungen gefragt. Von den Interviewern wurden dazu folgenden Begründungen angegeben:

Die Patienten waren insgesamt so wenig belastbar und eingeschränkt, dass es schien, als wäre die Frage nach Sexualität bereits eine Konfrontation mit einem Mangelzustand, die grenzüberschreitend wirken würde. Die Beziehung war zu distanziert, um eine so intime Frage zu gestatten. Aus den vorhergehenden Interviews war die Le-

${ }^{72}$ Siehe Zettl \& Hartlapp (1996); Curdt \& Kamm (1999). Zu sexuellen Störungen nach HDC siehe auch Andrykowski et al. 1995(b); McQuellon et al. (1995); Chao et al. (1992). 
benssituation bekannt: die Patienten lebten nicht in einer Beziehung, hatten auch vor der Therapie keine sexuellen Begegnungen.

Aus den Zitaten geht hervor, dass diejenigen, die befragt wurden, alle eine negative Veränderung zum Ausdruck bringen.

\begin{tabular}{|l|l|}
\hline \multicolumn{2}{|l|}{ Tabelle 44: Sexuelle Störungen } \\
\hline Textzitate (Patientennummer) & Kategorie \\
\hline $\begin{array}{l}\text { "Seit der HDC bin ich völlig impotent. Habe das auf die Strahlentherapie ge- } \\
\text { schoben..." (5) }\end{array}$ & Impotenz \\
\hline $\begin{array}{l}\text { "Sexualität hat sich sehr verändert. Fühle mich, als wäre ich keine richtige } \\
\text { Frau mehr. Mit ...Jahren schon in den Wechseljahren, ist doch auch nicht nor- } \\
\text { mal. Ich fühle mich wie eine alte Frau. Mein Mann hat Verständnis dafür, dass } \\
\text { wir nicht mehr miteinander schlafen." (24) }\end{array}$ & $\begin{array}{l}\text { weiblichen } \\
\text { Identität }\end{array}$ \\
\hline $\begin{array}{l}\text { "Das hat sich schon geändert... So wie früher ist es nicht mehr. Ich brauche } \\
\text { länger, das Vorspiel muss länger sein und manchmal geht es gar nicht. Es ist } \\
\text { nicht mehr so intensiv... aber impotent bin ich sicher nicht." (12) }\end{array}$ & Qualitätsverlust \\
\hline $\begin{array}{l}\text { "Das einzige ist, dass sich unsere sexuelle Beziehung verändert hat. Die hat } \\
\text { gelitten durch die Schleimhautschäden, die ich habe. Das tut einfach weh und } \\
\text { da kann ich nicht mit ihm schlafen. Sex ist schwieriger geworden. Am Anfang... } \\
\text { und jetzt ist es so, dass ich einfach keine Lust mehr dazu habe, kein Verlan- } \\
\text { gen." (17) }\end{array}$ & $\begin{array}{l}\text { Schmerzen } \\
\text { Minderung der }\end{array}$ \\
\hline
\end{tabular}

Bei den Männern stehen Einbußen an Potenz, bei den Frauen vor allem Minderung des sexuellen Verlangens sowie Schmerzen, im Vordergrund. Aus den Äußerungen zweier jüngerer Frauen lässt sich auch ein Verlust an Attraktivität und Störung weiblicher Identität heraushören, die auf die vorzeitigen Wechseljahre zurückgeführt werden (dicker geworden, Schweißausbrüche, keine Lust mehr). Zwei Frauen beklagen die Unmöglichkeit eines Gespräches mit dem Partner über die Problematik.

Bei drei Frauen war ein Mangel an sexueller Aktivität schon vor der HDC vorhanden und ist, wie zu erwarten, bestehen geblieben.

Legt man die Äußerungen der Patienten und die Beobachtungen der Interviewer zugrunde, dann zeigt sich deutlich, dass folgende Störungsebenen einen negativen Einfluss ausüben können:

- Die Ebene der existentiellen Bedrohung

- Die Ebene der Geschlechtsidentität

- Die Ebene der somatischen Veränderungen/Beeinträchtigungen

Es bedarf einer differenzierten (auch medizinischen) Diagnostik um diesen Patienten zu helfen. 


\subsubsection{Zusätzliche Belastungen}

Als zusätzliche, externe Belastungsfaktoren werden diejenigen bezeichnet, die sich nicht notwendigerweise durch die Therapie ergeben, sondern durch den Kontext in dem die Behandlung stattfindet.

\section{Tabelle 44: Externe Belastungsfaktoren}

\section{Textzitate (Patientennummer)}

„In drei Jahren sind Sie sowieso tot, da ist das mit den Nebenwirkungen und Spätfolgen eh kein Problem“. (Arztäußerung) „Na, das war schon hart" (2)

„Das Gravierendste ist, dass man die Visiten lockerer gestalten sollte. Hier hat doch keiner eine Jubeldiagnose. Da sollte man mehr mit als über den Patienten reden... habe ich auch ein ganz anderes Beispiel kennen gelernt. Dr. X ist ein Arzt, der diese Spielchen alle erkannt hat. Der ist ne echte Seele. Der schafft in Visiten von vorne herein eine andere Atmosphäre. Vielleicht weil der keine Angst hat vor mündigen Patienten." (22)

„Im rausgehen sagte sie (die Gastärztin) dann zu mir: sagen Sie mal, Sie haben doch bestimmt geraucht? ... Aber ich fand das so unverschämt, dass sie mir quasi im Vorübergehen die Schuld zuschiebt. Das hat mich lange geärgert." (22)

„Es kommt natürlich auch zu unterschiedlichen Lehrmeinungen...Gestern durfte der Arm nicht eingecremt werden, heute durfte er wieder, ... Das verunsichert mich generell." (26)

„... der ständige Wechsel der Ärzte, das hat mich genervt.“ (2)

„Natürlich wartet man auf sein Blutbild, kann aber doch nicht immer nachfragen." (32)

„Ich bin nie so richtig über die Verhaltensregeln in der Aplasie aufgeklärt worden..." (32)

„Die einzige Kritik, die ich habe ist der Umgang mit dem Mundschutz. Die Reinemachefrauen kommen ohne Mundschutz rein, das Pflegepersonal ebenfalls." (2)

„Ärzte, Schwestern und Pfleger vermitteln wenig Zuversicht“ (1)

„Mit Dr. X habe ich Probleme. Der will wohl Macht über die Patienten ausüben... behauptet jedes Mal wenn er mich sieht, dass diese Therapie nur ein Experiment wäre." (22)

„Dann als ich herkam mit Zahnschmerzen. Der Arzt in der Zahnklinik sagt, es ist nichts, obwohl eine deutliche Aphte zu sehen war... Spräche nicht gegen die Hochdosis. Dann sagt er mir, wenn die Schmerzen nicht aufhören, muss ich den Zahn eben in den nächsten Tagen ziehen... Das hat bei mir den Eindruck erweckt als wüsste er überhaupt nicht was Hochdosis ist." (3)

„...mit Ärzten habe ich wenig schlechte Erfahrungen... Aber mit der Organisation hier... Abläufe stimmen nicht,... umsonst hergefahren, oder habe stundenlang gewartet oder die Bilder oder Akten waren weg." (32)

„....war so frustrierend, ...Akte war weg. Und die Ärzte waren unvorbereitet." (17)

„Besser aufklären in Bezug auf das, was getan wird, nicht so in Bezug auf Nebenwirkungen." (5)

„Manchmal hat man das Gefühl, dass man sich hier zuviel selber kümmern muss, damit alles funktioniert." (8)

„....dass ich noch immer im Nest liegen würde... dann klingelt man gar nicht, ...weil man Angst hat gleich so doof behandelt zu werden." (6)

„Wissen Sie, es ist schon komisch, wenn man eine Perücke trägt und ein Arzt fragt nach Haarausfall." (3)

\begin{tabular}{|l|}
\hline Kategorie \\
\hline Angst wird geschürt \\
$\begin{array}{l}\text { Visiten sind nicht Patienten } \\
\text { zentriert } \\
\text { wangelnde Zu- } \\
\text { Bendung }\end{array}$ \\
\hline Schuldzuweisung \\
\hline $\begin{array}{l}\text { Meinungsdifferenzen im Be- } \\
\text { handlungsteam }\end{array}$ \\
\hline Arztwechsel \\
\hline $\begin{array}{l}\text { Information muss erfragt wer- } \\
\text { den }\end{array}$ \\
\hline $\begin{array}{l}\text { Mangelnde Aufklärung über } \\
\text { Schutzvorschriften }\end{array}$ \\
\hline $\begin{array}{l}\text { Umgang mit Schutzvor- } \\
\text { schriften }\end{array}$ \\
\hline $\begin{array}{l}\text { Zweifel am Erfolg der Behand- } \\
\text { lung }\end{array}$ \\
\hline Verunsicherung \\
\hline $\begin{array}{l}\text { Fachlich nicht ausreichend } \\
\text { informiert }\end{array}$ \\
\hline Ärzte unvorbereitet \\
\hline Art der Aufklärung ist nicht gut \\
\hline Selber kümmern \\
\hline Mangelnde Aundliches Verhalten \\
\hline
\end{tabular}


Die von den Patienten genannten Belastungsfaktoren lassen sich zweierlei Kategorien zuordnen:

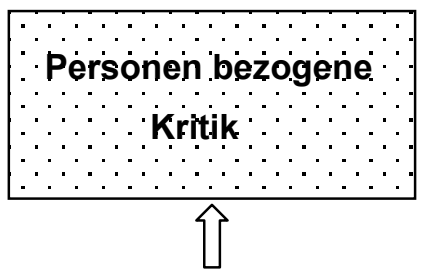

Unfreundliches Verhalten Unhöflichkeit

Mangelnde Aufmerksamkeit

Angst wird geschürt

Schuld wird zugewiesen

Meinungsdifferenzen werden deutlich

Verunsicherung durch uneindeutiges

Verhalten

Fachlich nicht ausreichend informiert

Machtausübung

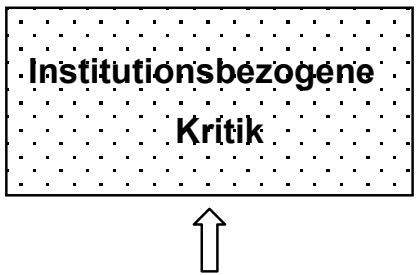

Sich selber kümmern müssen Visiten sind nicht Patienten zentriert Häufiger Arztwechsel Information muss erfragt werden Nicht nachvollziehbarer Umgang mit Schutzvorschriften Meinungsdifferenzen der Ärzte Schlechte Organisation Ärzte sind unvorbereitet Art der Aufklärung

Für die Patienten sind Äußerungen, die den Erfolg der Therapie in Frage stellen, entmutigen und Ängste schüren, am schwierigsten zu verkraften. Deutlich wird das daran, dass auch zu späteren Zeitpunkten solche Äußerungen in den Interviews wieder auftauchen. Äußerungen, die Patienten als mangelnde Achtung bzw. als kränkend erleben, haben auf Patienten ebenfalls eine sehr nachhaltige Wirkung. Derartige Bemerkungen „brennen“ sich in das Gedächtnis ein und es entsteht das Bedürfnis nach wiederholtem „darüber Sprechen“, um sich emotional zu entlasten. Aus den angeführten Beispielen wird auch deutlich, dass sachlich richtige Bemerkungen, wie der Hinweis darauf, dass es sich um eine experimentelle Therapie handelt, in diesem Kontext destruktive Effekte haben (können). Wenn der Behandlung zugestimmt wurde, der „Zug bereits fährt“, müssen Patienten zur Bewältigung der Belastungen auf Hoffnung setzen und dürfen nicht vom medizinischen Personal verunsichert werden.

Widersprüchliche oder auch unverständliche Aussagen führen auf dem Boden der bestehenden Verunsicherung durch die Erkrankung zur Steigerung von angstvollen Phantasien. Auch wenn es sich um medizinische „Kleinigkeiten“ handelt, sind sie für Patienten von großem Gewicht. Die Tendenz des Personals, sich in der Kommunikation an medizinischen Problemen zu orientieren, ist für Patienten oft eine zusätzliche Quelle von Unsicherheit. Patienten vermeiden in der Regel viele Fragen, wenn sie das Gefühl haben, das Personal ist sehr beschäftigt. Schweigen auf Seiten des Personals ist für Patienten unterschiedlich interpretierbar, lässt Spielraum für 
angstvolle Phantasien und wird selten in der gleichen Weise aufgefasst wie vom medizinischen Team, für das einfach nichts Auffälliges vorliegt. Positive, unspektakuläre Ergebnismitteilungen werden gewünscht, sie ermutigen und machen schwierige Behandlungssituationen erträglicher.

Problematisch für Patienten kann der Kontakt mit Konsiliarärzten sein, die nicht informiert sind. Das ist in großen Institutionen vermutlich nicht vermeidbar. Kein Arzt kann über die neuen Verfahren in den unterschiedlichsten Abteilungen auf dem aktuellen Stand sein, so lange es nicht üblich ist, vor der Durchführung des Konsils vom behandelnden Arzt informiert zu werden.

Einer der oben zitierten Patienten gibt wichtige Hinweise für die Gestaltung der Aufklärung/Information vor und im Behandlungsverlauf. Nicht das Aufzählen aller möglichen Komplikationen sollte im Vordergrund stehen, sondern eine Beschreibung, die Abläufe verständlich und durchschaubar macht. Nicht zu wissen was weiter passiert, schürt ebenfalls Ängste.

Die Patienten leiden häufig unter den Auswirkungen struktureller Probleme in der Klinikorganisation. Koordination und Kommunikation stehen dabei im Zentrum der Kritik. Eine Patientin formuliert: „Jeder für sich recht nett, alle zusammen aber furchtbar.“ (3) oder „Manchmal reicht der Informationsfluss innerhalb des Teams nicht, da weiß der eine nicht, was der andere tut." (32)

Die oben beschriebenen Erfahrungen sind kein Einzelfall. Besonders problematisch wird die Koordination zwischen den Abteilungen erlebt. Innerhalb der Station haben Patienten in der Regel jemanden den sie ansprechen können und der innen im Zweifelsfall weiterhilft. Außerhalb des geschützten stationären Bereiches fühlen sich Patienten hilfloser und ausgelieferter. Die Kritik an der Organisationsstruktur konzentriert sich vor allem auch auf die nachstationäre Zeit. Hier spielt der häufige Arztwechsel in der Nachsorge eine entscheidende Rolle. Arztwechsel stellt für Patienten eine hinlänglich bekannte zusätzliche Belastung dar. Für Patienten ist das der wichtigste Grund, warum sie die Nachsorge nicht mehr im Klinikum machen lassen.

Der Umgang mit Schutzvorschriften wurde ebenfalls von einigen Patienten erwähnt. Die Patienten schätzen ihre Gefährdung durch Infekte während der Aplasiephase als sehr hoch ein. Wenn Schutzvorschriften unterschiedlich praktiziert und begründet werden, kommt es zur Verunsicherung, im ungünstigsten Fall zu der Vermutung, mit der Sicherheit würde zu sorglos umgegangen. Patienten, in ihrer Angst und mit ihrer geschärften Wahrnehmung entdeckten sofort logisch inkonsistente Begründungszusammenhänge. Für Unruhe hat auch gesorgt, wenn Patienten sich mit einer Bettnachbarin/einem Bettnachbarn das Zimmer teilen mussten. Deren Besucher wurden aufmerksam beobachtet, um Anzeichen von Infekten zu entdecken. Die Ansteckungsgefahr 
wurde überschätzt. Zusätzliche Informationen und Diskussionen mit den betreffenden Patienten wurden notwendig.

Die Visitenproblematik ist aus unzähligen Darstellungen bekannt. Im zitierten Beispiel reflektierte ein Patient seine Erfahrungen. Andere äußern, dass sie ihre Fragen aufheben, bis sie Gelegenheit haben, mit dem Stationsarzt allein zu sprechen, dass sie lateinische Wörter nicht verstehen und sich nicht trauen nachzufragen. Beziehungsasymmetrie, Machtgefälle, mangelnde Kommunikation mit dem Patienten bestimmen nach wie vor den Charakter von Visiten.

Die Frage der Ernährung spielt für Patienten, die Chemotherapie bekommen in der Regel eine zentrale Rolle, da häufig Appetit und Nahrungsaufnahme gestört sind.

Von unserer Patientenstichprobe äußern sich ca. 50\% nicht spontan zum Essen. Explizit danach gefragt wurde nicht. Spontan kritisiert wurden folgende Punkte:

\section{Tabelle 45: Kritik an der Verpflegung in der Klinik}

\begin{tabular}{|c|c|c|}
\hline Textzitate (Patientennummer) & Kategorie & $\mathrm{N}=18$ \\
\hline $\begin{array}{l}\text { „Der erste Punkt ist das Essen. Da muss ich wirklich Kritik üben. } \\
\text { Die Teller waren immer so voll, das alleine hat schon Übelkeit aus- } \\
\text { gelöst. Und dann soviel Fleisch - das kann man ganz vergessen... } \\
\text { Sonst hat man immer ein schlechtes Erlebnis beim Essen und denkt } \\
\text { schon vorher: bald kommt das Essen wieder, das schaff ich wieder } \\
\text { nicht, oh je, wie krieg ich das bloß weg! Dann sagt man sich selber } \\
\text { und die anderen wieder, du musst aber essen und nicht so viel auf } \\
\text { dem Teller lassen. Der Misserfolg ist programmiert. Es wäre auch } \\
\text { schön, wenn man Wünsche äußern könnte, aber das ist wohl zuviel } \\
\text { verlangt." (6) }\end{array}$ & $\begin{array}{l}\text { Essen ist der } \\
\text { Situation nicht } \\
\text { angemessen }\end{array}$ & 16 \\
\hline „Das Essen ist von sehr guter Qualität.“ (22) & $\begin{array}{l}\text { Essen ist in } \\
\text { Ordnung }\end{array}$ & 2 \\
\hline
\end{tabular}

2 Patienten äußern sich positiv und 16 (45\%) kritisieren die Verpflegung.

Die negativen Kritiken lassen sich in folgender Hinsicht unterscheiden:

\begin{tabular}{|l|l|l|}
\hline \multicolumn{2}{|l|}{ Tabelle 46: Kritikpunkte - Ernährung } \\
\hline Textzitate (Patientennummer) & Kategorie & $\mathrm{N}=16$ \\
\hline $\begin{array}{l}\text { "Später, als der Mund kaputt war, war das Essen (Chemokost) völlig } \\
\text { ungeeignet: Volllkornbrot, hartes Gemüse, zähes Fleisch. Wenig auf } \\
\text { die Patientensituation zugeschnitten.“ (4) }\end{array}$ & $\begin{array}{l}\text { Nicht auf den } \\
\text { Zustand ab- } \\
\text { gestimmt }\end{array}$ & 4 \\
\hline "Das Essen ist aber eine Katastrophe...hat keinen Geschmack. Die & $\begin{array}{l}\text { Generell } \\
\text { schlecht }\end{array}$ & 5 \\
\hline $\begin{array}{l}\text { "Dasammenstellung ist unmöglich.“ (5) } \\
\text { nur den Teller sehe, Ich habe manchmal versucht, weniger zu } \\
\text { bestellen, kleine Portionen, aber sie sagen mir, dass das nicht geht } \\
\text { wegen dem Computer. Oben auf der Station ging es aber.“ (17) }\end{array}$ & $\begin{array}{l}\text { Nicht indivi- } \\
\text { duell }\end{array}$ & 4 \\
\hline „Das Essen war püriert. Das ging.“ (25) & Akzeptabel & 2 \\
\hline $\begin{array}{l}\text { „... Wenn ich länger hier wäre, wäre es mir nicht vitaminhaltig ge- } \\
\text { nug." (26) }\end{array}$ & Ungesund & 1 \\
\hline
\end{tabular}


Hauptkritikpunkt bleibt, dass die besondere Krankheitssituation keine Berücksichtigung findet. Die Frage der Ernährung spielt in den Interviews eine geringere Rolle als im normalen Klinikalltag. Das mag damit zusammenhängen, dass Patienten auf die Schwierigkeiten mit der Nahrungsaufnahme vorbereitet waren, zum Teil auch künstlich ernährt wurden und vor allem auch die Angehörigen einen Teil der Versorgung übernahmen und die gewünschten Nahrungsmittel mitbrachten.

Die modifizierte Isolation in der Aplasiephase wurde von keinem der Patienten als zusätzliche Belastung empfunden. Im Gegenteil, die meisten Patienten begrüßten die Möglichkeit, alleine liegen zu können (siehe oben) und waren froh, dass es gute Gründe, gab nicht so viele Besucher zu zulassen.

\section{Belastende Interaktionen}

Neben den Belastungen, die durch die medizinische Umwelt hervorgerufen werden, gibt es eine Reihe von negativen Erfahrungen, die sich auf die Interaktion mit Familienangehörigen, Bekannten und Freunden beziehen. Da sie „typisch“ sind, sollen sie hier ebenfalls Erwähnung finden:

\begin{tabular}{|c|c|}
\hline Textzitate (Patientennummer) & Kategorie \\
\hline $\begin{array}{l}\text { „Meine Mutter macht mir Kummer. Sie könnte eigentlich mal } \\
\text { kommen, tut es aber nicht.“ (1) }\end{array}$ & Kontakt wird vermieden \\
\hline „Und wenn sie mit mir spricht, redet sie über Banalitäten.“ (1) & $\begin{array}{l}\text { Berührung mit dem Thema } \\
\text { Krankheit wird vermieden }\end{array}$ \\
\hline $\begin{array}{l}\text { "Meine Mutter hat am Anfang immer geweint. Ich habe ge- } \\
\text { sagt, dass ich das nicht will. Es macht mir das Leben nur } \\
\text { schwerer." (4) }\end{array}$ & $\begin{array}{l}\text { Traurigkeit der Angehörigen } \\
\text { wird als Belastung empfunden }\end{array}$ \\
\hline $\begin{array}{l}\text { "Ganz schwierig war und ist es mit den Bekannten. In so einer } \\
\text { kleinen Stadt wird viel geredet, jeder weiß was und gibt } \\
\text { Ratschläge so auf die Art: „Ich an deiner Stelle würde..." Der } \\
\text { Pastor sagt mir: „So und nun versuchen Sie es mit Chemie“. } \\
\text { In so einem vorwurfsvollem Tone..." (1) }\end{array}$ & $\begin{array}{l}\text { Unerbetene Ratschläge werden } \\
\text { erteilt }\end{array}$ \\
\hline $\begin{array}{l}\text { "Ja, auch das habe ich erlebt, Rückzug von Leuten von denen } \\
\text { ich es nicht erwartet hätte. Ganz furchtbar war eine Karte von } \\
\text { zwei früheren Schulkameradinnen. Auf der Karte schrieben } \\
\text { sie mir von Aufrichtiger Anteilnahme. Es fehlte nur noch der } \\
\text { Trauerrand. Ich hatte das Gefühl, es ist eine Beerdigungskar- } \\
\text { te. War so schockiert und entsetzt.“ (26) }\end{array}$ & $\begin{array}{l}\text { Aufgezwungene Konfrontation } \\
\text { mit dem möglichen Tode } \\
\text { Mangelnde Empathie }\end{array}$ \\
\hline
\end{tabular}

Die Antizipation des möglichen eigenen Todes durch andere, wie im letzten Beispiel beschrieben, ist für alle Patienten, ohne Ausnahme, eine Qual und wird immer wieder als belastend genannt. Patienten wünschen kein Mitleid! Der Wunsch „normal“, als lebendige Person behandelt zu werden, ist ein zentrales Anliegen. Rückzug von Angehörigen und Freunden wird ebenfalls als große Belastung und Kränkung erlebt. Man- 
gelnde Empathie erschwert die Kommunikation. Der „soziale Tod“ wird gefürchtet. Unterschiedlich sind Wünsche an die Art der Kommunikation. Einzelne Patienten wollen nicht mit dem Kummer und den Sorgen der Angehörigen konfrontiert werden, andere dagegen stört, wenn nur oberflächlich kommuniziert wird. Wenige Patienten sind in der Lage zu artikulieren, wie sie sich die Kommunikation wünschen. ${ }^{73}$ „Gute Ratschläge“ in Bezug auf Therapien und Verhaltensmaßregeln von Angehörigen, Freunden und Bekannten sind generell unerwünscht.

Der nächste Abschnitt beschäftigt sich mit den Faktoren, die Belastung auszugleichen bzw. zu mindern vermögen.

\subsubsection{Belastungsausgleichende Faktoren}

Umgang mit den Krankheits- und Therapiebelastungen

- „Krankheitsbewältigung“ -

In diesem Abschnitt werden alle in den Interviews erfragten Themenbereiche behandelt, die sich im engeren und weiteren Sinne unter dem Stichwort der „Krankheitsbewältigung" einordnen lassen. Die Gliederung wird unter den folgenden Gesichtspunkten durchgeführt:

1. Entscheidungsfindung

2. Motivation zur Durchführung der Therapie - Hoffnung

3. Modus der Vorbereitung auf die HDC

4. „Anpassung“ („Abwehrmöglichkeiten“ - eigene Ressourcen - soziales Netz)

5. Rehabilitationsmaßnahmen

6. Unterstützung durch das medizinische Personal und die Institution Krankenhaus

\subsubsection{Entscheidungsfindung}

Die Frage, wie die Entscheidung zur HDC gefallen ist, wirft einen Blick darauf, welche Möglichkeiten der Entscheidungsfindung den Patienten in einer lebensbedrohlich erlebten Situation zur Verfügung stehen. Es wird davon ausgegangen, dass Patienten, die sich ja nicht mehr im „Diagnoseschock“ befinden und die sich schon längere Zeit (z. Teil schon sehr lange; siehe S. 133) mit der Krankheit auseinandersetzen, ihre Entscheidung sorgfältig abwägen, mit Angehörigen sprechen und dann eine Entscheidung treffen.

\footnotetext{
${ }^{73}$ Dieser Aspekt sollte im Rahmen von psychosozialer Unterstützung berücksichtigt werden.
} 
Die Antworten auf diese Frage lassen sich auf zwei Ebenen betrachten:

Wer entscheidet?

Welche Argumente fließen in die Entscheidung ein?

Die Frage, wer entscheidet lässt sich sehr eindeutig beantworten. Der überwiegende Teil der Patienten stimmt, ohne Diskussion, dem Vorschlag eines der behandelnden Ärzte zu.

\begin{tabular}{|l|l|ll|}
\hline \multicolumn{2}{|l|}{ Tabelle 48: Entscheidungsfindung } \\
\hline Textzitate (Patientennummer) & Kategorie & N & $(\%)$ \\
\hline „Der Arzt hat es vorgeschlagen."(10) & Ärztlicher Rat & 25 & $(74)$ \\
\hline "Ich habe das ganz allein entschieden.“ (34) & Eigene Entscheidung & 9 & $(26)$ \\
\hline
\end{tabular}

Die Betrachtung der Argumente zeigt tendenziell mehr Variationsbreite an, obwohl hier das Argument der fehlenden Alternativen vom größten Teil der Patienten genannt wird.

\begin{tabular}{|c|c|c|}
\hline \multicolumn{3}{|c|}{ Tabelle 49: Begründungen für die Entscheidung (Mehrfachnennungen möglich) } \\
\hline Textzitate (Patientennummer) & Kategorie & $\mathrm{N}$ \\
\hline „Es gibt keinen anderen Weg mehr.“ (Patient 2) & Letzte Möglichkeit & 21 \\
\hline $\begin{array}{l}\text { "Man hatte mir auch angeboten Interferon zu sprit- } \\
\text { zen. Aber, ich habe mir gedacht, lieber gehe ich } \\
\text { einmal durch die Hölle." (Patient } 32 \text { ) }\end{array}$ & Auswahl zwischen Alternativen & 6 \\
\hline $\begin{array}{l}\text { "Wenn ich nicht alles medizinisch Mögliche mache, } \\
\text { komme ich anschließend (wenn die Krankheit wieder } \\
\text { kommt) nicht zurecht." (Patient 13) }\end{array}$ & Alles medizinisch Mögliche tun & 1 \\
\hline $\begin{array}{l}\text { "Mein Arzt war von dieser Idee erst nicht begeistert, } \\
\text { hat aber dann doch mit Prof. X gesprochen." (20) }\end{array}$ & Durchgesetzt gegen den Arzt & 1 \\
\hline $\begin{array}{l}\text { "Als ich nach der ersten Chemotherapie gegangen } \\
\text { bin, wollte ich die HD nicht machen." (4) }\end{array}$ & $\begin{array}{l}\text { Nach anfänglichen Zweifeln } \\
\text { zugestimmt }\end{array}$ & 4 \\
\hline $\begin{array}{l}\text { "Letztlich geht die Entscheidung doch nur über das } \\
\text { Vertrauen zum Arzt." (Patient 15) }\end{array}$ & Arztvertrauen & 4 \\
\hline „,...das ging eben seinen Gang.“ (5) & Passierte eben & 4 \\
\hline
\end{tabular}

Die Antworten auf diese Frage lassen sich bei der Betrachtung beider Ebenen im Hinblick auf die Beziehungsgestaltung zum Arzt kategorisieren bzw. im Hinblick auf die Wahrung der eigenen Autonomie interpretieren und gestatten einen Blick auf die von den Patienten in der Vorphase der Therapie gewählten Bewältigungsstrategien: 


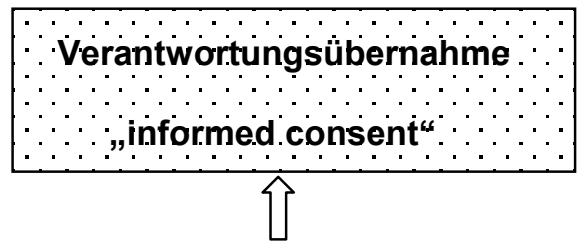

Rationale Begründung

Emotionale Begründung

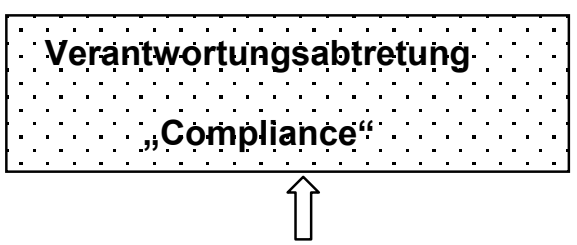

Kein eigener Einfluss

Auf der ersten Analyseebene wirkt die Entscheidung mehr von außen gesteuert, durch den Rat der Ärzte determiniert. Die Mehrzahl der Patienten begründet ihre Zustimmung zum ärztlichen Rat mit den oben angeführten Argumenten.

Eine Minderheit führt das Vertrauen zum Arzt, also eine emotionale Begründung für die Zustimmung zum Behandlungskonzept an. Beide Begründungen bedingen eine Zustimmung und ein sich einverstanden erklären und damit auch eine Teilhabe an der Verantwortung. Nur eine Minderheit verzichtet auf eigene Teilhabe an der Entscheidung: „Passierte eben“ und betrachtet die Entscheidungsfindung als zwangsläufig, schicksalhaft, ohne eigenes Zutun. Diese Minderheit überträgt ausschließlich dem Arzt die Verantwortung für die Entscheidung. Erstaunlich wenig Patienten, genau genommen nur zwei Patientinnen, haben zusätzliche Auskünfte eingeholt. Eine Patientin hat über Selbsthilfegruppen und Internet Informationen eingeholt, die andere hat sich von Patientinnen beraten lassen, die bereits eine HDC durchgemacht hatten. Die Begründung einer Patientin nimmt insofern eine Sonderstellung ein, als sie die Therapieeffektivität nicht überschätzt und von einer Position aus argumentiert, die perspektivisch eine Progredienz der Erkrankung einbezieht:

„Wenn ich nicht alles medizinisch Mögliche mache, komme ich anschließend (wenn die Krankheit wieder kommt, Anmerk. d. Verf.) nicht zu Recht.“ (Patient 13)

Die Beantwortung der Frage ist nicht nur im Hinblick auf die Entscheidungsfindung interessant, sondern sie wirft auch gleichzeitig einen Blick auf die von $74 \%$ der Patienten gewählte Bewältigungsstrategie: Sich auf den Arzt verlassen, nichts (wenig) hinterfragen, wird in dieser Situation von Bedrohung eindeutig präferiert. Kein einziger Patient problematisiert den - bekannten - experimentellen Charakter der Therapie.

Diese Strategie findet sich auch wieder bei der Frage nach den Motiven/Hoffnungen, die mit der HDC verbunden werden. 


\subsubsection{Therapiemotivation}

Hoffnung auf Heilung, auf Lebensverlängerung wird als der zentrale Faktor angesehen, der Patienten in die Lage versetzt, auch schwerste Belastungssituationen durchzustehen. In den Äußerungen, mit denen die Therapieentscheidung begründet wurde, fanden sich zahlreiche, die darauf hindeuten, dass die HDC als die wirksamere Therapiealternative aufgefasst wird. Bei der expliziten Nachfrage findet sich das gleiche Argumentationsmuster wieder:

\begin{tabular}{|c|c|c|}
\hline \multicolumn{3}{|l|}{ Tabelle 45: Hoffnungen } \\
\hline Textzitate (Patientennummer) & Kategorie & $\mathrm{N} \quad(\%)$ \\
\hline $\begin{array}{l}\text { "Ich erhoffe mir von der HDC dauerhafte Heilung. } \\
\text { Ich will die Heilung." (9) }\end{array}$ & Heilung & $19 \quad(56)$ \\
\hline $\begin{array}{l}\text { "Ich gewinne bis zu } 10 \text { Jahre, kann auch länger } \\
\text { sein. Eine Heilung gibt es aber nicht. Aber ich könn- } \\
\text { te halt länger leben." (17) }\end{array}$ & Lebensverlängerung & $10 \quad(29)$ \\
\hline „Lasse es auf mich zu kommen.“ (29) & Keine explizite Hoffnung & (15) \\
\hline
\end{tabular}

Mehr als die Hälfte der Patienten setzt auf eine Heilung, ein weiterer Teil zumindest auf eine Lebensverlängerung und nur eine kleine Minderheit vermeidet die Formulierung jeglicher Hoffnung. Ob sich die Patienten nicht trauen, Hoffnungen zu formulieren oder ob sie keine haben, lässt sich nicht mit Sicherheit bestimmen. Ein Teil dieser Gruppe gehört zu denjenigen, die für die Entscheidung keine Verantwortung übernehmen. Es liegt also durchaus nahe, bei diesen Patienten davon auszugehen, dass sie über geringe internale Kontrollmöglichkeiten verfügen. Möglicherweise deutet sich hier ein kausaler Zusammenhang an: Wenn keine Hoffnung formuliert wird, gibt es auch keinen Grund, der Therapie aktiv zuzustimmen. Hoffnung und Wirksamkeitserwartung in Bezug auf die Therapie stehen in enger Wechselbeziehung bzw. können als zwei Seiten derselben Medaille aufgefasst werden.
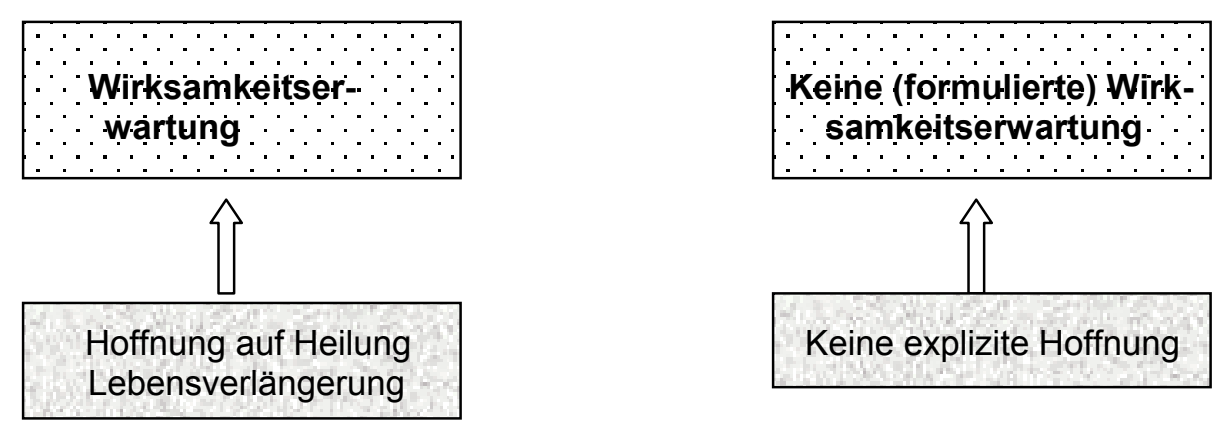

Durch die Begründungen für die HDC wird deutlich, wie leicht Nuancen in der Bewertung von Therapien „als besser“ die Entscheidung bestimmen. Wird die Prämisse der 
höheren Effektivität vom Patienten erst einmal akzeptiert, gibt es offensichtlich keine Entscheidungsspielräume mehr. Auch bei dieser Stichprobe zeigt sich das aus der Literatur bekannte Faktum der Wirksamkeitsüberschätzung von Chemotherapien (siehe S. 92) durch die Patienten.

\subsubsection{Vorbereitung auf die Behandlung}

Die Frage, wie sich Patientinnen und Patienten auf die HDC vorbereiten, wirft einen Blick darauf, ob Patienten auch in dieser Situation Handlungskompetenz aufrechterhalten können. Im Hinblick auf die Zielsetzung der Studie, Anhaltspunkte für die Unterstützung der Patienten zu gewinnen, sind v.a. diejenigen Aspekte von besonderer Bedeutung, die von den Patienten selber als hilfreich beschrieben werden.

Abgesehen davon, dass es von Interesse ist, zu erfahren, ob und welche Möglichkeiten der Vorbereitungen Patienten für sich gesehen haben, ermöglicht die Antwort auf diese Frage auch einen Blick auf die subjektiven Krankheitstheorien dieser Patientengruppe und darauf, wie sie ihre eigenen Kontrollmöglichkeiten einschätzen. Die Behandlungssituation unterscheidet sich deutlich von denen, die den Patienten bereits vertraut ist.

\begin{tabular}{|c|c|c|}
\hline \multicolumn{3}{|l|}{ Tabelle 46: Vorbereitung auf HDC } \\
\hline Textzitate (Patientennummer) & Kategorie & $\begin{array}{l}\text { Mehrfach- } \\
\text { nennungen }\end{array}$ \\
\hline $\begin{array}{l}\text { „Für die Zeit hier habe ich mich eigentlich nicht vorbe- } \\
\text { reitet." (2) }\end{array}$ & Gar nicht & 16 \\
\hline $\begin{array}{l}\text { Ich habe angefangen meine Geschichte in ein Buch zu } \\
\text { schreiben." (21) }\end{array}$ & $\begin{array}{l}\text { Ablenkung/Aktivität } \\
\text { Aufarbeitung }\end{array}$ & 20 \\
\hline $\begin{array}{l}\text { „.... und jetzt habe ich sehr viel Sojamilch getrunken } \\
\text { und Miso-Suppe gegessen. Man sagt, das bindet die } \\
\text { Strahlung im Körper und ich glaube daran...täglich Chi } \\
\text { Gong.. Außerdem habe ich eine Hypnotherapeutin } \\
\text { besucht, die sich auf Krebs spezialisiert hat. Die hat } \\
\text { mir viele Suggestionen auf Band gesprochen." (9) }\end{array}$ & $\begin{array}{l}\text { Gesundheitsförderung } \\
\text { durch Maßnahmen, } \\
\text { die dem „alternativen“ } \\
\text { Spektrum ent- } \\
\text { stammen }\end{array}$ & 6 \\
\hline „Ich habe mein Maskottchen dabei.“ (23) & Glücksbringer & 3 \\
\hline $\begin{array}{l}\text { "Meinem Sohn habe ich heimlich gesagt, wo er was } \\
\text { findet, wenn mir was passieren sollte. Mein Mann hat } \\
\text { genug Geld und das Testament ist gemacht..." (17) }\end{array}$ & $\begin{array}{l}\text { Angelegenheiten ge- } \\
\text { regelt }\end{array}$ & 2 \\
\hline
\end{tabular}

Die Frage nach der Vorbereitung auf die Behandlung war für einen Teil der Patienten offensichtlich unklar formuliert und musste durch Nachfragen präzisiert werden. Am häufigsten wurde von den Patienten genannt „ich mache nichts“ um dann im Nachdenken auf Dinge hinzuweisen, die meistens mit „sich beschäftigen in der Klinik“ zu tun haben. Trotz der erlebten Todesbedrohung, die von einigen Patienten direkt formuliert wurde, haben nur zwei Patientinnen konkrete Vorbereitungen für den Fall ihres Todes getroffen. Zwei Mal werden die Söhne eingeweiht und nicht die Ehemänner. Die Söhne 
sind diejenigen, die „Bescheid wissen“ sollen. Die Ehemänner werden geschützt, ihnen wird die Konfrontation mit einem möglichen Tod nicht zugetraut. Das Verhalten dieser beiden Patientinnen lässt sich ganz sicher nicht verallgemeinern! Hier waren sehr spezifische Familienkonstellationen ausschlaggebend. Eine dritte Patientin berichtet, dass sie unbedingt vor der Klinikaufnahme alles „aufgeräumt“ zurück lassen wollte: „Das war so ein richtiger Putzwahn, der mich da überkommen hat" (24). So, wie die Patientin es beschreibt, scheint es eher eine Form von Ablenkung oder Abwehr gewesen zu sein und keine bewusste Vorbereitung. Die Textzitate lassen sich auf drei Kategorisierungen verdichten, die wiederum auf völlig unterschiedliche Konfrontationen mit der Therapiebedrohung verweisen und Hinweise auf Bedrohungsabwehr bzw. auf den Grad an Reflexion über mögliche Zukunftsperspektiven liefern.



Testament Sohn über finanz. Angelegenheiten informiert
Vorberẹitụng dựch Überiẹgụngẹn wie die Klinîsżeit am besten praktisch bewăltiggt werden kannin

Eine Gruppe der Patienten bereitet sich vor im Sinne von „die Therapie unterstützen“. Hier wird der Einfluss von salutogenetischen Vorstellungen besonders deutlich. Das Spektrum der Komplementärtherapien ist hier genauso vertreten wie Vorstellungen aus dem engeren psychotherapeutischen Bereich. Eine Patientin und ein Patient führen vor der Behandlung ein „richtiges“ Fitnesstraining durch. Die große Gruppe, die sich ablenken und beschäftigen will, tut dies sehr bewusst und trifft entsprechende Vorkehrungen. Nicht hier einzuordnen sind die Maskottchen und Glücksbringer, die von Patientinnen und einem Patienten erwähnt werden. Vermutlich spielen magische Denkvorstellungen auch bei anderen Patienten eine Rolle, sie werden aber hier nur von drei 
Patienten direkt geäußert. ${ }^{74}$ Ein Patient kann mit der Frage nichts anfangen und bemerkt auf Nachfrage, er habe sich auf vier Wochen vorbereitet.

Wie aus den Nennungen hervorgeht, sind Aktivität und Ablenkung am wichtigsten. Weit mehr als die Hälfte der Patienten nimmt sich Materialien zur Beschäftigung mit. Einige bezeichnen das als "So weiter machen wie bisher", eine Strategie, durch die der veränderten Lebenssituation ein „Stück Normalität“ gegenübergestellt wird.

\subsubsection{9 „Anpassung“}

In diesem Abschnitt werden die Antworten zusammengestellt, die von den Patienten explizit angeführt werden als Beschreibung ihrer Möglichkeiten, mit den belastenden Krankheits- und Therapiebedingungen umzugehen. Hier wird der Begriff der Anpassung verwendet weil er mehr umfasst als Krankheitsbewältigung/verarbeitungsstrategien. ${ }^{75}$ Neben den selbstbeschreibenden, bewusstseinsnahen Strategien (siehe auch Exkurs Krankheitsverarbeitung/Coping S. 24f), auf denen in dieser Arbeit der Fokus liegt, werden ergänzend Fremdbeschreibungen herangezogen. Diese Fremdbeschreibungen erfolgen hier in der Terminologie des psychoanalytischen Abwehrkonzeptes und der klinischen Charakterisierung der „depressiven Verarbeitung“.

Ein früher Versuch, die Konzepte der tiefenpsychologischen Theorietradition mit nichttiefenpsychologischen Konzepten von Krankheitsverarbeitung zusammenzuführen, stammt von Kächele \& Steffens (1988). In „Bewältigung und Abwehr" werden die unterschiedlichen Konzepte von Copingforschung und psychoanalytischer Abwehrlehre integriert. Die Autoren betrachten Anpassung als den übergeordneten Beschreibungsbegriff, der ein sinnvolles Aufeinanderbeziehen beider Konzepte möglich macht. Die Abwehr von Verlust-, Bedrohtseins- und Todesängsten wird in diesem Konzept, in

\footnotetext{
${ }^{74}$ So „nebenbei“ werden diese Vorstellungen immer wieder deutlich, wenn Patientinnen (nach den eigenen Erfahrungen sind es mehr die Patientinnen) auf bestimmte Symbole verweisen die sie selber ausgewählt haben oder die innen für die Krankenhauszeit von Familie und Freunden geschenkt wurden. Ein sehr eindrückliches Beispiel, allerdings im negativen Sinne, als schlechtes Omen, beschreibt Patientin (3): Eine Klassenkameradin ihres Sohnes hatte kurz vor ihrem Tode dem Sohn einen Brief geschrieben, der über und über mit Engelsstickern beklebt war. Bei ihrer ersten Aufnahme in der Klinik klebte auf der Zimmertür des ihr zugewiesenen Zimmers ein Engelssticker. Diese Tatsache hat sie derartig beunruhigt, dass sie in ein anderes Zimmer verlegt werden musste. Der Hinweis der Mitpatientin, es handele sich um einen Schutzengel, konnte sie nicht aufnehmen.

${ }^{75}$ In der Arbeit von Hessel et al. 2000 werden fünf übergeordnete Komplexe, als Ergebnis der unterschiedlichsten Forschungsbemühungen zur Krankheitsbewältigung genannt:

- $\quad$ spezifische objektive/subjektive Belastungssituationen

- soziodemographische Parameter

- subjektive Krankheitstheorie

- persönlichkeitsstrukturell bedingte Fähigkeiten

- sozialer Rahmen
} 
Abhängigkeit vom erfahrenen Bedrohungsausmaß, als sinnvoll und überlebensnotwendig angesehen. Abwehr und Bewältigung sind keine sich ausschließenden Konzepte sondern bestehen nebeneinander. Abwehr wird in diesem Zusammenhang auch als eine Ich-Funktion verstanden, sie „unterliegt gewissermaßen einem Funktionswandel: Sie dient wie in der Kindheit dazu, die erreichte Differenzierung und Struktur vor einer Zerstörung zu schützen. Es leuchtet unmittelbar ein, dass in der Situation, in der konkrete Schritte zur vitalen Selbsterhaltung erforderlich sind, die Abwehr in erster Linie gegen eine Überflutung durch Affekte gerichtet ist [...] Die somato-psychische Wechselwirkung im Verlauf körperlicher Erkrankung erfordert geradezu eine Umkehr des geläufigen ätiopathogenetischen Denkens: nicht die seelischen Bedingungen sind es, die zu einer Symptombildung führen, sondern die eigenständigen somatischen Vorgänge erfordern eine psychologische Verarbeitung..." (ebenda S. 39/40). In diesem Modell sind vergangene traumatische Situationen in die aktuelle Situation eingebettet. Vom Ausmaß vergangener Traumatisierung und der aktuellen Situation, die je nach Lebensgeschichte und Bedrohungsausmaß jetzt auch als Retraumatisierung wirken können, wird die aktuelle Anpassungsmöglichkeit bestimmt. Die Autoren symbolisieren mit dem Bild des Januskopfes den zweizeitigen Bezug von Anpassung.

Die folgende Abbildung wurde von den o. g. Autoren adaptiert:

Abb. 35: Ineinandergreifen von Abwehr und Bewältigung (ebenda S.42)

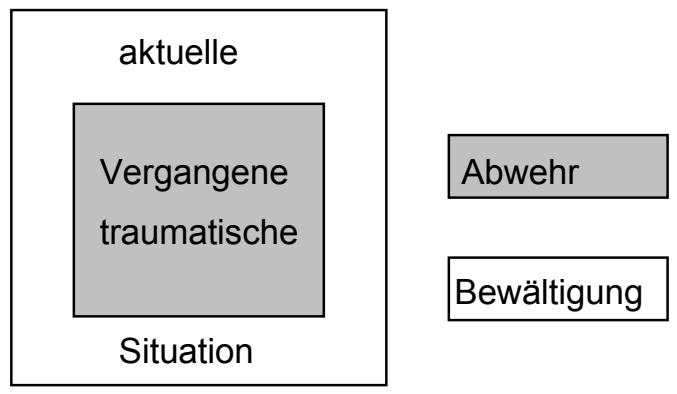

Dieses einfache Modell ermöglicht sowohl die Einordnung von Patientenäußerungen als auch die Einordnung von klinischen Beobachtungen.

In der folgenden Tabelle werden Textbeispiele aufgelistet, die sich auf die Frage beziehen, wie die Patienten es schaffen, die Krankheits- und Therapiebelastungen zu bewältigen. Die Beispiele und die daraus abgeleitete Kategorisierung beziehen sich auf die bewussten Bewältigungsanstrengungen. Die unbewussten Anpassungsvorgänge (Abwehr) werden im Anschluss beschrieben. 


\begin{tabular}{|c|c|}
\hline \multicolumn{2}{|l|}{ Tabelle 47: „Was hilft“ } \\
\hline Textzitate (Patientennummer) & Kategorie \\
\hline $\begin{array}{l}\text { „Ich rede mir immer gut zu: „du willst ja gesund werden“...lch } \\
\text { gebe mir immer selbst so einen Halt...Ich unterteile die ganze } \\
\text { Behandlung in einzelne Schritte und die hake ich dann innerlich } \\
\text { ab. Ich mache mir gute Gedanken. Ich stelle mir oft vor, wie ich } \\
\text { nach Hause komme...und habe meine Freude dran“ (6) }\end{array}$ & Optimismus \\
\hline $\begin{array}{l}\text { "Bei mir ist das ja wohl eine paradoxe Frage. Es ging mir ja die } \\
\text { ganze Zeit gut.“ (2) }\end{array}$ & Keine Belastung \\
\hline "Lesen, Kreuzworträtsel, auf dem Flur aufhalten.“ (12) & Beschäftigung/Ablenkung \\
\hline $\begin{array}{l}\text { "Meine Kraft...ich will unbedingt leben! Ich glaube auch an } \\
\text { Gott...liegt nicht in meiner Hand." (25) }\end{array}$ & $\begin{array}{l}\text { Religiöse Bindung } \\
\text { leben wollen }\end{array}$ \\
\hline „Ich nehme es wie es kommt. Mit Ruhe und Gelassenheit.“ (23) & Hinnehmen \\
\hline $\begin{array}{l}\text { "Chi Gong... Spülungen Ich passe schon auf - also behalte } \\
\text { die Kontrolle..." (9) }\end{array}$ & Kontrolle \\
\hline $\begin{array}{l}\text { "Das Wichtigste ist der Zuspruch von Schwestern und Angehö- } \\
\text { rigen. Anrufe helfen auch. Selber kann ich mir zurzeit schlecht } \\
\text { Mut zu sprechen..." (11) }\end{array}$ & Zuspruch \\
\hline $\begin{array}{l}\text { "Die Gespräche mit den Mitpatienten haben geholfen auch die } \\
\text { mit dem Pflegepersonal... (18) }\end{array}$ & Reden \\
\hline „Morphin hat mir die Schmerzen genommen.“ (14) & Medikamente \\
\hline $\begin{array}{l}\text { „Ich glaube, die Selbstdisziplin... Wenn Probleme auftreten, bin } \\
\text { ich ein ziemlich zupackender Typ... Das ist wie ein Krieg. Ich } \\
\text { lass mich von dem Krebs nicht in die Enge treiben." (22) }\end{array}$ & Eigene Stärke \\
\hline $\begin{array}{l}\text { "Ich behandele das gar nicht so, als wenn ich Krebs hätte, son- } \\
\text { dern mehr wie eine Mandelentzündung oder so etwas. Ich sehe } \\
\text { das nicht so negativ und manchmal ist es ganz weit weg. Ich } \\
\text { will auch keine Rücksichtnahme. Ich finde, man sollte ganz } \\
\text { normal mit der Krankheit umgehen." (6) }\end{array}$ & „Normalität" herstellen \\
\hline $\begin{array}{l}\text { "Der Stress ist also weg: ich kann Dinge tun, die mir Spaß ma- } \\
\text { chen. Ich will auch wieder malen und in Stein arbeiten." (3) }\end{array}$ & Veränderte Werte \\
\hline $\begin{array}{l}\text { "Habe den Kontakt nach draußen immer gehalten. ....dass mein } \\
\text { Vater immer am Bett saß, war existentiell notwendig.“ (13) }\end{array}$ & Nicht allein sein \\
\hline
\end{tabular}

Diese, den Betroffenen kognitiv zugänglichen Strategien, lassen sich in einem weiteren Abstraktionsschritt zusammenfassen zu einem aktiven und einem passiven Anpassungsmodus: ${ }^{76}$

\footnotetext{
${ }^{76}$ Heim et al. 1988 bevorzugt die Einteilung von Krankheitsbewältigung in kognitions-, emotions- und handlungsbezogene Form, denen die 28 Items der BEFO (Berner Bewältigungsformen) zugeordnet werden.

In der Arbeit von Hessel et al 2000 werden die mithilfe der BEFO an einer repräsentativen Bevölkerungsstichprobe gewonnen Formen faktorenanalytisch zusammengefasst zu: Bedrohungsminderndem Coping, Zuwendungsorientiertem Coping und Bedrohungsfokussierendem Coping.

Filipp \& Ferring (2002) diskutieren unter den Stichworten "changing the world“ und „changing the self" die unterschiedlichen Differenzierungen von Bewältigungsverhalten und stehen damit in der Tradition der Autoren, die von allo- und autoplastischer Bewältigung sprechen.
} 

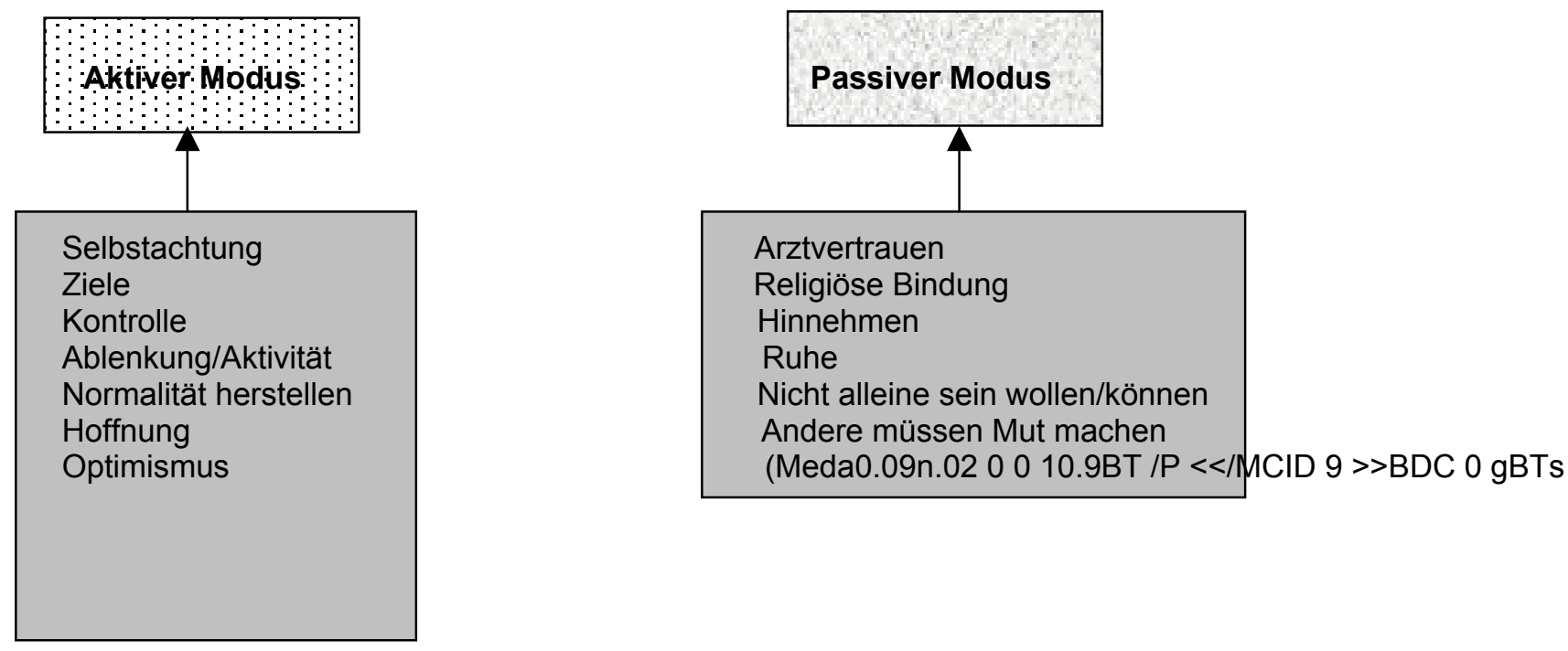


\begin{tabular}{|c|c|c|c|c|c|}
\hline \multicolumn{6}{|c|}{$\begin{array}{r}\text { Tabelle 48: Anpassungsstrategien } \begin{array}{r}\text { (Die Zahlen beziehen sich auf die jeweilige Patientennummer; } \\
\text { mit roten Zahlen sind die Rezidivpatienten gekennzeichnet) }\end{array}\end{array}$} \\
\hline & \multicolumn{5}{|c|}{ Zeitpunkte (Patientenanzahl) } \\
\hline Strategien & T1 $\quad(\mathrm{N}=34)$ & T2 $\quad(\mathrm{N}=34)$ & T3 $\quad(\mathrm{N}=34)$ & $\mathrm{T} 4 \quad(\mathrm{~N}=33)$ & T5 $\quad(\mathrm{N}=30)$ \\
\hline $\begin{array}{l}\text { Ziele haben } \\
\text { Eigene Stärke/ } \\
\text { Selbstachtung }\end{array}$ & \begin{tabular}{|llll}
5 & 12 & 18 & 25 \\
28 & 31 & 33 \\
34 & & &
\end{tabular} & $\begin{array}{llll}5 & 6 & 10 & 18 \\
21 & 22 & 29\end{array}$ & $\begin{array}{|llll|}5 & 12 & 15 & 18 \\
20 & 21 & 22 & 23 \\
25 & 28 & 29 & 31\end{array}$ & $\begin{array}{|lccc|}6 & 7 & 18 & 21 \\
22 & 23 & 25 & 28 \\
32 & 34 & & \end{array}$ & $\begin{array}{cccc}18 & 21 & 23 & 25 \\
27 & 31 & & \end{array}$ \\
\hline $\begin{array}{l}\text { Kontrolle } \\
\text { Aktivität/ } \\
\text { Ablenkung }\end{array}$ & \begin{tabular}{|lccc}
9 & 11 & 14 & 18 \\
20 & 21 & 23 \\
33 & &
\end{tabular} & 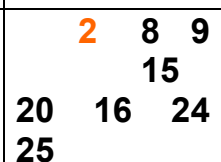 & $\begin{array}{lllll}2 & 3 & 8 & 9 & 10 \\
15 & 19 & 24 & 26 \\
32 & & & & \end{array}$ & $\begin{array}{llll}3 & 10 & 15 & 21 \\
24 & 26 & & \end{array} \mid$ & 1019 \\
\hline $\begin{array}{l}\text { „Normalität“" } \\
\text { herstellen }\end{array}$ & 12 & 217 & & $\begin{array}{lll}5 & 12 & 31\end{array}$ & $\begin{array}{llccc}5 & 6 & 7 & 9 & 12 \\
13 & 15 & 23 & 31\end{array}$ \\
\hline Arztvertrauen &  & $4 \quad 34$ & 11 & 4 & \\
\hline $\begin{array}{l}\text { Hoffnung/ Op- } \\
\text { timismus }\end{array}$ & $\begin{array}{|lcc|}\mathbf{7} & \mathbf{1 6} & 22 \\
23 & 26 & 27 \\
\mathbf{2 8} & 32 & \\
\end{array}$ & 2330 & 6 & & $\begin{array}{llll}22 & 25 & 30 & 32 \\
33 & 34 & & \end{array}$ \\
\hline $\begin{array}{l}\text { Informations- } \\
\text { Suche }\end{array}$ & $\begin{array}{llll}1 & 3 & 9 & 12 \\
15 & 19 & \end{array}$ & 34 & & & 33 \\
\hline $\begin{array}{l}\text { Veränderte } \\
\text { Werte }\end{array}$ & & & 25 & 30 & 230 \\
\hline $\begin{array}{l}\text { Religiöse Bin- } \\
\text { dung }\end{array}$ & & 7 & 7 & 1634 & 16 \\
\hline $\begin{array}{l}\text { Hinnehmen } \\
\text { Ruhe }\end{array}$ & & $\begin{array}{lcc}14 & 19 & 23 \\
26 & 2732\end{array}$ & $\begin{array}{|ll|}4 & 27\end{array}$ & 1827 & \\
\hline Medikamente & & 26 & $\begin{array}{|lll|}10 & 16 & 17 \\
33 & & \end{array}$ & & \\
\hline Reden & 21 & 1331 & & 34 & \\
\hline $\begin{array}{l}\text { Nicht alleine } \\
\text { sein } \\
\text { Andere müssen } \\
\text { Mut machen }\end{array}$ & 30 & $\begin{array}{lll}11 & 12 & 13 \\
33 & & \end{array}$ & $13 \quad 16 \quad 34$ & 1320 & \\
\hline $\begin{array}{l}\text { Depressive } \\
\text { Verarbeitung }\end{array}$ & \begin{tabular}{|lll}
20 & 24 & 26 \\
25 & &
\end{tabular} & $\begin{array}{lll}27 & 34 & 14 \\
15 & & \end{array}$ & $\begin{array}{|llll|}1 & 13 & 14 & 25 \\
30 & & & \\
\end{array}$ & $\begin{array}{llll}1 & 9 & 11 & 13 \\
14 & 17 & 26 & 2 \\
8 & 17 & 19 & 28 \\
33 & 34 & & \end{array}$ & $\begin{array}{lccc}3 & 11 & 14 & 16 \\
20 & 24 & 26 & 30 \\
1 & 8 & 12 & 15 \\
28 & & & \end{array}$ \\
\hline
\end{tabular}

Aus der Zusammenstellung in der Tabelle wird deutlich, dass von der Mehrzahl der Patientinnen und Patienten sehr variable Muster genutzt werden, aber bei einer Minderheit auch stabilere Muster vorkommen können. Da in dieser Stichprobe die Variabilität größer ist als die Stabilität, kann dieses Ergebnis als Hinweis für den Prozesscharakter der Krankheitsanpassung gewertet werden. Allerdings ist mit Prozess kein Entwicklungsmodell im Sinne von Kübler-Ross (1980) oder Kast (1991) gemeint, also kein 
Phasenmodell, das über verschiedene Phasen mit unterschiedlichen Emotionen zur „Zustimmung“ oder zum „abschiedlichen Leben“ führt, wie die beiden Autorinnen formulieren, sondern ein Modell, in welchem die unterschiedlichen Anforderungen - abhängig von unterschiedlichen Bedingungen im Krankheits- und Therapieverlauf - zu jeweils anderen Bewältigungsstrategien herausfordern.

Vor Beginn der Behandlung wird „Arztvertrauen“ von vielen Patienten genannt, daneben spielen vor allem Strategien, die dem aktiven Modus zugerechnet werden wie „Eigene Stärke“, „Aktivität“, „Optimismus/Hoffnung“ und „Informationssuche“ eine wichtige Rolle. „Eigene Stärke“ bleibt auch in der Folgezeit bedeutsam, ähnlich wie "Aktivität“. Patienten mit und ohne Rezidiv unterscheiden sich bezüglich der Kategorie „Hoffnung“. Vor der Behandlung und nach einem Jahr sind es relativ gesehen mehr Patienten mit Rezidiv, die auf diese Strategie setzen. „Veränderte Werte“ und „religiöse Bindung" werden nur von einer Minderheit genannt. Vermutlich ist der Einjahreszeitraum zu kurz und die Patienten waren noch überwiegend mit der Rückkehr in den Alltag und der Bewältigung der somatischen Auswirkungen beschäftigt.

Während der Aplasiephase, in der Zeit der größten körperlichen Beeinträchtigung, wählen mehr Patienten einen passiven Modus.

Neben diesen bewussten Strategien gibt es eine weitere Anpassungsform in dieser Stichprobe, die, unter Vorbehalt, „depressiv“ genannt werden soll. Depressiv deshalb, weil Hadern, Selbstanklagen, Selbstzweifel, Schuld, Enttäuschung, Gefühle von Hilflosigkeit, Ausgeliefertsein und Angst, neben vielfältigen körperlichen Beschwerden, häufig vorkommen. Der Vorbehalt soll einerseits gemacht werden, weil depressiv hier nicht im Sinne psychiatrischer Etikettierung verwendet wird, sondern eine Befindlichkeit beschreibt, die angesichts der „Verluste“ durchaus noch als angemessen gelten kann. Andererseits soll der Vorbehalt gemacht werden, weil die Interviewsituation von Seiten der Patienten ausdrücklich so aufgefasst wird, dass diese „negativen“ Äußerungen „erlaubt“ sind. Außerhalb der geschützten Interviewsituation werden viele dieser Äußerungen zurückgehalten, weil Patienten so gut wie möglich funktionieren wollen. „Gut funktionieren“ wird sowohl in der Kliniksituation als auch später, nach der Entlassung, von der Umwelt gratifiziert. Anhaltende Klagen können hingegen bei den Interaktionspartnern zu guten Ratschlägen, der Demonstration von abnehmender Empathie und sozialem Rückzug führen.

Wie aus der Tabelle hervorgeht, zeigt sich eine zunehmende Tendenz zur depressiven Anpassung, in Abhängigkeit von der Zeit nach HDC, die zu T4 mehr als ein Drittel der Patienten und zu T5 sogar 50\% der Patienten umfasst. Bemerkenswerter Weise zeigt sich dieser Modus deutlich häufiger bei den Patienten, die zu T5 kein Rezidiv haben. Die Patienten, bei denen zu T5 ein Rezidiv bekannt ist, beziehen sich häufiger auf die 
„Eigene Stärke“ und auf „Hoffnung/Optimismus“. Warum sind es ausgerechnet die Patienten, bei denen zumindest bis zu diesem Zeitpunkt ja angenommen werden kann, dass die Behandlung erfolgreich war? An anderer Stelle wurde bereits darauf hingewiesen, dass die langandauernden Leistungseinbußen und die Persistenz von Beschwerden von den Betroffenen nicht erwartet wurden. Sie waren mehr auf aktuelle Nebenwirkungen eingestellt. Die langsame und mit möglicherweise dauerhaften Leistungseinbußen einhergehende Genesungszeit war so nicht antizipiert worden. Außerdem ist bei vielen Patienten die Angst vor einem Rückfall nach kurzer Zeit wieder präsent. Nur bei 3 Patienten zeigt sich depressive Verarbeitung als relativ konstantes Muster über drei Zeitpunkte. Die Zunahme „depressiver“ Muster nach der Klinikentlassung lässt sich auch dadurch erklären, dass sich die Patienten anderen Alltagsanforderungen stellen müssen und sich der Veränderungen erst jetzt schmerzhaft bewusst werden. Das heißt, die Phase des Eingeständnisses von Verlust, wahrgenommen als Leistungseinbuße, als vorzeitige Alterung, als erzwungene Akzeptanz von möglicherweise bleibenden körperlichen Symptomen, beginnt erst nachdem die aktuelle Bedrohung durch die HDC vorbei ist. Die Belastungen in der Klinikzeit waren gewissermaßen berechenbarer und vor allem auch zeitlich leichter einzugrenzen als die späteren. Bei drei Patientinnen zeigt sich allerdings eine manifeste Anpassungsstörung, gemessen an den diagnostischen Kriterien der ICD10. Diesen Patientinnen wurde eine psychotherapeutische (psychiatrische) Behandlung angeraten und sie wurden bei der Suche nach einem Therapieplatz unterstützt.

Umgekehrt muss gefragt werden, warum die Patienten mit bekanntem Rezidiv in ihrer Mehrzahl in dieser Stichprobe überwiegend einen aktiven Modus („Eigene Stärke“) wählen. Bei drei von vier Patienten zeigt sich hier ein stabiles Muster. Der Blick auf die medizinischen Daten zeigt, dass es sich bei allen drei Patienten um Patienten mit schlechter Prognose handelt, deren Diagnose erst wenige Monate vor der HDC gestellt wurde. Wider Erwarten sind es nicht die Patienten, die schon längere Zeit erkrankt sind und entsprechende Anpassungsleistungen schon in der Vergangenheit erbringen mussten und deshalb über entsprechende „Routine“ verfügen. Dass drei weitere Rezidivpatienten auf „Hoffnung“ setzen, ist dagegen völlig erwartungskonform.

Zum Abschluss dieses Teiles werden in der folgenden Übersicht Textbeispiele und Beobachtungen aufgeführt, die auf verschiedene Abwehrmechanismen (unbewusst) hinweisen. Regression, Bagatellisieren, Verleugnung und Rationalisieren sind in dieser Stichprobe am häufigsten. Für die Typologisierung werden die angeführten Beispiele hier isoliert aufgelistet. In der Realität zeigt sich eher eine Mischung aus verschiedenen Abwehrformationen, deren Zuordnung nicht immer leicht fällt. 


\begin{tabular}{|c|c|}
\hline \multicolumn{2}{|l|}{ Tabelle 49: Beispiele Abwehrmechanismen } \\
\hline Indikatoren: Äußerungen - Beobachtungen (Patientennummer) & Abwehrmechanismus \\
\hline $\begin{array}{l}\text { Patientin (13) zieht sich in der Aplasiephase zurück und wünscht nur } \\
\text { den Kontakt zu sichernden Personen. „Existentiell wichtig“ ist die An- } \\
\text { wesenheit des alten Vaters, der tagtäglich am Bett sitzt. Neben ihrem } \\
\text { Vater ist die Patientin auf Kontakte zu einer bestimmten Ärztin, der PT } \\
\text { und einer zusätzlichen PT, die sie aus einer früheren ambulanten } \\
\text { Therapie kennt, angewiesen. }\end{array}$ & Regression \\
\hline $\begin{array}{l}\text { Patient (5) macht einen ausgesprochenen lockeren Eindruck. Die } \\
\text { Therapie wird schon nicht so schwer werden. Er sorgt sich nur, dass } \\
\text { er in der Aplasiephase nicht seine tägliche Zeitung am Kiosk holen } \\
\text { kann. Besuch seiner Familie wünscht er nicht. Da seine Frau Diabeti- } \\
\text { kerin ist, will er sie schonen. Sie soll die Station nicht sehen. } \\
\text { In der Aplasiephase begegne ich dem Patienten zufällig nachmittags, } \\
\text { als er im Bett auf einem der Flure auf eine Untersuchung wartet. Ich } \\
\text { erinnere mich, dass er mir von seinem Hochzeitstag heute erzählt } \\
\text { hatte. Ich gratuliere ihm und mache eine Bemerkung, dass er sich } \\
\text { seinen Hochzeitsstag sicherlich anders wünschen würde. Völlig un- } \\
\text { vermittelt bricht er in Tränen aus und es braucht eine Zeit, bis er seine } \\
\text { Fassung wieder gewinnt. Dieser „Vorfall“ ist zwischen uns nicht wie- } \\
\text { der thematisiert worden. Er hält seine lockere, bagatellisierende Hal- } \\
\text { tung bis zum letzen Untersuchungszeitpunkt durch. }\end{array}$ & $\begin{array}{l}\text { Bagatellisieren } \\
\text { (Projektion) }\end{array}$ \\
\hline $\begin{array}{l}\text { „Ich sage nie Krebs, weil das Wort so negativ klingt... Eigentlich habe } \\
\text { ich keine Befürchtungen. Auch wenn die HD nicht den gewünschten } \\
\text { Effekt haben sollte, gibt es immer noch Möglichkeiten weiter zu ma- } \\
\text { chen...Bei mir ist die HD ja rasch und recht erfolgreich gelaufen... } \\
\text { Insgesamt waren die zwei Wochen wie ein Fingerschnippen..." (21) } \\
\text { Der Patient entwickelt nach T4 einen Progress. Die deutlichste Ver- } \\
\text { leugnung zeichnet sich ab, als er kurz vor seinem Tode steht. Er ist im } \\
\text { Göttinger Hospiz. Bei einem Telefonkontakt berichtet er, es ginge ihm } \\
\text { besser und er würde bald nach Hause }{ }^{78} \text { entlassen. }\end{array}$ & \begin{tabular}{|l|} 
Verleugnen \\
Bagatellisieren
\end{tabular} \\
\hline $\begin{array}{l}\text { Patient (10) fragt, ob denn HDC was besonders Schlimmes sei? „Alle } \\
\text { machen so ein Getue drum...Befürchtungen habe ich keine...Was sind } \\
\text { denn jetzt drei Wochen in Bezug auf ein ganzes Leben...Mein Körper } \\
\text { ist ja eigentlich fit, bis auf die Erkrankung.“ }\end{array}$ & Rationalisieren \\
\hline
\end{tabular}

Abwehr, wie sie in den Beispielen beschrieben wird, lässt sich bei ca. der Hälfte der Patienten demonstrieren. Sieht man einmal von den obigen Beispielen ab, dann äußert sich "Abwehr" in anderen Formen, die gerade für Patienten mit Krebserkrankungen „typisch“ sind und die bei allen Patienten dieser Stichprobe nachzuweisen sind. Diese Formen wurden beschrieben als „middle knowledge“ oder „doppelte Buchführung“ (Weisman 1972; Faller 1998). Patienten wechseln zwischen Akzeptieren und Verleugnen. Für den Beobachter erscheint das Verhalten uneindeutig. Es ist nicht eindeutig auszumachen, auf welchem Stand sie sich bezüglich ihrer Erkrankung befinden: ob sie Wissen aussparen, verzerren oder nicht zur Kenntnis nehmen wollen oder können. Noll (1984) erklärt diese Art des Umgangs mit lebensbedrohlicher Situation, wie er sie durch seine eigene Krebserkrankung erfährt: „Leben kann nicht nur, Leben will auch nicht den Tod kennen, kann es nicht wollen. Ohne diesen geheimnisvollen Zwang der Natur zum

${ }^{78}$ „Nach Hause“ ist hier im realen Sinne gemeint, nicht im symbolischen. 
Lebenwollenmüssen gäbe es längst kein Leben mehr. Der Lebensdrang wird umso stärker, je grösser Not und Gefahr sind“" (ebenda S. 13). ${ }^{79}$

In der Situation, in der sich die Patienten dieser Untersuchung befinden, müssen sie mit „weniger Abwehr“ bezüglich der Bedrohlichkeit ihres Gesundheitszustandes auskommen, da eine permanente Konfrontation mit der Erkrankung unausweichlich ist: „....hier im Krankenhaus füllt der Krebs das ganze Zimmer aus. Hier habe ich nichts zu tun, ich will das nicht, dem Krebs soviel Raum geben, aber hier bestimmt er 24 Stunden alles" (13). Abwehr im Sinne der „doppelten Buchführung“ zeigt sich darin, dass Patienten wenig nachfragen, bewusst Themen aussparen und nicht insistieren, wenn es um widersprüchliche Aussagen in Bezug auf Erfolgschancen der Therapie geht. In dieser Stichprobe gibt es nicht einen Patienten, der wissen möchte, wie groß der angenommene Therapieeffekt ist und in welcher Größenordnung er sich von konventioneller Therapie unterscheidet. Patienten geben sich mit der lapidaren Äußerung „ist besser als“ zufrieden.

Außerdem spielt hier der Faktor "Zeit“ eine wichtige Rolle. In der Behandlungsphase, die ja sehr stark als Bedrohung wahrgenommen wird, müssen Erfolgserwartungen beschworen werden. Nur bei einzelnen Patientinnen gelingt das nicht. Erst zu einem viel späteren Zeitpunkt werden Rezidivängste wieder ausgesprochen. Rezidivängste formulieren heißt gleichzeitig, am Erfolg der HDC zweifeln. Eine durchgängige Verleugnung des bedrohlichen Zustandes findet sich nur bei einem Patienten (21). Mit zunehmender Progredienz der Erkrankung und dem Näherrücken des Todes erscheint die Verleugnung bei diesem Patienten immer perfekter.

Die Frage danach, welcher Anpassungsrmodus adaptiv bzw. maladaptiv ist, lässt sich auch durch diese Studie nicht beantworten. Ein wichtiger Hinweis auf das, was ein großer Anteil der Patienten in einer extremen körperlichen Ausnahmesituation braucht, ist in der Hinwendung zur Nutzung des passiven Modus (vor allem Ruhe) in der Aplasiephase zu sehen. Die Notwendigkeit eines sichernden Anderen weist in die gleiche Richtung. Allerdings muss auch hier einschränkend bemerkt werden, dass diese Schlussfolgerung nicht für alle Patienten gilt. Im Kontakt mit dem Patienten stellt sich in der Regel schnell heraus, was er zur Sicherung seines Selbst, zur Aufrechterhaltung seines Kohärenzgefühls, benötigt.

Langfristig muss auch das reaktive Auftreten einer manifesten Anpassungsstörung mit depressiver und/oder Angstsymptomatik nicht zwangsläufig zu einer schlechten Anpassung führen (unter der Bedingung, dass diese Symptomatik mithilfe von Therapie

\footnotetext{
${ }^{79}$ Beachtet werden sollte, dass dieses „Lebenwollenmüssen“ wie Noll formuliert nicht automatisch Todesverdrängung impliziert. Im Gegenteil, gerade Noll zeigt wie bewusste Auseinandersetzung und „Lebenwollenmüssen“ zusammengehen.
} 
oder durch andere Faktoren überwunden wird). Zwei Patientinnen dieser Stichprobe, die nach der Entlassung aus dem Krankenhaus der psychiatrischen/psychotherapeutischen Hilfe bedurften, hatten sich zu T5 ausgezeichnet erholt und beurteilten sowohl ihren physischen als auch psychischen Zustand als gut. Zwei weitere Patientinnen, die sich in psychotherapeutische Behandlung begaben, wurden, nach einer zwischenzeitlichen relativ ausgeglichenen Phase, durch einen Krankheitsprogress bedroht und in neue Anpassungsschwierigkeiten gestürzt.

Durch die lange nach Ende der Studie weiter bestehenden Kontakte auch zu Rezidivpatienten wurde deutlich, wie schwierig Anpassung wird, wenn keine Zeit dafür bleibt. Krankheitsereignisse gefährden immer aufs Neue das wiedergewonnene relative Gleichgewicht. Die Anpassung an die sich ständig objektiv verändernde Realität kann auch diejenigen Patienten überfordern, in deren Geschichte sich keinerlei Hinweise für eine psychische Vulnerabilität finden.

\subsubsection{Wichtigste Unterstützung}

Hintergrund dieser Frage war die Suche nach den wichtigsten Ressourcen der Patienten als Gegengewicht zu den erlebten Belastungen mit bedrohlichen und negativen Gefühlen. Emotionale Unterstützung als Schutzfaktor ist in vielen Studien unter dem Stichwort der „Sozialen Unterstützung“ untersucht worden. Eine kurze Zusammenfassung der wichtigsten Aspekte findet sich bei Faller (1998 Kapitel 4.5). Er stellt den früheren, einhellig positiven Befunden auch neuere Befunde gegenüber, die auch auf problematische Einflüsse hinweisen. Er verweist auf das Dilemma der Patienten: Belastung muss deutlich gemacht werden, um Unterstützung zu erhalten, gleichzeitig können Belastungsäußerungen zum Rückzug der Interaktionspartner führen. In welcher Weise die Patienten dieser Stichprobe Unterstützung über den Zeitverlauf erfahren, wird aus den folgenden Auswertungen deutlich.

Wie aus den soziodemographischen Daten hervorgeht, haben die meisten Patienten dieser Stichprobe Familie, meist eine eigene Familie oder sind noch (oder wieder) eng mit der Ursprungsfamilie verknüpft. ${ }^{80}$

\footnotetext{
${ }^{80}$ Das trifft auf drei Patientinnen zu, die entweder geschieden/getrennt sind, oder nie eine eigene Familie hatten.
} 


\begin{tabular}{|l|l|l|}
\hline \multicolumn{2}{|l|}{ Tabelle 49: Wichtigste Unterstützung } \\
\hline Textzitate (Patientennummer) & Kategorie & $\begin{array}{l}\text { Mehrfach- } \\
\text { nennungen }\end{array}$ \\
\hline „...Familie, dass ist zentral wichtig und gibt mir Rückhalt.“ (18) & Familie & 31 \\
\hline $\begin{array}{l}\text { „Meine Freundin und deren Familie.“ (3) (Die Patientin weint als } \\
\text { sie davon erzählt und betont, dass sie vor Rührung und Dank- } \\
\text { barkeit immer weinen muss, wenn sie von dieser Freundin be- } \\
\text { richtet) }\end{array}$ & 11 \\
\hline $\begin{array}{l}\text { „Am meisten hat mir geholfen, dass ich bei meinen Anfragen } \\
\text { von vielen Abteilungsleitern persönlich telefonisch zurückgeru- } \\
\text { fen wurde..." (1) }\end{array}$ & $\begin{array}{l}\text { Medizin. } \\
\text { Personal }\end{array}$ & 5 \\
\hline
\end{tabular}

Für diese Stichprobe bestätigt sich die Wichtigkeit des Eingebundenseins in einen sozialen, meist familiären Kontext. Auch wenn konflikthafte Situationen vorhanden sind, und die finden sich auch in dieser kleinen Stichprobe, wird auf die anderen verwiesen, die „Kraft geben“, „Mut machen“ usw. Ein Vergleich mit isoliert lebenden, vereinsamten Patienten ist hier nicht möglich. Vermutlich gehören diese nicht zu dem Kreis, denen eine solche Therapie angeboten wird (positive Auswahl in Bezug auf Belastbarkeit durch die Ärzte, Selbstselektion durch „Erkämpfen“ der Therapie). Die Bedeutung von vertrauten Personen wurde auch dadurch offensichtlich, dass Patientinnen (in der Tat waren es die Frauen) regelrecht organisierten, dass sie möglichst wenig alleine waren. Das galt besonders für die Aplasiephase. Angehörige saßen oft stundenlang im Zimmer. Die bloße Anwesenheit zählte. Aus der Literatur ist bekannt, dass die Qualität, nicht die Quantität des sozialen Netzes darüber entscheidet, ob Patienten in ihrer Krankheitsanpassung unterstützt werden (siehe auch Faller, ebenda, Kap. 4.5).

Explizit wurde nach der wichtigsten Unterstützung gefragt. Als wichtigste Unterstützung über alle untersuchten Zeitpunkte wird von den Patientinnen und Patienten überwiegend die Familie genannt. 31 Patienten nennen die Familie, in der Regel den Partner oder die Partnerin, aber auch Vater, Kinder, Schwester als wichtigste Hilfe. „Meine Frau..., Freunde. Meine Familie ist da." (23)

Elf nennen Freunde, meist in Kombination mit Familie. Bei zwei Patientinnen stehen Freunde an erster Stelle. Eine dieser Patientinnen hat erhebliche Konflikte mit dem Partner, die zweite hat einen Mann, der schon seit langer Zeit krank ist und einen minderjährigen Sohn, den sie schützen will. Für fünf Patienten ist das ärztliche und/oder das Pflegepersonal die wichtigste Hilfe, die Betreuung und die gute Atmosphäre auf der Station: „Zuwendung des Pflegepersonals...“ (14) und „...tolle Ärzte im Klinikum“ (8), wie eine Patientin bemerkt. Für 3 Patienten ist die eigene Stärke die beste Hilfe.

Eine Patientin beantwortet die Frage mit „....das Ende ist absehbar.“ Wie bereits an anderer Stelle bemerkt, ist diese Stichprobe hinsichtlich der familiären Situation sicherlich eine Ausnahme. Nur bei zwei Paaren bestanden konflikthafte Beziehungen, die, wie zu 
erwarten, auch schwierig geblieben sind. Die positiven Erfahrungen mit Angehörigen überwiegen in dieser Stichprobe. ${ }^{81}$

\subsubsection{Teilnahme an Rehabilitationsmaßnahmen}

Überraschenderweise nimmt nur die Hälfte der Patienten an einer stationären Rehabilitationsmaßnahme teil. Bei den deutlichen Belastungseinbußen und persistierenden Symptomen wäre ein deutlich höherer Anteil zu erwarten gewesen.

\begin{tabular}{|c|c|c|c|}
\hline \multicolumn{4}{|c|}{ Tabelle 50: Teilnahme an Reha-Maßnahmen } \\
\hline Maßnahme & $\mathrm{N}=32$ (gesamt) $\quad(\%)$ & P & $\hat{0}$ \\
\hline teilgenommen & (43) & 12 & 2 \\
\hline Nicht teilgenommen & $(57)$ & 9 & 9 \\
\hline
\end{tabular}

\begin{tabular}{|c|c|c|}
\hline \multicolumn{3}{|l|}{ Tabelle 51: Gründe für die Nichtteilnahme } \\
\hline Textzitate (Patientennummer) & Kategorie & $\mathrm{N}=32$ \\
\hline $\begin{array}{l}\text { "Reha wäre für mich Blödsinn gewesen. Meine Re- } \\
\text { ha war mein Garten, meine Familie. Was Besseres } \\
\text { gibt es für mich nicht.“ (2) }\end{array}$ & $\begin{array}{l}\text { Bessere Erholungsmög- } \\
\text { lichkeit zu Hause }\end{array}$ & 7 \\
\hline $\begin{array}{l}\text { "Mein Arzt hält nichts davon... Ich wollte eigentlich, } \\
\text { aber er hat es mir ausgeredet." (1) }\end{array}$ & $\begin{array}{l}\text { Arzt will keine Reha ver- } \\
\text { ordnen }\end{array}$ & 1 \\
\hline $\begin{array}{l}\text { "Ich habe keine Zeit gehabt für eine Reha. Hier gibt } \\
\text { es immer viel Arbeit.“ (10) }\end{array}$ & Keine Zeit & 1 \\
\hline $\begin{array}{l}\text { "Ich habe keine Reha gemacht. Ich wollte nicht weg } \\
\text { von zu Hause, weil ich dann Angst gehabt hätte, } \\
\text { dass es Knatsch gibt zwischen meinem Mann und } \\
\text { den Kindern." (8) }\end{array}$ & $\begin{array}{l}\text { Werde zu Hause ge- } \\
\text { braucht }\end{array}$ & 2 \\
\hline $\begin{array}{l}\text { "Habe an keiner Reha teilgenommen...hat mich } \\
\text { keiner zu aufgefordert, auch nicht die Versicherung." } \\
\text { (5) }\end{array}$ & $\begin{array}{l}\text { Wusste nichts von der } \\
\text { Möglichkeit/wurde nicht } \\
\text { angeboten }\end{array}$ & 2 \\
\hline „War zu krank für eine Reha.“ (4) & $\begin{array}{l}\text { Schlechter Gesundheits- } \\
\text { zustand }\end{array}$ & 2 \\
\hline $\begin{array}{l}\text { "Eine Reha habe ich nicht gemacht. Ich habe so } \\
\text { viele Wartezeiten bei der Behandlung in Kauf neh- } \\
\text { men müssen...Ich wollte nirgendwo mehr Termine } \\
\text { haben.“ (31) }\end{array}$ & $\begin{array}{l}\text { Rückkehr zu einem nor- } \\
\text { malen Rhythmus }\end{array}$ & 1 \\
\hline -------- & Keine Begründung & 2 \\
\hline
\end{tabular}

Von den Patienten, die nicht teilgenommen haben, hat der größere Teil bewusst darauf verzichtet, zwei Patienten haben nicht von der Möglichkeit gewusst und bei einer Patientin hat der behandelnde Arzt die Maßnahme abgelehnt, obwohl sie selbst den

${ }^{81}$ Eine Patientin, die sich während der HDC von ihrem Partner gut unterstützt fühlte, wird zwei Jahre später mit dem Trennungswunsch des Partners konfrontiert. Die Patientin schildert die dadurch entstehenden Belastungen als so schwerwiegend, dass sie dagegen all das, was die Krankheit damals ausgelöst hatte in den Schatten stellt. 
Wunsch geäußert hatte. Eine Patientin vermutet, dass man ihr die Möglichkeit nicht angeboten hat, weil sie bereits Rentnerin ist. Zwei Patienten fühlten sich zu krank für eine Reha-Maßnahme. Zwei Patientinnen wollten ihre Verpflichtungen zu Hause wieder aufnehmen und haben eine Maßnahme deshalb abgelehnt. Von den sieben Patienten, die meinten sich zu Hause besser erholen zu können, waren die Männer $(N=6)$ in der Mehrzahl. Nur eine der älteren Patientinnen, die bereits berentet war, meinte sich ebenfalls besser zu Hause erholen zu können. Zwei Gruppen lassen sich unterscheiden:
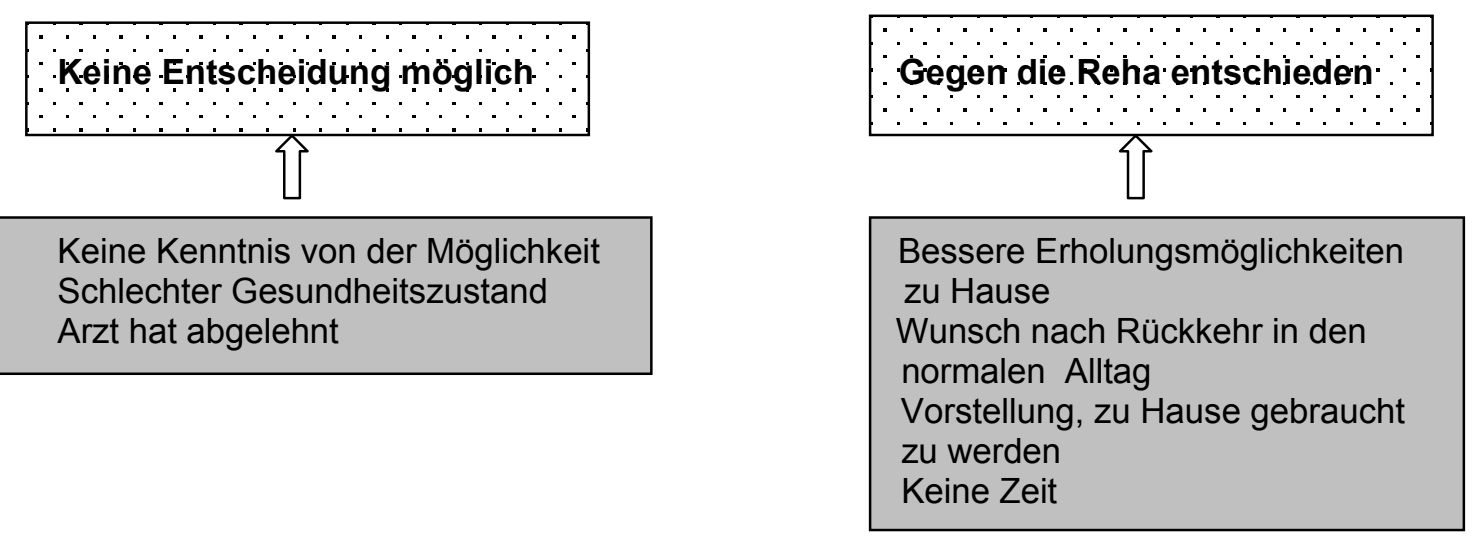

Von den drei Männern, die nach einem Jahr wieder in das Berufsleben integriert waren, hat keiner an der Rehabilitation teilgenommen. Die drei Frauen, die wieder berufstätig sind, haben an einer Rehabilitation teilgenommen.

Die Urteile der Patienten, die teilgenommen haben, fallen unterschiedlich aus:

\begin{tabular}{|c|c|c|}
\hline \multicolumn{3}{|c|}{ Tabelle 52: Patientenurteile über die Rehabilitationsmaßnahme } \\
\hline Textzitate (Patientennummer) & Kategorie & $\mathrm{N}=13$ \\
\hline $\begin{array}{l}\text { "Die Kur war ganz toll...Das hat mich unheimlich } \\
\text { aufgebaut. „(19) }\end{array}$ & Positive Auswirkungen & 7 \\
\hline „Insgesamt fand ich es so mittelprächtig.“ (22) & Bescheidene Auswirkungen & 2 \\
\hline $\begin{array}{l}\text { "...konnte die Klinik nicht mehr sehen. Bei den De- } \\
\text { pressionen, die ich hatte, dann noch auf der Onko- } \\
\text { logie zu liegen...das ist zuviel geworden. Da bin ich } \\
\text { eine Woche früher abgefahren." (9) }\end{array}$ & Negative & 3 \\
\hline
\end{tabular}

Die Begründungen, warum die Maßnahme positiv beurteilt wurde, beziehen sich auf Ernährung, Erholungsmöglichkeiten, Anwendungen und auch auf die Möglichkeit zum Austausch: „Es hat sich gelohnt! ...in der Reha haben wir Reden gelernt. Meiner Frau geht es jetzt dreimal besser." (21), berichtet ein Patient, der gemeinsam mit seiner Frau teilgenommen hat. Eine andere Patientin berichtet: „Ich war ja.... Da habe ich mich drauf gefreut und es war auch sehr schön...bei allen waren die Erkrankungen noch frisch. Natürlich haben wir uns davon erzählt. Zum Teil haben die mich aufgebaut. In dem Sinne: es ist manches anders 
geworden, aber das Leben geht weiter. Wir haben aber nicht nur über Krankheit gesprochen..." (15).

Zu eher mäßiger Beurteilung führt in einem Falle die mangelnde Betreuung des begleitenden Kindes, in einem anderen Fall die Konfrontation mit der Erkrankung: „Am Anfang war es ganz schrecklich. Mir war nicht so klar, dass ich mit lauter kranken Menschen zusammen war. Ich konnte es kaum ertragen immer über die Krankheit reden und sich mit ihr beschäftigen zu müssen...Nach zwei Wochen hatte ich mich arrangiert. Die Anwendungen haben auch gut getan und ich habe mich etwas erholen können." (14). Bei dieser Patientin ist im Verlauf der ersten beiden Wochen eine Bewertungsänderung erfolgt. Die Konfrontation mit der Erkrankung drängt die positiven Aspekte der Rehabilitationszeit nicht mehr in den Hintergrund.

Der dritte Patient führt keine Begründung an. Auch bei den Patienten, die den Aufenthalt ausschließlich negativ bewerten, steht die Konfrontation mit der Erkrankung als Begründung an erster Stelle: „....da waren nur Krebspatienten. Jeder hat von seiner Krankheit erzählt und da ging es einfach zu viel um Krebs. Und dann ist da noch eine Frau gestorben, eine ziemlich junge...Noch einmal würde ich da nicht hinfahren. Aber der Sport und die Anwendungen waren gut." (6)

"Das finde ich richtig deprimierend, wenn immer über die Krankheit geredet wird." (17).

Diese Begründung, die von einer Minderheit vorgetragen wird, stützt allerdings die häufig zu hörenden Bedenken von Ärzten und Patienten, dass genau aus diesem Grund lieber auf eine Rehabilitationsmaßnahme verzichtet werden sollte.

Auffällig ist die völlig unterschiedliche Inanspruchnahme von Reha-Maßnahmen bei Frauen und Männern. Frauen sind eher bereit, diese Möglichkeit in Anspruch zu nehmen, weil sie sich ihrer Rolle zu Hause noch nicht gewachsen fühlen. Da wo Hilfe vorhanden ist oder Verpflichtungen sich in Grenzen halten, lehnen auch einzelne Frauen ab und erholen sich zu Hause „lieber“. Bedauerlich ist, dass es für Frauen mit kleinen Kindern wenig adäquate Angebote gibt. Gerade diese Gruppe kann sich zu Hause den Anforderungen kaum entziehen und wird in der Rekonvaleszenzphase hoffnungslos überfordert.

\subsubsection{Einschätzung der Veränderungen nach einem Jahr}

In der Literatur, die sich mit der Bewältigung von Krebserkrankung beschäftigt, wird davon ausgegangen, dass Betroffene mindestens einen Zeitraum von einem Jahr benötigen, um die Ereignisse zu „bewältigen“, vorausgesetzt, es treten nicht wieder neue Krankheitsereignisse auf. Die psychometrischen Studien zur HDC legen den Schluss nahe, dass nach einem Jahr der Zustand wieder erreicht ist, den die Patienten in Bezug auf Funktionalität und Symptome, vor der Behandlung beschreiben. Allerdings 
muss an dieser Stelle beachtet werden, dass Kranksein den Ausgangszustand bestimmt. Die Patienten dieser Stichprobe wurden gefragt, ob sie an „ihr altes Leben“ anknüpfen konnten. Häufigkeiten werden nur für die Patientengruppe „ohne Rezidiv“ angegeben, da hier von allen Patienten Äußerungen vorliegen.

\begin{tabular}{|c|c|}
\hline \multicolumn{2}{|c|}{$\begin{array}{l}\text { Tabelle 53: } \\
\text { Veränderungen durch Krankheit } \\
\text { und Therapie } \quad \mathbf{N}=(22)\end{array}$} \\
\hline Überwiegend negativ & 5 \\
\hline Überwiegend positiv & 3 \\
\hline Keine & 2 \\
\hline Teils/teils & 12 \\
\hline
\end{tabular}

Ein knappes Viertel der Patienten beschreibt eindeutig negative Veränderungen ohne die Möglichkeit, auch einzelne positive Aspekte ihres jetzigen Lebens hervorheben zu können. Drei Patienten erleben ihre Situation im Vergleich zu früher positiv verändert. Zwei Patienten konstatieren keinerlei Veränderungen und die größte Gruppe beschreibt zwar negative Veränderungen, hebt aber gleichzeitig positive Aspekte hervor. Die Antworten auf diese Frage werden für die Gruppen, mit und ohne Rezidiv, getrennt dokumentiert: 


\begin{tabular}{|c|c|}
\hline Zitate (Patientennummer) & Kategorien \\
\hline $\begin{array}{l}\text { „Das ganze Leben ist völlig verändert, konnte durch Arbeit zwar anknüpfen } \\
\text { (an das alte Leben) aber... Bin sicherlich in bestimmten Dingen konsequen- } \\
\text { ter geworden aber in vielen auch empfindlicher. Meine Ernährung habe ich } \\
\text { umgestellt... nehme mir eine Putzhilfe, um zur Arbeit gehen zu können. Ich } \\
\text { habe ein Jahr gebraucht, um wieder für die Zukunft zu planen. Aber, dass } \\
\text { man sich mit der Erkrankung beschäftigt und denkt es könnte wieder kom- } \\
\text { men, dass wird wohl immer so gehen.“ (3) } \\
\text { „Ich bin überhaupt nicht belastbar. Es ist so geblieben wie vorher, kann } \\
\text { nicht mehr arbeiten... ansonsten leben meine Frau und ich wie immer. Ha- } \\
\text { ben viel Kontakt zu Freunden und machen mit, was geht. ... Fahre mit } \\
\text { Freunden weg. Kann nicht alles mitmachen aber komme auf jeden Fall mit.“ } \\
\text { (5) } \\
\text { „Es ist jetzt nicht so wie davor... Ich bin auch anders geworden. Ich habe } \\
\text { weniger Mitleid. Für die anderen ist alles so selbstverständlich. Wenn die } \\
\text { Kinder motzen, sage ich schon manchmal: seid froh, dass ich noch lebe.“ } \\
\text { (8) } \\
\text { „Seelische Probleme habe ich keine. Ich wäre allerdings froh, wenn es wie- } \\
\text { der so wäre wie früher.“ (25) } \\
\text { „Beruflich funktioniere ich voll... Nach der Arbeit bin ich platt... Im Umgang } \\
\text { mit anderen Menschen bin ich viel kompromissloser geworden. Manchmal } \\
\text { habe ich Angst, meine Liebe zu den Menschen zu verlieren... Ich habe } \\
\text { mehr Selbstbewusstsein bekommen durch die Krankheit. Ich kann auch } \\
\text { mal Nein sagen. Ich gebe die Vorgaben und die anderen müssen reagie- } \\
\text { ren.“ (16) }\end{array}$ & $\begin{array}{l}\text { Veränderung } \\
\text { Teils/teils }\end{array}$ \\
\hline $\begin{array}{l}\text { "Ich behandele das gar nicht so, als wenn ich Krebs hätte... Ich sehe das } \\
\text { nicht so negativ und manchmal ist es ganz weit weg. Ich will auch keine } \\
\text { Rücksichtnahme. Ich finde, man sollte ganz normal mit der Krankheit um- } \\
\text { gehen." (6) }\end{array}$ & $\begin{array}{l}\text { Keine Verän- } \\
\text { derung }\end{array}$ \\
\hline $\begin{array}{l}\text { „Hatte so gut wie vergessen, wie das vorher war. Jetzt habe ich ja einen } \\
\text { neuen Status. Ich lebe intensiver, verschwende keine Zeit mehr. Heute ist } \\
\text { meine Familie die Nr. 1, früher war es der Beruf. Man ärgert sich auch nicht } \\
\text { mehr über irgendwelche Kleinigkeiten, frisst es nicht mehr in sich rein, } \\
\text { guckt auch mal über was weg. ... Die Familie und die Freunde, das ist die } \\
\text { Hauptsache." (12) }\end{array}$ & $\begin{array}{l}\text { Positive } \\
\text { Veränderung }\end{array}$ \\
\hline $\begin{array}{l}\text { „Nein, so wie früher wird es wohl nie wieder. Für den Betrieb falle ich ganz } \\
\text { aus... Alles hat sich verändert, zum Negativen. In der Familie gibt es } \\
\text { Schwierigkeiten, mein Mann und meine Söhne verstehen das einfach } \\
\text { nicht.“ (14) }\end{array}$ & $\begin{array}{l}\text { Negative } \\
\text { Veränderung }\end{array}$ \\
\hline
\end{tabular}

Für die Kategorie „teils/teils“ wurden mehrere Beispiele aufgelistet, um die Bandbreite der Argumente zu demonstrieren. 

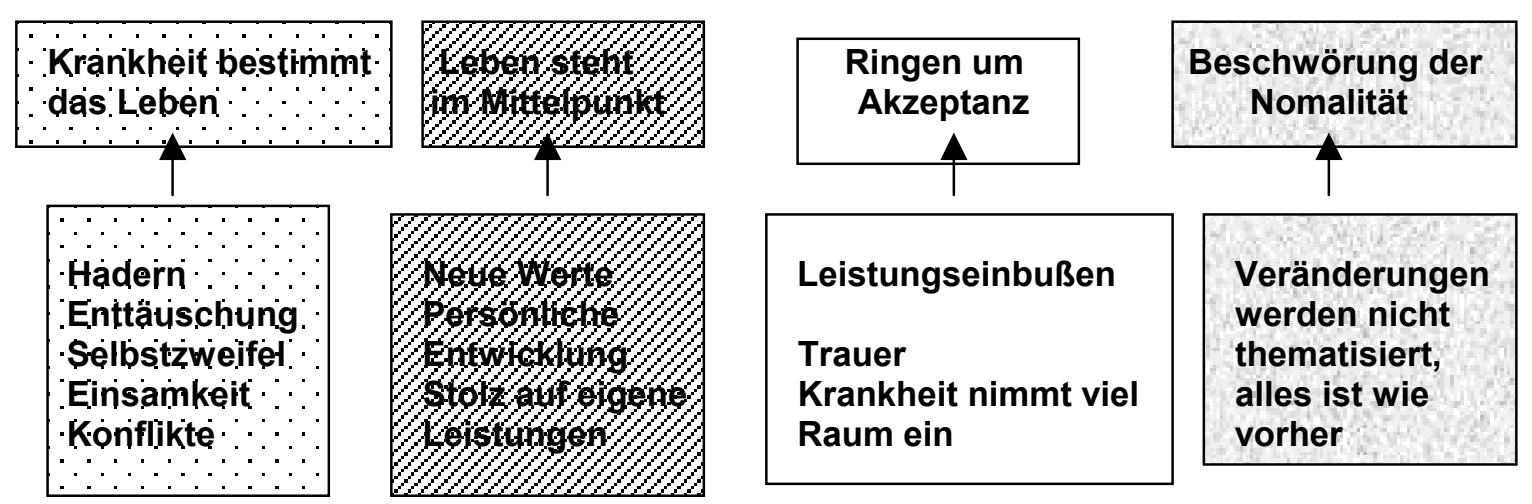

Die Patientengruppe, die positive Veränderungen beschreibt begründet diese mit der Fähigkeit, heute intensiver, bewusster Leben zu können, keine Zeit zu verschwenden und anderen Werten (Familie/Freunde) Vorrang einzuräumen. Gesteigertes Selbstbewusstsein und Stolz auf die eigenen Bewältigungsfähigkeiten klingen ebenfalls an. „Leben“ steht im Mittelpunkt, nicht Krankheit. Die Gruppe, die ausschließlich negative Veränderungen beschreibt, lässt sich diagnostisch als die Gruppe klassifizieren, die ein deutliches depressives Bewältigungsverhalten zeigt und die der psychotherapeutischen Hilfe bedarf.

Die kleinste Gruppe, die keine Veränderungen beschreibt, scheint erfolgreich Impulse von Trauer und Angst abzuwehren. Leistungseinbußen sind entweder nicht vorhanden oder werden von den Patienten nicht registriert. Die Patienten wirken außerordentlich gut angepasst. Krankheit und Therapie hatten nach ihrer Ansicht keine Auswirkungen auf ihre Lebensgestaltung.

Die größte Gruppe, die Veränderungen differenziert beschreibt, erscheint noch „mitten“ im Prozess der Bewältigung. Sehnsüchte nach dem alten Zustand, Trauer über Einbußen, die Kraftanstrengung, mit der gegenwärtigen Situation zu „leben“, werden deutlich. Angst vor einem Rezidiv spielt eine zentrale Rolle. Hervorgehoben werden eigene und familiäre Ressourcen. Einzelne Patienten trauen sich wieder, „Zukunft“ zu thematisieren. Für den größten Teil der Patienten dieser Stichprobe ist der Einjahreszeitraum zu kurz für eine physische und psychische Erholung. Auch in dieser Gruppe finden sich Patienten, denen eine psychosoziale Unterstützung angeraten wurde.

Unter dem Stichwort der Veränderung, wurde auch die Frage nach Reintegration ins Berufsleben bzw. nach der Berentung (auf Zeit oder dauerhaft) erfasst (siehe S. 133). In Bezug auf die soziale Rolle kommt dieser Frage eine große Bedeutung zu. 


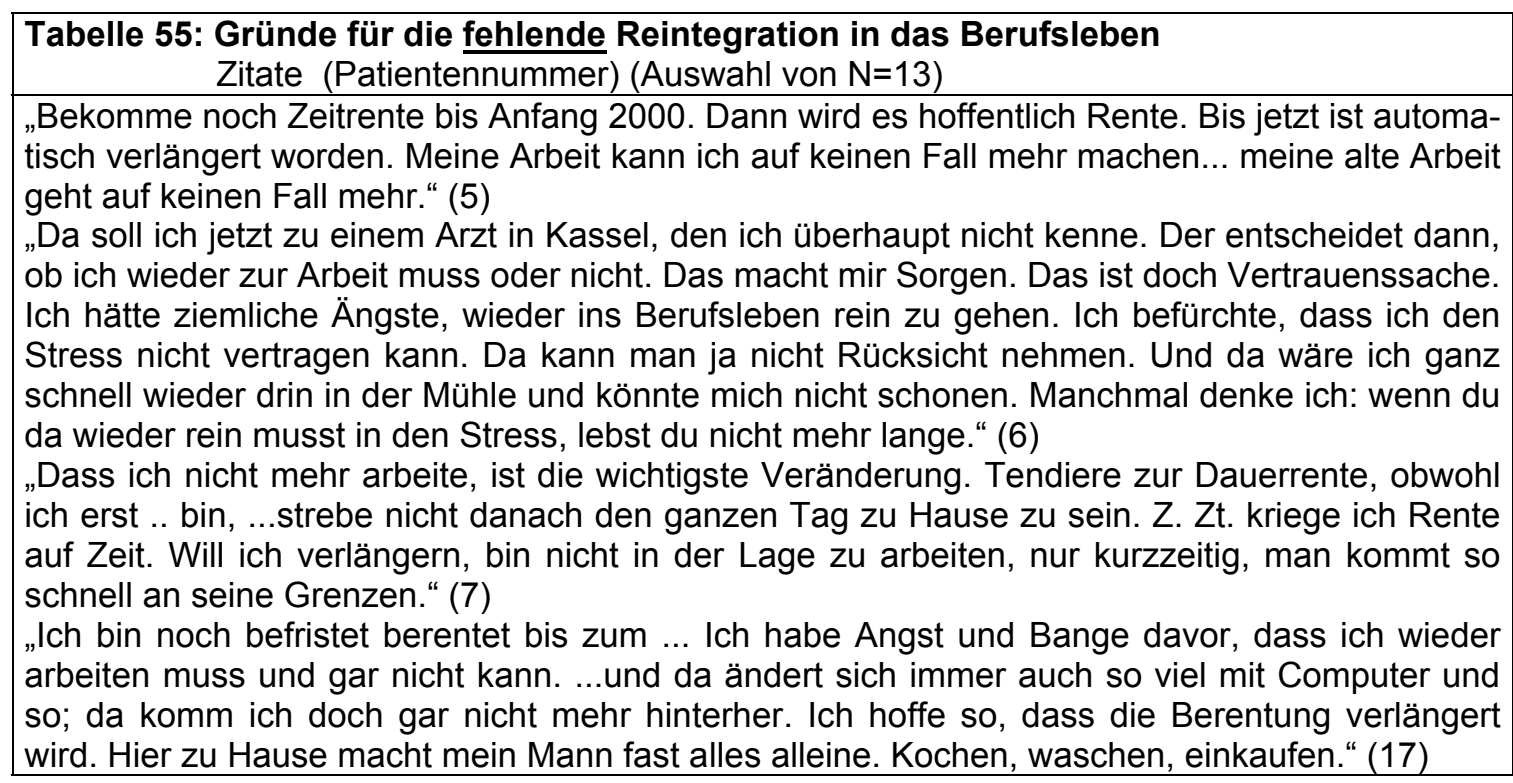

Zur Begründung, warum eine Reintegration nicht infrage kommt bzw. nicht infrage gekommen ist, führen ausnahmslos alle Patienten mangelnde physische Belastbarkeit, mangelnde Stresstoleranz und teilweise auch mangelnde Konzentrationsfähigkeit an. Sie haben Angst die erwarteten Leistungen nicht mehr zu erbringen und ihren Kollegen Mehrarbeit aufzubürden. Alle gehen selbstverständlich davon aus, dass mit dem Wiedereinstieg auch die Krankenrolle aufgegeben werden muss. Neben der Angst, die Leistungen nicht erbringen zu können, wird noch die Angst deutlich, die Arbeitsbelastungen könnten negative Auswirkungen auf den weiteren Krankheitsverlauf haben. Zwei Patienten berichten, dass diese Argumentation auch von ihren behandelnden Ärzten vertreten wird. Auf die Schwierigkeiten, mit denen sich Patienten bei der Reintegration konfrontiert sehen, verweist das folgende Zitat einer Patientin, die ihre Arbeit wieder aufgenommen hat aber nach einigen Monaten feststellt, dass sie den Anforderungen nicht mehr gewachsen ist und Rente beantragt:

„Frau Dr. X meint, dass ich so am Montag nicht wieder arbeiten kann. Aber an meiner Arbeitsstelle wissen sie nicht, dass ich es kaum noch schaffe...Auf die Arbeit habe ich mich gefreut... Das war zuviel. Habe Urlaub genommen, konnte aber keine Erholung finden so fertig war ich. Mir graut es vor der Arbeit am Montag, ich traue mich aber auch nicht zu sagen was wirklich los ist. Habe wahnsinnige Probleme an der Arbeit zuzugeben, dass ich nicht so kann. Mein Arzt meint ich kann es nicht schaffen, soll keine Überstunden machen. Das ist leicht gesagt, die Arbeit fällt an und ich will nicht, dass die anderen sagen sie müssten meine Arbeit mitmachen. Nach der Arbeit habe ich mich zu Hause nur noch hingelegt und geheult.

Nicht arbeiten kann ich mir auch nicht vorstellen. Dann grübele ich zuviel und Sie wissen ja, wie es bei mir hier zu Hause, mit meinem Mann aussieht... Wenn ich meine Arbeit durchhalte und nicht Zeitrente beantragen muss, nehme ich mir eine Putzhilfe, um zur Arbeit gehen zu können."

Die Patienten, denen eine Reintegration gelungen ist, beschreiben diese auf folgende Weise: 


\begin{abstract}
Tabelle 56: Reintegration in das Berufsleben
Zitate (Patientennummer) $(\mathrm{N}=7)$

„Der Übergang war nahtlos, ich bin gleich in die Arbeit eingestiegen. Die ersten 4 Wochen musste ich noch nicht zur Bestrahlung, da konnte ich richtig durchstarten. Das ist sowieso das Beste, was man machen kann. Wenn man zu Hause sitzen würde, kommt man nur ins Grübeln." (10)

„Aber, es hat wirklich auch ein Jahr gebraucht. Im Spätsommer habe ich wieder angefangen zu arbeiten. Erst mit drei Stunden täglich, dann 5, 7 und seit Dezember wieder ganztags. Ich habe aber auch immer gerne gearbeitet und wissen Sie, ich kann mir meine Arbeit auch so einteilen wie ich möchte. Was ich nicht gut ertragen kann, wenn soviel Leute ..., (13)

„Nur 6 Wochen war ich nach der Behandlung krank geschrieben. Dann habe ich mit 4-6-8

Stunden wieder angefangen... Die Arbeit schaffe ich, aber es ist schwer." (12)

„Ich hatte auch Probleme bei der Arbeit. Ich bin nicht auf meine 30 Std. gekommen. Wir müssen ja die reine Fahrzeit als Arbeitszeit aufschreiben... arbeite gerne und verstehe mich mit meinen Kollegen gut. Beruflich funktioniere ich voll. Da werde ich auch richtig gefordert und nicht geschont. Manchmal sage ich aus Spaß: Wisst ihr eigentlich, dass ich eine 80\%ige Behinderung habe. Aber darauf reagieren die gar nicht. Es hört sich fast ein wenig stolz an: ich bin wieder voll belastbar. Ich arbeite als Springerin und kriege völlig chaotische Touren. Nach der Arbeit bin ich platt." (16)

„Ich mache zwar beruflich, was ich vorher gemacht habe, aber unter völlig anderen Voraussetzungen. Mehr kann ich nicht leisten, früher war ich oft 11 Stunden unterwegs. Das ist vorbei. Ich kann nie mehr erreichen, was einmal war. ... Ich muss ja versuchen, meinen Umsatz zu erreichen. Ich habe vorher gut verdient. Jetzt ist es nur noch so viel, wie ich als Krankengeld bekommen habe. Da fragt man sich schon, ob sich das alles lohnt." (28)

„Ich arbeite seit vier Wochen jeden Tag drei Stunden. Das ist nicht so einfach. Ich bin immer noch sehr schnell erschöpft und an den Lärm und die Hitze in der Halle muss ich mich erst wieder gewöhnen." (24)

„Seit einem halben Jahr arbeite ich wieder, allerdings an einem neuen Arbeitsplatz, wo es weniger stressig zugeht. Darum hatte ich gebeten. Es würde mir wieder Spaß machen dort zu arbeiten. Allerdings gibt es dort manche extreme Situationen." (15)
\end{abstract}

Von den Patienten, die wieder integriert sind, äußern zwei Patienten keinerlei Belastungseinschränkungen. Die anderen registrieren die verminderte Belastbarkeit und stellen sich in ihrem Alltag darauf ein. Von den 7 Patienten dieser Gruppe sind zwei selbständig, eine in leitender Position als Angestellte, eine in untergeordneter Position als Angestellte und die anderen vier sind Arbeiter/innen, eine in Teilzeitarbeit. Für diese Stichprobe gilt nicht, dass diejenigen, die schwere körperliche Arbeit machen müssen, eher in die Frühberentung gehen. In dieser Gruppe sind es die Angestellten, die vorzeitig in die Berentung gehen. Für einige dieser reintegrierten Patienten stellt sich die Frage nach der Berentung nicht. Für sie ist die weitere Berufstätigkeit eine materielle Notwendigkeit. Für anderer spielt auch eine Rolle, dass sie gerne arbeiten, Berufstätigkeit einen hohen sozialen Stellenwert hat und eine positive Funktion übernimmt indem sie vom „Grübeln“ ablenkt.

Für die Patienten mit Rezidiv ergibt sich, wie zu erwarten, ein völlig anderes Bild. Alle Patienten dieser Gruppe sind krank geschrieben. 


\begin{tabular}{|c|c|}
\hline der Patienten mit Rezidiv & \\
\hline Zitate (Patientennummer) & Kategorie \\
\hline $\begin{array}{l}\text { "Meine Schaffenskraft bleibt geschmälert... Damit muss ich mich abfinden. } \\
\text { Das habe ich auch teilweise getan. Es ist nur noch ungewohnt. Ich habe doch } \\
\text { immer gearbeitet... Ich bin hier an einen Punkt gekommen, wo nichts mehr zu } \\
\text { gehen scheint... Ich lese hier manchmal den Panther von Rilke. So einge- } \\
\text { sperrt fühle ich mich auch.“ (18) } \\
\text { "Ich hadere mit meinem Schicksal. An die Krankheit denke ich } 24 \text { Stunden am } \\
\text { Tag." (1) } \\
\text { "Das Thema Krankheit steht an erster Stelle.“ (21) } \\
\text { "... Permanent. Man denkt einfach immer daran." (34) } \\
\text { "Bittere Enttäuschung. Aber jetzt wollen wir noch mal in den Süden, wenn es } \\
\text { klappt." (23) }\end{array}$ & $\begin{array}{l}\text { Negative Ver- } \\
\text { änderung: } \\
\text { Am Ende sein } \\
\text { Hadern } \\
\text { Krankheit be- } \\
\text { stimmt } \\
\text { Enttäuschung }\end{array}$ \\
\hline $\begin{array}{l}\text { Die Patientin hatte kurz nach T4 ein Rezidiv. Es ging ihr so schlecht, dass sie } \\
\text { glaubte, die angesetzte Nottrauung nicht mehr zu schaffen. Große Enttäu- } \\
\text { schung über die Wirkungslosigkeit der HDC. Aber, die Patientin ist stolz dar- } \\
\text { auf, ,,dem Tode von der Schippe gesprungen“ zu sein. Sie ist durch die Hölle } \\
\text { gegangen und hat es geschafft. Sie wirkt nicht ambivalent sondern ihre Aus- } \\
\text { sagen erscheinen stimmig. Angst vor dem was noch auf sie zukommen könn- } \\
\text { te hat sie keine mehr. (Bericht Interviewerin) (27) }\end{array}$ & $\begin{array}{l}\text { Jenseits von } \\
\text { Angst }\end{array}$ \\
\hline $\begin{array}{l}\text { "Die Hauptveränderung besteht darin, dass ich keinen Sport mehr treibe... } \\
\text { Gesund leben ist wichtiger geworden. Ich achte mehr als früher auf die Ernäh- } \\
\text { rung und trinke auch weniger Alkohol... Ich könnte ja auch morgen mit dem } \\
\text { Auto einen Unfall haben und tot sein. Darüber macht man sich normalerweise } \\
\text { ja auch keine Gedanken.“ (32) }\end{array}$ & $\begin{array}{l}\text { Nicht an den } \\
\text { Tod denken } \\
\text { Normalität }\end{array}$ \\
\hline $\begin{array}{l}\text { „Mittlerweile erschreckt mich auch das Sterben nicht mehr. Ich kann mich } \\
\text { ganz auf das Leben konzentrieren. Wenn ich wirklich in drei Monaten tot sein } \\
\text { sollte, dann brauche ich doch jetzt nicht mit dem Sterben anzufangen.“ (22) }\end{array}$ & $\begin{array}{l}\text { Positive Ver- } \\
\text { Änderung: } \\
\text { Konzentration } \\
\text { auf Leben }\end{array}$ \\
\hline
\end{tabular}

Die Äußerungen der Patienten mit Rezidiv, die zum Zeitpunkt T5 vorliegen, lassen drei unterschiedliche Muster erkennen:
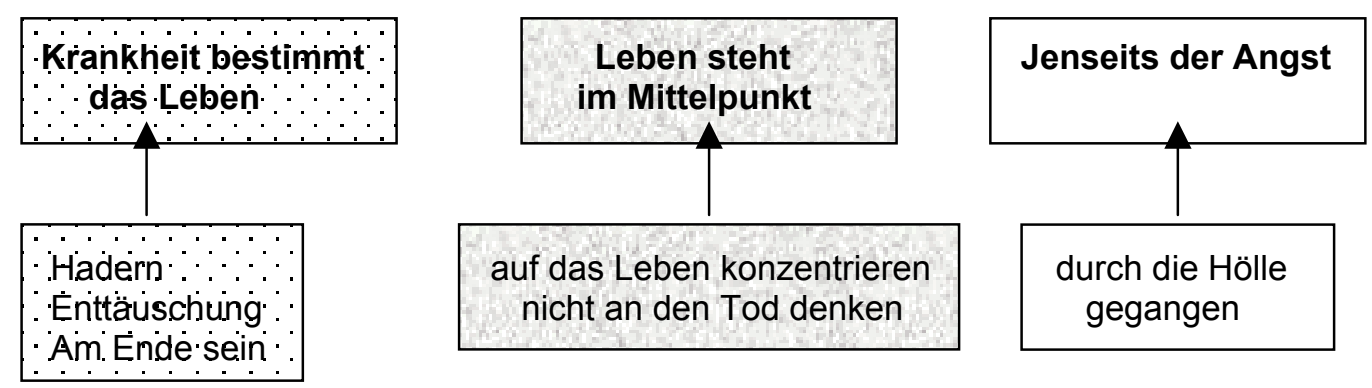

Von den acht Patienten sind fünf überwiegend mit ihrer Erkrankung beschäftigt. Sie beschreiben ihr Leben als von der Krankheit bestimmt. Zwei Patienten konzentrieren sich auf „leben“, lassen sich nicht von der Krankheit „unterkriegen“. Einer dieser beiden Patienten war in der Zeit als junger Erwachsener alkoholkrank und hat seine Sucht überwunden. Aus dem Überwinden der Sucht hat er viel an Stärke und Selbstbewusstsein gewonnen. Der zweite Patient war medizinisch schon vor der Therapie „aufgegeben“ worden. Er hat sich mit dem „Schlimmsten“ schon konfrontieren müssen. Die Patientin, die sich als angstfrei beschreibt ist, im wahrsten Sinne des Wortes, durch die 
Hölle gegangen. Die Symptomatik hatte sich so dramatisch verschlechtert, dass eine Nottrauung im Heimatkrankenhaus organisiert wurde. Wider Erwarten verbesserte sich ihr Zustand leicht, so dass sie nach Göttingen verlegt wurde. Sie bringt Verbitterung und Enttäuschung über die erfolglose Therapie zum Ausdruck, wirkt aber gelassen in Bezug auf den vermutlich nahen Tod. Sie hat das Schlimmste schon hinter sich gebracht.

Der Versuch, die unterschiedlichen „Geschichten“ der Patienten mit Rezidiv zusammenzufassen führt zu folgenden Aussagen:

Die prognostische Situation vor der Behandlung wird als ungünstig eingeschätzt und diese Einschätzung wird den Patienten auch vermittelt. Daraus ergeben sich zwei verschiedene Muster. Eine Gruppe bezieht gerade daraus Hoffnung und gibt sich mit wenig Erreichtem zufrieden. Für die andere Gruppe wird diese Einschätzung zur Quelle permanenter Angst, die nicht abgewehrt oder kontrolliert werden kann. Lange bevor das Fortschreiten der Erkrankung klinisch manifest wird, scheinen die Patienten zu ahnen, dass ihre Erwartungen an die Therapie enttäuscht werden. In diesem Zusammenhang soll nochmals auf das Ergebnis der „Physischen Funktion“ bei der psychometrischen Untersuchung hingewiesen werden: Die Rezidivgruppe schätzt vor Beginn der Behandlung ihre „PF“ deutlich schlechter ein, ohne dass diese Einschätzung von den Fremdbeurteilern geteilt wird. Dieses Ergebnis ist deshalb bemerkenswert, weil die „PF“ als wichtiger Prädiktor für die Überlebenszeit gilt. Was genau die späteren Rezidivpatienten zu ihrer negativeren Einschätzung bringt, ist nicht auszumachen. Gemessen an den Verhaltensmerkmalen gibt es diesen Unterschied zur anderen Gruppe nicht.

Ein gemeinsames Beschwerdemerkmal dieser Gruppe ist eine übergroße Erschöpfung, die vor allen anderen Beschwerden genannt wird und auch nach der Entlassung weiter bestehen bleibt.

Eine Hälfte der Patienten leidet sehr unter den Nebenwirkungen der Therapie in der stationären Phase, die andere Hälfte übersteht die Klinikzeit vergleichsweise gut.

Alle reagieren, verständlicherweise, mit Enttäuschung, Mutlosigkeit und Angst auf die Rezidivnachricht. Ganz unterschiedlich ist die weitere psychische Anpassung an den veränderten Zustand. Einzelnen gelingt es, neue Hoffnung zu schöpfen, andere leiden, ohne einen Ausweg zu finden.

Allen gemeinsam ist, dass sie von ihrem sozialen Umfeld gut unterstützt werden und das auch zum Ausdruck bringen. Tragfähige Beziehungen werden zum wichtigsten Halt. Von den wenigen Patienten dieser Stichprobe mit problematischen, konflikthaften Beziehungen ist keiner in der Rezidivgruppe. Das ist sicherlich als Zufallsbefund zu werten. 
Die Zuordnung "traditioneller“ Verarbeitungsstrategien ist für diese Stichprobe schwierig. Am ehesten gelingt die Zuordnung zu den Kategorien emotions- und kognitionsorientiertes Coping. „Fighting spirit“ oder „denial“ bietet sich nur für wenige Patienten dieser Stichprobe an.

\subsubsection{Unterstützung durch das medizinische Personal}

Die Patienten wurden dazu befragt, welche Unterstützungsmöglichkeiten das medizinische Personal hat. Dieser Aspekt ist besonders wichtig, da hier im Sinne einer optimalen Patientenversorgung, zusätzlich Einfluss genommen werden kann.

\begin{tabular}{|l|l|}
\hline \multicolumn{2}{|l|}{ Tabelle 58: Unterstützung durch das Behandlungsteam } \\
\hline Textzitate (Patientennummer) & Kategorie \\
\hline $\begin{array}{l}\text { „"Ich konnte auch oft fragen und war überrascht über das fundierte Wis- } \\
\text { sen. Besonders X, der weiß einfach alles.. alle haben hier ein hohes } \\
\text { Fachwissen. (18) }\end{array}$ & Hohes Fachwissen \\
\hline „Ich habe hier schon ... eine gewisse Kundenorientierung erlebt.“ (32) & Kundenorientierung \\
\hline $\begin{array}{l}\text { "Die Station ist 100\%ig. Das ist gar keine Station, das ist wie eine Fami- } \\
\text { lie ... kein Arzt oder Schwester wird ausfallend... reden auch mal privat } \\
\text { mit einem." (20) }\end{array}$ & $\begin{array}{l}\text { Familiäre Atmo- } \\
\text { sphäre }\end{array}$ \\
\hline $\begin{array}{l}\text { Einzelne haben sich viel Zeit genommen... Das hat mir den Rücken } \\
\text { gestärkt und ich habe mich nicht so alleine gefühlt.“ (1) }\end{array}$ & Zeit für Patienten \\
\hline $\begin{array}{l}\text {.,Die Atmosphäre hier, na wie soll ich das sagen, ist einfach toll. Die } \\
\text { geben sich alle viel Mühe es einem Recht zu machen. Das Pflegeper- } \\
\text { sonal sowieso aber die Ärzte auch.“ (2) }\end{array}$ & $\begin{array}{l}\text { Zugewandte Hal- } \\
\text { tung }\end{array}$ \\
\hline $\begin{array}{l}\text { "Die Atmosphäre und der Umgang sind gut, da braucht sich nichts zu } \\
\text { ändern... Es wird jede Hilfe gewährt. In aller Freundlichkeit.“ (18) }\end{array}$ & Freundlichkeit \\
\hline $\begin{array}{l}\text { "Die Leute reißen sich hier auf gut Deutsch gesagt den Arsch auf. Wer } \\
\text { hier anfängt und meckert, der hat von der Welt noch nicht viel gesehen. } \\
\text { Die gehen ganz selbstlos vor... Und wenn man sich dann meldet, dann } \\
\text { stehen sie auch Gewehr bei Fuß.“ (21) }\end{array}$ & Sofortige Reaktion \\
\hline $\begin{array}{l}\text { „Ich schätze, das hier ein höherer Zeitaufwand in Patientengespräche } \\
\text { investiert wird.“ (26) }\end{array}$ & Zeit für Gespräche \\
\hline $\begin{array}{l}\text { "Das Pflegepersonal ist sehr nett und zurückhaltend. Die bedrängen } \\
\text { einen nicht, sind aber sofort da, wenn was ist. Und helfen. Die Ärzte } \\
\text { sind o.k.“ (22) }\end{array}$ & $\begin{array}{l}\text { Zurückhaltung } \\
\text { Empathie }\end{array}$ \\
\hline $\begin{array}{l}\text { "Meine Hausärztin hat mir unheimlich geholfen. Sie hat mich sehr gut } \\
\text { aufgeklärt und mit mir über meine Angst gesprochen." (4) }\end{array}$ & Gute Aufklärung \\
\hline
\end{tabular}

Liest man die Äußerungen, so wird deutlich, was Patienten wünschen: Achtung, Rücksicht, Zeit, individuelles Eingehen, Höflichkeit, Verfügbarkeit in Notsituationen, eine Beziehung, die etwas „mehr“ an Nähe ermöglicht („auch mal was Privates austauschen können“). All das macht atmosphärisch ein Klima aus, in dem man gesunden kann.

Zusammenfassend sind zwei Aspekte therapeutischer Kompetenz auszumachen, die von Patienten genannt werden: 

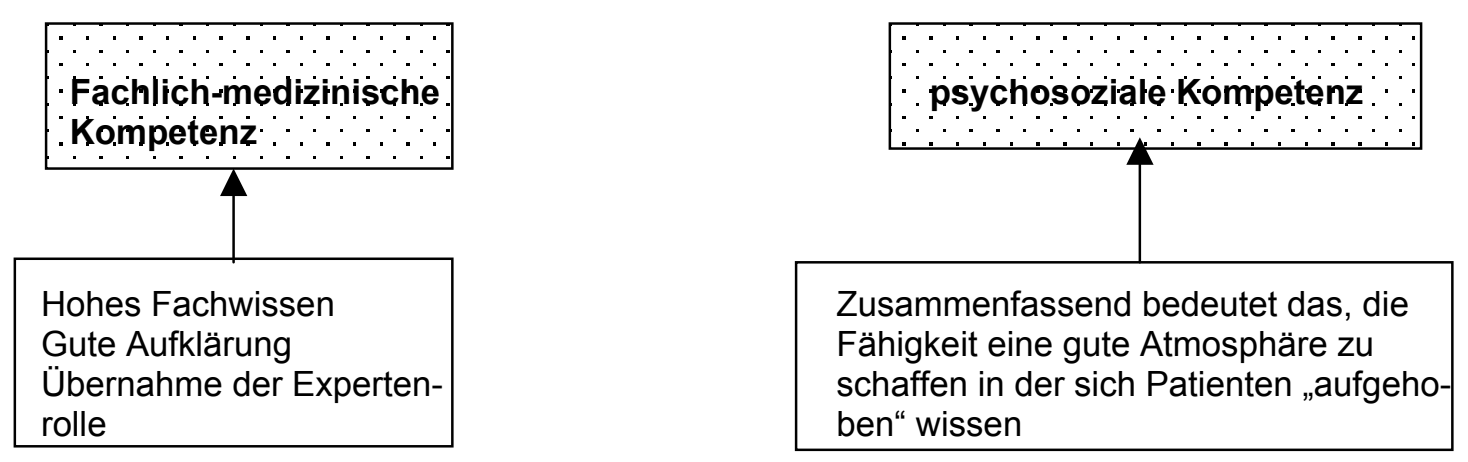

Zur fachlichen Kompetenz gehört neben dem Fachwissen auch eine „handwerkliche“ (instrumentelle) Kompetenz. Der Wunsch danach kommt indirekt zum Ausdruck, wenn Patienten davon sprechen, dass sie z. B. einen „fertigen“82 Arzt wünschen, der ihnen Braunülen legt. Dieser Aspekt wurde von mehreren Patienten hervorgehoben.

Geht man von der Wichtigkeit der beiden Komponenten aus, so liegt das Übergewicht bei der psychosozialen Kompetenz. Zu den unterschiedlichen Befragungszeitpunkten wird in fast allen Interviews auf die Atmosphäre, vermittelt durch die Beziehung zu den handelnden Personen, Bezug genommen. Positiv erwähnt wurden einige Besonderheiten, die als „unüblich“ erlebt wurden:

- eine Organisation mit weniger rigide Zeitplänen (morgens länger schlafen können)

- die Lage der Station (unmittelbar aus den Zimmern in den Park gehen können)

- das Angebot des Pflegepersonals, sich zu Duzen (macht den familiären, symmetrischen Beziehungsaspekt deutlich)

Im letzten Teil des Kapitels erfolgt eine Bewertung der HDC durch die Patienten und die Äußerungen zur Behandlungszufriedenheit werden wiedergegeben.

\subsubsection{Bewertung der Therapiebelastungen durch die Patienten}

In diesem Abschnitt der Interviewauswertungen sollen die Ergebnisse der Fragen, die sich direkt oder indirekt mit der aktuellen oder retrospektiven Bewertung der Therapie befassen, zusammengestellt werden. Die Antworten sind den drei letzten Interviews entnommen.

82 „fertig“ ist hier im Sinne von fertig ausgebildet gemeint. Unerfahrene Ärzte in Ausbildung oder Studenten sollen an weniger belasteten Patienten „üben“. 


\subsubsection{Soll anderen Patienten diese Therapie angeraten werden?}

Die Frage nach dem „Rat" an andere Patienten soll sowohl die Reflexion der eigenen Belastungserfahrungen als auch eine implizite Bewertung des Nutzens der Therapie einschließen.

\begin{tabular}{|c|c|c|}
\hline Textzitate (Patientennummer) & Kategorie & $\mathrm{N}=30 \quad(\%)$ \\
\hline $\begin{array}{l}\text { "Ich nicht. Nur experimentell, ohne die Überzeu- } \\
\text { gung, dass es deutlich besser ist, da würde ich es } \\
\text { lassen." (14) }\end{array}$ & Abraten & (7) \\
\hline „Ja, wenn man jung ist.“ (24) & $\begin{array}{l}\text { Mit Einschränkungen } \\
\text { zuraten }\end{array}$ & $(16)$ \\
\hline $\begin{array}{l}\text { "In jedem Fall machen. Es ist eine große Chance." } \\
(22)\end{array}$ & Bedingungslos zuraten & $(50)$ \\
\hline $\begin{array}{l}\text { "Ich möchte auf keinen Fall mit jemandem sprechen, } \\
\text {...wüsste nicht, was ich empfehlen soll." (13) }\end{array}$ & Keinen Rat erteilen & $(26)$ \\
\hline
\end{tabular}

Mehr als die Hälfte der Patienten würden, ohne Einschränkungen, anderen Patienten zuraten. Ein weiteres Drittel würde zuraten, wenn es keine anderen Möglichkeiten gibt, wenn man jung ist usw. Nur zwei Patienten würden definitiv abraten. Von diesen beiden hat einer ein Rezidiv erlitten, die andere Patientin ist zu diesem Zeitpunkt krankheitsfrei d.h. bis zu diesem Zeitpunkt ist die Behandlung erfolgreich gewesen. Der Grund, warum sie nachträglich die Behandlung in Frage stellt, findet sich sowohl in ihren Interviewäußerungen als auch im Fragebogenscreening: bei der Patientin liegt eine behandlungsbedürftige depressive Störung vor. Auch die meisten Patienten, bei denen die Therapie nicht erfolgreich war, würden zuraten. Die Therapie wird als Chance gesehen, deren Ausgang nicht vorhersehbar ist. Drei Patienten mit Rezidiv würden zuraten, aber mit der Einschränkung: „unter günstigeren Bedingungen“. Welches günstige Bedingungen sind, wurde nicht näher spezifiziert. Wie zu erwarten, würde die Mehrzahl der Patienten ohne Rezidiv bedingungslos zuraten, da die Therapie als erfolgreich eingestuft wird. Der größte Teil der Unentschiedenen hat kein Rezidiv. Hier scheinen die als sehr schwerwiegend erlebten Behandlungsbelastungen und die Behandlungsfolgen ausschlaggebend zu sein. Immerhin ein Drittel der Patienten, nimmt man diejenigen die abraten und diejenigen, die unentschieden sind zusammen, stellt die frühere Entscheidung zumindest ansatzweise in Frage. 


\subsection{4 Übereinstimmung der erwarteten Belastungen mit den tatsächlich erleb- ten Belastungen}

Die Diskrepanz zwischen den phantasierten erwarteten Belastungen und den tatsächlichen sollte mit der Frage: Waren die Belastungen so, wie sie sich das vorgestellt haben? erfasst werden.

Diese Frage ist nicht nur interessant im Hinblick auf die Erfahrungen der Patienten, sie wirft auch indirekt ein Licht auf die Informationen, die von den Patienten vor der HDC wahrgenommen wurden.

\begin{tabular}{|c|c|c|}
\hline \multicolumn{3}{|l|}{ Tabelle 60: Belastungserwartungen } \\
\hline Textzitate (Patientennummer) & Kategorie & $\mathrm{N}=34(\%)$ \\
\hline $\begin{array}{l}\text { "Nein, habe es mir noch schlimmer vorgestellt. Es ist } \\
\text { für Laien ja auch unbegreiflich, wenn er hört, Sie } \\
\text { haben dann keine Leukos mehr. Ich dachte, das sind } \\
\text { doch wichtige Lebensbestandteile, in der Zeit kannst } \\
\text { du ganz schnell sterben." (3) }\end{array}$ & Nicht so schlimm & $\begin{array}{ll}21 & (71)\end{array}$ \\
\hline $\begin{array}{l}\text { "So waren meine Erwartungen ähnlich wie das, was } \\
\text { ich hier erlebt habe..." (10) }\end{array}$ & Wie erwartet & (9) \\
\hline $\begin{array}{l}\text { "Aber dass es so schlimm ist, kann man sich kaum } \\
\text { vorstellen." (27) }\end{array}$ & Schlimmer & $(18)$ \\
\hline „Konkret konnte ich mir vorher nicht vorstellen...”(13) & Keine Vorstellung & (2) \\
\hline
\end{tabular}

Die Tatsache, dass die Mehrheit der Patienten schlimmere Belastungen erwartet hat, ist ein wichtiger Aspekt zur Beurteilung der Angemessenheit der Aufklärung. Dass eine weniger Angst erzeugende „Art“ der Aufklärung erreicht werden kann, macht die Äußerung einer Patientin deutlich: „Die allerschlimmste Befürchtung haben Sie mir durch das Gespräch neulich schon genommen. Dadurch habe ich wieder Boden unter die Füße bekommen“ (15). Ein anderer Patient formuliert, dass man „sich einige Sorgen und Ängste (hätte) ersparen können" (12). Eine noch ausführlichere Aufklärung, der Hinweis auf die bei vielen Patienten auftauchenden Todesängste und eine Relativierung eben dieser Ängste (mit Hinweisen auf die sehr geringe Mortalität) könnte eine erhebliche Erleichterung für die Patienten bedeuten.

\subsubsection{Retrospektive Beurteilung der Belastungen}

Im Rückblick wird die Belastung durch die HDC gravierender eingeschätzt als unmittelbar nach der Behandlung. Die Patienten verwenden unterschiedliche Metaphern und Beschreibungen um die Therapie zu charakterisieren: 


\begin{tabular}{|c|c|}
\hline \multicolumn{2}{|l|}{ Tabelle 61: Charakterisierung der Behandlungsbelastungen } \\
\hline Textzitate (Patientennummer) & Kategorie \\
\hline $\begin{array}{l}\text { "Es ist schon die aggressivste Therapie, die es heute gibt. War } \\
\text { schon hart, auf alle Fälle happig, obwohl ich gut durchgekom- } \\
\text { men bin." (5) }\end{array}$ & Aggressiv \\
\hline $\begin{array}{l}\text { "Sowieso, wenn ich an die Station da unten denke, da krieg ich } \\
\text { eine Gänsehaut. Da wird mir richtig kalt. Das ist so geblieben, } \\
\text { das hat sich nicht verändert...Wenn ich ein paar Treppen hoch } \\
\text { gehe auf die andere Station, wird es wärmer. ..Vielleicht ist mir } \\
\text { damals nicht bewusst gewesen, wie dreckig es mir eigentlich } \\
\text { ging. Da will ich nicht wieder hin." (6) }\end{array}$ & Unheimlich \\
\hline $\begin{array}{l}\text { "Also, dass wünsche ich meinem ärgsten Feind nicht! Obwohl, } \\
\text { mir ging es ja gut. Kein kaputter Mund, konnte meine Pizza } \\
\text { essen, kein Fieber. Aber ich kann schon verstehen, dass die } \\
\text { Station hier unten ist und nicht im 6. Stock. Da würde ich auch } \\
\text { aus dem Fenster springen." (10) }\end{array}$ & $\begin{array}{l}\text { Schlimmste Strafe } \\
\text { (Selbst-) mörderisch }\end{array}$ \\
\hline „Lieber nicht, will nicht so viel erinnert werden“ (7) & $\begin{array}{l}\text { So schlimm, dass sie } \\
\text { ausgeblendet werden } \\
\text { müssen }\end{array}$ \\
\hline "....es war doch eine ganz schöne Bombe..." (12) & Bombe \\
\hline „Die Belastungen waren extrem...“ (33) & Extrem \\
\hline $\begin{array}{l}\text { "Es war schon eine harte Sache. Manchmal wusste ich nicht, ob } \\
\text { ich weiter machen kann." (16) }\end{array}$ & $\begin{array}{l}\text { Hart } \\
\text { Fast nicht zu schaffen }\end{array}$ \\
\hline „Es war die Hölle.“ (13) & Hölle \\
\hline
\end{tabular}

Keine der Patientinnen und keiner der Patienten äußert zu T5, dass es eine "leichte“ Behandlung war. Die „Höllenmetapher“ wird rückblickend von mehreren Patientinnen und Patienten zur Beschreibung der durchlebten Erfahrungen verwendet. Einige äußern auch, dass sie eine solche Behandlung ein zweites Mal nicht schaffen würden. Wie gravierend die Belastungen waren und welche physischen Extremsituationen durchzustehen waren, machen die Interviewäußerungen deutlich.

\subsubsection{Behandlungszufriedenheit}

Behandlungszufriedenheit ist einer der Faktoren, der dazu beiträgt, schwerwiegende Therapiebelastungen erträglich zu machen. Patienten, die nicht zufrieden sind, sich schlecht behandelt fühlen, kein Vertrauen haben und deren Beziehung zum Behandlungsteam konflikthaft ist, werden in ihren Bewältigungsanstrengungen erheblich beeinträchtigt. Dieses Postulat, das für klinische Beobachter offensichtlich ist, wird von der empirischen Fragebogenforschung nicht bestätigt. ${ }^{83}$

${ }^{83}$ Behandlungszufriedenheit wird in dieser Arbeit implizit thematisiert. Es wird nicht wie in der Zufriedenheitsforschung üblich ein Fragebogen verwendet. Auf die Problematik dieser Forschung verweisen Jacob \& Bengel (2000) in ihrer Arbeit: „Das Konstrukt Patientenzufriedenheit: eine kritische Bestandsaufnahme“. Fragebogenuntersuchungen ergeben stets extrem hohe Zufriedenheitswerte. „Grundsätzlich werden Zufriedenheitsitems von mehr als $80 \%$ aller Patien- 
Die Frage der Zufriedenheit ist, aus der Sicht des Krankenhauses, ein wichtiges Bewertungskriterium im Prozess der Qualitätssicherung.

Die in der folgenden Tabelle aufgeführten Häufigkeiten sind den Interviewtexten entnommen, in denen explizit oder implizit über Behandlungszufriedenheit gesprochen wurde. Auffällig ist, dass die Zufriedenheitsbeschreibungen der einzelnen Patienten über die Zeitpunkte relativ konstant sind. Die überwältigende Mehrheit ist zufrieden und bewertet gelegentlich mit „eingeschränkt zufrieden“. Nach der Entlassung wird retrospektiv die Behandlungszufriedenheit bewertet. Das Urteil fällt etwas ungünstiger aus.

\begin{tabular}{|c|c|c|c|}
\hline \multicolumn{4}{|c|}{ Tabelle 62: Behandlungszufriedenheit } \\
\hline Kategorie & T2 & T3 & $\mathrm{T} 4$ \\
\hline & $\mathrm{N}$ & $\mathrm{N}$ & $\mathrm{N}$ \\
\hline Sehr zufrieden & 9 & 8 & 5 \\
\hline Zufrieden & 23 & 20 & 17 \\
\hline $\begin{array}{l}\text { Mit Einschrän- } \\
\text { kungen zufrieden }\end{array}$ & 5 & 11 & 9 \\
\hline Nicht zufrieden & 1 & 1 & - \\
\hline
\end{tabular}

(Mehrfachnennungen kommen dadurch zustande, dass Patienten in einzelnen Fällen zwischen ärztlicher und pflegerischer Behandlung unterscheiden)

Mit einer Ausnahme waren alle (!) Patientinnen und Patienten während der stationären Zeit sehr zufrieden/zufrieden/eingeschränkt zufrieden mit der Behandlung. Sowohl mit der ärztlichen als auch mit der pflegerischen Seite. Generell waren die Äußerungen der Patienten sehr differenziert: Kritik an Abläufen und Strukturen, am Klinikessen und aufgetretene Konflikte mit einzelnen Mitarbeitern hatten keinen Einfluss auf die positive Gesamtbeurteilung.

\begin{tabular}{|l|l|}
\hline Tabelle 63: Gründe für Zufriedenheit \\
\hline Textzitate (Patientennummer) & Kategorie \\
\hline „Die Atmosphäre hier unter den Ärzten der Abteilung ist sehr gut.“ (8) & Sehr zufrieden \\
\hline „Alle haben Zeit zum Gespräch...." (7) & zufrieden \\
\hline „Mit denen (Ärzten) habe ich nicht so viel Kontakt... ist mir peinlich.“ (7) & $\begin{array}{l}\text { Eingeschränkt } \\
\text { zufrieden }\end{array}$ \\
\hline "Pflegerisch fand ich nicht so gut dass, ... (13) & Nicht zufrieden \\
\hline
\end{tabular}

Als wesentliche Kriterien für die Zufriedenheit wurden hervor gehoben: ärztliche Kompetenz, schnelle Verfügbarkeit in Krisensituationen, persönlicher und interessierter Umgang und die Kompetenz des Personals, mehr Zeit als üblich für Gewerden dagegen mehr kritische Aspekte genannt (Fitzpatrick, 1991, zitiert nach Jacob \& Bengel ebenda, S. 287). 
spräche und die gute Stationsatmosphäre. Die Ergebnisse bestätigen die klassischen Komponenten „medizinisches Können“ und „Therapeut-Patient-Beziehung“ (Jacob \& Bengel 2000).

Mehr Kritik wurde geäußert zu den Zeitpunkten T4/T5, nach der Entlassung. Diese Kritik bezieht sich auf die Erfahrungen mit der Nachsorge:

\begin{tabular}{|l|l|}
\hline \multicolumn{2}{|l|}{ Tabelle 64: Kritische Anmerkungen zur Nachsorge } \\
\hline \multicolumn{2}{|l|}{ Textzitate (Patientennummer) } \\
\hline $\begin{array}{l}\text { "Das war insofern schlimm, weil jedes Mal ein anderer Arzt da ist. Diesen } \\
\text { hatte ich noch nie gesehen ...hab auch das Gefühl, der hat die Akte gar } \\
\text { nicht gelesen." (11) }\end{array}$ & Arztwechsel \\
\hline $\begin{array}{l}\text { "Ich war bisher nur einmal in Göttingen. Es war so frustrierend, dass ich das } \\
\text { eigentlich nicht wiederholen wollte." (32) }\end{array}$ & Keiner zuständig \\
\hline $\begin{array}{l}\text { "Gehe nicht mehr nach Göttingen... Wissen Sie, hier (beim niedergelasse- } \\
\text { nen Arzt) erfahre ich sofort das Ergebnis. Das ist der Hauptgrund für den } \\
\text { Wechsel. Die Wartezeiten in der Uni sind wirklich Nerven zerreibend.“ (3) }\end{array}$ & Spätes Ergebnis \\
\hline $\begin{array}{l}\text { "Mit den Akten das funktioniert manchmal nicht so ganz gut. Die sind dann } \\
\text { einfach verschwunden und keiner fühlt sich zuständig." (17) }\end{array}$ & $\begin{array}{l}\text { Verschwundene } \\
\text { Akten }\end{array}$ \\
\hline
\end{tabular}

Die wichtigsten Kritiken beziehen sich auf wechselnde Ärzte, Wartezeiten generell und Warten auf Befunde, verschwundene Akten (werden von mehreren Patienten erwähnt), lange Zeiten bis die Hausärzte informiert werden usw., also im wesentlichen auf organisatorische Probleme, die sich aus der Struktur einer Universitätsklinik ergeben können. Vor allem Arztwechsel, der in dieser Stichprobe für die Nachsorgezeit als Kritikpunkt genannt wird, kann, wie auch andere Studien belegen, als wichtiger Prädiktor für Patientenzufriedenheit einerseits und als Prädiktor für einen Arztwechsel andererseits gelten. Negative Erfahrungen mit dem medizinischen System, die ebenfalls als Determinanten von Zufriedenheit genannt werden (schlechte Erfahrungen bedingen weitere schlechte Erfahrungen und wirken sich negativ auf Zufriedenheit aus), lassen sich für diese Stichprobe nicht bestätigen. Im Gegenteil, einige Patienten, die vorherige negative Erfahrungen beschreiben, sind positiv überrascht in Bezug auf die Versorgung, die sie in der stationären Phase erleben.

Im Hinblick auf organisatorisch verursachte Probleme werden auch andere Erfahrungen gemacht. Einzelne engagierte Ärzte/Teammitarbeiter können durch Engagement und Aufmerksamkeit strukturelle Probleme ausgleichen: 


\begin{tabular}{|l|l|}
\hline Tabelle 65: Positive Erfahrungen in der Nachsorge \\
\hline \multicolumn{2}{|l|}{} \\
\hline Textzitat (Patientennummer) \\
\hline $\begin{array}{l}\text { "Dr. X ist die zentrale Stelle (Klinik), wo alle Untersuchungen zusammen- } \\
\text { laufen." (18) }\end{array}$ & Ansprechpartner \\
\hline $\begin{array}{l}\text { "Einmal alle vierzehn Tage gehe ich zu meinem Hausarzt... Der hat auch } \\
\text { schon Kontakt gehabt zu Dr. X von Station als es mir so schlecht ging. } \\
\text { Also das läuft ganz gut." (20) }\end{array}$ & $\begin{array}{l}\text { Kooperation mit } \\
\text { Hausarzt }\end{array}$ \\
\hline
\end{tabular}

Diese Erfahrungen sind nicht die Regel. Fünf Patienten äußern sich kritisch über die Nachsorge durch den Hausarzt. 9 äußern sich kritisch über die Nachsorge in der Klinik. Vier Patienten erwähnen ausdrücklich die hohe Kompetenz ihres niedergelassenen Arztes, sieben ausdrücklich die der Klinikärzte. Trotz der Kritiken bleibt der größte Teil der Patienten wegen der vorausgesetzten höheren fachlichen Kompetenz in einer Universitätsklinik auch zur Nachsorge in den entsprechenden Abteilungen.

Den Abschluss der Darstellung der Interviewergebnisse bilden drei ausgewählte Kasuistiken.

\subsubsection{Kasuistiken}

Um die Integration verschiedener Aspekte zu verdeutlichen, werden an dieser Stelle Patientenbeispiele vorgestellt. Für diesen Teil hat sich die Frage gestellt, nach welchen Gesichtspunkten Ausschnitte aus Lebensgeschichten ausgewählt werden sollten. Die Einzigartigkeit und das „Ungewöhnliche" trifft auf jeden einzelnen der Patienten dieser Stichprobe zu. Die Orientierung am Kontrast kann sich prinzipiell auf die verschiedensten, in der Studie untersuchten Aspekte beziehen. Im Hinblick auf das übergeordnete Thema „Lebensqualität“ wurde folgende Auswahl getroffen:

Zwei Patienten der Gruppe „ohne Rezidiv“ wurden ausgewählt nach dem Kriterium des höchsten/niedrigsten LQ-Wertes zu T5 (Patientinnen 15 und 16) im QLQ-C30, also danach, wie die Patientinnen selber ihre LQ bewerten. Das die Wahl auf zweit Patientinnen gefallen ist, war nicht beabsichtigt, ist aber, wegen der Extremwerte, auch kein Zufall.

Eine dritte Kasuistik wurde ausgewählt aus den Patienten der Rezidivgruppe: Die Geschichte der Patientin (33) erscheint auf den ersten Blick „extrem“ im Hinblick auf Erkrankungs- und Therapieverlauf. Trotzdem lässt sich anhand dieser Geschichte etwas demonstrieren, das in der klinischen Praxis typisch ist. ${ }^{73}$

\footnotetext{
${ }^{73}$ Einschränkend soll hinzugefügt werden, dass hier die klinische Praxis einer Universitätsklinik gemeint ist, also einer Einrichtung der Maximalversorgung, von der sich die Patienten mehr Therapiemöglichkeiten erhoffen.
} 
Zum Überblick die verschiedenen Verläufe der QL-Werte im Vergleich mit dem Gesamtmittelwert der Gruppe ohne Rezidiv:

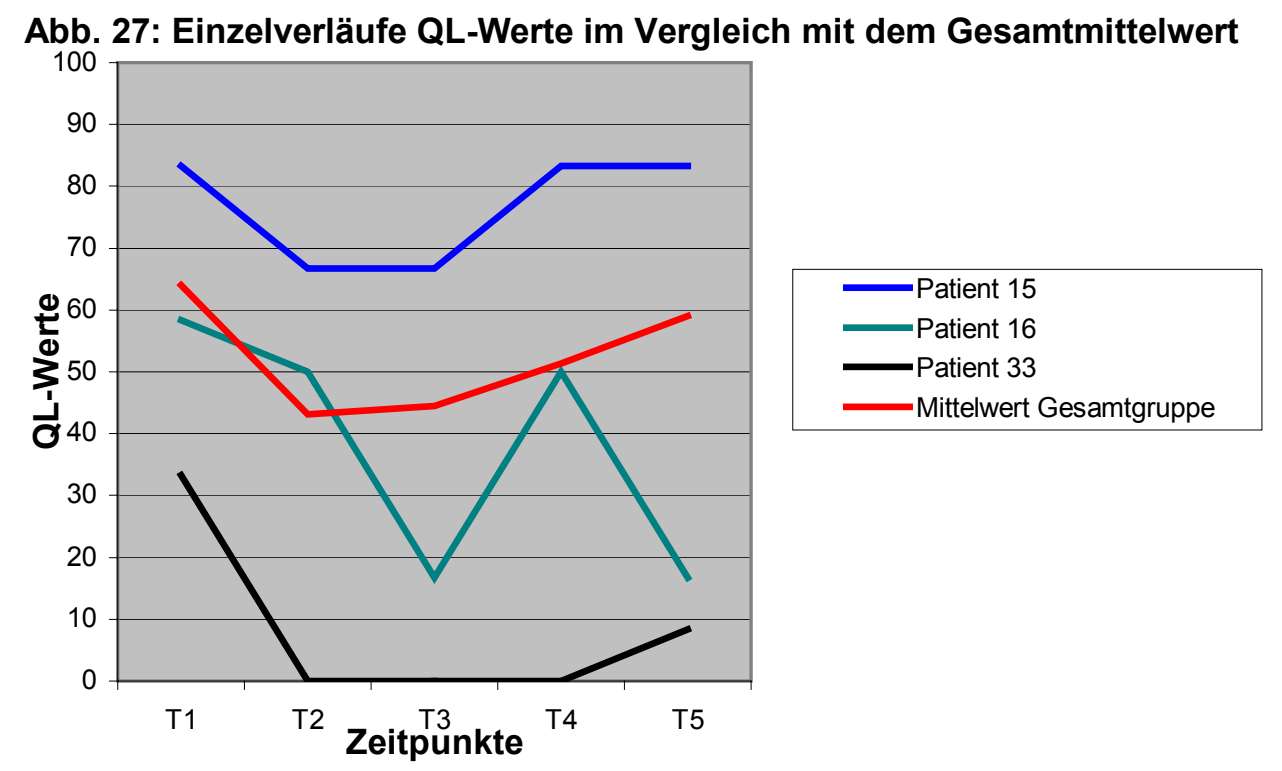

Die Kasuistiken orientieren sich an den folgenden Gliederungsgesichtspunkten:

1. Persönliche/familiäre Situation

2. Krankheitsanamnese

3. Belastungen durch die HDC und Zustand nach einem Jahr

4. Ressourcen

5. Kommentar

\section{Kasuistik der Patientin 15}

Die Patientin ist Anfang fünfzig, verheiratet und hat zwei erwachsene Töchter, eine der Töchter lebt noch zu Hause. Wegen der Kinder war sie eine Zeit zu Hause, hat sich dann weitergebildet und hat seit einigen Jahren eine anspruchsvolle Ganztagsstelle als Klinikssekretärin. Ihr Mann ist ebenfalls berufstätig, hat aber alle Freiheiten, sie bei Bedarf zu unterstützen und sie in der Klinik zu besuchen. Die Patientin hatte mehrere schwere Lebensereignisse zu verkraften: ihr 12jähriger Neffe ist durch einen Unfall zu Tode gekommen und ihr Schwiegervater hat sich vor Monaten erhängt. Die Mutter der Patientin macht ihr Sorgen, sie kann mit der Erkrankung ihrer Tochter nicht umgehen, weint häufig und signalisiert ihre Überforderung. Die Patientin erwähnt, dass ihre Mutter die eigene Mutter bereits im Alter von 11 Jahren verloren hat. Als die Patientin 18 Jahre alt war ist ihre 39jährige Tante sehr plötzlich an einem Gehirnschlag verstorben. 
Verluste von Menschen im Verwandtenkreis durchziehen als „roter Faden“ ihre Lebensgeschichte

Die Patientin hatte den Knoten in ihrer Brust selber entdeckt. Der erste behandelnde Arzt hat sie sofort von der Diagnose Mammakarzinom in Kenntnis gesetzt. Der Knoten war so groß, dass eine Amputation notwendig wurde. Die Induktionstherapien haben ihr sehr große Probleme bereitet. Sie schildert ausführlich ihre schrecklichen Erfahrungen mit der Chemotherapie: Kreislaufprobleme und andauernde Übelkeit hat sie in schlimmster Erinnerung. Zwei der vier Therapien waren sehr qualvoll mit tagelangem Erbrechen. Die Therapie wurde aus diesem Grunde in der Dosierung reduziert. Vor der Aufnahme zur HDC wurde sie vom behandelnden Arzt wegen ihrer ausgeprägten Ängste vorgestellt. Sie fürchtet an der Therapie sterben zu können und sie fürchtet, dass die Chemotherapie ihr wieder so zu schaffen macht.

Die HDC verkraftet die Patientin ausgesprochen gut. Sie kann aufstehen, macht draußen ihre Spaziergänge und kann unbeeinträchtigt essen. Ihre Ängste sind deutlich weniger geworden. Die Patientin schläft gut, sie geht mit Mundschutz auf den Flur und sucht den Kontakt zu anderen. Die Patientin fühlt sich geschwächt und müde, aber es geht ihr gut. Sie ist in der Lage zu lesen, fern zu sehen und auch zu handarbeiten. Die Konzentration reicht zwar immer nur für eine Stunde, aber sie ruht sich eben wieder aus. Die früheren Grübeleien sind nicht mehr notwendig. Die Zeit nach der Entlassung bewältigt sie mit Hilfe der Familie ohne Probleme. Sie muss viel ausruhen, hat aber den Haushalt so geplant, dass sie allein zu Recht kam. Nach zwei Treppen war eine Ruhepause nötig, und sie hat sich nur in der ersten Woche mittags hingelegt. Auch die anschließende Strahlentherapie hat ihr keine Mühe bereitet. Nach einem Jahr beschreibt sie ihren Zustand so: „Ich habe körperlich nicht die Belastbarkeit wie früher erreicht. Nach 5 Stunden Arbeit bin ich völlig erschöpft...Seelisch geht es mir ganz gut, bis auf einige Tiefs." Die Patientin arbeitet wieder, allerdings auf einem weniger anstrengenden Arbeitsplatz. In der Rehabilitation hat sie, trotz ihrer Bitten, keine Möglichkeit zu psychologischer Unterstützung erhalten. Ihre Ärztin hat ihr eine psychoonkologische Therapie wegen ihrer Ängste und Grübeleien vorgeschlagen. Im Rückblick bezeichnet sie die Therapie als „heftig“, am schlimmsten waren die Ängste.

Die Patientin ist ausgesprochen angenehm, freundlich, offen, voll des Lobes und auf der Station sehr beliebt. Sie hat in ihrer Familie einen sicheren Rückhalt. Freundschaften haben sich verfestigt, auch wenn es einzelne negative Beispiele von Rückzug gegeben hat, die kränkend und enttäuschend waren. Ihr Mann kommt jeden Tag nach der Arbeit zu Besuch. Die Patientin hat sich Lesestoff und Handarbeiten mit- gebracht, um sich „ein wenig zu Hause“ ins Krankenhaus zu holen. Die Patientin äußert sich ausgesprochen positiv zu allen Aspekten der Krankenhauszeit (bis auf das Essen und das 
harte Toilettenpapier). Sie sagt von sich selbst, dass ihre „Selbstzufriedenheit“ die größte Hilfe im Umgang mit der Situation ist. Durch die gesamte Zeit zieht sich die Frage, „wie geht es weiter?...Bin ich auf ewig geheilt?" Die Patientin hat phasenweise große Mühe, ihre Ängste unter Kontrolle zu halten: „Ausgerechnet abends, wenn wir ins Bett gehen, dann fangen meine Grübeleien wieder an...Und warum gerade ich? Für meinen Mann ist das natürlich auch nicht einfach, der tröstet mich dann zwar, aber nimmt die Sorgen doch mit in die Nacht." Die Patientin hatte große Probleme mit dem veränderten Aussehen durch die Amputation. Der Kontakt mit anderen Betroffenen in der Rehabilitation hat ihr geholfen. Wieder zu Hause, fühlt sie sich isoliert, sie vermeidet es, ihrer Gymnastikgruppe von ihrer Scheu sich ihnen zu zeigen, zu berichten und zieht sich stattdessen zurück. Nach ihrer Ansicht hat sich im Alltag nicht viel verändert. Allerdings denkt sie nicht mehr nur an die Arbeit, kann Dinge liegen lassen und ist in der Lage mit ihrem Mann spontan angenehme Dinge zu tun. Diesen Aspekt von Veränderung bewertet sie positiv. Ihr Fazit: „Es ist schon viel Arbeit mit all dem seelisch fertig zu werden. Ja, die Sorgen sind schwer abzuschütteln. Aber es hat sich schon geändert und ist in diesem Jahr besser geworden. Zurzeit habe ich die Grübeleien nur ein bis zweimal im Monat."

Wie in der Abbildung zum Ausdruck kommt, zeigt die Patientin eine hohe Zufriedenheit, deutlich über dem Durchschnitt in Bezug auf den "QL-Wert“. Bei dieser Patientin scheint es so, als wäre ihr Urteil konstant und, außerhalb der Kliniksphase, entkoppelt von Beschwerden. Zu den Zeitpunkten nach der HDC fällt ihr Urteil zwar schlechter aus, liegt aber immer noch deutlich über dem Durchschnittswert. Die Interviews erwecken keineswegs den Eindruck, als würde es der Patientin sehr gut gehen. Ihre Ängste, gerade auch auf dem Hintergrund ihrer Lebensgeschichte, die geprägt ist von Verlusten, stehen zu allen Zeitpunkten im Vordergrund. Sie hat allerdings auch die Erfahrung gemacht, dass Gespräche über die Angst, sowohl mit der Psychotherapeutin als auch mit anderen Betroffenen, Angst mindernd wirken und steht deshalb einer Psychotherapie offen gegenüber. Die schlechtere "QL" in der stationären Phase steht im Gegensatz zu dem, was sie im Interview zum Ausdruck bringt: vor der Behandlung ging es ihr am schlechtesten, sie hatte schreckliche Angst. Sie ist ausgesprochen positiv überrascht, dass die HDC mit weniger einschneidenden Belastungen verbunden war als die Chemotherapien vorher. In der Tat war bei dieser Patientin die HDC ausgesprochen glimpflich verlaufen. Keine andere Frau mit Mammakarzinom konnte die ganze Zeit spazieren gehen und wurde so wenig von Symptomen geplagt. Bei ihr liegt die Vermutung nahe, dass die schlechtere Bewertung der „QL“ in der stationären Phase eine sozial erwünschte Antwort darstellt. Die drei anderen Bewertungen sind konstant. Durch ihre Äußerung von der harten seelischen Arbeit bringt sie deutlich zum 
Ausdruck, dass trotz des Eintauchens in die Normalität noch viel Kraft benötigt wird, um Sorgen und Grübeleien unter Kontrolle zu halten.

\section{Kasuistik der Patientin 16}

Die Patientin ist fünfzig Jahre, sie hat zwei erwachsene Kinder und zwei Enkelkinder. Aus „Dummheit“ ist sie mit 19 Jahren schwanger geworden. Vom Vater ihrer Kinder ist sie geschieden worden. Sie berichtet davon, dass sie sich in den letzten Jahren viel mit Sterbebegleitung beschäftigen musste: Ihre Mutter und ihre Schwester, ihr geschiedener Mann und ihr späterer Partner sind in ihren Armen gestorben. Deswegen konnte sie ihren Beruf als Altenpflegerin nicht mehr ausüben, hat umgeschult und ist Busfahrerin geworden. Sie kümmert sich auch um ihren alten Vater, der 78jährig noch sein Geschäft leitet. Sie lebt allein, wünscht sich allerdings eine andere Wohnsituation mit einem Partner. Ein Versuch ist, wegen der Krankheit ihres Katers, gescheitert: „Mein Kater ist mein Partner. Das ist schon pervers, dass wir Menschen uns auf solche intensiven Beziehungen mit Tieren einlassen, statt mit Menschen zusammenzusein." Die Erkrankung hat sie in große finanzielle Nöte gestürzt, die sie sehr belasten.

Die Patientin leidet an einem NHL Ilb. Durch die Vorbehandlung mit Chemotherapie ist sie in eine komplette Remission gekommen. Nach einem Widerauftreten wurde ihr HDC vorgeschlagen. Sie hat sehr unter Übelkeit nach der HDC gelitten, dann standen heftigste Schmerzen im Vordergrund (Hals, After). Die Patientin musste einige Tage mit einem Morphinperfusor versorgt werden. „...das habe ich mir nicht so schrecklich vorgestellt“. Es gab außerdem eine Episode mit hohem Fieber und Schüttelfrost, „das war ein solches Elend, das kann man sich gar nicht vorstellen... Rotz und Wasser habe ich geheult... Ohne Medikamente würde ich es nicht aushalten." Die Erholungszeit nach der Entlassung hat nach ihren Vorstellungen sehr lange gedauert. Finanzielle Sorgen bestimmen nach wie vor ihren Alltag. Nach drei Monaten kommen Geschmack und Appetit langsam wieder. Nach einem Jahr funktioniert sie beruflich wieder „voll“. Nach der Arbeit ist sie „platt“. Sie hat wieder begonnen Sport zu treiben (walking, joggen, skaten).

Die Patientin ist offen und angenehm im Umgang. Ihre „depressive“ Beziehungsgestaltung ist davon geprägt, niemals zu viel zu verlangen, sich zu bedanken und sich für alles mögliche zu entschuldigen. In der Klinik ist das mit Sicherheit von Vorteil, sie ruft keine Konflikte hervor, sie ist beliebt und wird vom Personal umsorgt. Im Privatleben und im Beruf sieht das allerdings anders aus. Sowohl mit dem Arbeitgeber als auch mit einer Kollegin gibt es heftige Konflikte, in denen sie anfangs in eine „Opferrolle“ gerät, die sie kränkt und viel Energie kosten. Den Konflikt mit dem Arbeitgeber löst sie durch (Mehr-) Leistung, den mit der Kollegin durch Beziehungsabbruch. Insgesamt wird sie sowohl von der Familie als auch von vielen Freunden und Arbeitskollegen unterstützt. 
Sie ist stolz auf ihre Leistungsfähigkeit und legt Wert darauf, „Voll“ zu funktionieren. Kennzeichnend sind die folgenden Episoden: Sie wünscht sich, aus Gesundheitsgründen und wegen des Geldes, mit dem Rauchen aufzuhören. Sie ist aber schon ein paar mal mit ihren Versuchen gescheitert. Einmal hat sie sich Zigaretten mit dem Taxi bringen lassen, weil sie Fieber hatte und sich nicht raus traute. Als die Zigaretten gebracht wurden, hat sie nur noch geheult: „Dass ich so schwach bin und es nicht schaffe.“ Einmal war sie voller Schadenfreude über Missgeschicke einer Kollegin. Am Nachmittag hat sie dann "gleich“ einen Unfall verursacht. „Das passiert mir sonst nie. Das war die Strafe." In der Phase, als es ihr schlecht ging, hat sie viel gebetet. Dass sie jetzt abends zum Beten zu müde ist, macht ihr ebenfalls ein schlechtes Gewissen. Sie beschreibt allerdings auch Tendenzen persönlicher Veränderung: „Im Umgang mit anderen Menschen bin ich viel kompromissloser geworden. Manchmal habe ich Angst, meine Liebe zu den Menschen zu verlieren. Ich merke auch, dass ich nicht so abhängig davon bin, geliebt zu werden. Ich habe mehr Selbstbewusstsein bekommen durch die Krankheit. Ich kann auch mal Nein sagen. ... Man muss stark bleiben und darf auf keinen Fall die Hoffnung aufgeben." Ihre negative Bewertung der „QL“ zum Zeitpunkt T5 erklärt die Patientin mit einer kurzfristigen „Winterdepression“. Sie sei so richtig in ein Loch gefallen. Heute, einen Tag nach dem Ausfüllen der Fragebögen, gehe es aber schon besser.

Die Erklärung der Patientin, dass ihr „QL-Wert" durch ein depressives „Loch“ zustande gekommen sei, trifft sicher zu. Sie beschreibt zwar persönliche Entwicklungstendenzen in Richtung Autonomie, vermittelt der Zuhörerin aber deutlich, dass sie sich einsam fühlt, zeitweilig unglücklich ist und ihr Leben unbefriedigend verläuft. Familie und Freundeskreis werden von ihr zwar als stützend beschrieben, es mischt sich aber immer wieder der Unterton ein, dass sie diejenige ist, die versorgt und nicht diejenige, die genug bekommt. Auch ihre materielle Lage bleibt ein Risikofaktor in Bezug auf die weitere Anpassung.

\section{Kasuistik der Patientin 33}

Die Patientin ist 33 Jahre alt, verheiratet, keine Kinder. Sie stammt aus einer türkischen Familie und hat noch eine Schwester und einen Bruder. Ihr Vater ist vor zwei Jahren an Lungenkrebs gestorben. Durch ihre Sprachkompetenz nimmt sie die Funktion des Familienoberhauptes ein. Ihren Mann hat sie mit Anfang zwanzig kennengelernt. Durch die Erkrankung hat sich ihre Beziehung vertieft und sie fühlt sich in der Beziehung aufgehoben. Ihre Ursprungsfamilie bedeutet ihr sehr viel. Der Kontakt zu den anderen Familienmitgliedern ist eng und die Geschwister nehmen sich Urlaub, um bei ihr in der Klinik sein zu können. Ihr Mann unterstützt sie ebenso. Die Familie wechselt sich, ab um sie nicht allein zu lassen. Auch zu den Schwiegereltern hat sie eine gute Bezie- 
hung. Die Schwiegereltern haben dem Paar ein Haus gekauft, das z. Z. renoviert wird und nach dem Krankenhausaufenthalt bezogen werden soll. Die Patientin studiert Psychologie. Ihre erste Ausbildung als Lehrerin hat sie durch ein auftretendes Rezidiv kurz vor dem Ende des Referendariats abgebrochen.

Die Patientin ist mit 17 Jahren an einem Sarkom erkrankt. Nach einer Unterschenkelamputation hat sie sich der ersten HDC unterzogen. Danach war sie 7 Jahren rezidivfrei. Seit dem 25. Lebensjahr sind erneut 6 Rezidive aufgetreten. Sie hat sich wiederholt Operationen unterzogen. Die Metastase, die jetzt der Anlass für die HDC ist, wurde bei der letzten Operation von den Chirurgen übersehen. Der behandelnde Arzt wollte es so belassen. Die Patientin hat sich damit nicht zufrieden gegeben und sich selber die HDC organisiert.

Die erste HDC 1983 war „so grauenvoll“, dass keine Steigerung mehr möglich war. Nach der ersten HDC hat die Patientin jahrelang Essstörungen entwickelt. Die Nebenwirkungen jetzt erlebt die Patientin zwar auch als schlimm, aber längst nicht so schlimm wie beim ersten Mal. Übelkeit ist da, wird aber medikamentös besser unter Kontrolle gehalten als bei der ersten HDC. Die Patientin bekommt eine ausgeprägte Haarwurzelentzündung, die eine Gesichtshälfte anschwellen lässt. Sie fühlt sich sehr schlapp, „wie durch den Wolf gedreht" und hat zeitweilig starke Bauchschmerzen. Der Mund war ebenfalls kaputt. Die Patientin hat sich in ihr Bett zurückgezogen und wollte nicht gestört werden. Sie empfindet die Belastung als extrem, vor allem die Ängste in der stationären Zeit. Eine Bekannte von ihr ist hier in der Klinik, während der HDC an einem Infekt gestorben. Die Stammzellrückgabe hat sie wegen des schlechten Geschmacks und Geruchs als sehr unangenehm erlebt. Nach der Entlassung hat die Patientin noch Wochen unter Übelkeit gelitten. Auch über Schmerzen klagt sie noch: Kopfschmerzen, starke Rückenschmerzen und Gelenkschmerzen. Die Patientin leidet an Hitzewallungen und Schlafstörungen, die „sehr schlimm“ sind. Der Geschmack ist noch verändert. Schlappheit und mangelnde Ausdauer führen bei Belastungen dazu, dass die Patientin anfängt zu zittern und sich hinlegen muss. Nach T4 bekam die Patientin erneut eine HDC. Danach wurde eine Lungenoperation (ein Lungenlappen musste wegen Metastasen entfernt werden) durchgeführt und kurz vor T5 war die Patientin erneut in der Orthopädie zur Operation wegen eines Hüftbefundes, der sich dann histologisch allerdings nicht als Metastase bestätigt hatte. Das Interview zu T5 erfolgt telefonisch, da die Patientin wieder zu Hause ist. Es geht ihr besser als letzte Woche, sie muss aber Schmerzmittel nehmen.

Die Patientin macht einen offenen, anziehenden Eindruck. Sie spricht ausgezeichnet Deutsch. Ihre Möglichkeiten des Umgangs mit der Krankheit beschreibt sie in der folgenden Weise: „Ich versuche natürlich, die Angst zu verdrängen. Vor 13 Jahren hat es ja auch 
geholfen. Ich denke positiv... Ich bin auch sehr gläubig. Mittlerweile kann ich mir kein Leben ohne Krankheit vorstellen. Ich glaube, ich habe auch eine andere Schmerzgrenze als andere Menschen. Manche jammern... Ich freu mich, dass ich lebe. Ich könnte längst tot sein. Außerdem habe ich doch alles erreicht, was ich wollte. Wenn ich da andere ansehe - die sind in meinem Alter noch nicht so weit. Mir ist wichtig: ich muss die Überhand haben, nicht die Krankheit. Mir haben Menschen immer weiter geholfen..." In ihren Äußerungen kommt zum Ausdruck, dass sowohl ihre Fähigkeiten als auch die Hilfe von nahestehenden Menschen aber auch von Ärzten, die sie seit Jahren kennt, für sie eine wichtige Stütze sind. „Die Bedeutung der Familie ist riesengroß." Kennzeichnend für sie ist eine Episode, die sie beschrieben hat: Auf Anraten von anderen hat sie an einem Seminar der Dt. Krebshilfe für junge Krebspatienten teilgenommen. Das Seminar war überhaupt nichts für sie, sie hat dort „als glücklicher Mensch“ nicht hingepasst, ihr war nichts anzusehen und sie hat dann ihren Mann davon überzeugt, dass sie dort nicht hingehört. Um mit der Krankheit klar zukommen, muss man „sich selber akzeptieren, alles andere bringt nichts“. Sie beschäftigt sich in der stationären Zeit mit ihrem Haus, plant und freut sich auf die Zeit danach. Sie ist „kein Typ, der Entspannung schätzt“, sondern bevorzugt Aktivität. Sie umgibt sich mit Menschen die ihr gut tun, anderen geht sie aus dem Wege. Sie hat gelernt „Nein“ zu sagen und sich durchzusetzen. Die Vorstellung vom „Leben nach der Krankheit“ hilft ihr. In Bezug auf die Zeit in der Klinik kritisiert sie nur das rauchende Personal. An dieser Stelle kann sie sich außerordentlich ereifern und hält flammende Reden für den Schutz der Nichtraucher. Die Zeit nach der HDC meistert sie durch ihre „lange Krankheitserfahrung". Sie sagt von sich, dass sie an erster Stelle kommt, egoistisch ist und nur tut, was gut für sie ist. Sie hat den Maler alles umändern lassen, da es nicht nach ihren Vorstellungen war: „Das war eine gute Ablenkung“. Sie beschreibt, dass sie gegen den Willen ihres Mannes, mit Mundschutz, einkaufen gegangen ist. „Es war mir auch egal, wenn die Leute guckten. Wahrscheinlich bin ich etwas schwer zu bändigen." Trotz ihres schlechten körperlichen Zustandes hat sie mit ihrem Mann eine Reise in die Türkei unternommen. Es war viel zu heiß und sie mussten früher zurückkehren. Trotzdem war es so in Ordnung. Obwohl es erneut zum Progress gekommen ist, hält die Patientin eisern an der HDC fest. Sie hat wieder mehr Hoffnung. Beruhigt hat sie ihr Besuch in der Tübinger Universitätsklinik: „der (Arzt) kannte sich aus, obwohl auch dort keine entsprechenden Fälle bekannt waren“. Die Logik hinter dieser Äußerung lautet, wenn keine entsprechenden Erfahrungen da sind, dann gibt es natürlich auch keine schlechten Erfahrungen und keine Arztäußerung, die sie entmutigt.

Die Äußerungen der Patientin sprechen für sich und erübrigen fast weitere Kommentare. Sie verfügt über ein ausgesprochen gutes Selbstbewusstsein und hat die Fähigkeit, die Bedrohlichkeit der Erkrankung zu verdrängen. Kraft gibt ihr das phantasierte Leben 
nach der Krankheit, sie spricht von Wechseljahresbeschwerden, die sie sonst eben mit 50 so erleben würde, ohne dass Zweifel deutlich werden, ob sie je dieses Alter erreichen wird. Aus der Besonderheit ihres Krankheitsverlaufes zieht sie Hoffnung für eine erfolgreiche Krankheitsbekämpfung. Trotz der teilweise sehr abgeklärt wirkenden Äußerungen braucht sie Therapieaktivitäten, um Hoffnung aufrecht halten zu können. Trotz der greifbaren Erfolglosigkeit der Therapie zum jetzigen Zeitpunkt, hält sie unbeirrbar an ihrer Überzeugung fest und würde vermutlich, so sich ein Arzt fände, auch die vierte HDC durchführen lassen. Die negative Beurteilung ihrer "QL“ im Fragebogen wird, wie aus den Äußerungen hervorgeht, durch die körperliche Beeinträchtigung bestimmt, keinesfalls durch ihr Selbstwertgefühl. Die Vorstellung, wegen der erheblichen Therapiebelastungen auf weitere derartige Therapien zu verzichten, würde sie vermutlich für völlig abwegig halten. In einem fiktiven Dialog mit dieser Patientin über den „Preis“ den sie zahlt, würden folgende Argumente enthalten sein: Ohne die Therapien wäre ich schon längst tot. Leben lohnt sich immer!!! Was gibt es denn für Alternativen? Abwarten, nichts tun bis der Tod kommt? Dann kann ich mir gleich einen Strick nehmen. Mein Leben ist reich und lebendig. Ich will noch weiter leben. Ich habe meine Familie. Und „der Mensch kann viel aushalten“.

\section{Kommentar}

Versucht man ein Fazit dieser drei „LQ“-Geschichten, so wird deutlich, dass ganz unterschiedliche individuelle Einflüsse eingehen und zur inhaltlichen Bestimmung beitragen. LQ kann ein stabiles Persönlichkeitsmerkmal sein, das kurzfristig durch gravierende Einflüsse verändert wird um dann wieder seinen „eigentlichen Zustand“ zu erreichen, LQ kann ausschließlich auf funktionale Aspekte des eigenen Lebens bezogen werden (im entsprechenden Referenzrahmen), ohne dass die Qualität, die dem eigenen Leben zugeschrieben wird auch nur tangiert wäre oder LQ kann Ausdruck einer depressiven Episode sein. In keinem dieser Fälle sagt LQ irgendetwas über die medizinische Behandlung aus. Eine Reihe von Autoren geht davon aus, dass sich der negative Pol des Kontinuums „LQ“ mit Depressivität gleich setzen lässt. Klinisch spricht sehr vieles für diese Auffassung. Allerdings ist eine Aussage nur dann möglich, wenn die Urteile im Zeitverlauf betrachtet werden. Die Patientin 33 liefert keine klinischen Anhaltspunkte für eine Klassifikation als „depressiv" (außer man würde ihre Therapieaktivitäten als Depressionsabwehr deuten). Deutlich wird, dass einzelne „QL-Werte“ ohne weitere Kenntnisse kaum klinisch interpretiert werden können.

Die systematische Verallgemeinerung der Ausschnitte aus Lebensgeschichten über alle Zeitpunkte führt zu folgenden Kernaussagen: 
- (fast) alle Patienten erleben sich in der Rolle von Menschen, deren Leben durch die Krankheit bedroht ist.

- Auch Patienten die nach einem Jahr krankheitsfrei sind kreisen noch um die Frage, „Bin ich geheilt oder kommt die Krankheit wieder?"

- Diejenigen, die sich in der Rolle des/der chronisch Kranken akzeptieren (definiert durch die Leistungseinschränkungen: „Werde nie mehr die/der Alte“) streben in ihren sozialen Beziehungen und im Alltagshandeln „Normalität“ an.

- Wichtig bleibt, alles zur Bekämpfung der Krankheit getan zu haben.

- Zum standardisierten Vokabular gehören:

$$
\begin{aligned}
& \text { Hoffnung (auf Heilung) } \\
& \text { Angst (mehr vor der Krankheit als der Therapie) } \\
& \text { Belastungsbeschränkung (Leistungseinschränkung) } \\
& \text { Unterstützung durch Familie }
\end{aligned}
$$

\subsubsection{Bewertung der Hypothesen und Zusammenfassung}

„Das Wort Lebensqualität wollen wir mal gar nicht in den Mund nehmen.“ (Patientin 13)

Die Hypothese, dass die Therapiebelastungen in Kauf genommen werden zugunsten einer erhöhten Chance auf Heilung/Besserung kann uneingeschränkt als bestätigt gelten. Die erwarteten Belastungen durch die Therapie sind für die meisten Patienten kein Entscheidungskriterium und kein Hinderungsgrund.

Die nachträgliche Bewertung der Therapie ist weitgehend unabhängig von realen Belastungen und dem Erfolg der Therapie.

Angst, in unterschiedlicher Ausprägung und inhaltlicher Orientierung, spielt zu allen Zeitpunkten eine wichtige Rolle.

Diese Hypothese kann nur für einen Teil der Patienten bestätigt werden und ist abhängig vom Zeitpunkt der Untersuchung. Eine große Minderheit kann Angst offensichtlich gut abwehren. Das gilt insbesondere für die Männer dieser Stichprobe. Aber auch eine Minderheit der Frauen berichtet keine Ängste. Besonders vor und während der Therapie scheint Angstabwehr ein wichtiger Adaptatiosmechanismus zu sein. Ein Jahr nach Abschluss der Therapie ist die Bereitschaft dieser Patienten über Ängste zu sprechen größer.

Neben der Hoffnung auf Heilung sind soziale Beziehungen (Familie, Klinikmitarbeiter) wichtig für die Bewältigung der Therapiebelastungen. 
Für diese Stichprobe kann die Hypothese uneingeschränkt als bestätigt gelten. Die Familie steht an erster Stelle. Daneben wird die Behandlungsatmosphäre von der Mehrzahl der Patienten als bedeutsam erwähnt.

Ein Jahr nach Abschluss der Therapie sind die Patienten, die bis zu diesem Zeitpunkt nicht wieder erkrankt sind, zur Normalität zurückgekehrt.

Diese Annahme hat sich nicht bestätigt. Auch bei den Patienten, die äußerlich einen „Normalzustand“ erreicht haben, spielt die Krankheit noch eine zentrale Rolle. Alle Berufstätigen fühlen sich in ihrer Leistungsfähigkeit eingeschränkt. Viele befinden sich noch im Prozess der Trauer um Verlorenes. Der Zeitraum von einem Jahr ist ganz offensichtlich zu kurz für eine weitgehende psychische und physische Rehabilitation.

\section{Zusammenfassung der Ergebnisse}

\section{Methodische Aspekte}

1. Die Art der qualitativen Datenerhebung wird uneingeschränkt von den beteiligten Patienten geschätzt. Die Vollständigkeit, auch die der Fragebogendaten, ist diesem Vorgehen geschuldet.

2. Durch den wiederholten und engen Kontakt mit den Patienten wurde im Gespräch gelegentlich das Ausmaß der Angst unterschätzt: Selbst- und Fremdurteil deckten sich bei drei Patienten nicht. Die hohen Werte im HADS-D waren Anlass zur Überprüfung des Intervieweindrucks. Ob das mit „Gewöhnungseffekten" auf Interviewerseite oder „Verstecken“ auf Patientenseite zu tun hat, ist nicht auszumachen.

3. Die Informationen aus den Interviews unterscheiden sich hinsichtlich Ausführlichkeit und „Tiefe“ sehr erheblich von Patient zu Patient.

4. Nicht von allen Patienten liegen ausführliche biographische Informationen vor. Da keine ausführliche biographische Anamnese vorgesehen war, blieb es den Einzelnen überlassen, in welchem Umfang sie sich dazu äußern wollten.

5. Bei den Interviewdaten kommt es möglicherweise zu einer Verzerrung in negativer Richtung (Erlaubnis zum Klagen). Die Gefahr von Antworten in Richtung „Sozialer Erwünschtheit“ scheint hier nicht sehr ausgprägt.

\section{Inhaltliche Aspekte}

1. HDC ist eine Behandlung, die für ca. zwei Drittel der Patienten dieser Stichprobe mit ausgeprägten Nebenwirkungen einhergeht. Diese Nebenwirkungen sind teilweise so ausgeprägt und schwer zu verkraften, dass von vielen Patienten retrospektiv die Metapher der „Hölle“ oder eine ähnlich lautende Beschreibung verwendet wird. 
2. Ein Drittel der Patienten hat vergleichsweise wenig belastende akute Nebenwirkungen, beschreibt aber die Behandlung im Rückblick trotzdem als gravierend.

3. Nach einem Jahr beklagen die meisten Patienten ohne Rezidiv noch deutliche Leistungseinbußen, die sich sowohl im Arbeitsalltag als auch im Privatleben bemerkbar machen.

4. Für die meisten Patienten ist der Übergang von der Klinik in die häusliche Umgebung außerordentlich schwierig. Bestehende Symptome, Schmerzen, Probleme mit der Nahrungsaufnahme und Fatigue bestimmen den Alltag.

5. Hoffnung auf Heilung ist der wichtigste Faktor für die Akzeptanz der Therapie und ihrer Nebenwirkungen.

6. Angst spielt zu allen Zeitpunkten eine wichtige Rolle: vor der Therapie ist die Bedrohungsangst am größten, im Laufe der Zeit wird sie abgelöst von der Angst vor einem Rezidiv.

7. Für den größten Teil der Patienten (ob mit oder ohne Rezidiv) steht der Sinn einer HDC überhaupt nicht zur Debatte.

8. Für fast alle Patienten sind Angehörige die wichtigste Unterstützung.

9. Die Beurteilung der Qualität der Behandlung fällt sehr differenziert aus: trotz verschiedener Kritikpunkte, die sich sowohl auf persönliche (seltener) als auch auf organisatorische Aspekte (häufiger) beziehen, ist das Gesamturteil positiv.

Werden nur die Patienten mit Rezidiv betrachtet, so lassen sich folgende, zusammenfassende Aussagen treffen:

1. Die prognostische Situation vor der Behandlung wird noch ungünstiger eingeschätzt und diese Einschätzung wird den Patienten auch vermittelt. Daraus ergeben sich zwei verschiedene Umgangsmuster. Eine Gruppe bezieht gerade daraus Hoffnung und gibt sich auch mit wenig Erreichtem zufrieden (,man könnte ja schon tot sein"). Für die andere Gruppe wird diese Einschätzung zur Quelle permanenter Angst, die nicht abgewehrt oder kontrolliert werden kann.

2. Ein gemeinsames Beschwerdemerkmal ist eine übergroße Erschöpfung, die weiter bestehen bleibt.

3. Alle reagieren, verständlicherweise, mit Enttäuschung, Mutlosigkeit und Angst auf die Rezidivnachricht. Unterschiedlich ist dann die psychische „Anpassung“ an den veränderten Zustand: Einzelnen gelingt es, neue Hoffnung zu finden, andere leiden, ohne einen Ausweg gefunden zu haben.

4. Alle Patienten der Rezidivgruppe werden von ihrem sozialen Umfeld gut unterstützt und bringen diesen Punkt auch zum Ausdruck. Von den wenigen Patien- 
ten der Gesamtstichprobe mit problematischen, konflikthaften Beziehungen ist keiner in dieser Gruppe.

Bemerkenswert ist, dass, bis auf die in der Überschrift zitierte Patientin (und die nur in negierender Art und Weise), kein einziger Patient in den Interviews das Wort Lebensqualität zur Charakterisierung seiner Situation verwendet, obwohl die Studie von Untersucherseite so genannt wurde.

\subsubsection{Vergleich der Fragebogendaten mit denen der Daten im Interview}

Vergegenwärtigt man sich den Verlauf der „QL“-Daten des QLQ-C30, so entsprechen die gemittelten Werte von Funktions- und Symptomskalen denen allgemeiner Erwartungen (Einschränkungen siehe Kap. 9.1.3). Auch der Verlauf von Karnofsky-Index und den Skalenwerten D und A des HADS ist plausibel zu erklären. Beim folgenden Vergleich soll sowohl die individuelle als auch die gruppenstatistische Ebene berücksichtigt werden.

\section{Übereinstimmungen in den Daten}

Beim Vergleich der Daten aus Fragebögen und Interviews ergeben sich für die Symptomwerte und deren Verlauf gute Übereinstimmungen. Allerdings fällt auf, dass die Höhe der Symptomwerte, gemessen an den zum Teil dramatischen Schilderungen in den Interviews eher moderat ausfallen und in der Ausprägung nicht wesentlich höher liegen als die Werte, die von Patientenstichproben zu Beginn einer Rehabilitationsmaßnahme genannt werden. Bei der gruppenstatistischen Betrachtung der A und D Skalenwerte und deren Verlauf zeichnet sich eine relativ gute Übereinstimmung ab. Auf der individuellen Ebene lassen sich hohe D bzw. A Skalenwerte durch die Interviewaussagen stützen. Patienten, die ausgeprägte Angst (oder depressive Äußerungen) im Interview zum Ausdruck bringen, haben entsprechend auffällige Werte im HADS-D. Auch wenn das nicht mit der Studie intendiert war, lassen sich diese Ergebnisse im Sinne einer Validitätsaussage für die HADS-D verwenden. Die ausgeprägten individuellen Unterschiede im psychischen und physischen Symptombereich spiegeln sich deutlich in den Varianzen der Fragebogendaten und werfen die Frage auf, ob eine Regression zur Mitte überhaupt noch aussagekräftig ist oder ob die zwangsläufige Nivellierung nicht jede sinnvolle Interpretation zunichte macht.

\section{Differierende Ergebnisse}

Schwieriger als im Symptombereich lässt sich eine Übereinstimmung der jeweiligen Daten im Funktionsbereich belegen. Hier fällt auf, dass die Fragebogenergebnisse der 
Funktionsskalen des QLQ-C30 und Interviewäußerungen nicht in Deckung zu bringen sind. Besonders deutlich ist das zum Zeitpunkt T1, T4 und T5. Zu T1 spielt eine offensichtlich hohe Erwartungsangst eine wichtige Rolle, wie dies von vielen Patienten auch artikuliert wird. Die emotionale Funktionsskala vermag diesen Aspekt nicht abzubilden. Während im QLQ-C30 zu den beiden letzten Zeitpunkten die Werte deutlich angestiegen sind und das im Sinne der erwarteten Genesung interpretiert werden kann, zeigen sich in den Interviewäußerungen noch erhebliche Probleme. Der höhere Wert zu T4 spiegelt nicht die reale Situation nach der Entlassung wider. Von fast allen Patienten wird die erste Zeit nach Entlassung als sehr schwierig beschrieben. Trotz der niedrigen Werte im QLQ-C30 vor der Entlassung, kommt in den Interviews der Patienten deutlich eine emotionale Entlastung zum Ausdruck: Stolz auf die eigene Leistung, die Zeit durchstanden zu haben, Freude auf die Entlassung und eine Zukunft zugewandte Perspektive. Nach der Entlassung wird die eigene Morbidität erst richtig bewusst. Der Schonraum Klinik fällt weg und die Mehrzahl der Patienten merkt, dass die eigenen Kräfte völlig überschätzt wurden. Teilweise werden heftige emotionale Einbrüche beschrieben. Allerdings sind zum vierten Messzeitpunkt diese kritischen ersten Wochen bereits geschafft und die unterschiedliche Zeitperspektive trägt zu den divergenten Eindrücken aus Interview und Fragebogen bei. Zum letzten Erhebungszeitpunkt stimmen der positive Eindruck aus den Fragebogendaten und der sehr gedämpfte Eindruck aus den Interviewäußerungen ebenfalls schlecht überein. Die Mehrzahl der Patienten macht immer noch den Eindruck, belastet zu sein und den Rehabilitationsprozess noch nicht überstanden zu haben. Ähnlich, wenn auch nicht ganz so ausgeprägt, spiegeln sich auch hier die individuellen Differenzen in den Varianzen der Fragebogendaten. Der Karnofsky-Index zu T5 stimmt mit dem Intervieweindruck überein.

Die Leistungseinschränkungen und damit auch der Zwang zu veränderten Rollen durch Frühberentung oder Krankschreibung zeigen sich weniger im QLQ-C30 als beim Vergleich der Berufstätigkeit vor HDC und nach einem Jahr.

Die Betrachtung der QL-Werte zeigt Folgendes: Eindeutig ist der Zusammenhang zwischen den wenigen Patienten der Stichprobe, die sich als depressiv beschreiben und einem niedrigen QL-Wert. Dieser klinische Eindruck wird gestützt durch die hohen Korrelationen von D im HADS-D und dem QL-Wert des QLQ-C30. Eindeutig ist auch der Zusammenhang zwischen niedrigem QL und einem erlittenen Rezidiv.

Während niedrige QL-Werte durch die Interviewausführungen leicht erklärt werden können und fast immer auch auf Interventionsbedarf hinweisen, ist das bei durchschnittlichen und hohen QL-Werten anders. Hier kann oft nur erahnt oder interpretativ auf die inhaltliche Bedeutung geschlossen werden. 


\section{Schlussfolgerungen für die Patientenversorgung}

Aus den Interviewäußerungen ergibt sich eine Reihe von Anregungen, die der Verbesserung der Patientenversorgung dienen können. In der folgenden Zusammenstellung sind die wichtigsten Punkte berücksichtigt, die sich unmittelbar aus den Patientenäußerungen ergeben. Für Einzelheiten sei auf die entsprechenden Interviewauswertungen verwiesen.

1. Wenn sich Patienten für die Behandlung entschieden haben, darf von Seiten des Personals keine Skepsis in Bezug auf die Wirksamkeit deutlich werden. Notwendig ist ein Bejahen von Hoffnung. Ambivalente Äußerungen steigern Ängste. Patienten benötigen ernstgemeinte, empathische Aufmunterung und Trost. Neben der Aufklärung über Nebenwirkungen sollte all das, was positiv gegen die Erkrankung und durch die Therapie verursachten Nebenwirkungen getan werden kann, in den Mittelpunkt gerückt werden.

2. Die Beziehungen zum Behandlungsteam sind während der Klinikphase von herausragender Bedeutung. Patienten registrieren jedes Interesse, das innen als Person und nicht nur als Patienten gilt. In der Kommunikation wird Reversibilität gewünscht, Freundlichkeit, Achtung und verständliche Sprache. Konstante Bezugspersonen (Ärzte) werden in der Behandlungsphase sehr geschätzt und nach der Entlassung am dringendsten gewünscht. Loyalität im Team ist wichtig, Spannungen sind für Patienten belastend.

3. In Bezug auf medizinische Aspekte wird Komplementarität gewünscht. Patienten brauchen den kompetenten Experten, der Ruhe ausstrahlt, sein „Handwerk“ beherrscht und auch schwierige Situationen souverän meistert. Angst wird durch professionelles Handeln verringert. Hervorgehoben wurde auch immer wieder die schnelle Verfügbarkeit von Hilfe in kritischen, medizinischen Situationen. In Bezug auf die Beziehungsgestaltung zu den Mitgliedern des Behandlungsteams wird der Wunsch nach Symmetrie deutlich.

4. Das Team sollte „mit dem Kopf des Patienten denken“. Ergebnisse von Untersuchungen, auch unauffällige Routinebefunde, sollten unaufgefordert und schnell mitgeteilt werden. Für Patienten ist es quälend, auf Nachrichten, Befunde etc. zu warten. Viele trauen sich nicht nachzufragen, wenn sie Zeitdruck beim Gegenüber erleben.

5. Der größte Teil der Patienten nutzt intuitiv „Aktivität“ und „Ablenkung“ zur Anpassung an die Belastungen in der stationären Phase. Diese Strategien könnten bewusst gefördert werden durch Anleitung zu und Informationen über prophylaktische Maßnahmen zum Schutz der Mundschleimhaut, angepasstes 
Training zur Verringerung von Fatigue, Steigerung/Erhaltung der körperlichen Leistungsfähigkeit und Maßnahmen zur Spannungsreduktion. Als wichtiger Effekt ergibt sich daraus der Zugewinn an Handlungskontrolle und die Überwindung von Hilflosigkeit.

6. Obwohl sich fast alle Patienten auf die Entlassung freuen, stellt sich die Zeit danach, retrospektiv beurteilt, für viele als sehr belastend dar. Nach der engmaschigen Betreuung in der Klinik sind Patienten auf sich gestellt. Sie vermissen die Sicherheit der medizinischen Versorgung, zumal bei einem Teil noch ausgeprägte Beschwerden bestehen. Der Wunsch nach Informationen und Verhaltensvorschriften bezüglich der Rehabilitation wird geäußert. Wichtig wären ein dezidiertes Abschlussgespräch und möglichst auch eine schriftliche Information nicht nur für den Hausarzt, sondern auch für den Patienten. Personelle Konstanz auch in der Nachsorge ist unbedingt notwendig! In der Zeit unmittelbar nach der Klinikentlassung gibt es eine Versorgungslücke hinsichtlich der weiteren Betreuung. Hausärzte sind mit den Therapiefolgen nicht immer vertraut und auf die Information durch die Klinik angewiesen. Mehr Vernetzung zwischen Klinik und Hausärzten ist nötig!

7. Fatigue ist die am längsten und intensivsten persistierende Folge der HDC. Auch im Sinne der Prävention wären rehabilitative Maßnahmen zur Steigerung der körperlichen Leistungsfähigkeit unmittelbar an den Klinikaufenthalt erforderlich. Einzelne Patienten klagen über langanhaltende Probleme bezüglich der Nahrungsaufnahme und Ausscheidung. Auch Schmerzen und heftige allergische Reaktionen (bei einer kleinen Minderheit) sind noch zu verzeichnen. Therapeutische Hilfe ist hier nötig.

8. Schwierig sind die Schlussfolgerungen bezüglich der Informationsvermittlung vor der Therapieeinwilligung. Auf die komplexen Schwierigkeiten des „informed consent" bei der Anwendung von (noch) nicht etablierten Therapieverfahren kann hier nicht eingegangen werden. Betrachtet werden nur die Wünsche der Patienten an die aufklärenden Ärzte: Wichtig sind alle Informationen, die der Tendenz, die Gefährlichkeit der Therapie zu überschätzen, entgegen wirken, ohne dass die Risiken bagatellisiert werden. In bezug auf das Ausmaß der Informationen haben Patienten sehr unterschiedliche Bedürfnisse. Sowohl „Informationssuche“ als auch „Verleugnen“ kann eine wichtige Bewältigungsform sein. Ein auf den Patienten abgestimmtes differenzielles Vorgehen ist unerlässlich. Selbstverständlich gelten die Regeln, die auch sonst für eine gelungene Aufklärung stehen: Aufklärung hat Prozesscharakter und ist in ihrer Qualität an die Arzt-Patient-Beziehung gebunden. Einig sind sich die Patienten darin, dass 
nicht so sehr Listen von Nebenwirkungen aufgezählt werden sollten, sondern stärkeres Gewicht auf die Beschreibung der Prozeduren, der einzelnen Behandlungschritte usw. gelegt werden sollte, die mehr Transparenz des klinischen Alltags vermitteln, Unsicherheiten und Überraschungen vermeiden und auf diese Weise Angst mindern.

9. Für die Zustimmung zur Therapie ist der Faktor „Hoffnung“ entscheidend. Auch wenn Ängste vor den Therapiefolgen wahrgenommen und beschrieben werden, hindern sie Patienten nicht daran, ihre Zustimmung zu geben, sofern die Behandlung als Erfolg versprechend eingeordnet wird. Die Angst vor der Krankheit ist größer als die Angst vor der Therapie. Für die Patienten gilt die Maxime, „ich will leben“. Daraus ergibt sich die große Gefahr der Manipulierbarkeit von Patienten in Angst. Den aufklärenden Ärzten wird eine nur schwer zu lösende Aufgabe gestellt. Trotz informiertem Konsens bleibt die Verantwortung in der Hand des Arztes.

10. Ist die Zustimmung erfolgt, werden unterschiedliche psychische Mechanismen zur Angstkontrolle eingesetzt. An die Betreuer ist der Wunsch gerichtet, dass sie die individuellen Muster der Hoffnungs-Gefahr-Balance erkennen, respektieren und unterstützen. 


\section{Teil III}

\section{Zusammenfassung und Diskussion}

Im folgenden Kapitel werden die wichtigsten Ergebnisse zusammengefasst und kritisch diskutiert.

\subsection{Zusammenfassung und Diskussion der Ergebnisse}

Bei der vorliegenden Untersuchung handelt es sich um eine prospektive Längsschnittstudie mit fünf Untersuchungszeitpunkten an 34 Patienten (23 Frauen, 11 Männer), die mit Hochdosistherapie und autologer Blutstammzelltransplantation behandelt wurden. Bei der Stichprobe handelt es sich um eine inhomogene Patientengruppe hinsichtlich der Diagnosen. Patienten mit Non-Hodgkin-Lymphomen und Patientinnen mit Mammakarzinom überwiegen. Nach einem Jahr waren 22 der Patienten krankheitsfrei. Von den Patienten mit Krankheitsprogress sind vier zum letzten Erhebungszeitpunkt verstorben. 30 Patienten nahmen an allen fünf Erhebungszeitpunkten teil, wobei die Datenerhebung für die wiedererkrankten Patienten teilweise modifiziert wurde (Telefoninterviews). Erfasst wurden sowohl körperliche Belastungen und Einschränkungen als auch psychologisch relevante Faktoren im Verlauf von Therapie und Rehabilitation. Der Untersuchungszeitraum betrug ca. 13 Monate. Die Daten wurden unmittelbar vor Beginn der HDC, nach der Stammzellretransfusion, vor der Entlassung und 3 bzw. 12 Monate nach Therapie erhoben. Neben den psychometrischen Instrumenten, die am häufigsten in onkologischen Studien eingesetzt werden, wurden auch semistrukturierte Interviews zur Datenerhebung genutzt. Unter dem Begriff der „Lebensqualität" wurden verschiedene Aspekte von Belastung und Bewältigung untersucht, die in der Literatur als bedeutsam genannt werden (Aaronson \& Beckman 1987; Neuser 1989; Korth 1994; Faller 1998). Ziel war neben der genauen Beschreibung des Belastungsprofils die Ermittlung von moderierenden Faktoren. Daraus abgeleitet wurden Vorschläge für eine Verbesserung der Patientenversorgung.

Das Belastungsprofil wurde erfasst mit dem Lebensqualitäts-Fragebogen (QLQ-C30) der EORTC (European Organization of Research and Treatment of Cancer), einem Screeninginstrument bezüglich Depressivität und Angst (HADS-D) und dem KarnofskyIndex, der, im Vergleich mit den erstgenannten Instrumenten, aus der Fremdbeobachterperspektive den körperlichen Funktionszustand beschreibt. Mit den psychometrischen Instrumenten wurden folgende Aspekte der Belastung erfasst:

- Symptome (Selbstbeurteilung)

- Funktionseinschränkungen und allgemeine Lebensqualität (Selbstbeurteilung)

- Angst/Depressivität (Selbstbeurteilung) 
- Körperlicher Funktionszustand bezogen auf Selbständigkeit im Alltag (Fremdbeurteilung)

Durch die Interviews sollten drei Fragenkomplexe untersucht werden:

- Belastende Faktoren, insbesondere die Qualität der Belastung und ihre Bedeutung zu unterschiedlichen Zeitpunkten

- Entlastende Faktoren, die es den Patienten ermöglichen die Behandlungsbelastungen zu bewältigen

- Bewertung der Therapie

Folgende Hypothesen sollten überprüft werden:

Die Therapiebelastungen werden unabhängig von erwarteten Belastungen in Kauf genommen zugunsten einer erhöhten Chance auf Heilung/Besserung.

Die Bewertung des Therapienutzens ist unabhängig von realen Belastungen und dem Ergebnis der Therapie.

Angst, in unterschiedlicher Ausprägung und inhaltlicher Orientierung, spielt zu allen Zeitpunkten eine wichtige Rolle. Vor Beginn der Therapie ist die psychische Belastung am höchsten. ${ }^{84}$

Neben der Hoffnung auf Heilung ist soziale Unterstützung (Familie, Freunde, Klinikmitarbeiter) wichtig für die Bewältigung der Therapiebelastungen.

Krankheitsanpassung wird mitbestimmt von situativen Einflüssen und den Möglichkeiten der internalen und externalen Kontrolle dieser Einflüsse.

Ein Jahr nach Abschluss der Therapie sind die Patienten, die bis zu diesem Zeitpunkt nicht wieder erkrankt sind, zur Normalität zurückgekehrt.

Die Auswertung der Fragebogendaten ergibt, in Übereinstimmung mit den Vorannahmen, dass es nach der Therapie zu einem Absinken der Werte für die Skala „Physische Funktion“, „Soziale Funktion“, „Rollenfunktion“ und „Allgemeine Lebensqualität“ und einer deutlichen Zunahme von Symptomwerten (Fatigue, Übelkeit/Erbrechen, Kurzatmigkeit, Schlafstörungen, Appetitverlust, Durchfall und Schmerzen) kommt. In der nachstationären Phase folgt die Erholung kontinuierlich, bis dann, nach einem Jahr, der Ausgangszustand wieder erreicht ist. Einzig die Symptomskala „Verstopfung“ verändert sich nicht signifikant. Dieser Befund ist zu erwarten, da bei dieser Art der Chemotherapie (und der Bestrahlung für die Gruppe der Patienten mit Non-HodgkinLymphomen) Durchfall eher zu erwarten ist.

Ausnahmen zeigen sich bei zwei Skalen:

\footnotetext{
${ }^{84}$ Nur in einer HDC-Studie beschreiben Mammakarzinompatientinnen ein niedrigeres Angstniveau vor der Behandlung. Dieser Befund ist wenig plausibel.
} 
Die kognitive Funktion zeigt keine signifikanten Veränderungen im Zeitverlauf, gleichwohl kommt es zu einer tendenziellen Verschlechterung mit nachfolgender Erholung. Die Verschlechterung ist aus der Klinik bekannt, viele Patienten beklagen eine Abnahme ihrer Gedächtnisfunktionen und Konzentrationsleistungen, ohne dass diese Einbußen in Performanztests objektiviert werden können. Warum subjektive Einschätzung und Objektivierbarkeit nicht deckungsgleich sind, kann nur vermutet werden. Konkrete Aufträge, wie sie in Performanztests vorgegeben werden, erleichtern die Fokussierung der Aufmerksamkeit während „Vergeßlichkeit“ im Stationsalltag durch konfligierende Arbeitsspeicheransprüche erklärt werden kann. Milde/moderate Funktionseinbußen sind als Langzeitfolge nachgewiesen (U.a. Harder et al 2002), zeigen sich aber in dieser Untersuchung wegen des zu kurzen Nachbeobachtungszeitraumes nicht.

Auch die emotionale Funktion wird von der Gesamtgruppe der Patienten als weitgehend zeitstabil beschrieben, wenn auch tendenzielle Unterschiede im Verlauf zu beobachten sind (Umgekehrt U-förmiger Verlauf). Die in anderen Untersuchungen gefundene hohe Erwartungsangst zeigt sich nur bei einem Teil der Stichprobe (s. u.). Sie wird in der entsprechenden Skala zwar abgebildet - der emotionale Funktionswert ist vor der Behandlung am niedrigsten und unmittelbar vor der Entlassung am höchsten aber die Unterschiede sind nicht signifikant. Der Verlauf bestätigt den Eindruck aus den Interviews, dass die Angstwahrnehmung/der Angstausdruck interindividuell sehr unterschiedlich ist. Für die Patienten, denen die Bedrohungsabwehr gar nicht oder nur partiell gelingt, ist die psychische Belastung vor der Therapie am größten. Einem Teil der Stichprobe - fast allen Männern - gelingt eine weitgehende Angstabwehr bzw. Angst wird nicht mitgeteilt. Im Interview reagieren fast alle Patienten auf das Überstehen der Therapie und die bevorstehende Entlassung mit emotionaler Entlastung.

Das Symptom Fatigue ist nach einem Jahr signifikant ausgeprägter als vor der Behandlung. Dieses Ergebnis ist erwartungskonform da Fatigue das persistierende Symptom lange nach Abschluss der Therapie ist (siehe Studienübersicht) Insgesamt deckt sich die Höhe der Funktionswerte mit den Ergebnissen anderer Untersuchungen bzw. die Funktionswerte dieser Studie sind geringfügig besser wenn die entsprechenden Zeitpunkte verglichen werden (U. a. Hensel et al. 2002).

Die Betrachtung der Werte des Fragebogens für "Angst und Depressivität“ ergibt folgende Ergebnisse:

Bestätigt wird die Hypothese, dass Angst zu allen Zeitpunkten eine größere Rolle spielt als Depressivität. Diese Annahme wird begründet durch die Besonderheit der Stichprobe (hohe Belastbarkeit der Patienten wird bei der HDC von den Ärzten vorausge- 
setzt). Bestätigt wird auch, dass die Angst vor Beginn der Therapie am größten ist (Fife et al. 2000; Carlson et al. 2001), mit der Einschränkung (wie bei der emotionalen Skala), dass dieses Ergebnis in erster Linie auf die Werte der Frauen zurückgeführt werden kann. Die Kurvenverläufe für Männer und Frauen sind zeitweise gegenläufig: bei den Frauen nimmt die Angst in der Aplasiephase (unmittelbar nach der HDC - Zeit der stärksten körperlichen Belastung) ab, bei den Männern nimmt sie zu. Nach einem Jahr liegt der Angstwert der Frauen deutlich unter dem Ausgangsniveau, während der Angstwert der Männer auf gleichem Niveau liegt wie ihr Ausgangswert. Zum Zeitpunkt der Entlassung ist der Mittelwert der Angst für beide Geschlechter am geringsten und steigt dann auf ein Niveau an, welches über dem der Vergleichspopulation liegt. Ein für lange Zeit erhöhtes Angstniveau ist charakteristisch für Patienten mit Krebserkrankungen und spiegelt die Unsicherheit bezüglich des weiteren Verlaufes der Erkrankung wider. Ärzte sprechen bei vielen Krebserkranken erst jenseits der Fünfjahresgrenze von Heilung. Bezogen auf Brustkrebserkrankungen muss dieser Zeitraum noch wesentlich weiter ausgedehnt werden.

Unterschiede in der Angstausprägung gemessen mit dem HADS-D müssen sowohl für den Faktor Geschlecht als auch für den Faktor Alter angenommen werden. Der Alterseffekt konnte in dieser Stichprobe nicht überprüft werden (Stichprobengröße) und ist auch weniger bedeutsam (Gruppe der 40-60jährigen überwiegt). Vergleicht man die Mittelwerte dieser Studie mit der Normpopulation (Gesunde) von Hinz \& Schwarz (2001), so sind die Werte für die Gruppe der Frauen zu allen Zeitpunkten deutlich höher (MW 5.2 vs. 9.3 (T1) - 6.8 (T3) - 7.9 (T5)); für die Gruppe der Männer ergibt sich ein völlig anderes Bild: der MW liegt mit Ausnahme zu T2 (MW 5) unterhalb des Vergleichswertes (MW 4.4 für die Gruppe der 40-60jährigen Männern) der Normstichprobe. Auch die Betrachtung der relativen Häufigkeiten von erhöhten Angstscores (bei Hinz \& Schwarz sind doppelt so häufig Frauen betroffen, in dieser Stichprobe ist der Anteil noch wesentlich höher) bestätigt den klinischen Eindruck, dass Männer anders mit bedrohlichen Krankheitssituationen umgehen. Zusätzlich könnte eine Rolle spielen, dass bei ungünstiger Diagnose Angst stärker abgewehrt werden muss. Zu diesem Befund „passt“, dass nur von wenigen Patienten zusätzliche Informationen über die Nebenwirkungen der HDC eingeholt wurden. Die Verarbeitungsstrategie „Informationssuche" scheint dann vermieden zu werden, wenn sie Angst auslösende Resultate erbringen kann. Auf die Notwendigkeit der Angstabwehr bei körperlich Schwerkranken hat Faller (1997) hingewiesen. Offensichtlich nutzen Frauen und Männer unterschiedliche Adaptationsstrategien: während Frauen Angst wahrnehmen, beschreiben und Entlastung erfahren durch "Gespräche gegen die Angst", sprechen Männer nicht über die Angst, konzentrieren sich auf andere Aspekte wie Problembewältigung in der aktuellen 
Situation und suchen nicht das Gespräch über die Angst. Wie immer bei geschlechtsstereotypen Annahmen gibt es selbstverständlich Ausnahmen: Auch unter den Frauen dieser Stichprobe gab es eine kleine Gruppe die eher „männliches“ Verhalten zeigte, während die Angstabwehr bei den Männern im Verlauf der Zeit durchlässiger wurde und Angstinhalte stärker thematisiert wurden.

Neben den Unterschieden für „Angst und Depressivität“ finden sich auch signifikante Geschlechtsunterschiede für weitere Skalen des QLQ-C30: Für die emotionale Funktion, die kognitive Funktion, die soziale Funktion, die allgemeine Lebensqualität und für Fatigue. Bei allen anderen Skalen gibt es tendenzielle Unterschiede in der Weise, dass die Frauen niedrigere Funktions- und höhere Symptomwerte aufweisen. Die Kurvenverläufe sind ähnlich. Diese Effekte widersprechen den Ergebnissen anderen Studien (U. a. Hensel et al. 2002; Weis et al. 2001).

Übereinstimmung herrscht in Bezug auf das Ergebnis, dass Frauen sich besser erholen (siehe auch Weis et al. 2001). In allen Skalen zeigt sich ein tendenziell ungünstigerer Ausgangswert für Symptome und Funktionen, ausgeprägtere Schwankungen bei der Beurteilung aber eine deutlichere Erholungstendenz nach einem Jahr für die Gruppe der Frauen. Warum sich mit Ausnahme von Fatigue (Fatigue muss laut Faktorenanalyse eher dem Funktions- als dem Symtpombereich zugeordnet werden) die Geschlechtsunterschiede im Funktions- und nicht im Symptombereich abbilden kann nur spekuliert werden. Möglicherweise spielt für das Selbstbild der Frauen Abhängigkeit, Angewiesensein auf andere eine größere Rolle bei der Funktionsbeschreibung. Möglicherweise wirken sich auch die tendenziell stärker wahrgenommenen Symtome auf die Funktionsbeschreibung aus.

Vermutlich liefert die unterschiedliche Erholungstendenz der Geschlechter die Erklärung für die widersprüchlichen Studienergebnisse hinsichtlich des Geschlechtseffektes: Werden Daten, vor allem Querschnittsdaten, verglichen, kann der Zeitpunkt der Datenerhebung die Unterschiede verwischen. Nach einem Jahr sind die Unterschiede offensichtlich weniger ausgeprägt. Würde in dieser Studie nur der letzte Zeitpunkt im Querschnitt erfasst, wäre das Ergebnis deckungsgleich mit denen anderer Studien, die keinen Geschlechtseffekt finden.

Auch beim Karnofsky-Index zeigt sich ein signifikanter Geschlechtseffekt. Der Kurvenverlauf entspricht dem oben skizzierten der LQ-Skalen.

Eine Diskrepanz bezüglich der Beobachterperspektive zeigt sich auch in dieser Untersuchung, wenn man die Ergebnisse der Fragebögen zugrunde legt. Patienten schätzen eine Jahr nach Abschluss der Therapie ihren körperlichen Funktionszustand im LQFragebogen besser ein, als die Untersucher im Fremdrating mit dem Karnofsky-Index. 
Im Fremdrating zeigt sich eine signifikante Einbuße an körperlichen Fähigkeiten im Vergleich zum Ausgangszustand. Dieses Ergebnis hat sich auch in früheren Untersuchungen gezeigt (U. a. Korth 1994). Interpretiert wird dieses Ergebnis mit Hinweis auf die unterschiedliche Wahrnehmung vor und nach belastendem Therapieereignis. Das erfolgreiche Durchstehen und der positive Behandlungseffekt bei den Patienten, die nicht wieder erkrankt sind, beeinflussen die Bewertung.

Interessanterweise deckt sich die Interviewauswertung mit dem Fremdrating des Karnofsky-Index. Die Interviewdaten zeigen, dass die Patienten in der Mehrheit noch erhebliche Probleme haben und ihren Funktionszustand, wie vor der Therapie, nicht wieder erreichen konnten. Im Interview äußern Patienten mehr Beschwerden und der Vergleich mit dem früheren Zustand fällt ungünstiger aus. Vermutlich gehen in die Fragebogendaten Urteilsangleichungen mit ein, außerdem auch Antworten im Sinne von „Sozialer Erwünschtheit“ („Die Behandlung muss sich doch gelohnt haben“). In diese Richtung deuten auch die Antworten der Patienten, deren Behandlung nicht erfolgreich war und die wieder erkrankt sind. Der Nutzen der Therapie wird auch hier nur von wenigen Patienten in Frage gestellt. Die Hypothese, dass Patienten retrospekiv die Behandlung unabhängig von den durchgestandenen Belastungen und auch unabhängig vom Therapieerfolg als sinnvoll ansehen, kann als weitgehend bestätigt gelten.

Während die Ergebnisse des LQ-Fragebogens den Schluss zu lassen, dass der Zeitraum von einem Jahr für eine weitgehende Erholung nach HDC ausreicht (Ausnahme: Fatigue-Syndroms, Sexualität, Körperbild) (Chao et al.1992; Macquart-Moulin et al. 2000), lassen die Interviewauswertungen diesen Schluss nicht zu. Leistungseinschränkungen und Symptome werden noch von der Mehrheit der Patienten beklagt. Der Zeitraum von einem Jahr ist zur kurz für die Genesung und die diesbezüglich formulierte Hyptothese kann nicht bestätigt werden.

Patienten sind noch mitten im Trauerprozess um Verlorenes und die Krankheit ist bei den meisten, die nicht wieder erkrankt sind, noch Lebensmittelpunkt.

Erst recht trifft die Aussage, über die Krankheit als Lebensmittelpunkt, auf diejenigen Patienten zu, bei denen die Therapie nicht zum gewünschten Erfolg geführt hat, die wieder erkrankt sind bzw. deren Krankheit fortgeschritten ist.

Bemerkenswert ist in dieser Studie der niedrige Anteil derjenigen Patienten, denen die berufliche Rehabilitation nach einem Jahr gelungen ist. Während nach Kornblith (1998) drei Viertel aller überlebenden Krebspatienten wieder in den Beruf integriert werden, sind es für die Überlebenden nach einer HDC ca. zwei Drittel (Weis et al. 2001) denen die Reintegration gelingt. Auch Weis et al. (ebenda) finden in ihrer deutschen Stich- 
probe eine, im Studienvergleich deutlich niedrigere Rate beruflicher Reintegration. Die Autoren erklären diesen Effekt damit, „dass es sich um eine Negativselektion von Patienten handelte, die nach Abschluss der Transplantation noch eine stationäre Nachsorgebetreuung benötigten und daher möglicherweise deutlich vermehrte körperliche Probleme oder somatische Komplikationen hatten“ (S. 143). Die Motive der Inanspruchnahme von Rehabilitationsmaßnahmen in dieser Stichprobe stützt nicht die Annahme, dass es sich um die am meisten beeinträchtigten Patienten handelt. Die Daten legen nahe, dass es neben der eingeschränkten körperlichen Leistungsfähigkeit auch um Fragen der sozialen Absicherung geht, die sich auf eine Reintegration auswirken. Schlussfolgerungen sollten nur mit großer Vorsicht gezogen werden. Möglicherweise kommen die Ergebnisse der Studien aufgrund des heterogenen Diagnosespektrums zustande: sowohl in dieser Studie als auch in der von Weis et al. sind Patienten mit ganz unterschiedlichen Krebserkrankungen zusammengefasst worden.

Die Studie von Hensel et al. (2002) erbringt ebenfalls ein überraschendes Ergebnis: "The most striking observation of our study was the lack of difference in the re-employment rate or QOL scores among those patients who participated in rehabilitation programmes and those who did not" (S. 214). In beiden Studien handelt es sich um retrospektive Datenerhebungen. Der Schluss beider Autorengruppen auf einen negativen somatischen Selektionseffekt bleibt daher spekulativ.

Begründet wird die mangelnde Reintegration von den Teilnehmern dieser Studie mit Leistungseinbußen, daneben klingt bei einigen Frauen an, dass sie fürchten, durch den Arbeitsstress, die erneute Erkrankung zu begünstigen. Wenn Frauen von einer psychologischen Ursachenattribuierung ausgehen, dann ist verständlich, dass sie diesen Faktor zur zukünftigen Krankheitskontrolle ausschließen wollen.

Es ist zu vermuten, dass gerade diese Ergebnisse von den Kostenträgern der Rehabilitationsmaßnahmen mit Interesse verfolgt werden. Die Klärung muss prospektiven Studien mit größerer Teilnehmerzahl vorbehalten bleiben.

Aus der Literatur und der Klinik ist bekannt, dass Patienten gravierende Behandlungsbelastungen in Kauf nehmen, wenn sie von einer lebensbedrohlichen Erkrankung betroffen. Auch erhebliche Belastungen werden gut bewältigt. Für Patienten, die sich bedroht fühlen durch die Erkrankung, wird jede Behandlung, die mehr Hoffnung auf Heilung verspricht, akzeptiert. Diese Hypothese wird durch die Ergebnisse dieser Untersuchung ebenfalls bestätigt. Kaum einer der Patienten erkundigte sich nach Erfolgsbelegen der als vielversprechend akzeptierten Therapie und nur ein Teil holte Informationen über bevorstehende Nebenwirkungen der Therapie ein. Einerseits wollen sich Patienten vor den bevorstehenden Strapazen nicht verunsichern lassen, andererseits 
zeigt sich gerade in dieser Behandlungsphase ein großes Bedürfnis, den behandelnden Ärzten in einem umfassenden Sinne zu vertrauen. Beide Gründe stützen das „Prinzip Hoffnung“, das für die Phase der stationären Behandlung den stärksten emotionalen Belastungsausgleich darstellt. Gestützt wird diese Interpretation durch die Arbeitsergebnisse von Faller (1998), der in seinem Buch „Krankheitsverarbeitung bei Krebskranken“ auf die Notwendigkeit von „illusionären Räumen“ für die Bewältigung hinweist. Von Korth (1994) wird die zu beobachtende Realitätsverzerrung als „eine Art instinktive Notfallreaktion“ bezeichnet. In einigen Interviews dieser Studie berichten Patienten, dass es für das Durchstehen der Behandlungsbelastungen am schwierigsten sei, wenn vom Personal bestehende Restzweifel am Behandlungserfolg geschürt würden.

Hier werden die Schwierigkeiten deutlich, im Spannungsfeld „informed consent“/ „Erfolgserwartung/Hoffnung“ und „Verantwortungsabgabe bzw. -übernahme“, die ArztPatient-Beziehung sowie die Beziehung Pflegepersonal-Patient stützend und sensibel zu gestalten, ohne unlautere Erfolgsversprechungen abzugeben.

Eine Hochdosis-Chemotherapie wird - trotz der hohen Behandlungsbelastung - bezüglich ihrer Auswirkungen auf die "Lebensqualität" nicht schlechter beurteilt als eine langandauernde konventionelle Chemotherapie (u.a. Bonomi et al. 2000). Entscheidend ist der subjektive Gewinn für die Patienten - das Gefühl die wirksamste Behandlung zu bekommen. Die wirksamste Therapie wird häufig mit einschneidender Belastung und „neu sein“ assoziiert. Gestützt wird diese Auffassung mit Daten aus einer ganz anderen Richtung, die gleichzeitig die große Bedeutung des subjektiven Gewinnes für Patienten unterstreichen: Chemotherapieregimes bringen, wenn sie neu angewendet werden, höhere Überlebenszeitraten mit sich, als bei ihrer später Anwendung im Routinebetrieb (Fossati et al. 2001)! Vermutlich wird in der Patienten-ArztInteraktion ein Mehr an Hoffnung vermittelt, das sich in „harten“ Daten ausdrückt. Hoffnung ist denn auch die wichtigste Strategie der Krankheitsverarbeitung. Einen interessante Forschungsansatz liefert dazu Porzsolt (Vortrag auf dem Kongress für Palliativmedizin, 2000, Göttingen), der die Wirksamkeit von Chemotherapien im Hinblick auf Plazeboeffekt respektive Arzt-Patient-Beziehung untersucht und darauf aufmerksam gemacht hat, dass diese Effekte regelhaft in Therapievergleichsstudien vernachlässigt werden.

Hoffnung auf Heilung ist für die Patienten dieser Stichprobe die wichtigste interne Kontrollstrategie, die sich externer Mittel, nämlich der Behandlung mit HDC, bedient. 
Krankheitsverarbeitung gilt als Moderatorvariable bei der Beurteilung der LQ. Patienten passen ihre Überzeugungssysteme der veränderten Krankeitsrealität an. Aus den Interviewtexten wurde indirekt auf die Strategien der Krankheitsverarbeitung geschlossen. Ein wichtiges Ergebnis dieser Untersuchung ist, dass die Mehrheit der Studienteilnehmer variable Strategien nutzt. Vor der Behandlung spielen aktive Strategien (Kontrolle, Aktivität, Ablenkung), Arztvertrauen und Hoffnung die größte Rolle, in der stationären Phase stehen, neben regressiven Strategien (Rückzug/Ruhe, Anwesenheit eines Familienmitgliedes, Medikamente), ebenfalls aktive Strategien im Vordergrund. Zu den letzten beiden Zeitpunkten (3 Monate und 1 Jahr nach Therapie) beschreibt nahezu die Hälfte der Patienten depressive Strategien. Überraschenderweise werden depressive Strategien weniger von den Patienten genutzt, die inzwischen wieder erkrankt sind. Die eigenen klinischen Erfahrungen sprechen gegen eine Verallgemeinerung dieses Befundes. Vielmehr scheinen hier Besonderheiten der Stichprobe eine Rolle zu spielen. Der größere Anteil der wieder erkrankten Patienten nutzt Zielsetzungen/Eigene Stärke und Hoffnung/Optimismus als Bewältigungsstrategie. Möglicherweise sind hier Persönlichkeitsvariablen von Bedeutung. Die Patienten haben auf externe Kontrolle gesetzt und "verloren“. Wirksamere Therapieoptionen als die HDC stehen nicht mehr zur Verfügung. Es bleibt nur der Rückgriff auf interne Kontrollstrategien. Als Beispiel soll hier die Aussage eines Patienten dienen der, nach tiefer Enttäuschung durch ein Rezidiv, seine weitere Konzetration auf das lenkte, was inm noch blieb und der nach kurzer Zeit den Mut fand, sein Ziel für den Alltag zu formulieren: „....wenn ich schon sterben muss, dann werde ich jetzt noch nicht damit anfangen“.

Depressive Verarbeitungsstrategien müssen deutlich unterschieden werden von klinisch manifesten Depressionen im Sinne einer psychiatrischen Klassifikation. Nur wenige Patientinnen und keiner der Patienten wurden hier nach der Behandlung als depressiv eingestuft.

Depressive Verarbeitung kann gewertet werden als Ausdruck des Trauerprozesses um verlorene Gesundheit, verlorene Leistungsfähigkeit und einen veränderter Altag, der immer noch geprägt ist von der Erkrankung. Vielleicht auch als Reflex auf die Erkenntnis der existentiellen Unsicherheit und der Verletzlichkeit des Körpers.

„Normalität“ wird angestrebt, aber nicht erreicht. Die negativ bewertete Verarbeitungsstrategie „Hadern/Ruminieren“ kam allenfalls in abgeschwächter Form vor und wurde der Kategorie „depressive Verarbeitung“ zugeordnet. Religiöse Bindung spielte nur für drei Patienten eine stützende Rolle. Eine ausdrückliche Veränderung ihres Wertesystems benannten ebenfalls nur drei Patienten.

Indirekt zeigt sich eine Veränderung des Wertesystems darin, dass die Bedeutung von Arbeit und Leistungsfähigkeit relativiert wurde und Beziehungen einen größeren Stel- 
lenwert erhielten. Diese Veränderung ist darauf zurückzuführen, dass die große Mehrheit der Patienten dieser Studie uneingeschränkt die familiäre Unterstützung als wichtigste Ressource im Umgang mit Kranheit und Therapie beschreibt und damit auch die Notwendigkeit von Unterstützung anerkennt.

Wenn auch der Einfluss von „social support" heute differenzierter gesehen wird und einzelne Studien auch negative Einflüsse ausmachen konnten (siehe Übersicht von Faller 1998), so überwiegt doch der positive Einfluss auf Krankheitsbewältigung und damit auch auf die Bewertung der Lebensqualität, wie kürzlich wieder in einer prospektiven Studie an Mammakarzinompatientinnen von Michael et al. (2002) gezeigt wurde: Das Niveau der sozialen Integration ist ausschlaggebend für die Bewertung der gesundheitsbezogenen Lebensqualität und zwar unabhängig von Tumorcharakteristiken.

Neben der familiären Unterstützung wird eine gute Stationsatmosphäre als hilfreich empfunden. Gute Atmosphäre bedeutet das Erleben von persönlichem Interesse: „Keine Nummer sein“. Daneben spielt eine hohe fachlich-medizinische Kompetenz der Mitglieder des Behandlungsteams eine herausragende Rolle. Dem behandelnden Arzt kommt eine besondere Rolle zu. Patienten wünschen zu ihrer psychosozialen Unterstützung personelle Konstanz der Ärzte und führen als einen der wichtigsten Kritikpunkte für die nachstationäre Zeit, die häufigen Arztwechsel an.

Trotz Kritik, vor allem an strukturbedingten Mängeln der Krankenhausversorgung (schlechte Organisation, mangelnde Informationen, Arztwechsel, Wartezeiten) und der Kooperation zwischen Krankenhaus und niedergelassenen Ärzten, sind die meisten Patienten mit der Behandlung sehr zufrieden. Dieses Urteil ändert sich auch im Rückblick nach einem Jahr nicht.

Retrospektiv findet allerdings eine Bewertungsänderung der Belastungen durch HDC statt. Nach einem Jahr werden die Belastungen von vielen Patienten mit Metaphern wie z. B. „Hölle“ und „Bombe“ beschrieben während unmittelbar nach der HDC die Belastungen, gemessen an den schlimmen Erwartungen, als weniger gravierend empfunden wurden. Diese Relativierung der Behandlungsbelastungen steht vermutlich auch im Dienste der Bedrohungsabwehr. Der Sinn der Therapie wird kaum in Frage gestellt, auch nicht von den Patienten, die wieder erkrankt sind. 


\subsection{Ungelöste Probleme}

\subsubsection{Methodische Probleme}

Trotz psychometrischer Überprüfung, Stadardisierung der Prozeduren und Auswertungsvorschriften bleiben zahlreiche methodische Probleme bestehen. Auf einige wichtige Faktoren soll hier verwiesen werden.

Wie im Abschnitt „Konzeptualisierung von LQ“ ausgeführt wurde, können Daten, die mit unterschiedlichen Lebensqualitätsinstrumenten oder Instrumenten zur gesundheitsbezogenen Lebenszufriedenheit erhoben werden, nicht verglichen werden. Bei der Beschränkung der Ergebnisinterpretation auf ein Instrument (hier der Lebensqualitätsfragebogen der EORTC) kommen zumindest keine widersprüchlichen Studienergebnisse vor, auch wenn in Abhängigkeit von den Besonderheiten einzelner Stichproben Unterschiede auftreten.

Die Ergenisse des LQ-Fragebogens können dann sinnvoll interpretiert werden, wenn folgende Punkte berücksichtigt werden:

- Querschnittsuntersuchungen bei denen Patienten in unterschiedlichen Krankheits- und Rehabilitationsstadien unter einen einzigen Zeitpunkt subsummiert werden, wie dies aus erhebungsökonomischen Gründen heute vielfach üblich ist, haben wenig Aussagekraft.

- Die Symptomskalen scheinen weniger „verzerrungsanfällig“ zu sein als die Funktionsskalen. Wenn in verschiedenen Untersuchungen die Funktionsskalen keine Zeiteffekte aufweisen, ist das ein Hinweis auf die Veränderungen des Überzeugungssystems. Patienten verändern ihre Bewertungen in Relation zu Krankheits- und Therapieereignissen. Deutlich wurden diese Veränderungen, wenn Patienten beim Ausfüllen der Fragebögen rückfragen ob sie ihr Urteil im Vergleich mit "früher" oder im Vergleich mit situationsabhängigen Erwartungen fällen sollen. Das Gleiche trifft im Prinzip auch für Symptombeschreibungen zu, scheint sich aber nicht in gleicher Weise auszuwirken. Für diese Studie ist die Symptombeschreibung im Verlauf und ebenfalls die Ausprägung unterschiedlicher Symptome plausibel, d.h. aus dem klinischen Bild und den Therapienebenwirkungen zu erklären.

- Werden die Interviewdaten mitberücksichtigt, wird deutlich, dass den Symptomen im Zeitverlauf eine unterschiedliche Gewichtung in Bezug auf die allgemeine Lebensqualität beigemessen wird. Patientenäußerungen wie „Mir geht es gut, obwohl mir übel ist, ich Schmerzen habe, nicht essen kann usw ..." zeigen dies. Wenn Symptome lange nach Abschluss der Therapie bei Patienten, die nicht wieder erkrankt sind, auftreten, dann erhalten sie eine andere Bedeutung, werden unter Umständen als Verschlechterung des Gesamtgesundheitszu- 
standes interpretiert und können (nicht müssen!) dann maßgeblich die Bewertung der allgemeinen $L Q$ beeinflussen.

- Für den in dieser Untersuchung verwendeten LQ-Fragebogen kann als gesichert gelten, dass die Beurteilung der körperlichen Funktion eng mit der Beurteilung der allgemeinen LQ zusammenhängt. Auf die Identifikation von allgemeiner Lebensqualität und physischer Funktion hat bereits Sigle \& Porzsolt (1996) hingewiesen. Die Korrelationen zwischen beiden Skalen sind in dieser Studie für alle Zeitpunkte signifikant und erreichen auch die höchsten Korrelationen im Vergleich mit den Korrelationen der anderen Skalen. Vor der Entlassung ist der Zusammenhang relativ am schwächsten. Auch das ist plausibel, da in dieser Situation das Überstehen der HDC ganz im Vordergrund steht und körperliche Einschränkungen und Symptome weniger stark gewichtet werden, wie die Interviewtexte zeigen. Der Referenzrahmen für die LQ-Bewertung wird zusätzlich beeinflusst durch allgemeine Erwartungen, hier die Erwartung, dass die belastende Therapie zu Einbrüchen führen muss. Aber auch zu dieser generellen Tendenz gibt es Ausnahmen: bei der Betrachtung der individuellen Verlaufskurven gibt es einige wenige Patienten, die von einer Verbesserung der körperlichen Funktionswerte und der allgemeinen LQ-Werte unmittelbar nach der HDC berichten, im völligen Gegensatz zum Verlauf des Gesamtmittelwertes. Diese Patienten haben ausnahmslos eine schlechte Prognose, die sich im Verlaufe des Jahres nach HDC bestätigt. Hier scheint in der stationären Zeit die Tatsache, dass überhaupt behandelt wird, unabhängig von den realen Therapiebelastungen, das Urteil zu bestimmen. Die Beobachtung einer niedrigen LQ vor Behandlungsbeginn stützt die These des prädiktiven Wertes der allgemeinenLQ/der körperlichen Funktion im Hinblick auf den weiteren ungünstigen Krankheitsverlauf.

- Der Interpretationsrahmen der Fragebogendaten sollte sehr eng gehalten werden und darf nicht mit der umgangssprachlich weiten, unpräzisen Auffassung von Lebensqualität vermischt werden. Die Beurteilung der LQ wird vom Prozess der Krankheitsverarbeitung mitbestimmt und dieser Prozess wird beeinflusst von zahlreichen weiteren Variablen unter denen „Hoffnung“, „Schwere der Erkrankung/Prognose“, „Persönlichkeit“ und „Zeit“ die wichtigsten sind.

- Wenn es die Größe der Stichprobe erlaubt, müssen Gruppeneffekte berücksichtigt werden. Neben Gruppeneffekten, zeigt der Blick auf die ausgeprägten Varianzen, die sich in allen Studien finden, dass interindividuelle Differenzen sehr hoch bewertet werden müssen. 
- Leider bietet auch der Kontrollgruppenvergleich keine Patentlösung an, da es für die HDC keine adäquate Kontrollgruppe gibt. Der Faktor „Zeit“ spielt eine so gewichtige Rolle, dass die Behandlungsbedingungen einer kurzdauernden HDC kaum zu vergleichen sind mit einer, sich über Monate hinziehenden, konventionellen Chemotherapie. Langandauernde Chemotherapien können zur physischen und psychischen Erschöpfung führen, zum Ausgebranntsein und zur reaktiven „Erschöpfungsdepression“, die sich dann in einer geringen Bewertung der Lebensqualität ausdrückt.

- Vergleiche mit gesunden Populationen sind im Hinblick auf Funktionseinschränkungen und Symptome gestattet, im Hinblick auf allgemeine Lebensqualität wegen der Veränderungen der Wertesysteme unter der Bedingung einer lebensbedrohlichen Erkrankung aber unsinnig.

- Schlussfolgerungen, die sich als Fazit häufig finden und die sinngemäß lauten: „Die Lebensqualität wird durch die Therapie nicht beeinträchtigt“ oder „Die Lebensqualitätsmessung zeigt, dass die Therapie akzeptabel ist“, sollten in dieser Weise nicht gezogen werden.

\subsubsection{Problematik des LQ-Konstruktes aus klinischer Perspektive}

Für die Schlussbetrachtung der Problematik des LQ-Konstruktes wird hier ausdrücklich allein die klinische Perspektive gewählt. Gleichwohl kommt es auch bei der Konzentration auf die klinische Perspektive teilweise zu Überschneidungen bzw. Berührungen mit der ethisch-moralischen Perspektive.

Die LQ-Forschung ist mit zwei zentralen Ansprüchen angetreten:

1. Das enge biomedizinische Weltbild der Schulmedizin zu erweitern und psychosoziale Aspekte mit in den Blick zu nehmen.

2. In wichtigen Therapieentscheidungssituationen sowohl dem Kliniker als auch dem Patienten Hilfestellung zu leisten.

Auf beiden Ebenen ist die LQ-Forschung ihren Ansprüchen nicht gerecht geworden. Wenn im Folgenden eine abschließende die Betrachtung des Forschungsansatzes der gesundheitsbezogenen Lebensqualität (HRLQ) versucht wird, so bedeutet dies nicht, dass nicht Ergebnisse aus Studien, die auch eine Verbesserung der Patientenversorgung zur Folge hatten, gewürdigt werden. Noch immer gibt es nicht zuviel sondern eher zu wenig Dokumentation und Kenntnisnahme von belastenden Therapien, vor allen Dingen in der Klinikroutine und prinzipiell ist eine Dokumentation mit Hilfe von Selbstratings der Patienten außerordentlich sinnvoll. Vergleiche von Therapien und Kliniken außerhalb von Forschungsprojekten waren in der Vergangenheit eher selten. Hier wurde und wird in der Tat auch in Folge der LQ-Forschung ein Fortschritt für die 
Patienten erzielt - allein dadurch, dass Behandlungsergebnisse transparenter werden. Aber diese Art der Qualitätssicherung darf nicht mit LQ verwechselt werden - und für diese Verwechslung ist der Forschungsansatz LQ letztlich mitverantwortlich.

Die Erweiterung des biomedizinischen Weltbildes der Schulmedizin ist der LQForschung nur bedingt gelungen. Anstatt Veränderungen zu bewirken, hat sich die LQForschung eher selbst verändert und ist, wie gezeigt wurde, zum Bestandteil einer verengten biomedizinischen Forschung geworden.

LQ, wie sie in der psychometrischen Forschung aufgefasst wird, lässt zwangsläufig den interpersonellen Aspekt außer Acht, und genau in diesem Umstand liegt die große Gefahr einer falschen und damit für Missbrauch offennen Verwendung ihrer Ergebnisse. Auch diese Problematik ist in der Logik des Forschungsansatzes LQ selbst angelegt.

LQ wird in der psychometrischen Forschung zu einer messbaren Eigenschaft, einer Eigenschaft, die man hat oder nicht hat. Sie wird nicht verstanden als etwas, was maßgeblich im interpersonellen Bezug hergestellt wird bzw. sich herstellt.

Der Begriff LQ ist, wie an anderer Stelle gezeigt wurde, nicht klar abgrenz- bzw. definierbar, sondern er wird, je nach Fragestellung und Behandlungssituation, mit jeweils unterschiedlichen Inhalten gefüllt. Gerade im Zusammenhang mit einer lebensbedrohlichen Erkrankungsphase können aber unmöglich messbare Aspekte wie Funktionalität gemeint sein. Hilfreicher sind hier die Ausführungen von Habermas, der den Begriff der Menschenwürde verwendet und schreibt, dass Menschenwürde „im streng moralischen und rechtlichen Verstande an die Symmetrie von Beziehungen gebunden (sei) und nicht eine Eigenschaft, die man von Natur aus besitzen kann wie Intelligenz oder blaue Augen" (Habermas 2001 S.62). Diese Diskussion bietet auch für unseren Diskussionszusammenhang eine geeignete Grundlage.

In der Situation von Hinfälligkeit und lebensbedrohlicher Krankheit ist die „Symmetrie von Beziehungen“ zunächst zwangsläufig außer Kraft gesetzt, der Patient ist in eine fundamentale Form von Abhängigkeit geraten und auf die Empathie seines Gegenübers angewiesen. Denn: „Die Abhängigkeit vom Anderen erklärt die Verletzbarkeit des Einen durch den Anderen. Die Person ist Verwundungen in den Beziehungen am schutzlosesten ausgesetzt, auf die sie zur Entfaltung ihrer Identität und zur Wahrung ihrer Integrität am meisten angewiesen ist" (ebenda S. 63).

In der Situation von lebensbedrohlicher Erkrankung sind dies, zumindest phasenweise, hauptsächlich der Arzt und nachgeordnet auch andere Mitglieder des Behandlungsteams, auf die der Patient angewiesen ist. Und in diesem Kontext wird das mitkonstituiert, was Würde, respektive Lebensqualität ausmacht. Insofern ist Habermas auch zuzustimmen, wenn er in diesem Zusammenhang weiter ausführt, dass er „moralisches 
Verhalten“ versteht „als konstruktive Antwort auf Abhängigkeit und Angewiesenheiten, die in der Unvollkommenheit der organischen Ausstattung und der fortbestehenden Hinfälligkeit der leiblichen Existenz (besonders in Phasen von Kindheit, Krankheit und Alter) begründet sind“ (ebenda S. 62).

Eine „konstruktive Antwort auf Abhängigkeit und Angewiesenheit“ muss im hier diskutierten Spannungsfeld auch darauf gerichtet sein, die zwangsläufige Asymmetrie von Beziehungen im Sinne einer "gelungenen Begegnung“ - und Behandlung ist immer auch ge- oder misslungene Begegnung - aufzulösen.

Bei einer Erkrankung wie „Krebs“, wo der Körper, die „Hülle des Ich“ (ebenda), zum Feind werden kann bzw. geworden ist, ist es besonders wichtig, sich zu vergegenwärtigen, dass die Symmetrie der Beziehungen nicht vom Patienten hergestellt werden kann. Er ist vielmehr elementar darauf angewiesen, dass dies von ärztlicher Seite aus geschieht. Hier soll nicht die Bedeutung einer gelungenen pflegerischen oder psychotherapeutischen Behandlung unterbewertet werden, aber in lebensbedrohlichen Situationen ist die Hoffnung von Patienten in erster Linie auf die Wirksamkeit ärztlicher Kunst gerichtet und darauf, dass der behandelnde Arzt die Verantwortung für Therapieentscheidungen übernimmt.

Diese, auch durch die Interviews der vorliegenden Studie belegbare Patientenerwartung, scheint von ärztlicher Seite zunehmend in Frage gestellt zu werden. Als Beispiel möge hier ein Auszug aus der Stellungnahme der deutsch-österreichischen hämatologischen Gesellschaft dienen: „Die Medizin und ganz besonders die Onkologie befindet sich in einer revolutionären Phase ... Dies betreffe nicht nur die medizinische Entwicklung, die durch das Verstehen molekularer Zusammenhänge eine ganz neue Ebene erreicht habe, sondern auch das Verhältnis zum Patienten. Der Arzt verliere seine dominante Position und werde immer mehr zum Mediator und Berater in einem Team von Gesundheitsanbietern“ (Junker, 2002 S. 196). Das hier beschriebene Rollenverständnis vom Arzt als „Mediator und Berater in einem Team von Gesundheitsanbietern“, als Einer unter Anderen, scheint einen Ausweg zu bieten aus dem heute schwer erträglichen Balanceakt zwischen traditioneller, als überholt geltender „paternalistischer Verantwortungsübernahme“ und "(post)moderner Verantwortungsdelegation“. Die Verfasserin ist davon überzeugt, dass Stellungnahmen wie die o. g. in eine falsche Richtung weisen, da sie nahe legen, sich aus der Verantwortung und Zuständigkeit für den "ganzen“ Patienten zurückzuziehen und die Sorge für die seelische Not des Patienten an dafür ausgebildete Spezialisten Therapeuten oder Seelsorger (als Mitglieder eines „Teams von Gesundheitsanbietern“) - zu delegieren. 
Diese Spaltung berührt die ureigenste Frage der Psychosomatik, und die Ergebnisse der vorliegenden Studie zeigen, dass diese Teilung des Behandlungsauftrages von Patienten nicht gewünscht wird.

Auch wenn die praktischen Erfahrungen der Verfasserin und die dokumentierten Interviewäußerungen zeigen, dass psychotherapeutische Unterstützung wichtig ist und auch angenommen wird, so kann dies nicht darüber hinweg täuschen, dass sich in der Akutbehandlung die Patientenerwartung, gerade auch an seelische Unterstützung, auf den Arzt richtet.

Daher scheint es geboten, die interdisziplinäre Kompetenz im Sinne von Behandlung und Achtung von Leib und Seele für alle am Behandlungsprozess Beteiligten zu fördern. Ein in diesem Sinne verändertes Rollenverständnis könnte auch „Rollendissoziationen“, wie sie vor mehr als einem Vierteljahrhundert von Richter $(1995,1985)$ aus einem ganz anderen Blickwinkel beschrieben wurden, entgegenwirken.

Richter hat darauf hingewiesen, dass die Institution Krankenhaus die Aufteilung in die klassischen Stereotypen männlich/weibliche Medizin präformiert. „Weibliche Medizin“ bedeutet Verstehen, Mitfühlen, Mittragen, Geborgenheit vermitteln und Fürsorge und ist an die nicht-ärztlichen Mitarbeiter delegiert. „Den Ärzten, den Vertretern der männlichen Medizin obliegt es Distanz zu wahren, sachlich die Funktionssysteme des Organismus zu analysieren und vielerlei kunstvolle Tätigkeiten auszuführen" (S.77). Diese nach Auffassung der Verfasserin weiterhin gültige Charakterisierung Richters beschreibt die derzeitige Klinikswirklichkeit jedoch nur noch bedingt zutreffend, erfährt doch der „weibliche“ Anteil von Medizin auch im Rahmen von Pflege nicht mehr die frühere Würdigung und ist immer weiter zurückgedrängt worden. Es würde den Rahmen dieser Arbeit sprengen, differenziert auf das veränderte Pflegeverständnis einzugehen. In der Situation von Hilfsbedürftigkeit und Angewiesensein ist es für Patienten nahezu unerträglich wenn erlebt wird, dass elementare Pflegetätigkeit nicht mit der oben erwähnten „Moralität“ im Sinne von Habermas ausgeführt, sondern eher gering geachtet wird. Auch der Praxisbereich Pflege ist im Zuge von „Verwissenschaftlichung“ umstrukturiert worden. Auf der einen Seite wächst „die legitimierende Kraft der im weitesten Sinne klinischen Handlungsformen" - jede Technologie, jede Mechanisierung, jede Form von Apparatemedizin wird mit Patientenwohl und der Steigerung von LQ im wahrsten Sinne des Wortes verkauft - während „ihre gesellschaftliche Relevanz schwindet“ (Habermas ebenda S. 82) - Grundpflege gilt als anspruchlose Tätigkeit und wird schlecht bezahlt - die krassesten Missstände finden sich im (Alten)Pflegebereich.

Ein im Sinne des unbestritten hohen und schwer erfüllbaren Anspruchs an „moralisches Verhalten als konstruktive Antwort auf Abhängigkeit und Angewiesenheiten" der Patienten hätte eine Auffassung von Behandlung zur Folge, die sich grundlegend von 
dem Verständnis der oben zit. Hämatologischen Gesellschaft unterscheidet. Diese geht von einem Team von Gesundheitsanbietern aus - eine gerade im Zusammenhang mit onkologischen Erkrankungen zynische Wortwahl.

Nimmt man in dem Spannungsfeld Arzt/Patient/Therapie/Pflege/Psychotherapeut die Patientenseite in den Blick, dann spielen, neben diesen interpersonellen Beziehungen und Abhängigkeiten weitere Faktoren eine wichtige Rolle, wenn es um die Erfassung von LQ geht.

Von theoretischer Seite wird immer wieder betont, dass LQ nicht mit „lebenswert", respektive „lebensunwert“ gleichgesetzt werden darf, sondern ausschließlich im eingeschränkten Sinne der HRLQ gelten soll. Dies hindert Patienten nicht daran, LQ in genau diesem Sinne aufzufassen, und die in diesem Zusammenhang (noch) gültigen gesellschaftlichen Tabus nicht nur mental sondern auch verbal zu brechen. Aufschluss geben hier die Arbeiten, die sich mit den Euthanasiewünschen von Patienten befassen. Das Leben ist in der eigenen Betrachtung oder in den Augen der Bezugspersonen nichts mehr wert, es ist lebensunwert und verdient nicht mehr aufrechterhalten zu werden. Oder es ist zur Qual geworden, Hoffnungslosigkeit überwiegt im Moment oder längerfristig und Sterben wird als die gnädigere Perspektive aufgefasst.

Aus der Frage des "Lebenswertes" ergibt sich inhaltlich das größte Problem der LQMessung; unter anderem deshalb weil es nicht möglich ist LQ in einen bestimmbaren Zusammenhang mit unterschiedlichen Gesundheitszuständen, Schweregraden von Beeinträchtigungen, Symptomen sowie krankheit- und therapiebedingten Leiden zu bringen.

Problematisch wird LQ als Kriteriumsvariable dann, wenn sie als Evaluationskriterium in der Onkologie eingesetzt wird.

Folgendes Beispiel möge zur Verdeutlichung dieses Problems dienen: Es ist zweifelsfrei belegt, dass die Mehrheit der Patienten Wirksamkeit in Bezug auf Lebensverlängerung zum entscheidenden Behandlungs- und Erfolgskriterium macht, und vor allem im Zustand akuter Bedrohung kaum nach LQ fragt. Die Patienten honorieren die Tatsache, dass überhaupt behandelt wird und erleben dies unabhängig von Nebenwirkungen und Belastungen auch als verbesserte LQ. Nur so ist zu erklären, dass aktive Behandlung im Vergleich mit der besten supportiven Behandlung positiver abschneidet. Fremdurteile fallen hier deutlich anders aus - Nicht-Betroffene schätzen die Therapiebelastungen in dieser Situation als besonders hoch ein. ${ }^{85}$ Patienten formulieren in die-

${ }^{85}$ Die Verfasserin konnte dieses Phänomen wiederholt beobachten. Einer Patientin mit weit fortgeschrittener Erkrankung die sich in palliativer Chemotherapie befindet, kommt in drei Wochenabständen in die Tagesklinik. Die Patientin leidet unter den Auswirkungen der Chemothe- 
sem Zusammenhang: „So lange behandelt wird herrscht Hoffnung“. Herrscht diese Überzeugung vor, müssen Patienten die Beurteilung ihrer LQ bewusst oder unbewusst an der Frage ausrichten, ob behandelt wird oder nicht. Abbruch aktiver Behandlung bedeutet: „Es besteht keine Hoffnung mehr“.

Dass hier auch sachlich sinnvoll begründete Behandlungskonzepte - nicht zuletzt vor dem Hintergrund von Sparzwängen - bei Patienten auf Misstrauen und Ablehnung stoßen, möge folgendes Beispiel verdeutlichen:

Bis vor wenigen Jahren wurde in der Nachsorge von Mammakarzinompatientinnen routinemäßig eine ausgedehnte apparative Technik eingesetzt, um so früh wie möglich Metastasen aufzuspüren und eine Therapie einzuleiten. LQ-Argumente spielten für das Untersuchungs- und Behandlungskonzept keine Rolle.

Da inzwischen nachgewiesen ist, dass ein früher Metastasennachweis und damit entsprechend frühere chemotherapeutische Behandlung keinen Überlebensvorteil erbringen, sehen die derzeit gültigen Nachsorgeregelungen vor, das Gewicht auf die klinische Untersuchung zu legen, um Patientinnen nicht immer wieder zu beunruhigen und darüber ihre $L Q$ zu beeinträchtigen.

Patientinnen können dieser Argumentation in der Regel nicht folgen, sondern fordern geradezu bildgebende Verfahren - „zur Beruhigung“.

Dass es für Patientinnen äußerst schwer ist, die Sinnhaftigkeit der veränderten Nachsorgerichtlinien anzuerkennen ist mehr als nachvollziehbar, heißt dies doch auch anzunehmen, dass sich die Krankheitsentwicklung einem „linear begründeten Behandlungskonzept" - möglichst frühe Krankheitserkennung und/oder möglichst hoch dosierte Therapie unter Inkaufnahme gravierender Nebenwirkungen garantieren größt möglichen Behandlungserfolg - entziehen kann. Argwohn gegenüber diesem Behandlungskonzept und dahinter vermuteten Sparmaßnahmen bieten hier bisweilen auch einen Ausweg aus seelischer Not.

Wir haben gesehen, dass die Beurteilung von HRQL nicht nur durch methodische Probleme im engeren Sinne bestimmt, sondern auch von aktuellen gesundheitspolitischen Debatten und Entwicklungen, sowie daraus resultierenden Ängsten, beeinflusst wird.

Die größte Gefahr besteht nach Ansicht der Verfasserin derzeit darin, dass die Ergebnisse von LQ-Fragebögen in der medizinischen Diskussion unkritisch als Synonym für

rapie, Übelkeit und Fatigue sind sehr ausgeprägt, die Fahrten zur $30 \mathrm{~km}$ entfernten Klinik erlebt sie als Belastung. Trotzdem fällt das Ergebnis des Fragebogens, der den Patienten in der Tagesklinik regelmäßig vorgelegt wird und der nach Beschwerden fragt, außerordentlich positiv aus. Die Verfasserin, die mit der Patientin gerade wegen ihres bedrohlichen physischen und psychischen Zustandes in regelmäßigem Telefonkontakt steht, vermochte schriftliche und mündliche Äußerungen nicht in Einklang zu bringen. 
einen viel weiteren, eher umgangssprachlich gefassten Begriff von LQ benutzt werden, und suggeriert wird, dem „humanistischen“ Aspekt in onkologischer Forschung und Therapie würde schon dadurch Genüge getan, dass LQ-Fragebögendaten zur Kenntnis genommen werden.

In einer Phase, in der Patienten die schmerzhafte Tatsache annehmen müssen, dass Behandlung „nur" noch in der Linderung von Symptomen bestehen kann, dass trotz hochspezialisierter Ärzte und hochentwickelter Apparate und Medikamente ein "Sieg“ über die Krankheit nicht mehr „machbar“ ist, fühlen sie sich oft zuwenig getragen und gestützt.

Eine Stellungnahme des Rates der Konföderation evangelischer Kirchen in Niedersachsen - erarbeitet im Diskussionszusammenhang pränatale Diagnostik und genetische Beratung (nach Ansicht der Verfasserin aber durchaus auch auf andere Gebiete der Medizin und somit auch auf die Onkologie übertragbar) weist in eine Richtung, die die gesellschafts- und gesundheitspolitische Diskussion jenseits von Machbarkeits und Sparpolitik positiv beeinflussen könnte. Dort heißt es u. a. „Zur gegenwärtig stark ausgeprägten Ethik der Leidensbekämpfung muss eine Ethik des Umgangs mit dem Leid kommen...Ein gestörtes Gleichgewicht muss wieder hergestellt“" und die Einsicht muss gefördert werden, dass "Gesundheit nicht Abwesenheit von Störungen ist, sondern die Kraft mit ihnen zu leben“ (S. 22). Dabei zielt diese Stellungnahme nicht dahin, die „Ethik im Umgang mit dem Leid“ an die Stelle der „Ethik der Leidensbekämpfung“ zu setzen, es geht vielmehr um einen oben bereits erwähnten umfassenderen Behandlungsauftrag. Wenn tatsächlich eine Ethik des Umgangs mit dem Leid an die Seite der, die Klinikroutine bislang nahezu ausschließlich bestimmende Ethik der Leidensbekämpfung träte, dann hätte dies in der Tat nicht unerhebliche Auswirkungen auf das Behandlungsverständnis.

Umgang mit dem Leid ist eine Lebensaufgabe, der sich die von einer Krebserkrankung Betroffenen nicht entziehen können und für deren Bewältigung sie auf ihr gesamtes Umfeld - Angehörige und Freunde, Ärzte, Pfleger und Therapeuten - angewiesen sind.

Dieser Umgang mit dem Leid wird erschwert bzw. unmöglich gemacht, wenn die gesamtgesellschaftlichen Wertevorstellungen sich auf Gesundheit - Leistung - Erfolg Kampf - Machbarkeit konzentrieren. Individuelle Krankheitsbewertung und Krankheitsanpassung muss mit der Bürde der die Realität verzerrenden gesellschaftlichen Wertvorstellungen umgehen. Krankheit, Leiden, Alter wird verdrängt. Diese Verdrängung geht soweit, dass von „Gesundheitsanbietern“ gesprochen wird. Der Begriff der Krankheit wird sprachlich verbannt und es wird so getan als sei Gesundheit etwas, das man anbieten, annehmen oder zurückweisen kann. Wer Gesundheitsangebote zu- 
rückweist, ist selber schuld. Das treibt folgende, schon lange geübte Praxis auf die Spitze: Im Routinebetrieb werden erfolgreiche Therapien als Erfolg ärztlicher Kunst gewertet (durchaus zurecht), im Bereich von unsicheren Prognosen und geringer Erfolgserwartungen wird diese Verantwortung eher an die Patienten delegiert, nach dem Motto „Wir tun was wir können, jetzt liegt es an Ihnen“.

In einem Verständnis vom Arzt als Heilkundigem und Helfer wird ein völlig anderes Rollenverständnis transportiert, als im Begriff des Arztes als Gesundheitsanbieter. Anbietern stehen Konsumenten gegenüber, nicht Kranke, die der Hilfe bedürfen.

Für die Auseinandersetzung mit Krankheit ist die Art der Begriffsdegeneration oder Begriffsverwirrung vor allem für den Kranken fatal, ermöglicht doch nur die sprachliche Erfassung - das Übersetzen von Gefühlen, das Beschreiben von Geschehnissen und der Versuch Unsagbares, der Empathie kaum zugängliches, auszudrücken - die Krankheit als zum eigenen Leben zugehörig anzuerkennen. Mangelnde sprachliche Präzision ist nicht nur keine Hilfe, sondern verunsichert zusätzlich, wenn die eigene Krankheitsrealität nicht mit den Vorgaben der Anderen in Einklang zu bringen ist. Patienten können sich der Krankheitsrealität wenn überhaupt nur zeitweilig entziehen. Den Therapeuten allerdings bieten sprachliche Ungenauigkeiten Entzugs- und Verdrängungsmöglichkeiten an.

Wenn es jedoch gelingt, dass Patienten gerade in der Abhängigkeit mit den sie behandelnden, begleitenden und stützenden Personen im oben beschriebenen Sinne auf "gleicher Augenhöhe“ kommunizieren und sie so neben unterschiedlichen Arten von Hoffnung auch ihre Würde bewahren können, dann gilt die Aussage einer Patientin der Untersuchungsgruppe: „Der Mensch kann viel aushalten.“ 


\section{Literaturverzeichnis}

Aapro MS. (2001). Adjuvant therapy of primary breast cancer: a review of key findings from the 7th international conference, St. Gallen, February 2001. Oncologist, 6(4), 376-85.

Aaronson NK. (1990). Quality of Life Assessment in Cancer Clinical Trials. In Holland JC \& Zittoun R (Eds.). Psychosocial Aspects of Oncology (pp. 97-113). BerlinHeidelberg New York: Springer Verlag.

Aaronson NK, Ahmedzai S, Bergman B et al. (1993). The European Organization for Research and Treatment of Cander QLQ-C30: A Quality-of-Life Instrument for Use in International Clinical Trials in Oncology. Journal of National Cancer Institute, Vol.85, No.5, 365-376.

Aaronson NK \& Beckman J (Eds.). (1987). The Quality of Life of Cancer Patients. New York: Raven Press.

Aass N, Fossa SD, Dahl AA \& Moe TJ. (1997). Prevalence of anxiety and depression in cancer patients see at the Norwegian Radium Hospital. Eur J Cancer, 33, 1597-1604.

Abbey A \& Andrews FM. (1985). Modeling the psychosocial determinants of life quality. Social Indicators Research, 16, 1-34.

Ahles TA, Tope DM, Furstenberg C et al. (1996). Psychologic and neurologic impact of autologous bone marrow transplantation. J Clin Oncol, 14, 1457-1462.

Ahles TA, Tope DM, Pinkson B et al. (1999). Massage therapy for patients undergoing autologous bone marrow transplantation. J Pain Symptom Manage, 18 (3), 157-163.

Akechi T, Kugaya A, Okamura $\mathrm{H}$ et al. (1999). Fatigue and its associated factors in ambulatory cancer patients: A preliminary study. Pain Symptom Manage, 17, 42-48.

Alexander PJ, Dinesh N \& Vidyasagar MS. (1993). Psychiatric morbidity among cancer patients and its relationship with awareness of illness and expectations about treatment outcome. Acta Oncol, 32 (6), 623-626.

Andrykowski MA. (1994). Psychosocial factors in bone marrow transplantation: A review and recommendations for research. Bone Marrow Transplant, 13, 357-375.

Andrykowski MA, Altmaier EM, Barnett RI et al. (1990). Cognitive dysfunction in adult survivors of allogenic marrow transplantaton: relationship to dose of total body irradiation. Bone Marrow Transplant, 6, 269-276.

Andrykowski MA, Brady MJ, Greiner CB et al. (1995b). "Returning to normal" following bone marrow transplantation: outcomes, expectations and informed consent. Bone Marrow Transplant 15 (4):573-581.

Andrykowski MA, Curran SL \& Lighner R. (1998). Off-treatment fatique in breast cancer survivors: a controlled comparison. J Behav Med, 21, 1-18.

Andrykowski MA, Cordova MJ, Hann DM et al. (1999).Patients'psychosocial concerns following stem cell transplantation. Bone Marrow Transplant 24: 1121-1129

Andrykowski MA, Carpenter JS, Greiner CB et al. (1997). Energy level and sleep quality following bone marrow transplantation. Bone Marrow Transplant, 20 (8), 669-679.

Andrykowski MA, Greiner CB, Altmaier EM et al. (1995a). Quality of life following bone marrow transplantation: findings from a multicenter study. $\mathrm{Br} \mathrm{J}$ Cancer, $71,1322-1329$.

Andrykowski MA, Schmitt FA, Gregg ME et al. (1992). Neuropsychologic impairment in adult bone marrow transplant candidates. Cancer, 70, 2288-2297.

Angelino AF \& Treisman GJ. (2001). Major depression and demoralization in cancer patients: diagnostic and treatment considerations. Support Care Cancer, 9, 344-349.

Antman et al. (1995). High-dose chemotherapy with autologous hematopoietic stem cell support for breasth cancer in North America. Journal of Clinical Oncology, 15, 1870-1879.

Apolone G, Filiberti A, Cifani S et al. (1998). Evaluation of the EORTC QLQ-C30 questionnaire:a comparison with SF-36 Health Survey in a cohort of Italian long-survival cancer patients. Ann Oncol, 9 (5), 549-557.

Aragona M, Muscatello MR \& Mesiti M. (1997). Depressive mood disorders in patients with operable breast cancer. J Exp Clin Cancer Res, 16 (1), 111-118.

Arbeitsgruppe des Rates der Konföderation evangelischer Kirchen in Niedersachsen. Pränatale Diagnostik und genetische Beratung. Hausdruckerei des AfG.

Aulbert A \& Niederle N (Eds.). (1990). Die Lebensqualität des chronisch Krebskranken. Stuttgart, New York: Thieme Verlag.

Aulbert E (Ed.). (1993). Bewältigungshilfen für den Krebskranken. Stuttgart, New York: Thieme Verlag. 
Aulbert E. (1993). Hilfen bei der Gewinnung und Erhaltung von Lebensqualität. In Aulbert E (Ed.). (1993). Bewältigungshilfen für den Krebskranken (pp. 2-20). Stuttgart, New York: Thieme Verlag.

Aulbert E. (1998). Wie die Lebensqualitätsforschung die onkologische Praxis verändert hat. In Schneider E \& Keller M (Eds.), Guter Rat ist teuer. (pp. 96-105). Lengerich, Berlin, Düsseldorf: PABST.

Aulbert E \& Zech D (Eds.). (1997). Lehrbuch der Palliativmedizin. Stuttgart: Schattauer Verlag.

Aulbert E (1997). Lebensqualität bei inkurablen Krankheiten in der Palliativmedizin. In Aulbert E \& Zech D (Eds.). (1997). Lehrbuch der Palliativmedizin (pp82-98). Stuttgart: Schattauer Verlag.

Aymans P. (1992). Krebserkrankung und Familie: zur Rolle familiarer Ünterstützung im Prozeß der Krankheitsbewältigung. Bern, Toronto: Huber.

Balfour JL \& Kaplan GA (1998). Social class/sozio-öconomic factors. In Holland JC (Ed.), Psycho-oncology. New York: Oxford University Press

Baltrusch HJF \& Waltz EM. (1987). Theoretical Framework for Developing Measures of Quality of Life and Morale. In Aaronson NK \& Beckmann J (Eds.), The Quality of Life of Cancer Patients. (pp. 25-35) New York: Raven Press.

Bartsch HH \& Mertelsmann R (Eds.) (2001). Knochenmark und periphere Stammzelltransplantation. Freiburg, Basel: Karger

Bartsch HH, Moser MT \& Weis J. (2000). Prävalenz von Fatigue bei Krebspatienten in der stationären Rehabilitation: Verteilungsmuster und Einflußfaktoren. In Weis J \& Bartsch $\mathrm{HH}$ (Eds.), Tumortherapie und Rehabilitation (pp. 81-95). Basel, Freiburg: Karger.

Batel-Copel LM, Kornblith AB, Batel PC \& Holland JC. (1997). Do Oncologists Have an Increasing Interest in the Quality of Life of Their Patients? A Literature Review of the Last 15 Years. European J of Cancer, Vol. 33 No 1, 29-33.

Bausewein C \& Hartenstein R. (2000). Oncology and palliative care. Onkologie, 23 (6), 534537.

Baynes RD, Danser RD, Klein JL et al. (2001). High-dose chemotherapy and hematopoietic stem cell transplantation for breast cancer: past or future? Semin Oncol, 28(4), 377-388.

Beard SM, PC Lorigan \& FC Sampson. (2000). The cost-effectiveness of high dose chemotherapy in the treatment of relapsed Hodgkin's disease and non-Hodgkin's lymphoma. $\mathrm{Br} \mathrm{J}$ Cancer, 82 (1), 81-84.

Beckmann J \& Ditlev G. (1987). Quality of Life and Empirical Research. In Aaronson NK \& Beckmann J (Eds.), The Quality of Life of Cancer Patients (pp. 37-40). New York: Raven Press.

Berard RM, Boermeester F \& Viljoen G. (1996). Depressive disorders in an out-patient oncology setting: prevalence, assessment, and management. Psychooncology, 7 (2), 112-120.

Bergh J, Jonsson PE, Glimelius B \& Nygren P. (2001). A systematic overview of chemotherapy effects in breast cancer. Acta Oncol, 40(2-2), 253-81.

Berglund G, Bolund C, Fornander T et al. (1991). Late effects of adjuvant chemotherapy and postoperative radiotherapy on quality of life among breast cancer patients. European Journal of Cancer, 27 (9), 1147-1149.

Bernhard J, Gusset H \& Hürny C. (1995). Quality-of-life assessment in cancer clinical trials: an intervention by itself? Support Care Cancer, 3, 66-71.

Bernheim JL, Ledure G, Souris M \& Razavi D. (1987). Differences in Perception of Disease and Treatment Between Cancer Patients and Their Physicians. In Aaronson NK \& Beckmann J (Eds.), The Quality of Life of Cancer Patients (pp. 11-17). New York: Raven Press.

Bettex MC (Ed.). (1985). Sturz aus der normalen Wirklichkeit. Ergebnisbericht der 2. Jahrestagung der "Deutschen Arbeitsgemeinschaft für Psychoonkologie e. V." und der 6. Arbeitstagung des "Arbeitskreises Psychoonkologie". dapo Eigendruck.

Bezwoda WR, Seymour L, \& Dansey RD. (1995). High-dose chemotherapy with hematopoietic rescue as primary treatment for metastatic breast cancer: a randomised trial. $\mathrm{J}$ Clin Oncol, 13, 2477-2479.

Bierman PJ. (1994). Update and results of autologous bone marrow transplantation in lymphoma. Med Oncol, 11 (2), 35-41.

Bierman PJ, Vose JM, Armitagee JO et al. (1994). Autologous transplantation for Hodgkin`s disease: coming of age? Blood, 83 (5), 1161-1164. 
Bjellard J, Dahl AA, Haug Tt \& Neckelmann D (2002). The validity of the Hospital Anxiety and Depression Scale. An updated literature review. J of Psychosom Res, 52: 69-77.

Blazeby JM, Brookes ST \& Alderson D. (2000). Prognostic value of quality of life scores in patients with oesophageal cancer. Br J Surg, 87 (3), 362-373.

Blesch K \& Paice J. (1998). Correlates of fatigue in breast or lung cancer. Oncol Nurs Forum, 18, 81-87.

Bolund C. (1990). Crisis and Coping - Learning to Live with Cancer. In Holland JC \& Zittoun R (Eds.). Psychosocial Aspects of Oncology (pp. 13-25). Berlin Heidelberg New York: Springer Verlag.

Bolund C. (1990a). Suicide and Euthanasia. In Holland JC \& Zittoun R (Eds.). Psychosocial Aspects of Oncology (pp. 121-130). Berlin Heidelberg New York: Springer Verlag.

Bonomi P, Kim F, Fairclough D et al. (2000). Comparison of survival and quality of life in nonsmall-cell lung cancer patients treated with two dose levels of paclitaxel. J Clin Oncol, 18 (3), 623-631.

Borges V, Joyce R, Uhl L et al. (2000). Docetaxel and Filgrastim Provides a Safe and Effective Mobilization Regimen Prior to Hing-Dose Chemotherapy for Breast Cancer (Abstract). In ASCO (Ed.), 36th (Vol. 36th Annual Meeting, pp. 6). New Orleans, LA:

Bower JE, PA Ganz, KA Desmaon et al. (2000). Fatique in Breast Cancer Survivor: Occurrence, Correlates, and Impact on Quality of Life. J Clin Oncol, 18 (4), 743-753.

Brandt L, Kimby E, Nygren P et al. (2001). A systematic overview of chemotherapy effects in indolent non-Hodgkin`s lymphoma. Acta Oncol, 40 (2-3), 213-223.

Braun S, Kentenich C, Janni W et al. (2000). Lack of effect of adjuvant chemotherapy on the elimination of single dormant tumor cells in bone marrow of high-risk breast cancer patients. J of Clin Oncology, 18 (1), 80-86.

Bredart A, Didier F, Robertson C et al. (1999). Psychological distress in cancer patients attending the European Institute of Oncology in Milan. Oncology, 57 (4), 297-302.

Breitbart W. (1995). Identifying patients at risk for, and treatment of major psychiatric complications of cancer. Support Care Cancer, 3, 45-60.

Britten N. (1995). Qualitative interviews in medical research. BMJ, Volume 311, 251-253.

Broeckel JA, PB Jacobson, J Horton et al. (1998). Characteristics and Correlates of Fatique after Adjuvant Chemotherapy for Breast Cancer. J of Clinical Oncology, Vol 16 No 5, 1689-1696.

Broers S, Kaptein AA, Le Cessies et al. (2000). Psychosocial functioning and quality of life following bone marrow transplantation: a 3-year follow up. J Psych Res, 48 (1), 11-21.

Buchali A, Feyer P, Groll J et al. (2000). Immediate toxicity during fractioned total body irradiation as conditioning for bone marrow transplantation. Radiother Oncol, 54 (2), 157-162.

Bukeberg JB, Pennan D, Holland JC et al. (1984). Depression in hospitalized cancer patients. Psychosom Med, 46, 199-212.

Bullinger M. (1989). Forschungsinstrumente zur Erfassung der Lebensqualität bei Krebs - ein Überblick. In R Verres \& M Hasenbring (Eds.), Jahrbuch der Psychosozialen Onkologie (pp. 45-57). Berlin: Springer.

Bullinger M. (1991). Erhebungsmethoden zur Lebensqualität. Köln: Deutscher Ärzteverlag.

Bullinger M (Ed.). (1997). Lebensqualitätsforschung. Bedeutung - Anforderung - Akzeptanz. Stuttgart, New York: Schattauer.

Bullinger M. (1997). Entwicklung und Anwendung von Instrumenten zur Erfassung der Lebensqualität. In Bullinger M (Ed.), Lebensqualitätsforschung. Bedeutung - Anforderung - Akzeptanz. (pp. 1-6). Stuttgart, New York: Schattauer.

Bullinger M \& Pöppel E. (1989). Lebensqualität in der Medizin: Schlagwort oder Forschungsansatz? Dt. Ärzteblatt, 85, 436-443.

Bullinger M, Siegrist J \& Ravens-Sieberer U (Eds.) (2000). Jahrbuch der Medizinischen Psychologie. Göttingen, Bern, Toronto, Seattle: Hogrefe.

Bullinger M, Siegrist J \& Ravens-Sieberer U. (2000). Gesundheitsbezogene Lebensqualität in der Medizin - eine Einführung. In Bullinger M, Siegrist J \& Ravens-Sieberer U (Eds.), Jahrbuch der Medizinischen Psychologie (Vol. 18, pp. 11-21). Göttingen, Bern, Toronto, Seattle: Hogrefe.

Bullinger M, Siegrist J \& Ravens-Sieberer U. (2000). Lebensqualitätsforschung aus medizinpsychologischer Perspektive. In Bullinger M, Siegrist J \& Ravens-Sieberer U (Eds.), Jahrbuch der Medizinischen Psychologie (Vol. 18). Göttingen, Bern, Toronto, Seattle: Hogrefe.

Bundesminister für Gesundheit (Eds.). (1998). Forschungsbericht Lebensqualität. Baden- 
Baden: Nomos Verlagsgesellschaft.

Burns JM, Tierney DK, Long GD et al. (1995). Critical pathway for administering high-dose chemotherapy followed by peripheral blood stem cell rescue in the outpatient setting. Oncol Nurs Forum, 22 (8), 1219-1224.

Bush NE, M Haberman, G Donaldson \& Sullivan KM. (1995). Quality of Life of 125 Adults Surviving 6 - 18 Years after Bone Marrow Transplantation. Soc. Sci. Med., Vol.40, No. 4, 479-490.

Calman KC. (1987). Definitions and Dimensions of Quality of Life. In Aaronson NK \& Beckmann J (Eds.), The Quality of Life of Cancer Patients. New York: Raven Press.

Cameron JI, Franche R-L, Cheung AM \& Stewart DE. (2002). Lifestyle Interference and Emotional Distress in Family Caregivers of Advanced Cancer Patients. Cancer, 94/2, 521527.

Carelle N, Piotto E, Bellanger A et al. (2002). Changing Patient Perceptions of the Side Effects of Cancer Chemotherapy. Cancer, 95 (1), 155-163.

Carlson LE, Koski T \& Glück S. (2001). Longidtudinal effects of high-dose chemotherapy and autologous stem cell transplantation on quality of life in the treatment of metastatic breast cancer. Bone Marrow Transplantation, 27, 989-998.

Cella D. (1995). Methods and problems in measuring quality of life. Support Care Cancer, 3, 1122.

Cella D \& Tulsky DS. (1993). Quality of life in cancer: definitions, purpose and method of measurement. Cancer Invest, 11 (3), 327-336.

Cella D, Tulsky DS, Gray G et al. (1993). The Functional Assessment of Cancer Therapy Scale Development and Validation of the General Measure. Journal of Clinical Oncology, Vol 11 No 3, 570-579.

Cella DF, Peterman A, Passik S et al. (1998). Progress toward guidelines for the management of fatigue. Oncology (huntingt), 12, 369-377.

Chao NJ, Tierney DK, Bloom JR et al. (1992). Dynamic assessment of quality of life after autologous bone marrow transplantation. Blood, 80(3) 825-830, 825-830.

Chap L, Shpiner R, Levine M et al. (1997). Pulmonary toxicity of high-dose chemotherapy for breast cancer: a non-invasive approach to diagnosis and treatment. Bone Marrow Transplant, 20 (12) 1063-1067.

Coates A \& Gebski V. (1996). On the receiving end. VI. Which dimensions of quality-of-life scores carry prognostic information? Cancer Treatment Reviews, 22 (Suppl A), 63-67.

Coates A, Porzsolt F \& Osoba D. (1997). Quality of Life in Oncology Practice: Prognostic Value of EORTC QLQ-C30 Scores in Patients with Advanced Malignancy. Eur J of Cancer, 33 (7), 1025-1030.

Coates AS, Gebski V, Signorini D et al. (1992). Prognostic value of quality of life scores during chemotherapy for advanced breast cancer. Australian-New Zealand Breast Cancer Trials Group. J Clin Oncol, 10, 1833-1838.

Colon EA, Callies AL, Popkin MK \& McGlave PB. (1991). Depressed mood and other variables related to bone marrow transplantation. Psychosomatics, 32(4), 420-425.

Comeau TB, Epstein JB \& Migas C. (2001). Taste and semell dysfunction in patients receiving chemotherapy: a review of current knowledge. Support Care Cancer, 9, 575-580.

Cordova MJ, Studts JL, Hann DM et al. (2000). Symptom structure of PTSD following breast cancer. J Trauma Stress, 13 (2), 301-319.

Costantini M, Musso M, Viterbori P et al. (1999). Detecting psychological distress in cancer patients: validity of the Italian version of the Hospital Anxiety and Depression Scale. Support Care Cancer, 7, 121-127.

Courneya KS, Keats MR \& Turner AR. (2000). Physical exercise and quality of life in cancer patients following high dose chemotherapy and autologous bone marrow transplantation. Psychooncology, 9 (2), 127-136.

Crown J. (1995). High-dose chemotherapy of solid tumours. Annals of Oncology, 6 (supplement), 11-15.

Curd S \& Kamm M. (1999). Krebserkrankung und Sexualität. tägliche praxis, 4, 697-704.

Dam van FSAM, Schagen SB, Muller MJ et al. (1998). Impairment of cognitive function in woman recognition adjuvant treatment for high-rsik breast cancer: high-dose versus standard-dose chemotherapy. J Natl Cancer Inst, 90 (3), 210-218. 
Dancey J, Zee B, Osoba D et al. (1997). Quality of life scores: an independent prognostic variable in a general population of cancer patients receiving chemotherapy. Can Med Assoc.

Derogatis LR, Morrow GR, Fetting $\mathrm{J}$ et al. (1983). The prevalence of psychiatric disorders among cancer patients. Journal of the American Medical Association, 249, 751-757.

Detmar SB, Muller MJ, Schornagel JH. (2002). Role of Health-Related Quality of Life in Palliative Chemotherapy Treatment Decisions. J of Clin Oncology, 20 (4), 1056-1062.

Dilling $\mathrm{H}$, Mombour \& Schmidt MH (Eds.). (1995). Internationale Klassifikation psychischer Störungen ICD-10 (2. Auflage). Bern, Göttingen Toronto: Hans Huber.

Dimeo FC, Stieglitz RD, Novelli-Fischer U et al. (2000). Effects of physical activity on fatigue and psychologic status of cancer patients during chemotherapy. Cancer, 85 (10), 22732277.

Donnelly S, Rybicki L \& Walsh D. (2001). Quality of life measurement in the palliative management of advanced cancer. Support Care Cancer, 9, 361-365.

Engel J, Baumert J, Sauer H \& Hölzel D. (2001). Qualitätssicherung in der Onkologie am Beispiel des Mammakarzinoms der Feldstudie München. Onkologe, 7, 307-320.

Everson S, Goldberg D, Kaplan A et al. (1996). Hopelessness and Risk of Mortality and Incidence of Myocardial Infarcation and Cancer. Psychosmoatic Medicine, 58, 113-121.

Faller H. (1997). Beeinflussen psychologische Faktoren die Überlebenszeit bei Krebskranken? Teil I. PPmP, 47, 163-169.

Faller H. (1998). Krankheitsverarbeitung bei Krebskranken. Göttingen: Verlag für Angewandte Psychologie.

Faller H, Bülzebruck H, Schilling S et al. (1997). Beeinflussen psychologische Faktoren die Überlebenszeit bei Krebskranken? Teil II. PPmP, 47, 206-218.

Faller H, Lang H, Schilling S \& Otteni M. (1998). Positive und negative Effekte sozialer Unterstützung bei Krebspatienten. In Koch U \& Weis J (Eds.) Krankheitsbewältigung bei Krebs (pp. 141-160). Stuttgart New York: Schattauer.

Faller H, Lang H, Schilling S \& Wagner J. (1992). Krankheitsverarbeitung bei Bronchialkarzinom aus der Sicht der Patienten, ihrer Angehörigen und der Betreuer. Eine Fünf-Ebenen Untersuchung. PPmP, 42, 322-331.

Faller H, Lang H \& Schilling S. (1998). Coping bei Bronchialkrebs - Der Mehrebenenansatz. In Koch U \& Weis J (Eds.), Krankheitsbewältigung bei Krebs (pp. 37-48). Stuttgart New York: Schattauer.

Fayers P, Hopwood P, Harvey A et al. (1997). Quality of life assessment in clinical trialsguidelines and a checklist for protocol writers: the U.K. Medical research Council experience. Eur J Cancer, 33 (1), 20-28.

Feld R. (1995). Endpoints in cancer clinical trials: is there a need for measuring quality of life? Support Care Cancer, 3, 23-27.

Feyer H, Geismar D, Weiss M, Fritsche F \& Budach V. (2000). Fatigue bei Patienten mit fortgeschrittenen Kopf-Hals-Tumoren unter Radiotherapie und kombinierter Radiochemotherapie. In Weis J \& Bartsch HH (Eds.), Tumortherapie und Rehabilitation (pp. 69-80). Basel, Freiburg: Karger.

Fields KK, Elfenbein GJ, Perkins JB et al. et al. (1998). Defining the Role of Novel High-Dose Chemotherapy Regimens for the Treatment of High-Risk Breast Cancer. Seminars in Oncology, 25 (2), 1-6.

Fife BL, Huster GA, Cornetta KG et al. (2000). Longitudinal Study of Adaption to the Stress of Bone Marrow Transplant. J of Clinical Oncology, 18 (7), 1539-1594.

Filipp S.-H \& Ferring D. (2002). Vergleichsprozesse in der Auseinandersetzung mit schweren körperlichen Erkrankungen. PiD, 3, 2-12.

Flechtner H, Rüffer JU, Grotenthermen F \& Diehl V. (2000). Fatigue und Lebensqualität bei langzeitüberlebenden Patienten nach einer Morbus-Hodgkin-Erkrankung - erste Ergebnisse aus einer Fragebogen Validierungsstudie. In Weis J \& Bartsch HH (Eds.), Tumortherapie und Rehabilitation (pp. 52-68). Basel, Freiburg: Karger.

Flick U. (2000) (Ed.). Qualitative Forschung. Reinbek bei Hamburg: rowohlts enzyklopädie.

Flick U, Kardoff v. E, Keupp H, et al. (Eds.). (1995). Handbuch Qualitative Sozialforschung. Weinheim: Psychologie Verlags Union.

Ford S, Lewis S \& Fallowfield L. (1995). Psychological morbidity in newly referred patients with cancer. J Psychosom Res, 39 (2), 193-202. 
Fossa SD, Curran D, Aaronson NK et al. (2000). Quality of life of patients with newly diagnosed poor prognosis M1 prostate cancer undergoing orchiectomy without or with mitomycin C. Results from the EORTC Phase-III trial 30893. Eur Urol, 37 (5), 541-551.

Fossati R, Confalonieri C, Apolone G et al. (2002). Does a drug do better when it is new? Annals of Oncol 13:470-473.

Fox BH, Stanek EJ, Boyd SC \& Flannery JT. (1982). Suicide rates among cancer patients in Connecticut. J of Chronic Disease, 35, 85-100.

Frickhofen N. (1993). Hochdosistherapie mit Blutstammzell-Transplantation. Klinikarzt, 10/22, 28-34.

Fritsche K, Stein B, Herzog T et al. (1998). Psychococial Care of Oncological Inpatients. Onkologie, 21: 150-155.

Fromm K, Andrykowski MA \& Hunt J. (1996). Positive and negative psychosocial sequelace of bone marrow transplantation: implications for quality of life assessments. J Behav Med, 19 (3): 221-240.

Ganz PA, Lee JJ \& Siau J. (1991a). Quality of life assessment: An independent prognostic variable for survial in lung cancer. Cancer, 67, 3131-3135.

Ganz PA, Schag CAC, Lee JJ et al. (1992). Breast conservation versus mastectomy: Is there a difference in psychological adjustment or quality of life in the year after surgery? Cancer, 69, 1729-1738.

Gerdes N. (1989). Desiderate an die künftige psychoonkologische Forschung. Verres R \& Hasenbring M (Eds.). In Psychosoziale Onkologie - Jahrbuch der Medizinischen Psychologie (Vol. 3). Berlin, Heidelberg, New York: Springer.

Gerdes N (1985). Der Sturz aus der normalen Wirklichkeit. In Bettex MC (Ed.). Sturz aus der normalen Wirklichkeit. Ergebnisbericht der 2. Jahrestagung der "Deutschen Arbeitsgemeinschaft für Psychoonkologie e. V." und der 6. Arbeitstagung des "Arbeitskreises Psychoonkologie". dapo Eigendruck.

Gianni AM, Bregni M, Siena S et al. (1996). Is high-dose better than standarddoseChemotherapy as initial treatment of poor-risk large cell lymphomas? A critical analysis from available randomized trials. Ann Oncol, 7 (Suppl 3), 12.

Gilbert CJ. (1996). Peripheral blood progenitor cell transplantation for breast cancer: pharmacoeconomic considerations. Pharmacotherapy, 16 (3 Pt 2), 101-108.

Gill TM \& Feinstein AR. (1994). A criical appraisal of the quality-of-life measurements. JAMA, 272, 619-626.

Gisselbrecht C. (1994). Chemotherapy dose intensity facilitated by use of lenograstim - implications for quality of life and survival. Eur J Cancer, 30A (Suppl 3), 30-33.

Glaeske G. (1997). Klinische Wirksamkeit, patientenbezogener Nutzen und Lebensqualität aus der Sicht der Krankenkassen. In Bullinger M (Ed.). Lebensqualitätsforschung. Bedeutung - Anforderung - Akzeptanz (pp. 29-35). Stuttgart, New York: Schattauer.

Glatzer W \& Zapf W. (1994). Lebensqualität in der Bundesrepublik. Objektive Lebensbedingungen und subjektives Wohlbefinden. Frankfurt: Campus.

Glaus A. (1998). Fatigue in Patients with Cancer. Analysis and Assessment. Berlin: Springer.

Glaus A. (2000). Das Konzept Fatigue in der Onkologie: Definitionen, Hintergründe. In Weis J \& Bartsch HH (Eds.), Tumortherapie und Rehabilitation (pp. 1-13). Basel, Freiburg: Karger.

Glick HA, Shpall EJ, LeMarstre CF et al. (1998). Empirical criteria for the selection of quality-oflife instruments for the evaluation of peripheral blood progenitor cell transplantation. Int J Technol Assess Health Care, 14 (3), 419-430.

Gold MR, Patrick DL,Torrance GW et al. (1996). Identifying and Valuing Outcomes. In Gold MR, Siegel JE, Russell LB \& Weinstein MC (Eds.), Cost-Effektiveness in Health and Medicine (pp. 82-123). New York, Oxford: University Press.

Gold MR, Siegel JE, Russell LB \& Weinstein MC (Eds.). (1996). Cost-Effektiveness in Health and Medicine. New York, Oxford: University Press.

Goldhirsch A, Gelber RD, Simes RJ et al. (1994). Costs and benefits of adjuvant therapy in breast cancer: A quality adjusted survival analysis. J Clin Oncol, 7, 36-44.

Grady D. (2000). Breast cancer researcher admits falsifying data. New York Times, Febr 5, 9.

Graydon JE. (1994). Women with breast cancer: their quality of life following a course of radiation therapy. J Adv Nurs, 19 (4), 617-620.

Groopman. J (1998). A Healing Hell. Annals of Medicine, Oct 19, 34-39 
Groot Uyl-de CA, Hagenbeck A, Verdonck LF et al. (1995). Cost-effectiveness of ABMT in comparison with $\mathrm{CHOP}$ chemotherapy in patients with intermediate - and high-grade malignant non-Hodgkin`s lymphoma (NHL). Bone Marrow Transplant, 16 (3):463-470.

Gründel M. (1995). Gemeinsames Sorgerecht - Erfahrungen geschiedener Eltern. Freiburg im Breisgau: Lambertus.

Haas R, Möhle R, Frühauf S et al. (1994). Patient characteristics associated with successful mobilizing and autografting of peripheral blood progenitor cells in malignant lymphoma. Blood, 83, 3787-3794.

Habermas J (1973). Erkenntnis und Interesse. Frankfurt am Main: stw

Habermas J (2001). Die Zukunft der menschlichen Natur: Auf dem Weg zu einer liberalen Eugenik? Frankfurt am Main: Suhrkamp Verlag.

Härter MC. (2000). Psychische Störungen bei körperlichen Erkrankungen. PPmP, 50, 274-286.

Haes de JCM \& Knippenberg van FCE. (1985). The quality of life of cancer patients: A review of the literature. Soc. Sci. Med, 20, 809-817.

Haes de JCM \& Knippenberg van FCE. (1987). Quality of Life of Cancer Patients: Review of the Literature. In Aaronson NK \& Beckmann J (Eds.), The Quality of Life of Cancer Patients (pp. 168-181). New York: Raven Press.

Haes de JCM \& Stiggelbout AM. (1996). Assessment of values, utilities and preferences in cancer patients. Cancer Treatment Reviews, 22 (Suppl A), 13-26.

Hann DM, PB Jacobson, SC Martin et al. (1997). Fatigue in women treated with bone marrow transplantation for breast cancer: a comparison with women with no history of cancer. Support Care Cancer, 5, 44-52.

Hardman A, Maguire P \& Crowther D. (1989). The recognition of psychiatric morbidity on a medical oncology ward. J Psychosom Res, 33 (2), 235-239.

Harrer ME, Mosheim R, Richter R et al. (1993). Coping und Lebenszufriedenheit bei Patienten mit M. Hodgkin in Remission. PPmP, 43, 121-132.

Harrison CN, Gregory W, Hudson GV et al. (1999). High-dose BEAM chemotherapy with autologous haemopoietic stem cell transplantation for Hodgkin`s disease is unlikely to be associated with a major increased risk of secondary MDS/AML. British J of Cancer, 81 (3), 476-483.

Hautzinger M \& PL Janssen. (1995). Psychotherapeutische Grundlagen der psychosozialen Onkologie. Psychotherapeut, 40, 313-323.

Hayes JR. (1991). Depression and chronic fatigue in cancer patients. Prim Care, 18, 327-339.

Hecht JR, Lembo T \& Chap L. (1997). Prolonged nausea and vomiting after high dose chemotherapy and autologous peripheral stem cell transplantation in the treatment of high risk breast carcinoma. Cancer, 79 (9), 1698-1702.

Helmchen (1990). „Lebensqualität" als Bewertungskriterium in der Psychiatrie. In Schölmerich P \& Thews G (Eds.). "Lebensqualität" als Bewertungskriterium in der Medizin (pp.93115).Stuttgart; New York: G. Fischer Verlag.

Heim E. (1988). Coping und Adaptivität: Gibt es geeignetes oder ungeeignetes Coping? PPmP, 38, 8-18.

Henon P, Donatini B, Eisenmann JC et al. (1995). Comparative survival, quality of life and costeffectiveness of intensive therapy with autologous blood cell transplantation or conventional chemotherapy in multiple myeloma. Bone Marrow Transplant, 16 (1), 19-25.

Henrich G \& Herschbach P. (1998). Objektive Bedingungen subjektiver Lebensqualität. In KochU \& Weis J (Eds.). Krankheitsbewältigung bei Krebs (pp. 77-87). Stuttgart New York: Schattauer.

Henry -Amar M \& Joly F. (2001). Fatigue in Cancer Survivors (Abstract). Onkologie, 24 (Suppl 2), 8.

Hensel M, Egerer G, Schneeweiss H, Goldschmidt H \& Ho AD. (2002). Quality of life and rehabilitation in social and professional life after autologous stem cell transplantation. Annals of Oncology, 13, 209-217.

Herrmann C, Buss U \& Snaith R (1995). Psychologisches Screening von Patienten einer kardiologischen Akutklinik mit einer deutschen Fassung der "Hospital Anxiety and Depression" (HAD-)Skala. PPmP, 41, 83-92.

Herrmann C, Buss U \& Snaith R (1995). Hospital Anxiety and Depression Scale (HADS-D). Deutsche Version. Bern: Huber. 
Herrmann C, Kaminsky B, Rüger U \& Kreuzer H. (1999). Praktikabilität und klinische Relevanzeines routinemäßigen Screenings von Patienten internistischer Allgemeinstationen. PPmP, 49, 48-54.

Herrmann C, Scholz KH \& Kreuzer H. (1991). Psychologisches Screening von Patienten einer kardiologischen Akutklinik mit einer deutschen Fassung der "Hospital Anxiety and Depression" (HAD-)Skala. PPmP, 41, 83-92.

Herschbach P. (2002). Das "Zufriedenheitsparadox" in der Lebensqualitätsforschung. Wovon hängt unser Wohlbefinden ab? PPmP, 52 (3-4): 141-150.

Hessel A, Heim E, Greyer M \& Brähler E. (2000). Krankheitsbewältigung in einer repräsentativen Bevölkerungsstichprobe. PPmP, 50, 311-321.

Hiddemann W, Unterhalt M \& Sack H (1997). Aktueller Stand in der Therapie von follikulären Keimzentrumslymphomen und Mantelzell-Lymphomen. Der Internist, 38, 122-134.

Hillner BE, Smith TJ \& Desch CE. (1992). Efficacy and cost-effectiveness of autologous bone marrow transplantation in metastatic breast cancer. Estimates using decision analysis while awaiting clinical trial results. JAMA, 267 (15), 2055-2061.

Hinz A \& Schwarz R. (2001). Angst und Depression in der Allgemeinbevölkerung. PPmP, 51, 193-200.

Hjermstad MJ, Evensen SA, Kvaloy SO et al. (1999). Health-Related Quality of Life 1 Year After allogenic or autologous stem-cell transplantation: a prospective study. J Clin Oncol, 17 (2), 706-718.

Hjermstad MJ, Holte H, Evensen S et al. (1999). Do patients who are treated with stem cell transplantation have a health-related quality of life comparable to the general population after 1 year? allogenic or autologous stem-cell transplantation: a prospective study. Bone Marrow Transplant, 24 (8), 911-918.

Hoekman K \& Vermorken JB. (1996). Incidence and prevention of nonhaematological toxicity of high-dose chemotherapy. Ann Med, 28 (2), 175-182.

Hoff van DDGM, Clark m \& Weiss GR. (1986). Use of invitro dose response effects to select antineoplastics for high-dose or regional administration: An alternative approach. Journal of Clinical Oncology, 4, 1827-1834.

Holland JC (Ed.). (1998). Psycho-oncology. New York: Oxford University Press

Holland JC \& Zittoun R (Eds.). (1990). Psychosocial Aspects of Oncology. Berlin Heidelberg New York: Springer Verlag.

Holland JC. (1990). Diagnosis and Management of Symptoms from a Psychological Perspective. In Holland JC \& Zittoun R (Eds.). Psychosocial Aspects of Oncology (pp. 49-57). Berlin Heidelberg New York: Springer Verlag.

Hopf C. (1995). Qualitative Interviews - ein Überblick. In Flick U, Kardoff v. E, Keupp H, et al. (Eds.). Handbuch Qualitative Sozialforschung. (pp. 349-360). Weinheim: Psychologie Verlags Union.

Horton R. (2000). After Bezwoda. Lancet, 355 (18), 942-943.

Hosaka T \& Aoki T. (1996). Depression among cancer patients. Psychiatry Clin Neurosci, 50 (6), 309-312.

Hossfeld DK (1998). Was bringt die Hochdosis-Chemotherapie mit Stammzellsupport den Patienten mit Lymphomen und soliden Tumoren? www.alc.de/hodgkin/hochdos.htm 22.6.1998

Hudis CA \& Münster PN. (1999). High-Dose Therapy for Breast Cancer. Seminars in Oncology, 26 (1), 35-47.

Hürny C. (1997). Psychische und soziale Faktoren in Entstehung und Verlauf maligner Erkrankungen. InUexküll T v et al. (Eds.). Psychosomatische Medizin (Vol. 5. Auflage). München, Wien, Baltimore: Urban und Schwarzenberg.

Hürny C, Bernhard J, Coates A et al. (1994). Timing of baseline quality of life assessment in an international adjuvant breast cancer trial: Its effect on patient self estimation. Ann Oncol, $5,65-74$.

Ibbotson T, Maguire P, Selby $\mathrm{P}$ et al. (1994). Screening for anxiety and depression in cancer patients: the effects of disease and treatment. Eur J Cancer, 30A, 37-40.

Jacob G \& Bengel J. (2000). Das Konstrukt Patientenzufriedenheit: Eine kritische Bestandsaufnahme. Zt f Klinische Psychologie, Psychiatrie und Psychotherapie, 48 (3), 280-301.

Jacobsen PB, Widows MR, Hann DM et al. (1998). Posttraumatic stress disorder symptoms after bone marrow transplantation for breast cancer. Psychosm Med, 60 (3): 366-371.

Jacoby LH, Maloy B, Cirenza E et al. (1999). The basis of informed consent for BMT patients. Bone Marrow Transplant, 23:711-717. 
Jenkins PL \& Roberts DJ. (1991). Suicidal behavior after bone marrow transplantation. Bone Marrow Transplant, 7, 159-161.

Jerkeman M, Anderson H, Cavallin-Stahl E et al. (1999). CHOP versus MACOP-B in aggressive lymphoma - a Nordic Lymphoma Group randomised trial. Ann Oncol, 10 (9), 1079-1086.

Jones DR, Fayers PM \& Simons. (1987). Measuring and Analyzing Quality of Life in Cancer Clinical Trials: A Review. In Aaronson NK \& Beckmann J (Eds.), The Quality of Life of Cancer Patients (pp. 41-61). New York: Raven Press.

Junker A (2002). DGHO 2001. Onkologe, 8: 194-197.

Kaasa S, Bjordal K, Aaronson N et al. (1995). The EORTC core quality of life questionnaire (QLQ-C30): validity and reliability when analysed with patients treated with palliative radiotherapy. Eur J Cancer, 31A (13-14), 2260-2263.

Kaasa S, Mastekaasa A \& Lund E. (1989). Prognostic factors for patients with inoperable nonsmall cell lung cancer, limited disease. Radiotherapy and Oncology, 15, 235-242.

Kächele H \& Steffens W (Eds.). (1988). Bewältigung und Abwehr. Berlin Heidelberg New York: Springer Verlag.

Kächele H \& Steffens W. (1988). Abwehr und Bewältigung - Mechanismen und Strategien. Wie ist eine Intergration möglich? In Kächele H \& Steffens W (Eds.). Bewältigung und Abwehr (pp. 1-50). Berlin Heidelberg New York: Springer Verlag.

Kaiser UL, Trümper L, Pfreundschuh et al. (1997). Behandlung hochmaligner Non-Hodgkin Lymphome. Der Internist, 38, 135-142.

Kapfhammer. (1996). Depression und Suizid bei Krebskranken. TW Neurologie Psychiatrie, 10 (4), 281-283.

Karnofsky DA, Abelmann WH, Craver LF \& Burchenal JH. (1948). The Use of the Nitrogen Mustards in the palliative Treatment of Carcinoma. Cancer, Nov, 634-656.

Kast V. (1991). Trauern. Stuttgart: Kreuz Verlag.

Kaufman S. (2001). The Emerging Role of Health-Related Quality-of Life Data in Clinical Research - Part 1: The Increasing Importance of Quality-of-Life Research. Clinical Researcher, Vol.1 No 5, 18-22.

Keller M (2001). Effekte psychosozialer Interventionen auf LQ und Krankheitsverlauf von Krebspatienten. Onkologe, 7: 133-142.

Kennedy MJ. (1995). High-dose chemotherapy of Breast Cancer: Is the Question Answered? J Clin Oncol, Vol.13, No. 10, 2477-2479.

Kerekjarto M v, Schulz KH, Kramer C et al. (1989). Grundlegende Aspekte zum Konzept der Lebensqualität. In Verres R \& Hasenbring M (Eds.), Psychosoziale Onkologie. Jahrbuch der Medizinischen Psychologie (Vol. 3). Berlin, Heidelberg, New York: Springer.

Kimby E, Brandt L, Nygren P et al. (2001). A systematic overview of chemotherapy effects in aggressive non-Hodgkin`s lymphoma. Acta Oncol, 40 (2-3), 198-212.

Kleining B \& Schumacher A (Eds.) (2001). Psychotraumatologie in der Onkologie. Lengerich, Berlin, Riga: PABST.

Koch U \& Weis J (Eds.). (1998). Krankheitsbewältigung bei Krebs. Stuttgart New York: Schattauer.

Köhle K, Simons C \& Kubanek B. (1997). Zum Umgang mit unheilbar Kranken. In Uexküll T v et al. (Eds.),Psychosomatische Medizin (Vol. 5. Auflage). München, Wien, Baltimore: Urban und Schwarzenberg.

König H-H, Hoffmann C, Schulenburg Graf von der J-M \& Leidl R. (1999). Gemeinsame Effektmaße in medizinischen und gesundheitsökonomischen Studien? Medizinische Klinik, 94 (12), 665-672.

Kollmannsberger C, Hartmann JT, Kranz L \& Bokemeyer C. (1998). Risk of secondary myeloid leukemia and myelodysplastic syndrom following standard-dose or high-dose chemotherapy with stem cell support in patients with potentially curable malignancies. J Cancer Res Clin Oncol, 124 (3-4), 207-214.

Kornblith AB (1998). Psychosocial adaption of cancer survivors. In Holland JC (Ed.), Psychooncology. New York: Oxford University Press.

Korth EE. (1995). Psychologisch relevante Faktoren in der Bewältigung einer autologen Knochenmarkstransplantation bei Morbus Hodgkin. In Europäische Hochschulschriften Reihe IV Psychologie (Vol. 480). Frankfurt am Main, Bern, Berlin: Peter Lang.

Krischke NR \& Petermann F (2000). Depression und Lebensqualität in der Rehabilitation Krebskranker. In Bullinger M, Siegrist J \& Ravens-Sieberer U (Eds.), Jahrbuch der Medizinischen Psychologie (Vol. 18, pp. 25-42). Göttingen, Bern, Toronto, Seattle: Hogrefe. 
Krishan A, Bhatia S, Slovak ML et al. (2000). Predictions of therapy-related leukemia and myelodysplasia ollowing autologous transplantation for lymphoma: an assessment of risk factors. Blood, 95 (5), 1588-1593.

Kübler-Ross E. (1980). Interviews mit Sterbenden. Gütersloh: Güterloher Verlagshaus Mohn.

Küchler T. (1991). Interviewverfahren in der Lebensqualitätsforschung. In Schwarz R (Ed.), Lebensqualität in der Onkologie. München, Bern, Wien: Zuckschwerdt Verlag.

Küchler T, Flechtner H \& Herschbach P. (2000). Zum Stand der Lebensqualitätsmessung in der Onkologie. Forum, 5, 34-39.

Küchler T \& Schreiber HW. (1989). Lebensqualität in der Allgemeinchirurgie - Konzepte und praktische Möglichkeiten der Messung. $H A B, 43,246-250$.

Küchler Th, Flechtner H \& Herschbach P. (2000). Zum Stand der Lebensqualitätsmessung in derOnkologie. Forum, 5/2000, 34-39.

Kuupelmaki M \& Lauri S. (1998). Cancer patients' reported experiences of suffering. Cancer Nurs, 21 (5), 364-369.

Larsen J, Gardulf A, Nordstrom G et al. (1996). Health-related quality of life in women with breast cancer undergoing autologous stem-cell transplantation. Cancer Nurs, 19 (5), 368-375.

Lazarus HM. (1998). Hematopoitic progenitor cell transplant in breast cancer: current status and future directions. Cancer Invest, 16 (2), 102-126.

Lazarus RS \& Folkman S. (1984). Stress, appraisal and coping. New York: Springer.

Leitgeb C. (1998). The development of the hematopietic growth-factors had a major influence on the treatment of malignant diseases. Quality of life gets increasingly important in the setting of high-dose chemotherapy and stem cell transplantation. Wien Med Wochenschr, 148 (18), 224-226.

Leopold KA, Ahles TA, Walch S et al. (1998). Prevalence of mood disorders and utility of the PRIME-MD in patients undergoing radiation therapy. Int J Radiat Oncol Biol Phys, 42 (5), 1105-1112.

Levy SM, Lee J, Bagley L \& Lippman M (1988). Survival hazards analysis in first recurrentbreast cancer patients: seven-year follow-up. Psychosom Med, 50: 520-528.

Linch DC, Winfield D, Goldstone AH et al. (1993). Dose intensification with autologous bone marrow transplantation in relapsed and resistent Hodgkin's disease: results of a $\mathrm{BNCl}$ randomised trial. Lancet, 34, 1051-1054.

Lippman ME. (2000). High-Dose Chemotherapy plus Autologous Bone Marrow Transplantation for Metastatic Breast Cancer. N Engl J Med, 342 (15), 11191120.

Lucey C \& Westphal JR. (1998). New approach to administrative medical decision-making: evidence-based medicine using high dose chemotherapy/bone marrow transplant for breast cancer. South Med J, 91 (2), 196-201.

Macquart-Moulin G, Viens P, Palangie T et al. (2000). High-dose sequential chemotherapy with recombinant granulocyte colony-stimulating factor and repeated stem-cell support for inflammatory breast cancer patients: does impact of quality of life jeopardize feasibility and acceptability of treatment? J Clin Oncol, 18 (4), 754.

Maguire P. (1990). Employing Specialist Workers to Detect Psychological and Social Morbidity. In Holland JC \& Zittoun R (Eds.). Psychosocial Aspects of Oncology (pp. 59-72). Berlin Heidelberg New York: Springer Verlag.

Markman M. (1996). Chemotherapy-associated neurotoxicity: an important side effect - impacting on quality, rather than quantity, of life. J Cancer Res Clin Oncol, 122, 511-512.

Marmot M \& Wilkinson R.(2001). Psychosocial and material pathways in the relation between income and health: a response to Lynch et al. Brit Med J, 322:1233-1236

Martinelli G, Clinieri S, Ferrucci P et al. (2000). Multiple High-Dose Chemotherapy (HDCT) with Docetaxel (D), Epirubicin (E) and Cyclophosphamide (C), (HD-TEC) for High Risk Breast Cancer (HRBC) (Abstract). In ASCO (Ed.), 36th (Vol. 36th Annual Meeting, pp. 8). New Orleans, LA:

Mast ME. (1998). Correlates of fatique in survivors of breast cancer. Cancer Nurs, 21, 136-142.

Mattson T, Arvidsom K, Heimdahl A et al. (1992). Alterations in taste acuity associated with allogeneic bone marrow transplantation. J Oral Pathol Med, 21, 33-37.

Maughan TS, James RD, Kerr DJ et al. (2002). Comparison of survival, palliation, and quality of life with three chemotherpay regimens in metastatic colorectal cancer: a multicentre randomised trial. The Lancet, 359, 1555-1563. 
Mayring P. (1995). Qualitative Inhaltsanalyse. In Flick U, Kardoff v. E, Keupp H, et al. (Eds.). Handbuch Qualitative Sozialforschung (pp. 468-475).Weinheim: Psychologie Verlags Union.

McLachlan SA, Devins GM \& Goodwin PJ. (1998). Validation of the European Organization for Research and Treatment of Cancer Quality of Life Questionnaire (QLQ-C30) as a measure of psychosocial functioning in breast cancer patients. Eur J Cancer, 34 (4), 510-517.

McQuellon RP, Craven B, Russell GB et al. (1995). Quality of life in breast cancer patients before and after autologous bone marrow tra nspn Stion $f$ 
Nitz U (2000). Hochdosis-Chemotherapie des Mammakarzinoms. Dt Ärzteblatt, 97 (5): B212B217

Noll P. (1984). Diktate über Sterben \& Tod. Zürich: pendo.

Osoba D. (1996). Rationale for the timing of health-related quality of life HQL) assessments in ocological palliative therapy. Cancer Treat Rev, 22, 69-73.

Osoba D. (2000). Health-related quality-of-Life assessment in clinical trials of supportive care in oncology. Support Care Cancer, 8, 84-88.

Osoba D, Zee B, Warr D et al. (1997). Effect of postchomotherapy nausea and vomiting on health-related quality of life. The Quality of Life and Symptom Control Comittees of the National Cancer Institut of Canada Clinical Trials Group. Supp Care Cancer, 5, 307313.

Palangie T, Roche H, Viens $\mathrm{P}$ et al. (2000). Taxotere (TXT) in Sequential Association with Multiple Cycles High-Dose Doxorubicine (D) and Cyclophosphamide (C) as First Line Therapy in Inflammatory Breast Cancer French Colloborative FNCLL Study. In ASCO (Ed.), 36th (Vol. 36th Annual Meeting, pp. 9). New Orleans, LA.

Passik SD, Dugan W, McDonald MV et al. (1998). Oncologists 'Recognition of Depression in Their Patients with Cancer. J of Clinical Oncology, Vol 16, No 4, 1594-1600.

Pater J, Osoba D, Zee B et al. (1998). Effects of altering the time of administraion and the time frame of quality of life assessment in clinical trials: an example using the EORTC QLQC30 in a large anti-emetic-trial. Qual Life Res, 3, 273-278.

Pedersen-Bjergaard J, Andersen MK \& Christiansen DH. (2000). Therapy-related myelodysplasia (t-MDS) and akute myeloid leukemia (t-AML) after high-dose cchemotherapy (HDCT) and autologuous stem cell transplantatin (ASCT). Blood, 95 (11), 3273-3279.

Philip T, Guglielmi C, Hagenbeck A et al. (1995). Autologous bone marrow transplantation as compared with salvage chemotherapy in relapses of chemotherapy-sensitive nonHodgkin`s Lymphome. N Engl J Med, 333:1540-1545

Pinder KL, Ramirez AJ. Black ME et al. (1993). Psychiatric disorder in patients with advanced breast cancer: Prevalence and associated Factors. Eur J Cancer, 29, 224-227.

Pinedo HM. (1995). Drug Resistance, Illustration of the Complexity of Translational Research. Steiner Award Lecture 1995. Int. J. Cancer, 65, 561-566.

Pirl WF \& Roth AJ. (1999). Diagnosis and treatment of depression in cancer patients. Oncology (Huntingt), 13 (9), 1293-1301.

Poppelreuther M (2001).Neuropsychologische Diagnostik nach Hochdosis-Therapie. In Bartsch HH, Finke J \& Mumm A (Eds.), Hämatopoetische Stammzelltransplantation. Basel: Karger.

Pope C \& Mays N. (1995). Reaching the parts other methods cannot reach: an introduction to qualitative methods in health and health services research. BMJ, Volume 311, 42-45.

Porzsolt F. (1995). Wirksamkeit und Nutzen diagnostischer und therapeutischer Maßnahmen. Onkologe, 1, 241-245.

Porzsolt F. (1996). Klinische Ökonomik - ein Leitfaden für ökonomisches Arbeiten in der Klinik und Praxis. Medizinische Klinik, 91 (8), 531-535.

Porzsolt F. (2000). Therapie fortgeschrittener inkurabler Erkrankungen: Nutzen und Kosten (Abstract). Zeitschrift für Palliativmedizin, 1. Jahrgang, 5.

Porzsolt F \& Rist C. (1997). Lebensqualitätsforschung in der Onkologie: Instrumente und Anwendung. In Bullinger M (Ed.), Lebensqualitätsorschung (pp. 19-21). Stuttgart, New York: Schattauer.

Preiß J, W Dordoff, FG Hagmann \& A Schmieder. (1998). Empfehlungen zur Therapie- Onkologie 1998/99. Onkolog. Arbeitsgemeinschaft Saar-Pfalz-Mosel

Priestman TJ \& Baum M. (1976). Evaluation of Quality of Life in Patients Receiving Treatment for Advanced Breast Cancer. The Lancet, April 24, 899-900.

Prins JB, Bleijenberg G, Bazelmans E et al. (2001). Cognitive behaviour therapy for chronic fatigue syndrome: a multicetre randomised controlled trial. Lancet, 357, 841-847.

Pukrop R. (1997). Theoretische Explikation und empirische Validierung des Konstruktes Lebensqualität unter besonderer Berücksichtigung psychopathologischer Subgruppen und facettentheoretischer Methodik. Dissertation, Universität Bielefeld.

Radbruch L \& Zech D. (1997). Definition, Entwicklung und Ziele der Palliativmedizin. In Aulbert E \& Zech D (Eds.), Lehrbuch der Palliativmedizin. Stuttgart: Schattauer Verlag.

Raisch DW. (2000). Understanding Quality-Adjusted Life Years and Their Application to Pharmacoconomic Researche. Annals of Pharmacotherapy, 34, 906-914. 
Raspe H-H. (1990a). "Lebensqualität" in der Medizin. Ethik in der Medizin, 2 (1), 1-4.

Raspe H-H. (1990). Zur Theorie und Messung der "Lebensqualität" in der Medizin. In Schölmerich P \& Thews G (Eds.), "Lebensqualität" als Bewertungskriterium in der Medizin (pp. 23-40). Stuttgart; New York: G. Fischer Verlag.

Ratsak G. (1993). Angst und Angstbewältigung bei der Krebskrankheit. In Aulbert E (Ed.), Bewältigungshilfen für den Krebskranken (pp. 77-91). Stuttgart, New York: Thieme Verlag.

Razavi D \& Stiefel F. (1994). Common psychiatric disorders in cancer patients. I. Adjustment disorders and depressive disorders. Support Care Cancer, 2, 233-237.

Rehse B. (2001). Metaanalytische Untersuchungen zur Lebensqualität adjuvant psychoonkologisch betreuter Krebsbetroffener. Aachen: Shaker.

Reincke M, Lutz A, Slawik M \& Beuschlein F (2001). Endokrine Probleme nach allogener hämatologischer Stammzelltransplantation. In Bartsch HH, Finke J \& Mumm A (Eds.), Hämatopoetische Stammzelltransplantation. Basel: Karger.

Reuter K \& Härter M. (2000). Differentialdiagnose von Fatigue und depressiven Störungen bei Tumorerkrankungen. In Weis $\mathrm{J} \&$ Bartsch $\mathrm{HH}$ (Eds.), Tumortherapie und Rehabilitation (pp. 36-51). Basel, Freiburg: Karger.

Richter G (1997). Ethische Probleme der Pharmaökonomie an einem Beispiel aus der Onkologie. Ethik Med, 9: 3-14

Richter HE. (1985). Die Chance des Gewissens. Düsseldorf: ECON Taschenbuch Verlag.

Rohatiner AZ, Freedman A, Nadler L et al. (1994). Myeloablative therapy with autologous bone marrow transplantation as consolidation therapy for follicular Lymphoma. Ann Oncol, 5 (Suppl 2), 142-146.

Ruckedeschel JC, Piantadose S and the Lung Cancer Study Group. (1991). Quality of Life Assessment in Lung Surgery for Bronchogenic Carcinoma. Theoretical Surgery, 6, 201201.

Rüffer JU \& Flechtner H. (2001). Intervention bei Fatigue: Ein mögliches Modell (Abstract). Onkologie, Suppl 2, 14.

Sass H-M. (1990). Behandlungsqualität oder Lebensqualität? Ethische Implikationen von "Lebensqualität als Bewertungskriterium in der Medizin. In Schölmerich P \& Thews G (Eds.) "Lebensqualität" als Bewertungskriterium in der Medizin (pp. 225-256). Stuttgart; New York: G. Fischer Verlag.

Schara J. (1990). Was bedeutet Lebensqualität bei Krebs? In Aulbert A \& Niederle N (Eds.), Die Lebensqualität des chronisch Krebskranken (pp. 1-14). Stuttgart, New York: Thieme Verlag.

Schepank H. (1987). Erkrankungen in der Stadtbevölkerung. Eine tiefenpsychologischepidemiologische Feldstudie in Mannheim. Berlin, Heidelberg New York: Springer.

Schipper H, Clinch J, McMurray A \& Levitt M. (1984). Measuring the Quality of Life of Cancer Patients: The Functional Living Index-Cancer: Development and validation. Journal of Clinical Oncology, Vol. 2 No 5, 472-483.

Schipper H, Goh CR \& Wang TL. (1995). Shifting the Cancer Paradigm: Must we kill to Cure. Journal of Clinical Oncology, 13 (4), 801-807.

Schlömer-Doll U \& Schlömer D. (2000). Information und emotionale Unterstützung. Deutsches Ärzteblatt, 97 (46), 2597-2600.

Schmitt A. (2000). Posttraumatische Belastungsstörung bei Krebserkrankungen - Diagnostik und Epidemiologie. Zsch. psychosm. Med., 46, 35-56.

Schmitz N, Sextro M, Pfistner B et al. (1999). High-dose therapy(HDT) followed by hematopoietic stem cell transplantation (HSCT) for relapsed chemosensitive Hodgkin`s disease (HD): final results of a randomized GHSG and EBMT trial (HD-R1). Proc Am Soz Clin Oncol, 18:2a

Schmitz N. (1997). High-dose chemotherapy and autologous stem cell transplantation in nonHodgkin`s lymphoma. Schweiz Rundsch Med Prax, 87, 25-26.

Schmitz N. (1997). Rezidivtherapie hochmaligner Non-Hodgkin Lymphome. Der Internist, 38, 143-149.

Schneeweiss A, Goerner R, Hensel MA et al. (2001). Tandem high-dose chemotherapy in highrisk primary breast cancer: a multivariate analysis and a matched pair comparison with standard therapy. Biol Blood Marrow Transplant, 7(6), 332-42.

Schneider E \& Keller M (Eds.). (1998). Guter Rat ist teuer - Lebensqualität und Qualitätsmanagement in der Psychoonkologie. Lengerich, Berlin, Düsseldorf: Pabst Science Publishers. 
Schneider E \& Wittdorf S (Eds.). (2000). Psychosoziale Onkologie. Lengerich, Berlin, Düsseldorf: Pabst Science Publishers.

Schölmerich P \& Thews G (Eds.). (1990). "Lebensqualität" als Bewertungskriterium in der Medizin. Stuttgart; New York: G. Fischer Verlag.

Schouten HC, Colombat DH, Verdonck LF et al. (1994). Autologous bone marrow transplantation or low-grade non-Hodgkin's lymphoma: The European Bone Marrow Transplant Group experience. Ann Oncol, 5 (Suppl 2), 147-149.

Schüßler G \& Leibing E (Eds.). (1994). Coping. Verlaufs- und Therapiestudien chronischer Krankheit. Göttingen: Hogrefe.

Schulz H, Winzer A, Stump S \& Koch U. (2001). Beeinflussung der Lebensqualität von Tumorpatienten durch psychoonkologische Interventionen. Onkologe, 7, 157-166.

Schumacher A. (2001). "Posttraumatic Stress Disorder" bei Krebspatienten - eine neue Mode in der psychoonkologischen Forschung? In Kleining B \& Schumacher A (Eds.) Psychotraumatologie in der Onkologie (pp. 66-78). Lengerich, Berlin, Riga: PABST.

Schwarz R (Ed.). (1991). Lebensqualität in der Onkologie. München, Bern, Wien: Zuckschwerdt Verlag.

Schwarz R. (2001). Psyche und Krebsentstehung. Onkologe, 7, 124-132.

Schwarz R, Krauß \& Hinz A (2001). Fatigue in verschiedenen Bevölkerungsgruppen (Abstract).Onkologie (Suppl 2): 14.

Schwarz R \& Hinz A. (2000). Müdigkeit und Erschöpfung in der Normalbevölkerung - Ergebnisse einer repräsentativen Bevölkerungsumfrage. In Weis J \& Bartsch HH (Eds.), Tumortherapie und Rehabilitation (pp. 30-35). Basel, Freiburg: Karger.

Schwarz R \& Krauß O. (2000). Palliativmedizin - psychologische Therapie. Der Internist, 7, 612626.

Sellschopp A \& Herschbach P. (1998). Lebensqualitätsmessung - ein Paradigmenwechsel in der onkologischen Forschung. In Bundesminister für Gesundheit (Ed.), Forschungsbericht Lebensqualität (pp. 101-104). Baden-Baden: Nomos Verlagsgesellschaft.

Shapiro CL \& Henderson IC. (1994). Adjuvant therapy of breast cancer. Hematol Oncol Clin, 8 (1), 213-231.

Sherman AC, Plante TG, Simonton S et al. (2000). A multidimensional measure of religious involvement for cancer patients: the Duke Religious Index. Support Care Cancer, 8, 102-109.

Sigle J \& Porzsolt F. (1996). Practical aspects of quality-of-life measurement: design and feasibility study of the quality-of-life recorder and the standardized measurement of quality of life in an outpatient clinic. Cancer Treatment Reviews, 22 (Suppl A), 75-89.

Slevin ML, Plant H, Lynch D et al. (1987). Who should measure quality of life, the doctor or the patient? Br J Cancer, 57, 109-112.

Slevin ML, Stubbs L, Plant HJ et al. (1990). Attitudes to chemotherapy: Comparing views of patients with cancer with those of doctors, nurses, and general public. BMJ, 300, 14581460.

Smith EP \& Nademanee A. (1995). Bone marrow transplantation: the city of hope experience. Clin Transpl, 291-310.

Spiegel D, Bloom JR, Kraemer HC et al. (1989). Effect of psychosocial treatment on survival of patients with metastatic breast cancer. Lancet, October 14, 888-891.

Spiegel D, Sands S \& Koopman C. (1994). Pain and depression in patients with cancer.Cancer, $74,2570-2578$.

Spiegel et al. (1994). Common psychiatric disorders in cancer patients II. Anxiety and confusional states. Supp Care Cancer, 2, 233-237.

Spitzer OW, Dobson AJ, Hall J et al. (1981). Measuring the Quality of Life of Cancer Patients A concise QL-Index for use by physicians. J. Chron Dis, Vol 34, 585-597.

Sprangers MAG. (1996). Response shift bias: A challenge to the assessment of quality of life in cancer clinical trials. Cancer Treat Rev, 22 (Suppl A), 55-62.

Stadtmauerer EA, O'Neill A, Goldstein L et al. (2000). Conventional-Dose Chemotherapy Compared With High-dose Chemotherapy Plus Autologous Hematopoietic Stem-cell Transplantation For Metastatic Breast Cancer. N Engl J Med, 342 (15), 1069-1076.

Stiefel F, Trill MD, Berney A et al. (2001). Depression in palliative care: a pragmatic report from the Expert Working Group of the European Association for Palliative Care. Support Care Cancer, 9, 477-448. 
Stommel M, Given BA \& Given CW. (2002). Depression and Functional Status as Predictors of Death among Cancer Patients. Cancer, 94/10, 2719-2727.

Stone P, Richards M, A'Hern R \&Hardy J. (1999). A study to investigate the prevalence, severity and correlates of fatigue among patients with cancer in comparison with a control group of volunteers without cancer. Ann Oncol, 11, 561-567.

Storm HH, Christensen N, Jensen OM et al. (1992). Suicides among danish patients with cancer: 1971-1986. Cancer, 69, 1507--1512.

Strittmatter G \& Mawick R (Eds.). (1995). Ethik in der Onkologie. Münster: Tosch Verlag.

Sugarbaker PH, Barofsky I, Rosenberg SA et al. (1982). Quality of life assessment of patients in extremity sarcoma clinical trials. Surgery, 91 (1), 17-23.

Sweetenham SW. (2000). Economics of stem cell transplantation for lymphoma: counting the cost of living. Br J Cancer, 82 (1), 4-6.

Syrjala KL, Chapko MK, Vitalino PP et al. (1993). Recovery after allogenic bone marrow transplant: prospective study of predictors of long-term physical and psychosocial functioning. Bone Marrow Transplant, 11, 319-327.

Szekely J, Fabry I, Forgacs $\mathrm{G}$ et al. (1999). Total body irradiation before bone marrow transplantation. Technique and acute toxicity. Strahlenther Onkol, 175 (12), 606-610.

Szucs TD. (1996). Was ist medizinische Ökonomie? Medizinische Klinik, 91 (1), 49-53.

Tan AR \& Swain SM. (2001). Adjuvant chemotherapy for breast cancer: an update. Semin Oncol, 28(4), 359-76.

Tan BR \& Bartlett NL. (2001). Treatment advances in non-Hodgkin' s lymphoma. Expert Opin Pharmacother, 1 (3), 451-66.

Tasmuth T, Smitten von K, Hietanen P, Kataja M \& Kalso E. (1995). Pain and other symptoms after different treatment modalities of breast cancer. Annals of oncology, 6, 453-459.

Thomas SF, Glynne-Jones R, Glait J \& Marks DF (1997). Anxiety in long-term cancer survivors influences the acceptability of planned discharge from follow-up. Psychooncology, 6 (3): 190-196.

Torrance GW, Siegel JE \& Luce BR. (1996). Framing and Designing the Cost-Effectiveness Analysis. In Gold MR, Siegel JE, Russell LB \& Weinstein MC (Eds.). CostEffektiveness in Health and Medicine (pp. 54-81). New York, Oxford: University Press.

Trümper L. (1998/99). Knochenmark- und Blutstammzelltransplantation. In Preiß et al. (Ed.),Empfehlung zur Therapie, Onkologie 98/99. Onkolog. Arbeitsgemeinschaft SaarPfalz-Mosel.

Tschuschke V (2002). Psychoonkologie. Stuttgart: Schattauer.

Uexküll T v et al. (Eds.). (1997). Psychosomatische Medizin (Vol. 5. Auflage). München, Wien, Baltimore: Urban und Schwarzenberg.

Vahdat L \& Antman K. (1997). High-dose chemotherapy with autologous stem cell support for breast cancer. Curr Opin Hematol, 4 (6), 381-389.

Valente SM \& Saunders JM. (1997). Diagnosis and treatment of major depression among people with cancer. Cancer Nurs, 20 (3), 168-177.

Valentine AD, Meyers CA, Kling MA et al. (1998). Mood and cognitive side effects of interferonalpha therapy. Semin Oncol, 25 (1 Suppl 1), 39-47.

Vellenga E, Agthoven van M, Croockewit AJ et al. (2001). Autologous peropheral blood stem cell transplantation in patients with relapsed lymphoma results in accelerated haematopoietic reconstitution, improved quality of life and cost reduction compared with bone marrow transplantation: the Hovon 22 study. Br J Haematol, 114 (2), 319-329.

Verres R (1990). Psychologische Hilfen für die Betreuer Krebsbetroffener. In Aulbert A \& Niederle N (Eds.), Die Lebensqualität des chronisch Krebskranken (pp. 167-182). Stuttgart, New York: Thieme Verlag.

Verres R. (1986). Krebs und Angst. Subjektive Theorien von Laien über Entstehung, Vorsorge, Früherkennung, Behandlung und die psychosozialen Folgen von Krebserkrankungen. Berlin, Heidelberg, New York: Springer.

Verres R \& Hasenbring M (Eds.). (1989). Psychosoziale Onkologie. In Jahrbuch der Medizinischschen Psychologie (Vol. 3). Berlin, Heidelberg, New York: Springer.

Viehues H. (1990). Zur Mentalitätsgeschichte der "Lebensqualität". In Schölmerich P\& Thews G (Eds.), "Lebensqualität" als Bewertungskriterium in der Medizin (pp. 13-22). Stuttgart; New York: G. Fischer Verlag.

Wall van der E, Beijnen JH \& Rodenhuis S. (1995). High-dose chemotherapy regimen for solid tumours. Cancer Treatment Reviews, 21, 105-132. 
Walshe R \& Diehl V. (1998). Ökonomische Evaluationen im Rahmen klinischer Studien. Internist, 39, 943-954.

Weber HJ. (1997). Lebensqualitätsforschung als Aufgabe und aus der Sicht der pharmazeutischen Industrie. In Bullinger M (Ed.). (1997) Lebensqualitätsforschung. Bedeutung - Anforderung - Akzeptanz (pp. 23-28). Stuttgart, New York: Schattauer.

Weeks JC, Cook EF, O’Day SJ et al. (1998). Relationship between cancer patients predictions of prognosis and their treatment preferences. JAMA, 279, 1709-1714.

Weis J \& Bartsch HH. (2000). Fatigue bei Tumorpatienten. In Weis J \& Bartsch HH (Eds.), Tumortherapie und Rehabilitation. Basel, Freiburg: Karger.

Weis J \& Bartsch HH (Eds.) (2000). Tumortherapie und Rehabilitation. Basel, Freiburg: Karger.

Weis J, Bartsch HH \& Woltemate I. (2000). Fatigue und ihre Bedeutung für die Rehabilitation von Tumorpatienten: Rehabilitationsbedarf, Interventionen und Forschungsaufgaben. In Weis J \& Bartsch HH (Eds.), Tumortherapie und Rehabilitation. (pp. 103-107). Basel, Freiburg: Karger.

Weis J, Moser MT, Fachinger D et al. (2001). Lebensqualität als Evaluationskriterium in der stationären onkologischen Rehabilitation. In Bullinger M, Siegrist J \& Ravens-Sieberer U (Eds.), Jahrbuch der Medizinischen Psychologie (Vol. 18, pp. 43-58). Göttingen, Bern, Toronto, Seattle: Hogrefe.

Weisman AD. (1972). On dying and denying. New York: Behavioral Publications.

Weiss M. (1999). Sinn-Erleben als Kriterium der Lebensqualität bei Krebspatienten? Report Psychologie, 11-12, 26-27.

Wenz F, Steinvorth S, Lohr F et al. (1999). Acute central nervous system (CNS) toxicity of total body irradiation (TBI) measured using neuropsychological testing of attention function. Int J Radiat Oncol Biol Phys, 44 (4), 891-894.

Wettergren L, Langius A, Bjorkholm M \& Bjorvell H. (1999). Post-traumatic stress symptoms in patients undergoing autologous stem cell transplantation. Acta Oncol, 38 (4), 475-480.

Wettergren L, Languis A, Bjorkholm M \& Bjorvell H. (1997). Physical and psychosocial functioning in patients undergoing autologous bone marrow transplantation - a prospective study. Bone Marrow Transplant, 20 (6), 497-502.

Wilcynsky SW, Erasmus JJ, Petros WP et al. (1998). Delayed pulmonary toxicity syndrome following high-dose chemotherapy and bone marrow transplantation for breast cancer. Am J Respir Crit Care Med, 152 (2), 565-573.

Winer EP, Lindley C, Hardeee M et al. (1999). Quality of life inpatients surviving at least 12 months following high dose chemotherapy with autologous bone marrow support. Psychooncology, 8 (2), 167-176.

Wingard JR, Curbow B, Baker F et al. (1992). Sexual satisfaction in survivors of bone marrow transplantation. Bone Marrow Transplantation, 9(3), 185-190.

Wisloff F, Gulbrandsen N \& Nord E. (1999). Therapeutic options in the treatment of multiple myeloma: pharmaecoeconomoic and quality-of-life considerations. Pharmacoeconomics, 16 (4), 329-241.

Woo B, Dibble SL, Piper BF et al. (1998). Differences in fatigue by treatment methods in women with cancer. Oncol Nurs Forum, 25, 915-920.

Zettl S \& Hartlapp J. (1997). Sexualstörungen durch Krankheit und Therapie. Berlin, Heidelberg, New York: Springer.

Zierhut D, Lohr F, Schraube P et al. (2000). Cataract incidence after total-body irradiation. Int J Radiat Oncol Biol Phys, 46 (1), 131-135.

Zigmond AS \& Snaith RP. (1983). The Hospital Anxiety and Depression Scale. Acta Psychiatrica Scandinavica, 67, 361-370. 


\section{Anhang}

Einverständniserklärung und Patienteninformation.

$262 / 263$

Interviewleitfaden............................................ 264

Verzeichnis: Tabellen und Abbildungen......................... 265-267

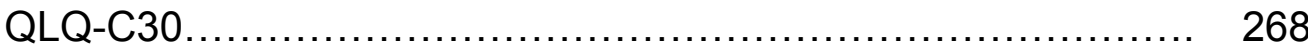

HADS-D ..................................................... 270

Karnofsky-Index................................................ 271 


\section{Einverständniserklärung}

Lebensqualität bei Patienten während und nach Hochdosistherapie mit nachfolgender autologer Blutstammzelltransplantation

Name:

Geburtsdatum:

Ich wurde über Sinn und Ablauf der Studie von

Name-

Anschrift-

Telefon-

Aufgeklärt. Ich habe das Informationsblatt gelesen und verstanden. Mir ist bekannt, dass ich die Einwilligung jederzeit widerrufen kann und mir hierdurch oder durch die Weigerung an der Studie teilzunehmen, keine Nachteile in der ärztlichen, pflegerischen und psychosozialen Betreuung entstehen.

Ich habe keine weiteren Fragen und bin bereit, an dieser Studie teilzunehmen.

Datum und Unterschrift der Patientin/des Patienten

Datum und Unterschrift des/der aufklärenden psychosozialen Mitarbeiters/Mitarbeiterin 


\section{Patienteninformation}

\section{Befragung von PatientInnen zur Ermittlung der Lebensqualität während und nach Hochdosistherapie mit nachfolgender autologer Blutstammzell- transplantation}

Sehr geehrte Patientin, sehr geehrter Patient,

Ein psychosozialer Mitarbeiter unserer Abteilung hat Ihnen vorgeschlagen, an dieser Studie teilzunehmen. Wir möchten Sie im Folgenden näher darüber informieren.

Wegen Ihrer Erkrankung werden Sie unter anderem mit Chemotherapie behandelt. Wir wissen aus Erfahrung, dass diese Behandlungen häufig sehr wirksam sind in der Bekämpfung von Tumorzellen. Wir wissen jedoch auch, dass sie Nebenwirkungen haben können, die für viele unserer PatientInnen eine Belastung darstellen. Die Studie, bei der wir um Ihre Unterstützung bitten, hat zum Ziel, eben jene Belastungen und auch die entsprechenden Entlastungen genauer zu erfahren. Und zwar aus Ihrer Sicht. Wir wollen also kein blut abnehmen und nichts messen, sondern Ihnen einige Fragen stellen und mit Ihnen reden über Ihre Erfahrungen während und nach der Behandlung. Wir wollen Ihre Meinungen sammeln und auswerten, so dass wir die Behandlung neben den Erfordernissen der medizinischen Therapie auch so gut wie möglich am Wohl unserer PatientInnen ausrichten können.

Wie man sich fühlt, ist natürlich auch abhängig davon, in welcher Phase der Chemotherapiebehandlung man gerade ist. Vor Beginn der Therapie beschäftigen einen andere Fragen und man wünscht sich andere Unterstützung als während der Zeit auf Station oder nachdem man die Therapie beendet hat und wieder in den Altag findet. Daher wollen wir Sie zu verschiedenen Zeitpunkten befragen.

- vor Beginn der Therapie geht es bei den Fragen vor allem um die Hoffnungen und Befürchtungen, die sich für Sie mit dem baldigen Behandlungsbeginn verbinden;

- während der Therapie interessieren wir uns für die belastenden und die entlastenden Bedingungen Ihres Aufenthaltes. Dazu zählen die Nebenwirkungen der Therapie, aber auch wie gut oder nicht gut Ihrer Meinung nach der Stationsalltag organisiert ist und wie Sie sich mit den Schwestern und Pflegern und den Ärztinnen und Ärzten verständigen können;

- nach der Therapie geht es darum, aus der Rückschau das im Krankenhaus Erlebte noch einmal in Ruhe zu betrachten und einzuordnen. Zudem interessiert uns, wie Sie Ihre Rückkehr in den Alltag bewerkstelligen.

Die Fragebögen, die wir Ihnen zum Ausfüllen geben, sind nicht sehr zeitaufwendig und auch nicht anstrengend. Die Gespräche werden etwa eine halbe bis 1 1 $1 / 2$ Stunden dauern. Ihre Gesprächspartnerlnnen sind für diese Aufgabe geschult und nehmen selbstverständlich darauf Rücksicht, wie es Ihnen gerade geht. Einen Termin kurzfristig zu verschieben ist in der Regel kein Problem.

Ihre Teilnahme ist freiwillig. Sie können jederzeit ohne Angabe von Gründen und ohne Nachteile für Ihre Behandlung befürchten zu müssen sich gegen weitere Befragungen entscheiden.

Die Beteiligten an der Untersuchung unterliegen der Schweigepflicht. Ihre Daten und die Inhalte der Gespräche werden natürlich streng vertraulich behandelt, auch gegenüber den behndelnden Ärztinnen und Ärzten und dem Pflegepersonal.

Wir danken Ihnen, wenn Sie an der Befragung teilnehmen. Sie leisten damit einen wichtigen Beitrag dazu, dass künftige PatientInnen von Ihren Erfahrungen profitieren können. 


\section{Interviewleitfaden}

\section{T1}

1. Was hat Sie veranlasst an der HDC teilzunehmen?

2. Welche Hoffnungen und Befürchtungen verbinden Sie mit der Therapie?

3. Welche Erfahrungen haben Sie im Vorfeld der Behandlung mit Menschen aus Ihrem privaten Umfeld und mit medizinischen Fachleuten gemacht?

4. Bereiten Sie sich in irgendeiner Weise auf die Behandlung und die Zeit im Krankenhaus vor?

\section{T2 T3}

1. Wie geht es Ihnen körperlich und seelisch?

2. Sind die Behandlungsbelastungen so, wie Sie es sich vorgestellt haben?

3. Wie schaffen Sie es, die Behandlung so gut wie möglich durchzustehen?

4. Was können andere tun um Sie zu unterstützen (Verwandte, Freunde, wir hier)?

5. Wie erleben Sie die Atmosphäre und den Ablauf auf der Station?

6. Was schätzen Sie am Verhalten des Personals, was ist eher schwierig?

T4

Haben Sie sich von der Behandlung erholt?

2. Was hat zu Ilhrer Erholung beigetragen bzw. trägt noch zur Erholung bei?

3. Wie haben Sie den Übergang vom Krankenhaus in die häusliche Umgebung gemeistert?

4. Wie ist der ambulante Kontakt zum Krankenhaus?

5. Wie beurteilen Sie im Rückblick die durchgestandenen Belastungen?

6. Was würden Sie anderen Patienten raten, die vor der Therapieentscheidung stehen?

7. Was raten Sie uns, wie können wir, das Personal, die Behandlung so verträglich wie möglich gestalten?

T5

1. Wie geht es Ihnen körperlich und psychisch?

2. Wie war die Zeit nach der Entlassung aus der Klinik, wenn Sie zurück schauen? (Beschwerden, seelisches Befinden, Sexualität, köperliche und seelische Entwicklungsphasen)

3. Haben Sie an einer Rehabilitationsmaßnahme teilgenommen? (Welche Erfahrungen, welche Angebote, wie hilfreich)

4. Konnten Sie an Ihr „altes“ Leben vor der Therapie anknüpfen? Was hat sich durch Erkrankung und Therapie verändert? Was ist neu in Ihrem Leben?

5. Beschäftigen Sie sich auch heute noch mit Ihrer damaligen Erkrankung?

6. In welcher Verbindung stehen Sie noch zum Arzt/Krankenhaus?

7. Wie würden Sie sich zum heutigen Zeitpunkt gegenüber anderen Patienten äußern in Bezug auf HDC? 


\section{Tabellenverzeichnis}

Tabelle 1 Übersicht LQ-Erhebungsinstrumente in der Onkologie.................. 18

Tabelle $2 \quad$ Krankheitsverarbeitung........................................... 25

Tabelle $3 \quad$ Anwendungsbereiche von Kosten-Nutzen-Analysen..................... 49

Tabelle $4 \quad$ Nutzwertbestimmung................................................ 50

Tabelle $5 \quad$ Beispiele von Nutzwerten.......................................... 51

Tabelle $6 \quad$ Rangliste für ausgewählten medizinische Interventionen................ 54

Tabelle $7 \quad$ Phase-III-Studien und Phase-II-Studien zur adjuvanten HDC........... 70

Tabelle $8 \quad$ Inzidenz von Nebenwirkungen....................................... 94

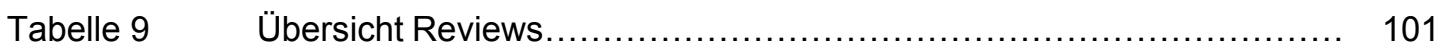

Tabelle $10 \quad$ Studien zu HDC und LQ......................................... 103

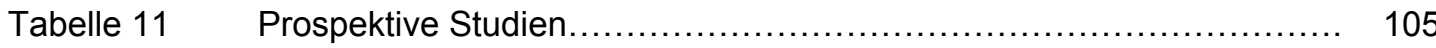

Tabelle 12 Gegenüberstellung qualitativer und quantitativer Wissenschaft.......... 112

Tabelle $13 \quad$ Messzeitpunkte...................................................... 114

Tabelle 14 Messzeitpunkte und zugehörige Instrumente der Datenerhebung........ 115

Tabelle $15 \quad$ Standardisierte Datengewinnung..................................... 117

Tabelle 16 Verfahren der Textinterpretation: Kodierung und Kategorisierung........ 124

Tabelle $17 \quad$ Altersmittelwere und Standardabweichungen............................ 129

Tabelle $18 \quad$ Verteilung der Diagnosen............................................. 130

Tabelle $19 \quad$ Krankheitsdauer......................................................... 131

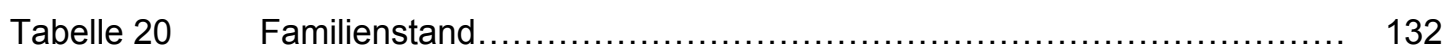

Tabelle $21 \quad$ Schulbildung und Berufsausbildung.................................... 132

Tabelle $22 \quad$ Berufsgruppe......................................................... 132

Tabelle $23 \quad$ Verteilung der Rezidivpatienten.................................... 132

Tabelle $24 \quad$ Veränderung der Beschäftigung....................................... 133

Tabelle $25 \quad$ Symptome/beschwerden............................................ 133

Tabelle $26 \quad$ Reliabilitätsanalyse................................................... 134

Tabelle $27 \quad$ Korrelationsmatrix der QL................................................ 136

Tabelle $28 \quad$ Korrelationsmatrix Karnofsky-Index - PF.............................. 136

Tabelle $29 \quad$ Korrelationsmatrix Skalen HADS - EF............................... 137

Tabelle $30 \quad$ Ergebnisse VA....................................................... 141

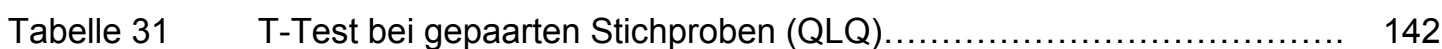

Tabelle $32 \quad$ T-Test bei gepaarten Stichproben (HADS) ............................ 147

Tabelle 33 Anzahl der Patienten in den verschiedenen Kategorien des HADS..... 149

Tabelle $34 \quad$ T-Tests Gruppenunterschiede „Sex“..................................... 152

Tabelle $35 \quad$ Ängste............................................................... 162

Tabelle $36 \quad$ Bewertung der Befindlichkei T2...................................... 164

Tabelle $37 \quad$ Seelische befindlichkeit T3........................................... 165

Tabelle $38 \quad$ Körperliche Befindlichkeit........................................... 165

Tabelle $39 \quad$ Befindlichkeit T4.................................................. 166 
Tabelle 40

Befindlichkeit T5

Tabelle 41

Einflussfaktoren Befindlichkeit.

Tabelle 42

Angaben über Fatigue. 169

Tabelle 43

Charakter von Fatigue. 169

Tabelle 44 Sexuelle Störungen. 171

Tabelle 45

Externe Belastungsfaktoren. 172

Tabelle 46

Kritik an der Verpflegung in der Klinik. 175

Tabelle 47 Kritikpunkte - Ernährung. 175

Tabelle 48

Belastende Interaktionen 176

Tabelle 49

Entscheidungsfindung 178

Tabelle 50

Begründungen für die Entscheidung. 178

Tabelle 51

Hoffnungen 180

Tabelle 52

Vorbereitung auf HDC 181

Tabelle 53

„Was hilft?“ 185

Tabelle 54

Anpassungsstrategien 187

Tabelle 55

Beispiele „Abwehrmechansimen“..... 190

Tabelle 56

Wichtigste Unterstützung 193

Tabelle 57

Teilnahme an Reha-Maßnahmen.... 194

Tabelle 58 Gründe für die Nichtteilnahme. 195

Tabelle 59

Patientenurteile über die Rehabilitationsmaßnahme.

Tabelle 60 Veränderung durch Krankheit und Therapie - Patienten ohne Rezidiv.. 197

Tabelle 61 Gründe für die fehlende Reintegration in das Berufsleben 200

Tabelle 62 Reintegration in das Berufsleben. 201

Tabelle 63 Veränderungen der Patienten mit mit Rezidiv. 202

Tabelle 64

Unterstützung durch das Behandlungsteam. 204

Tabelle 65 Was würden Sie anderen Patienten raten? 206

Tabelle 66

Belastungserwartungen 207

Tabelle 67

Charakterisierung der Behandlungsbelastungen. 208

Tabelle 68 Behandlungszufriedenheit. 209

Tabelle 69 Gründe für Zufriedenheit 209

Tabelle 70

Kritische Anmerkungen zur Nachsorge. 210

Tabelle 71 Positive Erfahrungen mit der Nachsorge 


\section{Abbildungsverzeichnis}

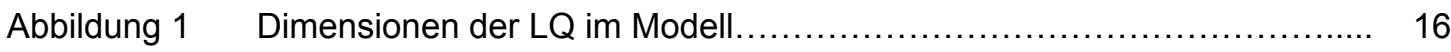

Abbildung 2 Modulares Modell am Beispiel Mammakarzinom.......................... 21

Abbildung 3 "Lebensqualität": verschiedene Erkrankungen und deutsche Norm........ 23

Abbildung $4 \quad$ Umgang mit Bedrohung............................................ 26

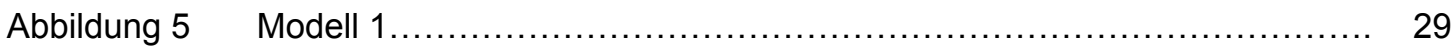

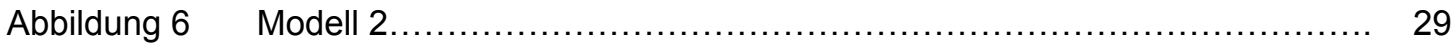

Abbildung $7 \quad$ Darstellung von Qualy................................................... 53

Abbildung $8 \quad$ Überschneidung von Depression und Fatigue.......................... 88

Abbildung $9 \quad$ Entwicklungsmodell Fatigue ............................................ 89

Abbildung 10 Verbindung von Sozialpsychologischen Konzepten mit dem LQ-Konzept. 110

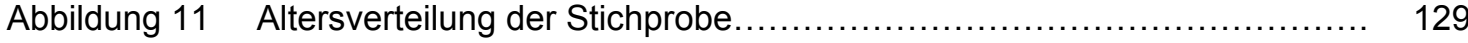

Abbildung 12 Diagnosen und ihre Verteilung........................................... 130

Abbildung 13 QLQ-C30 Funktionsskalen.......................................... 143

Abbildung 14 QLQ-C30 Allgemeine Lebensqualität................................... 144

Abbildung 15 QLQ-C30 Symptomskalen............................................ 145

Abbildung 16 Verlauf des Karnofsky-Index........................................... 146

Abbildung 17 HADS-D Angst und Depressivität........................................ 148

Abbildung 18 SF QLQ-C30 (Frauen, Männer)........................................ 153

Abbildung 19 CF QLQ-C30 (Frauen, Männer)....................................... 154

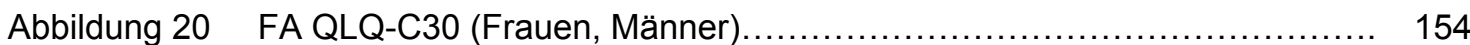

Abbildung 21 QL QLQ-C30 (Frauen, Männer).................................... 155

Abbildung 22 Karnofsky-Index (Frauen, Männer) ...................................... 155

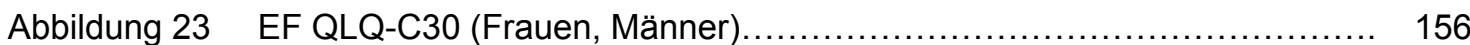

Abbildung 24 Verlauf des HADS-D Wertes „Depression“ (Frauen, Männer).............. 156

Abbildung 25 Verlauf des HADS-D Wertes „Angst“ (Frauen, Männer)..................... 157

Abbildung 26 Ineinandergreifen von Abwehr und Bewältigung......................... 184

Abbildung 27 Einzelverläufe QL des QLQ-C30........................................ 212 


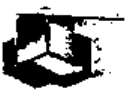

\section{EORTC QLQ-C30: (version 3.0)}

Wir sind an einigen Angaben interessiert, die Sie und thre Gesundteit betreffen.. Bitte beantworten Sie die foigenden Fragen selbst, indem Sie die Zahi ankreuzen; die am besten auf Sie zutrifft: Es gibt keine- "richtigen", oder "falschen" Antworten: Ihre Angaben werden streng vertrautich behandelt

Bitte tragen Sie: lhre Initialen ein:-

Ihr Geburtstag (Tag, Monat, Jahr):

Das. heutige Datuint (Tag. Monat, Jahr):
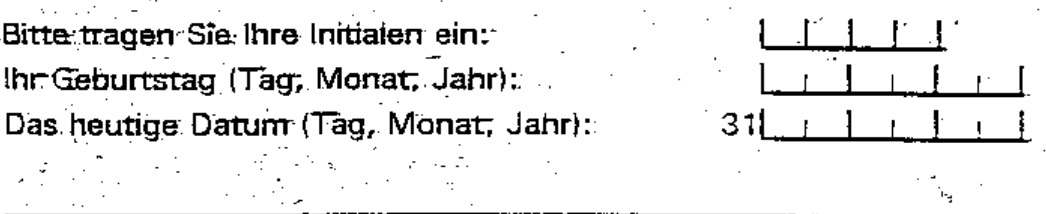

überhaupt
nicht: wenig mäßig sehr

1. Bereitetas /hnerr Schwierigkeiten sich körperlich anzustrengen ( $2 B$. aine: schwere Enkaufstasche oder einen Koffer zur tragen?)

2. Bereitet es lhnen Scfwierigkeiten, einen längeren Spaziergang ztumachen?

3. Bereitet es Ihner Schwierigkeiten, eine kurze

Strecke außier Haus «u gehen?

$1 \quad 2 \quad 3 \quad 4$

Müssen Sie tagsüber im Bettliegen oder in einem

Sessel sitzen?

Brauchen Sie Hilfe baim Essen, Anziehen, Waschen oder Benutzen der Tojlette?

\section{Während derletzten Woche:}

überhaupt
nicht ' wenig mäßig sehr

6. Waren Sie bei Ihrer Arbeit oder bei anderen tagtäglichen Beschärtigungen eingeschränkt?

$\begin{array}{llll}1 & 2 & 3 & 4\end{array}$

7. Warert Sie bei Ihren Hobbys oder anderen Freizeitbeschäftigungen eingesctiränkt?

8... Waren Sie kurzatmig?

9. Hatter Sie Schmerzen?

to. Mußten Sies sich ausnthern?

$\begin{array}{llll}1 & 2 & 3 & 4 \\ 1 . & 2 & 3 & 4 \\ 1 & 2 & 3 & 4 \\ 1 & 7 & 3 & 4\end{array}$




\section{Während der letzten Woche:}

15. Haben Sie.erbrochen?

16. Hatten Sie Verstopfung?

17. Hatten Sie Durchfall?

18. Waren Sie müde?

19. Fühlten Sie sich durch Schmerzen in Ihrem alltäglichen Leben beeinträchtigt?

20. Hatten Sie Schwierigkeitert sich auf etwas zu konzentrieren, z.B. auf das Zeitungiesen oder das Fernsegen?

21. Fühlten Sie sich angespannt?

22. Haben Sie sich Sorgen gemacht?

23. Waren Sie reizbar?

24. Fühiten Sie sich niedergeschlagen?

25. Hatten Sie Schwierigkeiten, sich an Dinge zu erinnern?

26. Hat thr körperlicher Zustand oder Ihre medizinische Behandlung thr Familienlebenn beeinträchtigt?

27. Hat ihr körperlicher Zustand oder Ihre medizinische Behandlung lhr Zusammensein oder Ihre gemeinsamen Unternehmungen mit anderen Menschen beeinträchtigt?

28. Hat lhr körperlicher Zustand oder thre medizinische Behandlung für Sie finanzielle Schwierigkeiten mit sich gebracht?

\section{überhaupt \\ nicht wenig mäßig sehr}

$\begin{array}{llll}1 & 2 & 3 & 4 \\ 1 & 2 & 3 & 4 \\ 1 & 2 & 3 & 4 \\ 1 & 2 & 3 & 4\end{array}$

$\begin{array}{llll}1 & 2 & 3 & 4\end{array}$

$\begin{array}{lll}1 & 3 \\ 4 & 4\end{array}$

$\begin{array}{llll}1 & 2 & 3 & 4\end{array}$

$\begin{array}{llll}1 & 2 & 3 & 4\end{array}$

$\begin{array}{llll}1 & 2 & 3 & 4\end{array}$

$\begin{array}{llll}1 & 2 & 3 & 4\end{array}$

$\begin{array}{llll}1 & 2 & 3 & 4\end{array}$

$\begin{array}{llll}1 & 2 & 3 & 4\end{array}$

$\begin{array}{llll}1 & 2 & 3 & 4\end{array}$

Bitte kreuzen Sie bei den folgenden Fragen die Zahl zwischen 1 und 7 an, di am besten auf Sie zutrifft

29. Wie würden Sie insgesamt lhren Gesundheitszustand während der letzten Woche einschätzen?
1.
2.
3
4
5
6 7

sehr schlecht

30. Wie würden Sie insgesamt Ihre Lehensqualität während der letzten Woche einschätzen?
1
2
3.4
5
6
7

sehr schlecht

ausgezeichnet

- Copyright 1995 EORTC Study Group on Quality of Life. Alle Rechte vorbehalten.

Version 3.0 


\section{H A D S - D}

Name, Vorname:

Geburtsdatum: Code-Nummer:

Sehr geehrte Patientin, sehr geehrter Patient!

Datum

199

Sie werden von uns wegen köperlicher Beschwerden untersucht und behandelt Zur volıständigen Beurteilung Ihrer vermuteten oder bereits bekannten Erkrankung bitten wir Sie im vorlegenden Fragebogen um einige persönliche Angaben. Man weiß heute daß korperliche Krankheit und seelisches Befinden oft eng zusammenhängen. Deshalb beziehen sich die Fragen ausdrücklich auf thre allgemeine und seelische Verfassung. Die Beantwortung ist selbstverständlich freiwillig. Wir bitten Sie jedoch, jede Frage zu beantworten, und zwar so, wie es für Sie persönlich in der letzten Woche am ehesten zutraf. Machen Sie bitte nur ein Kreuz pro Frage und lassen Sie bitte keine Frage aus! Überlegen Sie bitte nicht lange, sondern wähien Sie die Antwort aus, die Ihnen auf Anhieb am zutreffendsten erscheint! Alle thre Antworten unterliegen der ärztlichen Schweigepflicht

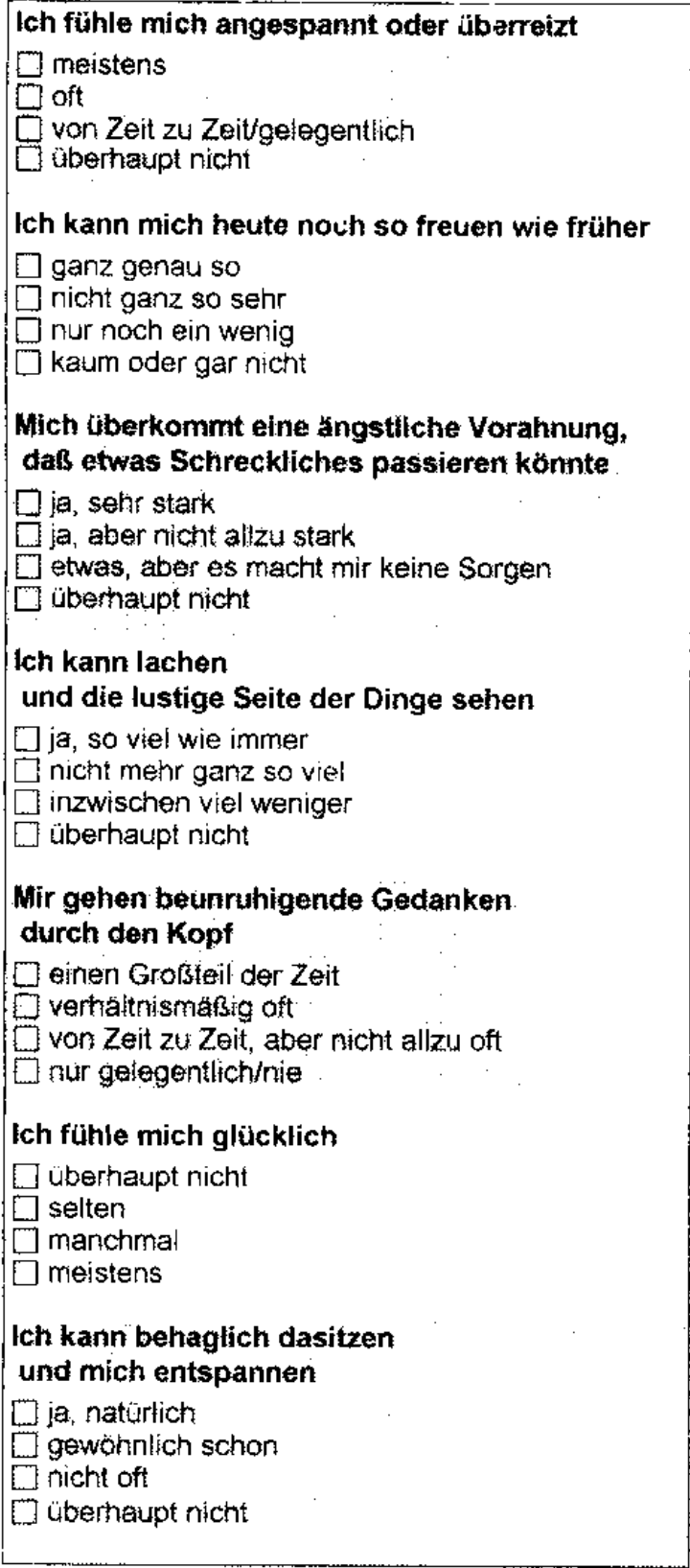

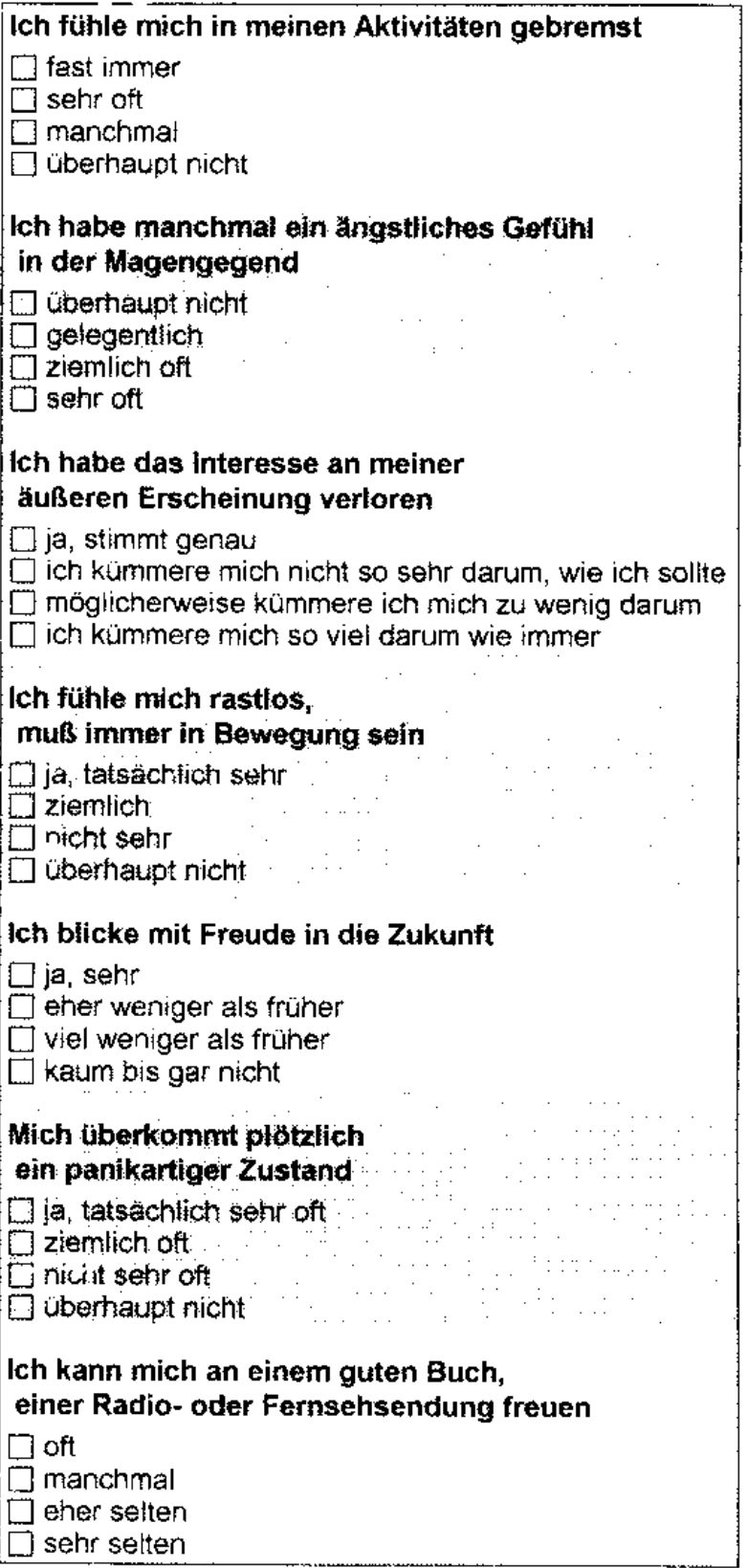

(c) für die deutsche Version Verlac Hars Huber, Bern 1995, 82587-0* (10) für die englische Originalausgabe NFEF Nelson, Windsor 1994 ) 


\section{Karnofsky-Index}

10 Normal, keine Klagen, keine Krankheitszeichen nachweisbar.

Ohne jegliche Einschränkungen. Der/die Patient/in erscheint vollkommen gesund.

$9 \quad$ Normale Aktivität, geringfügige Symptome oder Befunde.

Normale Aktivität ist ohne Einschränkungen möglich. Lediglich geringfügige Symptome oder Befunde sind erkennbar.

Normale Aktivität mit Anstrengung, beeinträchtigende Symptome oder Befunde.

Normale Aktivität mit Anstrengung. Beeinträchtigende Symptome/Befunde

7 Der Patient kann für sich selbst sorgen, wäre aber nicht in der Lage, zu arbeiten.

Der/die Patient/in könnte sich selbst versorgen (z. B. Waschen, kleine Einkäufe). Er/Sie könnte aber nicht arbeiten und/oder seine/ihre sozialen Aktivitäten in vollem Umfang wahrnehmen.

6 Gelegentliche Hilfe erforderlich, die meisten Bedürfnisse können selbst erledigt werden.

Der/die Patient/in würde in irgendeiner Weise Hilfe und Unterstützung bei der Bewältigung der alltäglichen Verrichtungen benötigen wenn er/sie entlassen würde

$5 \quad$ Regelmäßige Unterstützung erforderlich.

Der/die Patient/in würde zu Hause erhebliche Unterstützung bei der Bewältigung der alltäglichen Verrichtungen benötigen. Wenn er/sie die Station verlassen könnte, wäre eine Begleitperson notwendig. Er/sie kann sich auf der Station ohne Hilfe frei bewegen (wenn sein/ihr Immunstatus dies zulässt). Kann das Bett allein machen und die Morgenroutine allein bewältigen.

4 Regelmäßige besondere Pflege und Unterstützung erforderlich.

Der/die Patientin kann mit Anstrengung aufstehen, fühlt sich in der Lage z. B. sein/ihr Bett zu machen.

Belastungsdyspnoe (falls ersichtlich oder vom Pflegepersonal dokumentiert) bei alltäglichen Verrichtungen (z. B. Gang zur Toilette, Waschen, über den Flur gehen)

3 Stark geschwächt, medizinische Behandlung notwendig, noch nicht akut bedrohlich.

Der/die Patientin fühlt sich nicht in der Lage aufzustehen. Muß im Bett gewaschen werden.

2 Sofortige stützenden therapeutische Maßnahmen erforderlich, Intensivpflege.

Organfunktionen drohen außer Kontrolle zu geraten. Eine mögliche Verlegung auf die Intensivstation wird erwogen (z. B. wegen drohender Ateminsuffizienz) und/oder es besteht Gefahr, dass der/die Patient/in in einen akut lebensbedrohlichen Zustand fällt. Notfallmaßnahmen sind vorbereitet.

$1 \quad$ Moribund: letaler Prozeß, rasch fortschreitend.

Der/die Patientin wird intubationspflichtig, wird auf Intensivstation verlegt. 
